FINAL REPORT

U.S. Department of Energy

\title{
Electrically Driven Technologies for Radioactive Aerosol Abatement
}

\author{
Principal Investigator: David W. DePaoli \\ Institution: Oak Ridge National Laboratory \\ Principal Investigator: Ofodike A. Ezekoye \\ Institution: University of Texas at Austin \\ Collaborators: Costas Tsouris, and Valmor F. de Almeida \\ Institution: Oak Ridge National Laboratory \\ Project Number: 65328 \\ Grant Numbers: DE-FG07-98ER14937; OR-08SP24 \\ Grant Project Officers: Robert Price and Chester Miller \\ Project Duration: 08/12/98 through 5/30/02
}




\section{Table of Contents}

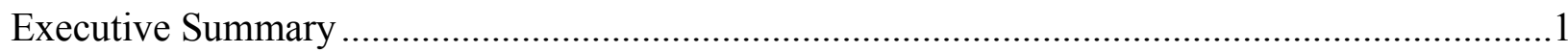

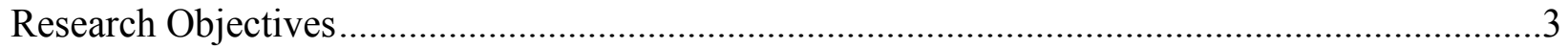

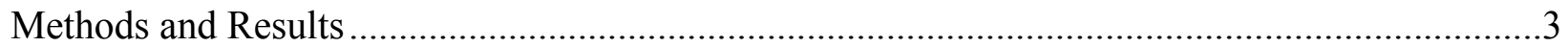

Relevance, Impact, and Technology Transfer .................................................................. 15

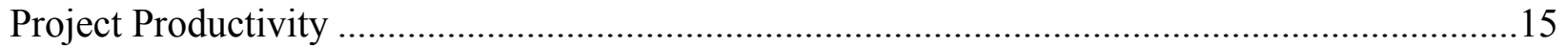

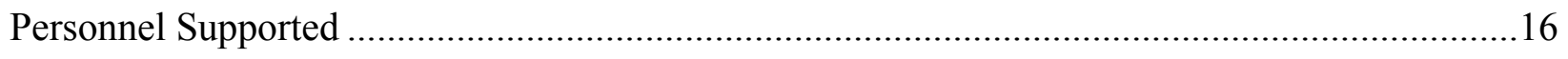

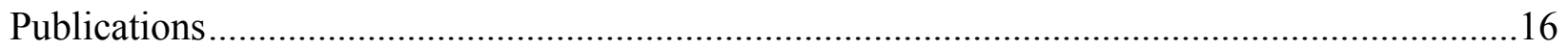

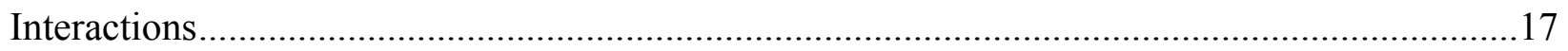

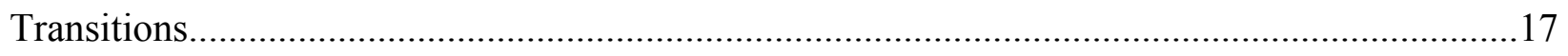

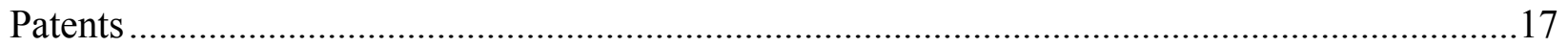

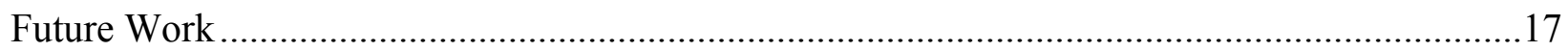

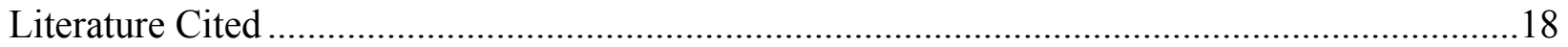

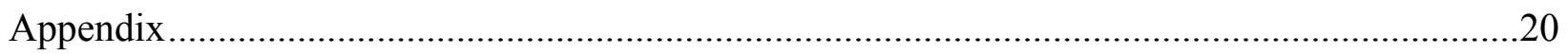




\section{Executive Summary}

The purpose of this research project was to develop an improved understanding of how electrically driven processes, including electrocoalescence, acoustic agglomeration, and electric filtration, may be employed to efficiently treat problems caused by the formation of aerosols during DOE waste treatment operations. The production of aerosols during treatment and retrieval operations in radioactive waste tanks and during thermal treatment operations such as calcination presents a significant problem of cost, worker exposure, potential for release, and increased waste volume.

There was anecdotal evidence in the literature that acoustic agglomeration and electrical coalescence could be used together to change the size distribution of aerosol particles in such a way as to promote easier filtration and less frequent maintenance of filtration systems. As such,

those electrically driven technologies could potentially be used as remote technologies for improved treatment; however, existing theoretical models are not suitable for prediction and design. To investigate the physics of such systems, and also to prototype a system for such processes, a collaborative project was undertaken between Oak Ridge National Laboratory (ORNL) and the University of Texas at Austin (UT). ORNL was responsible for the larger-scale prototyping portion of the project, while UT was primarily responsible for the detailed physics in smaller scale unit reactors.

It was found that both electrical coalescence and acoustic agglomeration do in fact increase the rate of aggregation of aerosols. Electrical coalescence requires significantly less input power than acoustic agglomeration, but it is much less effective in its ability to aggregate/coalesce aerosols. The larger-scale prototype showed qualitatively similar results as 
the unit reactor tests, but presented more difficulty in interpretation of the results because of the complex multi-physics coupling that necessarily occur in all larger-scale system tests. An additional finding from this work is that low-amplitude oscillation may provide an alternative, non-invasive, non-contact means of controlling settling and/or suspension of solids. Further investigation would be necessary to evaluate its utility for radioactive waste treatment applications.

This project did not uncover a new technology for radioactive waste treatment. While it may be possible that an efficient electrically driven technology for aerosol treatment could be developed, it appears that other technologies, such as steel and ceramic HEPA filters, can suitably solve this problem. If further studies are to be undertaken, additional fundamental experimentation and modeling is necessary to fully capture the physics; in addition, larger-scale tests are needed to demonstrate the treatment of flowing gas streams through the coupling of acoustic agglomeration with electrocoalescence.

Several students have received or are receiving graduate degrees related to work on the project. Copies of theses are presented in the appendix. 


\section{Research Objectives:}

The objective of this research program was to develop an improved understanding of how electrically driven processes, including electrocoalescence, acoustic agglomeration, and electric filtration, may be employed to efficiently treat problems caused by aerosols formed during DOE waste treatment operations. The production of aerosols during treatment and retrieval operations in radioactive waste tanks and during thermal treatment operations such as calcination presents a significant problem of cost, worker exposure, potential for release, and increased waste volume. Electrically driven technologies offer promise as remote technologies for improved treatment; however, existing theoretical models are not suitable for performance prediction and design.

The basis for the project is the general fact that for most particulate collection technologies, the marginal collection efficiency increases as the aerosol to be separated increases in size. Using this as a premise, we are investigating mechanisms for increasing the size of particles in an effluent stream as a preprocessing step. Our work is aimed at employing recent advances in theoretical approaches and experimental techniques to improve our understanding of how electrical and acoustic methods may be employed most efficiently alone or in tandem to tackle aerosol problems. The fundamental understanding achieved may provide the basis for development of innovative new approaches and for optimizing removal processes.

\section{Methods and Results}

This project included bench-scale, fundamental experiments of electrocoalescence and acoustic agglomeration conducted with non-flowing gas in unit reactors, larger-scale prototype experiments conducted with flowing gas streams, and modeling. Progress in each topic is described below.

\section{Electrocoalescence Unit Reactor}

To characterize the details of electrocoalescence, a unit reactor was constructed, as shown in Figure 1. The chamber has internal dimensions of approximately ten centimeters by ten centimeters by two centimeters. The chamber is made of acrylic with two copper plates forming the top and bottom surfaces. A high-voltage DC electric field is formed in the space between the copper plates. Jason Schmidt, who received a MSc on this project, conducted experiments to determine the sedimentation rate of aerosols suspended in the field. These experiments are primarily laser-based extinction methods that characterize the rate of change of the volume fraction of the aerosol as a function of the applied electrical field, aerosol size, elevation and initial concentration. 

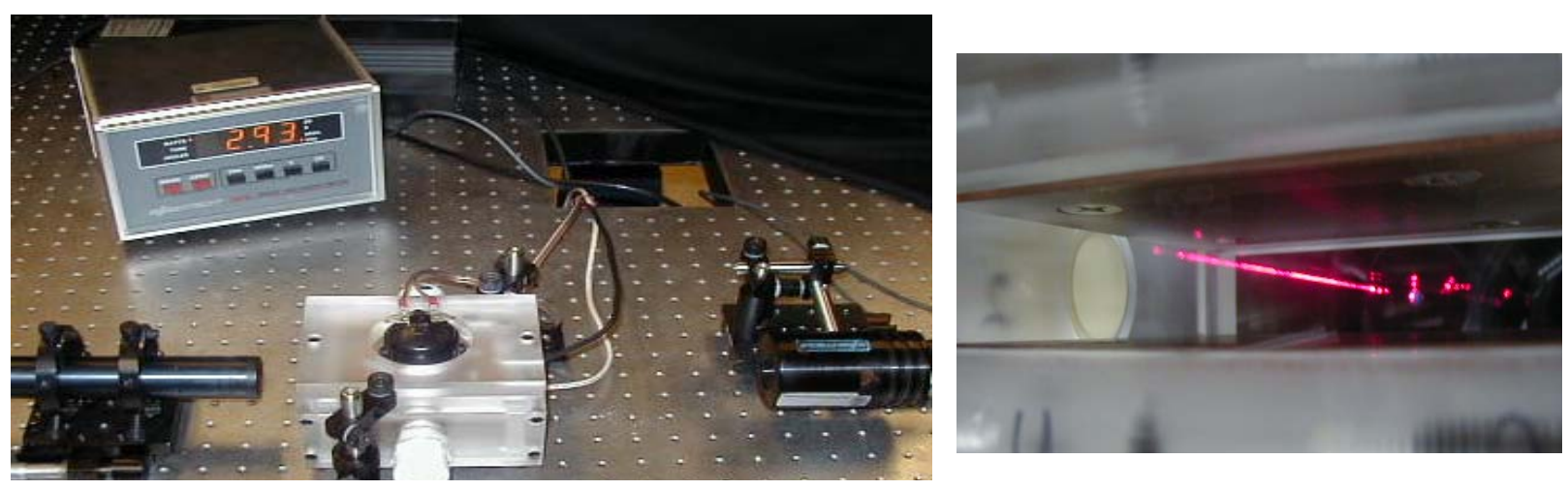

Figure 1. Unit reactor and laser scattering through unit cell filled with aerosol.

Experimental data for the aerosol loading were taken at various elevations and these data were then simulated using a sectional algorithm. Vinayak Barve, who is receiving a MS on this project, developed a computational procedure for characterizing the experiments performed by Schmidt. For modeling the settling process, a sectional code MAEROS, written by Gelbard and Seinfeld (1980) was used. This code calculates aerosol composition and mass concentration as a function of particle size and time. The processes incorporated are coagulation due to Brownian motion and gravity, and particle deposition due to gravitational settling. The numerical method of MAEROS is based on dividing the particle size domain into a number of sections and imposing conservation of mass for the processes given above. The coagulation coefficient is taken as the sum of the coagulation coefficients for Brownian motion and gravitational settling. These coagulation rates are integrated over time using the Runge-Kutta-Fehlberg method to give the particle concentrations.

When particles are settling due to gravity, the upper part of the chamber clears out faster than the lower part and stratification is observed. This occurs because the particles in the upper portion of the chamber move downwards and replace the particles in the lower portion that have already settled on the floor of the chamber. The supply of particles to the lower portions means that these portions take longer to clear out. To simulate this stratification, the MAEROS code was modified to account for the elevation effects (Ezekoye and Wiwobo 1999). As shown in Figure 2, the entire volume was divided into a number of equal horizontal compartments. The division was done such that each compartment had the same length and breadth as that of the entire volume, and only the height is modified. The conservation of mass equation was solved for each of these compartments with the coagulation process. In effect, each compartment had a source and a sink.

For each compartment, the general dynamic equation (GDE) was written as:

$\frac{\mathrm{dn}_{\mathrm{k}, \mathrm{c}}}{\mathrm{dt}}=\frac{1}{2} \sum_{\mathrm{i}+\mathrm{j}-\mathrm{k}} \beta\left(\mathrm{v}_{\mathrm{i}}, \mathrm{v}_{\mathrm{j}}\right) \mathrm{n}_{\mathrm{i}, \mathrm{c}} \mathrm{n}_{\mathrm{j}, \mathrm{c}}-\mathrm{n}_{\mathrm{k}, \mathrm{c}} \sum_{\mathrm{i}-1} \beta\left(\mathrm{v}_{\mathrm{i}, \mathrm{c}}, \mathrm{v}_{\mathrm{k}, \mathrm{c}}\right) \mathrm{n}_{\mathrm{i}, \mathrm{c}}-\mathrm{n}_{\mathrm{k}, \mathrm{c}} \mathrm{R}\left(\mathrm{v}_{\mathrm{k}}\right)+\mathrm{n}_{\mathrm{k}, \mathrm{c}-1} \mathrm{~S}\left(\mathrm{v}_{\mathrm{k}}\right)$

where the last term on the right hand side accounts for particles being added to the compartment $c$ from the upper compartment $c-1$. The uppermost compartment would have no source. The term before the last one on the right hand represents loss due to deposition to the lower compartment. The deposition occurring from the lowermost compartment would be the deposition to the floor. The other terms of the equation above are accumulation of particles of size $\mathrm{k}$ in compartment $\mathrm{c}$ 


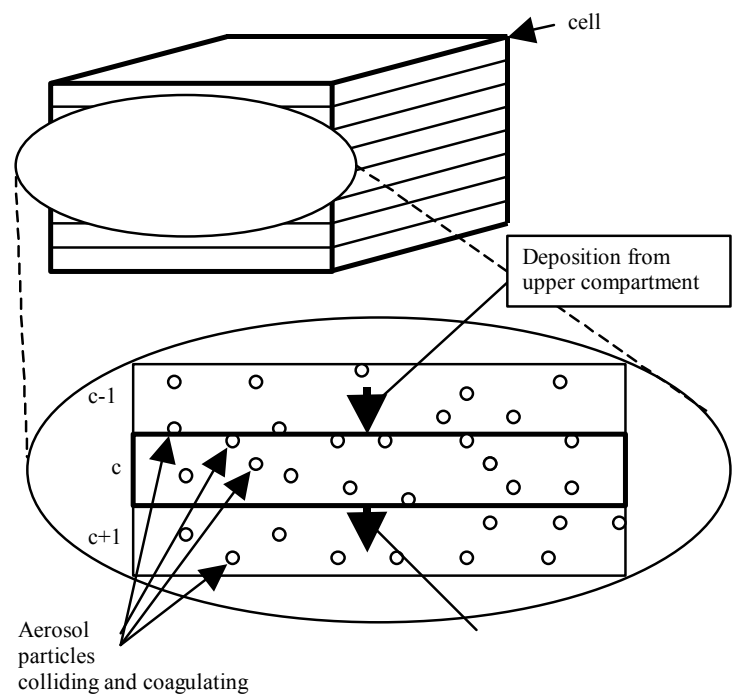

Figure 2. Schematic showing the subdivided cells for computation of unit cell.

on the left hand side, input of particles in the same size element due to aggregation of smaller particles in the same compartment (first summation term on the right hand side of the equation), loss of particles due to aggregation to form larger particles (second summation term on the right hand side). Breakup terms are neglected. In theory, the code should predict the particle concentration for different elevations within the chamber. This can be compared to the experimental laser-extinction measurements, which were taken at specific elevations.

Results of Calculation of Unit Cell Electrocoalescence Model: The sectional GDE code was used to simulate the settling process. The settings for the simulation were based on the experiments conducted by Schmidt (2000). For the modified (compartmentalized) MAEROS run, the volume fraction results for elevations of $3 \mathrm{~mm}, 7.5 \mathrm{~mm}$ and $11.5 \mathrm{~mm}$ from the upper copper plate are shown in Figure 3.

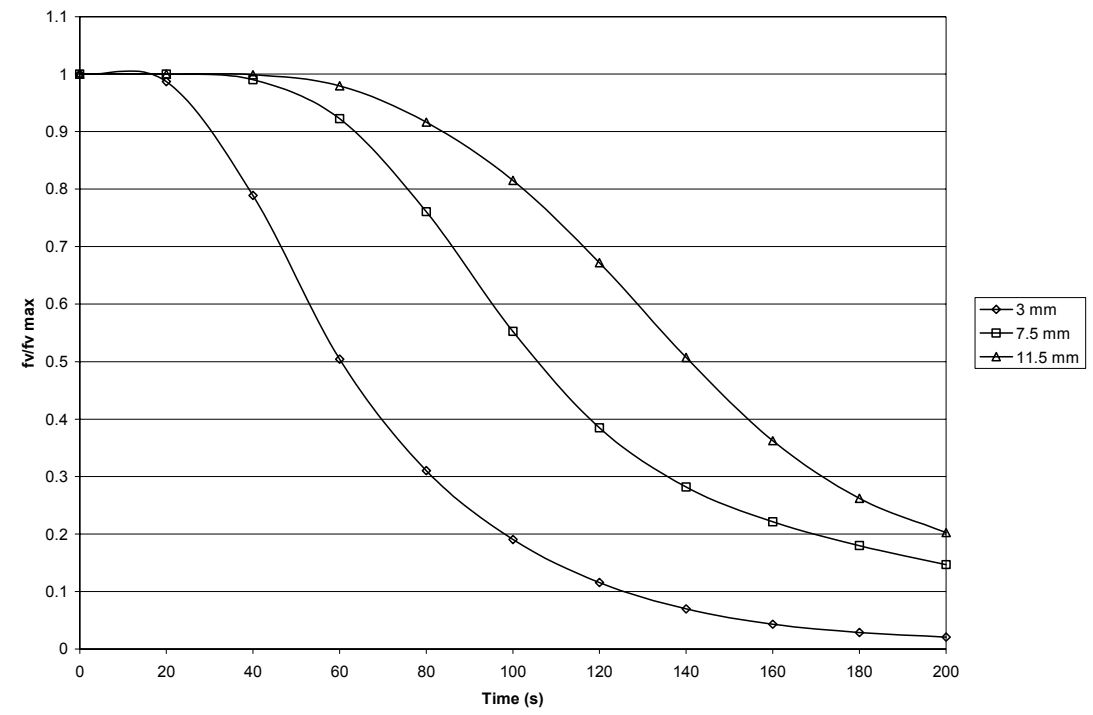

Figure 3. Volume fraction results for a compartmentalized code with 40 cells. 
Figures 4 and 5 are the volume fraction comparison plots of the computational model results with the experimental data from Schmidt (2000) under electric fields of $0 \mathrm{~V} / \mathrm{m}$ and 75000 V/m respectively. These conditions represent the extremes of the experiments.

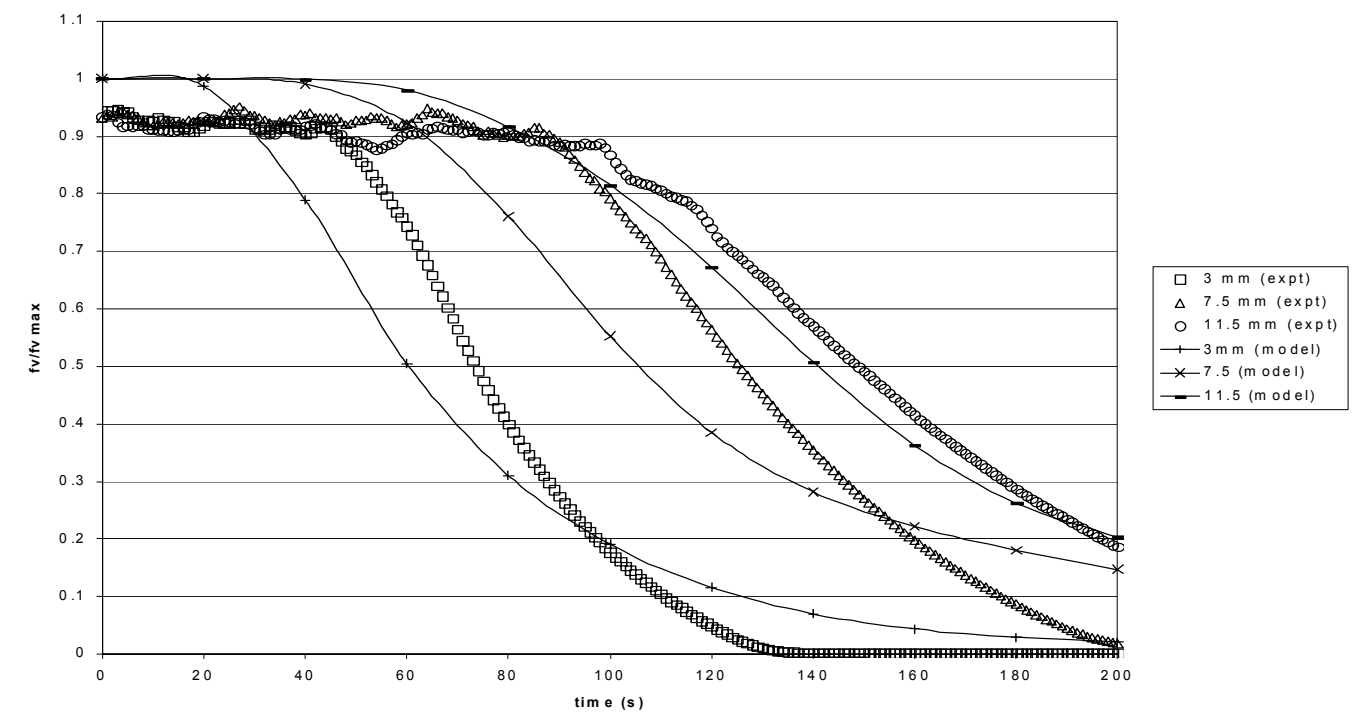

Figure 4: Experimental and model results of volume fraction loading for a field strength of $0 \mathrm{~V} / \mathrm{m}$.

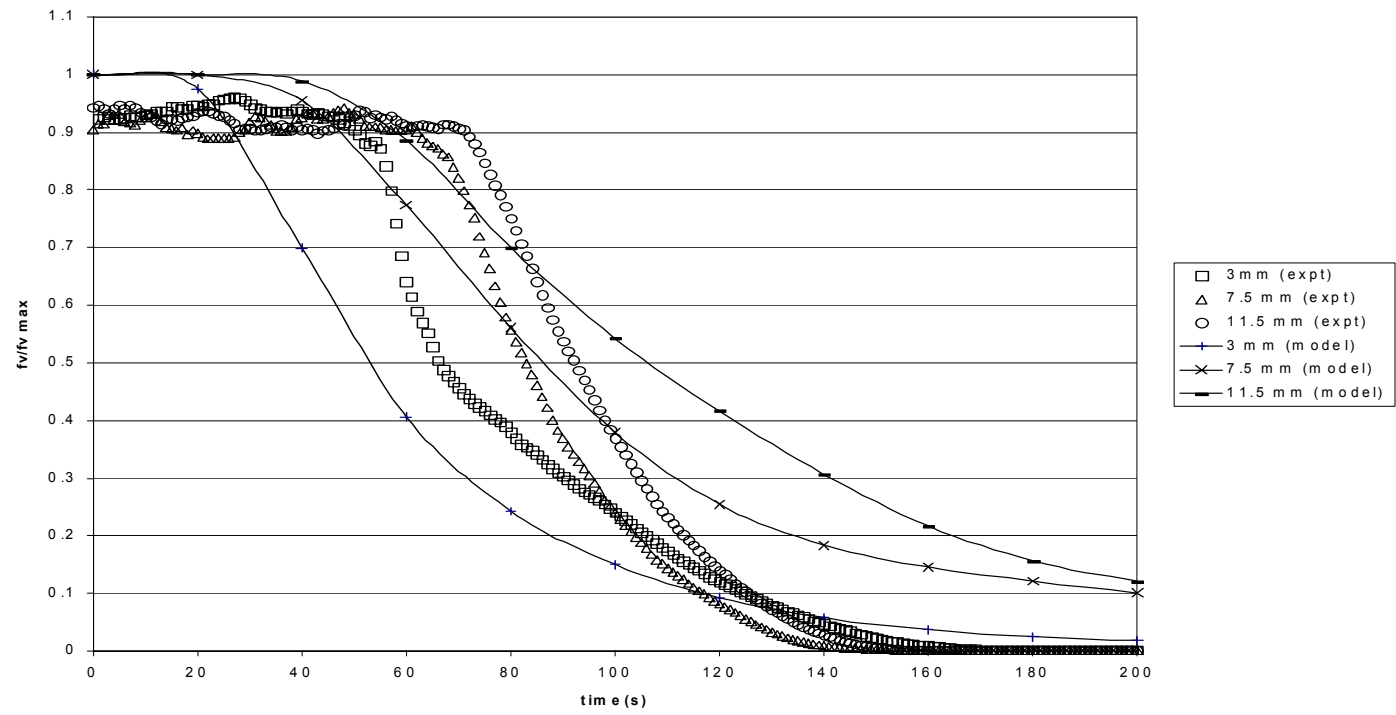

Figure 5: Experimental and model results of volume fraction loading for a field strength of $75 \mathrm{kV} / \mathrm{m}$.

The computational model and experimental data have similar settling trends. However, the model and the experimental data deviate from one another randomly as the electric field increases. The deviation is enhanced at later stages during the simulation. This can be observed from Table 1, which lists the percentage error of the model predictions for the times required to reduce to $50 \%$ of the initial loading at each of three elevations. 
Table 1: Errors in Model Predictions of Time Required to Reduce to 50\% of Initial Loading

\begin{tabular}{|c|c|c|c|}
\hline \multirow{2}{*}{$\begin{array}{c}\text { Electrical Field } \\
(\mathrm{V} / \mathrm{m})\end{array}$} & $3 \mathrm{~mm}$ & $7.5 \mathrm{~mm}$ & $11.5 \mathrm{~mm}$ \\
\cline { 2 - 4 } & +17.8 & +15.8 & -5.37 \\
\hline 0 & +10.8 & +1.0 & -12.7 \\
\hline 25000 & +10.9 & -4.3 & -29.0 \\
\hline 50000 & +18.2 & -3.6 & -17.4 \\
\hline 75000 & & & \\
\hline
\end{tabular}

The differences in the model and the experimental data could be due to the model accounting for the combination of Brownian and gravitational coagulation by adding them to give the total coagulation kernel. This is based on the premise that the velocity acquired by a particle due to Brownian diffusion is independent of the velocity due to gravitational acceleration. However, as particles settle, Brownian and gravitational forces act on the particles simultaneously and so the aerosol particles, which are moving randomly due to Brownian motion have a net downward velocity due to gravity. Thus, the gravitational field modifies the Brownian diffusion effects and thus changes the velocities acquired by the particles. Also, the modification of the gravitational agglomeration kernel to account for the electric field effects could have further magnified the errors in the model, thus increasing the error over time. This work indicates that electrocoalescence does modify the size distribution of aerosols, and that there is more research that must be done in i) coupling various coagulation kernels, and ii) testing the theoretical basis of electrocoalescence. In addition it needs to be determined whether droplets acquire electrical charge in the electric field. In the modeling work, noncharged droplets have been assumed.

\section{Acoustic Agglomeration Unit Reactor-Agitated Sedimentation System}

Through literature review and model testing of acoustic agglomeration dynamics of aerosol particles, we ascertained that there is still an incomplete picture of particle-particle interactions in the presence of external fields. Design codes for agglomeration processes will require more accurate models. Unfortunately, the dynamics of acoustic and electric agglomeration of the aerosols of interest occur on length scales that cannot be readily examined under realistic conditions. To address this problem, we designed and built a simple experiment involving spherical particles falling through a vibrating glycerin bath. Experimental conditions were designed to match those of acoustic agglomeration of aerosols. The apparatus was oscillated at a frequency of $10 \mathrm{~Hz}$ with peak-to-peak displacements on the order of $10 \mathrm{~mm}$. Particles of varying densities and diameters $(0.4 \mathrm{~mm}$ to $1.0 \mathrm{~mm})$ were placed into the oscillating flow. Video images of the particle dynamics were captured with both a personal video camcorder and high-speed digital camera. In parallel, computations were performed for the particle system in order to validate the experimental method and apparatus. Results from this experiment have produced one journal publication (Carter and Ezekoye, 2001) and a master's thesis for Jason Carter (Carter 2001). This apparatus proved considerably more valuable in later work on agitated sedimentation 
and suspended solids concentration measurements. That work was undertaken by Krishna Lakshminarasimhan, who is completing a MS on the topic. Lakshminarasimhan's work, although not directly covered by the initial scope of the project, is relevant to the high-level tank waste processing. Lakshminarasimhan's research involves characterizing resuspension and particle mixing in systems containing liquids mixed with solid particles. The system considered consists of a rectangular tank containing low Reynolds number particles suspended in a liquid. The issue of resuspension and solids characterization is important for DOE applications.

Experimental system and characterization of solids: A schematic of the setup used in these experiments is shown in Figure 6. A hydrophone, immersed in the suspension to be measured, emitted 10-microsecond pulses of 1 Megahertz ultrasound. This was received by a hydrophone separated from the transmitter by a distance of 8 inches. The received signal was found to have a very low signal-to-noise ratio. These pulses were observed on an oscilloscope. The ratio of the amplitude of the received pulses to the transmitted pulse can be used to find the extinction coefficient. This extinction coefficient is the sum of absorption by suspending liquid and that by the suspended particles. A simple Labview code was written to acquire the waveform from the oscilloscope into the computer. The waveform was then analyzed to determine features of the suspension. Measurement of suspended solids could be determined either through a correlation (Lakshimarasimhan 2002), or by direct calibration. Figure 7 shows the attenuation predicted by a correlation developed for this system for fly-ash suspensions, while Figure 8 presents results of a more direct calibration for a China Clay suspension.

To study the effects of agitation on the system, the container was oscillated, as shown in Figure 9, at approximately $10 \mathrm{~Hz}$. The results of the shaking on the settling of the suspension are dramatic. Shaking begins at $\mathrm{t}=0$ with the initial concentration of fly ash equal to $2 \%$ by mass. The concentration rapidly drops to $0.5 \%$ initially $(\mathrm{t}<100 \mathrm{~s})$ and then rises to an equilibrium value of $2 \%$.

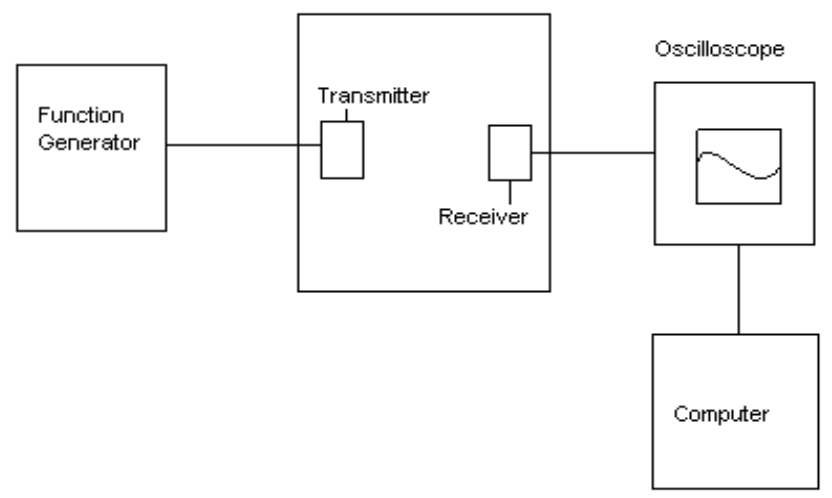

Figure 6. Experimental schematic for hydrophone measurements 


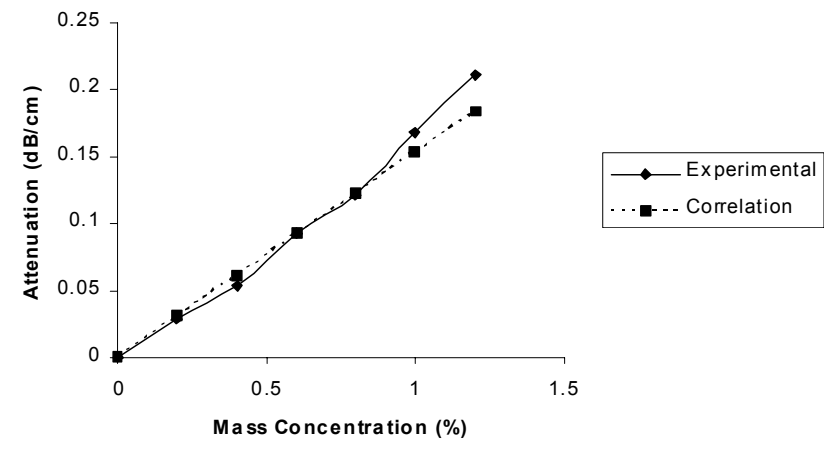

Figure 7. Attenuation predicted by correlation for fly-ash suspension.

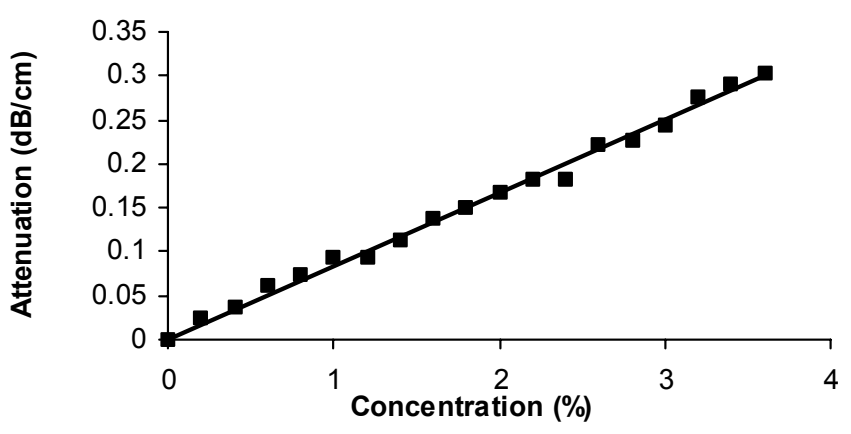

Figure 8. Calibration of ultrasonic transducer for particle studies.

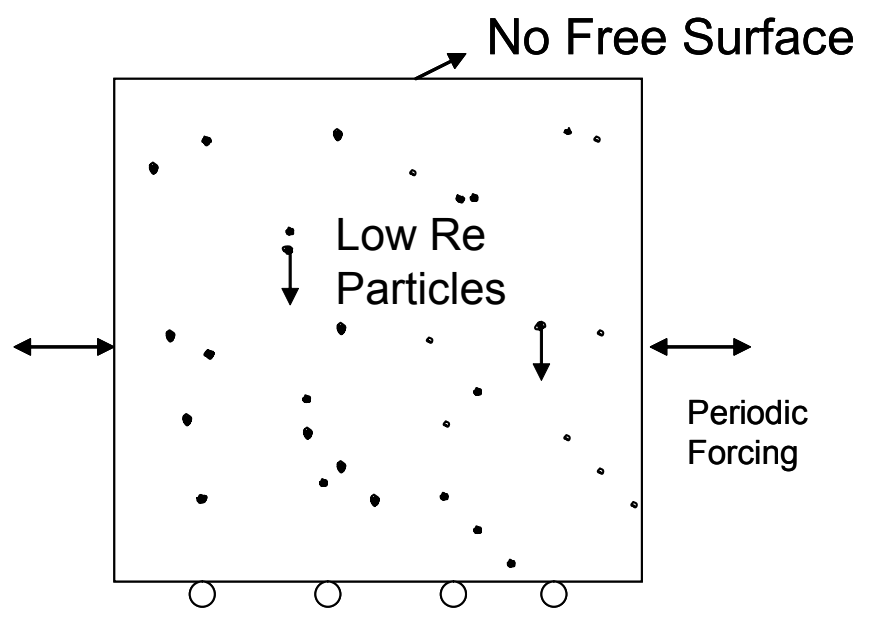

Figure 9. Schematic of oscillating system for sedimentation studies. 


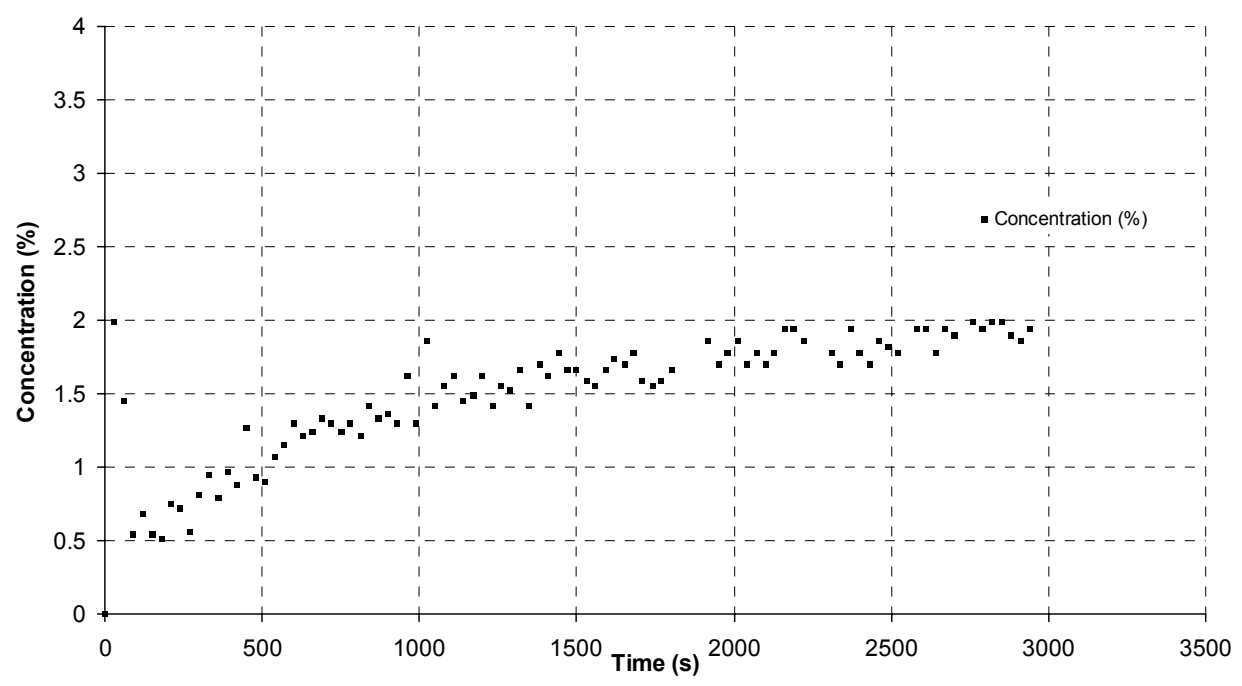

Figure 10. Data on sedimentation and resuspension for oscillating solidliquid system

The mechanism for the resuspension process was identified to be a result of internal pressure waves. The density stratification associated with the solids when acted upon by acceleration produces a vertical flow which serves to redistribute the solids. These results indicate that lowamplitude oscillation may provide an alternative, non-invasive, non-contact means of controlling settling and/or suspension of solids. Further investigation would be necessary to evaluate its utility for radioactive waste treatment applications.

\section{Prototype Study}

An apparatus for measuring the simultaneous effect of electric and acoustic fields on flowing aerosols was also constructed and operated. The apparatus, shown in Figure 11, allows continuous measurement of aerosol size distribution in the range of 0.4 to 200 micrometers along a 1-m length of a 10-cm square duct using a Malvern Spraytec RTS-5000 laser-scattering device. The test chamber is clear Lexan with a cross section of $10 \mathrm{~cm}$ x $10 \mathrm{~cm}$ and approximately $94 \mathrm{~cm}$ long mounted vertically, with the air flow from bottom to top through the unit. Two 9-cm x 60$\mathrm{cm}$ electrodes are mounted vertically approximately $8 \mathrm{~cm}$ apart on opposite sides of the test chamber. The applied electric potential gradients were thus normal to the gas flow direction. Six speakers were mounted on the sides of the chamber to allow simultaneous acoustic forcing; a limited number of tests conducted with acoustic forcing showed no measurable effect for the achievable frequencies $(1-50 \mathrm{kHz}$ ) and power levels (up to $135 \mathrm{~dB}$ ).

A series of experiments was conducted to quantify the effect of an applied direct-current electric potential on the water droplet content in moist air streams simulating aerosols generated by tank waste mobilization processes. The droplets were produced by an ultrasonic aerosol generator fed 
with distilled water; the water droplets in the mist ranged in diameter from a few micrometers to about 20 micrometers in each of the experiments. The mist-containing air streams were forced through the test chamber at Reynolds numbers whose nominal values ranged from 940 to 2200, which correspond to residence times in the test section of 7 seconds to 3 seconds. The droplet loadings in the inlet air streams, determined by condensation from a sample stream withdrawn isokinetically, ranged from nominal values of 8 to $24 \mathrm{~g}$ mass per cubic meter.
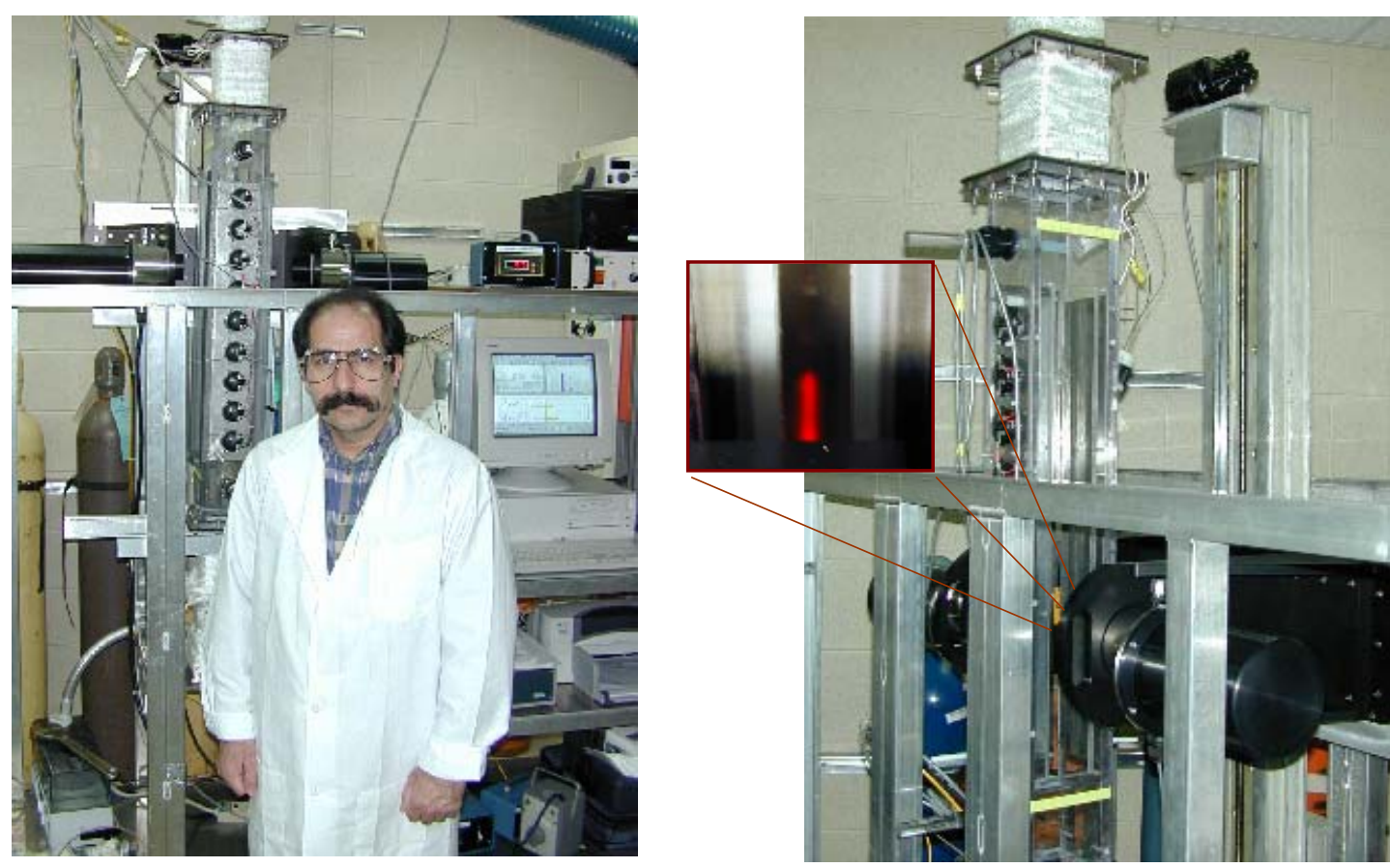

Figure 11. Apparatus for measuring the effect of electric fields on flowing aerosols

At zero voltage, the mist distribution was uniform throughout the test chamber. At a given nonzero voltage level, the water droplets were deposited principally on the lower end of the positive electrode. As the experiment progressed over time, droplet deposition was observed at the upper surfaces of both electrodes. The electric field caused the mist motion to change from a uniform pattern to a somewhat random pattern, with some jetting toward the positive electrode. This more or less chaotic droplet motion was especially intense in the voltage range of $20 \mathrm{kV}$ and above. In all cases, at high voltages, the droplets build up at the lower level of the test chamber, causing a short circuit between positive and negative electrodes, resulting in sparks and thereby shutting down the system. It was therefore necessary to limit the potential difference across the electrodes to $26 \mathrm{kV}$ in these experiments.

Typical results are shown in Figure 12, which presents data for the measured concentration of water droplets exiting the electrified chamber as a function of applied voltage at a constant gas flow rate for three different inlet concentrations. The data show that the application of an electric field could significantly reduce the aerosol loading. Other data collected for droplet size distribution showed only a small increase in the average diameter of the suspended droplets during these experiments. 


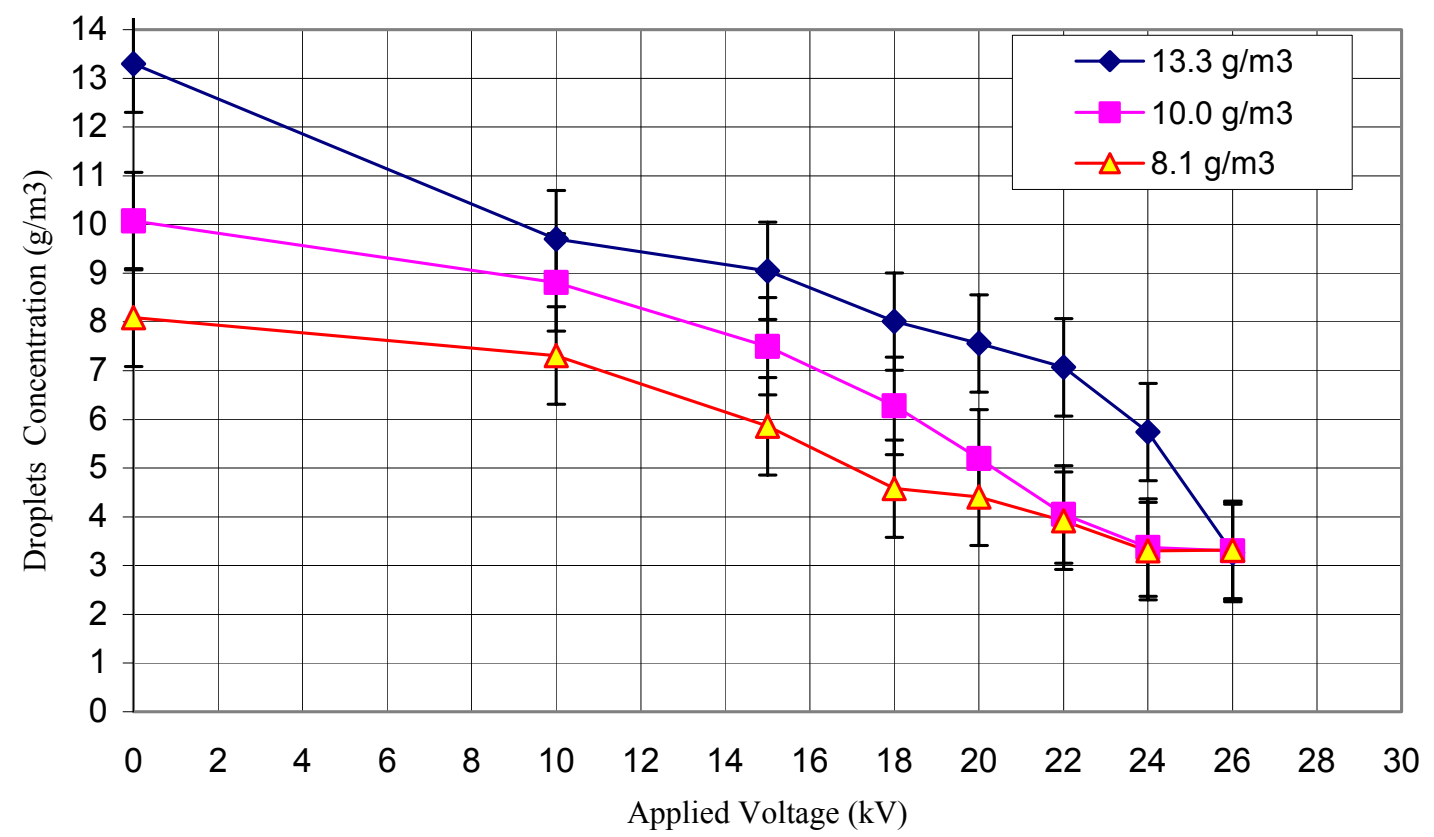

Figure 12. Aerosol concentration in outlet from chamber as a function of applied voltage; Reynolds number $=1950$.

The following observations were made from the experimental results with the prototype unit:

- The applied electric potentials reduced the mist content of the air exiting the test unit as the potential was increased from 0 to $26 \mathrm{kV}$, in all but one of the cases investigated. In that case there was no further reduction in water droplet content of the air stream when the potential was increased from 24 to $26 \mathrm{kV}$, suggesting a saturation effect. This saturation effect, seen in Figure 12, may limit the effectiveness of treatment of more dilute aerosols.

- The efficiency of the droplet removal process ranged from about $65 \%$ to $85 \%$ on a mass basis for the series of runs at a nominal Reynolds number of 1350, with the higher efficiencies corresponding to the higher nominal initial droplet loading of $24 \mathrm{~g}$ mass per cubic meter. The removal efficiency of $65 \%$ was achieved for a nominal droplet loading of $8 \mathrm{~g}$ mass per cubic meter.

- In a strict sense, the effect of the applied electric potential on the water droplet content of the air stream is not linear. However, fitting the data with a linear model may be useful for preliminary engineering design purposes.

- The water droplets migrate to the electrodes when the electric potential is applied across the plates, as evidenced by water accumulation on the plate surfaces. Although not quantified, it appeared that more water accumulated on the positive electrode than the negative one. Accumulation of mist on the plates results in draining of water down the plates and pooling at the bottom of the unit. (In a tank application, the device could be deployed to return the liquid directly to the tank.) This migration of mist from the air stream to the plates due to the influence of the electric potential is the principal mechanism of droplet removal from the air stream in these experiments. Quantification of charge on the droplets is necessary for further investigation of mechanisms. 
- Experiments show that the droplet content of the air stream decreases approximately linearly with distance up the test unit. This result suggests that further increases in removal efficiencies can be achieved by the use of electrode plates longer than the $60 \mathrm{~cm}$ used in these experiments.

Although the concentration of droplets decreased as the aerosol flowed through the duct between the electrodes, the size of the droplets that remained suspended in the gas flow did not increase markedly. There is limited evidence that the electric potential promoted droplet coalescence in the size range of approximately 10 to 20 micrometers, at least. The process could possibly improve droplet collection efficiency in a filter installed downstream of an electric potential demisting unit in an actual application, although sufficient data are not provided here for quantifying the extent to which the overall droplet collection efficiency may be improved.

\section{$\underline{\text { Modeling }}$}

Progress was made towards the development of modeling tools capable of simulating the interactions of aerosols suspended in a fluid under the effect of an externally applied electric field. The basis of the model is the continuous BGK-Boltzmann equation governing the evolution of the velocity distribution function through a simplified collision operator defined by a local equilibrium distribution function, and a single parameter which controls the relaxation time to the local equilibrium (Bhatnagar et. al, 1954). The solution method employs a discontinuous Galerkin finite element method to approximate the distribution function in the phase space as a combination of Hermite (velocity space) and Lagrange (configurational space) polynomials (a somewhat similar method was recently proposed by Tolke et. al, 2000). The incorporation of the motion of individual suspended aerosol particles follows the approach advanced by Ladd (1994a and 1994b) for a relatively small number of aerosols. Whereas the account of large quantities of aerosols follows a friction ansatz similar to Ahlrichs and Dunweg (1998). The resulting method allows for the use of conventional unstructured meshes accompanied by a choice of the either explicit, implicit, or hybrid solution of the algebraic system of equations which contrasts with conventional lattice Boltzmann methods.

Tests were made with 10 to 1000 circular particles in a 2-D box (figs. 13 and 14) by computing finite dimensional approximations for the distribution function. The computed fields can be subsequently combined to recover hydrodynamic fields such as mass density, velocity, and stress. The method captures accurately discontinuities in the distribution function and the spatial accuracy can be readily improved by increasing the order of the finite element Lagrange polynomials. Moreover in view of the compact support of the discontinous polynomials, advantage can be taken of parallel computer architectures for efficient implementation of the method.

It proved to be non-trivial to incorporate boundary conditions on the distribution function in terms of the hydrodynamic fields. For instance, the Dirichlet boundary condition for the distribution function is mathematically valid but it is not of physical interest. On the other hand, the simple no-slip boundary condition for the velocity field implies an involved integral constraint on the particle distribution function. Hence, fundamental work needs to be done on the 


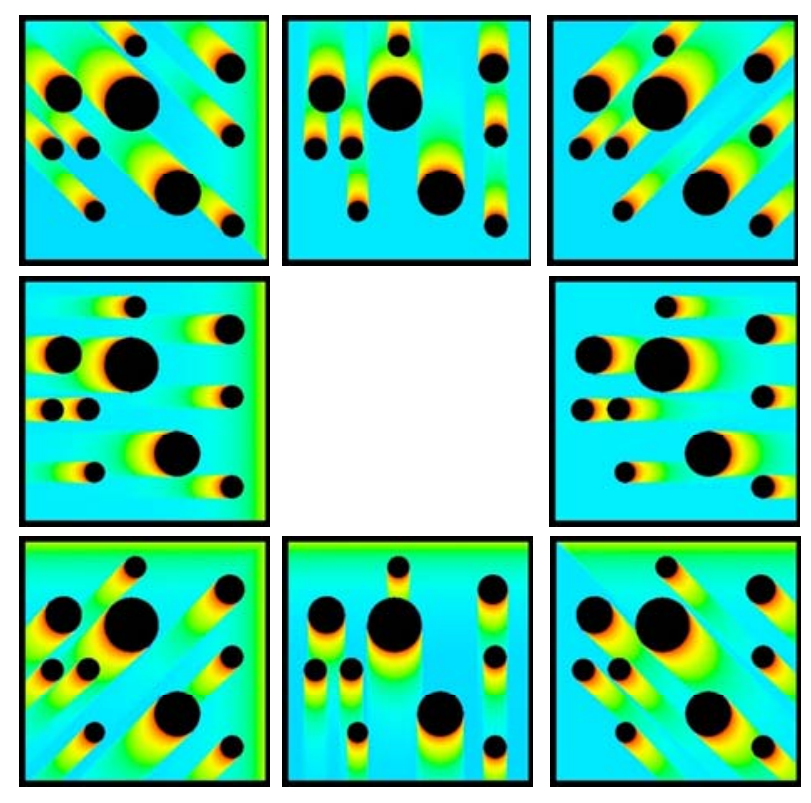

Figure 13. Computed finite dimensional distribution functions in 8 velocity directions for 10 fixed circular aerosol particles in a 2-D box; Dirichlet boundary conditions apply on admissible boundary segments.

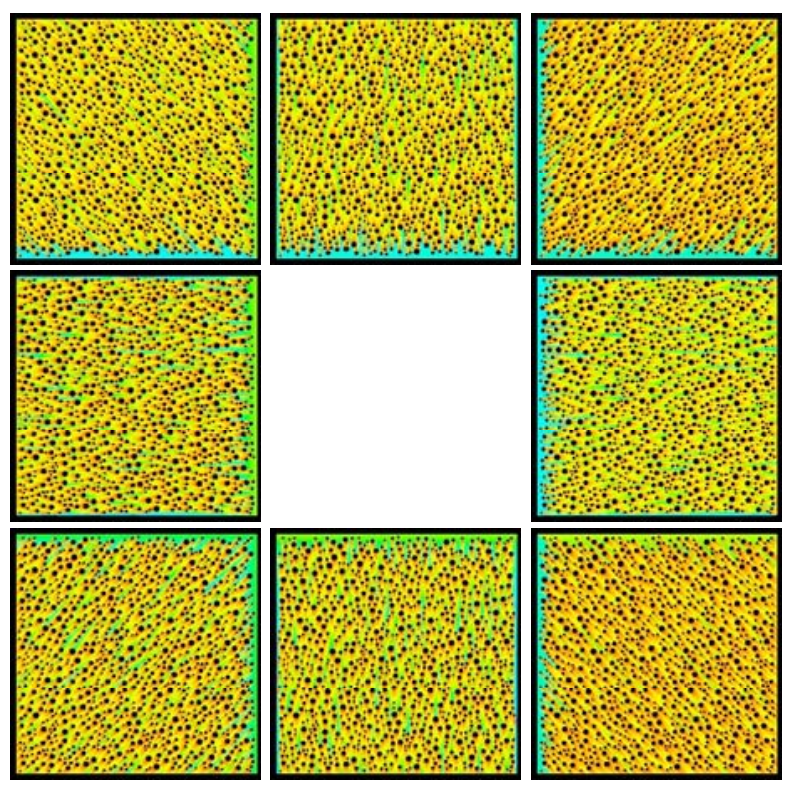

Figure 14. Results similar to figure 13 for 1000 particles.

implementation of boundary conditions before our proposed method can be used with confidence.A number of articles (Mei et. al, 1999, Zou and He, 1997, Maier et. al, 1996, Skordos 1993) have appeared in the literature covering this topic for the lattice Boltzmann method, 
therefore a critical review of different approaches should point to practical implementation of boundary conditions.

The model proposed applies to aerosols of colloidal size up to non-Brownian suspension of particles. To study coalescence of systems consisting of micron sized drops, substantial changes in the model are required to introduce surface tension force, interfacial charge, and mass transfer. The resulting mathematical problem may be exceedingly complex and require solution approaches radically different than what has been examined here.

\section{Relevance, Impact, and Technology Transfer:}

This project has focused on developing new scientific knowledge to apply towards critical DOE environmental management problems related to aerosol treatment. Aerosol-treatment issues are related to treatment and retrieval operations in radioactive waste tanks and to thermal treatment operations such as calcination, and present significant problem of costs, worker exposure, potential for release, and increased waste volume.

This project aimed to provide new scientific knowledge to bridge the gap between fundamental research and needs-driven applied technology development. The knowledge generated by this project could potentially improve the performance of technologies for waste treatment and cleanup; however, to this point, the fundamental studies have not advanced sufficiently to provide opportunities for new applications. Therefore, there is no direct way to identify opportunities to significantly reduce future costs, schedules, and risks and/or meet DOE compliance requirements. Larger-scale trials of this technology are not warranted. No other government agencies or private enterprises expressed interest in the project.

Technology now appears to be available to solve DOE aerosol-treatment problems. Significant progress has been made in development and testing of washable ceramic and fritted-metal HEPA filters. Those filters, which feature high filter-media strength, resistance to water damage, and capability to operate at elevated temperatures, have performed well in testing (Adamson et al. 2002) and appear to be suitable to meet DOE needs for aerosol treatment.

\section{Project Productivity:}

The project plan involved experimentation and modeling of acoustic agglomeration and electrocoalesence/electrofiltration separately for the first two years, followed by a year of modeling and experimental study of coupled interactions of acoustic and electric fields. The project schedule was lengthened with a no-cost extension. As shown below, the project was productive, particularly in generating knowledge and graduate student training. The full project plan was not completed; details of experimentation with single fields limited progress towards the coupled-field case. The work plan was revised to include the agitated agglomeration system to explore the dynamics of acoustic agglomeration under realizable length and time scales. That work uncovered a large effect of low-amplitude oscillation on sedimentation and resuspension that may be attractive as an alternative, non-contact means of controlling settling and/or suspension of solids. 


\section{Personnel Supported:}

The following professional personnel were partially supported by this project:

Valmor de Almeida

David DePaoli

Ofodike Ezekoye

Costas Tsouris

In addition, the following graduate students were fully supported by the research effort:

Vinayak Barve

Jason Carter

Krishna Lakshminarasimhan

Cyrus Riahi-Nezhad

Jonathan Schmidt

Yanuar Wibowo

\section{Publications:}

Papers published in peer-reviewed journals:

Carter, J., K. M. Martin, W. B. Campbell, N. A. Hall, and O. A. Ezekoye, " Design of an Oscillating-Flow Apparatus for the Study of Low-Reynolds-Number Particle Dynamics," Experiments in Fluids 30(5), 578-583 (2001).

Ezekoye, O. A., and Y. W. Wibowo, "Simulation of Acoustic Agglomeration Processes Using a Sectional Algorithm," Journal of Aerosol Science 30(9), 1117-1138 (1999).

Papers published in unreviewed publications (proceedings, technical reports, etc.):

Wibowo, Y.W. and Ezekoye, O. A, "Computations of Sedimentation Rates for Acoustically Enhanced Agglomeration," AIChE CCPS Annual International Conference and Workshop on Modeling Consequences of Accidental Releases of Hazardous Materials, San Francisco, CA, September, 1999.

Student theses:

Vinayak Barve, "Simulation of Gravitational Settling Under Electric Fields", M.S. thesis, The University of Texas at Austin, 2002

Krishna Lakshminarasimhan "Effects of Lateral Oscillation on Sedimentation of Particles in Suspensions", M.S. thesis, The University of Texas at Austin, 2002

Jason Carter, "Study of Fluid Oscillation and its Effect on Low Reynolds Number Particle Sedimentation," M.S. thesis, The University of Texas at Austin, 2000.

Cyrus Riahi-Nezhad, "The Effect of Electric Field on Moving Aqueous Aerosol Suspended in a Dynamic Fluid Chamber," M.S. thesis, University of Tennessee, Knoxville, December 2001.

Jonathan Schmidt, "Experimental Study of Electro-coalescence in a Unit Reactor", M.S. thesis, The University of Texas at Austin, 2000. 
Yanuar Wibowo, "Simulation of Acoustic Agglomeration Processes Using a Sectional Algorithm”, M.S. Report, The University of Texas at Austin, 1998.

\section{Interactions:}

This project was represented at meetings, workshops, conferences, seminars, etc.:

Schmidt, J. J., O. A. Ezekoye, C. K. Riahi-Nezhad, C. Tsouris, V. F. de Almeida, and D. W. DePaoli, "Electrically Driven Technologies for Radioactive Aerosol Abatement," presented at EMSP National Workshop, Atlanta, GA, April 27-27, 2000.

Wibowo, Y.W. and Ezekoye, O. A, "Computations of Sedimentation Rates for Acoustically Enhanced Agglomeration," AIChE CCPS Annual International Conference and Workshop on Modeling Consequences of Accidental Releases of Hazardous Materials, San Francisco, CA, September, 1999.

DePaoli, D. W., C. Tsouris, V. F. de Almeida, O. A. Ezekoye, "Progress in Electrically Driven Technologies for Radioactive Aerosol Abatement," presentation at EMSP program review, Oak Ridge, TN, September, 1999.

O. A. Ezekoye, particpation at EMSP/TFA Workshop in Richland, Washington, November, 1998.

The participants in this project performed no consultative and advisory functions to other laboratories and agencies as part of this work. Communications were made with:

- Multiple stakeholders at the EMSP/TFA Workshop in Richland (11/98), regarding tankwaste offgas treatment

- Mike Terry, Safety Technical Integration Manager for Tanks Focus Area, for possible application to aerosols in offgas of calcining operations

- Sam Ashworth, INEEL, for applicability to thermal treatment technologies, including melter offgas

\section{Transitions:}

Knowledge resulting from this project has not been used in a technology, technique, or processimprovement application.

\section{Patents:}

None.

\section{Future Work:}

A number of topics are worthy of study to better understand acoustic and electric agglomeration and removal of aerosols.

Attempts at modeling the unit reactor data using the most sophisticated theory present in the literature showed that there is still much that must be done in coupling aggregation kernels having different physics. There is at present no straightforward approach to rigorously combine Brownian and gravitational aggregation kernels. This fact results in significant errors in the 
computed aggregation dynamics when the particle size distribution is not in a limiting case where either process is dominant.

The modeling approach pursued in this project was relatively new, within the discipline of fluid mechanics, and incorporated, explicitly, the existence of individual aerosol particles within the suspending fluid. The corresponding solution method was selected to take advantage of state-ofthe-art parallel computing machines so that a meaningful number of the particles could be simulated. The model and solution method proposed still require additional tests and further development before they can be considered a predictive tool. First, as mentioned above there are open issues regarding boundary conditions that need to be addressed; these issues do not seem to be particularly difficult but do require careful attention. Second, a number of simulation consisting of a few particles should be performed and compared with continuum models of classical electrohydrodynamics. Last but not least, to facilitate the experimental validation of the model, contrived experiments need to be designed with and without electric field applied, so that bulk interaction of aerosols, and surface interaction of aerosols and electrode are distinguished; this may turn out to be a difficult task in view of the difficulty of measuring/controlling electrical charge in the system.

In the experiments conducted in this work using the ORNL prototype system, resuspension of droplets from the electrodes was observed. To eliminate this phenomenon in a continuous agglomeration system, it will be required to have a mechanism to continuously remove the collected droplets. In addition, the potential for removal of aerosols via dielectric filtration, by addition of dielectric media between electrodes, warrants additional study. Ideally the detailed modeling of aerosols under the effect of external fields can greatly improve the understanding of these dynamical systems.

At this point, no future work based on the results of these studies has been initiated.

\section{Literature Cited}

Adamson, D. J., D. B. Burns, and M. T. Terry, "Insitu Cleanable Alternative HEPA Filter Media," Westinghouse Savannah River Company Report WSRC-MS-2002-00431, available at <http://www.srs.gov/general/pubs/fulltext/ms2002431/ms2002431.html > (2002).

Ahlrichs, P., and Dunweg Burkhard, "Lattice Boltzmann Simulation of Polymer-Solvent Systems," International Journal of Modern Physics C 9(8), 1429-38 (1998).

Bhatnagar, P. L., Gross, E. P., and Krook, M., "A Model for Collision Processes in Gases. I. Small Amplitude Processes in Charged and Neutral One-Component Systems," Physical Review 94(3), 511-25 (1954).

Carter, J., K. M. Martin, W. B. Campbell, N. A. Hall, and O. A. Ezekoye, " Design of an Oscillating-Flow Apparatus for the Study of Low-Reynolds-Number Particle Dynamics," Experiments in Fluids 30(5), 578-583 (2001).

Carter, J. "Study of Fluid Oscillation and its Effect on Low Reynolds Number Particle Sedimentation", M.S. Thesis, The University of Texas at Austin. 2000.

Ezekoye, O. A., and Y. W. Wibowo, "Simulation of Acoustic Agglomeration Processes Using a Sectional Algorithm," Journal of Aerosol Science 30(9), 1117-1138 (1999). 
Gelbard and Seinfeld (1980) Simulation of Multicomponent Aerosol Dynamics, J. Colloid Interface Sci., 78, 485.

Ladd, A. J. C., "Numerical Simulation of Particles Suspensions via a Discretized Boltzmann Equation. Part 1. Theoretical Foundation," Journal of Fluid Mechanics 271, 285-309 (1994).

Ladd, A. J. C., "Numerical Simulation of Particles Suspensions via a Discretized Boltzmann Equation. Part 2. Numerical Results," Journal of Fluid Mechanics 271, 311-39 (1994).

Lakshimarasimhan (2002) "Effects of Lateral Oscillation on Sedimentation of Particles in Suspensions", M.S. thesis, The University of Texas at Austin, 2002.

Maier, R. S., Bernard, R. S., Grunau, D. W., "Boundary Conditions for the Lattice Boltzmann Method," Physics of Fluids 8(7), 1788-91 (1996).

Mei, R., Luo, L.-S., Shyy, W., "An Accurate Curved Boundary Treatment in the Lattice Boltzmann Method," Journal of Computational Physics 155, 307-30 (1999).

Schmidt (2000) "Experimental Study of Electro-coalescence in a Unit Reactor", M.S. thesis, The University of Texas at Austin.

Skordos, P. A., "Initial and Boundary Conditions for the Lattice Boltzmann Method," Physical Review E 48(6), 4823-42 (1993).

Tolke, J., Krafczyk, M., Schulz, M., Rank, E., "Discretization of the Boltzmann Equation in Velocity Space Using a Galerkin Approach," Computer Physics Communications 129, 91-9 (2000).

Zou, Q., He, X., "On Pressure and Velocity Boundary Conditions for the Lattice Boltzmann BGK Model," Physics of Fluids 9(6), 1591-8 (1997). 
Appendices 
Experimental Study of Electro-coalescence in a Unit Reactor

By

Jonathan Jacob Schmidt, B.S.

\author{
Thesis \\ Presented to the Faculty of the Graduate School \\ Of The University of Texas at Austin \\ in Partial Fulfillment \\ of the Requirements \\ for the Degree of
}

Master of Science in Engineering

The University of Texas at Austin

August, 2000 


\title{
Experimental Study of Electro-coalescence in a Unit Reactor
}

\author{
By \\ Jonathan Jacob Schmidt, M.S.E. \\ The University of Texas at Austin, 2000 \\ SUPERVISOR: Ofodike A. Ezekoye
}

\begin{abstract}
Experiments were conducted on electro-coalescence in a unit scale reactor to evaluate the physical basis of the phenomenon. A strong electric field was produced in a reactor of square cross-section and with a fixed small height/gap. Various types of aerosol particles were introduced into the reactor space and were subjected to different field strengths. The overall sedimentation and coalescence properties of the aerosols were monitored using a laser extinction measurement. Results of these measurements indicate that some aerosols are more likely to be affected by the electric fields. The results also show that a moderate decrease in the time to clear the chamber can be accomplished using a relatively small amount of electrical power, which shifts the particle size distribution towards larger sizes by a small amount.
\end{abstract}




\section{Table of Contents}

CHAPTER 1. INTRODUCTION.................................11

1.1. Conventional Filtration Systems.........................12

1.2. Electro-coalescence.................................13

1.3. Electro-Filtration...................................... 16

CHAPTER 2. EXPERIMENTS...................................18

2.1. Experimental Setup.................................18

2.2. Experimental Diagnostic.............................20

2.3. Experimental Diagnostic............................24

CHAPTER 3 - THEORY ..................................27

3.1. Electric Field Theory.................................27

3.2. Theory of electro-coalescence........................30

3.3. Particle Dynamics................................. 31

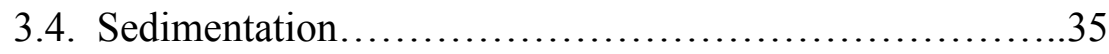

CHAPTER 4. RESULTS ................................... 37

4.1. Preliminary Results................................37

4.2. Mean Values......................................43

4.3. Size Distribution..................................52

4.4. Multiple Elevation Results...........................55

4.5. Multiple Voltage Results...........................58

4.6. Size Distribution Results...........................60

CHAPTER 5. CONCLUSIONS ...................................73

BIBLIOGRAPHY ............................................ 76

VITA ...................................................... 


\section{List of Tables}

Table 1.1. Collection system efficiencies and power requirements.............13

Table 1.2. Electro-coalescence systems and collection efficiencies.............25

Table 2.1. Experimental matrix for different aerosols........................24

Table 3.1. Dielectric constants of several materials.......................... 30

Table 3.2. Relative dipole coalescing forces vs. approximate emulsion content, cubic packing assumed............................. 35

Table 4.1. Comparison of procedures 2 and 3 to procedure $1 \ldots \ldots \ldots \ldots \ldots . . \ldots 44$

Table 4.2. Particle diameter statistics from the particle sizer...................54

Table 4.3. Normalized approximate sedimentation rates $(\mu \mathrm{m} / \mathrm{s})$ for all laser elevation and applied voltage experiments................55

Table 4.4. Mean particle diameters from size distribution results..............72 


\section{List of Figures}

Figure 1.1. Induced dipole in adjacent particles associated with a

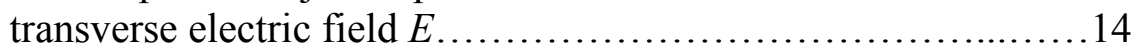

Figure 2.1. Experimental setup for the laser extinction measurement...........19

Figure 2.2. Dimensions and components of the unit reactor....................19

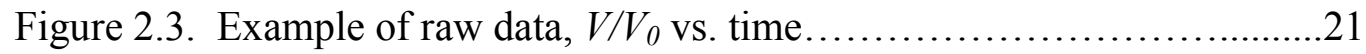

Figure 2.4. Example of volume fraction ratio data $f_{v} / f_{v, \max }$ vs. time.............23

Figure 3.1. Electric field between parallel plates with plate spacing, $d \ldots \ldots \ldots . .27$

Figure 3.2. Surface charge densities and force on two drops induced by an externally applied electric field for different values of angle $\psi$ between the electric field and the line joining centers of the two drops........................................34

Figure 4.1. Raw data for oil fog experiments with no applied voltage...........39

Figure 4.2. Raw data for oil fog experiments with an applied voltage of 1000 Volts....

Figure 4.3. Raw data for tissue paper experiments with no applied voltage

Figure 4.4. Raw data for tissue paper experiments with an applied voltage of 1000 Volts.

Figure 4.5. Raw data for Topps cigarette experiments with no applied voltage.

Figure 4.6. Raw data for Topps cigarette experiments with an applied voltage of 1000 Volts

Figure 4.7. Raw data for Maverick cigarette experiments with no applied voltage.

Figure 4.8. Raw data for Maverick cigarette experiments with an applied voltage of 1000 Volts.

Figure 4.9. Mean $f_{v} / f_{v, \max }$ vs. time plots using procedures 2 and 3

Figure 4.10. Average $f_{v} / f_{v, \max }$ vs. time plot for oil fog experiments with no applied voltage. 
Figure 4.11. Average $f_{v} / f_{v, \max }$ vs. time plot for oil fog experiments with an applied voltage of 1000 Volts.

Figure 4.12. Average $f_{v} / f_{v, \max }$ vs. time plot for tissue paper experiments with no applied voltage.

Figure 4.13. Average $f_{v} / f_{v, \max }$ vs. time plot for tissue paper experiments with an applied voltage of 1000 Volts

Figure 4.14. Average $f_{v} / f_{v, \max }$ vs. time plot for Topps cigarette experiments with no applied voltage.

Figure 4.15. Average $f_{v} / f_{v, \max }$ vs. time plot for Topps cigarette experiments with an applied voltage of 1000 Volts

Figure 4.16. Average $f_{v} f_{v, \max }$ vs. time plot for Maverick cigarette experiments with no applied voltage

Figure 4.17. Average $f_{v} f_{v, \max }$ vs. time plot for Maverick cigarette experiments with an applied voltage of 1000 Volts

Figure 4.18. Particle sizer results run 1 with a $10 \mathrm{~s}$ sample time................53

Figure 4.19. Particle sizer results run 3 with a 10 s sample time................53

Figure 4.20. Particle sizer results run 4 with a 30 s sample time................53

Figure 4.21. Particle sizer results run 5 with a $30 \mathrm{~s}$ sample time................53

Figure 4.22. $f_{v} / f_{v, \max }$ vs. time plots for all laser elevations with no applied voltage. .56

Figure 4.23. $f_{v} / f_{v, \max }$ vs. time plots for all laser elevations with an applied voltage of 500 Volts

Figure 4.24. $f_{v} / f_{v, \max }$ vs. time plots for all laser elevations with an applied voltage of 1000 Volts.

Figure 4.25. $f_{v} / f_{v, \max }$ vs. time plots for all laser elevations with an applied voltage of 1500 Volts.

Figure 4.26. $f_{v} / f_{v, \max }$ vs. time plots for all applied voltages at a laser elevation of $\mathrm{z}=3.05 \mathrm{~mm}$. .58

Figure 4.27. $f_{v} / f_{v, \max }$ vs. time plots for all applied voltages at a laser elevation of $\mathrm{z}=7.62 \mathrm{~mm}$ 
Figure 4.28. $f_{v} / f_{v, \max }$ vs. time plots for all applied voltages at a laser

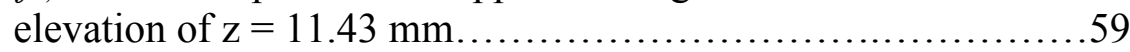

Figure 4.29. Monodisperse aerosol passing through a volume..................60

Figure 4.30. $f_{v}$ vs. $t$ at a specific elevation for a monodisperse aerosol..........61

Figure 4.31. Polydisperse aerosol passing through a volume......................61

Figure 4.32. $f_{v}$ vs. $t$ at a specific elevation for a polydisperse aerosol............61

Figure 4.33. Size distribution plots for the case where the applied voltage was 0 Volts at a laser elevation of $\mathrm{z}=3.05 \mathrm{~mm} \ldots \ldots \ldots \ldots .68$

Figure 4.34. Size distribution plots for the case where the applied voltage was 0 Volts at a laser elevation of $\mathrm{z}=7.62 \mathrm{~mm} \ldots \ldots \ldots \ldots . . .68$

Figure 4.35. Size distribution plots for the case where the applied voltage was 0 Volts at a laser elevation of $\mathrm{z}=11.43 \mathrm{~mm}$ 68

Figure 4.36. Size distribution plots for the case where the applied voltage was 500 Volts at a laser elevation of $\mathrm{z}=3.05 \mathrm{~mm}$

Figure 4.37. Size distribution plots for the case where the applied voltage was 500 Volts at a laser elevation of $z=7.62 \mathrm{~mm}$

Figure 4.38. Size distribution plots for the case where the applied voltage was 500 Volts at a laser elevation of $\mathrm{z}=11.43 \mathrm{~mm}$......69

Figure 4.39. Size distribution plots for the case where the applied voltage was 1000 Volts at a laser elevation of $\mathrm{z}=3.05 \mathrm{~mm} \ldots \ldots . .70$

Figure 4.40. Size distribution plots for the case where the applied voltage was 1000 Volts at a laser elevation of $\mathrm{z}=7.62 \mathrm{~mm}$.......70

Figure 4.41. Size distribution plots for the case where the applied voltage was 1000 Volts at a laser elevation of $\mathrm{z}=11.43 \mathrm{~mm} \ldots \ldots .70$

Figure 4.42. Size distribution plots for the case where the applied voltage was 1500 Volts at a laser elevation of $\mathrm{z}=3.05 \mathrm{~mm}$......71

Figure 4.43. Size distribution plots for the case where the applied voltage was 1500 Volts at a laser elevation of $\mathrm{z}=7.62 \mathrm{~mm} \ldots \ldots .71$

Figure 4.44. Size distribution plots for the case where the applied voltage was 1500 Volts at a laser elevation of $\mathrm{z}=11.43 \mathrm{~mm} \ldots \ldots .71$ 


\section{CHAPTER 1. INTRODUCTION}

Renewed interest in the role of particulate matter as a source of increased morbidity in many urban areas has prompted research in the capture and filtration of fine particulate matter. It is well known that small aerosol particles (diameter $<1$ micrometer) are linked towards respiratory illness in many populations. This has often been attributed to the ability of these small particles to bypass the natural filtration system of the human respiratory system. There are many sources of these types of particles, although combustion generated mechanisms tend to play a disproportionate role. The US EPA controls the regulations associated with monitoring the sources and the overall environmental impact associated with combustion mechanisms. While not nearly as ubiquitous, some focused applications exist for fine particle removal where the goal is for complete capture due to the nature of the aerosol particles. For example, the US DOE has significantly more stringent regulations than EPA on particle filtration from DOE complexes due to the possible toxicity and radioactivity of the particles. In cases where the particles are radioactive or extremely toxic, special care must be taken in the use of conventional filtration technology. For example, the frequency of maintenance of the filtration systems should necessarily be longer when dealing with hazardous aerosols given the potential human risk associated with maintenance and repairs to the systems. It is the goal of this research project to evaluate one method for post-processing an effluent stream containing aerosol particles with the aim of reducing the particulate loading of the stream. Prior to discussing the details of the technology under investigation (electro-coalescence) a discussion and comparison will be made of the potential benefits of this technology with other filtration and aerosol post-processing techniques. 


\subsection{Conventional Filtrations Systems}

The simplest particulate filtration system is the ordinary fiber filter. Fiber filters can be made from a variety of materials including cloths, paper, and even recently from ceramic and metallic materials. The advantage of filter-based capture is the relative ease of implementation of the system. The major problem with these systems is that the fiber filters typically must be manually removed and replaced after a loading period. Other problems with fiber filters are the potential pressure drops across the filters and the capture efficiency of the filter material. Interestingly, a trade-off often exists between pressure drop and capture efficiency. By stacking filters in series, it is often the case that a better capture efficiency will result when compared to a single filter, but there will accordingly be a greater pressure drop. Pressure drop is often expressed in practical systems in terms of specific power for the filtration system. One might compare the specific power requirements of a range of filtration systems in order to evaluate optimal systems for a given required capture efficiency. Four typical collection systems are shown in Table 1.1. where the fractional collection/capture efficiencies are shown along with the specific power of the capture system. One important point to note and the basis for the work that we are performing is that for very small particles the fractional collection efficiency always increases with increasing particle size. Thus, one obvious method for increasing the capture efficiency of a system is to shift the size distribution of aerosols towards larger sizes. Ideally, one would attempt to make this modification without incurring a significant power cost. Two methods for producing this shift that are noted in the literature are electro-coalescence and acoustic agglomeration. This thesis will focus on effects associated with electro-coalescence. 
Table 1.1. Collection system efficiencies and power requirements.

\begin{tabular}{|c|c|c|c|c|}
\hline \multicolumn{1}{|c|}{ Typical Fractional Collection Efficiencies and Specific Power } \\
Requirements for Particulate Control Equipment; Wark and Warner (1981) \\
\hline
\end{tabular}

\subsection{Electro-coalescence}

Electro-coalescence refers to the process by which particles (aerosols, hydrosols, and emulsions) are induced to coalesce based on the application of strong electric fields. Fundamentally, the strong electric fields induce a dipole in the aerosol particles. Adjacent particles experience an attractive force, which can eventually lead to agglomeration or coalescence (see Figure 1.1).

Literature on the use of electro-coalescence (EC) for separation processes of solids, liquids and gases extends to the late 1950's and early 1960's (Waterman 1965). Use of electro-coalescence has been particularly effective in separation of emulsions. Waterman presents a table of the collection efficiency of a specific EC system for a range of suspensions and emulsions (see Table 1.2). Contained in the table are results for other electrically based filtration systems. These include dipole coalescers (which we focus on as the fundamental EC system), a process called electrofining and a developmental process called (electro-filtration). A natural practical extension of EC is electro-filtration. More detailed discussion of the basic operating theory for electro-coalescence processes will be provided in 
the theoretical section, which follows. Overall, Waterman notes that the processes were quite versatile and effective in removal of water from oil, salts from oil and solids from fuel. Williams and Baily (1986) describe their experiments on the changes in the size distribution of a water-in-oil emulsion due to electro-coalescence. Zhang et al (1995) added theoretical justification and used a more rigorous approach to show the change in size distribution for a water-inoil dispersion.

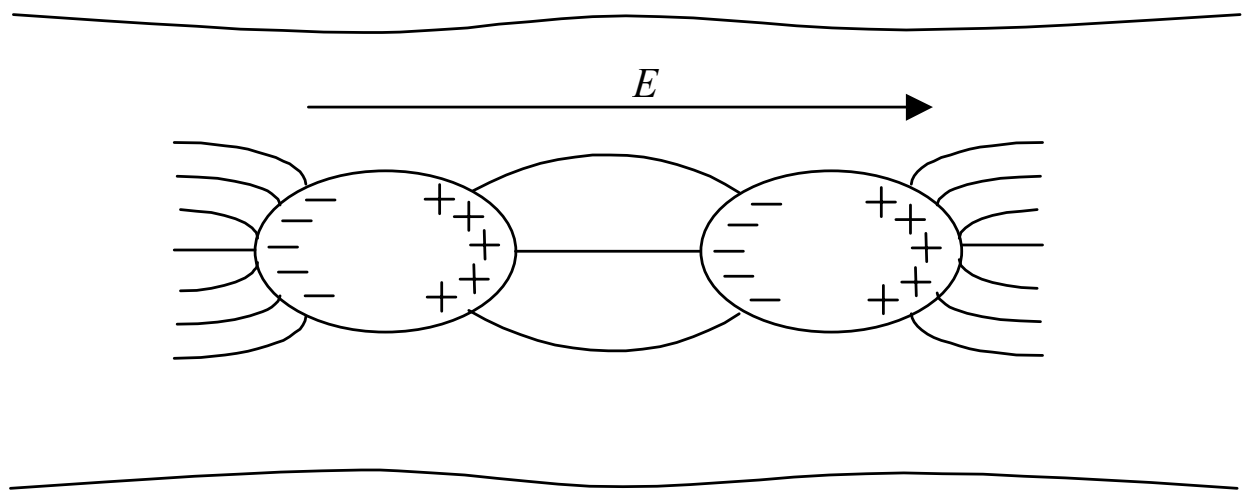

Figure 1.1. Induced dipole in adjacent particles associated with a transverse electric field $E$. 


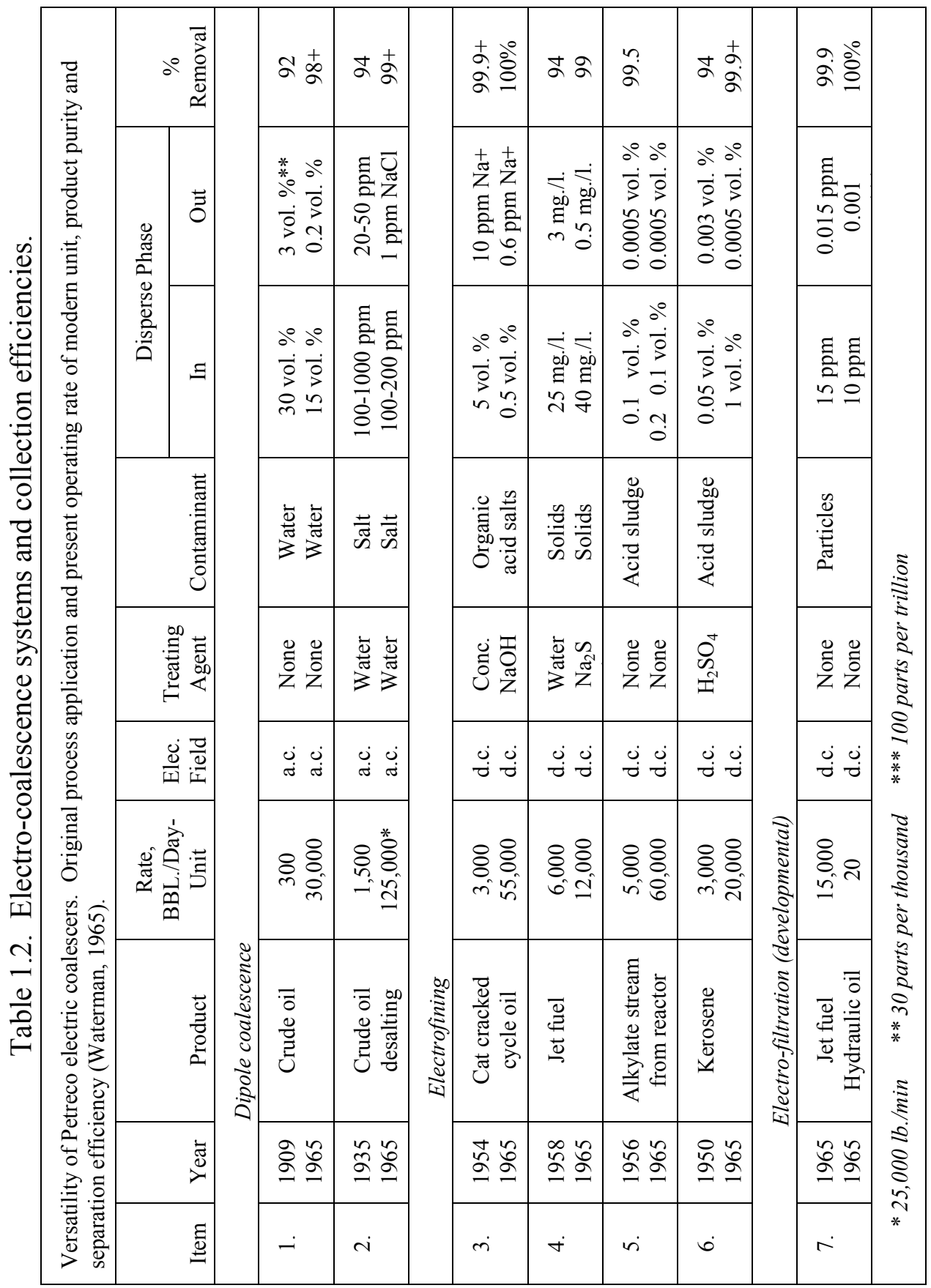




\subsection{Electro-Filtration}

The removal of small particles (diameter $<1 \mu \mathrm{m}$ ) from processed waste streams is expensive and often consumes a significant amount of energy. Granular filtration is mechanically simple and consumes relatively low energy, however, it relies principally on the mechanisms of diffusion, inertia, and surface forces, which yield low collision efficiency between particles and collectors. Thus, it is often inefficient, requiring the use of large filters. It is well known that the efficiency of aerosol filtration in fiber mats (Silverman et al., 1954) and in granular beds (Harriot and Saville, 1971) can be improved by the application of an electric field to the filter media. Filtration efficiencies of greater than $99 \%$ are reported in the literature (Waterman, 1965).

An electro-filter is essentially an electro-coalescer with the interelectrode space packed with a matrix for trapping particles. The matrix can be foam, fiber, or filament (Waterman, 1965). A granular electro-filter is a bed of coarse particles (diameter $1 \mathrm{~mm}$ ), with electrodes arranged so that a strong electric field can be applied to the bed. The electric field polarizes the packing granules and the combined electric field causes the charged suspended particles or drops to deviate from the fluid streamlines and be collected on the oppositely charged surface of the granules. In another concept of electro-filtration, the electric charge is directly applied to the granules. In this case, the granular electro-filter operates like an electrostatic precipitator, but with more complex flow paths. This type of electro-filtration is more suitable with less densely packed beds, such as fiber filled beds. Applied electric fields across fibrous rather than granular filters have enhanced performance of gas filtration (Henry and Ariman, 1986). The main differences between fibrous and granular filters are (i) the geometry of the collecting solids and (ii) in fibrous filtration of gaseous streams, the drag on suspending particles is dominated by inertial rather than viscous forces. 
In the case that the granules/fiber packing is nonconducting, the field lines divert around the granules or fibers. If the packing is a better conductor than the fluid, the field lines will be diverted toward the granules or fibers. Since electric forces on the particulates act along the electric field lines, it is desirable to have the lines diverted into (rather than around) the granules or fibers. This is similar to the concept of using conducting granules or fibers, which can be considered to have a very high dielectric constant. In general, it is desirable that the granules have a higher dielectric constant than the fluid. When both the fluid and packing are conductors, it is desirable for the granules or fibers to have a higher conductivity than the fluid. In summary, electric filtration is affected by many parameters including packing geometry and density, orientation and strength of the electric field, particle charge, dielectric constant and conductivity of the packing material and fluid, and particle size.

Various types of separators have been developed in the past for the separation of droplets from gas streams. The separators made of many layers of knitted mesh at various densities and geometries are shown to be most effective in removing drops above $5 \mu \mathrm{m}$ from gas streams of velocities up to $2 \mathrm{~m} / \mathrm{s}$ (Fabian et al., 1993). However, their efficiency drops significantly when the size of the drops is of order $1 \mu \mathrm{m}$. 


\section{CHAPTER 2. EXPERIMENTS}

To better clarify the physical processes relevant to EC, we undertook a study of EC in a unit reactor. Details of the experimental system follow.

\subsection{Experimental Setup}

The Experimental setup used for this study is shown in Figure 2.1.

A 0.1-milliwatt-class red laser pointer is powered by a Radio Shack 300 milliamp, variable voltage, DC power supply. The laser has a 650-680 nm wavelength. The laser was aimed through the windows of the unit reactor and into the photodiode of a Thorlabs DET110 photo detector. The photo detector has a rise time of 20 nanoseconds, a spectral range from 350 to $1100 \mathrm{~nm}$, and a maximum output voltage of 12 Volts. The photodiode converts the intensity of the laser (input) into a current (output). The current from the photodiode was converted into a positive voltage that would accommodate the 10 Volt input range of the computer. This conversion was accomplished using a terminating resistor circuit. This voltage was then sent to the computer using Labview data acquisition hardware and software. The software was set to acquire data at constant intervals for the duration of the experiment. The reactor was constructed out of Acrylic and two copper plates, and assembled using machine screws. A schematic of the reactor is shown in Figure 2.2. The internal dimensions are $10 \mathrm{~cm} \times 10 \mathrm{~cm} \times 2$ $\mathrm{cm}$, with the copper plates set into the inner top and bottom. A Bertan 205B highvoltage power supply was connected to the copper plates in order to generate an electric field within the reactor. The laser, reactor, and photo detector were mounted to an optical table. Several aerosol sources were used to fill the reactor. A Safex F2001 plus Nebelgerat-Fog Generator was used to generate the aerosol used in the oil fog experiments. 


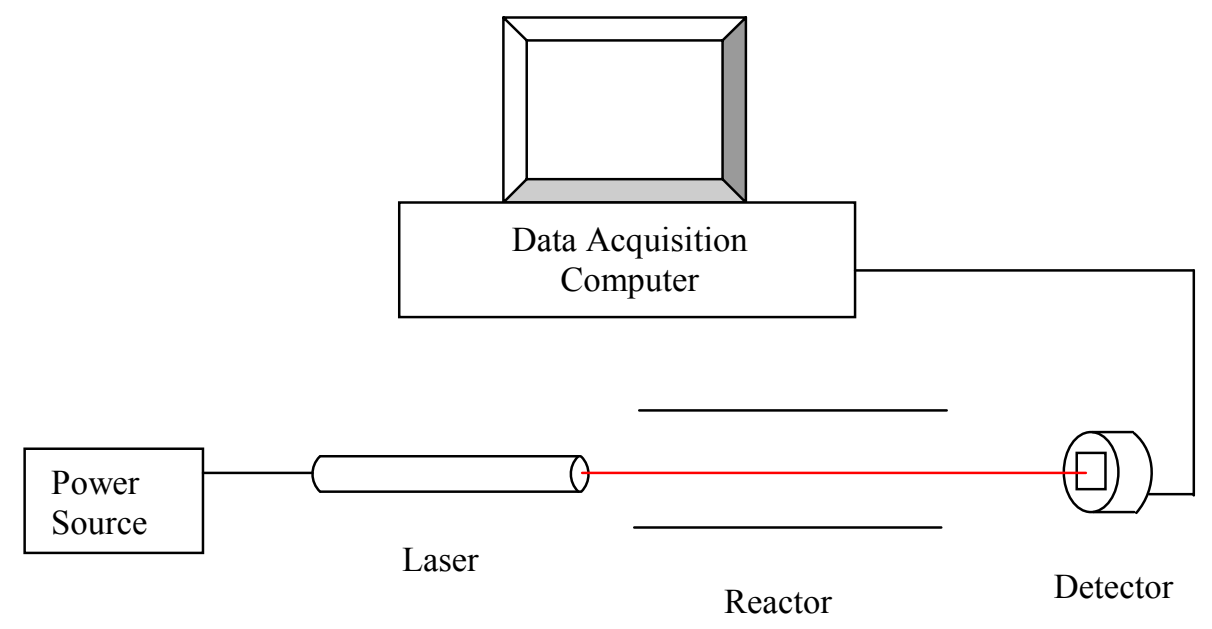

Figure 2.1. Experimental setup for the laser extinction measurement.

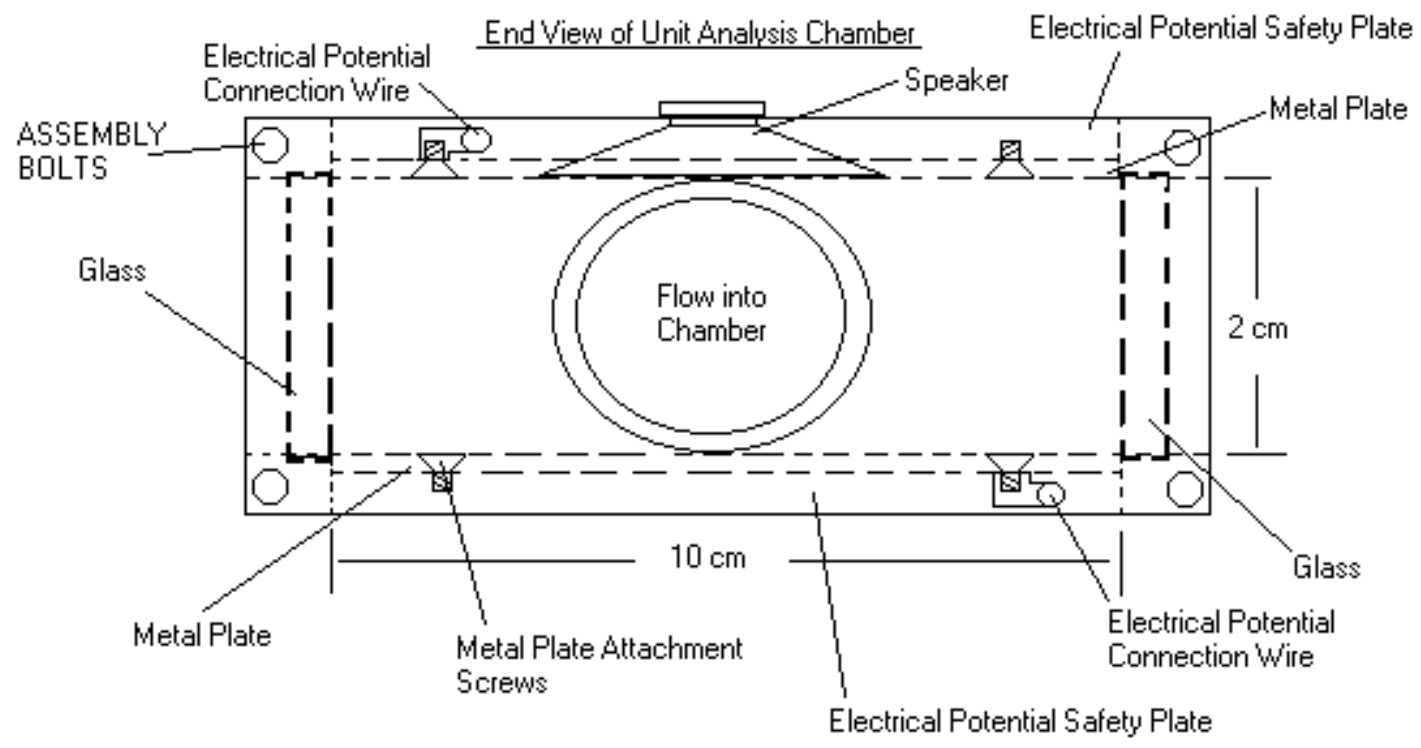

Figure 2.2. Dimensions and components of the unit reactor. 
For the other experiments, compressed air, attached to a Cole Parmer P03295-18 rotometer, was used to force the aerosol into the reactor. The rotometer controlled the volumetric flow rate of the compressed air, and was calibrated using a Brooks Instrument Volumeter. The flow rate was maintained constant at $1.2 \mathrm{~L} / \mathrm{min}$. A TSI 3320 Aerodynamic Particle Sizer and TSI 3302A Aerosol Diluter (at a dilution rate of 20:1) were used to evaluate each aerosol's characteristic size distribution.

\subsection{Experimental Diagnostic}

Experiments were conducted on electro-coalescence to evaluate the physical basis of the phenomenon. A strong electric field was produced in the reactor, and various types of aerosol particles were introduced into the reactor space. A laser extinction measurement was used to calculate the volume fraction of particles. The volume fraction as a function of time was used to monitor the overall sedimentation and coalescence properties of the aerosols. As the particles filled the reactor, the ones in the laser beam path absorbed and scattered the light from the laser. This diminished the intensity of the beam. The transmitted intensity of the laser $I_{\lambda}$ is directly proportional to $V$,

$$
I_{\lambda} \propto V
$$

Where $V$ is the voltage seen by the computer. This leads to the equation,

$$
\frac{I_{\lambda}}{I_{\lambda, 0}}=\frac{V}{V_{0}}
$$

Where $I_{\lambda, 0}$ and $V_{0}$ are the intensity and voltage associated with an empty reactor.

Figure 2.3 shows a representative experimental run where the aerosol was smoke from a Maverick cigarette; the laser was positioned $11.43 \mathrm{~mm}$ from the ceiling of the reactor, and no electric field was induced. $V / V o$ is shown as a function of time. 


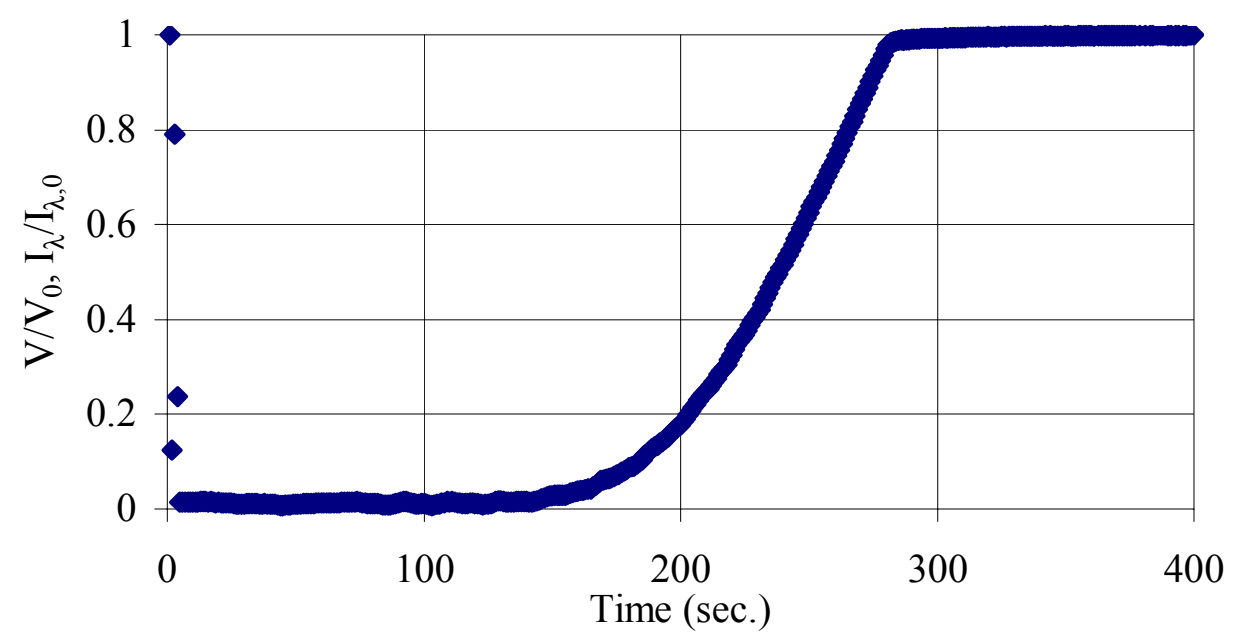

Figure 2.3. Example of raw data, $V / V_{0}$ vs. time.

The intensity, $I_{\lambda}$, of a monochromatic beam passing through a homogeneous polydisperse aerosol can be related to the initial intensity, $I_{\lambda, 0}$, by the Lambert-Beer law,

$$
\frac{I_{\lambda}}{I_{\lambda, 0}}=\exp \left(-a_{\lambda} L_{p a t h}\right)
$$

Where $L_{\text {path }}$ is the laser beam path length through the reactor, and $a_{\lambda}$ is the aerosol extinction coefficient defined by the absorption and scattering properties of the aerosol. This coefficient can be related to the aerosol volume fraction/loading, $f_{v}$, by

$$
a_{\lambda}=\frac{f_{v} k_{1}}{\lambda}
$$

Where $\lambda$ is the laser wavelength, and $k_{1}$ is a constant defined by the optical properties of the aerosol. The wavelength of the laser is approximately 660 nanometers, and a value of 5 is assumed for $k_{1}$ (Siegel and Howell, 1992). 
The loading can now be calculated from the intensity ratio by the following equation,

$$
f_{v}=\frac{-\lambda \ln \left(I_{\lambda} / I_{\lambda, 0}\right)}{k_{1} L_{p a t h}}
$$

Dividing the volume fraction at a given time by the max volume fraction for a given experiment normalizes the volume fraction, and has the benefit of canceling the wavelength, $\lambda$, and the constant $k_{1}$.

$$
\frac{\ln \left(I_{\lambda} / I_{\lambda, 0}\right)}{\ln \left(I_{\lambda, \min } / I_{\lambda, 0}\right)}=\frac{f_{v}}{f_{v, \max }}
$$

For the base line case presented above, we can show volume fraction ratio, $f_{v} / f_{v, \max }$, variation with time (Figure 2.4). 


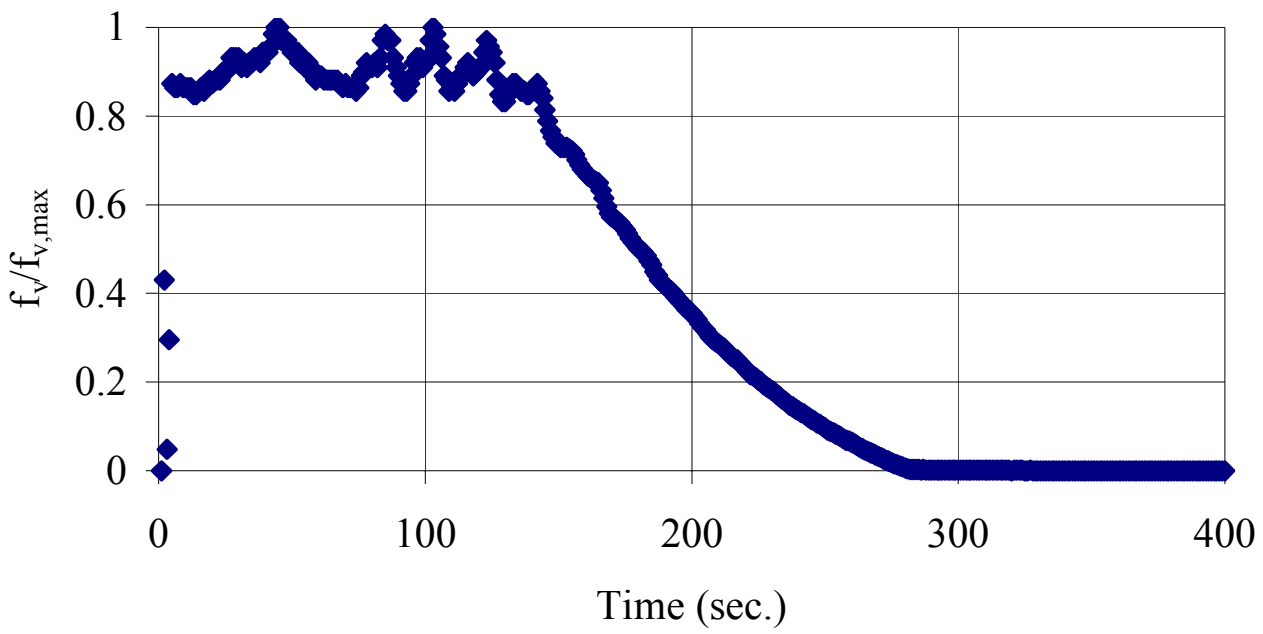

Figure 2.4. Example of volume fraction ratio data $f_{v} f_{v, \max }$ vs. time. 


\subsection{Experimental Conditions and Procedure}

In each experiment the reactor was filled with an aerosol. The experiment was run until the laser intensity seen by the photo detector approached its original intensity and stabilized. The aerosols were subjected to different field strengths by applying different voltages to the copper plates. The windows of the reactor were cleaned after each experiment. An experimental matrix is presented in Table 2.1 .

Table 2.1. Experimental matrix for different aerosols.

\begin{tabular}{|c|c|c|c|c|}
\hline $\begin{array}{c}\text { Aerosol } \\
\text { Type }\end{array}$ & $\begin{array}{c}\text { Voltage } \\
\text { Applied }\end{array}$ & $\begin{array}{c}\text { Observationally } \\
\text { Reproducible } \\
\text { Yes/No }\end{array}$ & $\begin{array}{c}\text { Laser } \\
\text { elevation } \\
\text { from ceiling } \\
\text { mm }\end{array}$ & $\begin{array}{c}\text { Number of } \\
\text { experiments } \\
\text { conducted }\end{array}$ \\
\hline Oil fog & $0 \mathrm{~V}$ & No & 11.91 & 27 \\
$1000 \mathrm{~V}$ & No & 11.91 & 6 \\
\hline Tissue paper & $0 \mathrm{~V}$ & No & 11.91 & 5 \\
smoke & $1000 \mathrm{~V}$ & No & 11.91 & 5 \\
\hline Cigarette & $0 \mathrm{~V}$ & No & 11.91 & 9 \\
smoke & $500 \mathrm{~V}$ & No & 11.91 & 5 \\
(Topps) & $1000 \mathrm{~V}$ & No & 11.91 & 5 \\
& $1500 \mathrm{~V}$ & No & 11.91 & 5 \\
\hline Cigarette & $0 \mathrm{~V}$ & Yes & 11.43 & 13 \\
smoke & & Yes & 7.62 & 8 \\
(Maverick) & \multirow{2}{*}{$500 \mathrm{~V}$} & Yes & 3.05 & 8 \\
& & Yes & 11.43 & 10 \\
& & Yes & 7.62 & 9 \\
& $1000 \mathrm{~V}$ & Yes & 3.05 & 8 \\
& Yes & 11.43 & 8 \\
& & Yes & 7.62 & 8 \\
& \multirow{2}{*}{$1500 \mathrm{~V}$} & Yes & 3.05 & 8 \\
& & Yes & 11.43 & 9 \\
& & Yes & 7.62 & 8 \\
& & Yes & 3.05 & 8 \\
\hline
\end{tabular}


Several different types of aerosols were used throughout the experiments. First an oil fog generator was used to supply the aerosol. A fog generator was attached to the inlet of the chamber. The fog generator was switched on and the chamber was filled by periodically partially capping the exit. The chamber was filled for fifteen seconds, and then the inlet and exit were capped. The computer was set to acquire scans at a rate of 10 scans per second. The experiments were run with 0 Volts and with 1000 Volts applied to the copper plates.

Next, the smoke from burning tissue paper was used. The computer was set to acquire scans at a rate of 1 scan per second. A quarter turn ball valve was installed at the inlet to control the flow into the reactor, and the exit was capped. The reactor was not sealed so the smoke leaked through the seams. This had the effect of filling up the reactor with a dense smoke. The smoke was generated by compressed air flowing through a copper tube filled with ignited tissue paper. The reactor was filled with smoke until the observed voltage dropped from its initial value to below 0.12 Volts. The valve was closed and the data acquisition software was initiated. The experiments were run with 0 Volts and with 1000 Volts applied to the copper plates.

After experiments were performed with oil fog and tissue paper, the experimental setup was moved to another optical table and placed under a vented hood as a safety precaution. The third source of smoke was a burning Topps brand cigarette. Topps cigarettes are hand rolled and do not have filters. A wire screen cylinder was constructed to hold the cigarette and keep the tobacco from migrating into the reactor. The smoke was generated by compressed air flowing at a constant rate through a small pipe containing the screen cylinder with a lit cigarette inside. The reactor was filled with smoke until the detector voltage dropped from its initial value to below 0.12 Volts. The experiments were run with $0,500,1000$, and 1500 Volts applied to the copper plates. 
The last source of smoke was a burning Maverick brand cigarette. Maverick cigarettes are factory rolled with a filter. A brass cylinder was machined to hold the cigarette such that all the smoke was forced through the filter. The smoke was generated by compressed air flowing through the brass cylinder containing a lit cigarette. The inlet valve was initially closed. The cigarette was ignited and the compressed air was attached to the brass cylinder. After a five second delay, the software was initiated and the inlet valve was opened. The valve was open for 25 seconds, and then closed. The experiments were run with $0,500,1000$, and 1500 Volts applied to the copper plates. These experiments were duplicated for three different laser beam heights to monitor the loading at different elevations within the reactor. The three positions of the laser were $11.43 \mathrm{~mm}, 7.62 \mathrm{~mm}$, and $3.05 \mathrm{~mm}$ from the upper copper plate. For all the previous experiments, the laser was positioned $11.91 \mathrm{~mm}$ from the upper plate. 


\section{CHAPTER 3. THEORY}

Theory for the various physical processes will be presented. First the basic electric field theory will be presented followed by discussions on EC and mechanisms for particle removal from a suspension.

\subsection{Electric Field Theory}

When a voltage is applied across a gap such as the one in the unit reactor, an electric field is setup within the gap (Figure 3.1).

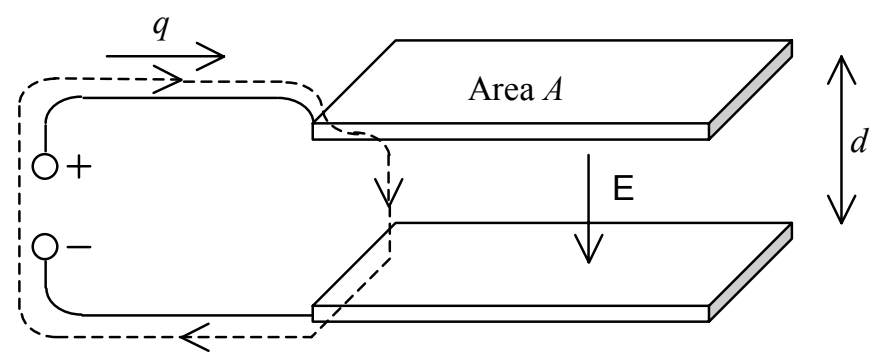

Figure 3.1. Electric field between parallel plates with plate spacing, $d$.

In the simplest sense, the electric field strength is the voltage potential divided by the gap spacing, d. More formally, the electric field can be specified by (Crowly, 1986),

$$
\oint \mathbf{E} \bullet d \mathbf{r}=\int_{\text {terminal }}^{\text {terminal }} \mathbf{E} \bullet d \mathbf{r}+\int_{\text {wires }} \mathbf{E} \bullet d \mathbf{r}+\int_{\text {gap }}^{\mathbf{E}} \bullet d \mathbf{r}=0
$$

Where $\mathbf{E}$ is the vector force experienced by a unit charge, or the electric field. The electric field inside good conductors is very small, so the integral inside the wires is negligible. The $\mathbf{E}$ field between the terminals can be described by the voltage across the terminals, as,

$$
V=-\int_{\text {terminal }}^{\text {terminal }} \mathbf{E} \bullet d \mathbf{r}
$$


This leaves,

$$
V=-\int_{\text {terminal }}^{\text {terminal }} \mathbf{E} \bullet d \mathbf{r}=\int_{g}^{\mathbf{g} a p} \mathbf{E} \bullet d \mathbf{r}
$$

Where,

$$
\int_{g a p}^{\mathbf{E}} \bullet d \mathbf{r}=E d
$$

Thus, in this example,

$$
V=E d
$$

For sufficiently large electric field strengths, breakdown may occur within the material. There are two limits when breakdown becomes possible, a voltage limit and an electric field limit. Generally, for breakdown both limits must be breached. For air, the breakdown electric field is known to be $3 \mathrm{MV} / \mathrm{m}$, and the breakdown voltage is 300 Volts (Crowley, 1986). The fact that a distance relates the electric field and the voltage implies that there is a characteristic distance for breakdown in air. Note that for parallel plate capacitors that $\mathrm{E}=\mathrm{V} / \mathrm{d}$. Thus, we find that $\mathrm{d}_{\mathrm{bd}}=0.1 \mu \mathrm{m}$. For capacitors with a gap distance less than $\mathrm{d}_{\mathrm{bd}}$, there can be very large field strengths, but breakdown will not occur until the voltage difference exceeds $300 \mathrm{~V}$. On the other side of the spectrum, for very large gaps, it is possible to have very large applied voltages, but breakdown will not occur until the field strength exceeds the critical value of $3 \mathrm{MV} / \mathrm{m}$. When these limits are reached and breakdown becomes possible corona and sparking become a problem. Ionization of air occurs when free electrons in the air move under the force of the electric field, and collide with other electrons in the molecules of the air breaking them loose and ionizing the molecules. These electrons in turn collide with other molecules, and so on. Breakdown actually occurs when the insulation properties of the material breakdown and the material acts like a conductor. If the air acts like a conductor the current can actually surge across the gap. This is known as sparking. A corona discharge is a partial breakdown that 
can occur near a conductor with sharp points, or a small radius of curvature. The application of electro-coalescence obviously requires that the field strength does not exceed the breakdown value for the fluid and for the particle. The presence of particles in the fluid may modify the properties of the electric field in a pure fluid system.

Observationally, various types of aerosols were tested in the experimental system. Of these, only cigarette smoke was tested over a wide range of conditions. However, it was noted early in the experimental design and testing stages that different aerosols responded differently to application of the voltage. It is not clear if the electrical properties of these aerosols modified their potential for coalescence. The literature does not appear to show any influence of the type of electrical properties of the particle on coalescence properties. Williams and Bailey (1983) note that when the permittivity of the particle is significantly larger than that of the fluid (continuous phase) then the inter-particle force depends only on the permittivity of the fluid and the size of the particle. Thus, based on the simplest theory associated with electro-coalescence, it appears that the most important properties that influence EC are the particle size, electric field strength, fluid permittivity and separation distance between particles.

Limiting case analysis of the effect of insertion of a material within a capacitor will be discussed. Take for example a parallel plate capacitor where the fluid between the plates is air. If a material with a higher dielectric constant than air, such as a solid or liquid insulator, is introduced, the electric field in the surrounding air is increased (Crowley, 1986 p. 20). Table 3.1 lists the dielectric constants of several materials. On the other end of the spectrum, if you add conducting material to a capacitor containing an insulator, the electric field in the insulator increases (Crowley, 1986 p. 130).

Table 3.1. Dielectric constants of several materials.

\begin{tabular}{|l|c|}
\hline Material & Dielectric Constant \\
\hline vacuum, and most gasses & 1 \\
\hline Teflon & 2.1 \\
\hline insulating oils & 2.2 \\
\hline Polystyrene & 2.5 \\
\hline Nylon & 3.4 \\
\hline
\end{tabular}




\begin{tabular}{|l|c|}
\hline Paper & 3.7 \\
\hline pyrex glass & 5.6 \\
\hline neoprene rubber & 6.7 \\
\hline Porcelain & 7 \\
\hline Water & 80 \\
\hline strontium titanate & 233 \\
\hline barium titanate & 1200 \\
\hline
\end{tabular}

\subsection{Theory of electro-coalescence}

Dipole electro-coalescence requires particles to grow through collisions under the action of an electric field. As a result of the particle growth, the sedimentation velocity of the particles increases dramatically. Note that the sedimentation velocity is quadratic in the particle diameter, and doubling particle diameter is a four-fold increase in sedimentation velocity. In the absence of sedimentation, the coalescence process will reduce the number concentration of particles but will not change the particle volume fraction. The rate of change of volume fraction is solely associated with removal of particles from the fluid. This may occur by deposition on the walls of the chamber and is most often driven by deposition to the floor of the chamber.

\subsection{Particle Dynamics}

The evolution of the number density of particles in the absence of sedimentation is given by the General Dynamic Equation (GDE). This equation is presented by Ezekoye and Wibowo (1999) as,

$$
\frac{d n_{k}}{d t}=\frac{1}{2} \sum_{i+j-k} \beta\left(v_{i}, v_{j}\right) n_{i} n_{j}-n_{k} \sum_{i=1} \beta\left(v_{i}, v_{k}\right) n_{i}
$$

The first term represents the rate of conversion of particles of size $i$ and $j$ into size $k$ such that the sum the volumes of the $i$ and $j$ particles sums to the volume of the $k$ particle. The second term represents the conversion of $k$ sized particles to larger 
particles through collisions with the rest of the distribution. The evaluation of $\beta$ for various types of collisional processes is essential to properly modeling and characterizing the aerosol dynamics. Typically, Brownian and gravitational coalescence/agglomeration processes are known to be quite important in modifying the number concentration. In general, the GDE depends on the collision rate of different sized particles, which can be formulated in terms of Beta or in terms of the relative velocity between particles.

$$
\beta n_{i} n_{j}=\pi r^{2} \underline{V}_{i j} n_{i} n_{j}=\pi r^{2}\left(V_{i}-V_{j}\right) n_{i} n_{j}
$$

The relative velocity, $\underline{V}_{i j}$, associated with electro-coalescence was shown by Zhang et al (1995) to be of the form,

$$
\underline{V}_{i j}=V_{i j}^{(0)}\left(-\underset{\text { gravity }}{L \cos \theta \underline{e}_{r}}+M \sin \theta \underline{e}_{\theta}\right)-\frac{D_{i j}^{(0)}}{k T}\left(G F_{E, j i}^{r} \underline{e}_{r}-H F_{E, i j}^{\theta} \underline{e}_{\theta}\right)-\frac{D_{i j}^{(0)}}{k T} G F_{V, i j} \underline{e}_{r}
$$

A discussion of various coefficients can be found in the original reference.

As two drops approach one another, the strength of the electric field in the region between their closest points can attain values many times that of the imposed electric field, as a result of the mutual interactions between the induced charges on the drop surfaces (Latham \& Roxburgh, 1966). A variable separation method for electrohydrostatic problems of two conducting spheres has been developed by Davis (1964) to solve the Laplace equation that governs the electric potential field outside the droplets. Variations in the disturbed electric field and the charges induced on the surfaces of two different sized droplets were determined under an arbitrary electric field and different separation of two drops. Electric field induced forces on two conducting droplets with radii of $a_{1}$ and $a_{2}$ can be decomposed into two components, along and normal to the line of centers of the droplets, $F_{E, 12}^{r}$ and $F_{E, 12}^{\theta}$, respectively, 


$$
F_{E, 12}^{r}=4 \pi \varepsilon a_{2}^{2} E_{0}^{2}\left(F_{1} \cos ^{2} \psi+F_{2} \sin ^{2} \psi\right)
$$

Where $E_{0}$ is the magnitude of the external electric field, $\psi$ is the angle between the electric field and the droplet center line, and $\varepsilon$ is the permittivity of the surrounding fluid. The force coefficients, $F_{i}(i=1,2, \ldots, 10)$, are complicated series of expressions that depend on the relative geometry of the two droplets (their size ratio and separation). The solutions for the force coefficients, $F_{i}$, diverge when the distance between two drops tends to zero, indicating a dramatic increase in the electric field induced forces between the two droplets, which has been shown experimentally by Latham \& Roxburgh (1966). Electrohydrodynamic interactions between two leaky dielectric spherical drops have been examined by Erker and Baygents (1996) using the bispherical coordinates technique to solve the Laplace equation for the electric potential and the Stokes equations for the velocity field. Their results showed that the electrical interaction induced between two drops by an imposed electric field is always attractive when the drops are in near contact.

$$
F_{E, 12}^{\theta}=4 \pi \varepsilon a_{2}^{2} E_{0}^{2} F_{3} \sin 2 \psi
$$

A simplified form of equation 3.10 is often used to analyze particle forces. Williams and Baily (1986) present one form:

$$
F=24 \pi \varepsilon\left(\frac{a}{l}\right)^{4} a^{2} E^{2}
$$

Where $l$ is the particle spacing (see Figure 3.2). This assumes that the permittivity of the continuous phase is much lower than that of the dispersed phase. Electrohydrodynamic problems of two charged, dielectric spheres have been recently studied by Keh \& Chen (1989a, b) for the electrophoretic motion along and normal to the drop joint center line and by Loewenberg \& Davis (1995) for near contact axisymmetric electrophoretic motion. Their results show that near 
contact electrophoresis motion is at least three times greater than buoyancy driven motion. Depending on their free charge amount (or the zeta potential) and polarity, two drops may respond differently to the electrohydrodynamic interactions. When the drops are near contact, the interaction between spheres can be very intense and one sphere may even change its direction of motion. Figure 3.2 is duplicated from Zhang et al (1995), and shows the interparticle reactions in an electric field. Figure 3.2c is of particular interest and suggests that when the electric field is perpendicular to line joining centers of the two drops, the interparticle forces are repulsive. 

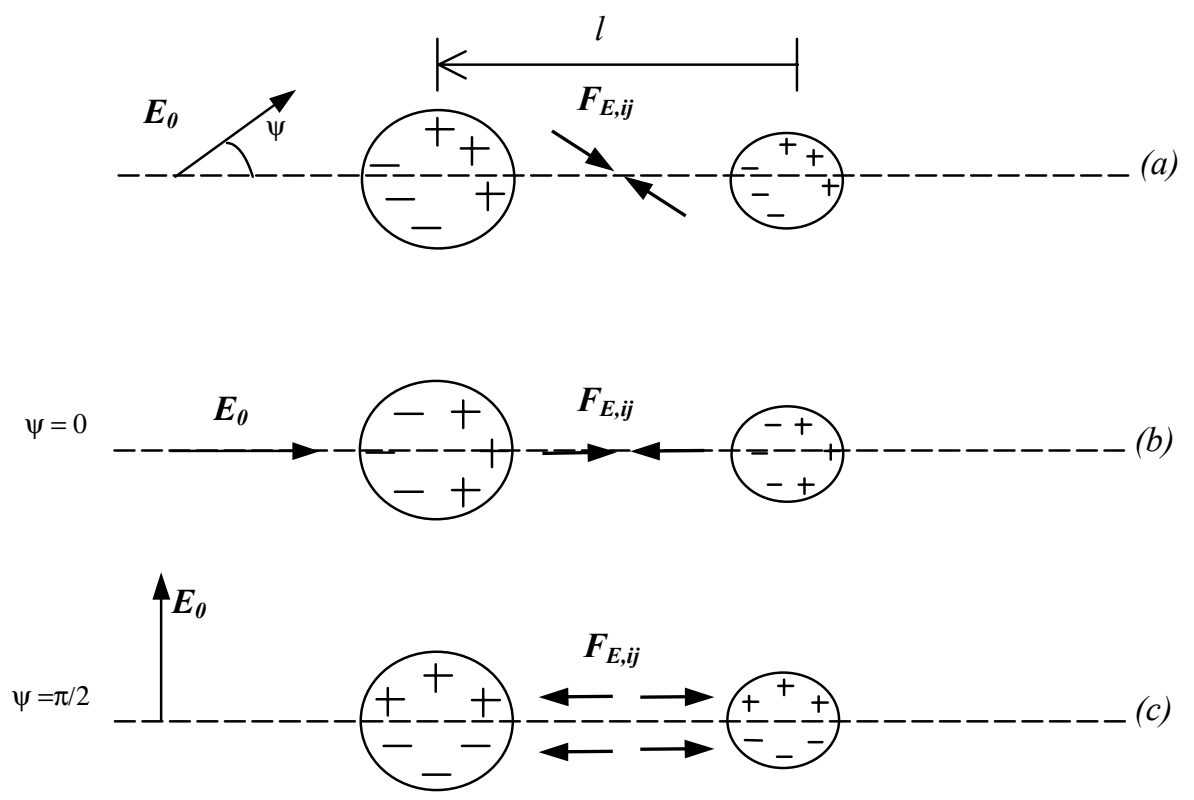

Figure 3.2. Surface charge densities and force on two drops induced by an externally applied electric field for different values of angle $\psi$ between the electric field and the line joining centers of the two drops (Zhang et. al.).

Williams \& Bailey $(1983,1986)$ make theoretical predictions of the effects of electric fields on drop coalescence, by using an approximate solution for electric field induced forces, which is not valid when two drops are close to one another. Moreover, they did not account for viscous interactions in their studies, which result in a considerable overestimation of the coalescence rate (cf. Zhang and Davis, 1991). To remedy this, Zhang et al. (1995) used a trajectory analysis method to predict the pairwise coalescence rate of spherical, conducting drops bearing zero net charge. By completely accounting for viscous and electrostatic interactions, their results show that the coalescence rate can be increased significantly by applying an electric field, particularly when the field acts in a direction perpendicular to gravity. 
Waterman (1965) presents a table showing the average force between drops of fixed diameters and spacing (Table 3.2). Recognizing that the EC force (Dipole) is a short-range force, it is not surprising to see that the interparticle force falls off quite sharply as the emulsion loading is decreased.

Table 3.2. Relative dipole coalescing forces vs. approximate emulsion content, cubic packing assumed.

\begin{tabular}{|c|c|l|}
\hline $\begin{array}{l}\text { Approx } \\
\text { emulsion } \%\end{array}$ & $\begin{array}{l}\text { Ratio of distance between drop } \\
\text { centers and drop diameters }(\mathrm{d} / 2 \mathrm{a})\end{array}$ & $\begin{array}{l}\text { Relative force } \\
\text { due to }(\mathrm{a} / \mathrm{d})^{4}\end{array}$ \\
\hline 52 & 1 & touching \\
\hline 7 & 2 & $\mathrm{~F}$ \\
\hline 2 & 3 & $\mathrm{~F} / 5$ \\
\hline 1 & 4 & $\mathrm{~F} / 16$ \\
\hline 0.1 & 8 & $\mathrm{~F} / 256$ \\
\hline
\end{tabular}

The assumption is that the emulsion is a spherical close cube packed system. This table, duplicated from Waterman (1965), suggests that an additional field is required to bring the particles together over the long length scales.

\subsection{Sedimentation}

The GDE is easily modified to take into account removal mechanisms such as plating on the walls and sedimentation to the floor of the reactor. The additional term to the GDE is the final term on the right hand side.

$$
\frac{d n_{k}}{d t}=\frac{1}{2} \sum_{i+j-k} \beta\left(v_{i}, v_{j}\right) n_{i} n_{j}-n_{k} \sum_{i=1} \beta\left(v_{i}, v_{k}\right) n_{i}-n_{k} R\left(v_{k}\right)
$$

The removal of the suspended particles from the gaseous medium in the enclosure occurs due to diffusion (Brownian), gravitational, thermophoretic, and diffusiophoretic depositions to the internal surfaces of the enclosure. It may also result from the leakage of the enclosure. When the temperature gradient and the 
water vapor gradient near the floor, the walls and the ceiling are small, the thermophoretic and diffusiophoretic depositions are negligible.

Brownian diffusion deposition (plating) is the removal of particles resulting from the diffusion of the suspended particles to the floor, the walls and the ceiling. The removal rates of particles due to Brownian diffusion, is given by,

$$
R_{B}\left(v_{k}\right)=\frac{k T A_{W}}{6 \pi \mu V \chi \Delta}\left[\frac{\alpha^{1 / 3}}{r_{k}}+\frac{C \lambda \alpha^{2 / 3}}{r_{k}^{2}}\right]
$$

Where $k$ is the Boltzmann constant, $T$ is the absolute temperature, $A_{W}$ is the area of the internal surfaces of the enclosure, $V$ is the volume of the enclosure, $\chi$ is the dynamic shape factor, $\Delta$ is the diffusion boundary layer thickness, and $r_{k}$ is the radius of the particles with the volume $v_{k}$. This expression shows that this process is effective in removing small particles.

Gravitational sedimentation (settling) is the sedimentation of the particles onto any available floor or horizontal surface. The sedimentation rate due to this process is given by,

$$
R_{G}\left(v_{k}\right)=\frac{2 g \rho A_{F}}{9 \mu V \chi}\left[\alpha^{1 / 3} r_{k}^{2}+C \lambda \alpha^{2 / 3} r_{k}\right]
$$

Where $g$ is the gravitational constant and $A_{F}$ is the area of the available floor or horizontal surface. A simplification of equation 3.14 is the approximation,

$$
R_{G}\left(v_{k}\right) \approx \frac{U_{s} A_{F}}{V}
$$

It is clear that the larger the particle, the more effective the gravitational sedimentation process. 


\section{CHAPTER 4. RESULTS}

The results for the EC experiments will be discussed. First the raw data results will be discussed followed by more meaningful results for loading and size distribution.

\subsection{Preliminary Results}

Raw experimental data was converted into a volume fraction ratio by the method presented in chapter 2. This ratio was plotted vs. time for each experiment. The plots for the oil fog experiments are shown in Figure 4.1 and Figure 4.2. Figure 4.1 includes data for 27 experiments where no voltage was applied to the copper plates. Figure 4.2 includes data for 6 experiments where the applied voltage was 1000 Volts. The plots were very random and the actual trends were not obvious.

Based on the experimental procedure, the data for the tissue paper experiments were plotted, starting at time $t\left(V_{0}\right)$, where,

$$
t\left(V_{0}\right)=t(0.20 \mathrm{~V})
$$

The data for these experiments are plotted in Figure 4.3 and Figure 4.4. Figure 4.3 includes data from 5 experiments with no applied voltage. Figure 4.4 includes data from 5 experiments where the applied voltage was 1000 Volts. Examining these figures, it appears that it may actually have taken longer for the particles to settle when an electric field was applied.

The data for the Topps cigarette experiments are plotted in Figure 4.5, and Figure 4.6. Figure 4.5 presents 9 experiments with no applied voltage. Figure 4.6 presents 5 experiments where the applied voltage was 1000 Volts. Experiments with applied voltages of 500 and 1500 Volts were also conducted, but are not discussed here. For Figure 4.5 and Figure 4.6, the data were still scattered, but it 
was clearly apparent that the applied voltage was causing the particles to settle out at a faster rate than when no voltage was applied.

The data for the Maverick cigarette experiments is plotted in Figure 4.7 and Figure 4.8. Figure 4.7 presents data for 13 experiments with no voltage applied. Figure 4.8 presents data for 8 experiments where 1000 Volts was applied. Many more experiments were conducted using Maverick cigarettes. These experiments are discussed later in this chapter. In Figure 4.7 and Figure 4.8 the data was much more reproducible, and the effects of the applied voltage were very evident. The loading within the reactor decreased at a much faster rate when 1000 Volts was applied. 


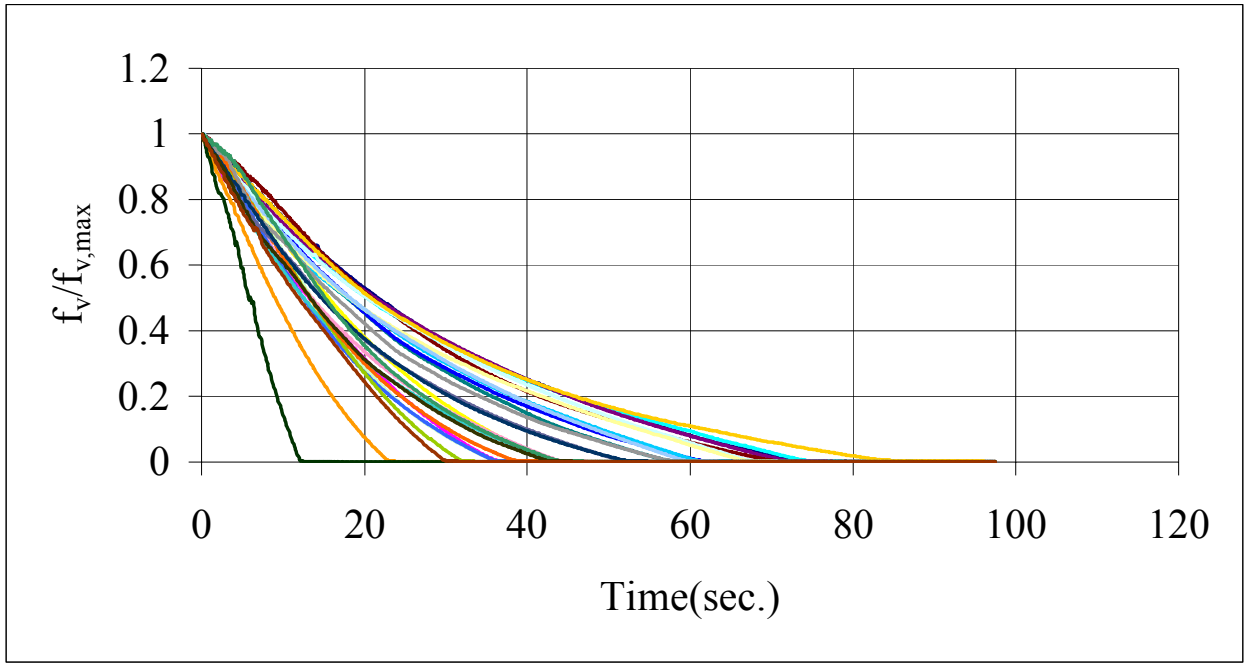

Figure 4.1. Raw data for oil fog experiments with no applied voltage.

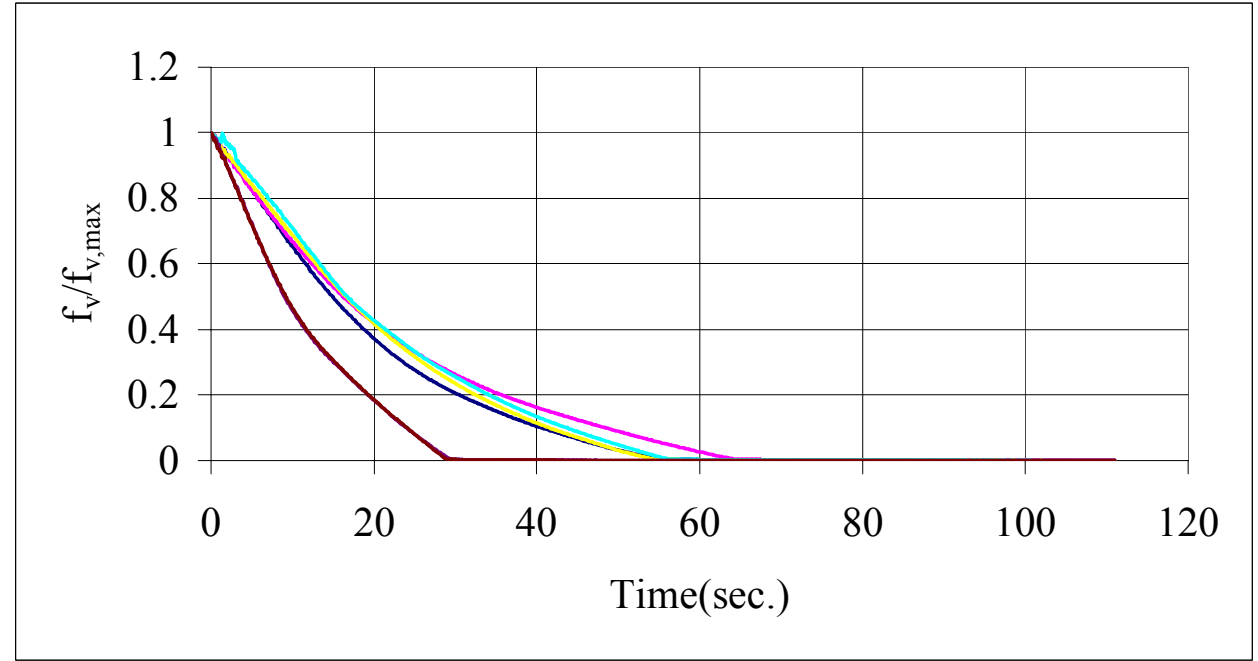

Figure 4.2. Raw data for oil fog experiments with an applied voltage of 1000 Volts. 


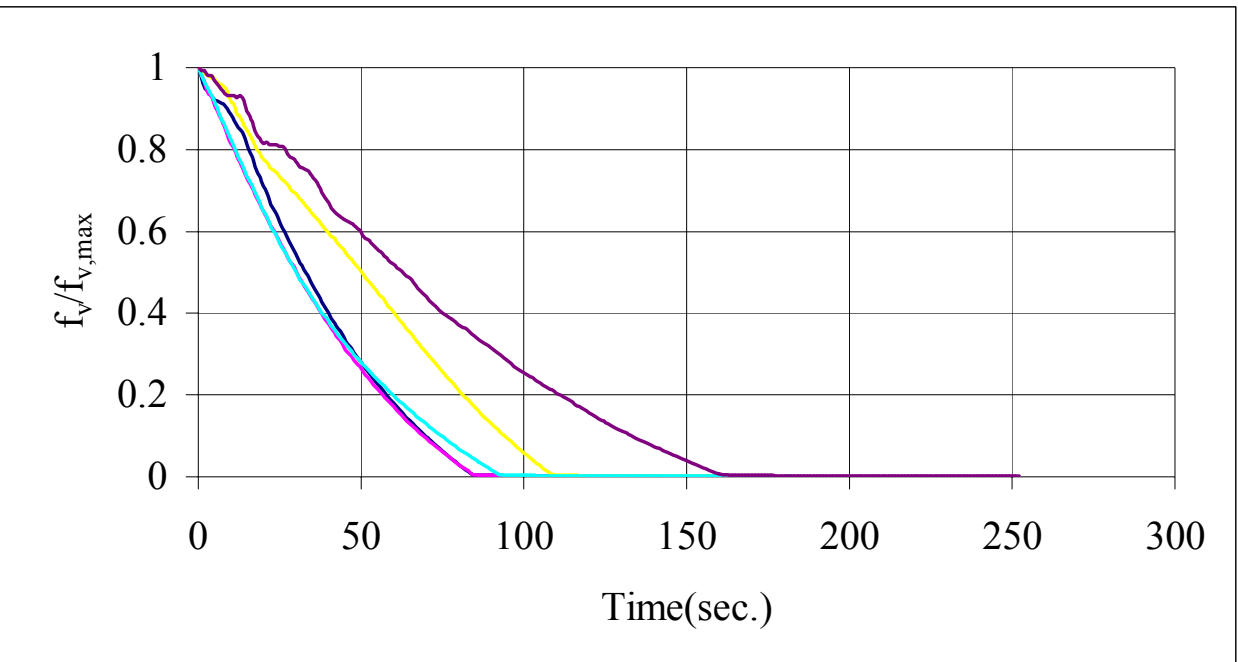

Figure 4.3. Raw data for tissue paper experiments with no applied voltage.

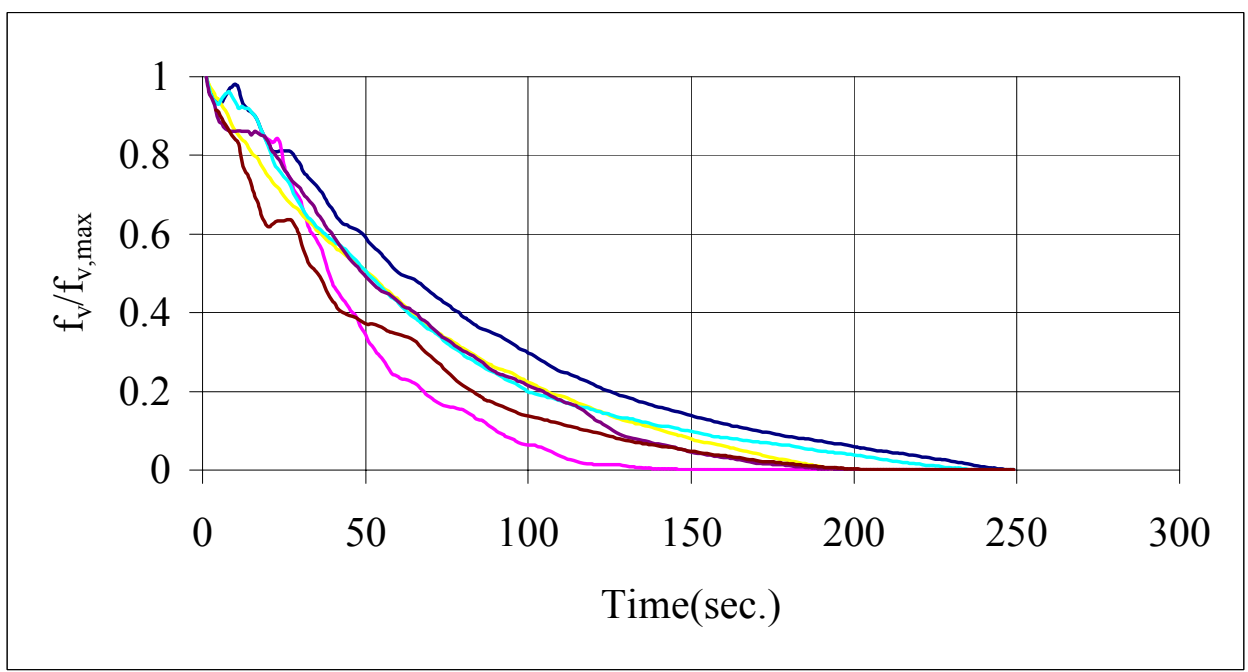

Figure 4.4. Raw data for tissue paper experiments with an applied voltage of 1000 Volts. 


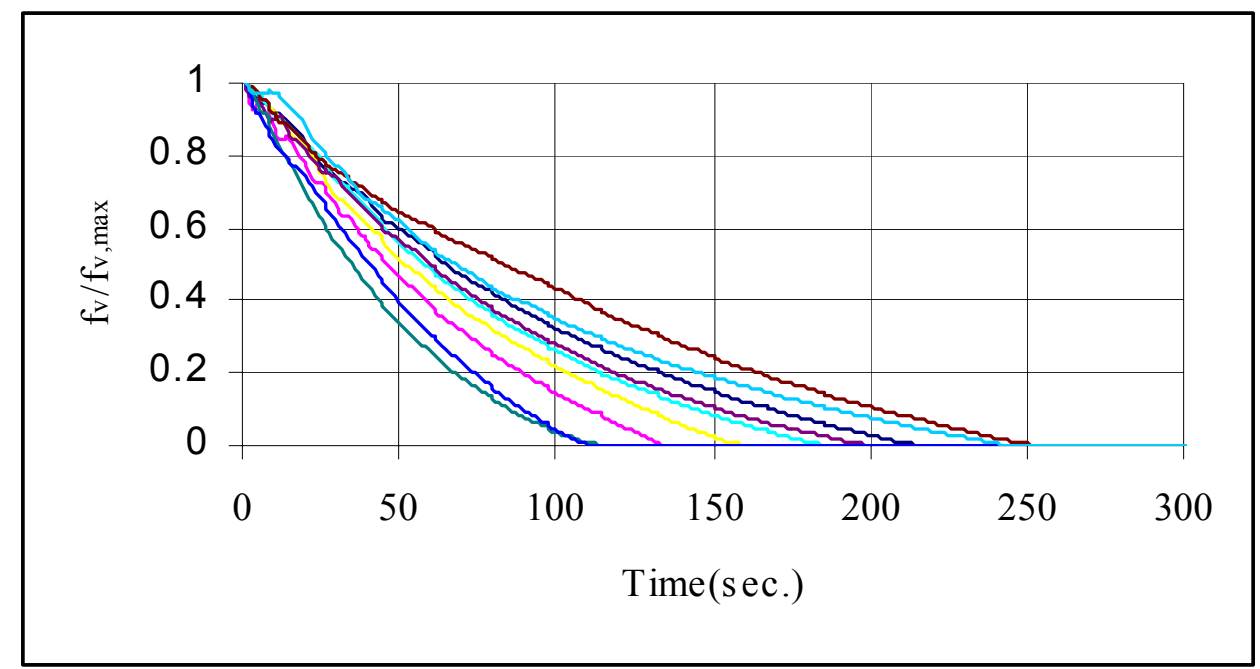

Figure 4.5. Raw data for Topps cigarette experiments with no applied voltage.

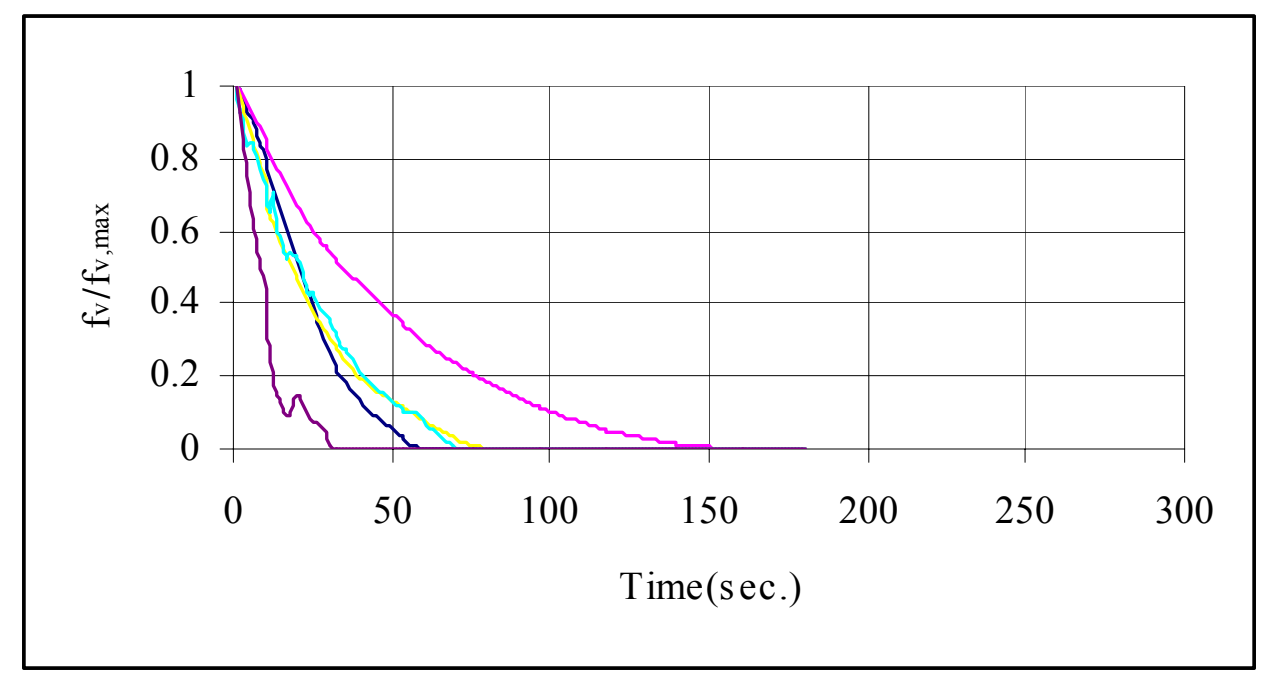

Figure 4.6. Raw data for Topps cigarette experiments with an applied voltage of 1000 Volts. 


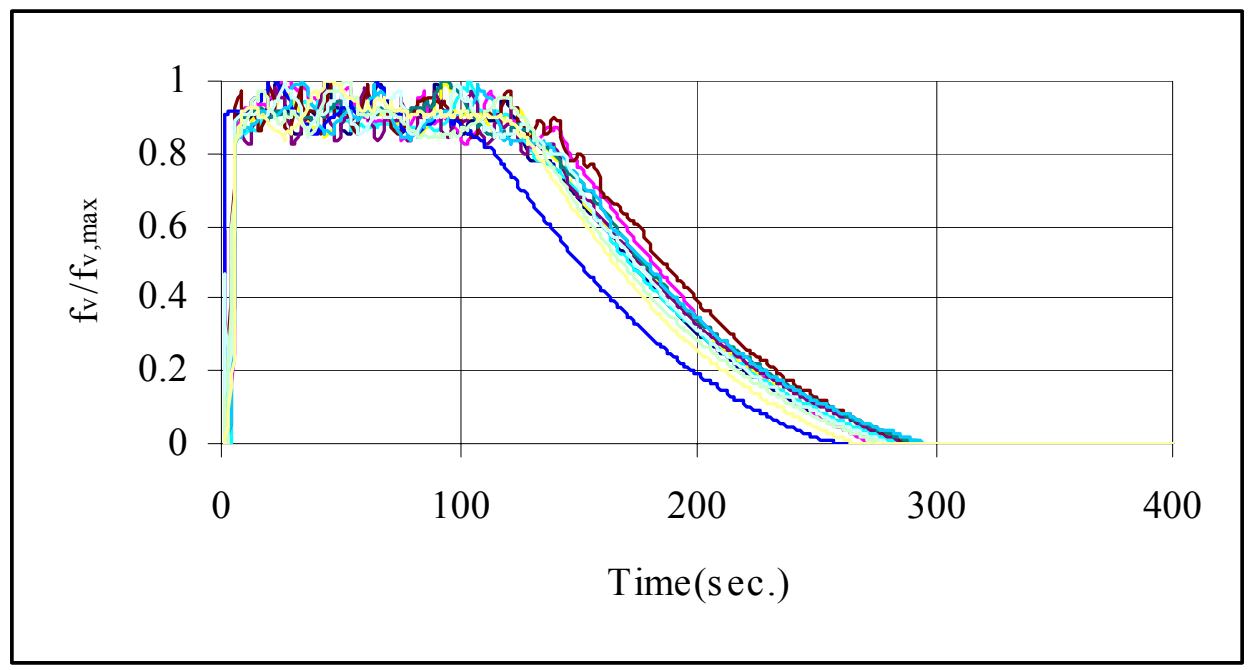

Figure 4.7. Raw data for Maverick cigarette experiments with no applied voltage.

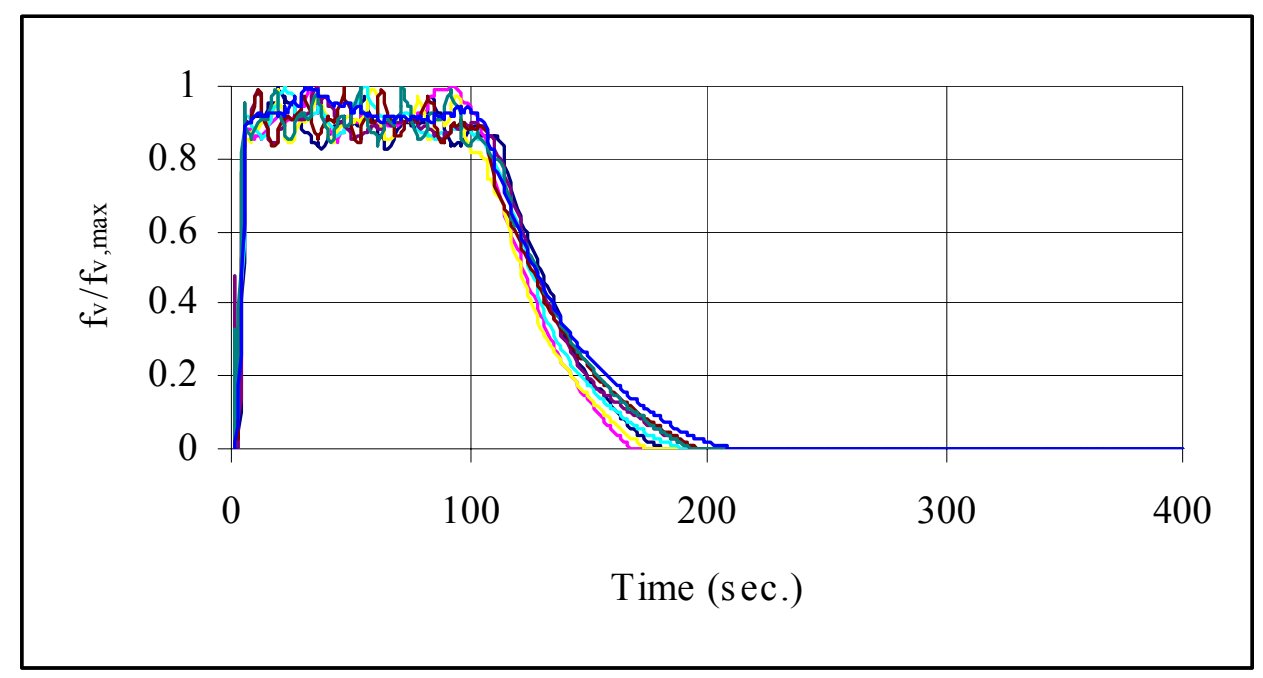

Figure 4.8. Raw data for Maverick Cigarette experiments with an applied voltage of 1000 Volts. 


\subsection{Mean Values}

In order to present the data in a conclusive manner an acceptable average must be calculated. The average volume fraction ratio, $f_{v} / f_{v}$, max , values for a representative set of experiments were calculated using two methods. These two methods were compared to a more rigorous procedure. This procedure, procedure 1 , is presented first. For each experiment in the representative set, the raw data were used to calculate the volume fraction ratio, $f_{v} / f_{v, \max }$, as a function of time. Two values were recorded from each experiment. These values are the times that correspond to $f_{v} / f_{v, \max }$ values of 0.50 and 0.05 . These times were averaged over the representative set. These two average times were used to evaluate the two methods presented next. One of these methods, procedure 2, involved taking the raw voltage data for each experiment, and averaging the voltages at each time interval. This average data set was used to calculate an average $f_{v} f_{v, \max }$ data set. The two values mentioned in the first procedure were recorded from this average $f_{v} f_{v, \max }$ data set. The other method, procedure 3 , is very similar to procedure 1 . For each experiment in the representative set, the raw data was used to calculate, $f_{v} f_{v, \text { max }}$, as a function of time. At each time interval, the $f_{v} / f_{v, \text { max }}$ value for each experiment was averaged. This produced an average set of $f_{v} / f_{v, \text { max }}$ as a function of time. This set and the average $f_{v} / f_{v, \max }$ data set from procedure 2 are plotted in Figure 4.9. Once again, the times when $f_{\vee} / f_{v, \max }$ equals 0.50 and 0.05 were recorded. Table 4.1 presents the times recorded for each procedure. Figure 4.9 shows that both procedure 2 and procedure 3 produced acceptable average plots. The only visible differences were within the first 130 seconds, where saturation occurred. Table 4.1 shows that values for procedure 3 are slightly closer to those for procedure 1 than those for procedure 2. Procedure 3 was used to calculate the average values presented next. 


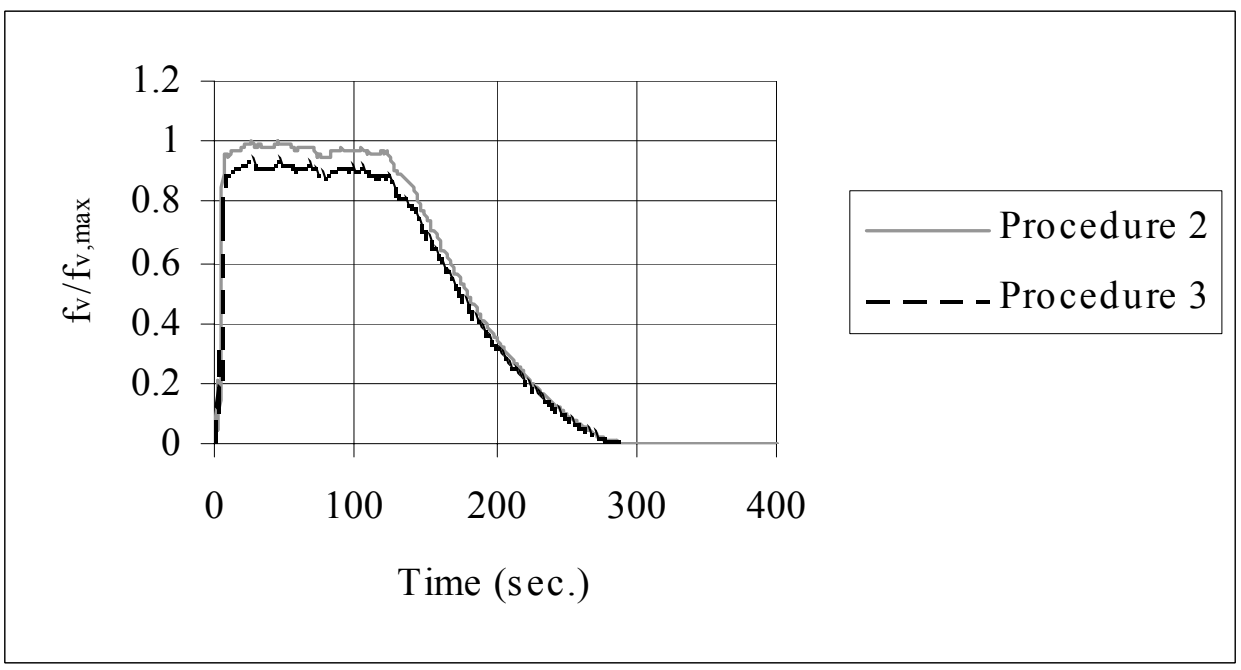

Figure 4.9. Mean $f_{v} / f_{v, \max }$ vs. time plots using procedures 2 and 3.

Table 4.1. Comparison of procedures 2 and 3 to procedure 1.

\begin{tabular}{|c|c|c|}
\hline Procedure & time where $f_{v} / f_{v, \max }=0.50$ & time where $f_{v} / f_{v, \max }=0.05$ \\
\hline 1 & $173.9 \mathrm{sec}$. & $263.6 \mathrm{sec}$. \\
\hline 2 & $180 \mathrm{sec}$. & $265 \mathrm{sec}$. \\
\hline 3 & $174 \mathrm{sec}$. & $263 \mathrm{sec}$. \\
\hline
\end{tabular}

The sample standard deviation, $S_{x}$, was used to characterize the error of the average plots presented next, and is given by,

$$
S_{x}=\sqrt{\frac{\left(\sum_{i=1}^{n} x_{i}^{2}\right)-n \bar{x}^{2}}{n-1}}
$$

And,

$$
\bar{x}=\sum_{i=1}^{n} \frac{x_{i}}{n}=\frac{x_{1}+x_{2}+\ldots+x_{n}}{n}
$$

Where $x$ is the magnitude of a particular measurement, and $\bar{x}$ is the sample mean. 
The standard deviation for a given set of experiments is represented in the form of error bars on the average $f_{v} / f_{v, \max }$ plot.

The average $f_{v} / f_{v, \max }$ plots, with standard deviation error bars for the oil fog experiments are presented in Figure 4.10 and Figure 4.11. Figure 4.10 presents the average for 27 experiments where no voltage is applied. Figure 4.11 presents the average for 6 experiments where 1000 Volts is applied. The electric field effect was very minimal. The standard deviation for these experiments was quite large, indicating poor repeatability conditions. The lack of repeatability of these experiments was attributed to a poor procedure for filling the reactor, and inconsistency in the rate that the fog generator produced fog. As repeatability is a very important issue for experimental based research, a different procedure for introducing particles into the reactor was necessary. The procedure for the oil fog experiments involved filling the reactor with oil fog for a specific amount of time. During the first half of the experiment the signal was saturated and nothing could be concluded from this part of the experiment. This was corrected by filling the chamber until saturation was achieved. In an attempt to improve the experimental procedure, the flow into the chamber was controlled by the addition of a valve and a rotometer (details listed in experimental procedure), and a new method of aerosol generation was used.

The average $f_{v} / f_{v, \max }$ plots, with standard deviation error bars, for the tissue paper experiments are presented in Figure 4.12 and Figure 4.13. Figure 4.12 presents the average for 5 experiments where no voltage is applied. Figure 4.13 presents the average for 5 experiments where 1000 Volts is applied. The standard deviation in these experiments shows that the repeatability improved, but was still not acceptable. Comparing Figure 4.12 to Figure 4.13, it was apparent that the electric field may have actually caused the particles to settle out slower than when no electric field was present. This was not the effect that we expected to see. 
This may have been due to the electrical properties of the tissue paper. To examine EC effects of an electric field on an aerosol, a different source of particles was used for the next set of experiments.

The average $f_{v} / f_{v, \max }$ plots, with standard deviation error bars, for the Topps cigarette experiments are presented in Figure 4.14 and Figure 4.15. Figure 4.14 presents the average for 9 experiments with no applied voltage. Figure 4.15 presents the average for 5 experiments where 1000 Volts was applied. Experiments with applied voltages of 500 and 1500 Volts were also conducted, but not presented due to the poor repeatability of these experiments. The standard deviation in these experiments was fairly large, indicating poor repeatability. The poor repeatability was attributed to three factors. One factor may have been due to the procedure used to fill the reactor with smoke. The procedure for the Topps cigarette experiments involved filling the chamber until the measured voltage/Intensity fell below a certain level. This process did not ensure that the same particle loading was achieved each time the experiment was conducted. This was corrected by using a very strict filling procedure, including a set duration of reactor filling. Another factor may have been due to the fact that Topps cigarettes are hand rolled, and are may not be packed with the same density each time (human error). This was corrected by using a cigarette that was factory rolled. The last factor was attributed to the fact that the Topps cigarettes did not contain a filter, and it may have been possible for small tobacco pieces to migrate through the screen chamber and into the reactor. This was corrected by using a cigarette with a filter, and forcing the smoke to go through this filter before entering the reactor. To ensure consistency, one cigarette was used per experiment.

A preliminary set of experiments (not shown) was conducted using Maverick cigarettes. When experiments with an applied voltage were conducted 
the experiments became very unrepeatable. The apparent solution was to delay applying the electric field for a specific amount of time. The new procedure for applying the electric field was to wait 50 seconds after filling the chamber, before applying a voltage. This had the effect of making the applied voltage experiments repeatable. And, the effect of the electric field on the settling rate of the particles was still very evident. The average $f_{v} / f_{v, \max }$ plots, with standard deviation error bars, for the Maverick cigarette experiments are presented in Figure 4.16 and Figure 4.17. Figure 4.16 presents the average for 13 experiments where no voltage was applied. Figure 4.17 presents the average for 8 experiments where 1000 Volts was applied. The standard deviation in these experiments was fairly small and these experiments were considered repeatable. The presence of an electric field seemed to increase the settling rate of the particles. This was attributed to EC. Since this was our best set of experiments, more experiments were conducted using the same procedure. This was the most widely experimented case. Experiments were conducted with applied voltages of 0, 500, 1000 , and 1500 Volts. These experiments were repeated for three different vertical locations of the laser (details in Table 2.1). 


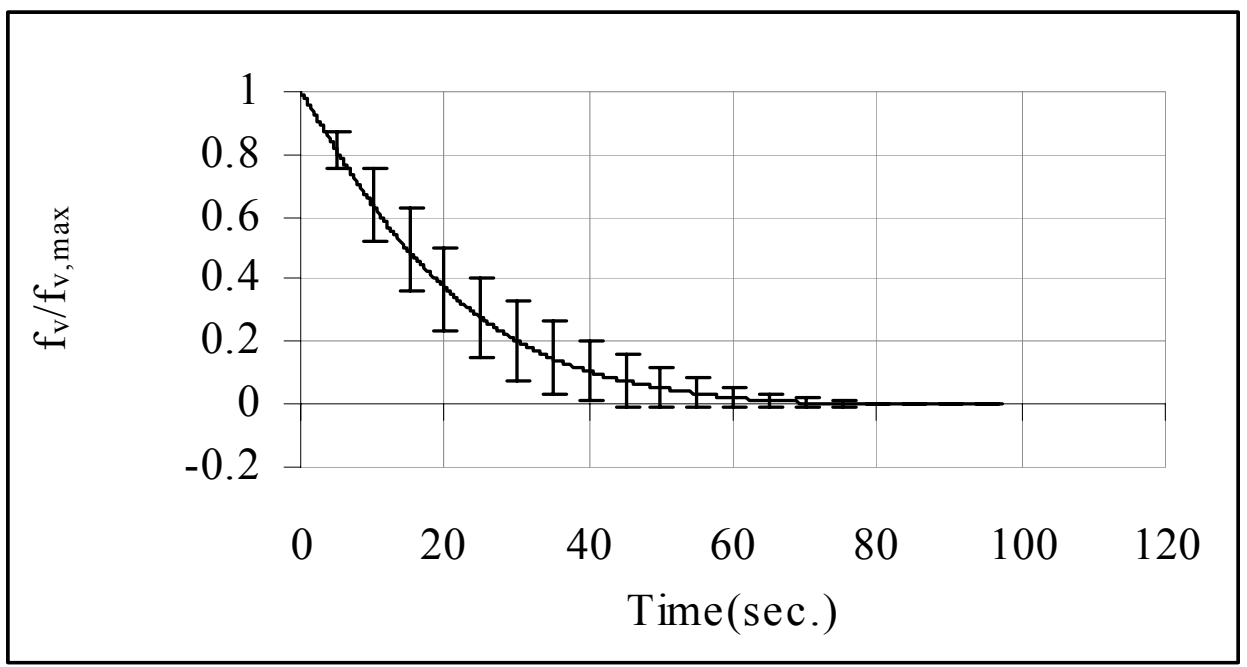

Figure 4.10. Average $f_{v} / f_{v, \max }$ vs. time plot for oil fog experiments with no applied voltage.

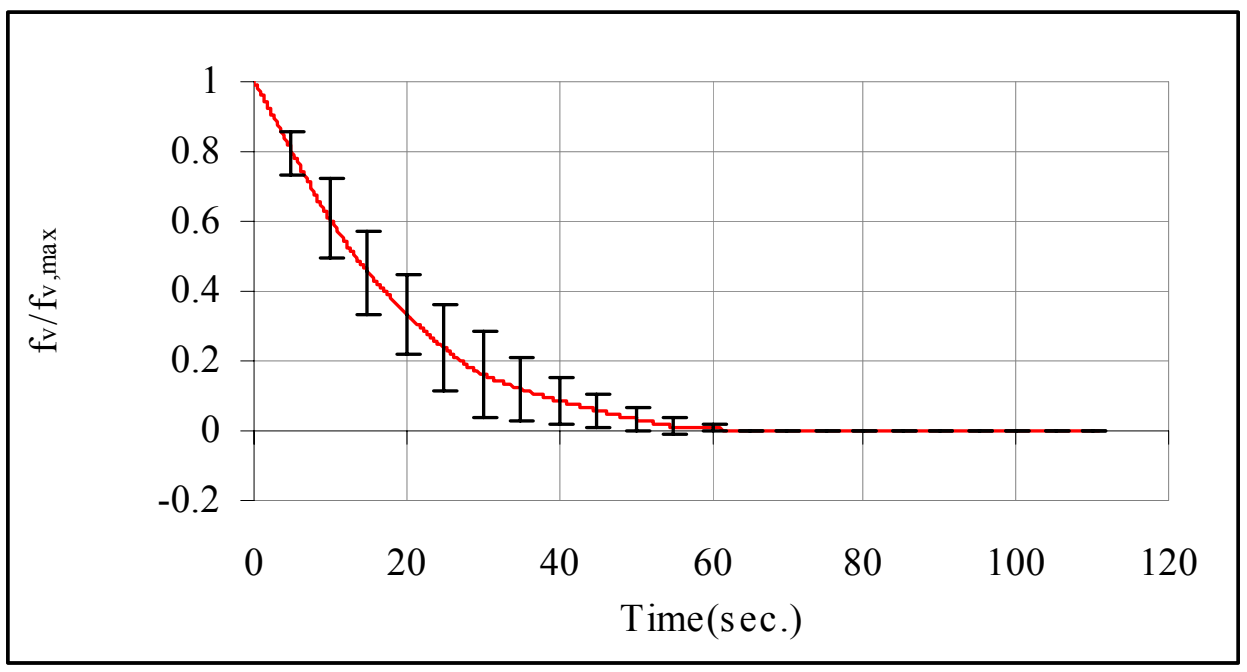

Figure 4.11. Average $f_{\sqrt{ }} / f_{v, \max }$ vs. time plot for oil fog experiments with an applied voltage of 1000 Volts. 


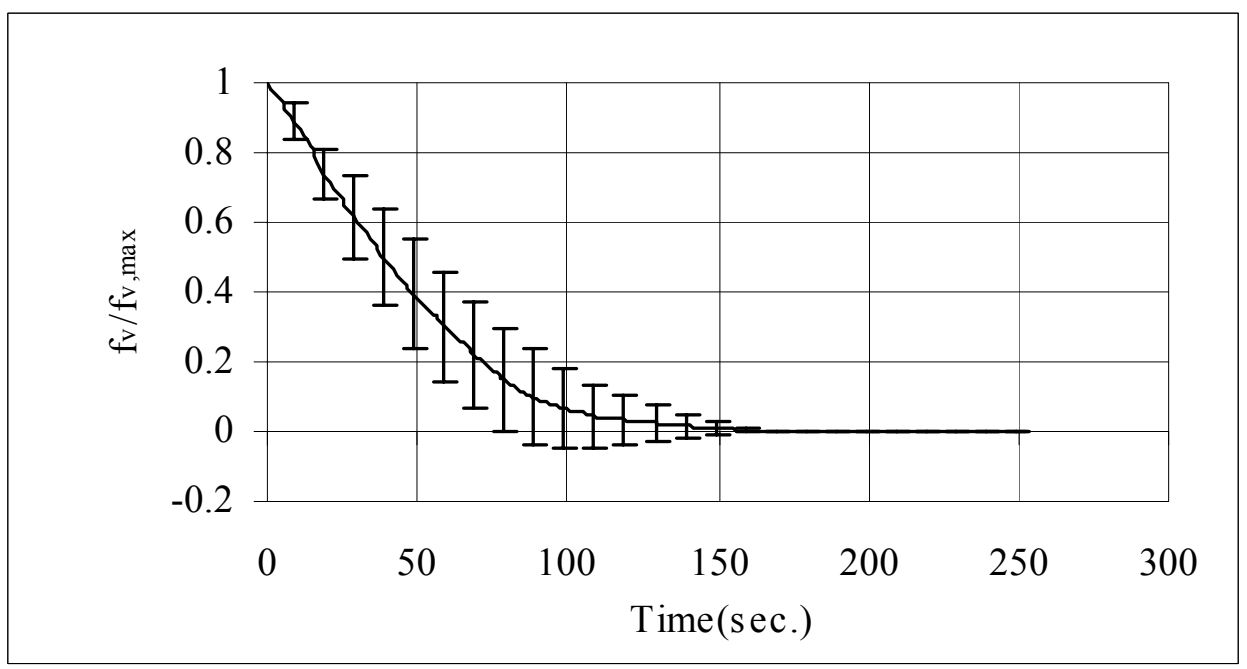

Figure 4.12. Average $f_{v} / f_{v, \max }$ vs. time plot for tissue paper experiments with no applied voltage.

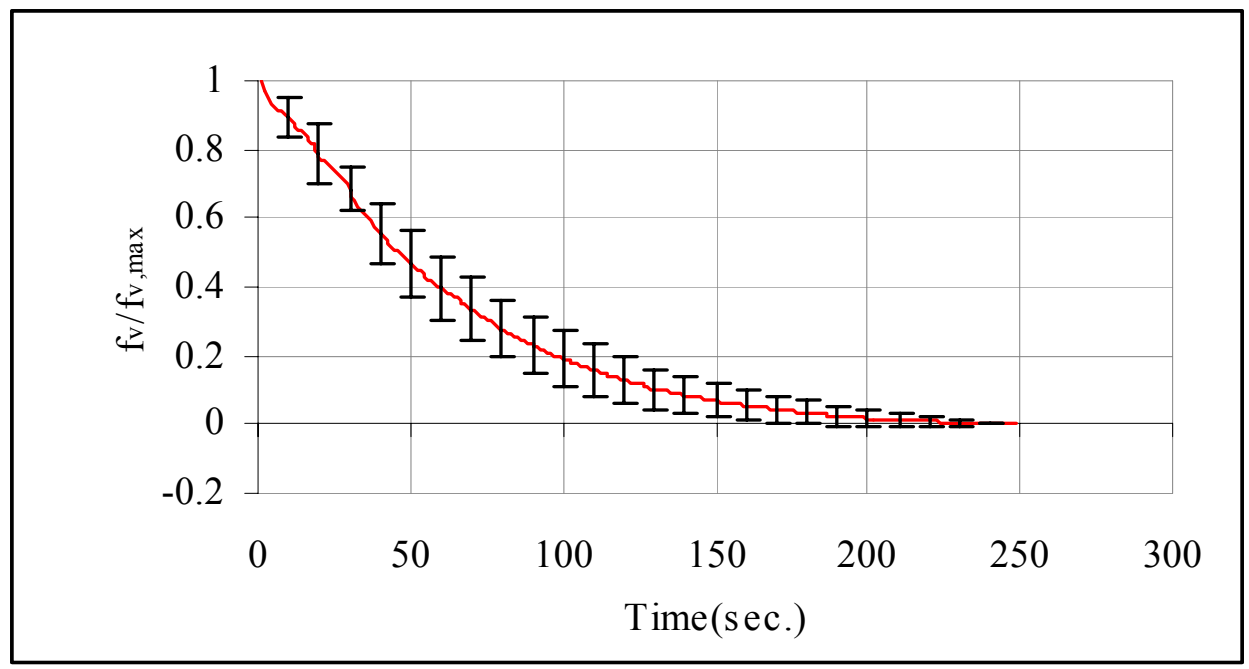

Figure 4.13. Average $f_{\sqrt{ }} / f_{v, \max }$ vs. time plot for tissue paper experiments with an applied voltage of 1000 Volts. 


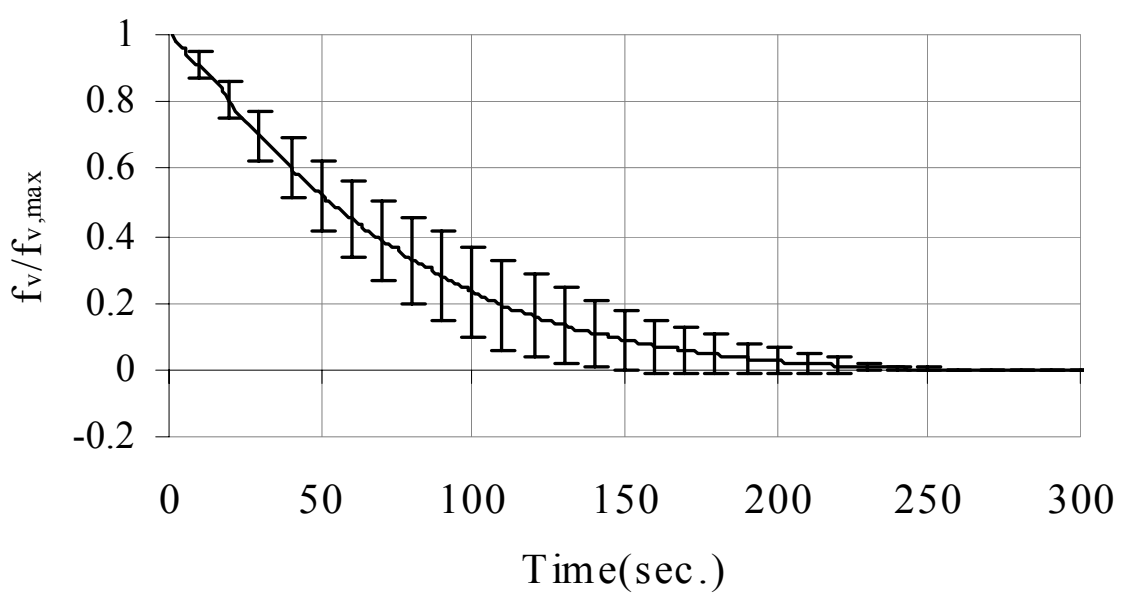

Figure 4.14. Average $f_{v} / f_{v, \max }$ vs. time plot for Topps cigarette experiments with no applied voltage.

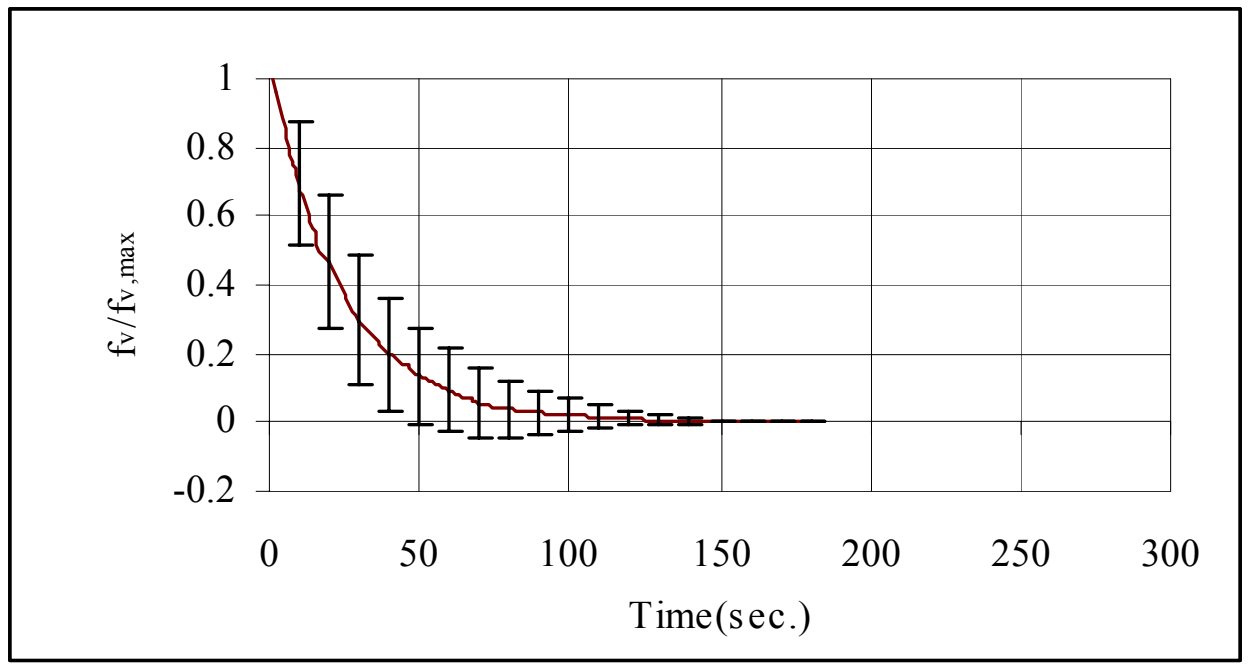

Figure 4.15. Average $f_{v} f_{v, \max }$ vs. time plot for Topps cigarette experiments with an applied voltage of 1000 Volts. 


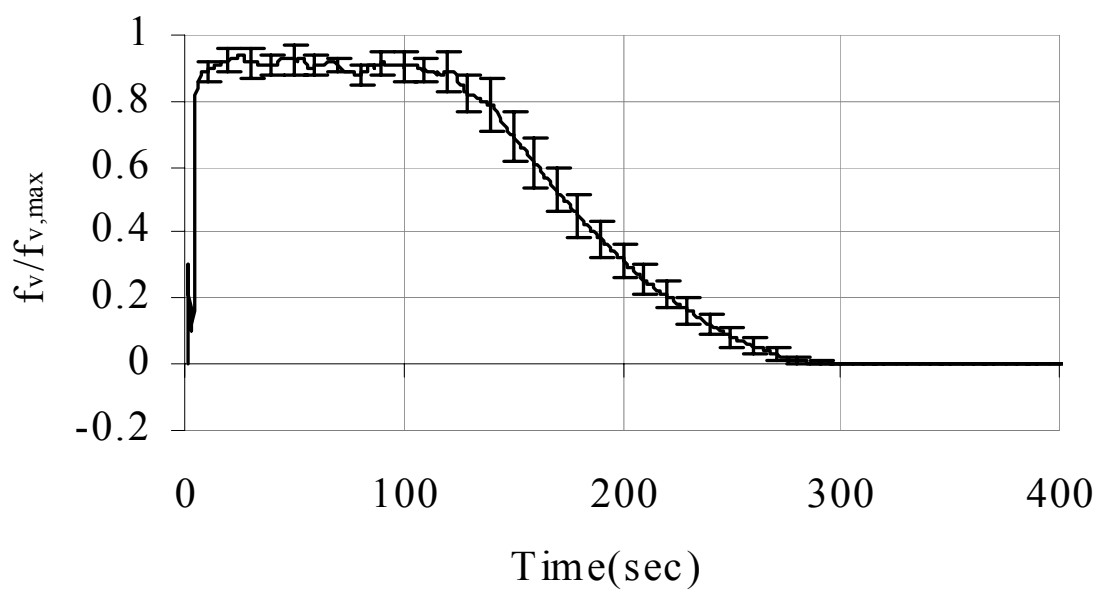

Figure 4.16. Average $f_{v} / f_{v, \max }$ vs. time plot for Maverick cigarette experiments with no applied voltage.

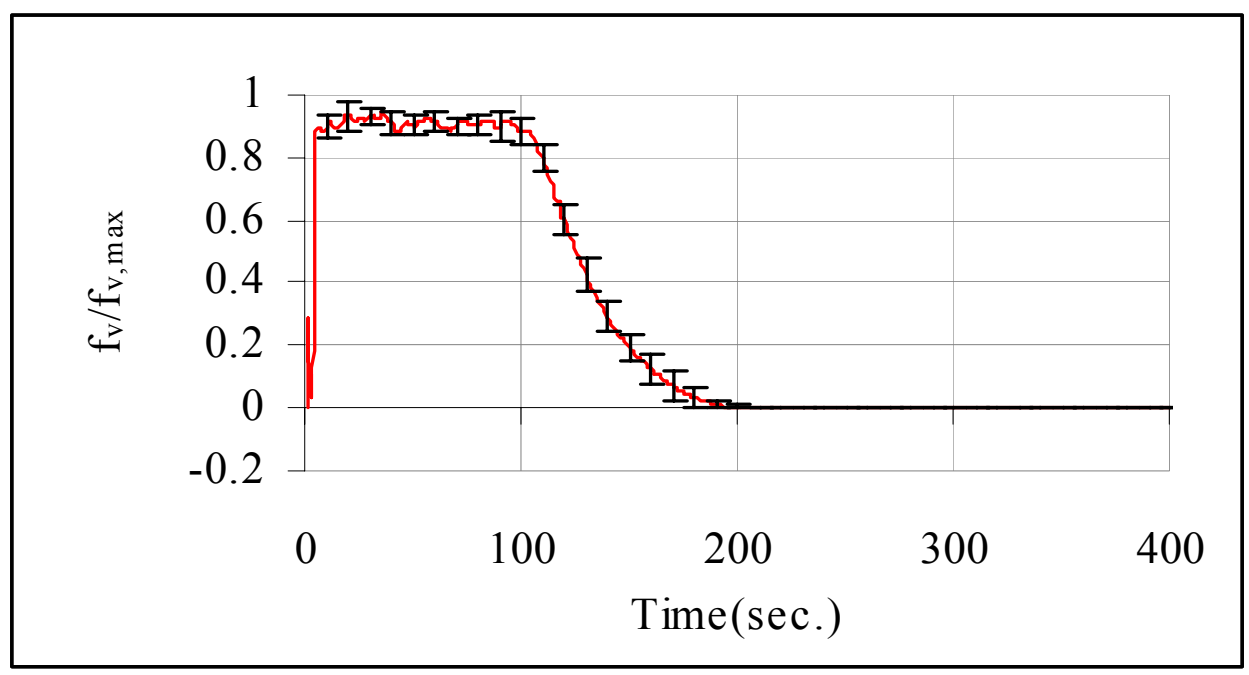

Figure 4.17. Average $f_{v} / f_{v, \max }$ vs. time plot for Maverick cigarette experiments with an applied voltage of 1000 Volts. 


\subsection{Size Distribution}

A particle sizer was used to examine the particle size distribution of smoke from a burning Maverick cigarette. The aerodynamic diameter and number concentration results from the particle sizer are shown in Figure 4.18, Figure 4.19, Figure 4.20, and Figure 4.21. The differences in these figures may be attributed to different burning rates. Figure 4.18, Figure 4.19, Figure 4.20, and Figure 4.21 all show the same size range and high concentrations at $0.7 \mu \mathrm{m}$. The particles sizer captures particles larger than $0.3 \mu \mathrm{m}$, but does not have the capacity to measure particles below $0.5 \mu \mathrm{m}$. The particle sizer groups all of the particles between 0.5 and $0.3 \mu \mathrm{m}$ in the $0.3 \mu \mathrm{m}$ bin. The sizer does not include these particles when it calculates the statistics. Table 4.2 shows statistics including median, mean, geometric mean, mode, geometric standard deviation, and total concentration of particle diameters for the data represented in these figures, based on the number of particles, particle surface area, and particle mass. The particle sizer software calculates these statistics over a log scale of diameters. This tends to shift the statistics towards larger values. A sample calculation of the mean particle diameter based on the number of particles was conducted for run 1. A value of $1.01 \mu \mathrm{m}$ was calculated. This value agrees very well $(0.8 \%$ difference) with the value of $1.002 \mu \mathrm{m}$ presented in Table 4.2. 


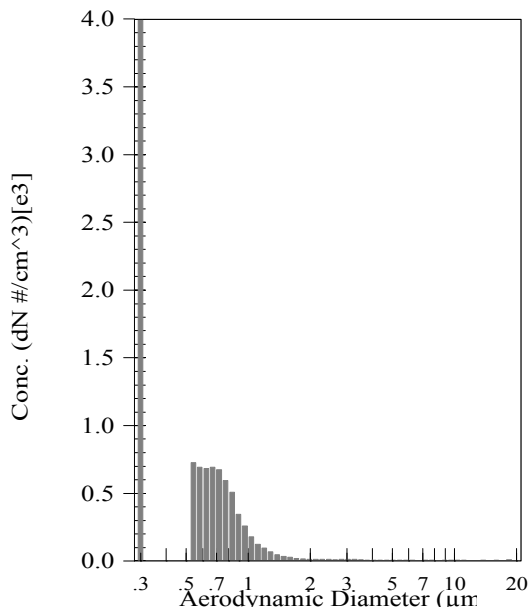

Figure 4.18. Particle sizer results run 1 with a $10 \mathrm{~s}$ sample time.

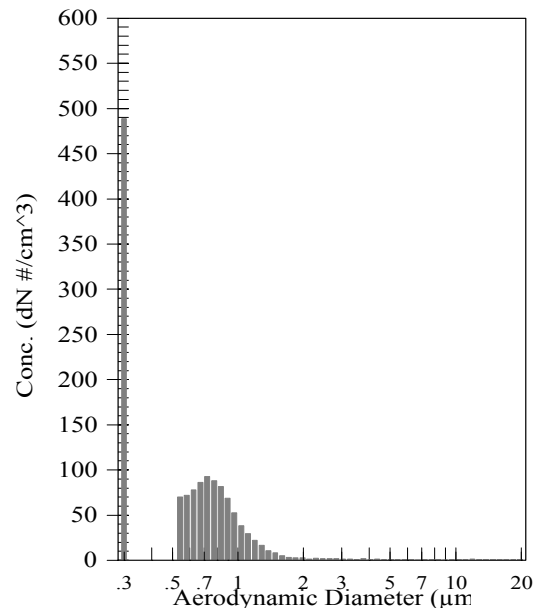

Figure 4.20. Particle sizer results run 4 with a $30 \mathrm{~s}$ sample time.

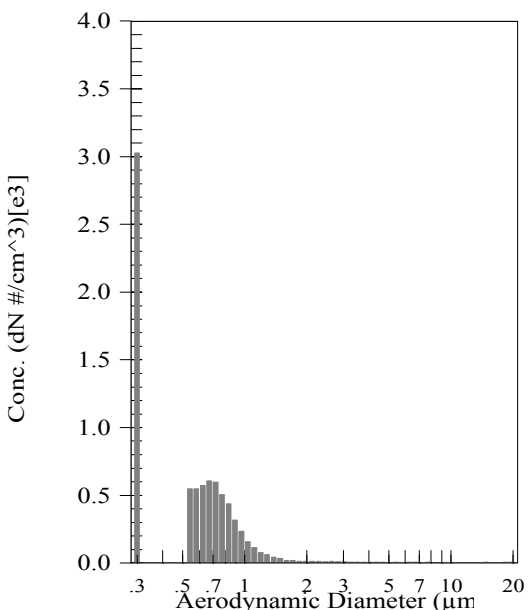

Figure 4.19. Particle sizer results run 3 with a 10 s sample time.

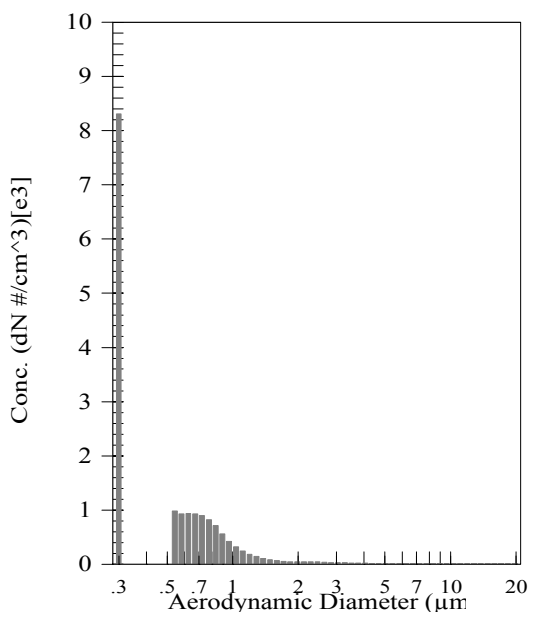

Figure 4.21. Particle sizer results run 5 with a 30 s sample time. 
Table 4.2. Particle diameter statistics from the particle sizer.

\begin{tabular}{|c|c|c|c|c|}
\hline Run & Statistic & $\begin{array}{c}\text { Number } \\
\text { Particle Size }\end{array}$ & Surface Particle Size & $\begin{array}{c}\text { Mass } \\
\text { Particle Size }\end{array}$ \\
\hline 1 & median & $0.7157(\mu \mathrm{m})$ & $13.21(\mu \mathrm{m})$ & $16.40(\mu \mathrm{m})$ \\
& mean & $1.002(\mu \mathrm{m})$ & $11.49(\mu \mathrm{m})$ & $15.24(\mu \mathrm{m})$ \\
& geom. mean & $0.7943(\mu \mathrm{m})$ & $7.877(\mu \mathrm{m})$ & $14.20(\mu \mathrm{m})$ \\
& mode & $0.5425(\mu \mathrm{m})$ & $18.843(\mu \mathrm{m})$ & $18.43(\mu \mathrm{m})$ \\
& goem. st. dev. & 1.612 & 3.027 & 1.589 \\
& total conc. & $37994.65\left(\# / \mathrm{cm}^{3}\right)$ & $75584.41\left(\mu \mathrm{m}^{2} / \mathrm{cm}^{3}\right)$ & $130\left(\mathrm{mg} / \mathrm{m}^{3}\right)$ \\
\hline 3 & median & $0.7234(\mu \mathrm{m})$ & $13.19(\mu \mathrm{m})$ & $16.21(\mu \mathrm{m})$ \\
& mean & $1.013(\mu \mathrm{m})$ & $11.52(\mu \mathrm{m})$ & $15.36(\mu \mathrm{m})$ \\
& geom. mean & $0.8036(\mu \mathrm{m})$ & $7.867(\mu \mathrm{m})$ & $14.28(\mu \mathrm{m})$ \\
& mode & $0.6732(\mu \mathrm{m})$ & $19.81(\mu \mathrm{m})$ & $19.81(\mu \mathrm{m})$ \\
& goem. st. dev. & 1.614 & 3.033 & 1.599 \\
& total conc. & $29351.45\left(\# / \mathrm{cm}^{3}\right)$ & $64065.09\left(\mu \mathrm{m}^{2} / \mathrm{cm}^{3}\right)$ & $110\left(\mathrm{mg} / \mathrm{m}^{3}\right)$ \\
\hline 4 & median & $0.7722(\mu \mathrm{m})$ & $13.40(\mu \mathrm{m})$ & $16.45(\mu \mathrm{m})$ \\
& mean & $1.089(\mu \mathrm{m})$ & $11.77(\mu \mathrm{m})$ & $15.37(\mu \mathrm{m})$ \\
& geom. mean & $0.8533(\mu \mathrm{m})$ & $8.237(\mu \mathrm{m})$ & $14.38(\mu \mathrm{m})$ \\
& mode & $0.7234(\mu \mathrm{m})$ & $19.81(\mu \mathrm{m})$ & $19.81(\mu \mathrm{m})$ \\
& goem. st. dev. & 1.6442 & 2.937 & 1.569 \\
& total conc. & $4776.54\left(\# / \mathrm{cm}^{3}\right)$ & $12319.73\left(\mu \mathrm{m}^{2} / \mathrm{cm}^{3}\right)$ & $220\left(\mathrm{mg} / \mathrm{m}^{3}\right)$ \\
\hline 5 & median & $0.7455(\mu \mathrm{m})$ & $13.79(\mu \mathrm{m})$ & $16.77(\mu \mathrm{m})$ \\
& mean & $1.205(\mu \mathrm{m})$ & $12.26(\mu \mathrm{m})$ & $15.53(\mu \mathrm{m})$ \\
& geom. mean & $0.8733(\mu \mathrm{m})$ & $9.132(\mu \mathrm{m})$ & $14.55(\mu \mathrm{m})$ \\
& mode & $0.5425(\mu \mathrm{m})$ & $19.891(\mu \mathrm{m})$ & $19.81(\mu \mathrm{m})$ \\
& goem. st. dev. & 1.796 & 2.648 & 1.544 \\
& total conc. & $75630.51\left(\# / \mathrm{cm}^{3}\right)$ & $177868.96\left(\mu \mathrm{m}^{2} / \mathrm{cm}^{3}\right)$ & $330\left(\mathrm{mg} / \mathrm{m}^{3}\right)$ \\
\hline \multirow{2}{*}{} & & &
\end{tabular}




\subsection{Multiple Elevation Results}

To get some idea of the stratification of smoke layers within the reactor, experiments were conducted with the laser positioned at three different heights, $11.43 \mathrm{~mm}, 7.62 \mathrm{~mm}$, and $3.05 \mathrm{~mm}$. The laser heights presented here were measured from the ceiling of the reactor where the positive direction was down. For example, the lowest measurement height was $11.43 \mathrm{~mm}$. Figure 4.22, Figure 4.23, Figure 4.24, and Figure 4.25 present average $f_{v} / f_{v, \max }$ plots at all three laser heights for applied voltages of $0,500,1000$, and 1500 volts respectively. It is not clear form these plots if the presence of an electric field changed the stratification of smoke layers within the reactor. The overall time that it took for most of the particles to fall out was not enough to determine the stratification characteristics within the reactor. A normalized sedimentation rate was calculated for each set of experiments by taking the laser elevation that the experiment was run at and dividing this number by the approximate time that it took for overall sedimentation to occur (determined graphically). These values are presented in Table 4.3. The only obvious trends that can be seen from this table are that the normalized sedimentation rate seemed to increase with increasing electric field strength and increase as the laser elevation approached the floor of the reactor.

Table 4.3. Normalized approximate sedimentation rates $(\mu \mathrm{m} / \mathrm{s})$ for all laser elevation and applied voltage experiments.

\begin{tabular}{|l|l|l|l|l|}
\hline \multirow{2}{*}{$\begin{array}{c}\text { Laser } \\
\text { Elevation }\end{array}$} & \multicolumn{4}{|c|}{ Applied Voltage } \\
\cline { 2 - 5 } $\mathrm{z}=3.05 \mathrm{~mm}$ & $19 \mu \mathrm{m} / \mathrm{s}$ & $14 \mu \mathrm{m} / \mathrm{s}$ & $15 \mu \mathrm{m} / \mathrm{s}$ & $16 \mu \mathrm{m} / \mathrm{s}$ \\
\hline $\mathrm{z}=7.62 \mathrm{~mm}$ & $32 \mu \mathrm{m} / \mathrm{s}$ & $35 \mu \mathrm{m} / \mathrm{s}$ & $36 \mu \mathrm{m} / \mathrm{s}$ & $42 \mu \mathrm{m} / \mathrm{s}$ \\
\hline $\mathrm{z}=11.43 \mathrm{~mm}$ & $39 \mu \mathrm{m} / \mathrm{s}$ & $48 \mu \mathrm{m} / \mathrm{s}$ & $54 \mu \mathrm{m} / \mathrm{s}$ & $64 \mu \mathrm{m} / \mathrm{s}$ \\
\hline
\end{tabular}




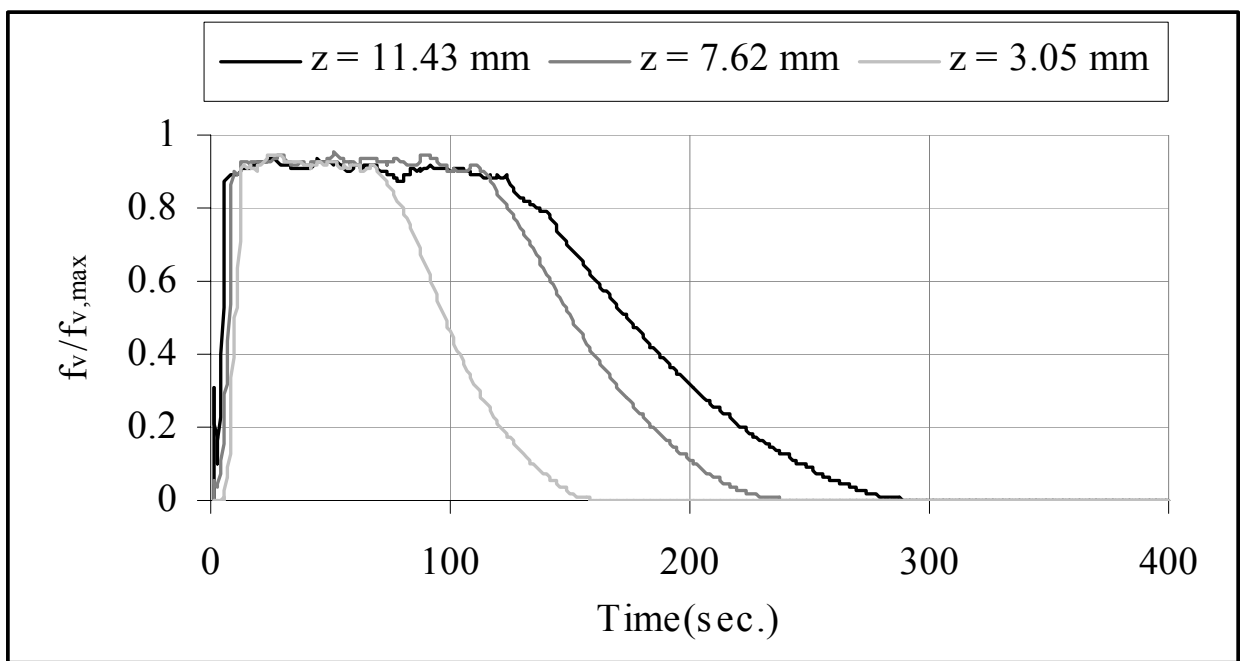

Figure 4.22. $f_{v} / f_{v, \max }$ vs. time plots for all laser elevations with no applied voltage.

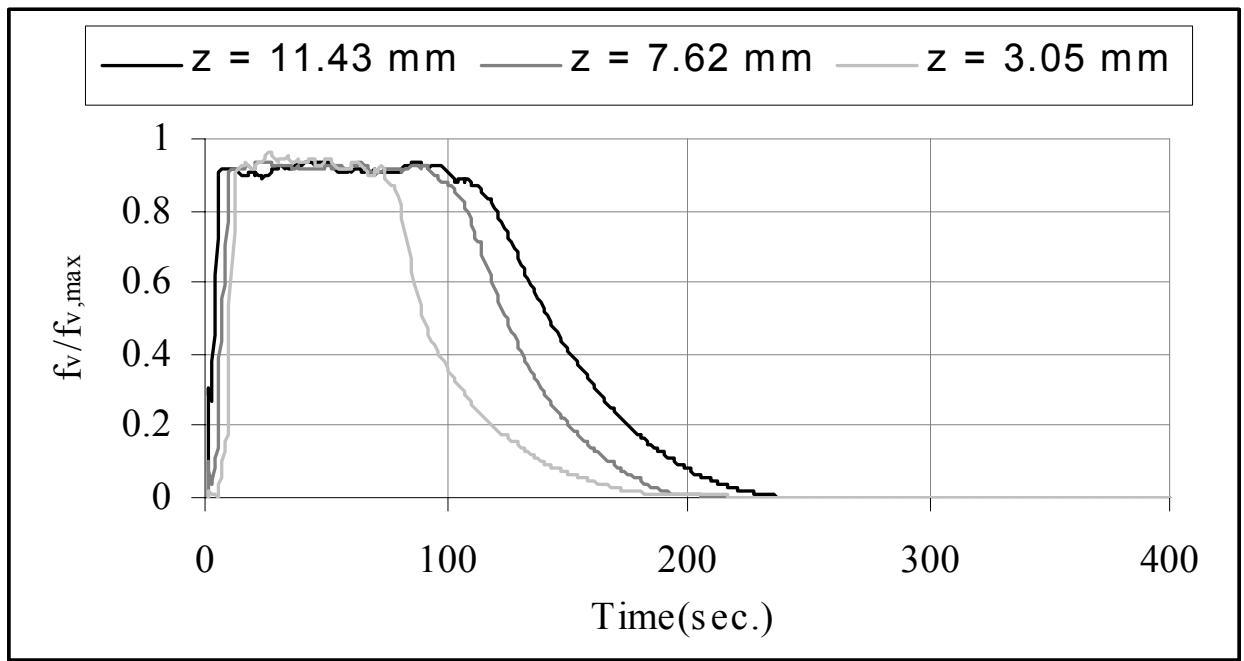

Figure 4.23. $f_{v} / f_{v, \max }$ vs. time plots for all laser elevations with an applied voltage of 500 Volts. 


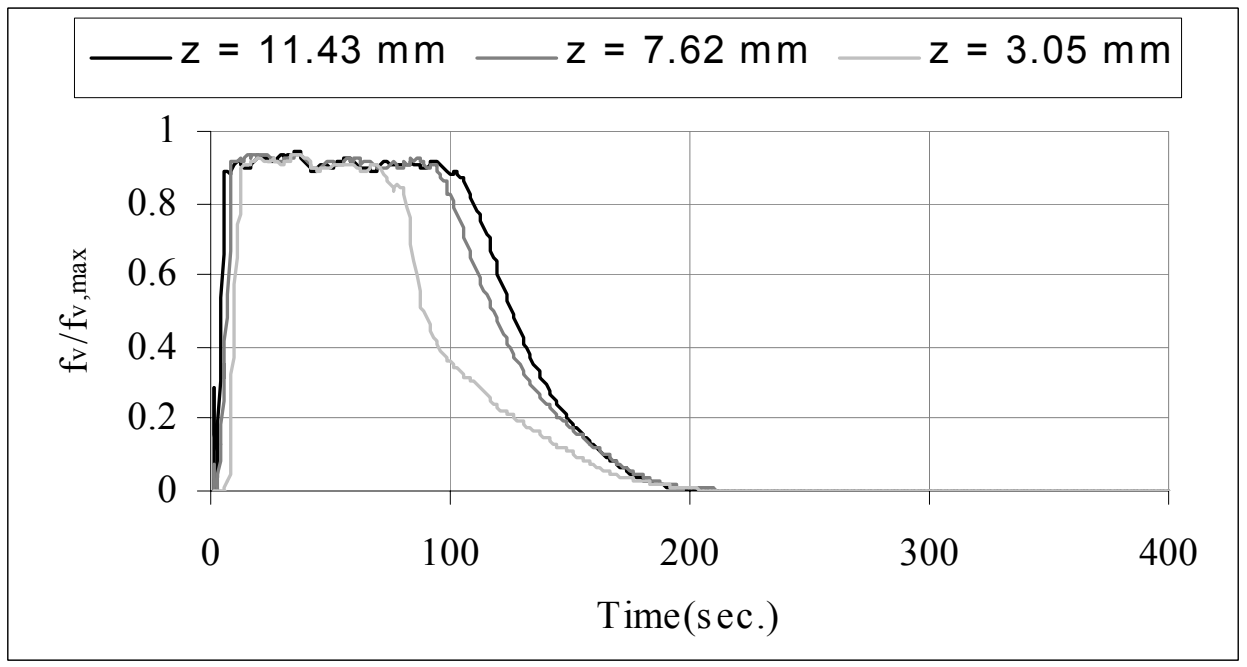

Figure 4.24. $f_{v} / f_{v, \max }$ vs. time plots for all laser elevations with an applied voltage of 1000 Volts.

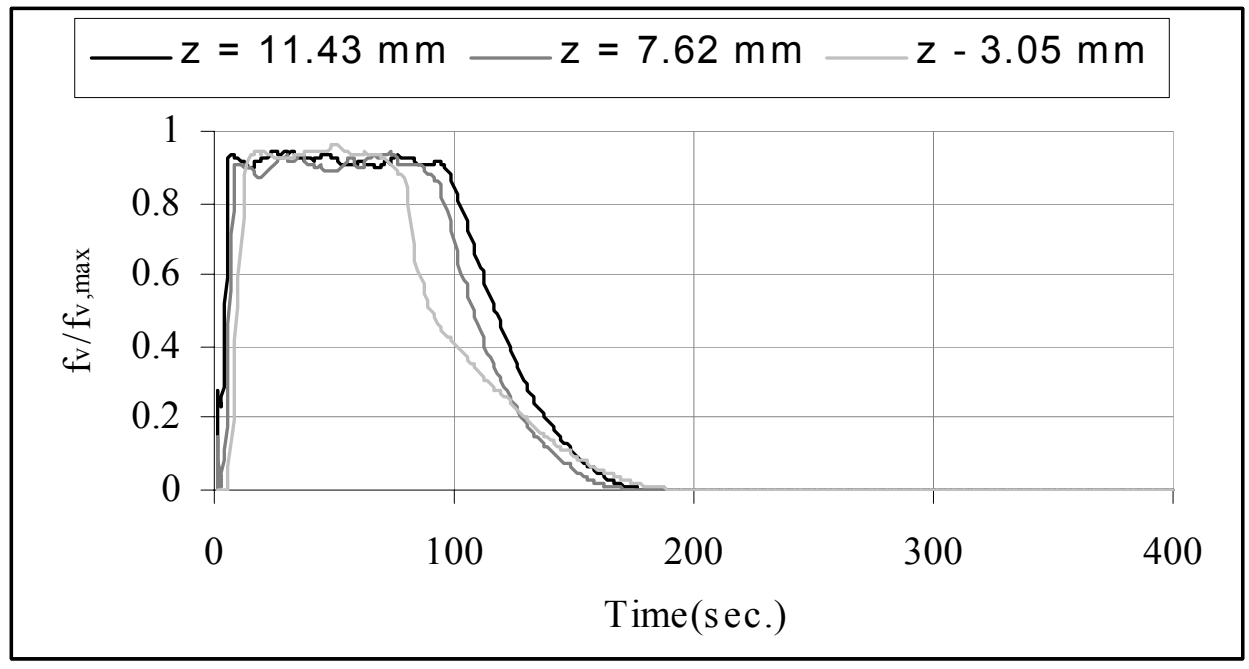

Figure 4.25. $f_{v} / f_{v, \max }$ vs. time plots for all laser elevations with an applied voltage of 1500 Volts. 


\subsection{Multiple Voltage Results}

To visualize the effect of different electric field strengths, experiments were conducted with applied voltages of $0,500,1000$, and 1500 volts. Figure 4.26, Figure 4.27, and Figure 4.28 present average $f_{v} / f_{v, \max }$ plots at all four applied voltages for laser heights of 3.05, 7.62, and $11.43 \mathrm{~mm}$ respectively. These plots clearly show that the effects of the electric field were more dominant near the bottom of the reactor. Near the top of the reactor (Figure 4.26), The electric field did not seem to affect the sedimentation rate of the particles. As the observation point was lowered (Figure 4.27 and Figure 4.28), the effects of the electric field increased. These figures clearly show that increasing the electric field decreases the amount of time that it takes for the reactor to clear. This corresponds to an increase in settling velocity. This makes sense if the electric field was causing the particles to agglomerate. The particles agglomerated as they fell, increasing the particle size and settling velocity.

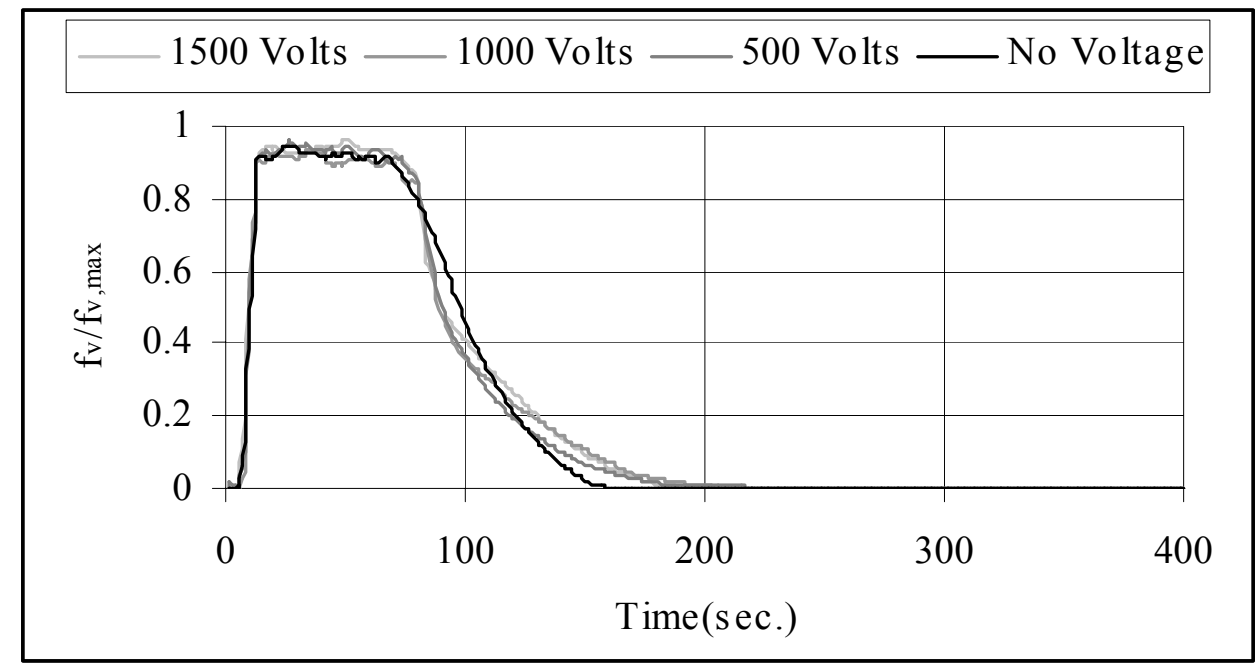

Figure 4.26. $f_{v} / f_{v, \max }$ vs. time plots for all applied voltages at a laser elevation of $\mathrm{z}=3.05 \mathrm{~mm}$. 


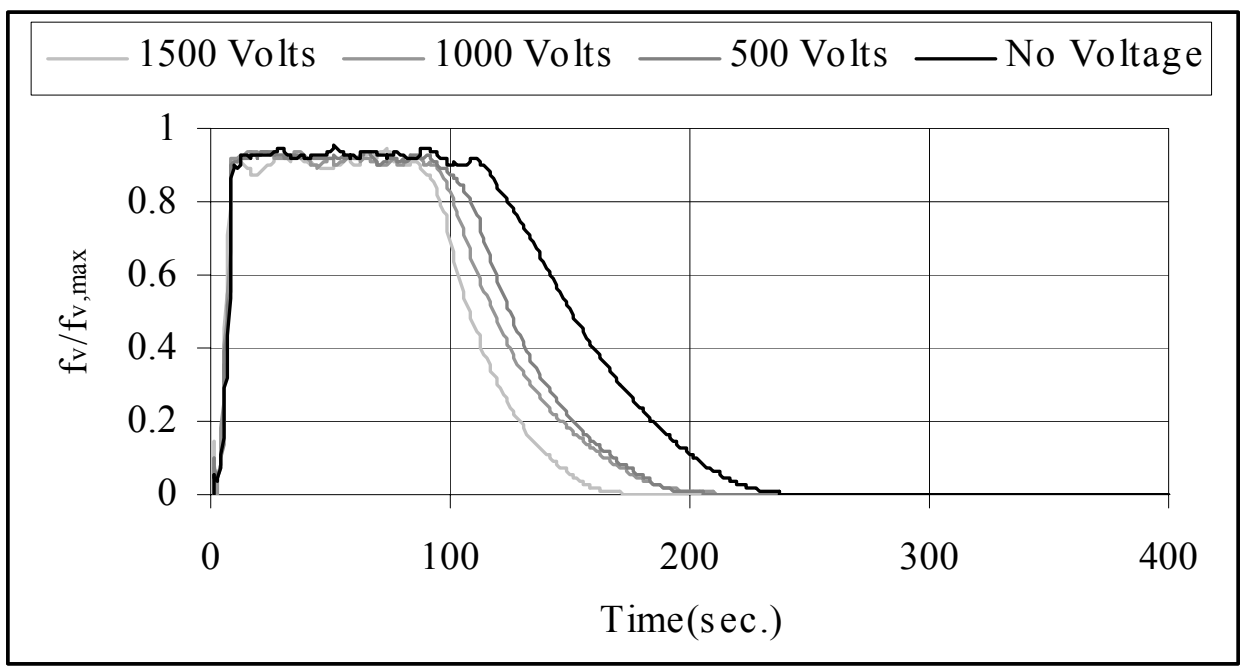

Figure 4.27. $f_{v} / f_{v, \max }$ vs. time plots for all applied voltages at a laser elevation of $\mathrm{z}=7.62 \mathrm{~mm}$.

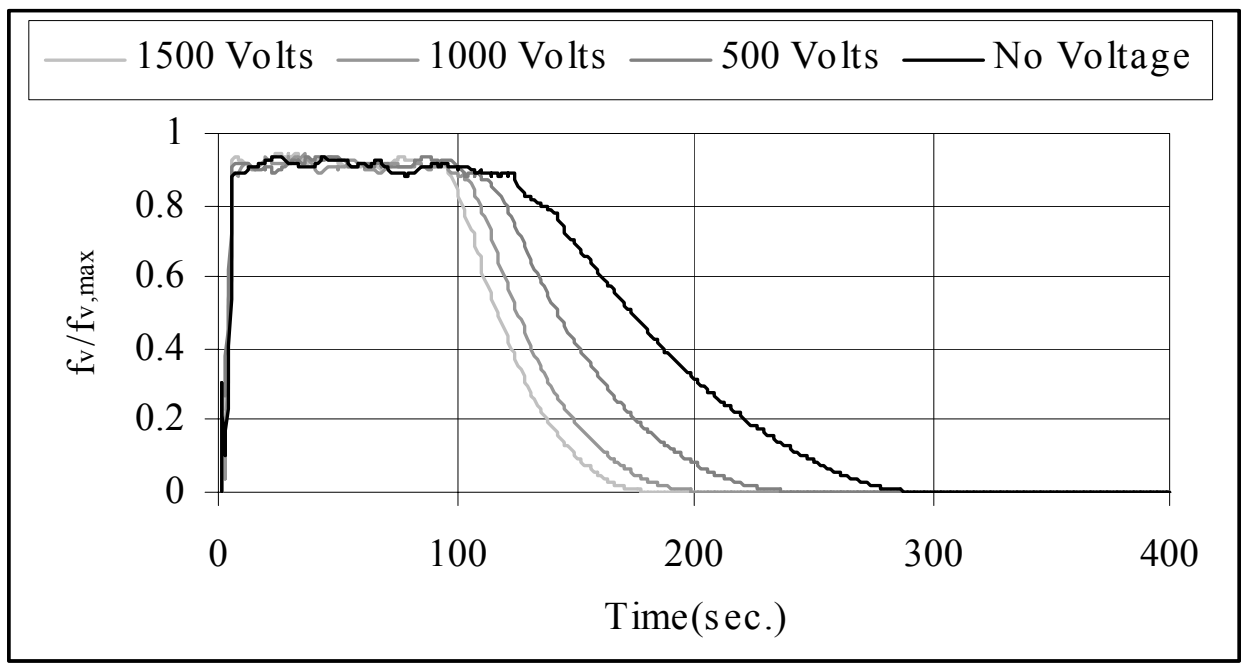

Figure 4.28. $f_{v} / f_{v, \max }$ vs. time plots for all applied voltages at a laser elevation of $\mathrm{z}=11.43 \mathrm{~mm}$. 


\subsection{Size Distribution Results}

An effective means of interpreting the results was to calculate the particle size distribution for each set of experiments. The cases with no voltage applied can be compared to the results, presented previously, from the particle sizer. The procedure for calculating the particle size distribution from the volume fraction ratio plot is presented below. The first assumption for this procedure is no agglomeration. If an aerosol is monodisperse, the particles leaving a specific volume will be replaced by particles entering the volume (see Figure 4.29). When there are no particles left to replace the particles leaving the volume, the volume begins to clear. This suggests that all of the particles will fall out past a certain elevation at the same time. Figure 4.30 shows what the $f_{v}$ vs. time plot would look like at a given elevation for a monodisperse aerosol.

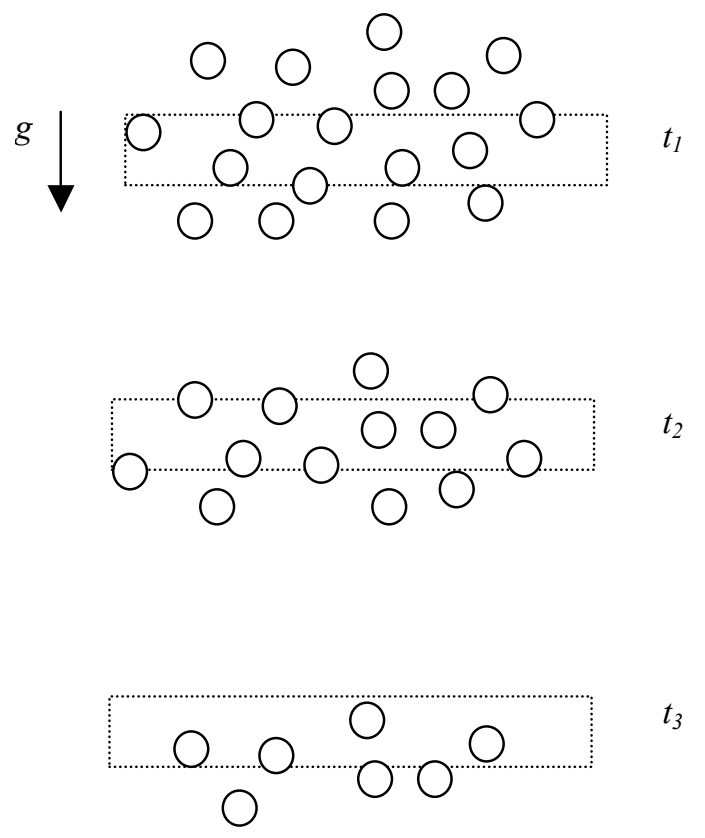

Figure 4.29. Monodisperse aerosol passing through a volume. 


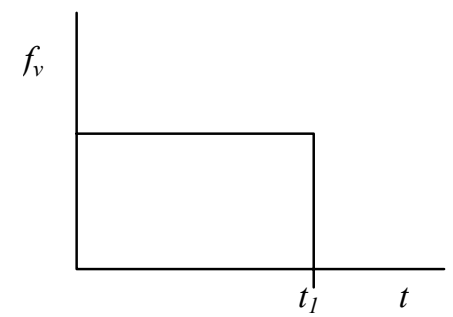

Figure 4.30. $f_{v}$ vs. $\mathrm{t}$ at a specific elevation for a monodisperse aerosol.

In Figure 4.30, at time $t_{1}$ all the particles have dropped below the given elevation, and there is no loading at or above this elevation. A similar idea applies to a polydisperse aerosol. In a polydisperse aerosol the largest particles are the heaviest and will clear the volume first, followed by the next size smaller

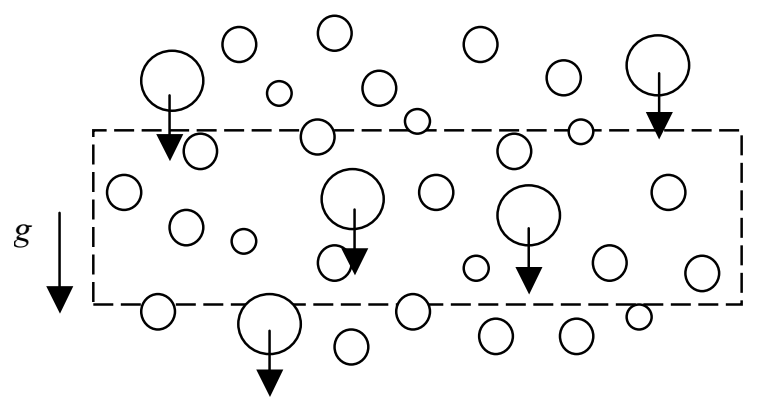

particles, and so on (see Figure. 4.31).

Figure 4.31. Polydisperse aerosol passing through a volume.

The corresponding $f_{v}$ vs. t figure for a polydisperse aerosol is presented as Figure 4.32.

Figure 4.32. $f_{v}$ vs. $\mathrm{t}$ at a specific elevation for a polydisperse aerosol.

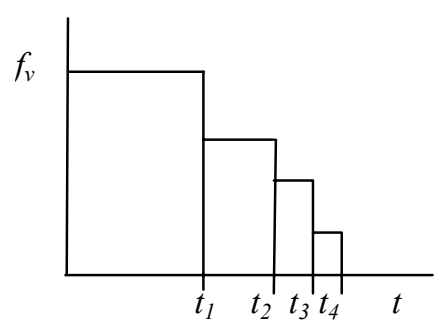


In Figure 4.32, $t_{1}$ indicates the time that it takes for the largest size particles to drop below the given elevation. At time $t_{2}$, the next size smaller particles have dropped below the given elevation, and so on for $t_{3}$ and $t_{4}$.

Given that a specific particle size will fall out past a given point by a specific amount of time, the diameter of that particle can be calculated from the sedimentation velocity of that particle. The sedimentation velocity, $U_{s}$, is said to be equal to the distance the particle fell divided by the time that it took for the particle to fall that distance or,

$$
U_{s}=\frac{h}{t}
$$

Where, $h$ is the distance from the measurement location (laser elevation) to the ceiling of the reactor. And $t$ is the time that it takes for the particles to reach $h$. The sedimentation velocity can be found by balancing the gravitational force with Stoke's drag law,

$$
3 \pi \mu_{a} U_{s} D_{p}=m_{p} g
$$

In this expression, $\mu_{a}$ is the viscosity of the surrounding fluid (air), $D_{p}$ is the diameter of a single particle, $g$ is gravity, and $m_{p}$ is the mass of the particle given as,

$$
m_{p}=\rho_{p} v_{p}
$$

The density and volume of the particle are $\rho_{p}$ and $v_{p}$ respectively, and $v_{p}$ is approximated as the volume of a sphere,

$$
v_{p}=v_{\text {sphere }}=\frac{1}{6} \pi D_{p}^{3}
$$

And, $\rho_{p}$ is estimated to be $2000 \mathrm{~kg} / \mathrm{m}^{3}$. 
The sedimentation velocity can now be presented as,

$$
U_{s}=\frac{\rho_{p} D_{p}^{2} g}{18 \mu}=\frac{h}{t}
$$

Solving for the diameter and substituting the equation for the sedimentation velocity gives,

$$
D_{p}=\sqrt{\frac{18 \mu h}{\rho_{p} t g}}
$$

This equation gives the diameter of particles at a given time. The diameter is calculated for every time during the experiment within a specific range of $f_{v} / f_{v, \max }$. This range covers values for $f_{v} / f_{v, \max }$ that are continually decreasing for every set of experiments examined in this section. The endpoints of this range are 0.85 and 0.0025 . This does not give the entire range of particle sizes rather, gives a good representation of the range of particle sizes.

The range of particle sizes is the first step in finding the particle size distribution. The next step is to find the number concentration of particles for each diameter in this range. The number concentration is related to the loading, $f_{v}$, by,

$$
f_{v}\left(\vec{x}_{0}, t\right)=\Sigma n_{i}(t) v_{i}
$$

Where $\vec{x}_{0}$ and $\mathrm{t}$ are the position and time, respectively, at which the measurement is taken. And, $n_{i}$ and $v_{i}$ are the number concentration and volume, respectively, of particles at a specific diameter. At a fixed position and time $t_{0}$, the loading in the chamber is,

$$
f_{v}\left(t_{0}\right)=n_{1} v_{1}+n_{2} v_{2}+\ldots+n_{n} v_{n}
$$

At the next time, $t_{0}$ plus some time step, $\Delta t$, the largest particles, $n_{l} v_{l}$, have already passed through the volume, and the loading is now,

$$
f_{v}\left(t_{0}+\Delta t\right)=0+n_{2} v_{2}+\ldots+n_{n} v_{n}
$$


Subtracting these two equations gives,

$$
f_{v}\left(t_{0}\right)-f_{v}\left(t_{0}+\Delta t\right)=n_{1} v_{1}
$$

Where,

$$
v_{1}=\frac{\pi D_{1}^{3}}{6}
$$

Solving for $n_{1}$ gives,

$$
n_{1}=6 \frac{f_{v}\left(t_{0}\right)-f_{v}\left(t_{0}+\Delta t\right)}{\pi D_{1}^{3}}
$$

More generally,

$$
n_{i+1}=6 \frac{f_{v}\left(t_{i}\right)-f_{v}\left(t_{i}+\Delta t\right)}{\pi D_{i+1}^{3}}
$$

Where $D_{i}$ is specified by equation 4.9. This equation is used to calculate the number concentration at each time in the range of $f_{v} / f_{v \max }$ discussed above.

The size distribution is presented for the multiple voltage and multiple elevation experiments. Figure 4.33, Figure 4.34, and Figure 4.35 show the size distribution results for all three elevations where no voltage was applied to the copper plates. Figure 4.33 presents results for the laser elevation closest to the ceiling of the reactor. This figure shows a representative size range from $0.6-$ $0.9 \mu \mathrm{m}$, with high particle concentrations at $0.7 \mu \mathrm{m}$. These results are similar to the results presented earlier from the particle sizer. Figure 4.34 presents results for a laser elevation near the vertical center of the reactor. This figure shows a representative size range from $0.75-1.1 \mu \mathrm{m}$, with high particle concentrations at 0.85 and $0.9 \mu \mathrm{m}$. Figure 4.35 presents results for the laser elevation closest to the floor of the reactor. This figure shows a representative size range from $0.9-1.35$ $\mu \mathrm{m}$, with high particle concentrations at $0.95,1$ and $1.05 \mu \mathrm{m}$. These figures suggest that the particles are growing as they fall. This is attributed to natural 
agglomeration processes, gravitational, and Brownian effects. An obvious decrease in the overall particle concentration near the floor of the reactor agrees with the idea of agglomeration.

The next set of figures present size distribution results for all three elevations where 500 Volts was applied. Figure 4.36 presents results for the laser elevation closest to the ceiling of the reactor. This figure shows a representative size range from $0.55-0.9 \mu \mathrm{m}$, with high particle concentrations at $0.9 \mu \mathrm{m}$. These results are similar to the results presented in Figure 4.33. Figure 4.37 presents results for a laser elevation near the vertical center of the reactor. This figure shows a representative size range from $0.8-1.15 \mu \mathrm{m}$, with high particle concentrations at 1.05 and $1.1 \mu \mathrm{m}$. Figure 4.38 presents results for the laser elevation closest to the floor of the reactor. This figure shows a representative size range from $1.0-1.3 \mu \mathrm{m}$, with high particle concentrations at $1.1 \mu \mathrm{m}$. Comparing these figures with the previous figures where no voltage is applied, there was a small overall shift in particle size distribution. It appears that the application of an electric field enhanced particle agglomeration.

The third set of figures presents size distribution results for all three elevations where 1000 Volts was applied. Figure 4.39 presents results for the laser elevation closest to the ceiling of the reactor. This figure shows a representative size range from $0.5-0.9 \mu \mathrm{m}$, with high particle concentrations at $0.85 \mu \mathrm{m}$. These results are similar to the results presented in Figure 4.33 and Figure 4.36. Figure 4.40 presents results for a laser elevation near the vertical center of the reactor. This figure shows a representative size range from $0.8-1.2$ $\mu \mathrm{m}$, with high particle concentrations at $1.05,1.1$, and $1.15 \mu \mathrm{m}$. Figure 4.41 presents results for the laser elevation closest to the floor of the reactor. This figure shows a representative size range from $1.0-1.4 \mu \mathrm{m}$, with high particle 
concentrations at $1.3 \mu \mathrm{m}$. Comparing these figures with the previous figures where 500 Volts was applied, there was again a small overall shift in particle size distribution. As the strength of the electric field increased the particle agglomeration appeared to increase.

The final set of figures present size distribution results for all three elevations where 1500 Volts was applied. Figure 4.42 presents results for the laser elevation closest to the ceiling of the reactor. This figure shows a representative size range from $0.55-0.9 \mu \mathrm{m}$, with high particle concentrations at $0.85 \mu \mathrm{m}$. These results are similar to the results presented in Figure 4.33, Figure 4.36, and Figure 4.39. Figure 4.43 presents results for a laser elevation near the vertical center of the reactor. This figure shows a representative size range from $0.9-1.25 \mu \mathrm{m}$, with high particle concentrations at $1.15 \mu \mathrm{m}$. Figure 4.44 presents results for the laser elevation closest to the floor of the reactor. This figure shows a representative size range from $1.05-1.45 \mu \mathrm{m}$, with high particle concentrations at $1.25,1.3$, and $1.35 \mu \mathrm{m}$. Comparing these figures with the previous figures where 1000 Volts was applied, there was another small overall shift in particle size distribution. The application of an electric field clearly increased the particle agglomeration. The effects of the electric field clearly increased as the electric field strength increased. Qualitatively, Figures 4.33, 4.36, 4..39, and 4.41 are very similar. This agrees with the idea that the affects of agglomeration are very small, if not negligible, near the ceiling of the reactor.

To examine these results quantitatively, an average diameter was calculated. A meaningful average diameter as presented by Reist (1993) is the mean particle diameter, $\bar{d}$, expressed mathematically as,

$$
\bar{d}=\frac{\sum n_{i} D_{i}}{\sum n_{i}}
$$


The mean particle diameters for all elevation, and all applied voltage experiments are presented in Table 4.3. The average increase in particle size between the no voltage case and the $1500 \mathrm{~V}$ case was 1.02 to 1.26 micrometers. Since sedimentation velocity scales like the particle diameter squared, one would expect an approximately $60 \%$ increase in the sedimentation velocity between these two extreme cases. Obviously, this would correspond to an approximately $40 \%$ decrease in the sedimentation time. When one examines figure 4.28 , it is apparent that the time for sedimentation at this bottom measurement location goes from a maximum of 290 seconds for no voltage to approximately 180 seconds for 1500 Volts. This corresponds to a $38 \%$ change in the sedimentation time. 


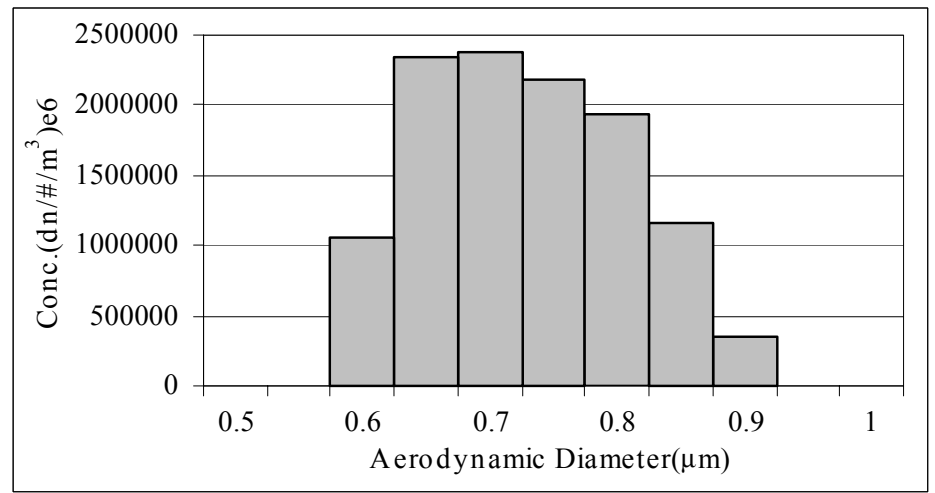

Figure 4.33. Size distribution plots for the case where the applied voltage was 0 Volts at a laser elevation of $z=3.05 \mathrm{~mm}$.

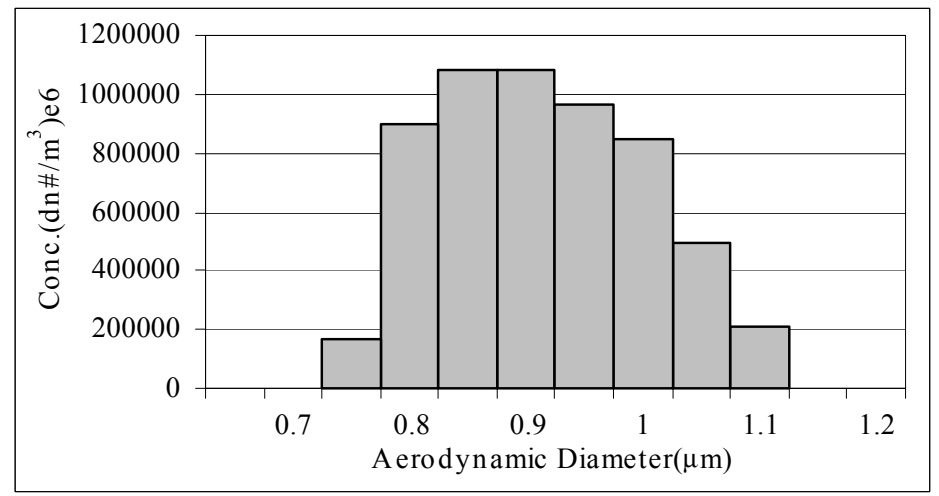

Figure 4.34. Size distribution plots for the case where the applied voltage was 0 Volts at a laser elevation of $z=7.62 \mathrm{~mm}$.

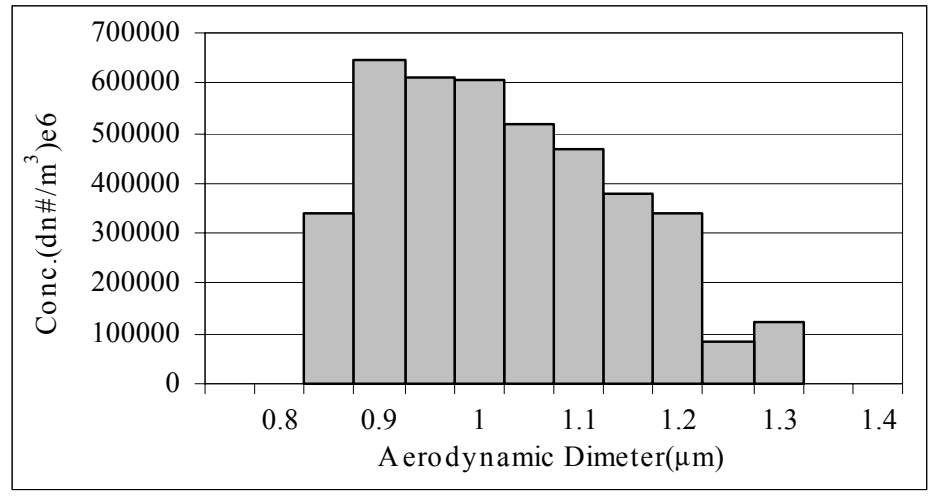

Figure 4.35. Size distribution plots for the case where the applied voltage was 0 Volts at a laser elevation of $z=11.43 \mathrm{~mm}$. 


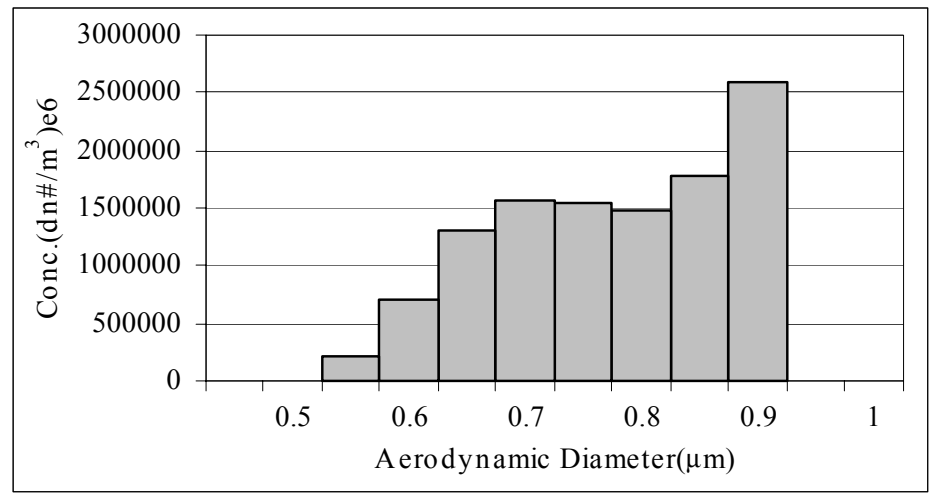

Figure 4.36. Size distribution plots for the case where the applied

voltage was 500 Volts at a laser elevation of $z=3.05 \mathrm{~mm}$.

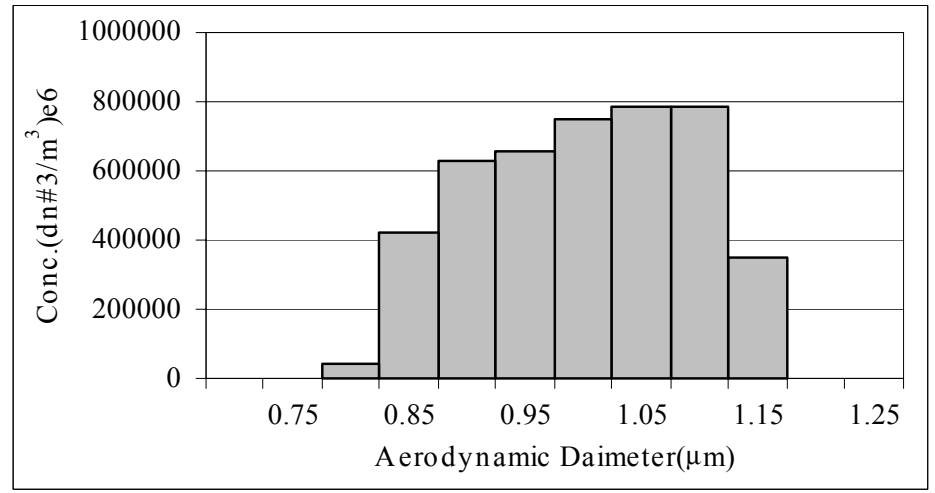

Figure 4.37. Size distribution plots for the case where the applied

voltage was 500 Volts at a laser elevation of $z=7.62 \mathrm{~mm}$.

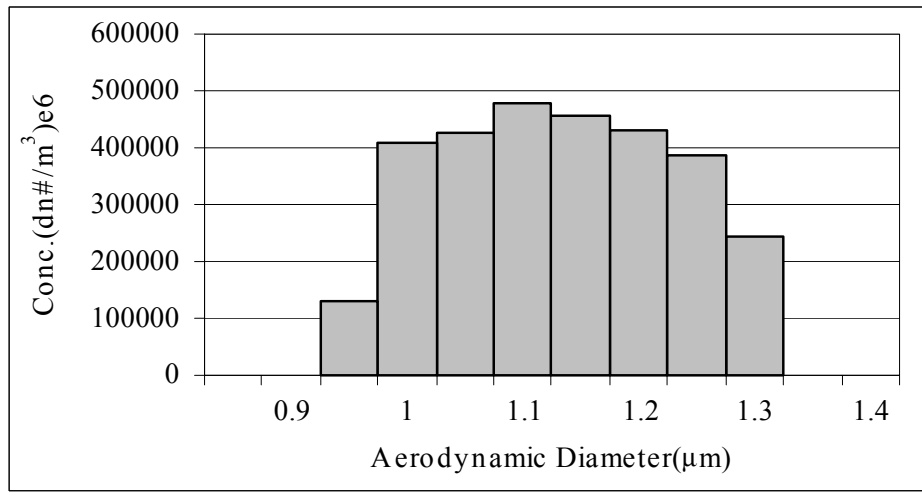

Figure 4.38. Size distribution plots for the case where the applied

voltage was 500 Volts at a laser elevation of $z=11.43 \mathrm{~mm}$. 


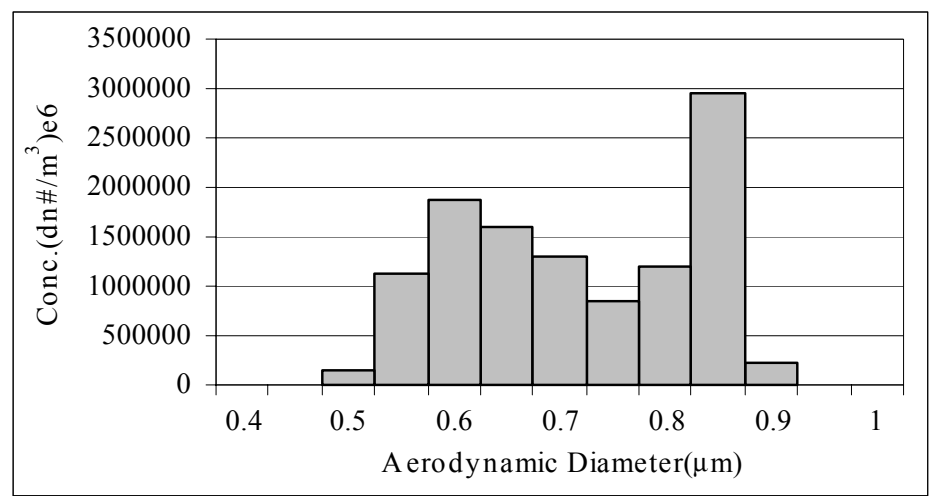

Figure 4.39. Size distribution plots for the case where the applied

voltage was 1000 Volts at a laser elevation of $\mathrm{z}=3.05 \mathrm{~mm}$.

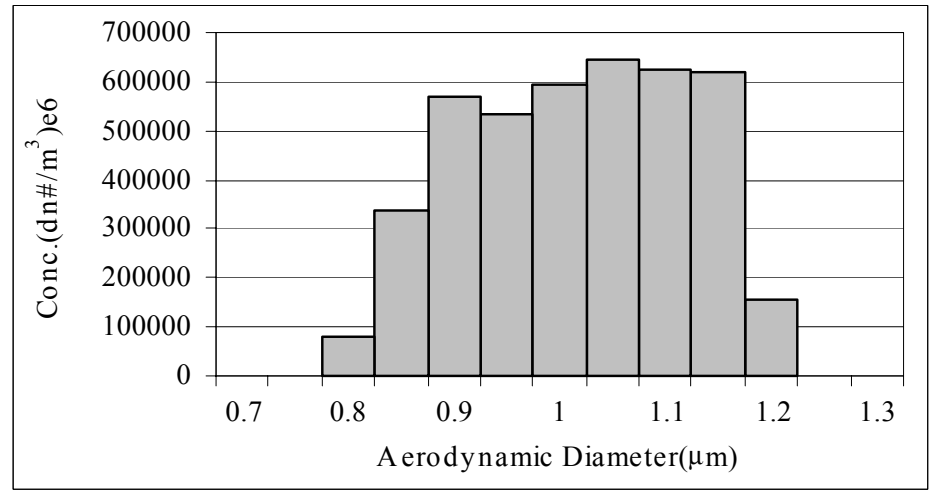

Figure 4.40. Size distribution plots for the case where the applied

voltage was 1000 Volts at a laser elevation of $z=7.62 \mathrm{~mm}$.

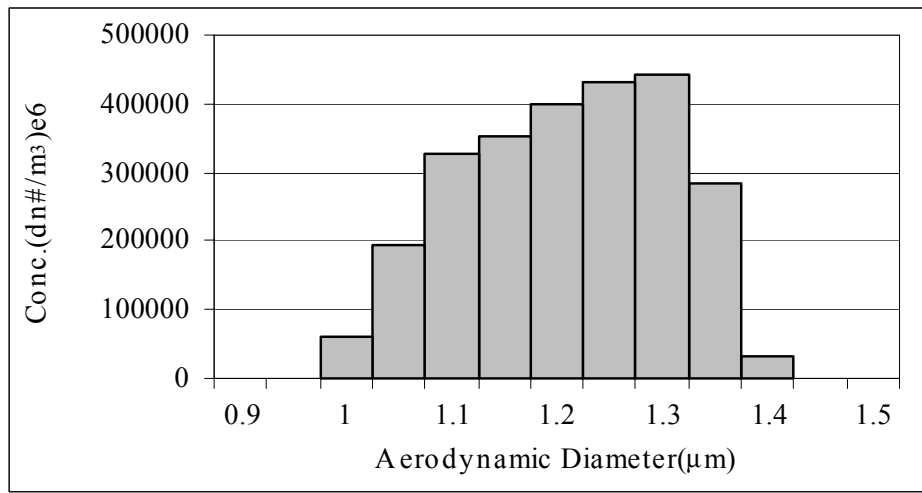

Figure 4.41. Size distribution plots for the case where the applied

voltage was 1000 Volts at a laser elevation of $\mathrm{z}=11.43 \mathrm{~mm}$. 


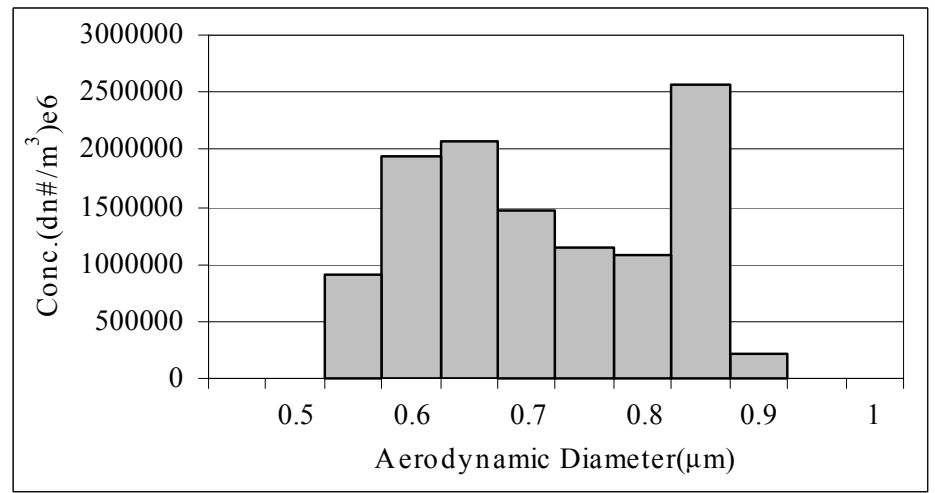

Figure 4.42. Size distribution plots for the case where the applied voltage was 1500 Volts at a laser elevation of $z=3.05 \mathrm{~mm}$.

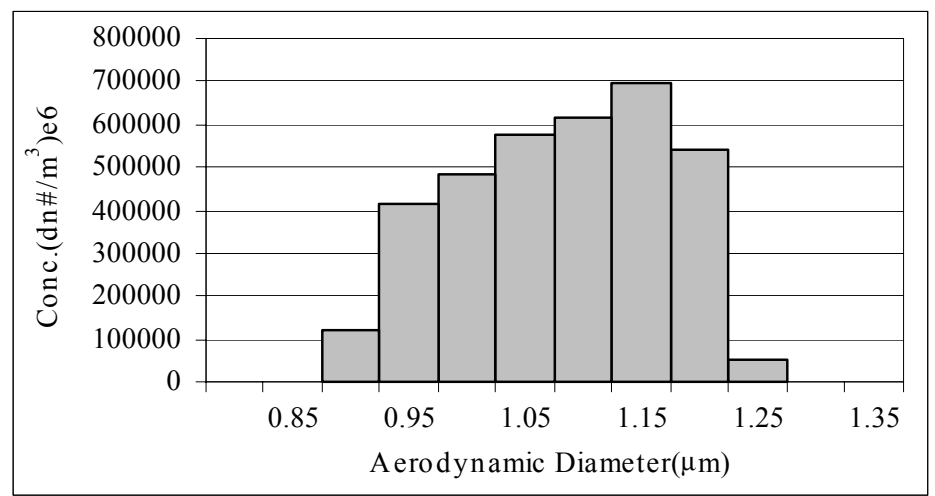

Figure 4.43. Size distribution plots for the case where the applied

voltage was 1500 Volts at a laser elevation of $z=7.62 \mathrm{~mm}$.

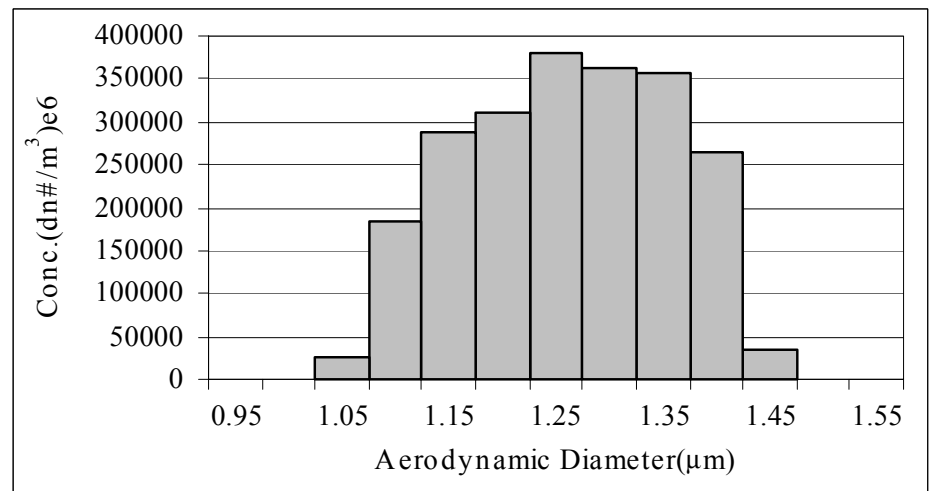

Figure 5.44. Size distribution plots for the case where the applied

voltage was 1500 Volts at a laser elevation of $\mathrm{z}=11.43 \mathrm{~mm}$. 
Table 4.4. Mean particle diameters from size distribution results.

\begin{tabular}{|c|c|c|}
\hline Applied Voltage (volts) & Laser Elevation & Mean Diameter $(\mu \mathrm{m})$ \\
\hline 0 & 3.05 & 0.728 \\
& 7.62 & 0.913 \\
& 11.43 & 1.02 \\
& 3.05 & 0.725 \\
& 7.62 & 1.00 \\
& 11.43 & 1.13 \\
\hline 500 & 3.05 & 0.717 \\
& 7.62 & 1.02 \\
& 11.43 & 1.21 \\
\hline 1000 & 3.05 & 0.715 \\
& 7.62 & 1.08 \\
& 11.43 & 1.26 \\
\hline
\end{tabular}




\section{CHAPTER 5. CONCLUSIONS}

The purpose of this thesis was to conduct experiments on electrocoalescence and determine the effects of an electric field on aerosol particle sedimentation processes. To see if, in fact, a strong electric field will cause particle agglomeration and an increase in overall size distribution. Various types of aerosol particles were introduced into the reactor and subjected to different electric field strengths. The overall sedimentation and coalescence properties of the aerosols were quite different. This may have had something to do with the electrical properties of the aerosol. The experimental procedure was changed until the experiments became repeatable. Once an acceptable procedure was reached, experiments were conducted with different electric field strengths. The electric field clearly affected the time that it took for particle sedimentation to occur. As the electric field increased, the rate of particle sedimentation increased, which decreased the overall sedimentation time. Examining Figure 4.28 the sedimentation times for experiments with applied voltages of $0,500,1000$, and 1500 Volts are approximately 290, 235, 200, and 180 seconds respectively. These times are for the lowest laser location.

In order to examine the EC effects at different elevations within the reactor, experiments were performed at three different laser elevations. When no electric field was present the particles sedimented much faster near the floor of the reactor than near the ceiling of the reactor. Examining Table 4.3 the normalized approximate sedimentation rates for the laser elevations near the top, middle, and bottom of the reactor are approximately 19,32 , and $39 \mu \mathrm{m} / \mathrm{s}$ respectively. When an electric field was applied, this sedimentation rate at the different elevations increased, except near the top of the reactor. As the electric field was increased, this sedimentation rate increased. Examining Table 4.3 the normalized approximate sedimentation rates for the laser elevations near the top, 
middle, and bottom of the reactor with an applied voltage of 1500 Volts are approximately 16,42 , and $64 \mu \mathrm{m} / \mathrm{s}$ respectively. The effects of the electric field on this sedimentation rate were much more evident near the floor of the reactor than near the ceiling. Near the ceiling the sedimentation rate was actually slightly larger $(19 \mu \mathrm{m} / \mathrm{s})$ with no electric field than when an electric field was present (16 $\mu \mathrm{m} / \mathrm{s})$. Near the floor, the sedimentation rate was much larger when an electric field was present $(64 \mu \mathrm{m} / \mathrm{s})$ than when no electric field was present $(39 \mu \mathrm{m} / \mathrm{s})$. The data was processed further to acquire the particle size distribution at different experimental conditions. The experiments with no induced electric field showed signs of natural agglomeration, gravitational, and Brownian effects. Examining the results at the three different heights showed a shift in the overall particle size distribution from the top laser elevation to the bottom laser elevation. For no applied electric field, Table 4.3 presents mean diameters for the top, middle, and bottom laser elevations of $0.728,0.913$, and $1.02 \mu \mathrm{m}$ respectively. This suggests that the particles were agglomerating as they fell. This effect was enhanced/increased with the application of an electric field. This effect was further increased with an increase in electric field strength. For experiments with an applied voltage of 1500 Volts, Table 4.3 presents mean diameters for the top, middle, and bottom laser elevations of $0.715,1.08$, and $1.26 \mu \mathrm{m}$ respectively. This suggests that the presence of the electric field enhanced particle coalescence and caused the particles to sediment faster. Electro-coalescence caused the particles to increase in size by agglomeration, which caused them to settle out of the reactor faster using very little power. An overall decrease in the time that it took for the chamber to clear was seen when an electric field was present.

The final experimental procedure produced acceptable results. However, several improvements could be made. A few suggestions to improve the 
repeatability of the experiments are to add an electrically actuated valve to the inlet, use a high resistant (nichrome) wire to ignite the cigarette electronically, and control the whole process utilizing input and output from LabView. It would be beneficial to find a better way (quicker) to clean the windows of the reactor after each experiment. The EC experiments could be evaluated on the basis of power consumption if the exact power used during the experiment was measured.

Future experiments should be conducted to examine the effects of an acoustic field and a combination of an electric field and an acoustic field in the unit reactor. These effects could then be compared to the EC effects presented in this thesis. Experiments should also be conducted using different types of aerosols with known electrical properties to see if EC is truly dependent on the electrical properties of the aerosol. 


\section{BIBLIOGRAPHY}

Berry, M.V., and Percival, I.C. (1986) "Optics of Fractal Clusters Such as Smoke," Optica Acta, 33, 577-591

Beckwith, T.G., Marangoni, R.D., and Lienhard, J.H. (1993) Mechanical Measurements, Addison-Wesley, Reading, Massachusetts.

Callen, H.B. (1985) Thermodynamics and an Introduction to Thermostatistics, John Wiley and Sons, New York

Cavicchi, T.J. (1993) Fundamentals of Electrical Engineering: Principles and Applications, Prentice Hall, Englewood Cliffs, New Jersey.

Crowley, J. M. (1986) Fundamentals of Applied Electrostatics, John Wiley and Sons, New York. Davis, M.H. (1964) Two Charged Spherical Conductors in a Uniform Electric Field: Forces and Field Strength. Quart. J. Mech. Appl. Math. 17, 499.

Erker, J.A. and Baygents, J.C. (1996) NASA Conference Publication 3338, 731

Ezekoye, O.A. and Wibowo, Y.W. (1999) Simulation of Acoustic Agglomeration

Processes using a Sectional Algorithm. J. Aerosol Sci. 30, 1117-1138

Fabian, P., Cusack, R., Hennessey, P., and Neuman, N. (1993) Chem. Eng.

$100(11)$ 148.

Farius, T.L., Carvalho, M.G., Köylü, Ü.Ö., and Faeth G.M. (1995) Computational

Evaluation of Approximate Rayleigh-Debye-Gans/Fractal-Aggregate

Theory for Absorption and Scattering Properties of Soot. J. Heat Tran. $117,152-159$

Harriot, G. M., and Saville, D.A. (1971) AIChE J., 26, 325.

Hect, E (1998) Optics, Addison-Wesley, Reading, Massachusetts

Henry, F. S., and Ariman, T. (1986) ASTM Committee F-21, Fluid Filtration:

Gas, 1, 13.

Hinds, W.C. (1982) Aerosol Technology: Properties, Behavior, and Measurement of Airborne Particles, John Wiley and Sons, New York, 315-344 Incropera, F.P. and DeWitt, D.P. (1996) Fundamentals of Heat and Mass

Transfer, John Wiley and Sons, New York

Keh, H.J. and Chen, S.B. (1989a) J. Colloid Interf. Sci. 130, 542.

Keh, H.J. and Chen, S.B. (1989b) J. Colloid Interf. Sci. 130, 556.

Latham, J. and Roxburgh, I.W. (1966) Proc. Roy. Soc. A 295, 84.

Loewenberg, M. and Davis, R.H. (1995) J. Fluid Mech. 89, 123. 
Manoucheri, M. and Ezekoye, O.A. (1995) Polystyrene Soot Agglomeration Enhancement in an Ultrasonic Acoustic Field. J. Haz. Waste Haz. Matls.

$13,121-$ 130

Manoucheri, M.P. (1995) Investigation of Acoustic Agglomeration in a Three

Dimensional Standing Wave Field. Master's Thesis, University of Texas at Austin

Martin, K.M. (1997) Acoustic Filtration in a Closed System. Master's Thesis, University of Texas at Austin.

Panton R.L. (1984) Incompressible Flow, John Wiley and Sons, New York

Ptasinski, K.J. and Kerkhof, P.J.A.M. (1992) Electric Field Driven Separations: Phenomena and Applications. Sep. Sci. Technol., 27, 995.

Reist, P.C. (1993) Aerosol Science and Technology, McGraw-Hill, New York. Serway, R.S. (1990) Physics for Scientists and Engineers with Modern Physics, Saunders College, Philadelphia

Seigel, R. and Howell, J.R. (1992) Thermal Radiation Heat Transfer, Hemisphere, Washington

Silverman, L., Billings, G.E., and Dennis, R. (1954) USAEC Report NYO 1592, Harvard University.

Stokes, C. A. (1950) Sonic Agglomeration of Carbon Black Aerosols. Chemical Engineering Progress Transactions, 46, 423-432.

Wark, K. and Warner, C.F. (1981) Air Pollution: Its Origin and Control, Harper and Row, New York

Waterman, L. C. (1965) "Electrical Coalescers", Chem. Eng. Prog., 61, 51-57.

Williams, T.J. and Bailey, A.G. (1983) Inst. Phys. Conf. Ser. 66-II, 39.

Williams, T.J. and Bailey, A.G. (1986) Changes in Size Distribution of a WaterEmulsion Due to Electric Field Induced Coalescence. IEEE Trans.

in-Oil 536.

Zhang, X. and Davis, R.H. (1991) The Rate of Collisions Due to Brownian or Gravitational Motion of Small Drops. J. Fluid Mech. 230, 479-504.

Zhang, X., Davis, R.H. and Ruth, M.F. (1993) J. Fluid Mech. 249, 227.

Zhang, X., Basaran, O.A. and Wham, R.M. (1995) Theoretical Prediction of Electric Field-Enhanced Coalescence of Spherical Drops. AIChE J. 41, 1629. 
Zhang, X. and Basaran, O.A. (1996) J. Fluid Mech. 326, 239. 


\title{
Effects of Lateral Oscillation on Sedimentation of Particles in Suspensions
}

\author{
by \\ Krishna Lakshminarasimhan, B. Tech.
}

\section{Thesis}

Presented to the Faculty of the Graduate School of

The University of Texas at Austin

in Partial Fulfillment

of the Requirements

for the Degree of

Master of Science in Engineering.

The University of Texas at Austin

December 2002 


\title{
Abstract \\ Effects of Lateral Oscillation on Sedimentation of Particles in Suspension
}

\author{
Krishna Lakshminarasimhan, M. S. E. \\ The University of Texas at Austin, 2002
}

Supervisor: Ofodike A. Ezekoye

Fly-ash particles in an aqueous suspension were found to resuspend on being shaken in an oscillating flow apparatus. The apparatus was resonantly driven at a frequency of $10 \mathrm{~Hz}$ with a peak to peak displacement of about 0.625 $\mathrm{cm}$. The mass loading of particles in the suspension at two different elevations were measured using $1 \mathrm{MHz}$ ultrasonic transducers. The ultrasonic transducers were calibrated for different concentrations with a well-mixed, non-shaking suspension of fly-ash and water. Experiments were conducted to find the effect of shaking on settling particles. It was found that the particles resuspended until particle concentration in the suspension reached an equilibrium vertical 
concentration variation. The experiments were then conducted with an aqueous suspension of china clay (kaolin) and the results were consistent with the previous results. The mechanism causing this phenomenon is not entirely clear. Internal gravity waves setup in the suspension due to sinusoidal forcing seems to be one of the factors contributing to the resuspension. A simple theoretical model of the apparatus containing two homogeneous liquids of different densities forming an interface based on internal gravity waves showed that cellular flow fields are being setup. Similar flow structures setup in the suspension could cause the particle resuspension. 


\section{Table of Contents}

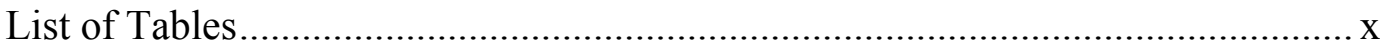

List of Figures ......................................................................................

Chapter 1: Literature Review. .................................................................. 1

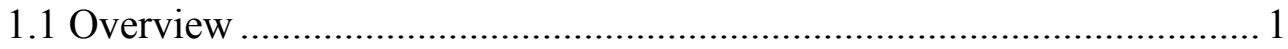

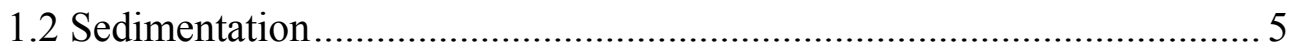

1.2.1 Sedimentation of fine particles.............................................. 6

1.2.2 Sedimentation of coarse particles......................................... 10

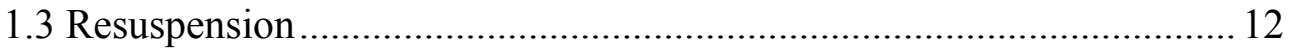

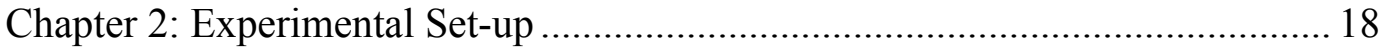

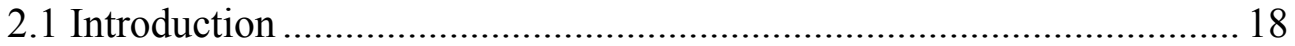

2.2 Structural and Design Considerations. ................................................. 19

2.3 Test Apparatus Construction............................................................... 20

2.4 Shaker Apparatus Performance....................................................... 22

2.5 Acoustics Measurement Techniques ................................................ 25

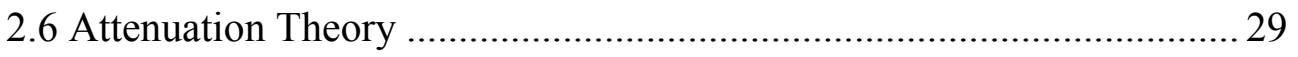

2.7 Ultrasonic Transducer Performance.................................................. 43

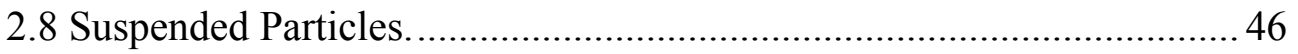

2.9 Calibration of Ultrasonic Transducers ............................................ 50

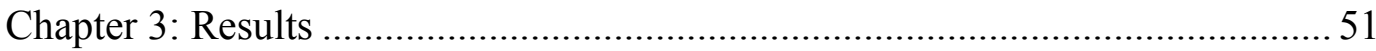

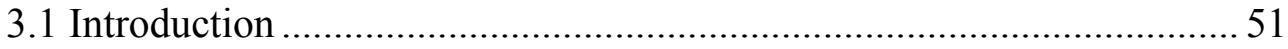

3.2 Sedimentation without Oscillation. ...................................................... 52

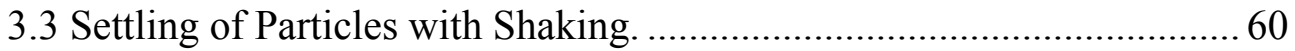

3.4 Shaking a Settled Bed of Particles. .................................................. 71

Chapter 4: Resuspension Mechanisms .................................................... 73

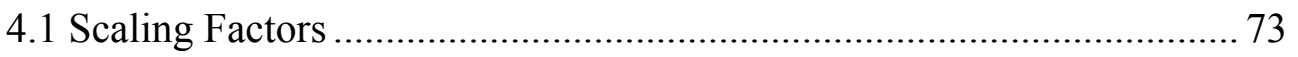




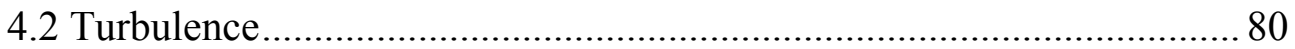

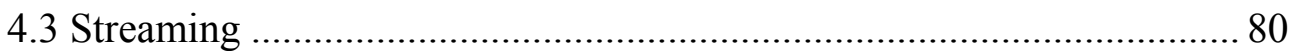

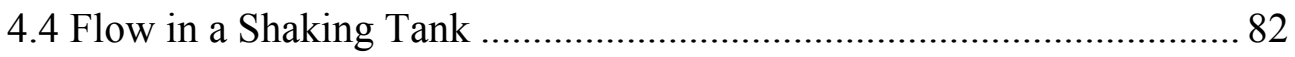

4.4.1 Analysis using Fluent. ........................................................... 82

4.4.2 Theoretical Analysis ...................................................... 88

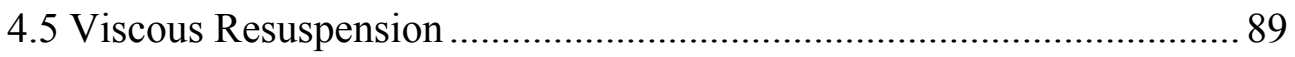

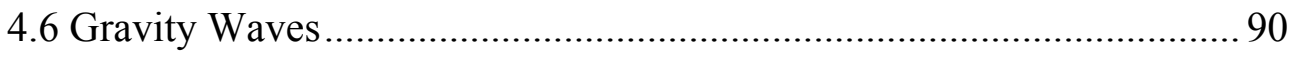

4.6.1. Superposed Fluids ............................................................. 91

4.6.2. Importance of Superposed Fluids........................................... 98

Chapter 5: Summary and Future Work …...................................................... 103

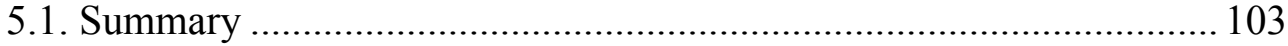

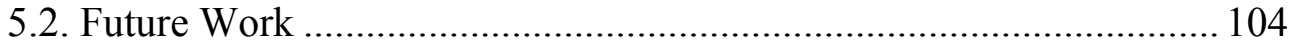

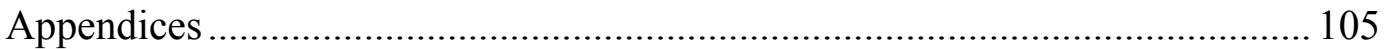

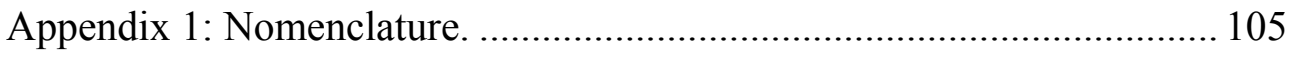

Appendix 2: Calculation of Ultrasonic Transducer Excitation. ................ 111

Appendix 3: Calibration of Ultrasonic Transducers. .............................. 112

A3.1 Experimental Procedure .................................................... 112

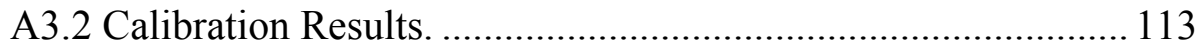

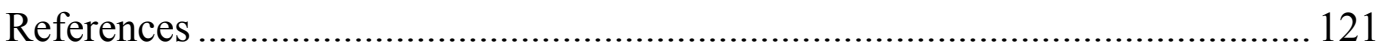

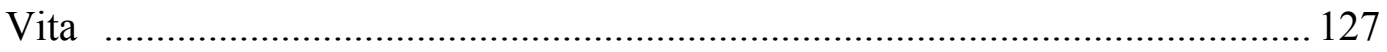




\section{List of Tables}

Table 2.1: $\quad$ Transducer excitation signal.......................................................... 28

Table 2.2: Experimentally obtained Physical Properties of Fly-ash and

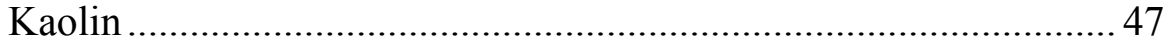

Table 2.3: $\quad$ Physical Properties of Kaolin and Fly-ash .................................... 49

Table 2.4: Chemical Properties of Kaolin and Fly-ash .................................. 49

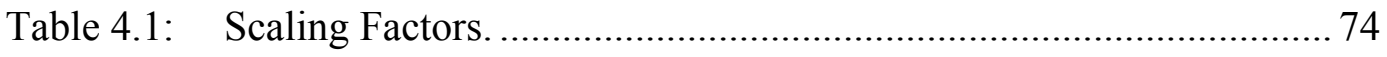

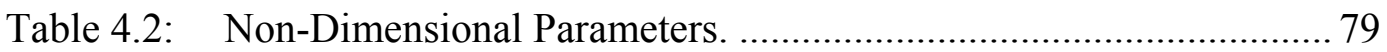




\section{List of Figures}

Figure 1.1: Schematic of a Multiphase system........................................... 2

Figure 1.2: Schematic of a Gravity Clarifier. ............................................... 3

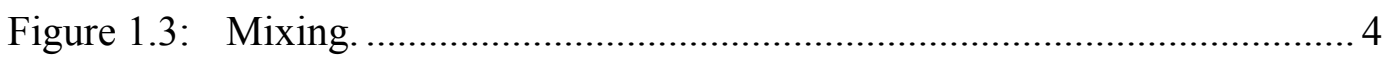

Figure 1.4: Sedimentation in concentrated suspensions (a) Sludge line settling (b) Selective Settling ...................................................... 7

Figure 1.5: Schematic of an Internal Gravity Wave. ....................................... 16

Figure 2.1: Schematic of the Experimental System........................................ 21

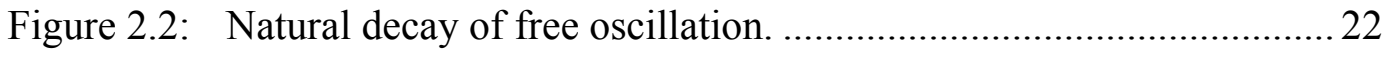

Figure 2.3: Shaker Frequency Response................................................. 24

Figure 2.4: Flow visualization in shaking tank. a) Before oscillation, b) 10 seconds after start of oscillation, c) 10 seconds of oscillation after the introduction of a free surface.................................................. 25

Figure 2.5: Experimental schematic for hydrophone measurements..................34

Figure 2.6: Cumulative PSD of Kaolin and Fly-ash..................................... 35

Figure 2.7: Experimentally determined attenuation for fly-ash and Kaolin

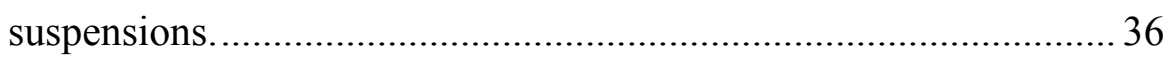

Figure 2.8: Integrals I1, I2, I3, and I4 for fly-ash suspension. ......................... 37

Figure 2.9: Attenuation results for fly-ash suspension. ................................. 38

Figure 2.10: Attenuation results for kaolin suspension. ................................... 38

Figure 2.11: Error in attenuation due to approximations for fly-ash

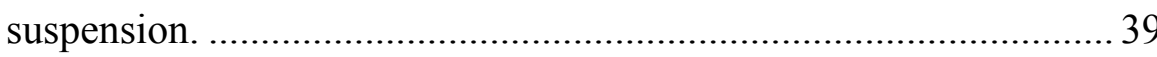

Figure 2.12: Error in attenuation due to approximations for kaolin suspension... 40 
Figure 2.13: Attenuation predicted by correlation (20) for fly-ash suspension.... 41

Figure 2.14: Attenuation predicted by correlation for Kaolin suspension. 41

Figure 2.15: Error in attenuation predicted by correlation for fly-ash suspensions 42

Figure 2.16: Error in attenuation predicted by correlation for kaolin suspensions 42

Figure 2.17: Top transducer response at no loading, no shaking. 44

Figure 2.18: Bottom transducer Response at no loading, no shaking .44

Figure 2.19: Top transducer response at no loading, $10 \mathrm{~Hz}$ shaking. 45

Figure 2.20: SEM image of Fly-ash Particles................................................ 48

Figure 2.21: Image of Kaolin (china-clay) Particles.......................................... 48

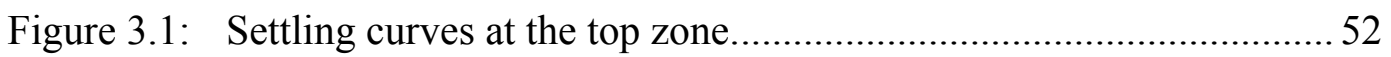

Figure 3.2: Settling curves at the bottom zone............................................ 53

Figure 3.3: Comparisons of Top and Bottom Zone Settling Curves. ................. 55

Figure 3.4: Settling rates at the top and bottom zones..................................56

Figure 3.5: Effect of Height of Suspension on Settling in the Top Zone. The curves correspond to $11,11.5$ and 12 inches of suspension height.

Figure 3.6: Effect of Height of Suspension on Settling in the Bottom Zone. The curves correspond to $11,11.5$ and 12 inches of suspension height. 59

Figure 3.7: Concentration for shaking experiments measured at Top Zone....... 61

Figure 3.8: Concentration for shaking experiments measured at bottom zone. .61 
Figure 3.10: Variation of Equilibrium Concentration $\left(\mathrm{C}_{\mathrm{f}}\right)$ with Initial Concentration $\left(\mathrm{C}_{0}\right)$ at the top and bottom zones. 64

Figure 3.11: Variation of Equilibrium Concentration (Cf) with $\mathrm{C}^{\prime}$ at the top zone. 65

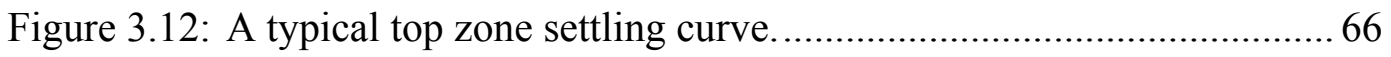

Figure 3.13: Power Spectral Analysis of top zone settling curve.......................67 67

Figure 3.14: Effect of height of suspension at the top zone. The curves correspond to $11,11.5$ and 12 inches of suspension height. 68

Figure 3.15: Effect of height of suspension at the bottom zone. The curves correspond to $11,11.5$ and 12 inches of suspension height. 69

Figure 3.16: Effect of amplitude of oscillation on settling of particles at the top zone.

Figure 3.17: Effect of amplitude of oscillation on settling of particles at the bottom zone. 70

Figure 3.18: Shaking a Settled Bed of Particles: Top Zone.............................. 71

Figure 3.19: Shaking a Settled Bed of Particles: Bottom Zone......................... 72

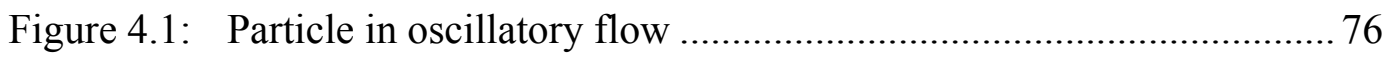

Figure 4.2: Contours of Stream Function at $\mathrm{t}=0.2$ seconds. ........................ 84

Figure 4.3: Contours of $\mathrm{x}$-velocity at $\mathrm{t}=0.2$ seconds.............................. 84

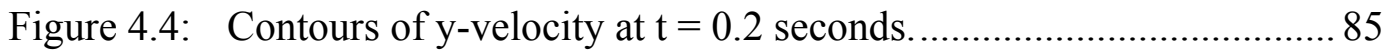

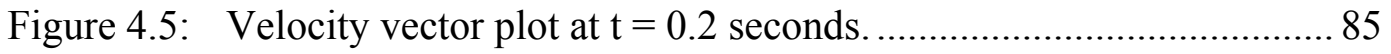

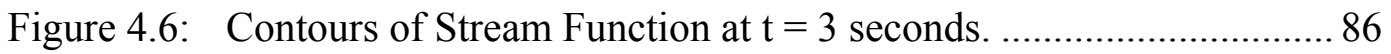

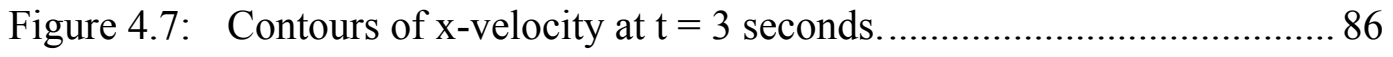




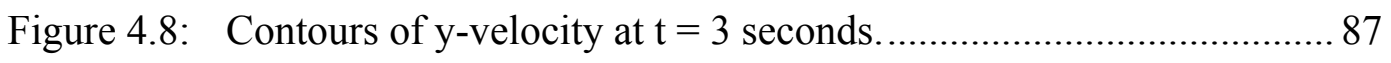

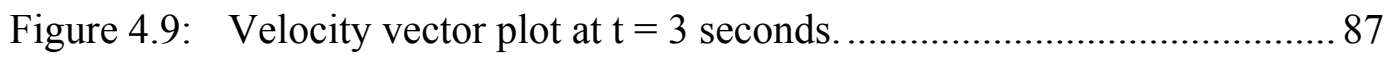

Figure 4.10: Contour plot of stream function for two shaking superposed

fluids with $\mathrm{R}=0.75$ and $\mathrm{H}=1$

Figure 4.11: Velocity vector plot for two shaking superposed fluids with $\mathrm{R}=$

0.75 and $\mathrm{H}=1$

Figure 4.12: Plot of Wave amplitude variation with time at the interface along the $\mathrm{x}$-axis for $\mathrm{R}=0.45$ and $\mathrm{H}=1.0$ 96

Figure 4.13: Plot of Wave amplitude at the interface for different modes with

$\mathrm{R}=0.45$ and $\mathrm{H}=0.11$.

Figure 4.14: Plot of wave amplitude variation with time at the interface along the $\mathrm{x}$-axis with $\mathrm{R}=0.45$ and $\mathrm{H}=0.11$.

Figure 4.15: Plot of wave amplitude for different modes with $\mathrm{R}=0.45$ and $\mathrm{H}$ $=0.11$ 98

Figure 4.16: 2-layer model. 100

Figure 4.17: Plot of interface position with time for $\mathrm{R}=0.45$ and initial settled layer height of $5 \mathrm{~cm}$. 101

Figure 4.18: Plot of interface position with time for $\mathrm{R}=0.45$ and initial settled layer height of $15 \mathrm{~cm}$. 101

Figure A3.1: Calibration curve for top transducer in fly-ash suspension. 113

Figure A3.2: Calibration curve for bottom transducer in fly-ash suspension...... 114

Figure A3.3: Calibration curve for top transducer in kaolin suspension............. 116

Figure A3.4: Calibration curve for bottom transducer in kaolin suspension....... 117 
Figure A3.5: Calibration curves for Top Transducer in fly-ash suspension........ 118

Figure A3.6: Calibration curves for Bottom Transducer in fly-ash suspension. . 119 


\section{Chapter 1: Literature Review.}

\subsection{OVERVIEW}

Multiphase flows are important in many areas of the chemical and process industries. Some of the important multiphase systems are:

- Mixtures of liquids with gas or vapor.

- $\quad$ Liquids mixed with solid particles.

- $\quad$ Gases carrying solid particle wholly or partly in suspension.

- $\quad$ Mixtures containing solids, liquids and gases.

This article will be restricted to systems containing liquids mixed with solid particles. The system considered consists of a rectangular tank containing low Reynolds number particles suspended in a liquid. The tank is completely filled with this suspension and there is no free surface at the top of the tank. The tank is mounted on a roller bearing and the system is subjected to a periodic forcing. Figure 1.1 shows the schematic of the multiphase system. It is observed that the stability of the multiphase system varies with the periodic forcing, that is, the sedimentation rate varies with the forcing and for some forcing, resuspension may occur. The analysis of the stability of such systems is important in many industrial applications. 


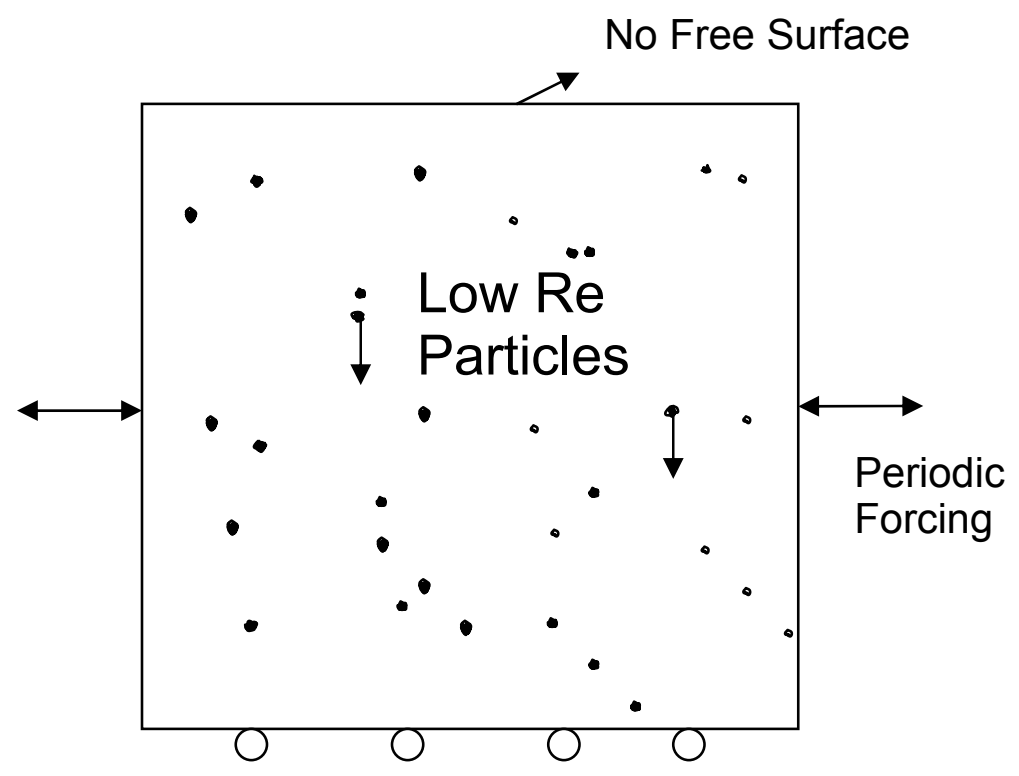

Figure 1.1: Schematic of a Multiphase system.

For example, the effectiveness of clarification of gravity clarifiers is affected by the stability of multiphase system. In a gravity clarifier, there is an inflow of liquids mixed with solids as shown in figure 1.2. The mixture is clarified by the sedimentation of particles. After clarification, clear liquid is taken from the top of the clarifier. However, flow disturbance sets up sloshing in the system, thereby, causing resuspension to occur. Thus, sloshing decreases the sedimentation rate and causes fluctuation in the concentration of clarified liquid. The clarification effectiveness is reduced as a result of sloshing set up due to instability of multiphase system. 


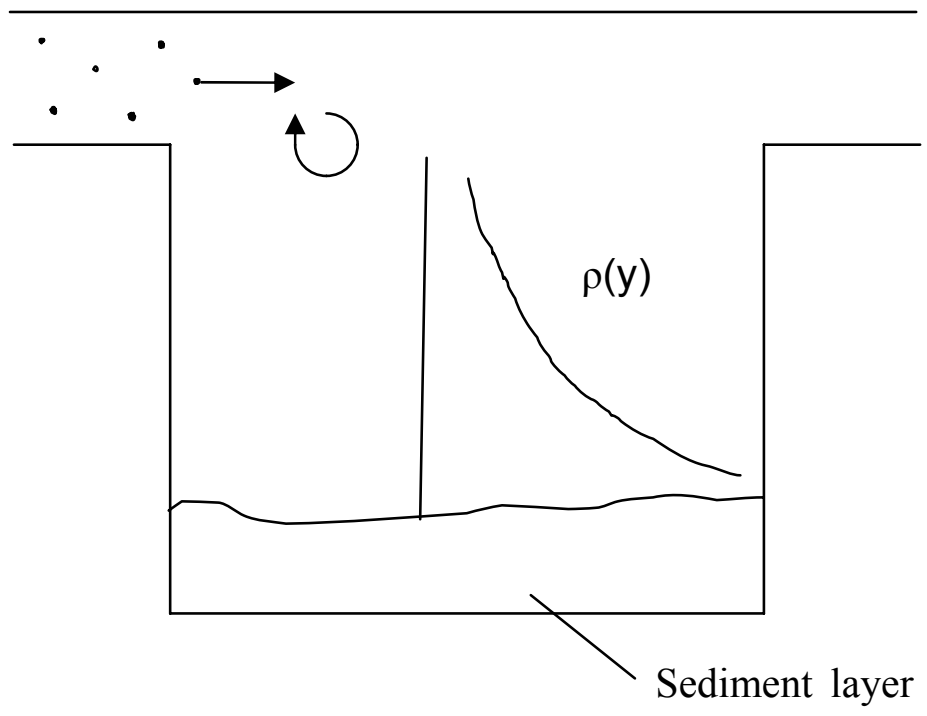

Figure 1.2: Schematic of a Gravity Clarifier.

Sometimes, unlike in a clarifier, mixing is performed in order to produce uniform mixtures. Mechanical agitation may be used to suspend particles in a liquid to promote chemical reaction or mass transfer. Liquids involved have low viscosity and usually the particles settle down as soon as the mechanical agitation ceases. Figure 1.3 shows mixing produced by propeller. The propeller resuspends the solid particles and maintains them in the suspension. Mixing is often constrained by the power requirements of the propeller. In this system, resuspension arising due to the instability of the system is of importance. 


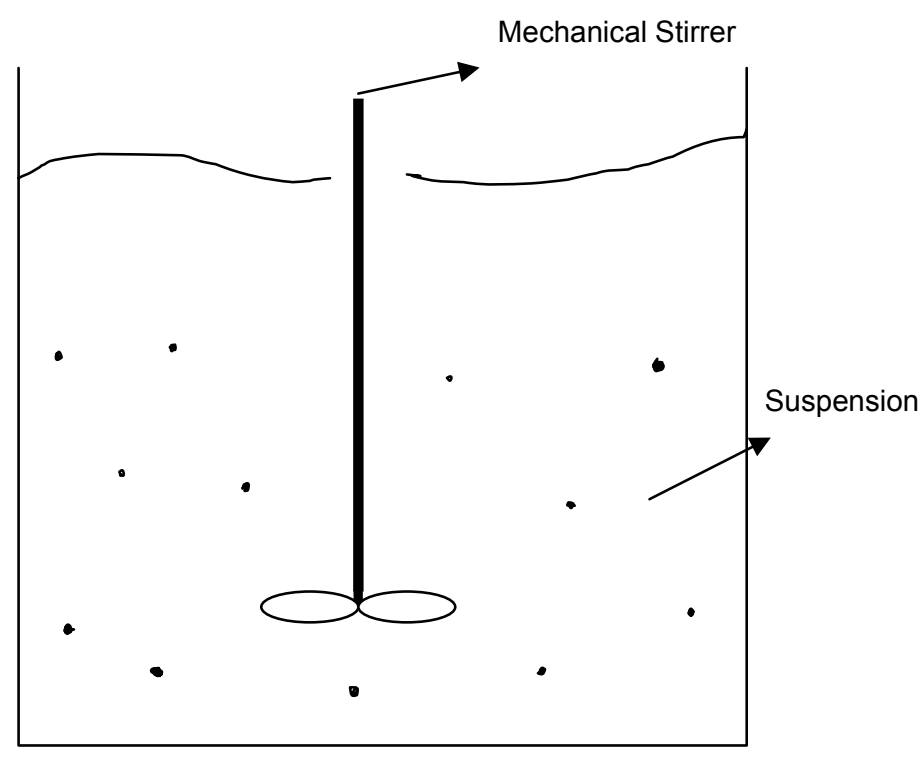

Figure 1.3: Mixing.

The system shown in figure 1.1 can be used in resonant mixing of solids in a suspension. If the forcing were done at the resonance frequency of the system, the power is required only to overcome the friction in the bearings and the disturbance caused due to the relative motion of particles. In the absence of particles, the fluid moves with the tank in a rigid body motion. The presence of particles causes a vortical flow leading to mixing.

The objective of this work is threefold, namely,

- $\quad$ To study the stability of a multiphase stratified liquid system as shown in figure 1.1, 
- $\quad$ To estimate the sedimentation rate of the settling particles, and

- $\quad$ To study the characteristics of internal gravity waves.

In the following sections, processes like sedimentation and resuspension, internal gravity waves and various techniques for characterizing a suspension are described briefly.

\subsection{SEDIMENTATION}

Sedimentation is the process in which solid particles move downwards due to the action of gravity while displacing an equal volume of liquid upwards. The liquid exerts a viscous drag force on the particles due to their relative motion. After a certain time period, the net gravitational force on the particle (weight buoyancy force) is balanced by the viscous drag force and settling particles attain a terminal settling velocity.

Sedimentation in concentrated suspensions is different from that of dilute suspensions. In concentrated suspensions, the interaction between particles is significant and the drag exerted by the liquid on the particles for a given velocity may be greatly increased due to the change in flow pattern. In some cases, hindered settling takes place. Thus, the sedimentation rate of particles in concentrated suspensions is considerably less compared to the terminal settling velocity of the particle under free settling conditions.

The particles in a suspension of fine particles settle differently than particles in a coarse suspension. This is due to the high degree of flocculation that may occur as a result of the high specific surface of the particle. 
Although the sedimentation velocity of particles tend to decrease as the concentration is increased, it was shown by Kaye and Boardman (1962) that particles in a concentrated suspension may settle at velocities up to 1.5 times their terminal velocities. This is attributed to the cluster of particles formed which settle in well defined streams.

In this section, sedimentation of fine and coarse particles as well as sedimentation in concentrated and dilute suspensions is discussed briefly. For a better understanding of the processes involved in sedimentation the author suggests reading the book by Coulson and Richardson (1991)

\subsubsection{Sedimentation of fine particles}

Coe and Clevenger (1916) studied the sedimentation in slimes and concluded that fine particles in concentrated suspension may settle in one of two ways.

- $\quad$ Sludge line settling.

- $\quad$ Selective settling. 


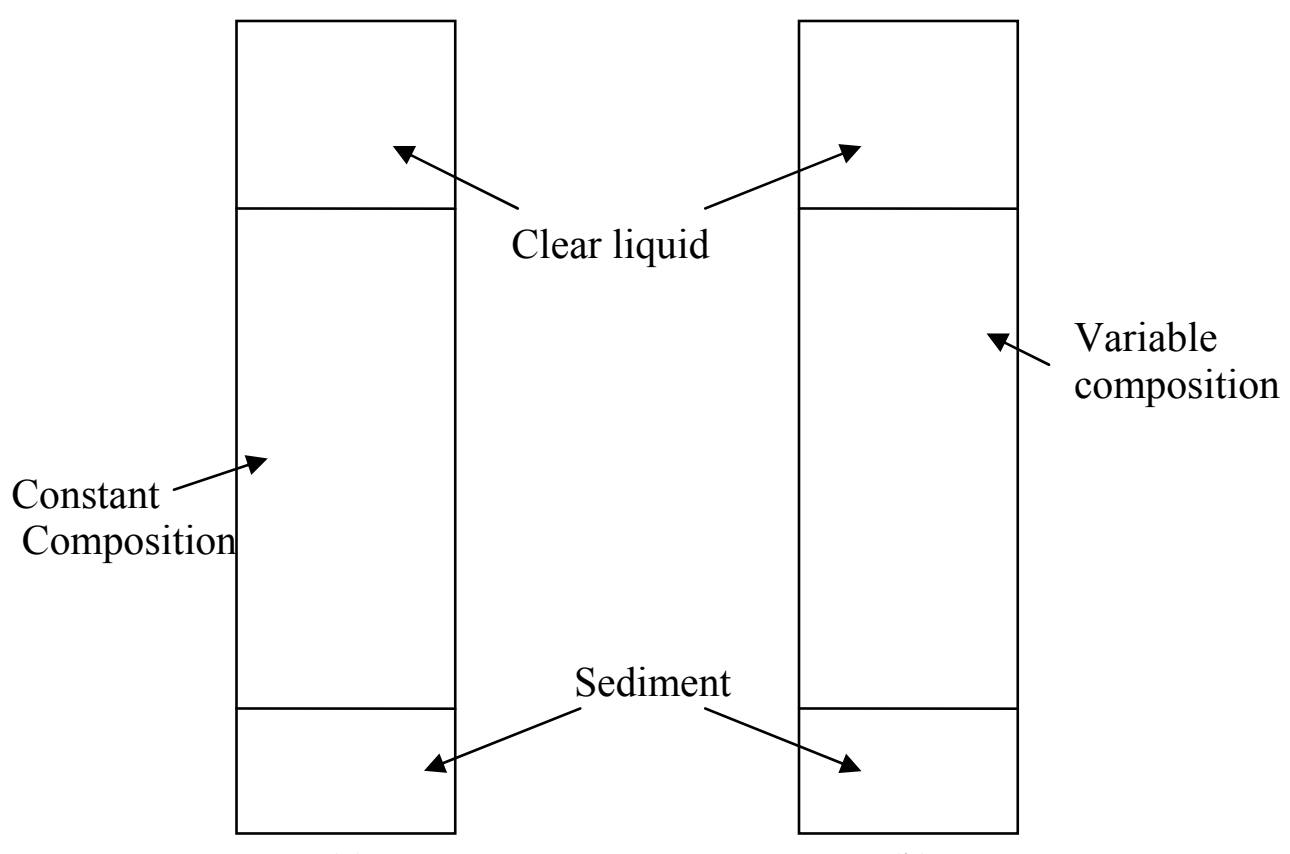

(a)

(b)

Figure 1.4: Sedimentation in concentrated suspensions (a) Sludge line settling (b) Selective Settling

Figure 1.5 shows the zones formed when suspensions undergo (a) sludge line settling, and (b) selective settling. In sludge line settling, there is a zone of clear liquid at the top below there is a zone of constant composition. Due to the presence of this constant composition zone, an interface can be clearly seen at the beginning of constant composition zone. In selective settling, below the clear liquid zone there is a variable composition zone. Due to the absence of a constant composition zone an interface is not seen at the beginning of variable composition zone. In both type of settling, there is sediment layer formed at the bottom of the tank. If the range of particle size is not more than $6: 1$, the concentrated suspension 
undergoes sludge line settling. Suspensions having particle size range more than 6:1 undergo selective settling. Robinson (1926) modified the Stokes' law in order to predict the apparent settling velocity of particles in concentrated suspensions. Instead of using the density and viscosity of the liquid, he used the density $\left(\rho_{c}\right)$ and viscosity $\left(\mu_{c}\right)$ of the suspension.

$$
u_{c}=\frac{K^{\prime \prime} d^{2}\left(\rho_{s}-\rho_{c}\right) g}{\mu_{c}}
$$

Robinson determined the viscosity of the suspension experimentally, but this can also be obtained using the formula given by Einstein (1906).

$$
\mu_{c}=\mu\left(1+k^{\prime \prime} C\right) .
$$

In the above equation, $k$ " represents a constant for a given shape (2.5 for spheres), $C$ represents the concentration of particles in the suspension, and $\mu$ is the viscosity of the fluid.

Other experimentalists [Steinour (1944), Hawksley (1950)] correctly assumed that the upward thrust acting on the particle is determined by the properties of the suspension rather than the fluid. It should be noted that this assumption is true only for large particle settling in a suspension of fine particles. In suspensions of uniform particles, the increased drag is attributed to the steepening of the velocity gradient rather than to a change in viscosity.

The rate of sedimentation of a suspension of fine particles depends on the degree of flocculation. Most fine suspensions flocculate in water and a deflocculating agent should be added to keep the particles individually dispersed. Another factor affecting the sedimentation rate is the degree of agitation of the suspension. A small degree of agitation produces an accelerating effect if the 
suspension acts like a non-Newtonian fluid for which the viscosity is a function of the rate of shear.

Other factors affecting the rate of sedimentation are the height of the suspension, the characteristic length of the vessel and the concentration of the suspension. The sedimentation rate may be reduced for small values of characteristic length due to the wall effects. The higher the concentration, the lower the sedimentation rate for sludge line settling since the upward velocity of the displaced liquid is greater and the steeper are the velocity gradients in the liquid. Egolf and McCabe (1937), Work and Kohler (1940) and others have given empirical relations for the sedimentation rate.

Kynch (1952) predicted the settling behavior of slurries, including the formation of concentration shocks in some instances, without specifying the force on the particles. Other notable works are Brenner's work on multi-particle systems (1983), Batchelor's derivation of the mean velocity of dispersion (1972), and the studies of multi-particle systems by Mazur and Van Saarloos (1982). Kermack et al (1929) derived the dependence of the mean velocity of dispersion on the solids concentration and their theoretical and experimental coefficients were close to modern values. Though some of Kynch's results have been cited by others, the description of his method was too complicated for others to be useful. For more information on the sedimentation of small particles, the author suggests reading Tory (1996). 


\subsubsection{Sedimentation of coarse particles}

Coarse particles have much lower specific surface compared to fine particles and consequently surface forces between particles are less significant than in the fine particle systems. Flocculation is absent and the particles do not influence the rheology of the liquid. The critical size is of the order of $0.1 \mathrm{~mm}$ or $100 \mu \mathrm{m}$.

Several studies have been made on the sedimentation of uniform particles. Richardson and Zaki (1954) noted that the drag force on a particle, in a concentrated suspension, is dependent on the relative velocity of the particle with respect to the liquid. The relative velocity is a function of the concentration of the suspension, or in other words, a function of voidage of the suspension. For a given suspension, they found that the log-log plot of sedimentation rate and the voidage is a straight line. The equation relating the settling velocity of particles in a concentrated suspension and the voidage in the suspension is given by the following equation:

$$
\log u_{c}=n \log e+\log u_{o}
$$

where $u_{c}$ is the settling velocity in the concentrated suspension, $e$ is the voidage, $u_{o}$ is the settling velocity of a single particle in an infinite fluid and $n$ is the Richardson-Zaki exponent.

Richardson and Zaki found the slope of this straight line experimentally. Several relations have been obtained for finding this slope [Rowe (1987), Khan and Richardson (1989)]. The relation for this slope (Richardson-Zaki exponent) as suggested by Richardson and Khan is given by the following equation: 


$$
\frac{4.8-n}{n-2.4}=0.043 G a^{0.57}\left[1-2.4\left(\frac{d}{d_{t}}\right)^{0.27}\right]
$$

where $G a$ is the Galileo number given by:

$$
G a=\frac{d^{3} g \rho\left(\rho_{p}-\rho\right)}{\mu^{2}},
$$

and $d / d_{t}$ is the particle diameter to vessel dimension ratio.

Various approaches to sedimentation in concentrated suspension are given in a paper by Khan and Richardson (1990).

In a suspension containing particles of two different sizes but same density, the large particles have higher settling velocities than the smaller particles. There are four zones formed instead of the three zones in sludge line settling, namely,

- Clear liquid,

- $\quad$ Suspension of fine particles,

- $\quad$ Suspension of mixed sizes, and

- $\quad$ Sediment layer.

Richardson and Shabi (1960) have shown that in suspensions of particles of mixed sizes, the total concentration rather than the individual concentration which controls the settling rate of each particle. Richardson and Meikle (1961) investigated the sedimentation of suspensions containing two different types of particles and experiments clearly showed that the tendency for segregation to occur in a two component mixtures increases with increase in total concentration. This behavior is in contrast to suspensions containing multi-sized particles of a given material where segregation reduces as the concentration is increased. 
The suspension to be discussed in this thesis has a maximum particle concentration of $2 \%$ by mass (less than $1 \%$ by volume). Also the particles used are fine having an average diameter of $4 \mu \mathrm{m}$. Therefore the sedimentation of particles in this system can be well described by equations (1.1) and (1.2).

Section 1.2 described sedimentation in dilute and concentrated suspensions. The opposite of sedimentation or settling is resuspension. Studies are going on to understand the various mechanisms of resuspension. Some of these are described in the following section.

\subsection{RESUSPENSION}

The phenomenon of resuspension is the process in which an initially settled layer of negatively buoyant particles is entrained into the bulk fluid and convected away. For resuspension to occur, the net gravitational force on the particle should be overcome by a lift force exerted by the liquid flow on the settled or settling particle. The study of resuspension has important industrial applications in the transport of particulates in the form of slurries through pipelines.

The conditions under which resuspension occurs is not clearly known. Typically this process has been associated with high Reynolds number and turbulent flows. The dispersion and resuspension of particles in turbulent flow has received much theoretical and experimental interest. The gravitational settling of aerosol particles in randomly oriented steady cellular flow fields with one length scale was studied by Maxey and Corrsin (1986). They found that the inertia of particles, though weak or moderate, played a significant role and caused the 
particles to get trapped in some isolated paths. Crisanti et al (1990) studied the Lagrangian motion of particles in a steady two-dimensional cellular flow where the motion of the fluid elements is regular. Particles slightly heavier than the fluid element but with strong inertia may induce chaotic motion and dispersion like phenomena in simple regular flows. For the same cellular flow, Shin and Maxey (1997) found that the settling motion of a non-spherical particle with large aspect ratio is chaotic even in the absence of particle inertia. More recently Wang et al (1992) found that under certain conditions of Stokes number and density ratio, the particle motion may be chaotic. The average settling velocity of small spherical particles under gravity through an infinite, periodic, non-divergent unsteady cellular flow field subject to the effects of the Stokes' drag and the inertia of the particles was studied numerically and experimentally by Fung (1997). He found that, for high Stokes terminal settling velocity of the particles, the particles accumulated at the boundary of the cells and that the tendency of particles to spend more time on the upward flow region of the cell increased.

The vortex flow can be caused by, instead of turbulence, streaming. Streaming flow is a steady vortex flow caused by the viscosity of the liquid. It characteristics feature is that the streaming velocity is independent of viscosity though it is created because of viscosity. This was first noted by Lord Rayleigh in 1883. Acoustic streaming has received a good deal of attention. This acoustic streaming occurs in the second approximation with respect to the wave amplitude. The theoretical considerations involved in acoustic streaming are detailed in Landau and Lifshitz (1959) and Hamilton and Blackstock [25]. 
The phenomenon of viscous resuspension, i.e., resuspension which occurs at sufficiently low Reynolds number such that inertial effects are insignificant and the flow is laminar. Viscous resuspension was first observed by Gadala-Maria (1980) during the course of his measurements of the rheological properties of suspensions of coal particles in viscous Newtonian fluids. Leighton and Acrivos (1986) demonstrated that the viscous resuspension results from a balance between the gravitational force and an upward flux arising from shear induced particle diffusion. Leighton and Acrivos (1987) determined the shear induced diffusion coefficients for the two directions of primary significance in parallel shear flows: the direction normal to the plane of shear and that parallel to gradients on fluid velocity within the plane of shear. These coefficients are important in determining the concentration profile of particles in suspensions undergoing shear flows. Also they emphasized that the drifts identified were viscous in nature, unlike wellknown sources of particle migrations such as the Segre-Silberberg effect for flow through tubes [Ho and Leal (1974)]. Schaflinger et al (1989) investigated viscous resuspension in a Hagen-Poiseuille channel flow and in a corresponding gravitydriven flow along an inclined plate. They found that, in the former case, the region occupied by the suspension cannot extend beyond the plane of zero stress, while for the film flow, a critical value for feed concentration is predicted beyond which steady state operation is no longer possible.

Resuspension and mixing could also be caused by other types of flow like the oscillatory flow. Levesley and Bellhouse (1997) reported observations in which some suspended neutrally buoyant particles are retained within a straight 
channel through which the suspension is flowing by adding an oscillatory component to the flow. They correlated the degree of retention of particles with the frequency and amplitude of the applied oscillation. They also noted that under certain conditions, single particles could be trapped in fixed positions within the channel. Andrieu et al (1992) measured the mean sedimentation rates of spherical and cylindrical particles in pulsating flow at different frequencies and amplitudes of motion of the suspending liquid. Their results show the effects of frequency and the amplitudes of oscillation, viscosity of the liquid and the shape of particles. Mackley et al (1993) reported experimental observation on the way particles that would normally sediment in a liquid, can be maintained in suspension by the use of oscillatory flow in baffled tubes. In one regime of operation, they found that a near uniform suspension of particles can be obtained and in another regime, the particle concentration profiles along the tube were observed.

There are other mechanisms that can lead to resuspension like the internal gravity waves (IGW). The phenomenon of IGW is discussed in the following paragraphs.

Gravity waves are the waves that are created due to the presence of a sharp density gradient at an interface. When there is a perturbation, two points at the same height have different pressures due to the density gradient. 


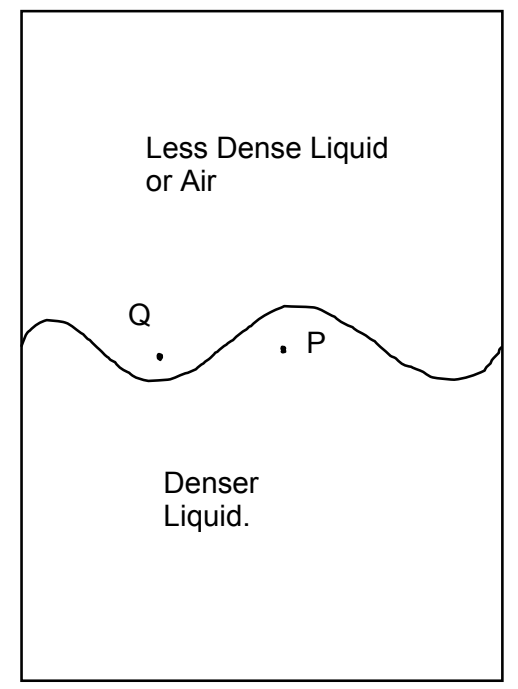

Figure 1.5: Schematic of an Internal Gravity Wave.

For example, in figure 1.6, point $\mathrm{P}$ is at a higher pressure than the point $\mathrm{Q}$ though they are at the same elevation. This difference in pressure causes the liquid near point $\mathrm{P}$ move towards point $\mathrm{Q}$. Thus the wave is propagated along the interface. A vortical flow is created due to the presence of a pressure gradient and a density gradient. This makes the particles near the interface to follow a circular or an elliptical path. When two liquids are separated by an interface, then the gravity waves produced at the interface are called Internal Gravity Waves (IGW) or sometimes simply internal waves.

Experimental and theoretical work is being conducted in order to understand the gravity waves. These waves are of special importance in many of the industrial processes like clarification processes. For example, the existence of internal waves in gravity clarifiers causes fluctuations in the output concentration of the clarifier. Lionel Catalan et al (1991) studied the locale of internal waves inside a clarifier tank and showed that the internal waves are present only in the 
upper third of the tank. They also found that there is an increase in clarification efficiency resulting from the use of flow contraction baffle and that this increase is not combined with the decrease in internal wave amplitude within the clarifier. In gravity clarifiers, the perturbation is caused by the inflow of the liquid to be clarified.

Resuspension was found in the experimental system that will be described in the next chapter. The reason for this resuspension could be due to mixing caused by IGW and the flow field it sets up in the participating fluid(s). IGW sets up a cellular flow field in the fluid and as mentioned already, cellular flow field can cause mixing. IGW also creates a zone where particles get mixed. This mechanism is discussed in detail in the fourth chapter of this thesis.

In this chapter, some of the important issues of the project to be discussed in this thesis were explained. A literature review of sedimentation and resuspension was provided earlier in this chapter. The next chapter will detail the experimental set up and the ultrasonic measurement technique used in measurement of concentration 


\section{Chapter 2: Experimental Set-up}

\subsection{INTRODUCTION}

The sedimentation of particles in a suspension is an example of Low Reynolds number fluid flow. A detailed review of literature on the sedimentation of particles has been given in the previous section. There are two conflicting phenomena that have been witnessed. On one hand, the particles tend to repel each other in a direction perpendicular to the axis of sedimentation. On the other hand, the particles can come together and agglomerate under the action of an external field. It has been found that particles tend to agglomerate under the action of acoustic field.

The purpose of this research and hence this experimental setup is to study the effects of lateral oscillation on the process of sedimentation of particles. The oscillatory system developed facilitates visualization experiments as well as concentration measurements using ultrasound. Water was chosen as the suspending fluid with an absolute viscosity of $10^{-3} \mathrm{~N}-\mathrm{s} / \mathrm{m}^{2}$. Fly-ash particles with an average size of $2 \mu \mathrm{m}$ and density of $2270 \mathrm{~kg} / \mathrm{m}^{3}$ were used in the experiments.

The test apparatus was subject to a sinusoidal forcing setting up an effective time scale of $1 / f$. Another time scale in a low Reynolds number flow is the Stokes time given by 


$$
\tau_{s t}=\frac{\rho_{p} d^{2}}{18 \mu}
$$

where $\rho_{p}$ is the density of the particles, $d$ is the particle diameter and the $\mu$ is the absolute viscosity of the suspending fluid (water). Stokes characteristic time scale is the time required for a particle to attain the velocity of the surrounding fluid. The test apparatus was designed to provide fluid-particle interactions with characteristic time between the limiting flow values.

The system had a drop time (the time required for a particle to complete its descent) of about 50 seconds and to provide a reasonable number of oscillations the frequency was set at $10 \mathrm{~Hz}$. The typical amplitude of the sinusoidal forcing was about $1.25 \mathrm{~cm}(1 / 2$ inch). The lateral dimension of the apparatus was chosen to be $1 \mathrm{ft}(30 \mathrm{~cm})$ so that the apparatus dimension was large compared to the particle size.

\subsection{STRUCTURAL AND DESIGN CONSIDERATIONS.}

The low Reynolds number oscillating sedimentation system was made of a tank or container $1 \mathrm{ft}$ in dimension. In order to reduce the mass of the system, the container was made of $1.3 \mathrm{~cm}(0.5 \mathrm{in})$ thick aluminum. Also, the front, the back and the top faces of the container consisted of $1.3 \mathrm{~cm}$ thick acrylic windows to allow particle visualization. The total mass of the system including the tank of overall dimension $0.33 \mathrm{~m}$ or 13 inches filled with water was about $41 \mathrm{~kg}$ or 90 lbm.

The force that had to be exerted by a shaker on the mass was found using Newton's second law. 


$$
F=-\omega^{2} M_{s y s} x
$$

where $F$ is the force to be exerted, $\omega$ is the angular frequency, $M_{s y s}$ is the mass of the system and $x$ is the displacement of the system. The maximum force to be exerted on the system for a $M_{\text {sys }}=41 \mathrm{~kg}, \mathrm{f}=10 \mathrm{~Hz}$ and $\mathrm{x}=6.3 \mathrm{~mm}\left({ }^{1} / 4\right.$ inch) was found to be $1.02 \mathrm{kN}(230 \mathrm{lbf})$. This force was far greater than the force that can be provided by an in-house shaker. In order to overcome this limitation, a spring-mass system was developed to make use of the resonance. The spring constant for the fore-mentioned specifications was found to be $161.1 \mathrm{kN} / \mathrm{m}$ at 10 $\mathrm{Hz}$ frequency. Eight springs were used to produce the desired stiffness and to distribute the load over the surface of the support frame evenly. At $10 \mathrm{~Hz}$, the spring stiffness was found to be $20.1 \mathrm{kN} / \mathrm{m}$. The number of springs could be increased in order to change the resonance frequency, although the structural limitations of the material would restrict this possibility. More details about this experimental setup could be found in Carter (2000).

\subsection{Test APparatus CONSTRUCTION.}

In this section the construction of the oscillation apparatus will be explained. A schematic of the apparatus is shown in figure 2.1 below. A KrohnHite 4400A function generator drives the Unholtz-Dickie 5PM magnetic shaker. When the polarity of the sinusoidal input changes, the large electromagnet inside the shaker starts to oscillate. The magnetic shaker is connected to the tank by means of a steel connecting rod. The cubic tank having a dimension of $1 \mathrm{ft}$ (300 $\mathrm{mm}$ ) is constructed using quarter inch thick aluminum. The tank was filled with a 
suspension of water and fly-ash. The front and back faces of the tank are fitted with acrylic windows to allow for visualization of particle interaction of the container.

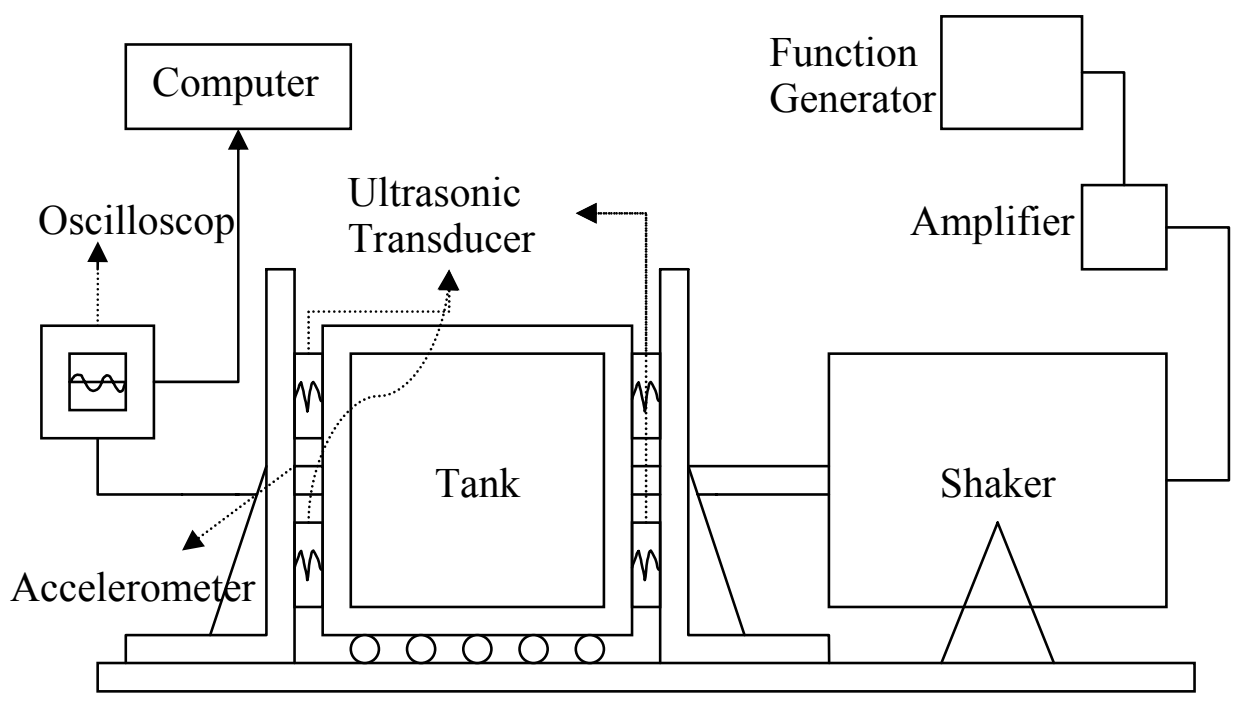

Figure 2.1: Schematic of the Experimental System.

The top face of the container is fitted with two circular acrylic lids. The lids do not have any slits in them in order to avoid free surface and air bubbles inside the tank.

Several types of diagnostics were used to characterize the global system dynamics as well as the local particle-fluid dynamics. The global systems were measured and analyzed using both accelerometer and optical diagnostics, while characterization of local dynamics posed a problem. After trying out different characterizing techniques, it was decided to use ultrasonic transducers in order to characterize the local dynamics. 
The side-mounted accelerometer output was fed through an amplifier into a PC running LabView where a virtual instrument (.vi) was written to obtain the desired information from the accelerometer output. The .vi performed a fast Fourier transform on the accelerometer output in order to obtain the frequency spectrum of the system response to the sinusoidal force exerted on the shaker apparatus.

\subsection{SHAKer APPaRatus PERformanCE.}

The free oscillation frequency and the natural damping coefficient of the shaker apparatus was found (Carter, 2000). This was done by displacing the tank from its equilibrium position and then releasing it. The accelerometer output was then captured on an oscilloscope. A typical accelerometer output is shown below.

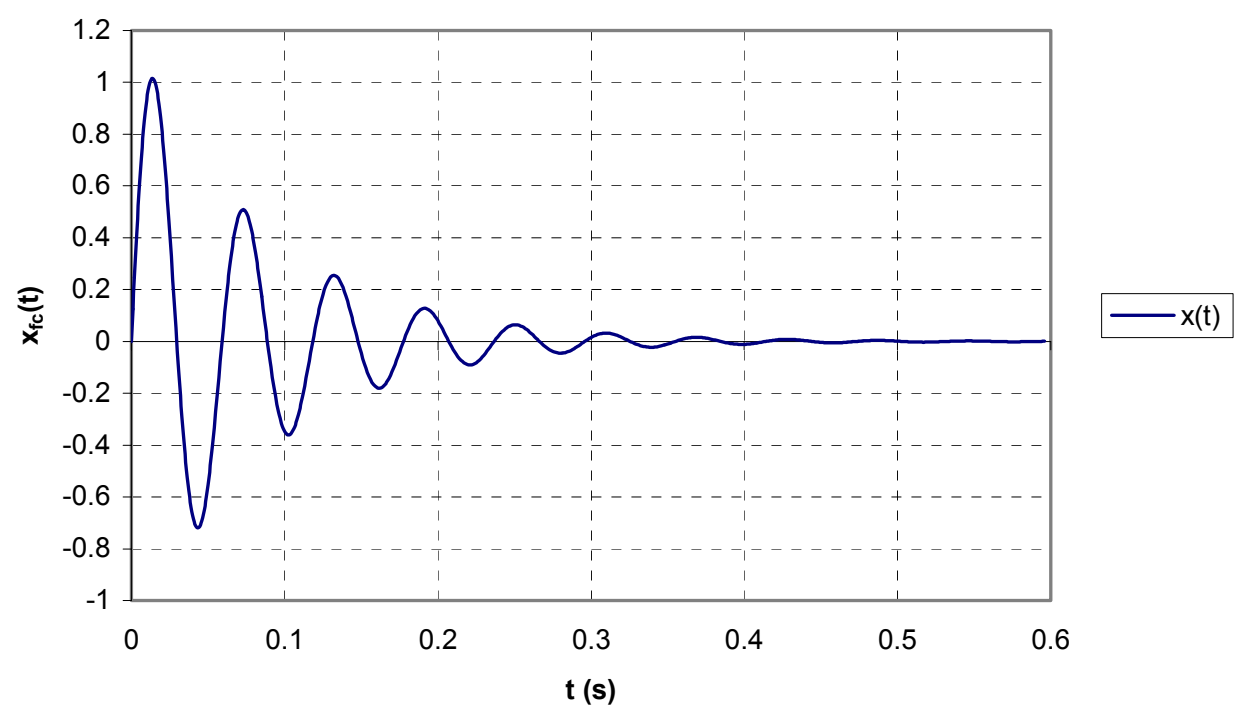

Figure 2.2: Natural decay of free oscillation. 
The decaying of the oscillation, nearly exponentially, indicates that there is a natural damping in the system. The motion of the shaker apparatus under free oscillation is given by the differential equation for the unforced response of a damped mass-spring system.

$$
m \ddot{x}+c \dot{x}+K x=0 .
$$

The damping ratio, $\zeta$, is defined as:

$$
\zeta=\frac{c}{2 m \omega} \text {. }
$$

The damping ratio can be found from the logarithmic decay of the system as seen in figure 2.2. If the logarithmic decrement is defined as

$$
\delta=\ln \frac{x_{f c}\left(t_{1}\right)}{x_{f c}\left(t_{1}+T\right)},
$$

the damping ratio can be found from the following relation:

$$
\zeta=\frac{\delta}{\sqrt{4 \pi^{2}+\delta^{2}}} .
$$

The damped natural frequency was determined to be $\zeta=0.11 \pm 0.01$. The natural frequency was determined to be $16.9 \pm 0.93 \mathrm{~Hz}$. This frequency is significantly different from the calculated natural frequency of $11 \mathrm{~Hz}$ based on the approximate weight and the spring constant.

A frequency sweep of the shaker was performed from $7 \mathrm{~Hz}$ to $15 \mathrm{~Hz}$. The constant power displacement response over this frequency range is given in figure 2.3 . 
Shaker Frequency Response (4.7 A)

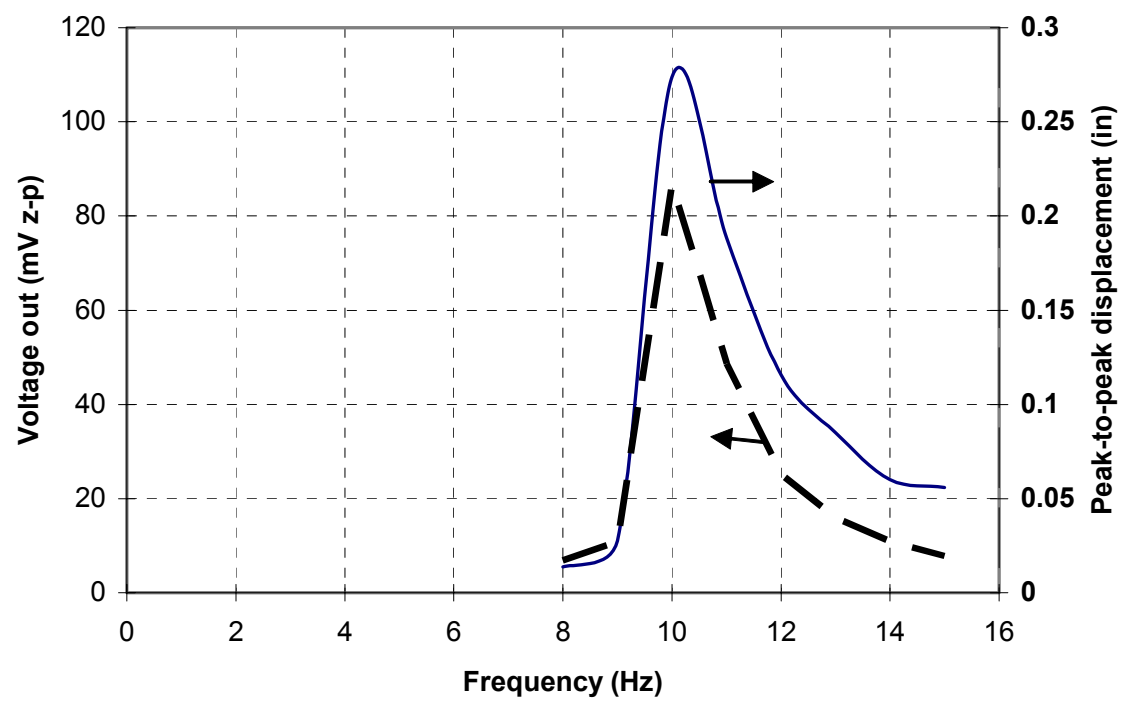

Figure 2.3: $\quad$ Shaker Frequency Response.

In figure 2.3, right ordinate axis corresponds to the dashed line and the left ordinate axis corresponds to the solid line. It is clear that the system displays resonance at a frequency of $10 \mathrm{~Hz}$ which is closer to the natural frequency found using the initial calculation based on apparatus mass and spring constant.

It is assumed that the fluid in the shaker apparatus undergoes a rigid-body motion when there is no free surface. In order to verify this, a simple test was performed. The container was sealed with water containing a small amount of buoyant ink and oscillated at $10 \mathrm{~Hz}$. Then the container lid was opened to introduce a free surface. Figure 2.4 shows three video frames of the ink: a) before oscillation, b) no free surface, 10 seconds after commencement of oscillation and c) 10 seconds after introduction of a free surface. The comparison shows that the 
ink undergoes rigid body motion when there is no free surface while the motion is chaotic when there is a free surface.

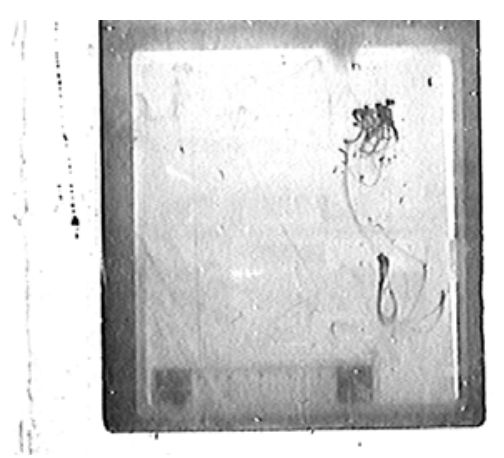

(a)

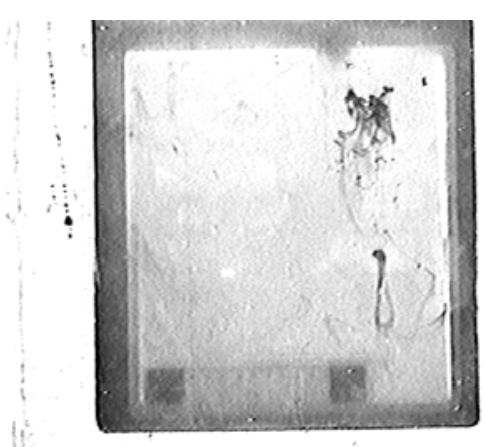

(b)

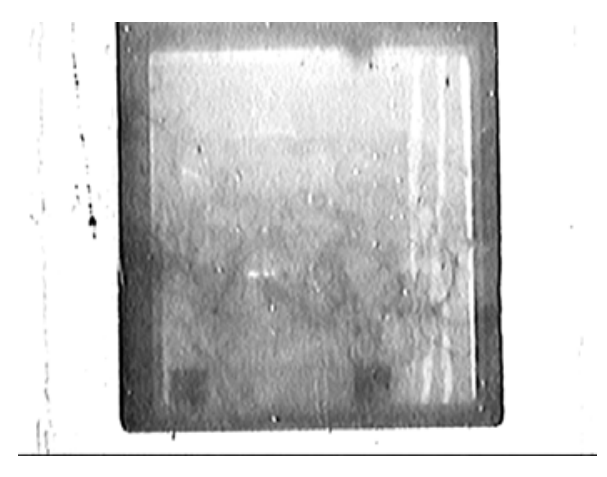

(c)

Figure 2.4: Flow visualization in shaking tank. a) Before oscillation, b) 10 seconds after start of oscillation, c) 10 seconds of oscillation after the introduction of a free surface.

\subsection{ACOUSTICS MEASUREMENT TEChNiQueS}

In this section, a brief overview of the measurement will be given. Subsequently, a detailed description of the ultrasonic transducers and the theory behind the measurements will be provided. 
Dispersed systems such as emulsions and suspensions play an important role in many industrial processes. Significant needs exist to develop measurement techniques for probing the physical and chemical properties of these systems and processes. Further, there is an increasing demand for online monitoring of these systems. Conventional methods, like laser diffraction, are limited to dilute system. This has led to the development of acoustic characterizing techniques.

Acoustic techniques have been used to find the concentration (particle loading) and particle size [Knudsen (1946); Urick (1948)]. Knudsen (1946) made absorption measurements in a water-fog system. Urick (1948) developed a robust theory for measuring particle loading and conducted loading measurements on aqueous sand and kaolin suspensions More recently, acoustic spectroscopy has been used to find the particle size distribution (Carlson, 1999). They made mass fraction measurements in a polydisperse suspension of iron-ore powder in water using a $3 \mathrm{Mhz}$ transmitter and two receivers. Significant contributions to developments in this area have been made by Cushman et al (1973), Riebel (1976) and Alba (1992).

The ultrasonic transducer should be selected according to the application. For the experimental system described in this thesis, the ultrasonic transducer was selected based on the following arguments.

- For a good measurement to be done, the ultrasonic beam should be directional. This means that the beam should propagate in a particular direction as opposed to propagating uniformly in all directions. This, in 
turn, suggests that the wave-front should be planar rather than spherical. For the beam to be directional, the following condition should be satisfied. $k a_{b} \succ \succ 1$

$k=$ wave-number

$a_{b}=$ beam diameter

- The measurement made is based on the attenuation of ultrasound. This viscous attenuation of ultrasound is proportional to the square of the ultrasonic frequency. For the attenuation caused by the particles to be significant enough, i.e., for the attenuation to be measured by an oscilloscope, the frequency of ultrasound should be high.

- The near-field distance or the Rayleigh distance should be greater than the water tank in order to apply the near field approximations. This imposes another condition on the frequency of the ultrasound.

$$
\frac{1}{2} k a^{2} \succ L
$$

$L=$ length of the water tank.

- The beam loss can be attributed to the attenuation which is an actual loss and to the scattering of beam by the particles. For attenuation to be the dominant loss mechanism rather than scattering effects, the viscous boundary layer should be several times smaller than the scatterer radius. The viscous boundary layer thickness is proportional to the square root of the ultrasonic frequency. This suggests that the frequency should not be too high. 
Based on the above-mentioned conditions, the frequency of ultrasound was chosen to be $1 \mathrm{MHz}$ and the beam diameter was chosen to be 1 inch. So, Panametrics A $302 \mathrm{~s}$ immersion transducers were selected for the application.

The concentration measurement in the experimental system was done using $1 \mathrm{MHz}, 1$ inch ultrasonic transducers. One transducer acts as the ultrasound source and the other transducer acts as the receiver. The details of the transducer excitation signal are as follows:

\begin{tabular}{|l|l|}
\hline Frequency & $1 \mathrm{MHz}$ \\
\hline Amplitude & $3.5 \mathrm{~V}$ \\
\hline Cycles per burst & 10 \\
\hline Time gap between bursts & $50 \mathrm{~ms}$ \\
\hline Waveform & Sinusoidal \\
\hline
\end{tabular}

Table 2.1: Transducer excitation signal.

A sample calculation for finding the transducer excitation is shown in the appendix. Based on the amplitude of signal received by the receiver and the source signal, the attenuation caused by the suspension is found. The attenuation caused by the suspension is directly proportional to the concentration of particles in the suspension. This measurement, though, is an average measurement since the longitudinal variation of concentration, i.e., the variation of concentration along the path of the ultrasonic beam is neglected. This concentration 
measurement is done at two different elevations in order to study the concentration variation in the direction of settling.

\subsection{ATtenUATION THEORY}

Urick (1946) developed a theory for the absorption of sound in aqueous suspensions of small spherical particles. In this theory, the greater part of the absorption was attributed to the viscous drag between the fluid and the particles in the sound field. The expression for viscous attenuation was given by

$$
\begin{aligned}
& 2 \alpha=C\left[\frac{1}{6} k^{4} a^{3}+k(\rho-1)^{2} \frac{s}{s^{2}+(\rho+\tau)^{2}}\right], \\
& \rho=\frac{\rho_{p}}{\rho_{f}} \\
& s=\frac{9}{4} \beta a\left(1+\frac{1}{\beta a}\right), \text { and } \\
& \tau=\frac{1}{2}+\frac{9}{4} \frac{1}{\beta a} .
\end{aligned}
$$

In these expressions, $\mathrm{C}$ is the concentration of particles in the suspension, $k$ denotes the compression wave number given by

$$
k=\frac{2 \pi}{\lambda}
$$

$\lambda$ being the wavelength and $a$ is the particle radius.

The first of the two terms in the expression for attenuation accounts for the scattering losses while the second term involves the viscous drag attenuation. The scattering losses, being small compared to the viscous drag loss, can be neglected. Hence the expression for attenuation simplifies to 


$$
\alpha=\frac{C}{2}\left[k(\rho-1)^{2} \frac{s}{s^{2}+(\rho+\tau)^{2}}\right]
$$

Hay and Mercer (1985) presented an expression for viscous absorption coefficients derived using viscosity modified phase shifts. This expression was, then, shown to reduce to the one derived by Urick in the high frequency end of the long wavelength region. This means that the particle size should small compared to the wavelength, but the frequency must be large enough that the viscous boundary layer thickness is less than the scatterer radius. This can be represented by the inequalities, $k a \prec \prec 1$ and $\beta a \succ \succ 1$, where $1 / \beta$ represents the viscous boundary layer thickness and is given by

$$
\beta=\left(\frac{\omega \rho_{p}}{2 \mu}\right)^{\frac{1}{2}} \text {. }
$$

It is clear from the expressions for attenuation that the effect of the particle size distribution (PSD) was not taken into consideration. Often times, the mean particle size, often defined in different ways, is used as the basic particle length scale used to compute the attenuation. The effect of PSD is critical since large particles cause significant scattering losses relative to small particles. In order to consider this effect, Cushman et al (1973) employed a mathematical model consisting of an empirical calibration between the attenuation at a single frequency and a single point in the PSD. Riebel (1986) postulated that the attenuation at each frequency could be predicted as a linear combination of a number of size fractions representing the PSD of the particles. Alba (1992) predicted that the attenuation spectrum for a particle population having a range of particle sizes was a linear function of the size fractions. By knowing a number of 
physical properties of both phases in the suspension, the attenuation can be predicted using fundamental physics.

The authors of this work present a simple expression for attenuation provided the PSD can be reasonably approximated to a log-normal distribution (Log-Normal Approximation). For any PSD with a frequency function $f(a)$, the attenuation is given by

$$
\alpha=\frac{2}{3} \pi k \frac{(\rho-1)^{2}}{V} \int \frac{s}{s^{2}+(\rho+\tau)^{2}} a^{3} f(a) d a
$$

Many PSD can be fit with the log-normal distribution since it has many of the basic properties of naturally observed size distributions and its mathematical form is convenient for dealing with the moment distributions and averages. The frequency function for a log-normal distribution is given by

$$
d f=\frac{1}{\sqrt{2 \pi} \ln \left(\sigma_{g}\right)} \exp \left[-\frac{\left(\ln (a)-\ln \left(a_{g}\right)\right)^{2}}{2 \ln ^{2}\left(\sigma_{g}\right)}\right] d(\ln (a)),
$$

where $a_{g}$ is the geometric mean particle size (GMD) given by

$$
\ln \left(a_{g}\right)=\frac{\sum n_{i} \ln \left(a_{i}\right)}{N_{0}}
$$

and the geometric standard deviation (GSD), $\sigma_{g}$, is given by

$$
\ln \left(\sigma_{g}\right)=\left(\frac{\sum n_{i}\left(\ln \left(a_{i}\right)-\ln \left(a_{g}\right)\right)^{2}}{N_{0}-1}\right)
$$

Using this frequency function, the expression for attenuation is:

$$
\alpha=\frac{2}{3} \pi \frac{\varpi}{C_{0}} \frac{N_{0}}{V} \frac{(\rho-1)^{2}}{\sqrt{2 \pi} \ln \left(\sigma_{g}\right)} \int_{0}^{\infty} \frac{s}{s^{2}+(\rho+\tau)^{2}} \exp \left[-\frac{\left(\ln (a)-\ln \left(a_{g}\right)\right)^{2}}{2 \ln ^{2}\left(\sigma_{g}\right)}\right] a^{2} d a
$$


While the total attenuation might be obtained by the integration of this expression, the process of integration can be simplified by making the following two approximations.

The function $F(s, \tau)$ given by

$$
\begin{array}{rlrl}
F(s, \tau)=\frac{s}{s^{2}+(\rho+\tau)^{2}} \text { is approximated by } & \\
1.96 \times 10^{9} a^{1.62} & 0 \prec a & \prec 0.38 \mu \mathrm{m} \\
0.88 & 0.38 \mu \mathrm{m} & \prec a & \prec 1.22 \mu \mathrm{m} \\
5.70 \times 10^{-6} a^{-0.88} & a & \succ 1.22 \mu \mathrm{m}
\end{array}
$$

The log-normal frequency function is approximated by

$$
\begin{aligned}
& f=\frac{1}{\sqrt{2 \pi} \ln \left(\sigma_{g}\right)}\left[1+\frac{\ln \left(a / a_{g}\right)}{\sqrt{2 \pi} \ln \left(\sigma_{g}\right)}\right] \quad a_{g} \sigma^{-\sqrt{2 \pi}} \leq a \leq a_{g} \\
& f=\frac{1}{\sqrt{2 \pi} \ln \left(\sigma_{g}\right)}\left[1-\frac{\ln \left(a / a_{g}\right)}{\sqrt{2 \pi} \ln \left(\sigma_{g}\right)}\right] \quad a_{g} \leq a \leq a_{g} \sigma^{\sqrt{2 \pi}}
\end{aligned}
$$

This approximates a log-normal distribution with a triangular distribution on logarithm of $a$.

Using these two approximations, we can get the expression for attenuation to be

$$
\begin{aligned}
& \alpha=\frac{2}{3} \pi N_{0} k(\rho-1)^{2}\left[I_{1}+I_{2}+I_{3}+I_{4}\right], \text { where } \\
& I_{1}=\int_{a_{g} \sigma^{-\sqrt{2 \pi}}}^{0.38} \frac{1}{\sqrt{2 \pi} \ln \left(\sigma_{g}\right)}\left[1+\frac{\ln \left(a / a_{g}\right)}{\sqrt{2 \pi} \ln \left(\sigma_{g}\right)}\right] 1.96 \times 10^{9} a^{3.61} d a
\end{aligned}
$$




$$
\begin{aligned}
& I_{2}=\int_{0.38}^{1.22} \frac{0.88}{\sqrt{2 \pi} \ln \left(\sigma_{g}\right)}\left[1+\frac{\ln \left(a / a_{g}\right)}{\sqrt{2 \pi} \ln \left(\sigma_{g}\right)}\right] d a, \\
& I_{3}=\int_{1.22}^{a_{g}} \frac{1}{\sqrt{2 \pi} \ln \left(\sigma_{g}\right)}\left[1+\frac{\ln \left(a / a_{g}\right)}{\sqrt{2 \pi} \ln \left(\sigma_{g}\right)}\right] 5.70 \times 10^{-6} a^{1.13} d a, \\
& I_{4}=\int_{a_{g}}^{a_{g} \sigma^{\sqrt{2 \pi}}} \frac{1}{\sqrt{2 \pi} \ln \left(\sigma_{g}\right)}\left[1-\frac{\ln \left(a / a_{g}\right)}{\sqrt{2 \pi} \ln \left(\sigma_{g}\right)}\right] 5.70 \times 10^{-6} a^{1.13} d a
\end{aligned}
$$

These are the four integrals that approximate the integral given in equation 2.32. These integrals are of the standard form and can be obtained from any standard calculus text.

\subsubsection{Experiments.}

It is important to compare the results of the approximation suggested in the previous section and the attenuation observed in a suspension. A hydrophone, immersed in the suspension to be measured, emitted 10 microsecond pulses of 1 $\mathrm{MHz}$ ultrasound. This was received by a hydrophone separated from the transmitter by a distance of 8 inches. The received signal was found to have a very low signal to noise ratio. These pulses were observed on an oscilloscope. The ratio of the amplitude of the received pulses to the transmitted pulse can be used to find the extinction coefficient. This extinction coefficient will be sum of the absorption of the suspending liquid and that of the suspended particles. A 
simple labview code was written to acquire the waveform from the oscilloscope into the computer. The waveform was then analyzed to determine features of the suspension. A schematic of the experimental setup is shown below.

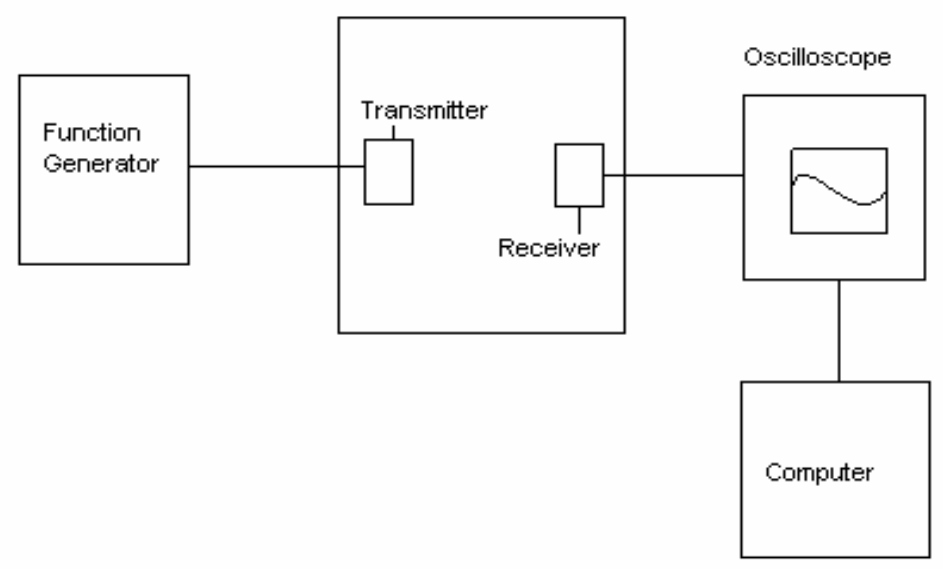

Figure 2.5: Experimental schematic for hydrophone measurements.

The particles used for the suspensions were fly-ash and kaolin. The study of these particles under a scanning electron microscope showed that the fly-ash particles were spherical and the kaolin particles were flat euphedral crystalline plates. 


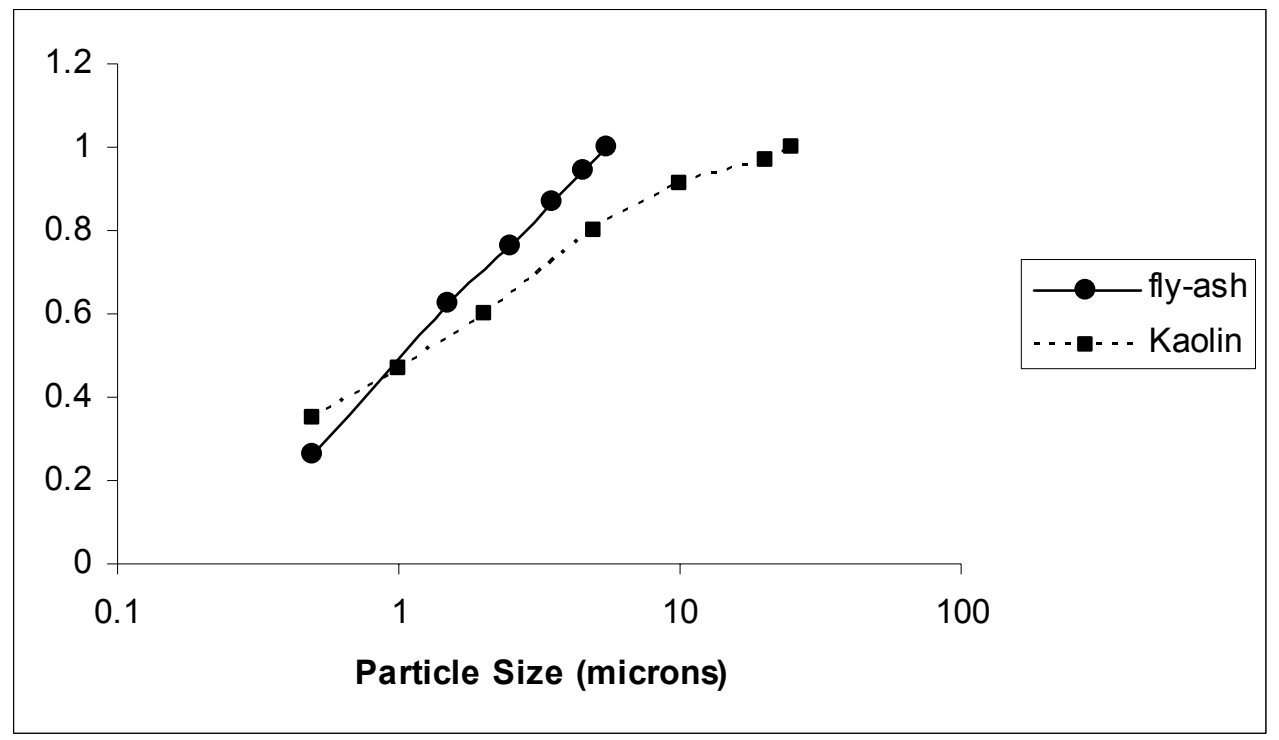

Figure 2.6: Cumulative PSD of Kaolin and Fly-ash.

The mean diameter of the fly-ash particles was found using SEM to be 2.05 microns while that of the kaolin particles was found to be 4.60 microns. The specific gravity of these materials was also measured using a simple experiment. The specific gravity of kaolin and fly-ash were found to be 2.53 and 2.27 respectively. The suspensions were made by thoroughly dispersing the material using a mechanical stirrer.

\subsubsection{Results.}

Figure 2.7 shows the extinction coefficient (attenuation) plotted against mass fraction for fly-ash and Kaolin particles. The absorption varies linearly with concentration which is in agreement with the theories. 


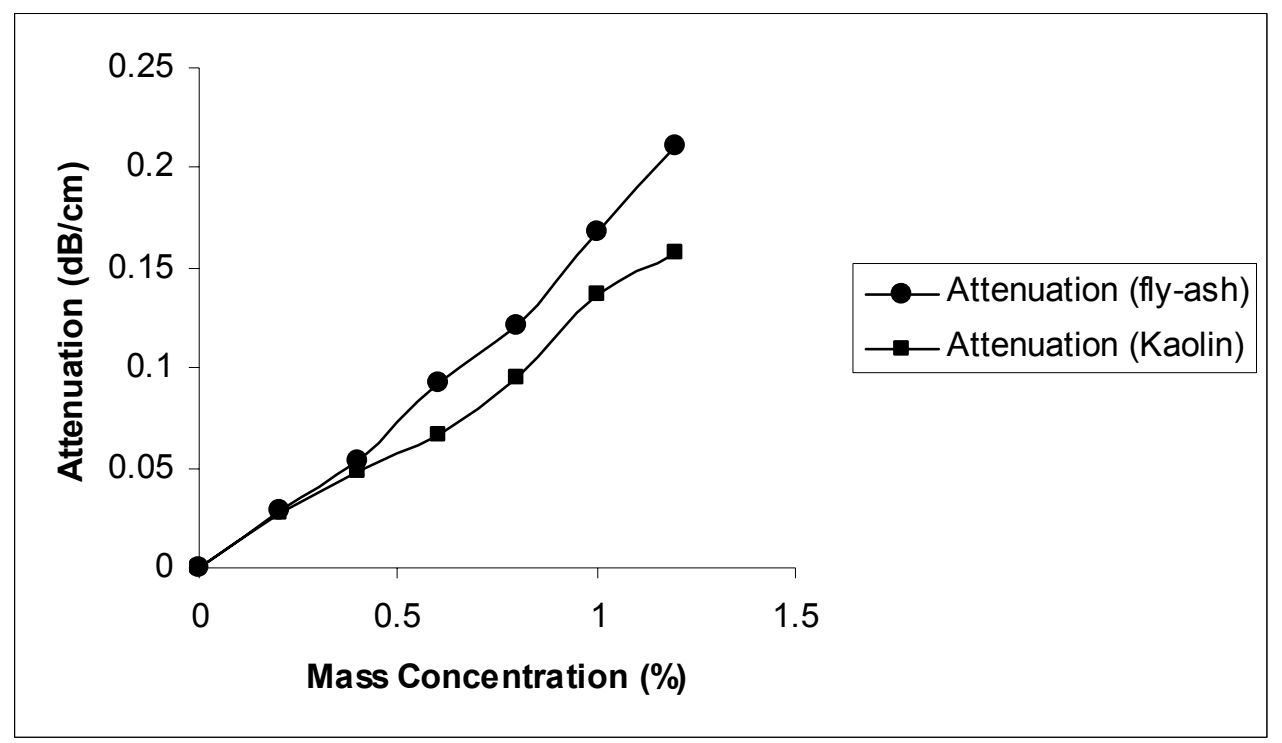

Figure 2.7: Experimentally determined attenuation for fly-ash and Kaolin suspensions.

The attenuation for fly-ash suspension predicted by equation 2.14 was calculated. Also, the attenuation was calculated using equation 2.25. The PSD of fly-ash was approximated by a log-normal distribution with the same mean particle size to carry out the calculation. The GSD for this distribution was found to be 2.2 while the GMD was found to be 1.54 microns. Figure 2.8 shows the values of integrals $I_{1}, I_{2}, I_{3}$, and $I_{4}$ found from equations (9)-(12) for various GSD. It was clear that the sum of the four integrals (Total Integral Approximation) could be approximated by integral $\mathrm{I}_{4}$ alone without much error $\left(\mathrm{I}_{4}\right.$ approximation $)$. Figure 2.9 shows the attenuation found using different approximations for fly-ash type of particles. It was clear that attenuation found using Urick's theory was off by a factor of 2 . It could be seen that while the attenuation found using log-normal approximation deviates from the experimental values substantially for small concentration, it is more accurate for higher concentration. The $\mathrm{I}_{4}$ approximation 
yields good agreement with experimental results for lower concentrations. For higher accuracy at lower concentrations, the total integral approximation could be used. These comments can be seen clearly from figure 2.11 which shows the error in attenuation due to different approximations as compared to the experimental results.

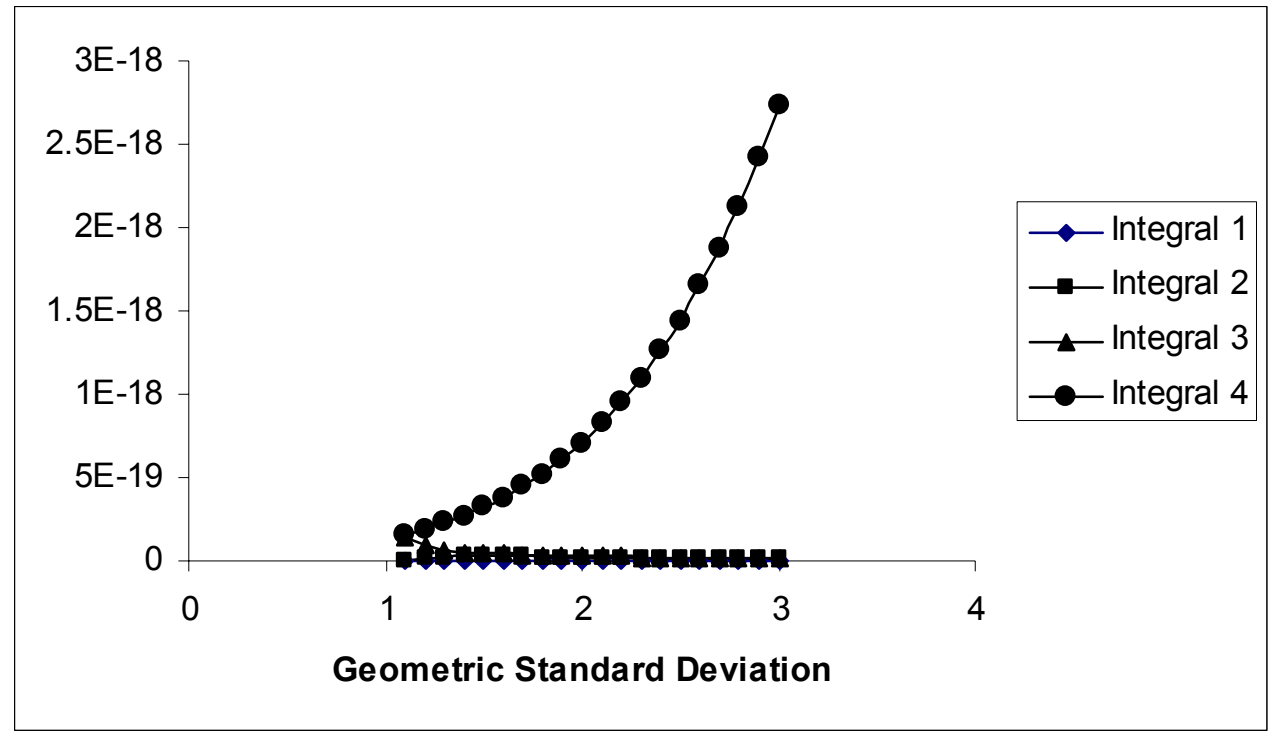

Figure 2.8: Integrals I1, I2, I3, and I4 for fly-ash suspension. 


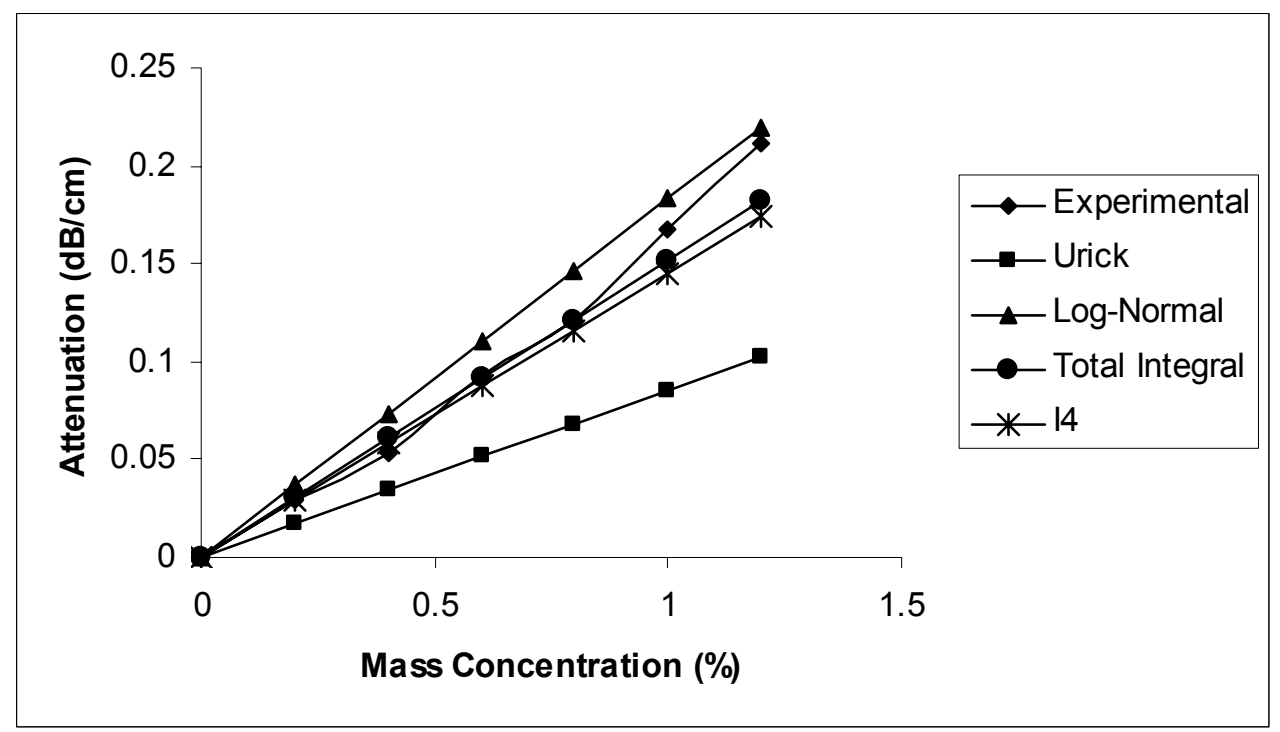

Figure 2.9: Attenuation results for fly-ash suspension.

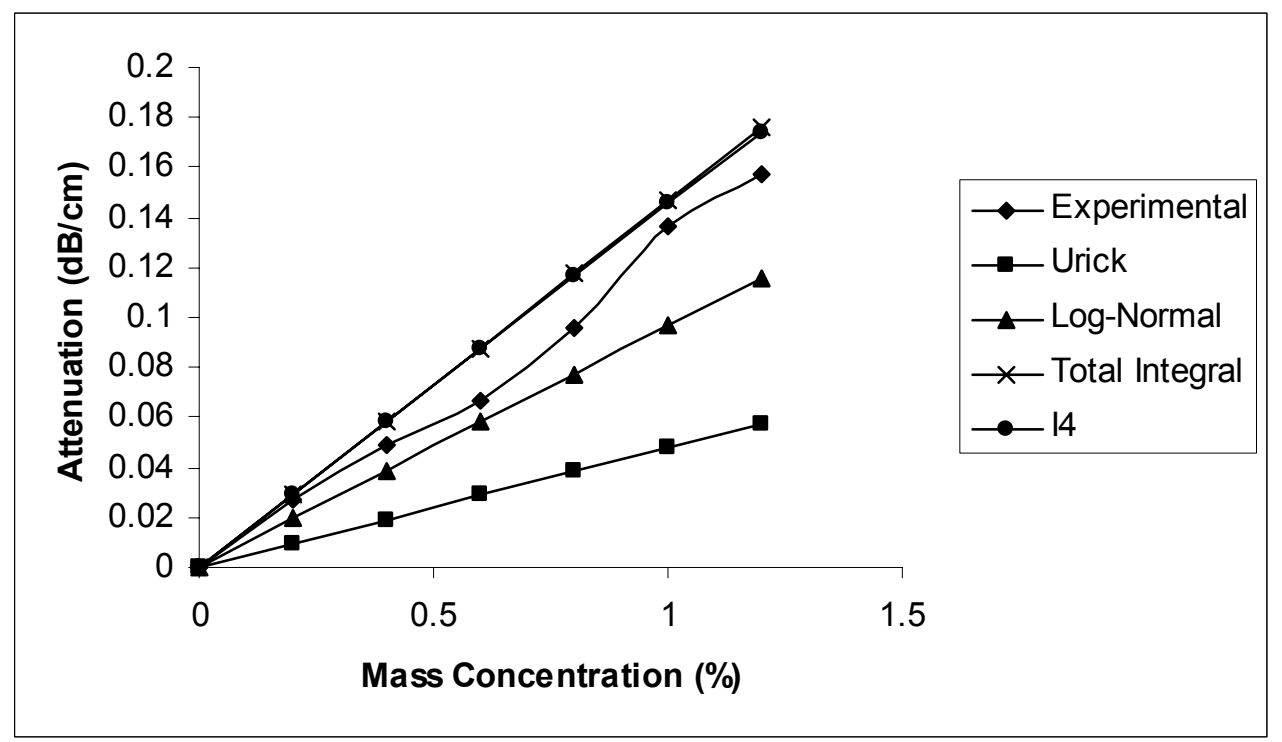

Figure 2.10: Attenuation results for kaolin suspension. 


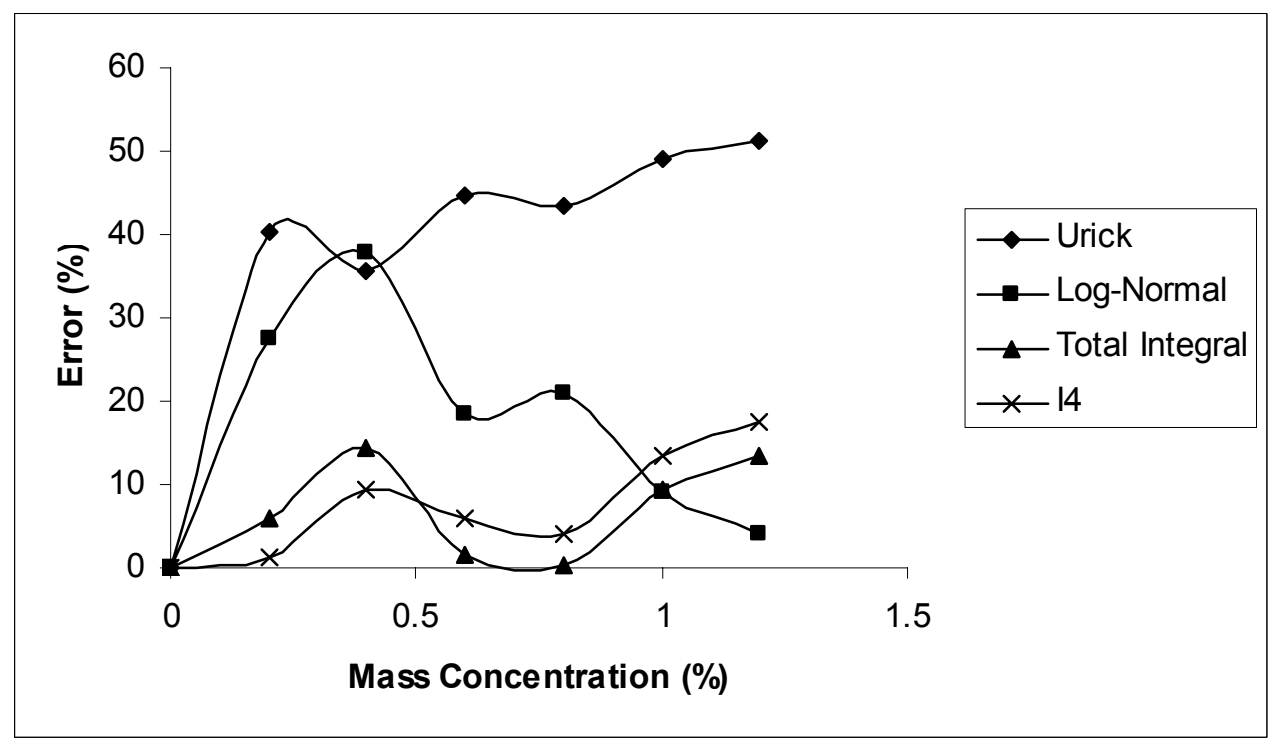

Figure 2.11: Error in attenuation due to approximations for fly-ash suspension.

Similar plots for attenuation and error in attenuation for kaolin suspension are shown in figures 2.10 and 2.12. It can be seen that the errors induced by the $\mathrm{I}_{4}$ is surprisingly the least on an average. A correlation was developed based on the $\mathrm{I}_{4}$ approximation to describe the acoustic attenuation. Equation 2.30 gives the correlation for the same.

$$
\alpha=3.5693 * 10^{-7} \frac{N_{0}}{V} \frac{\omega}{C_{0}}(\rho-1)^{2} a_{g}^{2.13} \sigma_{g}^{2.84}
$$




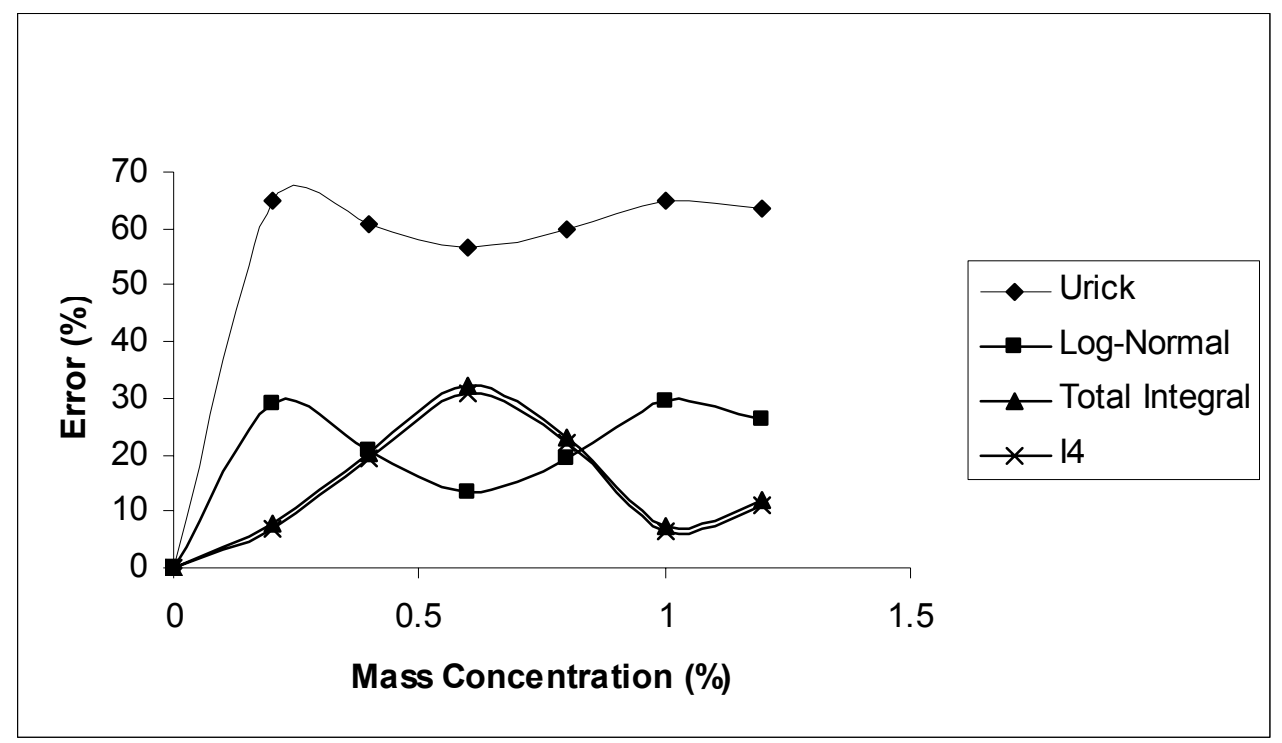

Figure 2.12: Error in attenuation due to approximations for kaolin suspension.

In correlation given by equation $2.30, a_{g}$ and $\sigma_{g}$ refer to the GMD and GSD of the log-normal distribution approximating the actual PSD. These values were calculated using the formula given in equations 2.19 and 2.20. Figures 2.13 and 2.15 show the attenuation predicted by the correlation and the error induced by the correlation compared to the experimental results respectively for fly-ash suspensions. 


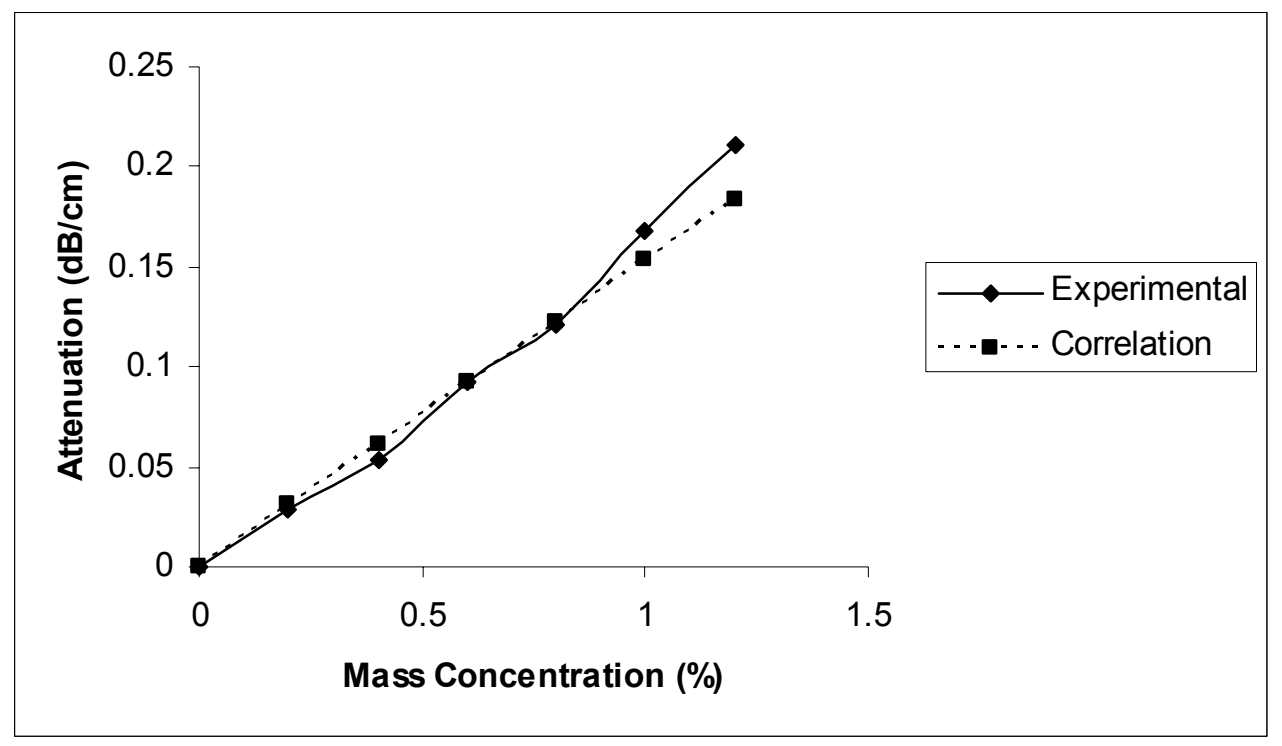

Figure 2.13: Attenuation predicted by correlation (20) for fly-ash suspension.

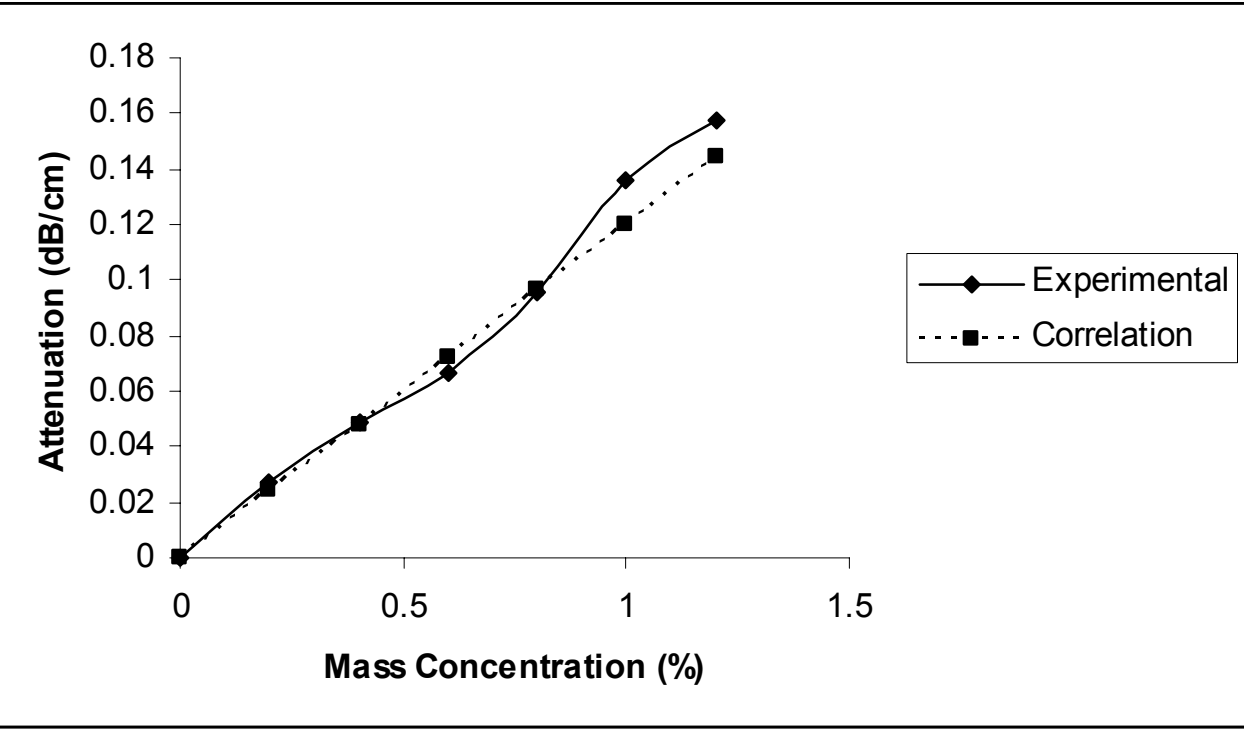

Figure 2.14: Attenuation predicted by correlation for Kaolin suspension. 


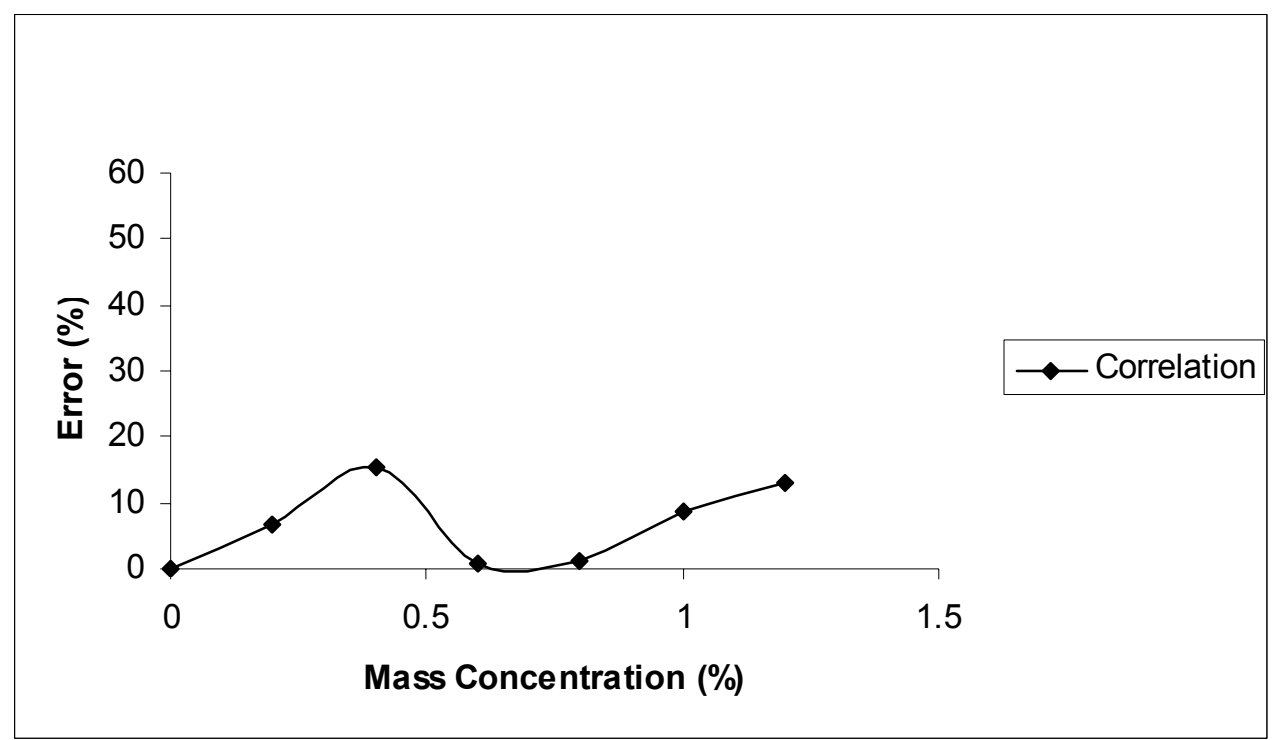

Figure 2.15: Error in attenuation predicted by correlation for fly-ash suspensions.

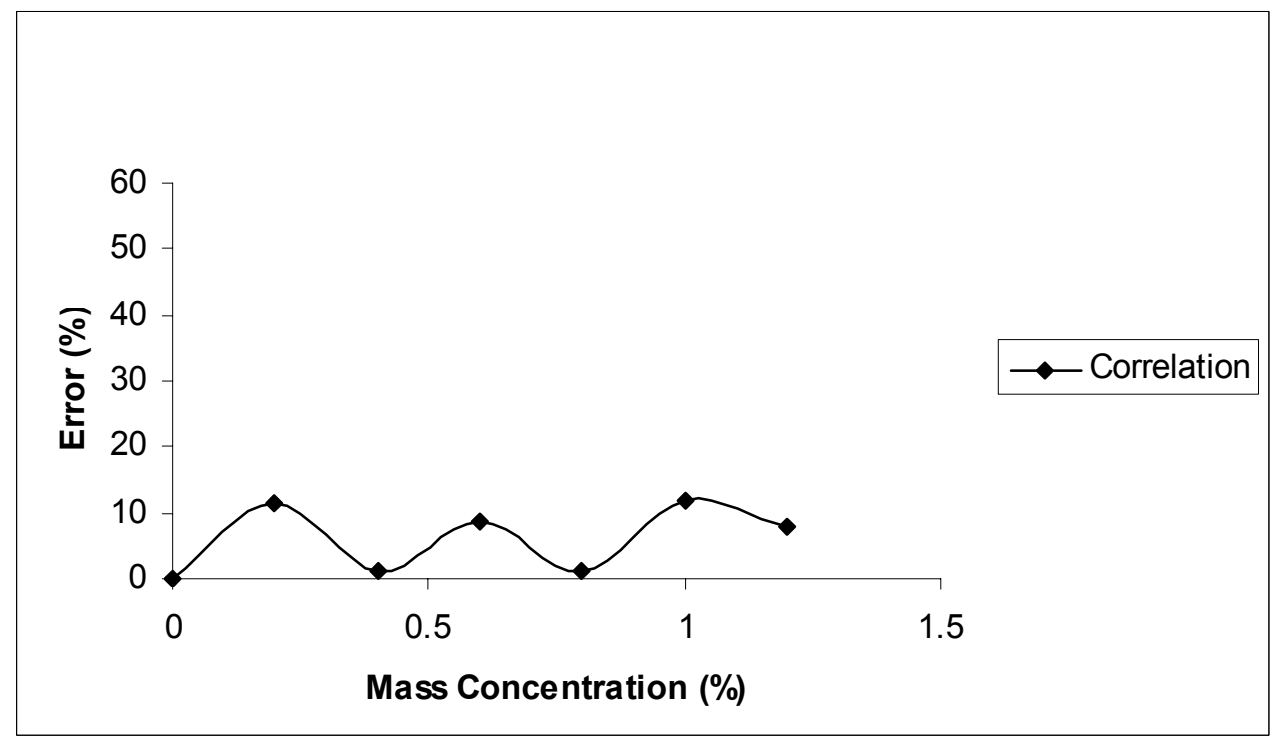

Figure 2.16: Error in attenuation predicted by correlation for kaolin suspensions. 
Figures 2.14 and 2.16 give similar plots for kaolin suspension. It could be seen that the maximum error induced by this correlation for both the suspensions compared to the experimental results is less than $15 \%$. It is also clear that the correlation gives, surprisingly, the least error compared to the other approximations.

\subsection{Ultrasonic Transducer Performance.}

The selected transducers were flush mounted to the side walls at two different elevations as shown in the schematic. The dynamics of the particles in the suspension is not changed due to mounting of these transducers. The transducers have to be checked for their performance under different conditions. Two experiments were conducted in order to evaluate the transducers and to find the error introduced in the measurement due to different operating conditions. First of all, the transducers were checked for repeatability under no loading and no shaking. In this experiment, the tank was filled completely with pure water (without any air gap). Then the transducer acting as the source was excited using a Hewlett-Packard frequency generator. The signal received by the receiver was delivered to an oscilloscope. Then a LabView code was run in order to acquire the signal from the oscilloscope onto a computer. This code converted the waveform into spreadsheet file containing time and corresponding voltage. The spreadsheet file was processed using Microsoft Excel to find the amplitude of the signal. This amplitude was measured at different instances in order to check for repeatability. This experiment was conducted for the top transducer as well as the bottom transducer. The figure below shows the result obtained from this experiment. 


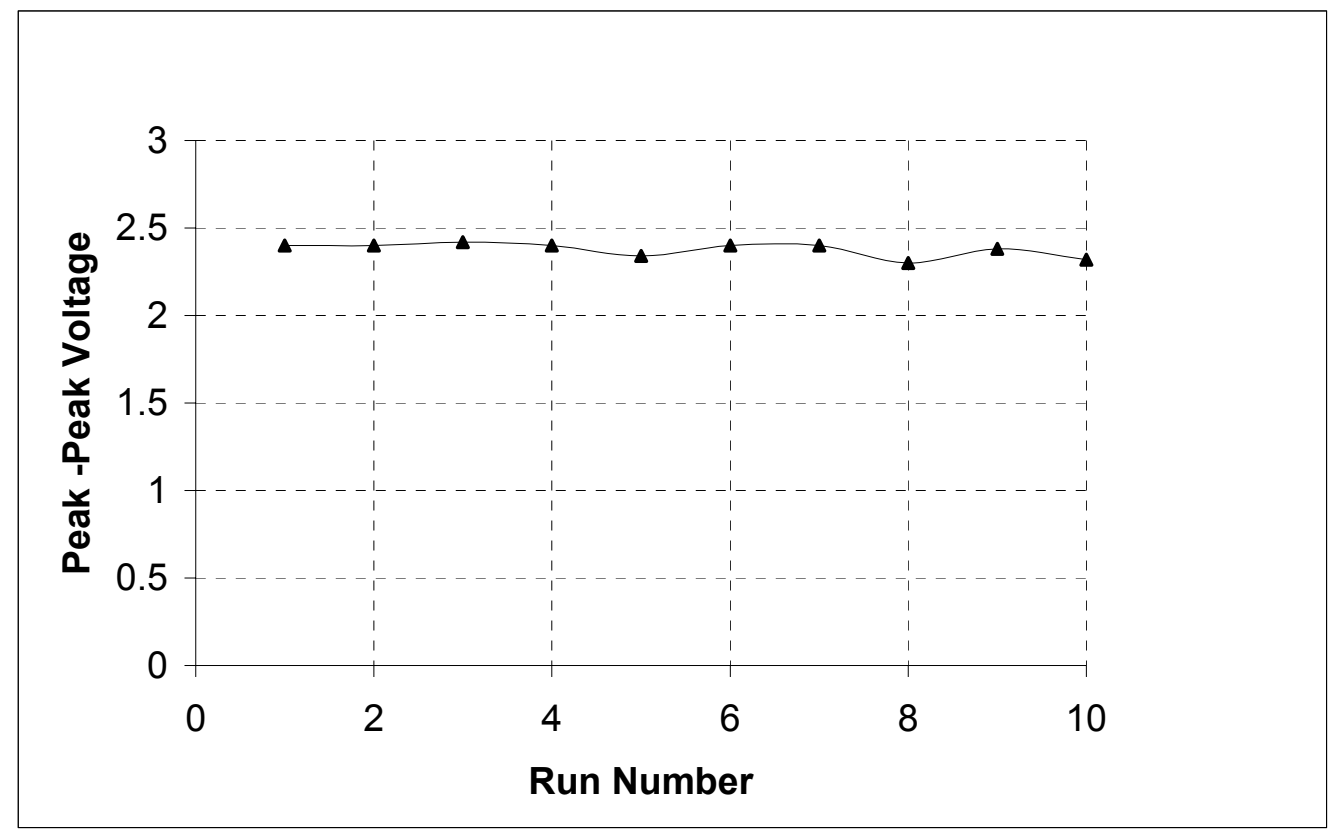

Figure 2.17: Top transducer response at no loading, no shaking.

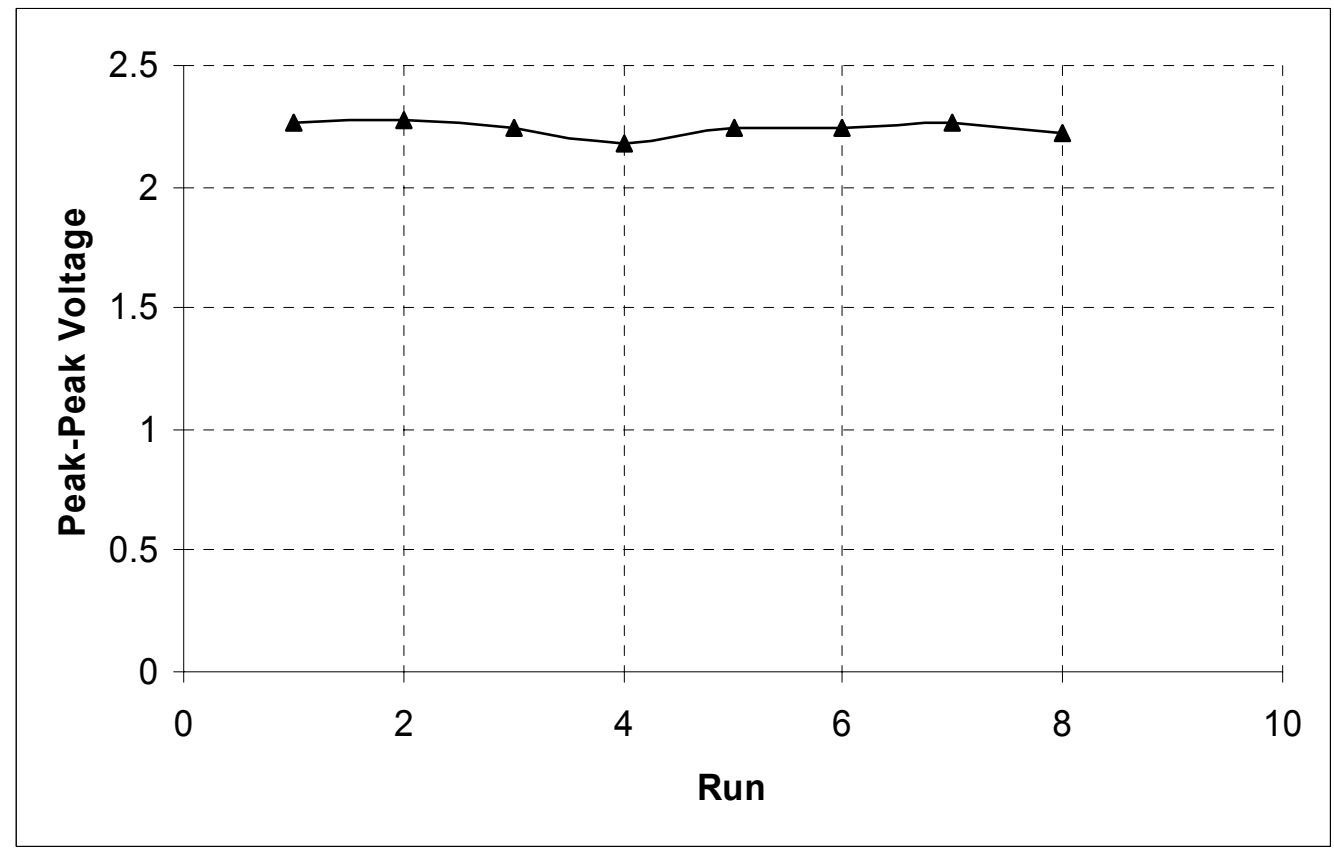

Figure 2.18: Bottom transducer Response at no loading, no shaking. 
Figure 2.17 shows the voltage output obtained from the top receiver and figure 2.18 shows the same for the bottom receiver. The fractional standard deviation of the voltage outputs from the top transducer was found to be per cent while that of the bottom transducer was 1.3 per cent. This shows that the voltage outputs from the receivers are repeatable.

The second experiment to be conducted was another repeatability experiment. This time the tank was shaken at a frequency of $10 \mathrm{~Hz}$. Again the transducers worked well giving consistent voltages as shown in figure 2.19. The data acquisition procedures were similar to that of the previous experiment.

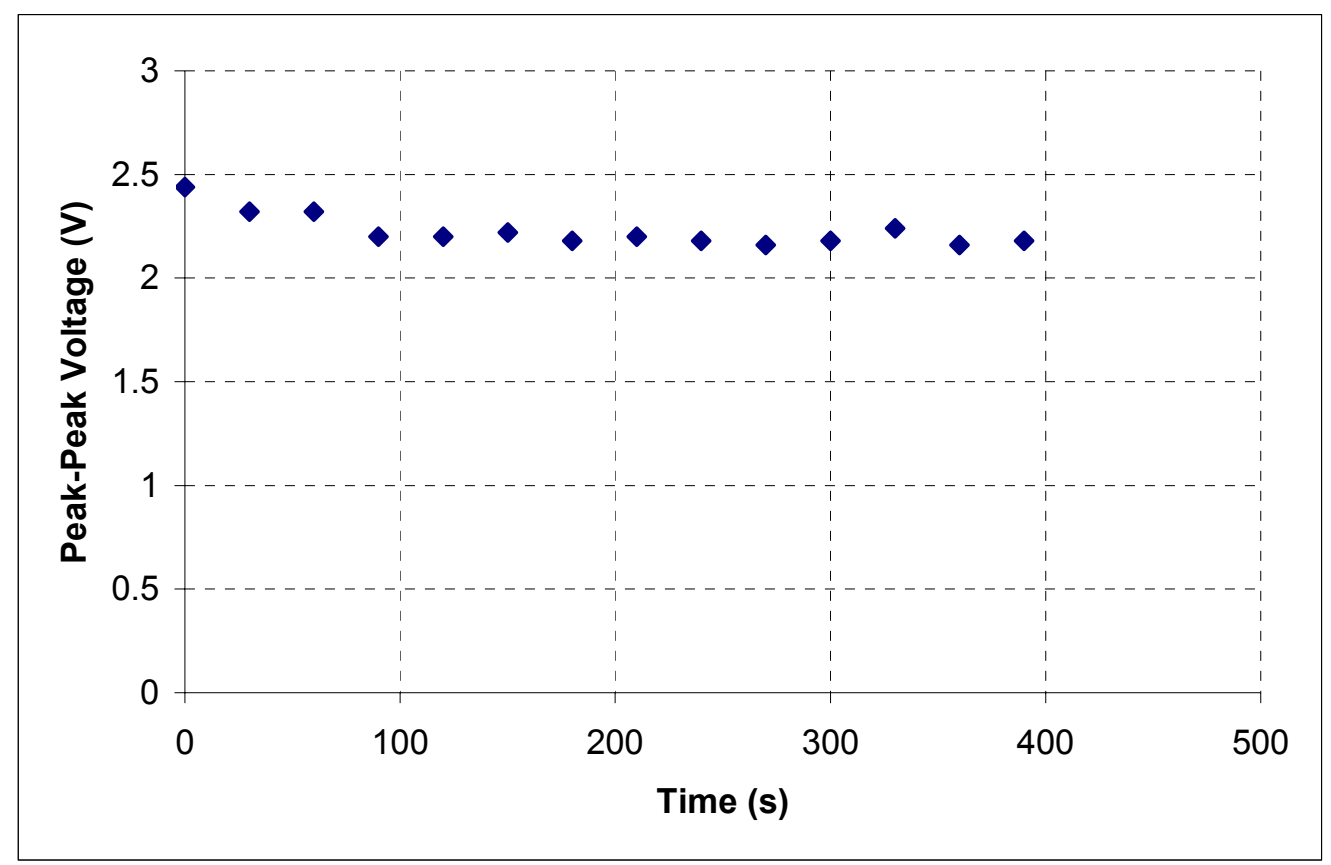

Figure 2.19: Top transducer response at no loading, $10 \mathrm{~Hz}$ shaking. 
Figure 2.19 shows the voltage outputs from the top transducer when the tank was shaking. The fractional standard deviation of the voltage outputs from the top transducers was found to be 3.5 per cent. This experiment was conducted for a time duration of 400 s (six and a half minutes approximately).

These experiments showed that this attenuation based ultrasound measurement technique is a reliable technique for the experimental system already described. Also, the error introduced due to shaking of the experimental set-up corresponds to a 0.2 per cent by mass (based on the calibration curves to be explained later in the chapter). Thus any concentration below 0.2 per cent by mass can not be measured accurately by this measurement technique. The reason for this error is not known.

\subsection{SUSPENDED PARTICLES.}

Two types of particles were used in the experiments conducted. These were fly-ash and kaolin particles. The reasons for selecting these particles were that they were non-reactive, relatively small particles and importantly, they were widely available. The amount of particles needed for these experiments were large and that made the use of particles like polystyrene beads, etc. cost ineffective. Some tests were conducted in order to verify the mean size of the particles and their densities.

In order to verify the mean size of these particles, a sample of these particles was viewed under an SEM (Scanning Electron Microscope). The images obtained were analyzed using image processing software in order to find the mean 
size of the particles. The mean size and standard deviation of PSD are shown in Table 2.2.

Then, tests were conducted to verify the densities of fly-ash and kaolin. A simple volume-displacement measurement was done in order to estimate the densities. In this test, a known mass of particles was added to a beaker containing a known volume of water. The difference between the final volume of particlewater mixture and the initial volume of water is equal to the volume of the particles added. The ratio of the mass of the particles added to the measured volume of particles gives an estimate of density of the particles. The results of these experiments are shown in Table 2.2.

Figures 2.20 and 2.21 show SEM images of fly-ash and kaolin respectively. It can be seen that the fly-ash particles are spheroidal in shape while the kaolin crystals are hexagonal.

\begin{tabular}{|c|c|c|}
\hline Properties & Fly-ash & China clay \\
\hline Size & 2.04 microns & 4.61 microns \\
\hline Density & $2.27 \mathrm{~g} / \mathrm{cc}$ & $2.53 \mathrm{~g} / \mathrm{cc}$ \\
\hline Shape & Spherical & Hexagonal \\
\hline
\end{tabular}

Table 2.2: Experimentally obtained Physical Properties of Fly-ash and Kaolin 


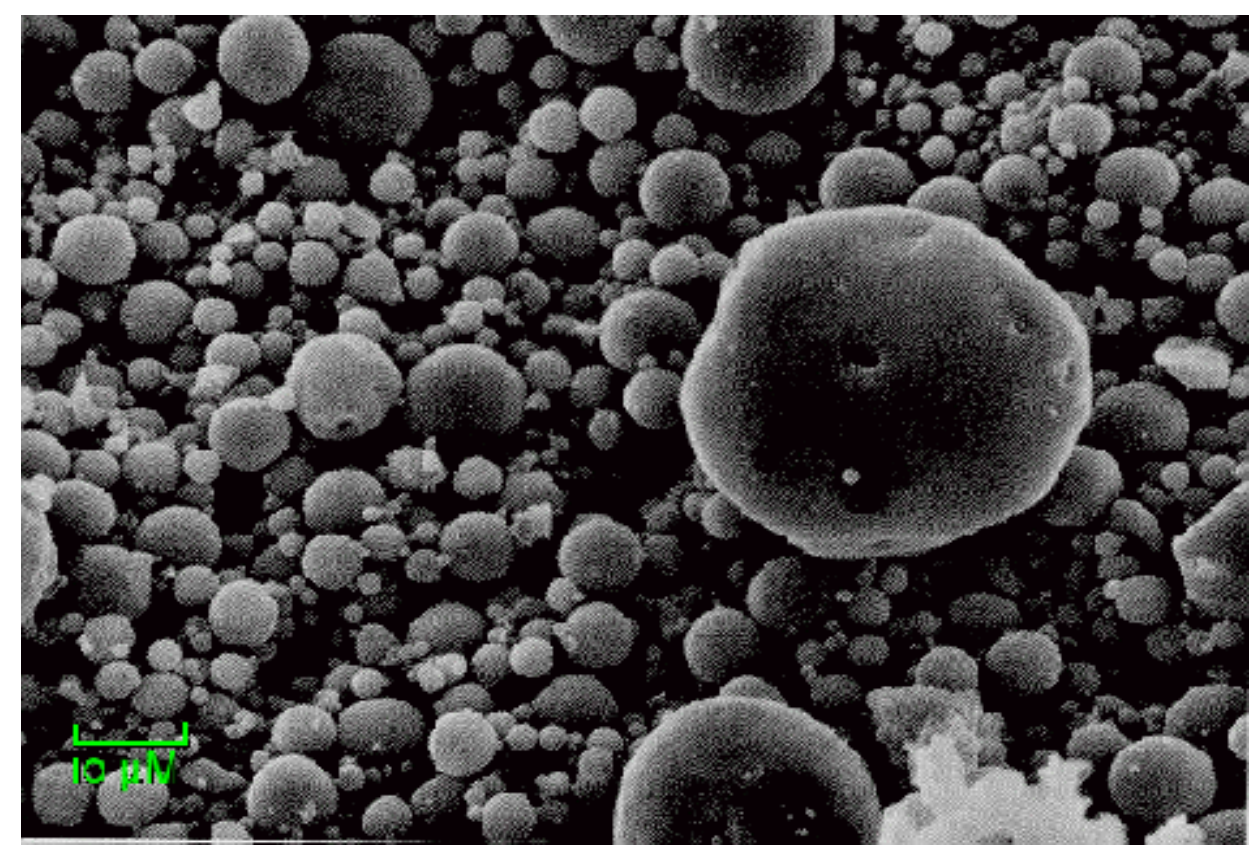

Figure 2.20: $\quad$ SEM image of Fly-ash Particles.

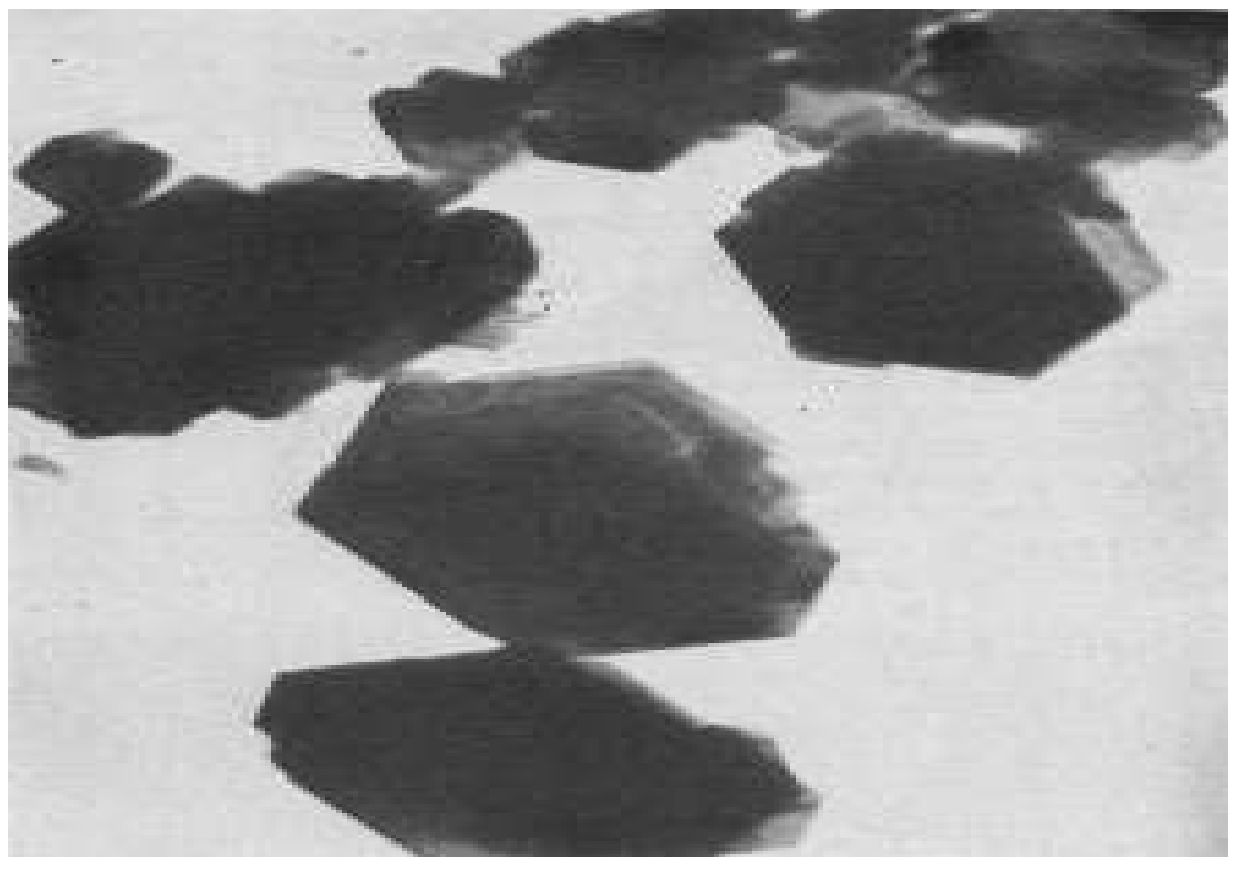

Figure 2.21: Image of Kaolin (china-clay) Particles. 
Also, literature survey was done in order to get information on the physical and chemical properties of fly-ash and kaolin. Some of the information are shown below in Table 2.3. and 2.4. Chemical properties show that kaolin and fly-ash contain large amounts of silicon dioxide $\left(\mathrm{SiO}_{2}\right)$ and aluminum oxide $\left(\mathrm{Al}_{2} \mathrm{O}_{3}\right)$.

\begin{tabular}{|l|l|l|}
\hline Properties. & Fly-ash. & Kaolin. \\
\hline Density & $2.2-2.8 \mathrm{~g} / \mathrm{cc}$ & $2.6 \mathrm{~g} / \mathrm{cc}$ \\
\hline Melting Point & $>1000^{\circ} \mathrm{C}$ & $>1600^{\circ} \mathrm{C}$ \\
\hline Shape & Spheroidal & Euphedrine \\
\hline Solubility & Insoluble & Insoluble \\
\hline Color & Light to Dark Grey & White \\
\hline
\end{tabular}

Table 2.3: Physical Properties of Kaolin and Fly-ash

\begin{tabular}{|l|l|l|}
\hline & Fly-ash & Kaolin \\
\hline $\mathrm{SiO}_{2}$ & $42-54 \%$ & $52-54 \%$ \\
\hline $\mathrm{Al}_{2} \mathrm{O}_{3}$ & $16-24 \%$ & $42-44 \%$ \\
\hline $\mathrm{Fe}_{2} \mathrm{O}_{3}$ & $16-24 \%$ & $1-1.4 \%$ \\
\hline $\mathrm{TiO}_{2}$ & $1-1.5 \%$ & $<3 \%$ \\
\hline
\end{tabular}

Table 2.4: Chemical Properties of Kaolin and Fly-ash 
The physical and chemical properties of fly-ash could change due to prolonged exposure to water. There are some chemicals like $\mathrm{Na}_{2} \mathrm{O}$ which dissolve in water changing the chemical composition. Therefore, a fresh sample should be used for every experiment.

\subsection{CALIBRATION OF Ultrasonic TRANSDUCERS}

A calibration for acoustic transducers measuring particle concentration is a curve of attenuation against concentration of particles in the suspension. Figure 2.9 in the previous section shows a typical calibration curve for the transducers. Using this curve, the mass concentration in the suspension can be found from the results of sedimentation experiments.

The ultrasonic transducers were calibrated for two different particles namely fly-ash and kaolin (china clay). The calibration curves for both the particles were linear with an $R^{2}$ value of not less than 0.91 . The slope of this curve, though, will vary according to the PSD of the particles used. Therefore calibration has to be performed before every sedimentation experiment in order to take care of the effects of varying PSD. A detailed account of the calibration tests is provided in the appendix.

In this chapter, the experimental set up was described in the first few sections. Then the ultrasonic measurement technique was detailed giving some information on the transducers and the criteria for selection of the same. In the next chapter the results obtained would be reported. 


\section{Chapter 3: Results}

\subsection{INTRODUCTION}

In this study of sedimentation, three different kinds of experiments were done. First, settling experiments without shaking were performed in order to make sure that the measurement technique is, indeed, suitable for the applications. Also, the results of these experiments were studied in detail in order to understand the sedimentation of particles in suspension better. Second, the tank was oscillated from the beginning. This means that the suspension was stirred and the tank was oscillated immediately. Third, the particles were allowed to settle completely and then the tank was oscillated.

Experimental procedure similar to the one used during calibration test was followed. First the data acquisition system was made ready. The LabView was used to acquire data at regular intervals. In most of the cases this interval was 20 seconds, though it was varied during certain experiments. Then fly-ash was added to water in the tank and stirred. In all the experiments, initial concentration of flyash was $2 \%$ by mass. As indicated before, calibration test was done for the sample first. Once calibration was completed, the suspension was stirred up in order to prepare a uniform suspension. In settling experiments without shaking, data acquisition was started immediately after this. In case of shaking experiments, the tank was closed with its lid and then the tank was oscillated. Simultaneously, data acquisition was also started. In the following sections the results of these experiments will be shown and analyzed. 


\subsection{SEDIMENTATION WITHOUT OSCILLATION.}

As explained earlier, in this experiment, the tank was not oscillated. The settling curves for the top and bottom zones are shown in figure 3.1 and 3.2 respectively.

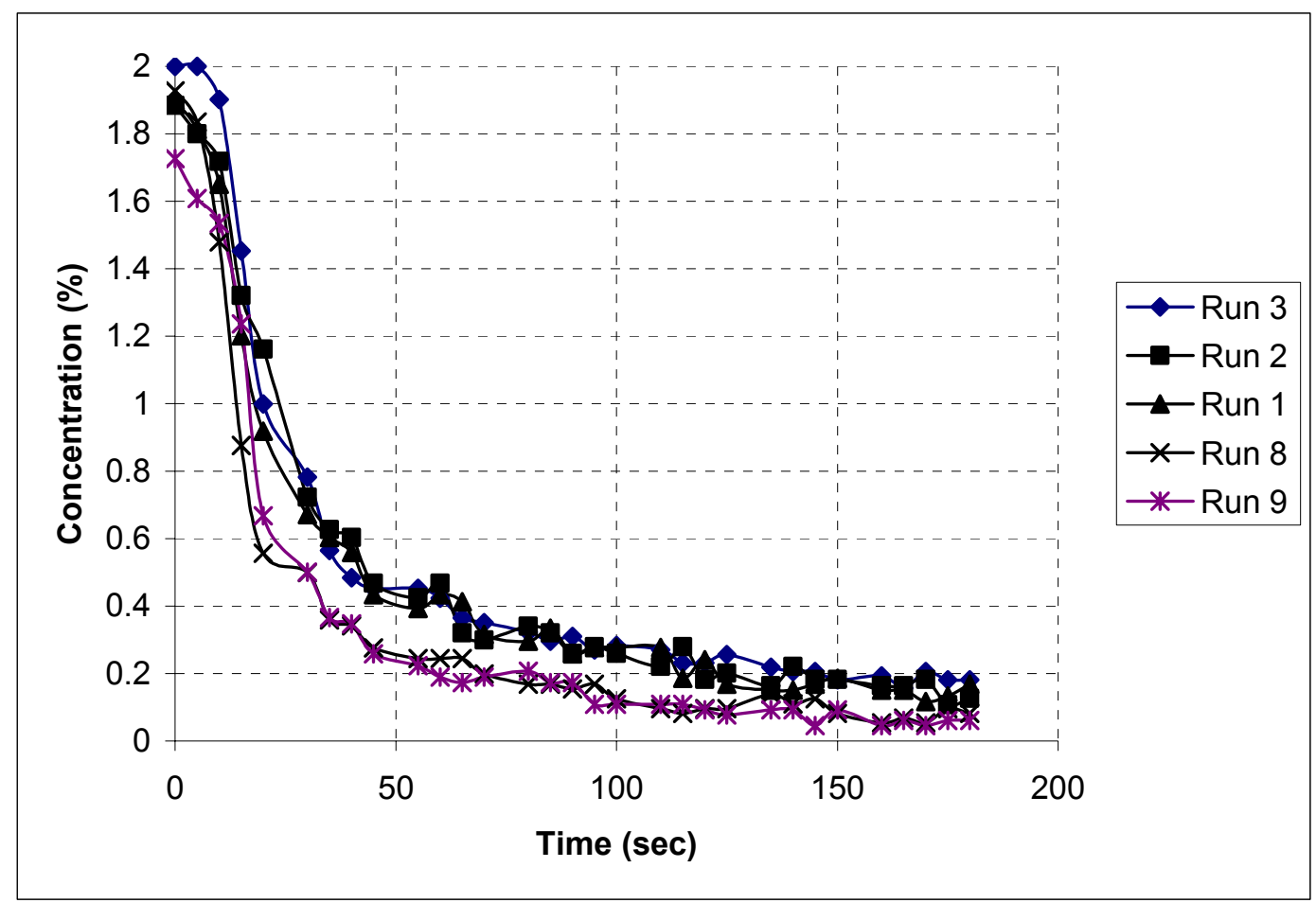

Figure 3.1: $\quad$ Settling curves at the top zone. 


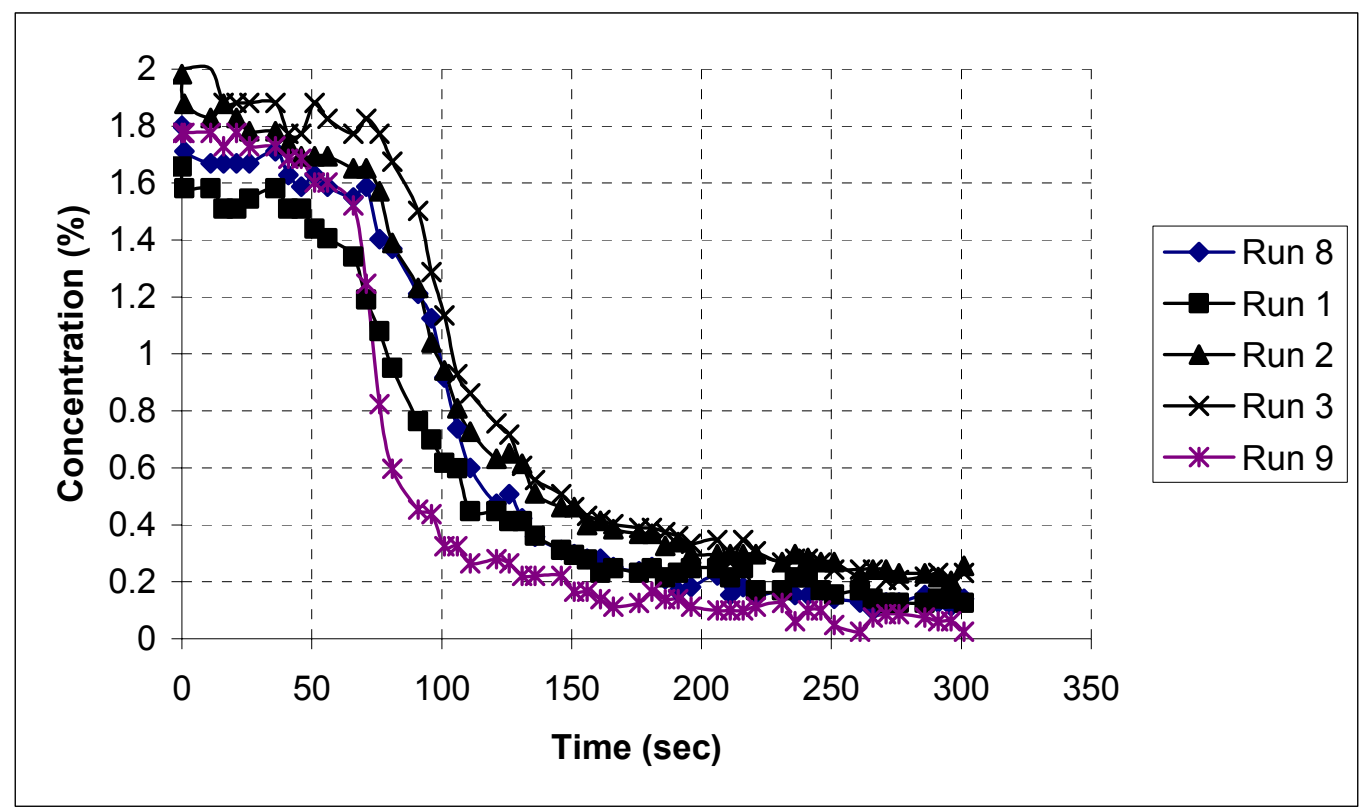

Figure 3.2: $\quad$ Settling curves at the bottom zone.

The figures show concentration of particles in the corresponding zones with time. Data was acquired at an interval of 8 seconds during these experiments. From figure 3.1 , it can be seen clearly that the settling rate is very high at the beginning of the experiment. Then the settling rate becomes slower and later, the curve becomes asymptotic. Another point to note is that the data acquisition has started at a concentration of about $1.9 \%$ by mass instead of the initial concentration of $2 \%$. This is due to the time gap (about 2-3 seconds) between the end of mixing and start of data acquisition. Particles settle down during this time gap. This argument is supported by the fact that settling rate is very high at the beginning. Another reason is improper mixing of suspension before the start of the experiment. 
Figure 3.2 shows settling of particles in the bottom zone. The large particles at the bottom settle out rapidly. This is one of the reasons for the concentration at time $\mathrm{t}=0$ being $1.65 \%$ instead of $2 \%$. At the same time, particles from the top zone move down towards the bottom zone at a rate nearly equal to that of settling rate at the bottom zone. This causes the concentration in the bottom zone to remain high for initial 40 seconds or so. After this time, the concentration of particles at the bottom zone starts to decrease. Again the settling rate between 40 seconds and 100 seconds is greater compared to the settling rate later. The comparison of top zone settling and bottom zone settling curves during the first 3 minutes is shown in figure 3.3. The top zone settling curve can be thought of as an exponentially decreasing curve while the bottom zone settling curve can be thought of as a sum of two exponentially decreasing curves. For a mono-disperse suspension, the settling curves at both the zones will look like a step function, that is, the concentration will be a constant ( $2 \%$ by mass) until a certain period of time and then will go to zero. As the suspension gets polydisperse, the concentration at either zones keeps decreasing with time (due to differential settling of the particles) unlike mono-disperse suspension. Figures 3.1 and 3.2 are reasonable since fly-ash particles are poly-disperse (see figure 2.6). 


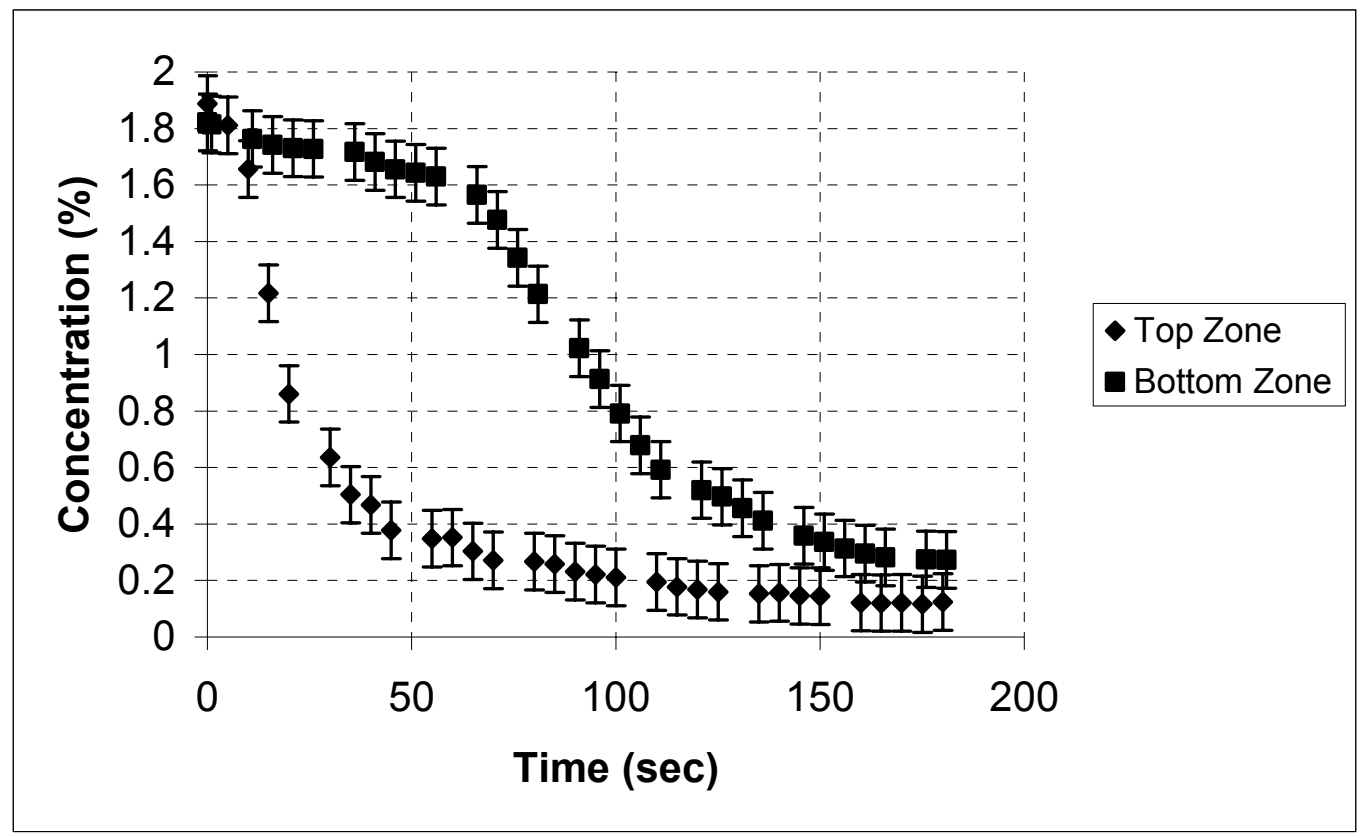

Figure 3.3: Comparison of Top and Bottom Zone Settling Curves.

Figure 3.3 was obtained by smoothing the individual curves in figure 3.1 and then averaging them. These settling curves for a mono-disperse suspension can be thought of as a step function. As explained in the first chapter, for monodisperse suspension undergoing settling, there is a zone of clear liquid, a zone of constant proportion and a settled layer. According to this theory, the transducer would be measuring $2 \%$ concentration by mass until the interface between the clear liquid and the suspension below the transducer. Once this interface passes below the transducer, the concentration measured would be zero. Thus the settling curve is similar to a step function. However, it is clear from figure 3.3 that the suspension used in the experiments is poly-dispersed. More information about the PSD can be found from these curves. This is explained in the following paragraphs. 
From the slope of these settling curves, the settling velocity of the particles can be found. The slope gives the rate of change of concentration of particles in the suspension. The settling rates at the top and bottom zones are shown in figure 3.4. It can be seen from figure 3.4 that the settling rate for the top zone is $-0.275 \mathrm{~kg} / \mathrm{m}^{2}$-s after 30 seconds while the settling rate for the bottom zone reaches a maximum of about $-0.09 \mathrm{~kg} / \mathrm{m}^{2}-\mathrm{s}$ at about a minute after the start of the experiment. The bottom zone settling rate reaches a maximum later than that of the top zone because particles are being fed into the bottom zone from the top zone.

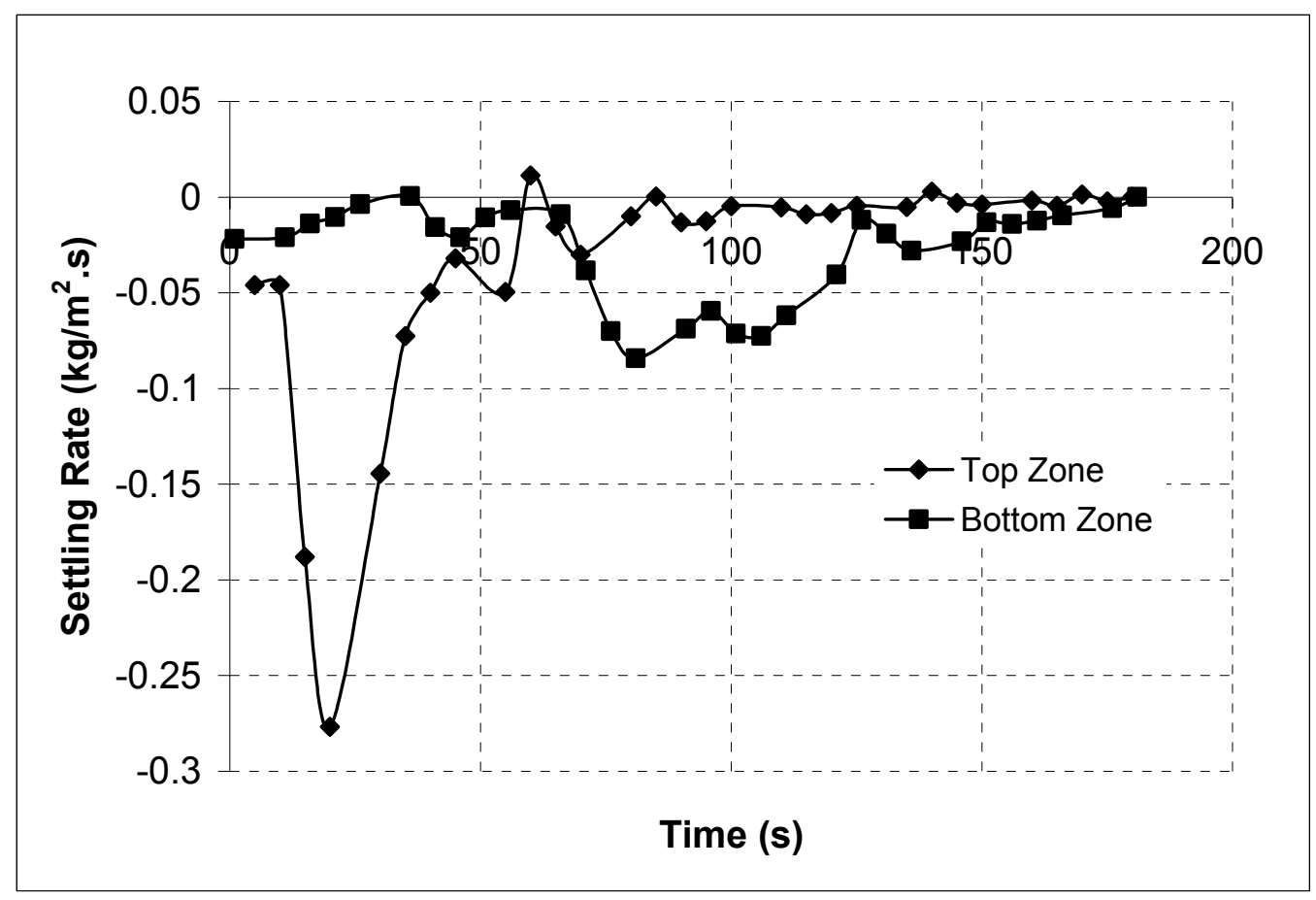

Figure 3.4: Settling rates at the top and bottom zones.

The slope of the settling curves in figures 3.2 and 3.3 is related to the settling velocity through the following equation: 
$\frac{d C}{d t}=\frac{1}{M} \rho A_{c s} u_{c}$

$M=$ total mass of water in the tank.

$A_{c s}=$ area of cross-section of the tank.

$u_{c}=$ settling velocity.

Knowing the settling velocity, if the modified Stokes law given by equation (1.1) is assumed correct, the mean diameter of the settling particles could be found. For example, slope of the settling curve in figure 3.1 (near the end) can be found as 0.00016 / sec. Using equation (3.4) we can find that the settling velocity, $u_{c}$, is $0.31 \mathrm{~mm} / \mathrm{sec}$. Making use of equation (1.1) we find that this settling velocity corresponds to a mean diameter of $3 \mu \mathrm{m}$. A sample of fly-ash used in this experiment was analyzed under a SEM and the mean diameter was found to be $2.06 \mu \mathrm{m}$. The particle sizes varied from $0.5 \mu \mathrm{m}$ to $15 \mu \mathrm{m}$. Thus the calculations made gave a reasonably good estimate of the mean diameter of the particles. It was found that the mean size found from the bottom zone measurements were a lot more accurate. The calculations made using the slope obtained from figure 3.2 showed the mean diameter to be $2.4 \mu \mathrm{m}$. If the mean diameter is known, then the validity of equation 1.1 can be ascertained. The calculations made above show that equation 1.1 gives a good estimate of the settling velocity.

These experiments were carried out a few times in order to check the repeatability of the data obtained. The results can be seen in figure 3.1 and figure 3.2. It can be seen that the curves are reasonably repeatable but for the starting 
concentration. The reasons for the discrepancy in the measured initial concentration have been discussed before.

The effect of suspension height was also studied. Three different cases were considered: a) no air-gap in the tank, b) half an inch of air-gap in the tank, and c) 1 inch of air-gap. This means that the total suspension height was 12 inches, 11.5 inches and 11 inches respectively. The results of these experiments are shown below in figures 3.5 and 3.6.

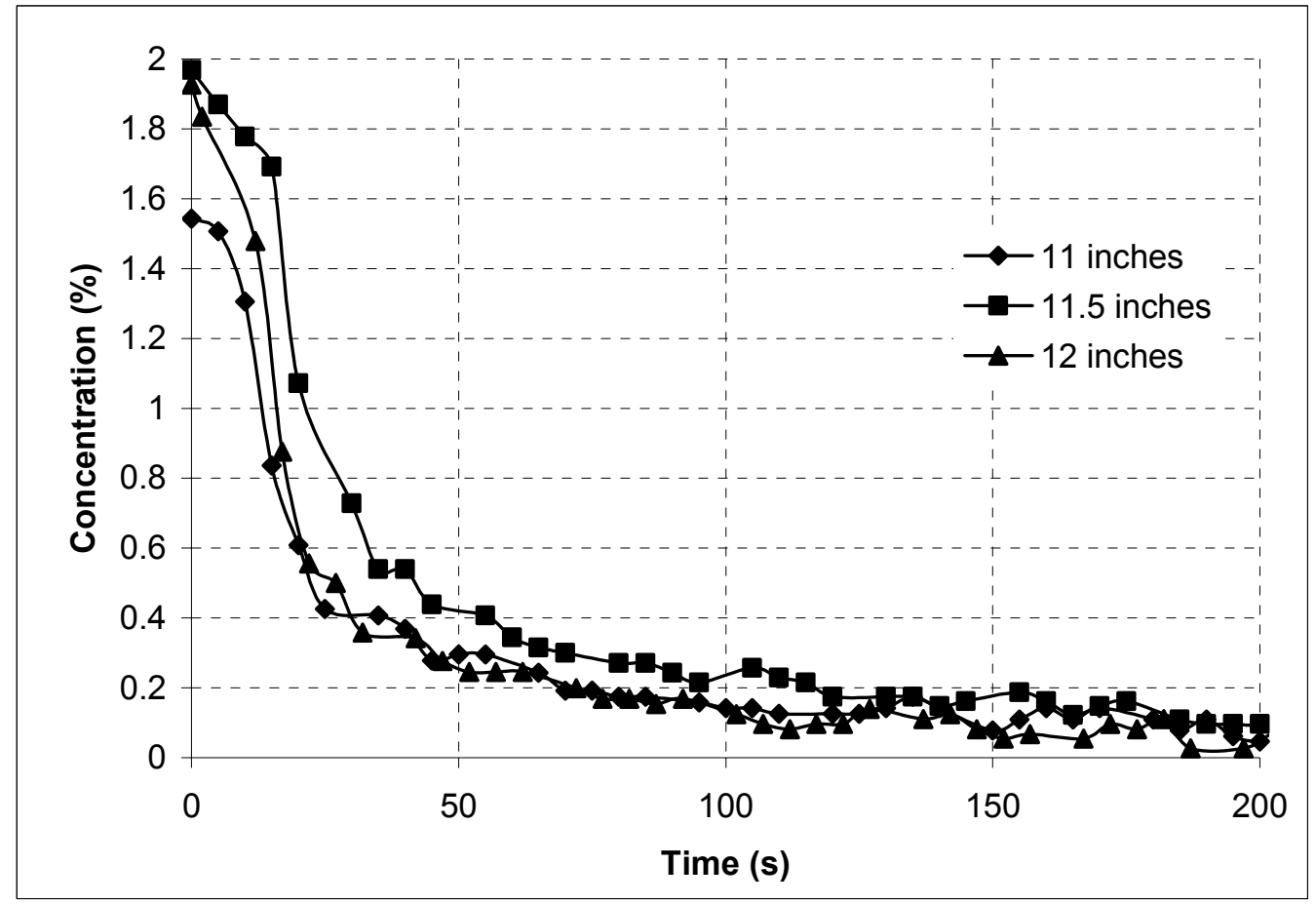

Figure 3.5: Effect of Height of Suspension on Settling in the Top Zone. The curves correspond to $11,11.5$ and 12 inches of suspension height. 


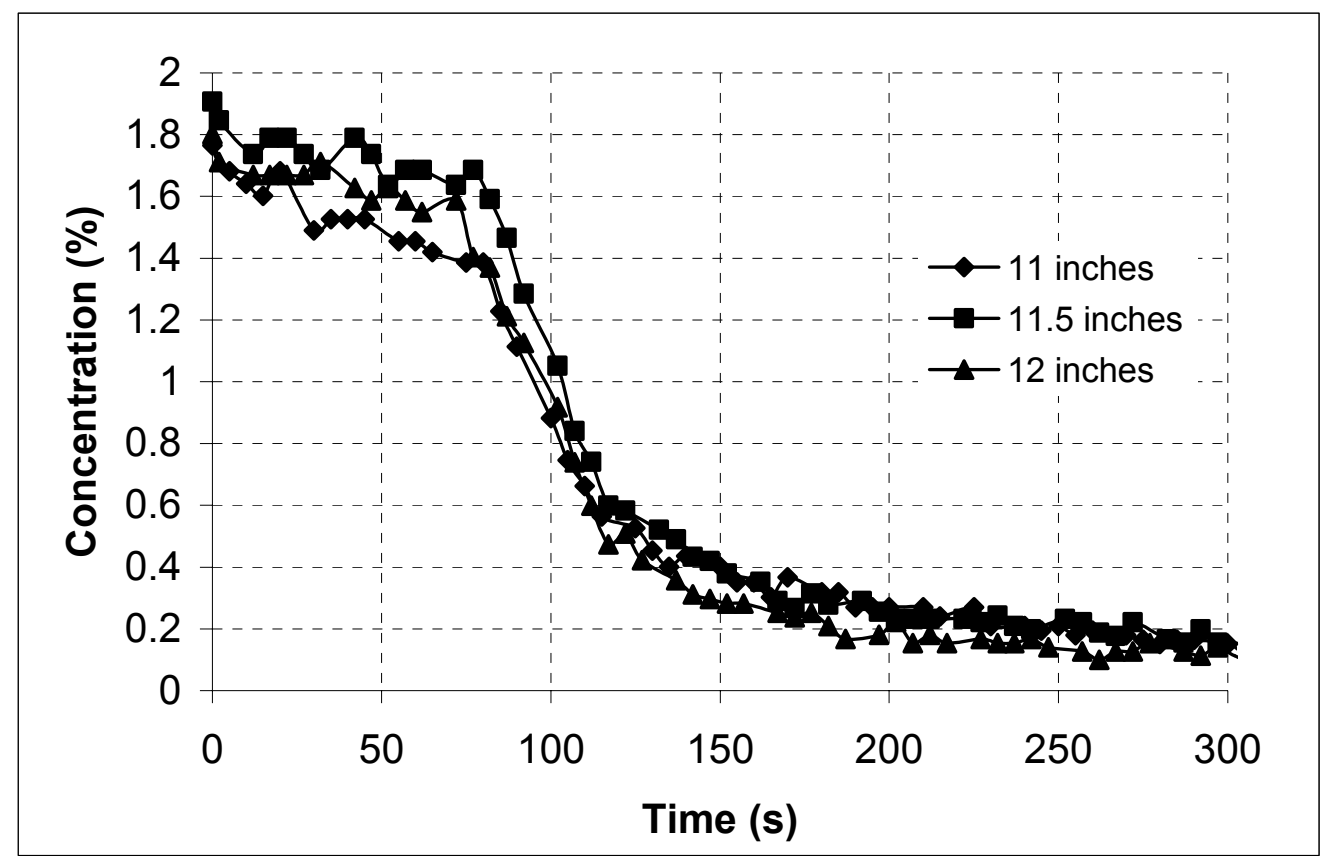

Figure 3.6: Effect of Height of Suspension on Settling in the Bottom Zone. The curves correspond to $11,11.5$ and 12 inches of suspension height.

Figure 3.5 shows the effect of height of suspension on the settling curve at the top zone. It can be seen that the curves are similar except for the initial concentration. The reason for the initial concentration not being the same has been discussed already. Also, there is no particular effect on settling due to difference in height of suspension. Figure 3.6 shows that there is no effect on sedimentation due to differences in the height of suspension at the bottom zone. Sedimentation theory, also, predicts that there be no effect on settling due to change in height of suspension. The height of suspension generally does not affect the rate of sedimentation or the consistency of the sediment layer obtained. The position of the sludge line (interface between clear liquid and zone of constant proportion) plotted versus time for two different heights of suspension are 
different. However the curves are similar and once one of the curves is obtained the other one can be obtained.

\subsection{Settling Of Particles With Shaking.}

In the following experiments, as mentioned before, the tank was oscillated once the mixing of the suspension was complete. The tank was shaken at a frequency of $10 \mathrm{~Hz}$ and at amplitude of $0.625 \mathrm{~cm}$ or quarter of an inch $(1 / 4 \mathrm{inch})$. There is a time gap of about 20 seconds between the end of mixing process and start of shaking. This time is required in order to start the function generator, and the power amplifier. More time on the order of 45 seconds were spent in closing the lid of the tank without leaving any air-gap in the tank. Totally there is a gap of about 65 seconds before shaking begins. During this time, the particles would settle out. So the particle concentration in the suspension when the shaking begins can not be controlled. This concentration can be found from the settling curves that show the variation of particle concentration with time. This experiment was repeated a few times in order to find a relation between the initial concentration $\left(\mathrm{C}_{0}\right)$ and the final equilibrium concentration $\left(\mathrm{C}_{\mathrm{f}}\right)$. Also, a relation between the

concentration when shaking begins $\left(C^{\prime}\right)$ and $C_{f}$ was sought. Figures 3.7 and 3.8 show the settling curves for the top zone and bottom zones respectively. As it was noted in the settling without shaking experiments, $\mathrm{C}_{0}$ varies based on PSD and amount of mixing. 


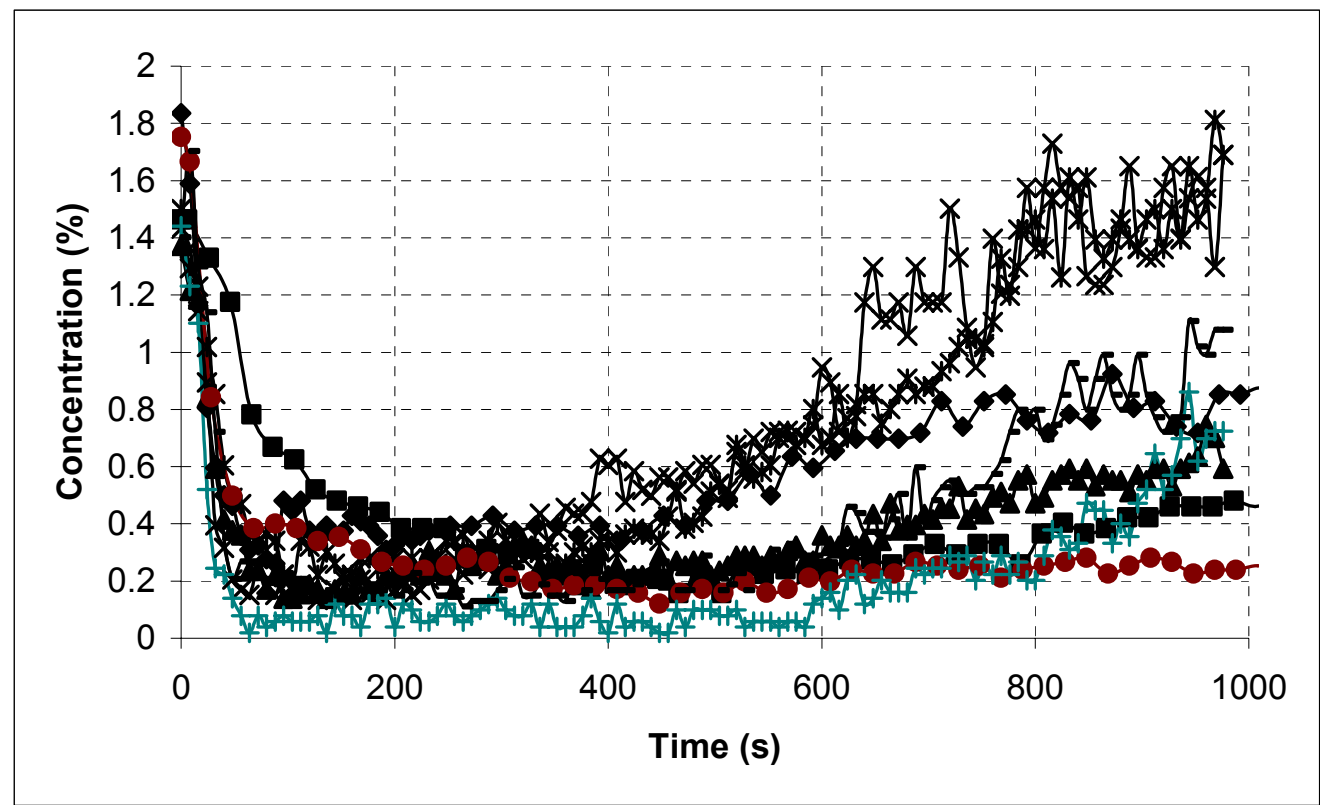

Figure 3.7: Concentration for shaking experiments measured at Top Zone.

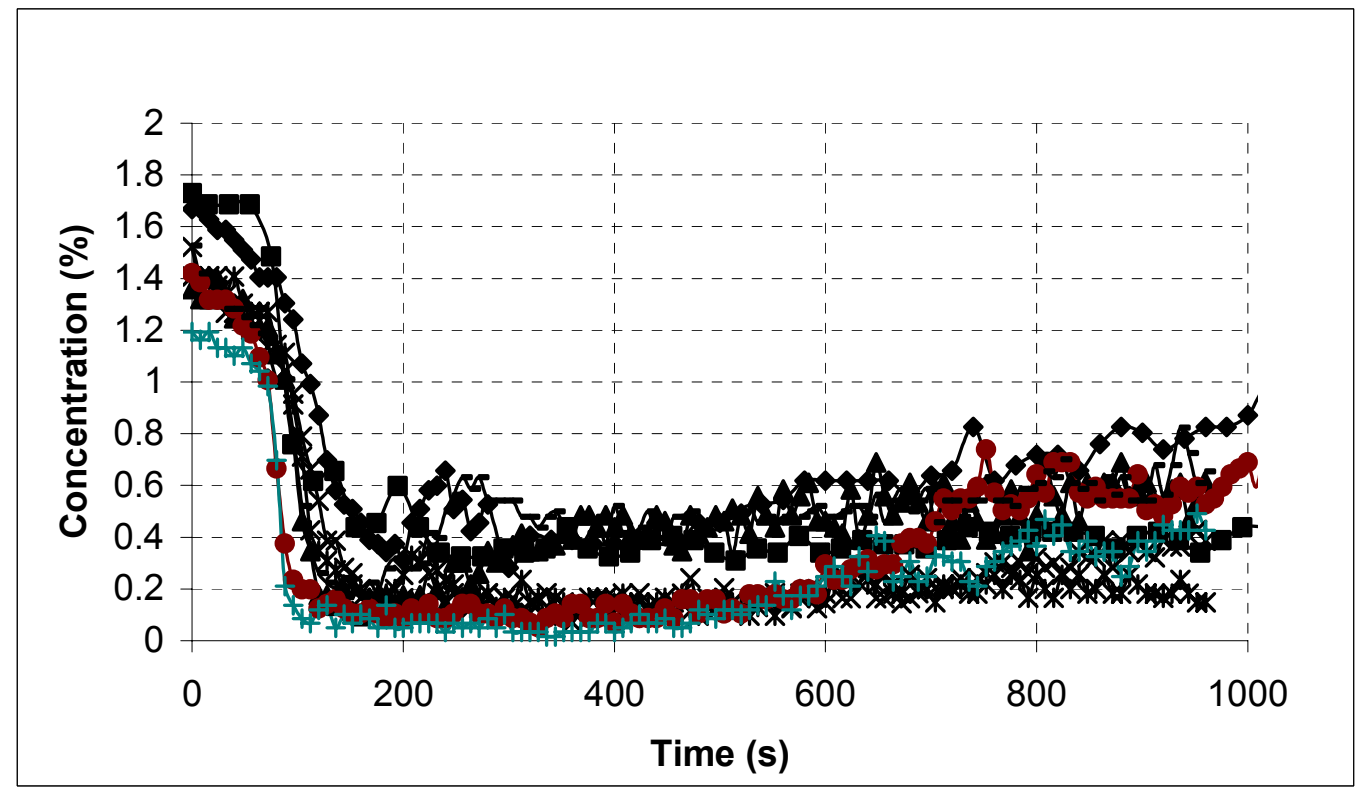

Figure 3.8: Concentration for shaking experiments measured at bottom zone. 
It can be seen from figures 3.7 and 3.8 that the equilibrium concentration varies even though the experimental conditions but for the particle sample was the same. The reason for this poor repeatability might be the PSD of the sample. The curves shown in figure 3.7 and 3.8 were averaged and smoothed to the curve shown in figure 3.9. As noted already, shaking begins 65 seconds after data acquisition is started. It can be seen that the particles settle during this 65 seconds delay. It can be seen that the concentration of particles in the suspension at the top zone is around $0.6 \%$ after 65 seconds while that of the bottom zone is around $1.2 \%$. At the top zone, the particles continue settling down for about 50 seconds after the beginning of shaking with the concentration reaching a minimum value of about $0.2 \%$ by mass. Then, the concentration of particles remains nearly a constant for about a hundred seconds after which the concentration starts to rise rapidly with time. The concentration of particles keeps increasing until it reaches an equilibrium concentration at the end. Though the attainment of an equilibrium concentration is not evident from figure 3.9 due to averaging, yet this is clear from figure 3.7 and figure 3.8 . The averaged curve shows that $C_{f}$ is about $1.1 \%$ by mass. 


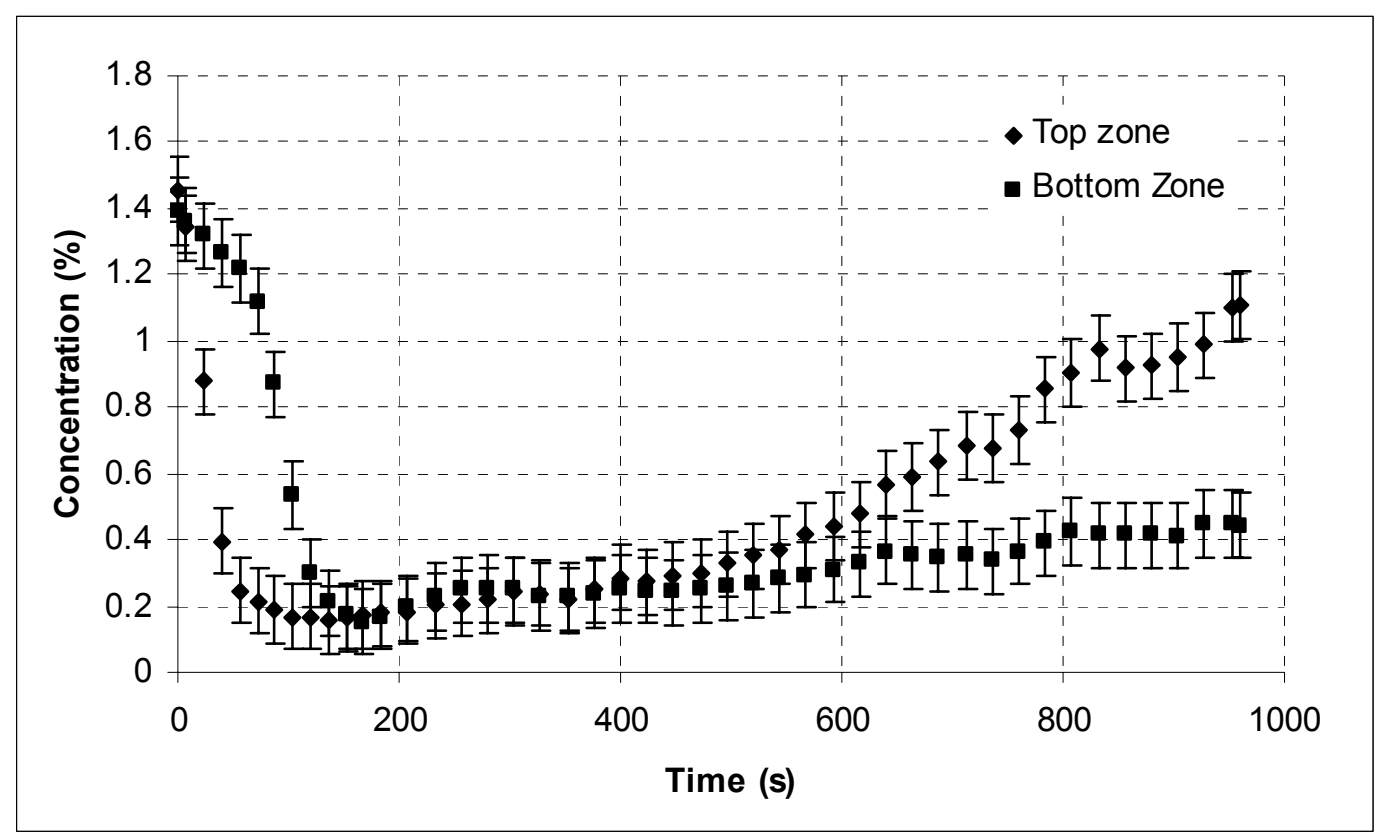

Figure 3.9: Average settling curves for top and bottom zones.

The settling trend is the same at the bottom zone as that of the top zone. The particles tend to settle out for about 200 seconds and the concentration reduces to a value of about $0.2 \%$ by mass. Then the concentration of particles in the suspension starts increasing until it reaches a final equilibrium concentration. The rate of increase in concentration at the bottom zone seems to be lesser as compared to that of the top zone. The equilibrium concentration is found to be about $0.5 \%$ by mass from figure 3.8 . This is about $0.75 \%$ less than the top zone equilibrium concentration.

The variation of $\mathrm{C}_{\mathrm{f}}$ with the initial concentration $\mathrm{C}_{0}$ for the top and bottom zone is shown in figure 3.10. The relation between $\mathrm{C}_{0}$ and $\mathrm{C}_{\mathrm{f}}$ is not quite clear at this moment. It is interesting to note that the equilibrium concentration reaches high values for low values of initial concentration. 


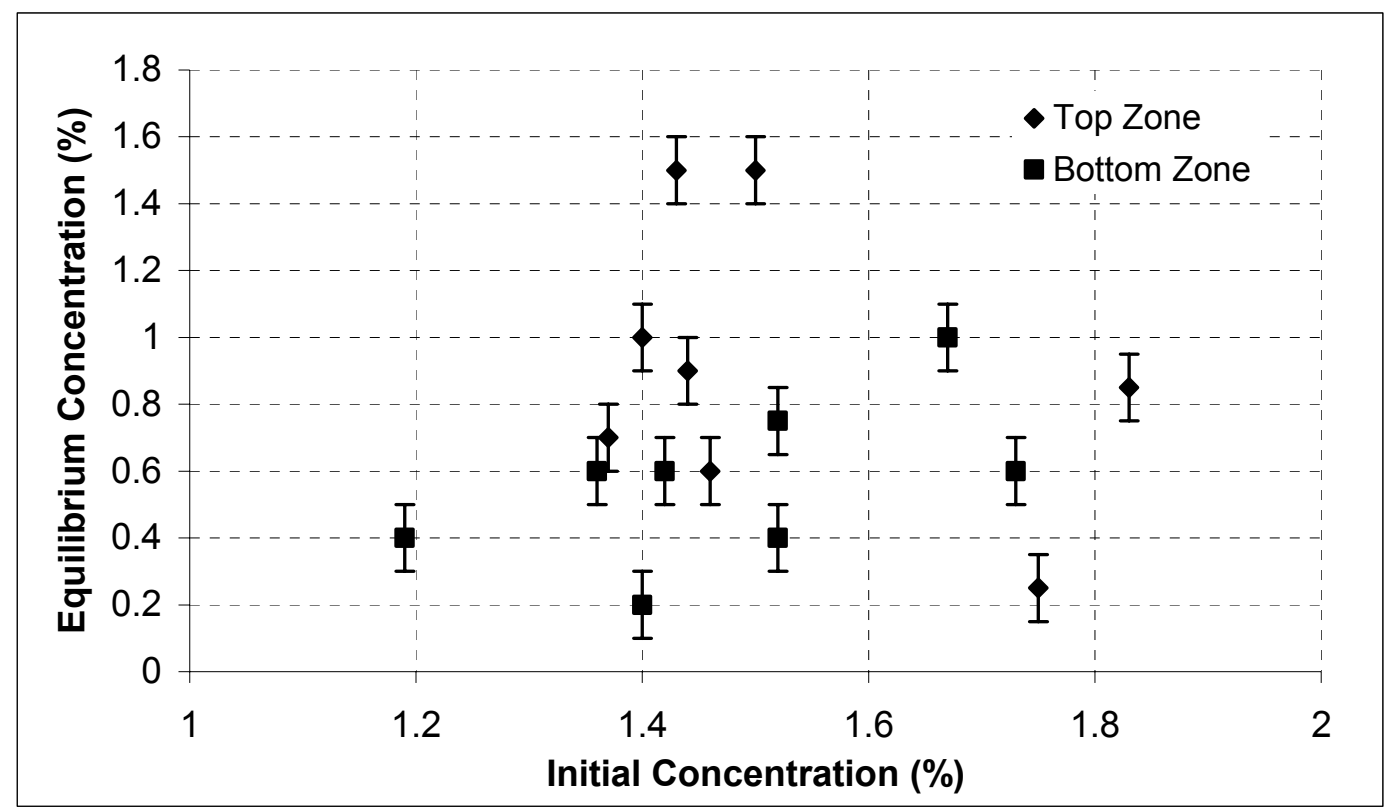

Figure 3.10: Variation of Equilibrium Concentration $\left(\mathrm{C}_{\mathrm{f}}\right)$ with Initial Concentration $\left(\mathrm{C}_{0}\right)$ at the top and bottom zones.

The variation of $\mathrm{C}_{\mathrm{f}}$ with $\mathrm{C}^{\prime}$ (concentration of particles in the suspension when shaking begins) for the top and bottom zones is shown in figure 3.11. Since shaking causes the resuspension that has been reported, the concentration at the beginning of shaking would be expected to determine the equilibrium concentration. Though a better correspondence between $\mathrm{C}_{\mathrm{f}}$ and $\mathrm{C}^{\prime}$ is expected as compared to that of $\mathrm{C}_{\mathrm{f}}$ and $\mathrm{C}_{0}$, the equilibrium concentration does not show any particular relation with $\mathrm{C}^{\prime}$. 


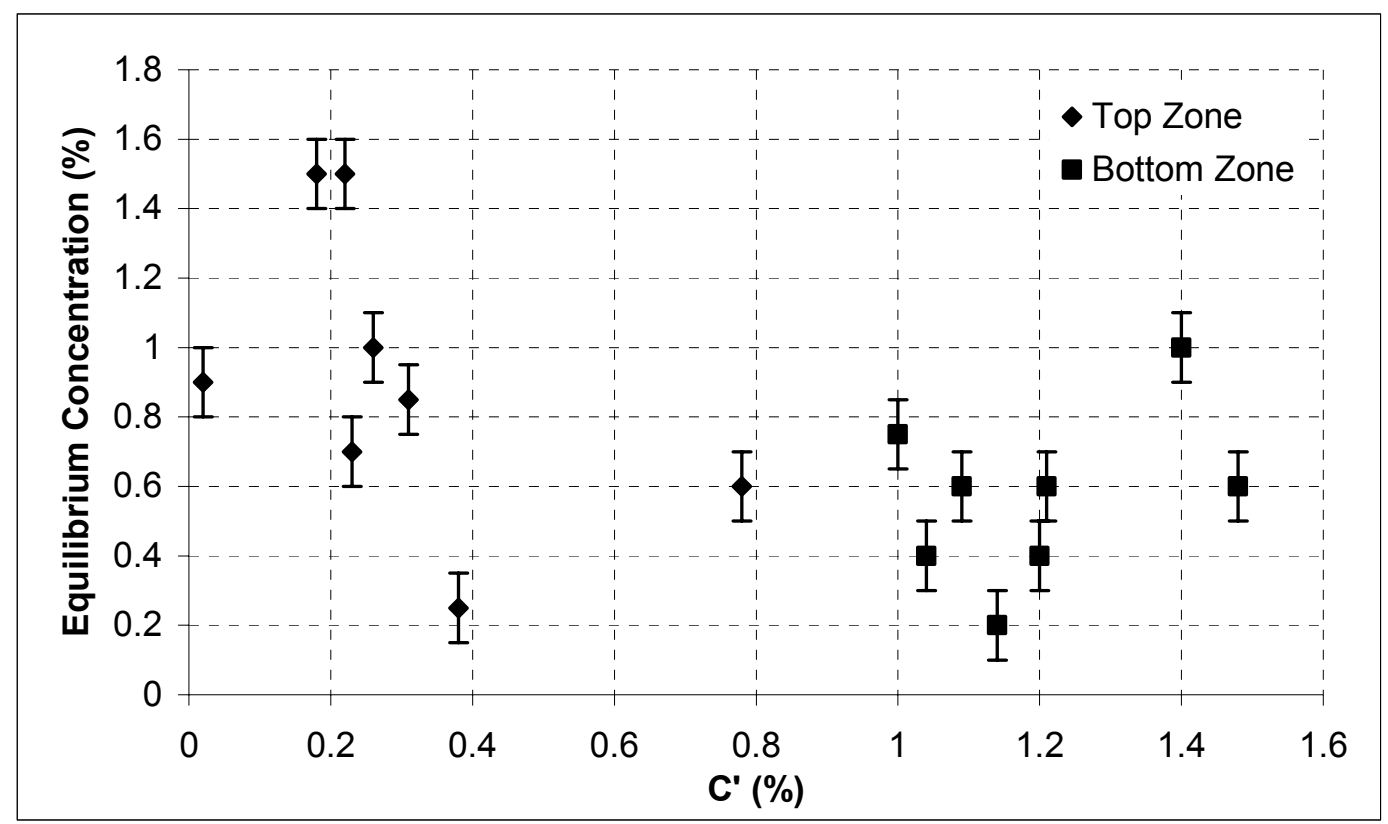

Figure 3.11: Variation of Equilibrium Concentration (Cf) with $\mathrm{C}^{\prime}$ at the top zone.

Figure 3.12 shows one of the settling curves from the top zone. It can be seen that the particle concentration oscillates up and down after a certain amount of time. In the case shown the fluctuations start after a hundred seconds. The resuspension mechanism could be the cause of these fluctuations also. The number of peaks in each interval of time (between two grid lines) about the same and in this case it is seven. A Fourier power spectrum analysis had to be done in order to find out the various frequencies making up the settling curve. The power spectral analysis of a top zone settling curve is shown in figure 3.13. The maximum spectral coefficient is obtained for zero frequency. This trivial peak value has been removed in order to look at some of the other peaks. There are two significant peaks at frequencies of $0.007 \mathrm{~Hz}$ and $0.025 \mathrm{~Hz}$. It was found that the peak frequencies are not the same for all settling curves. These two peak values 
might just be the noise frequencies. However, the actual reason behind these two peaks is not clear yet.

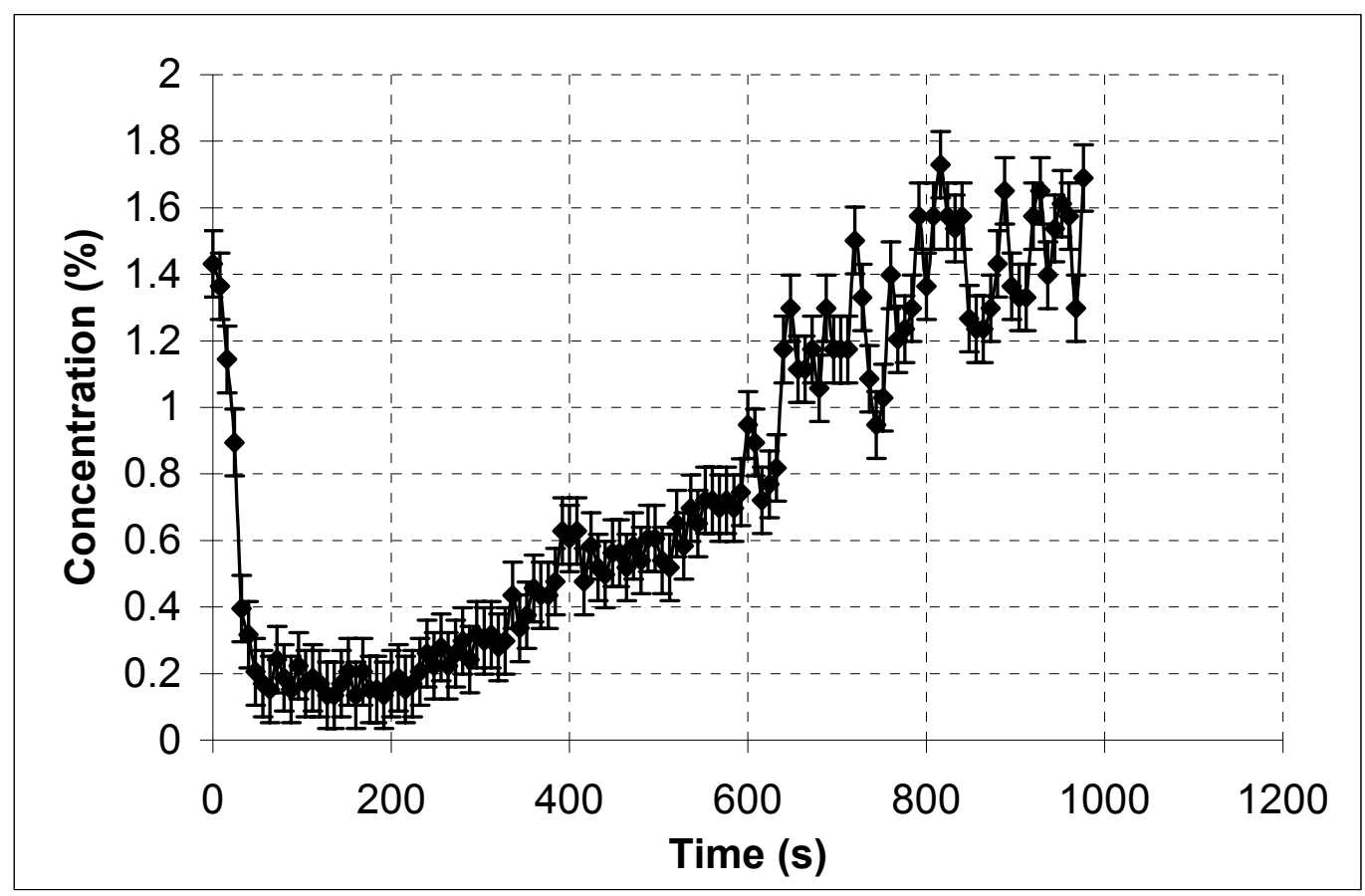

Figure 3.12: A typical top zone settling curve. 


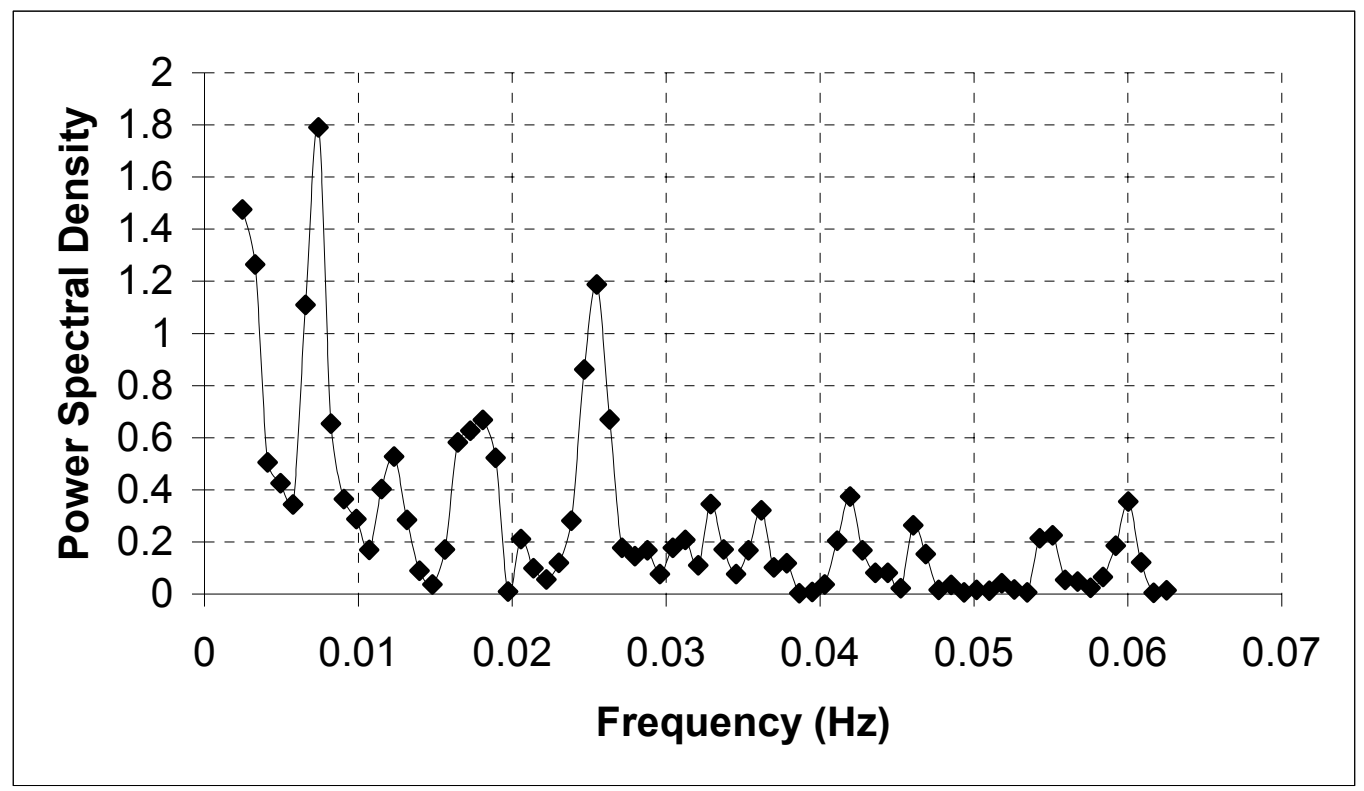

Figure 3.13: Power Spectral Analysis of top zone settling curve.

The reason for this resuspension or mixing is discussed in the next chapter. Some of the factors that could cause this kind of mixing were discussed in the first chapter of the thesis. More discussion of these factors pertaining to the shaking tank is provided in the following chapter. A superimposed fluids problem is discussed later in the thesis in order to support this stand. 


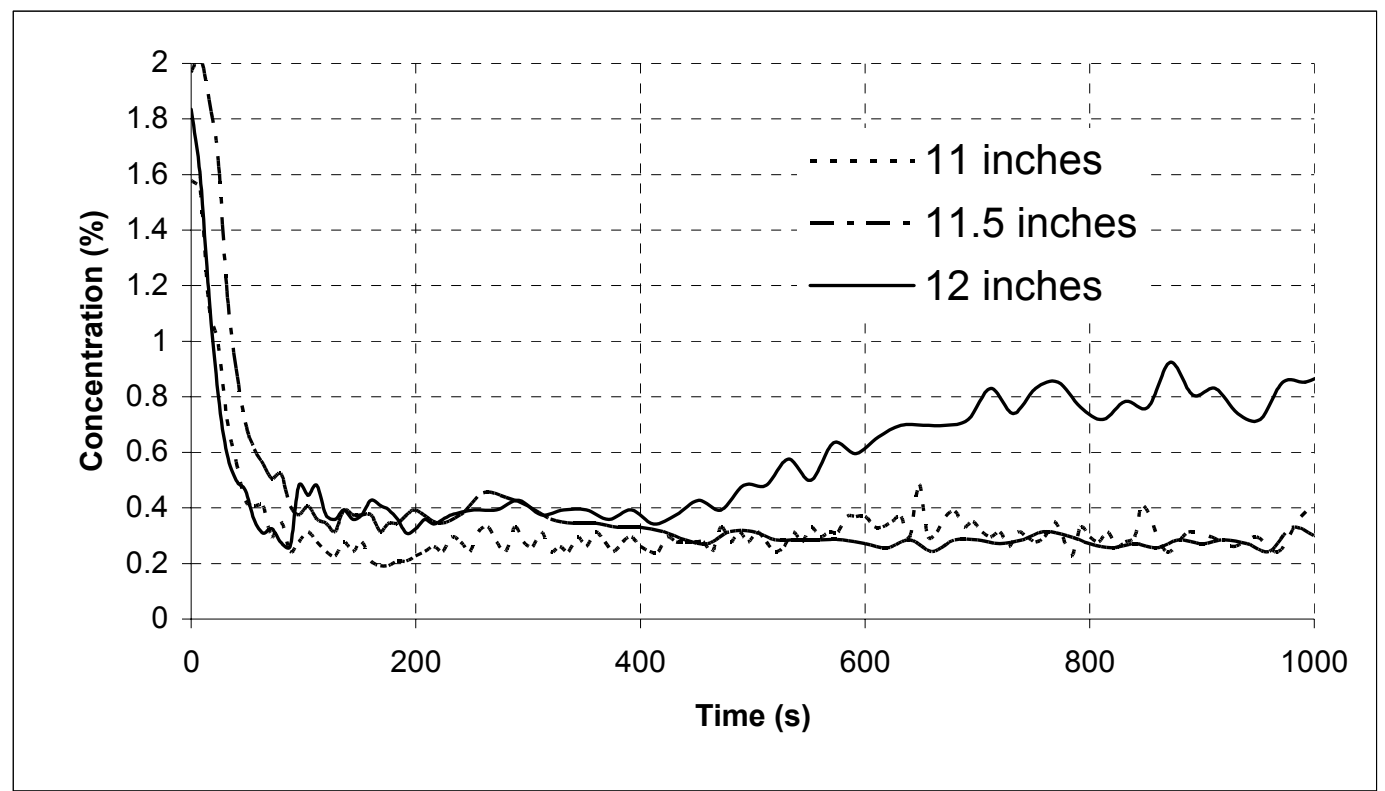

Figure 3.14: Effect of height of suspension at the top zone. The curves correspond to $11,11.5$ and 12 inches of suspension height.

The effects of the height of suspension were studied then. Surprisingly, the results showed in figures 3.14 and 3.15 indicated that there is nearly no resuspension when there is an air-gap. It has to be noted that 11 inch suspension height means 1 inch of air-gap and 12 inch suspension height corresponds to no air-gap. The solid line in both the figures corresponds to the case where the tank is completely filled. The dotted line corresponds to the case where there is an inch of air gap and we can see that there is no resuspension. The other line corresponds to the case where there is half an inch of air gap and again there is no resuspension. On the contrary, enhanced mixing was expected in the latter cases since the displacement of sloshing waves would be greater when there is an airgap. 


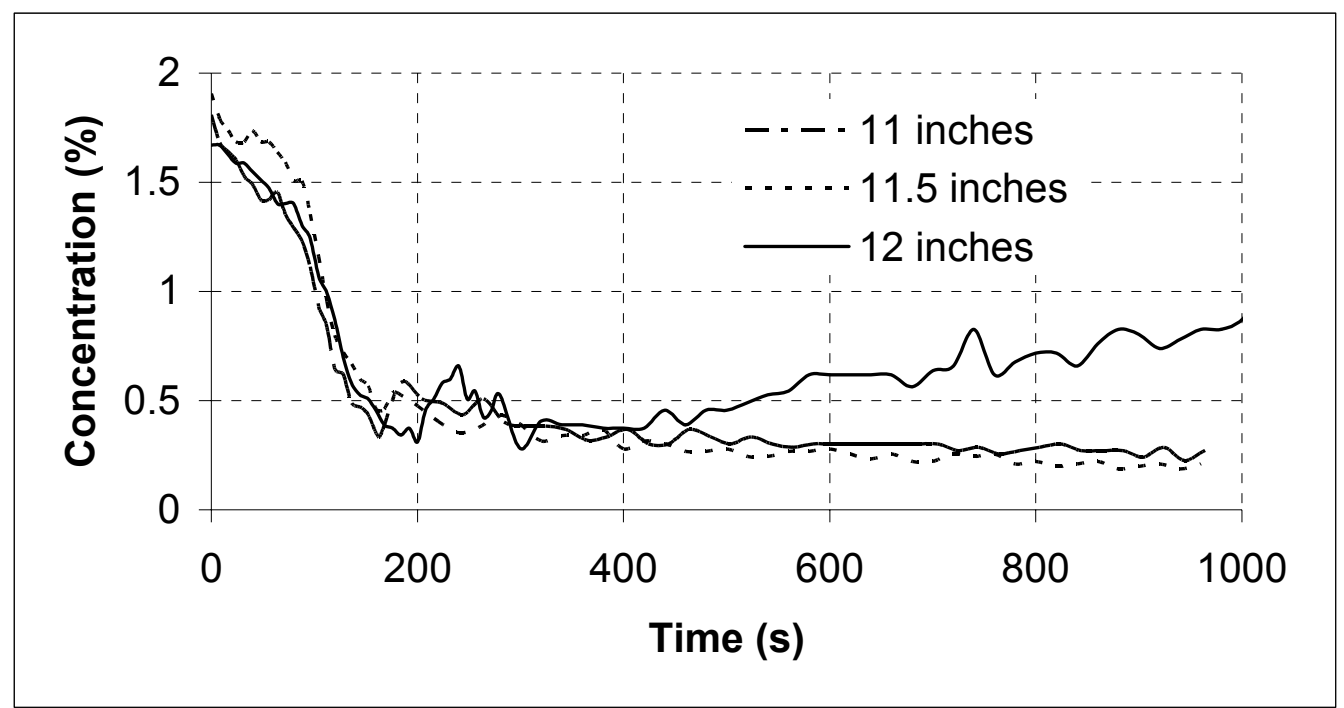

Figure 3.15: Effect of height of suspension at the bottom zone. The curves correspond to $11,11.5$ and 12 inches of suspension height.

Experiments were conducted in order to find the effect of amplitude of oscillations on the resuspension of particles. The amplitudes of oscillation used were 0.15 inch, 0.2 inch and 0.25 inch. Figures 3.16 and 3.17 show the effects of amplitude of oscillation at the top zone and bottom zone respectively. The solid lines in the two figures show settling with amplitude of quarter of an inch. The other two lines correspond to 0.15 inch and 0.2 inch amplitude of oscillation. It is clear from the figures that the resuspension is considerably larger when the amplitude of oscillation is 0.25 inch as compared to the other amplitudes of oscillation. The equilibrium concentrations for the latter cases are nearly equal to each other. 


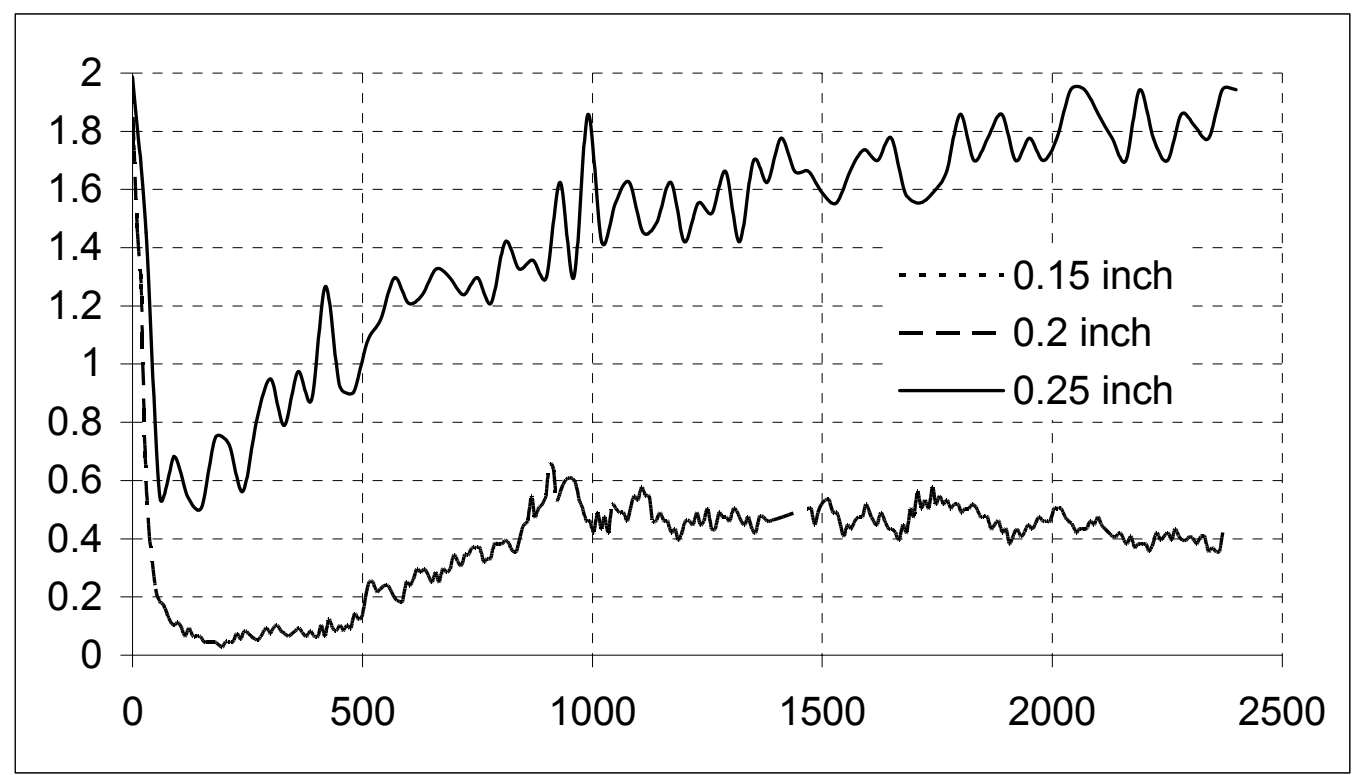

Figure 3.16: Effect of amplitude of oscillation on settling of particles at the top zone.

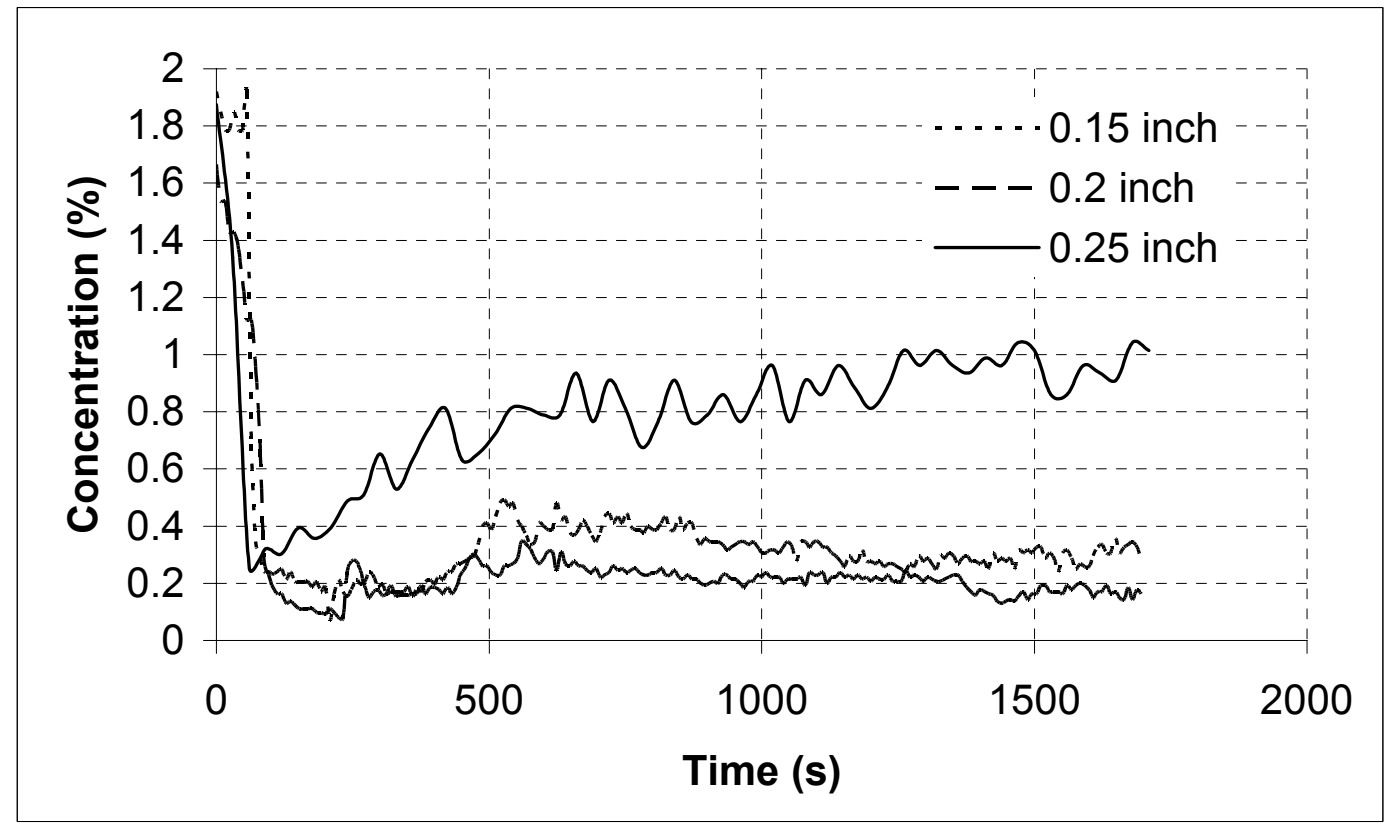

Figure 3.17: Effect of amplitude of oscillation on settling of particles at the bottom zone. 


\subsection{Shaking A SETTLEd Bed OF PARTiCles.}

In this set of experiments, the particles were allowed to settle down to the bottom before the tank was oscillated. Data acquisition was started only after the particles were completely settled. The results obtained for the top and bottom zones from this experiment are shown in figures 3.18 and 3.19 respectively.

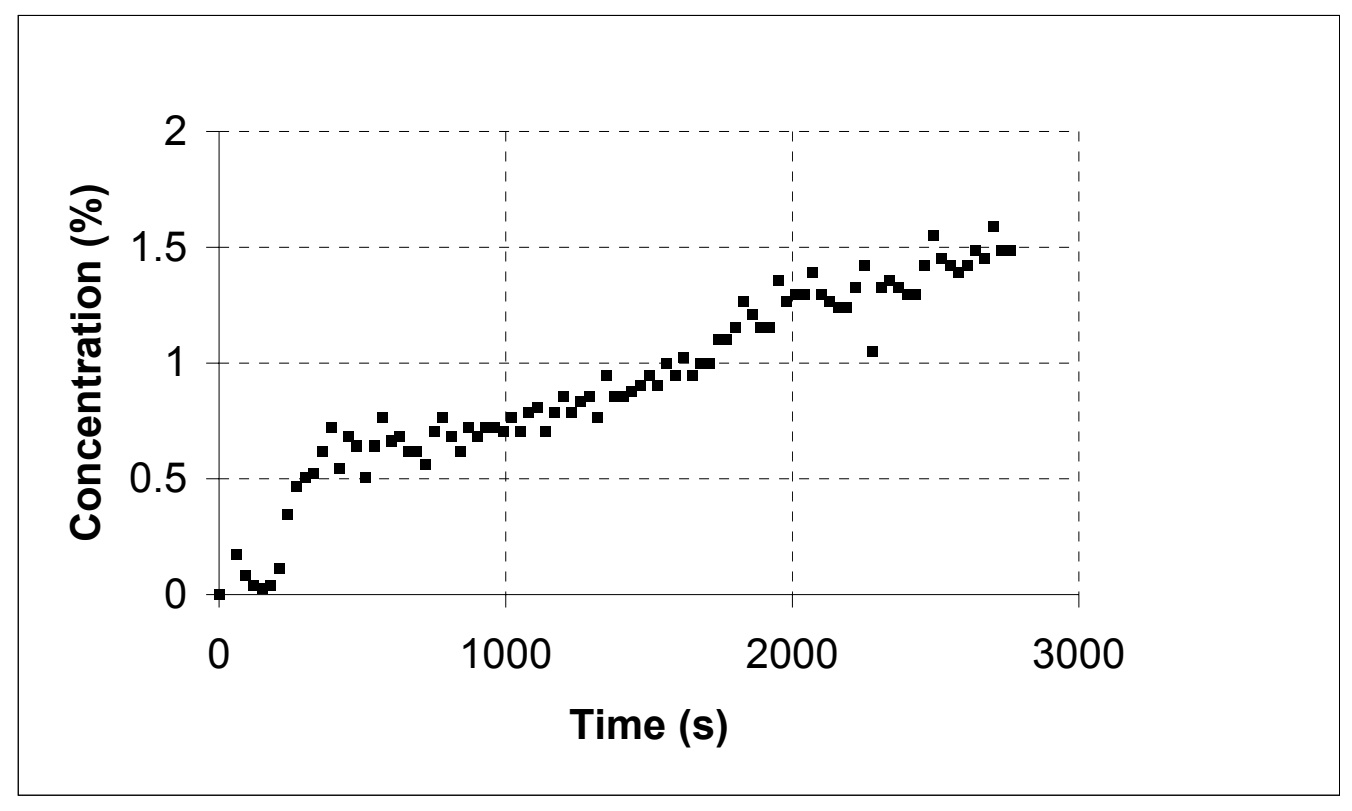

Figure 3.18: Shaking a Settled Bed of Particles: Top Zone. 


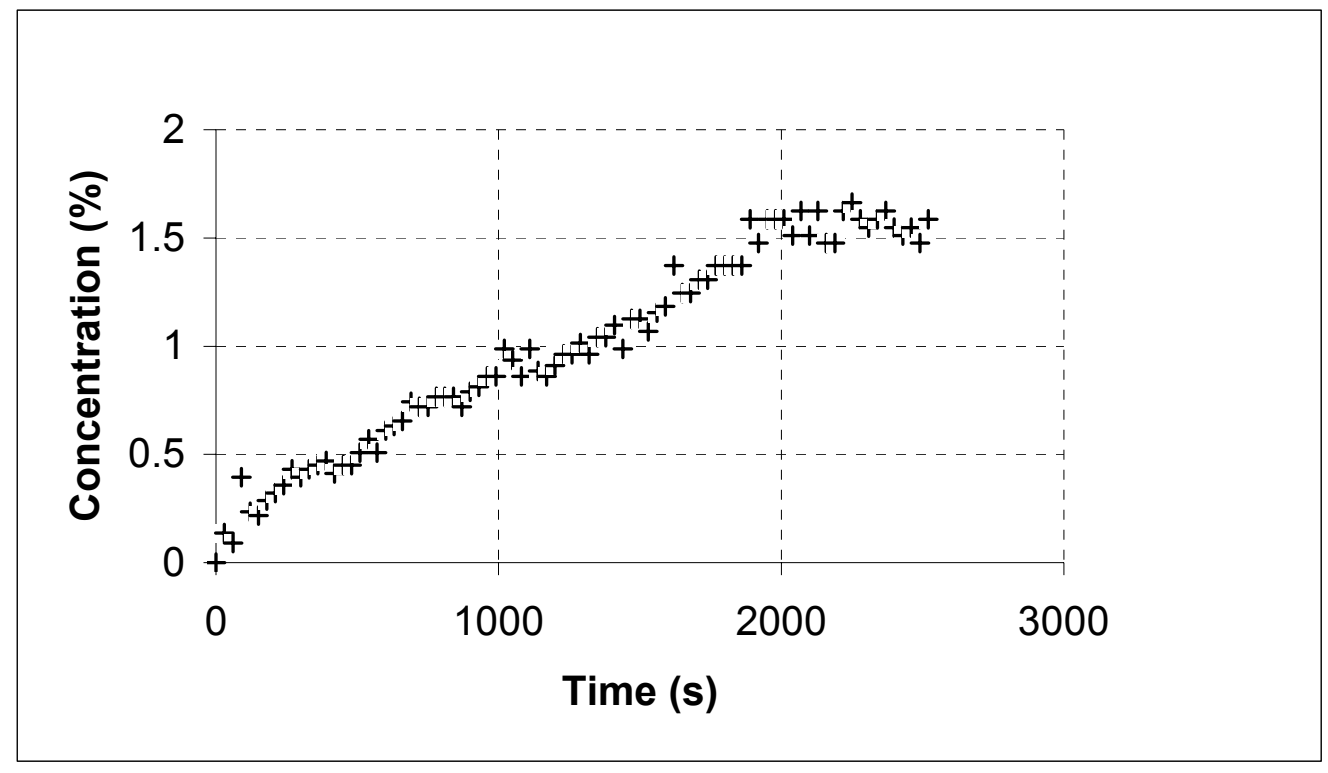

Figure 3.19: Shaking a Settled Bed of Particles: Bottom Zone.

From figures 3.18 and 3.19 , it is found that the particles mix and get resuspended when the tank begins to oscillate. The concentration of particles in the suspension increases steadily until it reaches an equilibrium value. This equilibrium concentration was about $1.5 \%$ in both the cases. The resuspension could have been enhanced by the repulsive forces existing between particles in the settled layer. Since the resuspension can be modified by factors other than the lateral oscillation in this case, this experiment was not repeated.

Some of the preliminary data obtained through the ultrasonic measurement have been shown and discussed in this chapter. The data show that there, indeed, is a resuspension of particles in the suspension when the tank is oscillated. Also the data shows that the amplitude of oscillation and air-gap in the tank has an effect on the resuspension of particles. 


\section{Chapter 4: Resuspension Mechanisms}

Results of the experiments conducted with the resonant shaking apparatus have been discussed in the previous chapter. It was found that resuspension of particles in the suspension occurs. The reason for the resuspension of particles is not clear. A literature review on some of the mechanisms that cause resuspension or mixing of particles in the suspension was given in the first chapter. More details on some of these mechanisms would be given in this chapter.

A few of the possible mechanisms for the resuspension reported in the previous chapter are given below:

- Turbulence,

- Viscous resuspension,

- $\quad$ Viscous streaming, and

- Internal gravity waves.

\subsection{SCALING FACTORS}

Three different length scaling factors can be identified for the system discussed. They are:

- $\quad$ Length of the tank, $L(=0.3 \mathrm{~m})$,

- $\quad$ Size of the particles, $d(4 \mu m)$, and

- $\quad$ Amplitude of oscillation, $A(6.35 \mathrm{~mm})$.

Similarly, the different velocity scales identified for the system are:

- Terminal settling velocity of the particles in the suspension, $u_{c}(0.1 \mathrm{~mm} / \mathrm{s})$, and 
- Velocity of oscillation, $u_{o}(0.4 \mathrm{~m} / \mathrm{s})$.

Following are the time scales identified for the system:

- $\quad$ Stokes time, $\tau_{s t}(2 \mu s)$, defined by

$$
\tau_{s t}=\frac{\rho_{p} d^{2}}{18 \mu} \text {, }
$$

- $\quad$ Time period of oscillation, $T(0.1 \mathrm{~s})$, given by $T=\frac{1}{f}$, where $f$ is the frequency of oscillation, and

- $\quad$ Time required for the particles to settle down to the bottom of the tank based on the terminal settling velocity, $\tau(3000 \mathrm{~s})$, given by

$$
\tau=\frac{L}{u_{c}}
$$

\begin{tabular}{|c|c|c|c|c|}
\hline \multirow{2}{*}{$\begin{array}{c}\text { Scaling } \\
\text { Factor }\end{array}$} & \multicolumn{3}{|c|}{ Dimension } & \multirow{2}{*}{ Value } \\
\cline { 2 - 5 } $\boldsymbol{L}$ & 0 & Mength & Time & \\
\hline $\boldsymbol{d}$ & 0 & 1 & 0 & $0.3 \mathrm{~m}$ \\
\hline $\boldsymbol{A}$ & 0 & 1 & 0 & $4 * 10^{-6} \mathrm{~m}$ \\
\hline $\boldsymbol{u}_{\boldsymbol{c}}$ & 0 & 1 & 0 & $6.35^{*} 10^{-3} \mathrm{~m}$ \\
\hline $\boldsymbol{u}_{\boldsymbol{o}}$ & 0 & 1 & -1 & $10^{-4} \mathrm{~m} / \mathrm{s}$ \\
\hline $\boldsymbol{\tau}_{\boldsymbol{s} t}$ & 0 & 1 & -1 & $0.4 \mathrm{~m} / \mathrm{s}$ \\
\hline $\boldsymbol{T}$ & 0 & 0 & 1 & $2 * 10^{-6} \mathrm{~s}$ \\
\hline $\boldsymbol{\tau}$ & 0 & 0 & 1 & $0.1 \mathrm{~s}$ \\
\hline
\end{tabular}

Table 4.1: $\quad$ Scaling Factors.

Table 4.1 gives the various scaling factors and their values. Based on these scaling factors, several non-dimensional numbers like the Reynolds number can 
be defined. Reynolds' number is the ratio of inertial forces to that the viscous forces. This means that a small Reynolds' number corresponds to viscous flow. For example, the particle Reynolds' number is defined as:

$$
\operatorname{Re}_{p}=\frac{u_{c} d \rho_{p}}{\mu} .
$$

The Reynolds number is based on the terminal settling velocity of the particle. This number is very small, on the order of $10^{-4}$. This means that the particles act like Stokes particles, that is, their inertia is negligible. Several other Reynolds number could be defined for this problem. For example, a Reynolds number can be defined based on the oscillatory velocity, $A \omega$, instead of the terminal settling velocity and the tank length instead of particle diameter. This non dimensional number is given by the following equation:

$$
\operatorname{Re}_{o}=\frac{A \omega \rho_{p} L}{\mu}
$$

The value of this Reynolds number, $R e_{o}$, turns out to be 272000 . This Reynolds number is significantly large and may suggest that the flow is turbulent. In the definition of $R e_{o}$, it has been assumed that the suspending fluid is moving with a velocity, $A \omega$, and that the particles are stationary. However, the particles in a fluid undergoing an oscillatory flow, as shown in figure 4.1, acquire a velocity that is very close to that of the fluid velocity. 

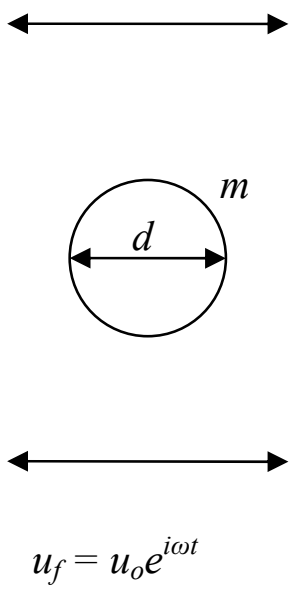

Figure 4.1: Particle in oscillatory flow.

Assuming that the only force acting on such a particle (particle in oscillatory flow) is the Stokes drag force, we find that the particle velocity is given by the following equation:

$$
u_{p}=\frac{u_{o}}{\left(1+\omega^{2} \tau_{s t}^{2}\right)}\left[\cos (\omega t)+\omega \tau_{s t} \sin (\omega t)-\exp \left(-\frac{t}{\tau_{s t}}\right)\right]
$$

From equation (4.5) it can be seen that the particle velocity, $u_{p}$, is nearly the same as the fluid velocity, $u_{f}$, as long as the product of angular frequency, $\omega$, and Stokes time, $\tau_{\mathrm{st}}$, is small compared to unity. For the problem considered in this thesis, this product is negligibly small compared to unity. This means that the particles would follow the fluid identically. Thus the Reynolds number based on the relative velocity of particles with respect to that of the fluid is negligibly small.

Another non-dimensional parameter that could be defined is the Stokes number. It is defined as: 


$$
S t=\frac{S}{d_{c}},
$$

where $S$ is the stopping distance of a particle and $d_{c}$ is the characteristic dimension of the obstacle. For example, for flow perpendicular to a cylinder of diameter $d_{c}$, the Stokes number is

$$
S t=\frac{\tau_{s t} U_{0}}{d_{c}},
$$

where $U_{0}$ is the undisturbed fluid velocity well away from the cylinder and $\tau_{s}$ is the relaxation time ( also, called Stokes time). The relaxation time is the time required for the particles to adjust or relax their velocities to a new condition of forces. The Stokes number is also the ratio of the particle relaxation time to the transit time past an obstacle, or the ratio of time $\tau_{s}$ it takes a particle to adjust to the time $d_{c} / U_{0}$ available for adjustment. When the Stokes number, St, is large compared to unity the particles continue moving along their trajectory when the fluid turns; when $S t$ is small compared to unity the particles follow the fluid streamlines perfectly. As the Stokes number increases, the particles resist changing their directions according to the change in fluid streamlines. Different Stokes number could be defined based on different characteristic dimensions of the system considered. For example, in the experimental system considered in this thesis, there are three different characteristic dimensions and hence three different Stokes number could be defined. First Stokes number is based on the particle size and is defined as follows:

$$
S t_{p}=\frac{A \varpi d \rho_{p}}{\mu} .
$$


The particle Stokes number is on the order of 1 meaning that the relaxation time and the time available are nearly the same. A similar Stokes number can be defined based on the characteristic dimension of the system as well. This number is defined as follows:

$$
S t_{s L}=\frac{\rho_{p} A \varpi d^{2}}{\mu L} \text {. }
$$

This number based on the length of the tank and is equal to $4.8^{*} 10^{-5}$. Such low value of Stokes number means that the particle has sufficient time to adjust to the flow field around it. The Stokes number based on the dimension of the tank is significantly smaller than the particle Stokes number. This means that the particle follows the flow field setup in the fluid. Thus the probability of the flow being turbulence is small. However, it is not clear if this Stokes number is the dominant non-dimensional parameter of this problem.

Another Stokes number can be defined based on the amplitude of oscillation as follows:

$$
S t_{s A}=\frac{\rho_{p} \varpi d^{2}}{\mu} .
$$

This number turns out to be $2.3 * 10^{-3}$ for oscillation amplitude of $0.625 \mathrm{~cm}$ or quarter of an inch. It can be seen from equation (4.8) that the Stokes number based on amplitude is independent of the amplitude, $A$. Resuspension or mixing was not seen for any oscillation amplitude less than or equal to $0.4 \mathrm{~cm}$. This means that the mixing is dependent on the amplitude of oscillation. Hence the Stokes number based on the amplitude of oscillation is not an important parameter. The Stokes number defined based on the amplitude of oscillation is an 
important non-dimensional parameter in the Stokes oscillating plate problem. The flow transitions from laminar to turbulent for Stokes number of 50000 and becomes completely turbulent for a Stokes number of 500000 [Hsu et al, (2000)]. However, it is clear from the particle Stokes number that the inertial term in the Navier-Stokes equations can not be neglected. A summary of all the nondimensional numbers identified can be found in table 4.2.

\begin{tabular}{|c|l|c|}
\hline $\begin{array}{c}\text { Non-Dimensional } \\
\text { Parameter }\end{array}$ & \multicolumn{1}{|c|}{ Description } & Value \\
\hline$R e_{p}=\frac{u_{c} d \rho_{p}}{\mu}$ & $\begin{array}{l}\text { Reynolds number based on particle settling } \\
\text { velocity and diameter. }\end{array}$ & $10^{-4}$ \\
\hline$R e_{o}=\frac{A \omega \rho_{p} L}{\mu}$ & $\begin{array}{l}\text { Reynolds number based on oscillatory velocity } \\
\text { and tank length. }\end{array}$ & 272000 \\
\hline$R e_{r e l p}=\frac{u_{r e l} d \rho_{p}}{\mu}$ & $\begin{array}{l}\text { Reynolds number based on relative velocity of } \\
\text { particle with respect to fluid and particle } \\
\text { diameter. }\end{array}$ & $10^{-12}$ \\
\hline$R e_{r e l L}=\frac{u_{r e l} L \rho_{p}}{\mu}$ & $\begin{array}{l}\text { Reynolds number based on relative velocity of } \\
\text { particle with respect to fluid and tank length. }\end{array}$ & $5^{*} 10^{-6}$ \\
\hline$S t_{p}=\frac{A \varpi d \rho_{p}}{\mu}$ & $\begin{array}{l}\text { Particle Stokes number. } \\
S t_{s L}=\frac{\rho_{p} A \varpi d^{2}}{\mu L}\end{array}$ & $\begin{array}{l}\text { Stokes number based on characteristic } \\
\text { dimension of the tank. }\end{array}$ \\
\hline$S t_{s A}=\frac{\rho_{p} \varpi d^{2}}{\mu}$ & $\begin{array}{l}\text { Stokes number based on amplitude of } \\
\text { oscillation. }\end{array}$ & $10^{-5}$ \\
\hline
\end{tabular}

Table 4.2: Non-Dimensional Parameters. 


\subsection{TurbulenCE}

Some of the characteristics of the turbulent flows have been witnessed in the shaking tank problem. For example, the flow in the shaking tank is irregular. It has been reported in the previous chapter that there is significant amount of mixing in the shaking tank problem.

Turbulence is inherently three dimensional and rotational. It is not clear from the experiments conducted on the shaking tank apparatus that the flow is three dimensional or rotational. It was assumed that the flow is two dimensional when the measurement technique was devised. Also, the Stokes number based on the particle size or the amplitude of oscillation is small to cause instabilities in the flow and hence cause the flow in the shaking tank to become turbulent. The Reynolds number based on the relative velocity of the particles with respect to suspending fluid is negligibly small. This means that the particles will follow the fluid and hence the presence of particles in the fluid does not cause turbulence.

From the above arguments, it is not unlikely that turbulence is causing the mixing reported.

\subsection{STREAMING}

A superimposed field of flow that occurs in the boundary layer, whose direction deviates from that of the external flow, is referred to as a secondary or streaming flow. For example, the secondary flow which accompanies near a solid wall can be clearly observed in a tea-cup. When tea in a cup is left to itself after vigorous stirring, a radial inward flow is formed at the bottom. The existence of 
this flow can be inferred from the fact that tea leaves settle near the centre at the bottom.

Small oscillations of a body in a fluid at rest induce characteristic flows whose nature is such that a steady motion is imparted to the whole fluid. This means that a potential flow which is periodic can induce a steady, secondary (streaming) motion at a large distance from the wall or body as a result of viscous forces. It should be noted, however, that the magnitude of the streaming velocity is independent of viscosity.

The secondary flow has its origin in the convective terms and is due to the interaction between inertia and viscosity. It should noted that simplifications in which the convective terms are neglected lead to flow without streaming and may give a misleading representation of the flow. Streaming, in general, appears only when the solution is carried to at least the second order approximation.

For oscillating external flows, the magnitude of the secondary flow velocity scales as follows [Schlichting, (1979)]:

$$
u_{2} \sim \frac{U_{r e l}^{2}}{L \omega} \text {, }
$$

where $U_{0}$ is the relative velocity of the fluid with respect to the moving body or wall, $\omega$ is the frequency of oscillation and $L$ is the characteristic dimension of the body or wall. In the oscillating tank problem, the relative velocity of water with respect to the tank walls is small. It was shown previously that a dye injected into the shaking tank moved along with the tank (like rigid body motion). This shows that the relative velocity should be small and hence the secondary flow velocity should be even smaller. 


\subsection{FLOW IN A SHAKING TANK}

\subsubsection{Analysis using Fluent.}

When a tank containing water completely starts shaking there is a body force acting on all the fluid elements. There is no shear stress on the fluid elements. There is no free surface present inside the tank. In the presence of free surface, there could be sloshing or similar motion at the surface of the water. This motion of the surface might set up a flow field in the fluid. Any of this motion is arrested by filling the tank completely with water. For this reason no relative motion was expected between the tank and the fluid inside.

An experiment was conducted in order to study the flow field inside a tank containing water completely. The details of this experiment were detailed in the second chapter of this thesis. It was found that the motion was a rigid body motion. In order to verify that motion was indeed rigid, simulations of the flow field in a shaking tank were conducted using Fluent. A UDF (user-defined function) had to be written in order to incorporate a couple of source terms in the $\mathrm{x}$ and $\mathrm{y}$ momentum equations. The details of these simulations follow.

First of all, the frame of reference was shifted from a stationary one to the shaking tank. This means that the following substitution was made.

$$
u^{\prime}=u-u_{0} \sin (\omega t) e_{x},
$$

where $u$ is the velocity with respect to the fixed frame of reference, $u$ ' is the velocity with respect to the moving reference frame, $u_{0}$ is the magnitude of the shaking velocity and $e_{x}$ is the unit vector along the positive x-direction. This 
expression for $u$ has to be substituted in the Navier-Stokes equation to get the following equation.

$$
\frac{\partial u^{\prime}}{\partial t}+u^{\prime} \cdot \nabla u^{\prime}+u_{0} \omega \cos (\omega t) e_{x}+u_{0} \sin (\omega t) e_{x} \cdot \nabla u^{\prime}=-\frac{1}{\rho} \nabla p+v \nabla^{2} u^{\prime} .
$$

The third term on the right hand side of equation (4.12) represents the inertial force acting on the fluid and the fourth term is due to convection of momentum by the oscillatory velocity. The other terms are the same as that of the regular terms in the Navier-Stokes equation.

These two terms had to be added to the x-momentum and y-momentum equations in Fluent. This was done by writing a UDF (user-defined function) in Fluent environment. Then the simulations were run with very small time-steps of about a micro-second. The results of these simulations at different times are shown in figure $4.2-4.9$. The results show the contour of stream functions, horizontal velocity, vertical velocity and finally, the vector plot of the velocity at the end of 0.2 seconds which is one-fifth of the time period of oscillation and at the end of 3 seconds which is 30 times of the time period of oscillation. 


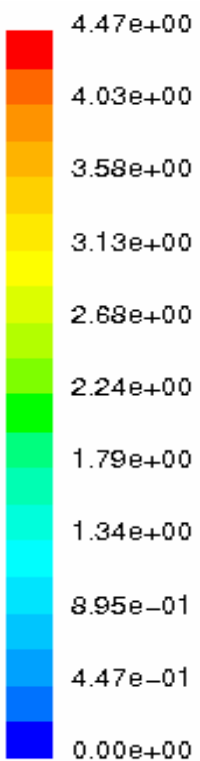

Figure 4.2: $\quad$ Contours of Stream Function at $t=0.2$ seconds.

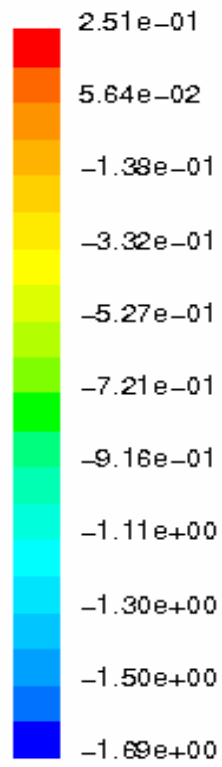

Figure 4.3: $\quad$ Contours of $\mathrm{x}$-velocity at $\mathrm{t}=0.2$ seconds. 

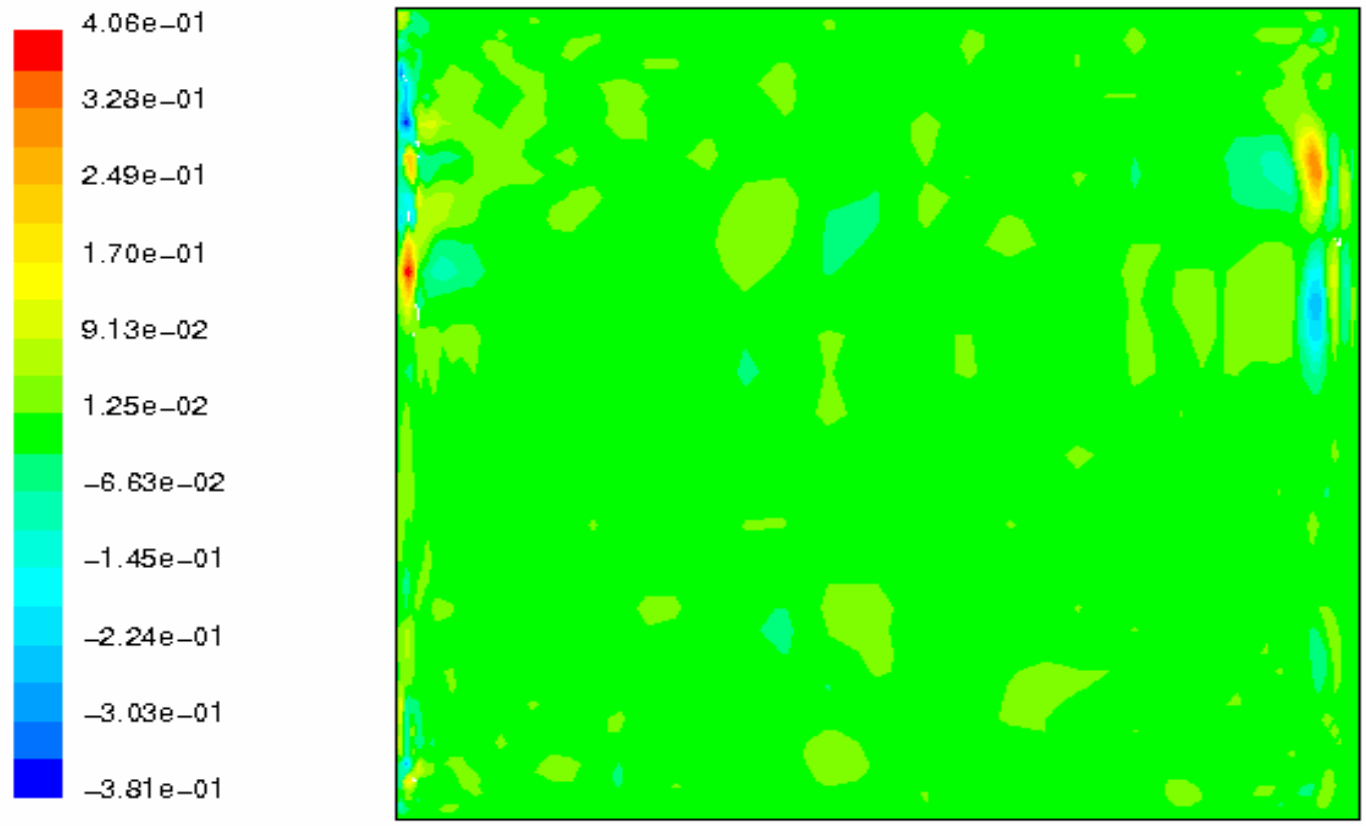

Figure 4.4: Contours of $\mathrm{y}$-velocity at $\mathrm{t}=0.2$ seconds.
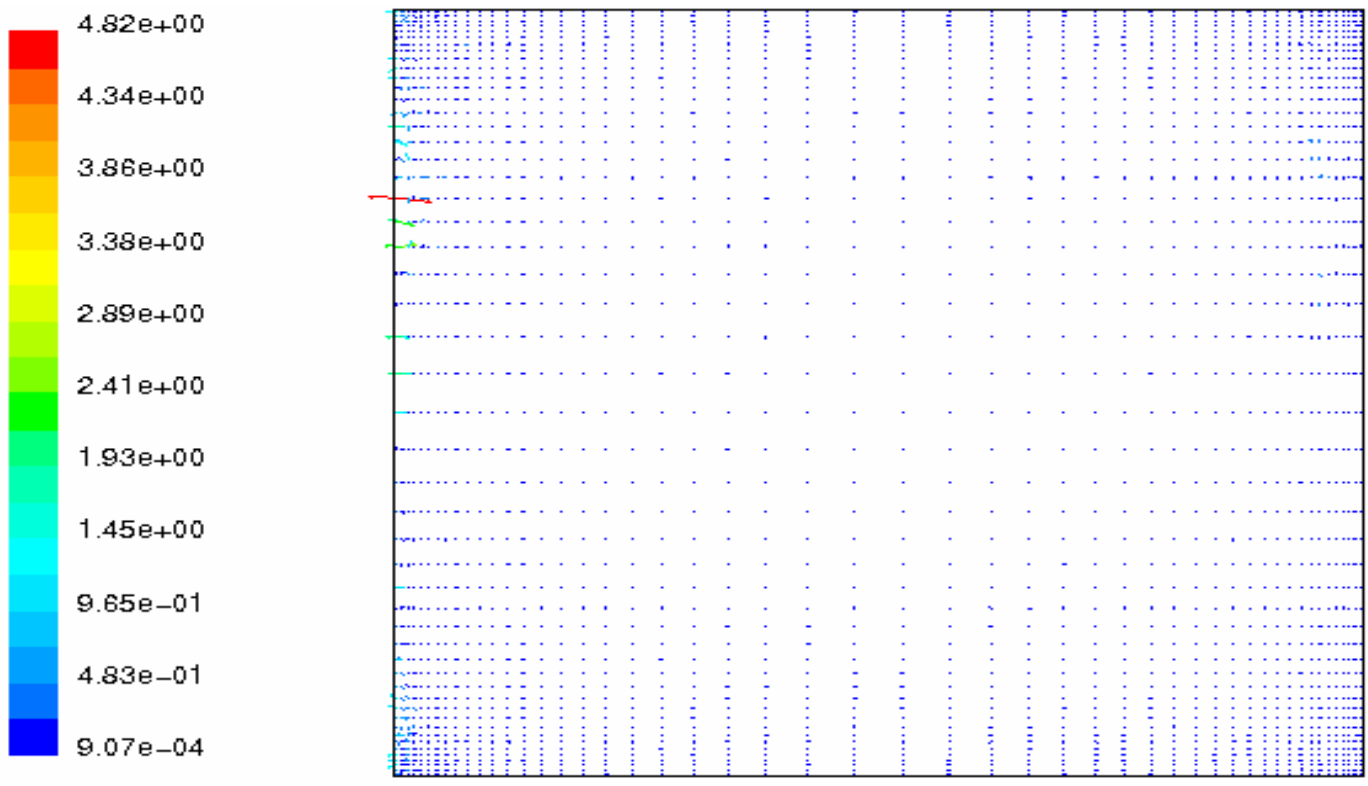

Figure 4.5: Velocity vector plot at $\mathrm{t}=0.2$ seconds. 


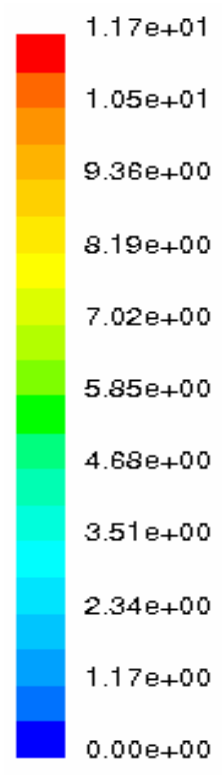

Figure 4.6: Contours of Stream Function at $\mathrm{t}=3$ seconds.

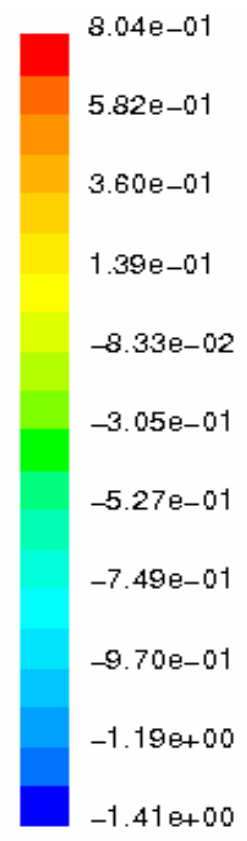

Figure 4.7: Contours of $\mathrm{x}$-velocity at $\mathrm{t}=3$ seconds. 


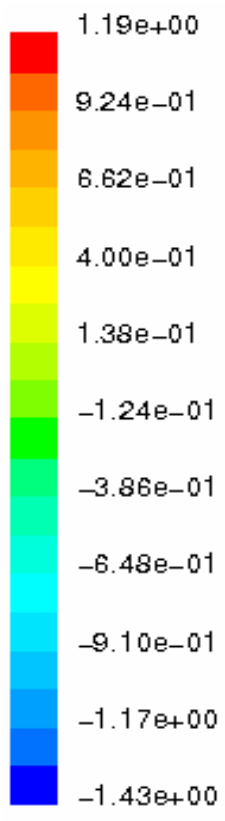

Figure 4.8: $\quad$ Contours of $\mathrm{y}$-velocity at $\mathrm{t}=3$ seconds.

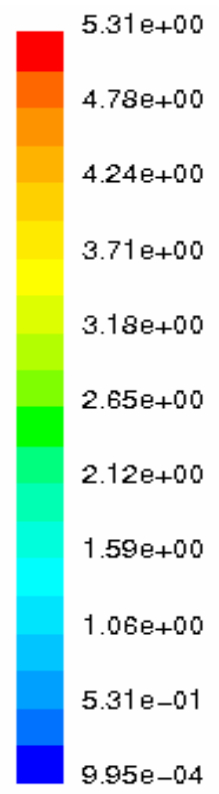

Figure 4.9: Velocity vector plot at $\mathrm{t}=3$ seconds.

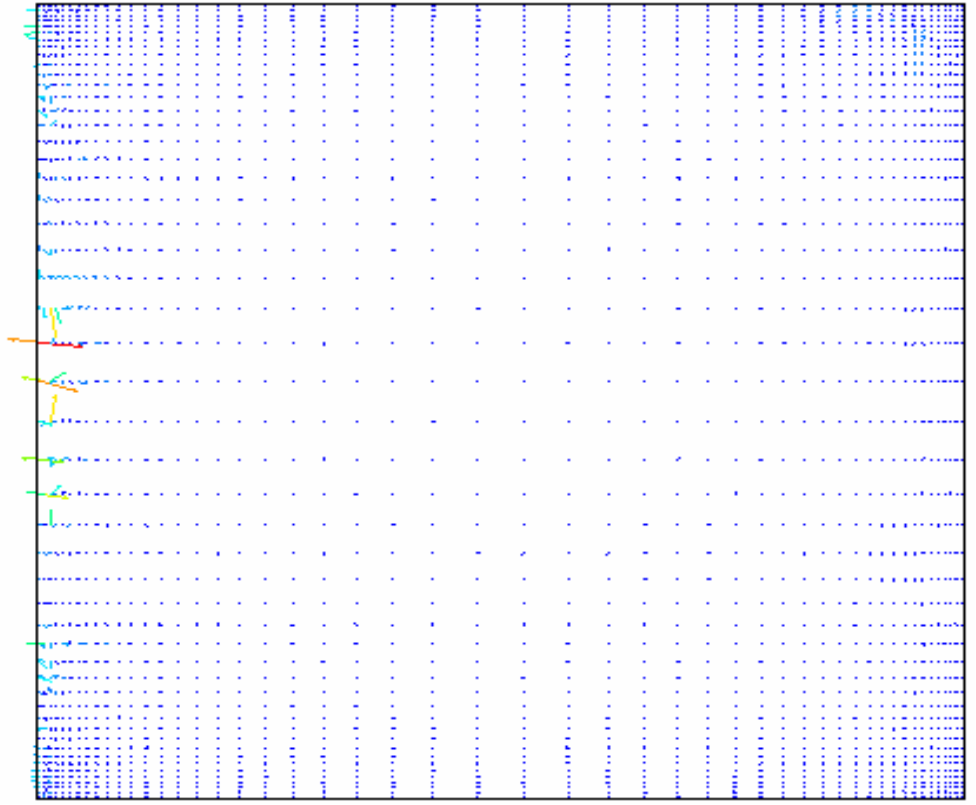


It can be seen from these results that the motion inside the shaking tank is not a rigid body motion. Instead, the velocities near the walls were found to be significant. This can be seen clearly from figure 4.5 and figure 4.9. It can also be seen that there are vortices formed near the corners at early time. These vortices disappear with time and the flow becomes more uniform. However, the velocities (horizontal and vertical) were not negligible even after a long time (10 seconds or 100 time periods of oscillation). The fluent simulations were done with very small time-step without which the simulations did not converge. Also, the residuals had to be on the order of 0.001 which is relatively large. A relatively coarse grid was used since the simulation is highly expensive in terms of execution time. This means that the error in the results obtained might be large. A theoretical analysis of this problem shows that there is no relative flow between the tank and the contained fluid.

\subsubsection{Theoretical Analysis}

In this section the same problem as above will be analyzed theoretically. If we assume that the flow is irrotational, i.e., the vorticity, $\Omega$, is zero, then the governing equation for the problem is given by the following equation:

$$
\nabla^{2} \psi=0
$$

where $\psi$ is the streamfunction. At all the boundaries we have the following boundary condition:

$$
\begin{aligned}
& \frac{\partial \psi}{\partial y}=u_{0} \cos (\omega t), \text { and } \\
& \frac{\partial \psi}{\partial x}=0
\end{aligned}
$$


Let the following substitution be made in order to take care of the inhomogeneous boundary condition:

$$
\psi=\phi+u_{0} \cos (\omega t) y
$$

The governing equation for $\phi$ is the same as that of $\psi$ while the boundary conditions are homogeneous. The boundary conditions are given by the following equations:

$$
\begin{aligned}
& \frac{\partial \phi}{\partial x}=0, \text { and } \\
& \frac{\partial \phi}{\partial y}=0
\end{aligned}
$$

The only solution for $\phi$ satisfying the above mentioned boundary conditions is the trivial solution, i.e., $\phi$ is zero everywhere. This means that the relative velocity of fluid with respect to the tank turns out to be zero. This is in accordance with the experiments detailed in chapter 2 of this thesis. This means that the results obtained through Fluent simulations might be erroneous due to coarse grid and some other reasons.

\subsection{VISCOUS RESUSPENSION}

Viscous resuspension is the resuspension which occurs at sufficiently low values of Reynolds number such that the inertial effects are insignificant and the flow is laminar. It was demonstrated by Acrivos et al (1985) that viscous resuspension, characterized by the equilibrium height achieved by the initially settled bed of particles expanding in the presence of applied shear, results from a balance of between the downward flux of particles due to gravity and an upward 
flux arising from shear induced particle diffusion. They found that the important dimensionless group given by

$$
\frac{v_{h} \mu}{\tau_{s s} \Delta h}
$$

where $v_{h}$ is the horizontal velocity at the top of the resuspended layer, $\mu_{0}$ is the viscosity of the suspending fluid, $\tau_{s s}$ is the applied shear stress and $\Delta h$ is the height of the resuspended layer. The dimensionless group indicated above in equation (4.19) is a measure of the concentration profile of the resuspended layer. If this parameter were close to unity, it would indicate that the bulk of the resuspended layer was at a low concentration, while small values of this parameter would result from a more concentrated resuspension layer. In the shaking tank problem discussed in this thesis, applied shear stress is very small. The magnitudes of $v_{h}$ and $\Delta h$ are on the same order. Any shear stress in the fluid is caused due to the presence of the particles and is otherwise equal to zero. So the dimensionless group is nearly equal to unity. This means that the bulk of the resuspended layer is at low concentration. The results of the experiments conducted on the shaking tank showed that the concentration in the resuspended layer is significant. Though viscous resuspension could be one of the reasons of the resuspension seen in the shaking tank problem, yet it is unlikely for the fore-mentioned reasons.

\subsection{GRAVITY WAVES}

One of the other reasons for the resuspension seen in the problem could be internal gravity waves. Internal gravity waves are created due to the presence of a density gradient in the fluid. These waves are created at an interface between 
higher density and lower density or at a free surface. Cellular flow structures are set up in the fluid below and above the surface of the interface. These cellular structures could cause mixing in the fluids. In this section, internal gravity waves in a superposed two fluids problem will be discussed. For more details on waves, the author suggests reading Lamb (1945).

\subsubsection{Superposed Fluids}

In this section, the oscillation of the common boundary (interface) of two superposed fluids contained in a shaking tank is considered. The tank is considered to oscillate at an angular frequency $\omega$. A few assumptions are made in order to get an analytical solution to this problem. First, it has been assumed that the flow is irrotational. Thus, it is assumed that a velocity potential can be defined for this flow. Second assumption is that non-linearity in this problem is negligible. This means that the convective term in the momentum equation has been neglected.

The continuity equation written in terms of $\phi$, the velocity potential forms the governing equation for this problem. The governing equation is given by the following equation:

$$
\nabla^{2} \phi=0 \text {. }
$$

This is the governing for both the fluids. The boundary conditions at the side walls are as follows:

$$
-\frac{\partial \phi}{\partial x}=u_{0} \cos (\omega t)
$$

At the top and bottom walls, the normal component of the velocity is zero. 


$$
-\frac{\partial \phi}{\partial y}=0
$$

The final boundary conditions to be satisfied are the interface condition. The first interface condition is that the normal component of velocity across the interface should be equal, i.e.,

$$
\frac{\partial \phi}{\partial y}=\frac{\partial \phi^{\prime}}{\partial y} \text {, at the interface }
$$

where $\phi$ is the velocity potential of the denser fluid and $\phi^{\prime}$ is the velocity potential of the fluid on top. The second condition is that the total pressure should be condition across the interface. This can be written as follows:

$$
P=P^{\prime}
$$

In order to find the pressure at any point, the momentum equations were used. The following expression was made use of, in order to find pressure in terms of the velocity potential $\phi$ :

$$
u=-\nabla \phi
$$

The above mentioned relations were used to get the following relation between pressure and the velocity potential.

$$
P=\rho\left(\frac{\partial \phi}{\partial t}-g y\right)
$$

As mentioned before the non-linear term, namely, $u \cdot \nabla u$ was neglected in order to get an analytical solution. In order to find the velocity potential, the following relation was used, without loss of generality:

$$
\phi(x, y, t)=X(x) Y(y) \cos (\omega t)
$$

The method of separation of variables was used to solve the governing equation. An expression was found for $X(x)$ and $Y(y)$ by making use of the 
corresponding boundary condition and making use of the fact that the sinusoidal functions are orthogonal. Thus the velocity potentials were found to be:

$$
\begin{aligned}
& \phi=\left(-x u_{0}+\sum_{i=1}^{\infty} A_{i} \sin \left(\lambda_{i} x\right) \cosh \left(\lambda_{i}(y+h)\right)\right) \cos (\omega t) \\
& \phi^{\prime}=\left(-x u_{0}+\sum_{i=1}^{\infty} B_{i} \sin \left(\lambda_{i} x\right) \cosh \left(\lambda_{i}\left(y-h^{\prime}\right)\right)\right) \cos (\omega t)
\end{aligned}
$$

where $\lambda_{i}$ is the eigenvalue given by

$$
\lambda_{n}=\frac{(2 n+1) \pi}{2 L}
$$

and the coefficients $A_{n}$ and $B_{n}$ are given by the following expression:

$$
\begin{aligned}
& A_{n}=(-1)^{n} \frac{8 L u_{0}(1-R)}{(2 n+1)^{2} \pi^{2}} \frac{\sec h\left(\lambda_{n} h\right)}{\left\{\left(1-\frac{p_{n}^{2}}{\omega^{2}}\right)+\frac{R \operatorname{coth}\left(\lambda_{n} h^{\prime}\right)}{\operatorname{coth}\left(\lambda_{n} h\right)}\left(1+\frac{p_{n}^{2}}{\omega^{2}}\right)\right\}} \\
& B_{n}=-A_{n} \frac{\sinh \left(\lambda_{n} h\right)}{\sinh \left(\lambda_{n} h^{\prime}\right)}
\end{aligned}
$$

In these expressions, $R$ refers to the density ratio of the two fluids given by:

$$
R=\frac{\rho^{\prime}}{\rho} .
$$

If the value of $R$ is greater than 1 , then the system is inherently unstable since the denser liquid is on top of the lighter liquid. The denser liquid would displace the lighter liquid from the bottom. $\mathrm{p}_{\mathrm{n}}$ and $p_{n}$ ' refer to the period of free waves of length $4 a /(2 n+1)$ in a channel of depth $h$ and $h$ ' respectively and are given by the following equations:

$$
\begin{aligned}
& p_{n}^{2}=g \lambda_{n} \tanh \left(\lambda_{n} h\right) \\
& p_{n}^{, 2}=g \lambda_{n} \tanh \left(\lambda_{n} h^{\prime}\right)
\end{aligned}
$$


The fluid velocity in the top and bottom fluids were found using equation (4.25). The expressions for the velocity potentials and velocities were evaluated using Matlab and were plotted. The results obtained are shown in figure 4.10 and 4.11 .

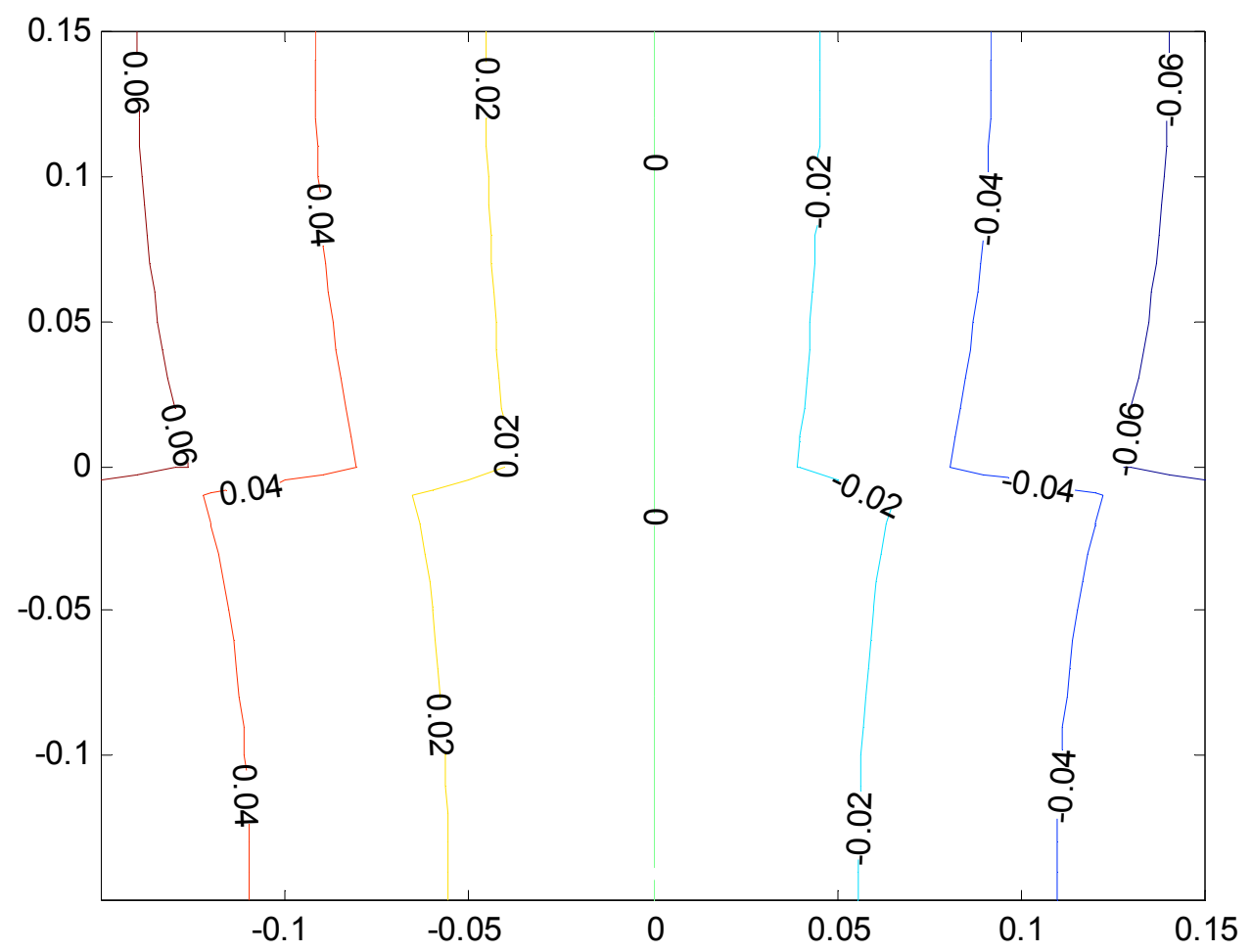

Figure 4.10: Contour plot of stream function for two shaking superposed fluids with $\mathrm{R}=0.75$ and $\mathrm{H}=1$. 


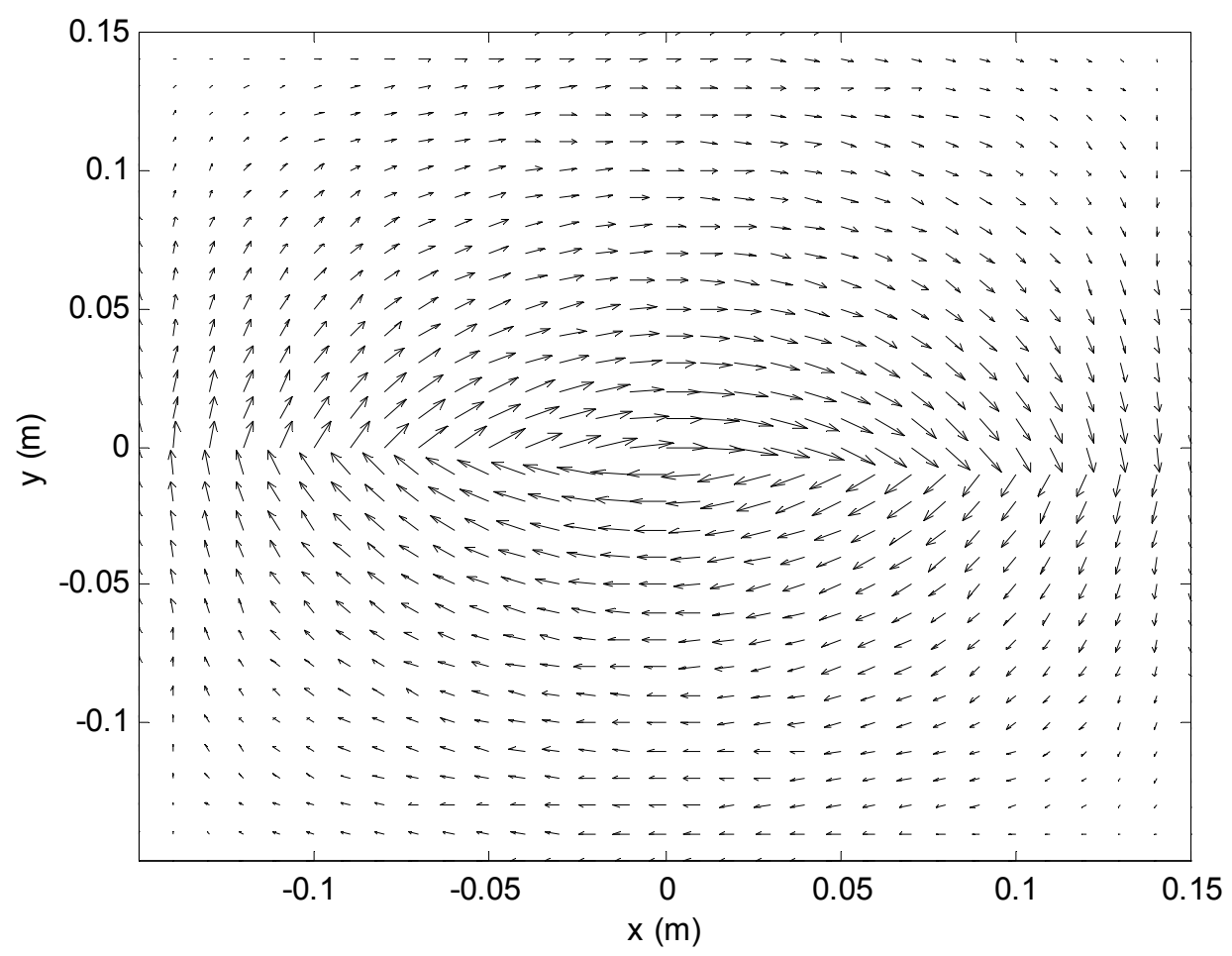

Figure 4.11: Velocity vector plot for two shaking superposed fluids with $\mathrm{R}=$ 0.75 and $\mathrm{H}=1$

Figure 4.10 shows the contour plot of the velocity potential in the shaking tank. The heights of the two fluid layers in the case shown are equal and the density ratio $R$ is equal to 0.75 . It can be seen from figure 4.10 that there is a discontinuity in the velocity potential at the interface $(y=0)$. However, the vertical velocity is continuous at the interface which is one of the boundary conditions. Figure 4.11 shows the velocity vector plot in the tank. It can be seen that a cellular flow is set up in the fluids above and below the interface.

From the expression for velocity potential, it was found that the dominant mode of oscillation is the zero order oscillation for the cases where the heights of the two fluid layers are comparable. The first and second order oscillations 
become important when one of the two fluid layers is shallow. This can be seen from the results shown in figure 4.12.

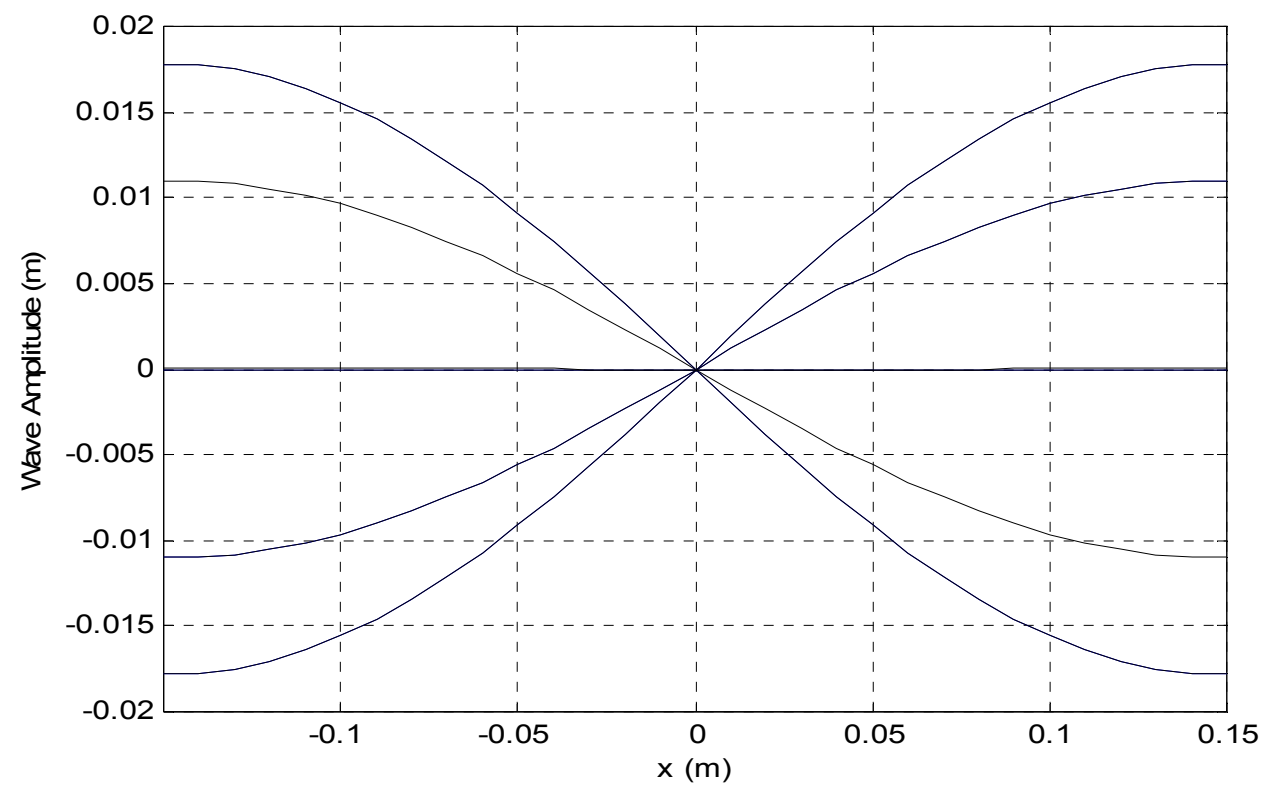

Figure 4.12: Plot of Wave amplitude variation with time at the interface along the $\mathrm{x}$-axis for $\mathrm{R}=0.45$ and $\mathrm{H}=1.0$

Figure 4.12 shows the interfacial wave profiles at different time for the case where the fluid layer depths are equal and the density ratio is 0.45 . Figure 4.13 shows the interfacial wave amplitude for different modes for the same case of $R=0.45$ and $H=1$. It can be seen that the zero order oscillation dominates when the heights of the two layers are comparable. Figure 4.14 shows the interfacial wave profiles for the case where the layer height ratio is 0.11 and figure 4.15 shows the wave amplitude for different modes for the same case. It can be seen the lower modes are starting to be comparable to the primary or zero order mode for this case. 


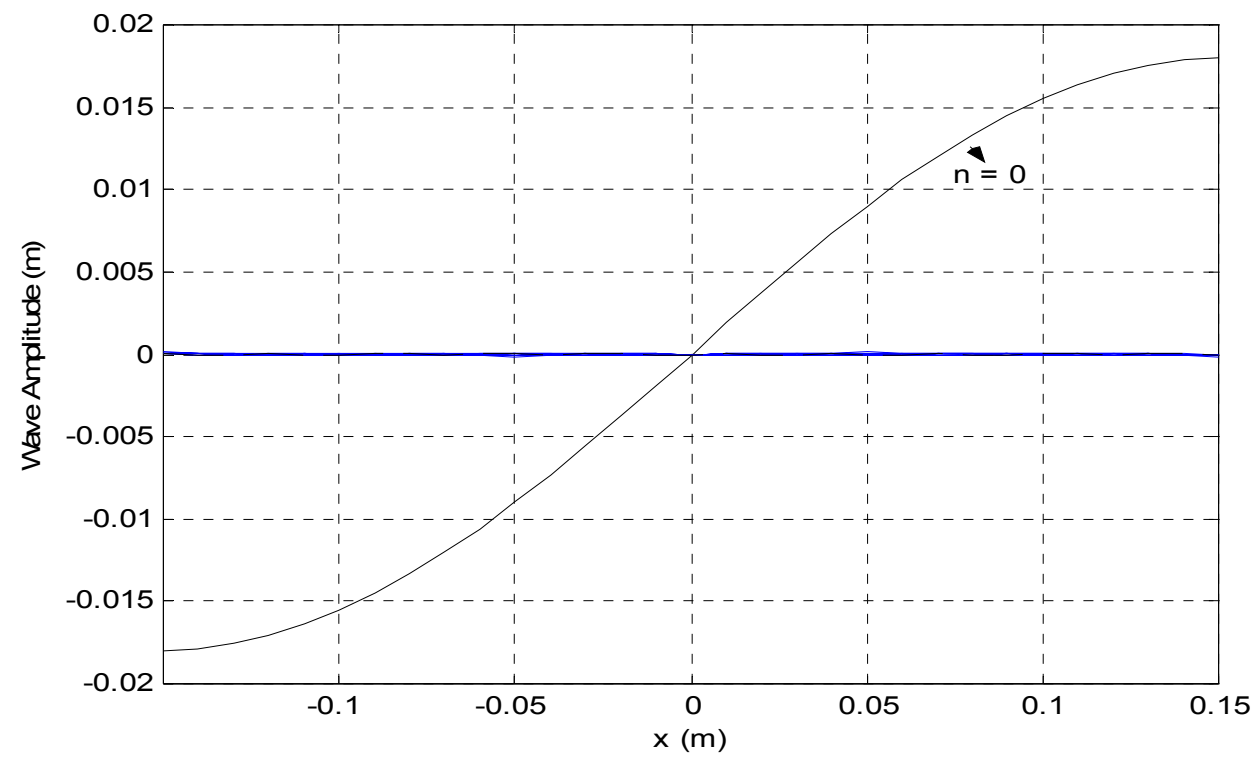

Figure 4.13: Plot of Wave amplitude at the interface for different modes with $\mathrm{R}$ $=0.45$ and $\mathrm{H}=0.11$.

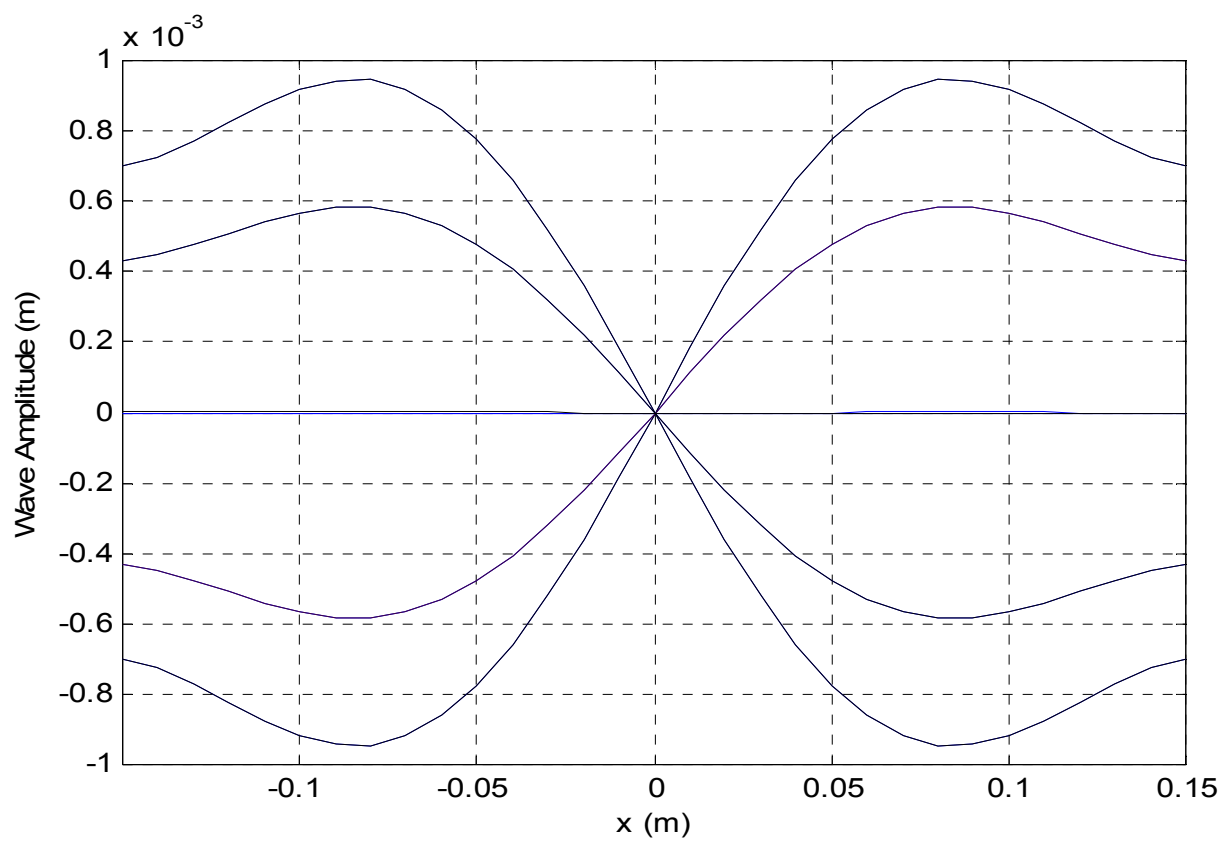

Figure 4.14: Plot of wave amplitude variation with time at the interface along the $\mathrm{x}$-axis with $\mathrm{R}=0.45$ and $\mathrm{H}=0.11$. 


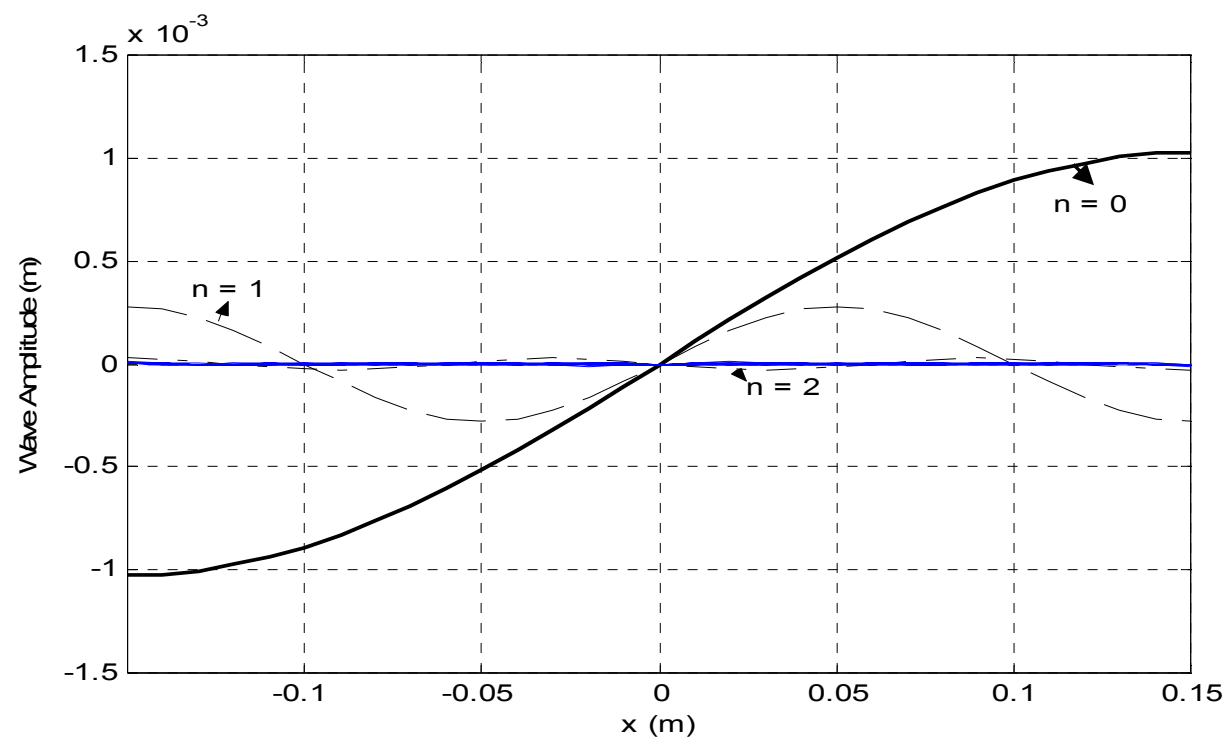

Figure 4.15: Plot of wave amplitude for different modes with $\mathrm{R}=0.45$ and $\mathrm{H}=$ 0.11 .

As the ratio of layer depths decreases, the lower order oscillations become more dominant than the zero order oscillation. This is because the wavelengths of these waves are comparable to the layer height. A point to note is that the amplitude of wave decreases as the height ratio decreases. This can be seen from figure 4.12 and figure 4.14. The wave amplitude in figure 4.12, for which the height ratio is 1 , is around $2 \mathrm{~cm}$ and in figure 4.14 the wave amplitude is reduced to $1.5 \mathrm{~mm}$, the height ratio being 0.11 . Also the amplitude increases with decrease in the value of $R$, the density ratio.

\subsubsection{Importance of Superposed Fluids}

In the previous section, the theory behind two superposed fluids problem was discussed. In this section, the relevance of this problem to the shaking tank problem discussed in this thesis will be discussed. 
First, since the measurements are taken at two different depths, the tank can be divided into a top zone containing less dense liquid and a bottom zone containing denser liquid. Second, the particles in the suspension settle down and form a dense layer at the bottom and a clear liquid on top. Actually, the suspension in the shaking tank is hypothesized to be stratified vertically, i.e., the density of the suspension increases continuously with depth. Analysis of two superposed fluids would be a good start to solve the continuously stratified suspension. Continuously stratified suspension could be thought of as multiple superposed fluids of infinitesimal layer heights. These are some of the important reasons for studying the two superposed fluids problem.

In the shaking tank problem, the particles in the suspension settle down and form a dense layer at the bottom. There are two fluid layer formed as a result. When these two layers are oscillated, gravity waves are set up at the interface between these two layers because of the sharp density gradient between the two layers. The gravity waves, as already mentioned, can cause cellular flow structures to be formed in the fluids. The cellular flow structures causes mixing in the two layers. It is hypothesized that the particles get trapped in some regions of the cellular flow and do not settle down. There can also be mixing in the fluids due to sloshing. 

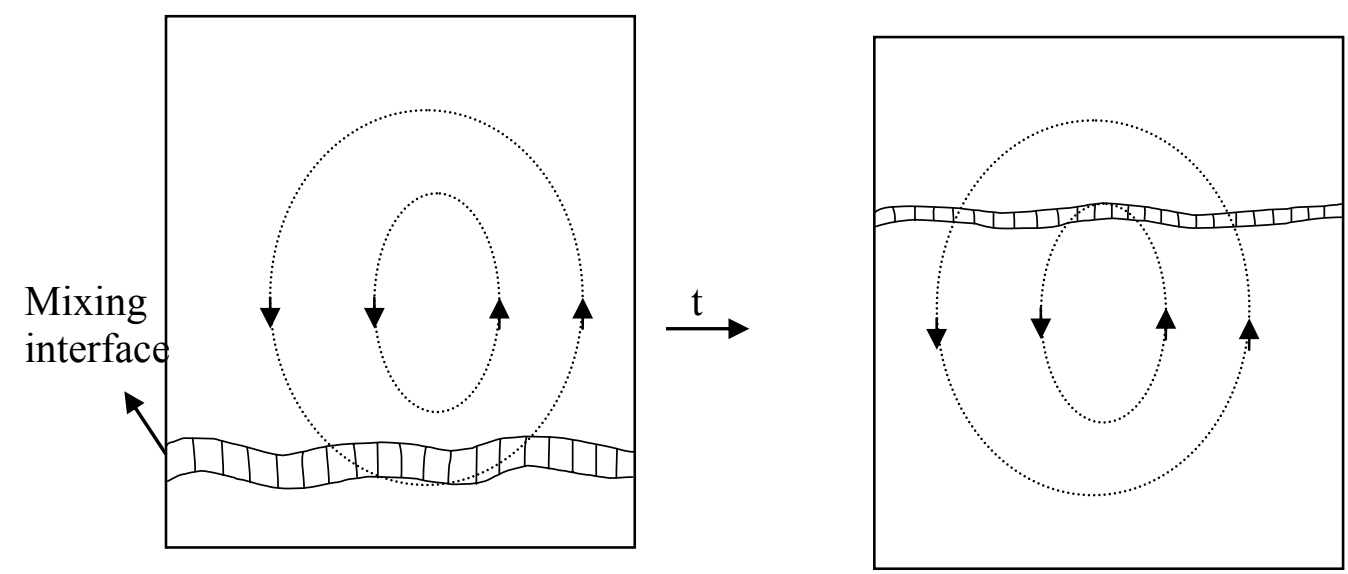

Figure 4.16: 2-layer model.

The interface between the top and bottom layers is thought of as a negligibly thin layer in order to find a solution to the velocity potential. However, the interface is not negligibly thin as is shown in figure 4.12 and 4.14. The thickness of the interface is twice the amplitude of the interfacial wave. This means that the bottom layer height or thickness keeps increasing by an amount equal to the amplitude of interfacial wave. Thus the interface of the bottom layer and top layer is moved up by a distance of one wave amplitude. Due to this translation of the interface, the density ratio of the top layer to that of the bottom layer decreases and the height ratio of the top layer to that of the bottom layer decreases. Again, waves are set up at the interface between the two layers though the wave amplitude decreases. As time progresses the height ratio and density ratio tend towards unity and the wave amplitude becomes negligibly small. Thus the interface reaches an equilibrium height. As shown in figure 4.16, the interface moves from towards the top of the tank. A simple simulation of the same was done using Matlab and the results are shown in figure 4.17 and 4.18. 


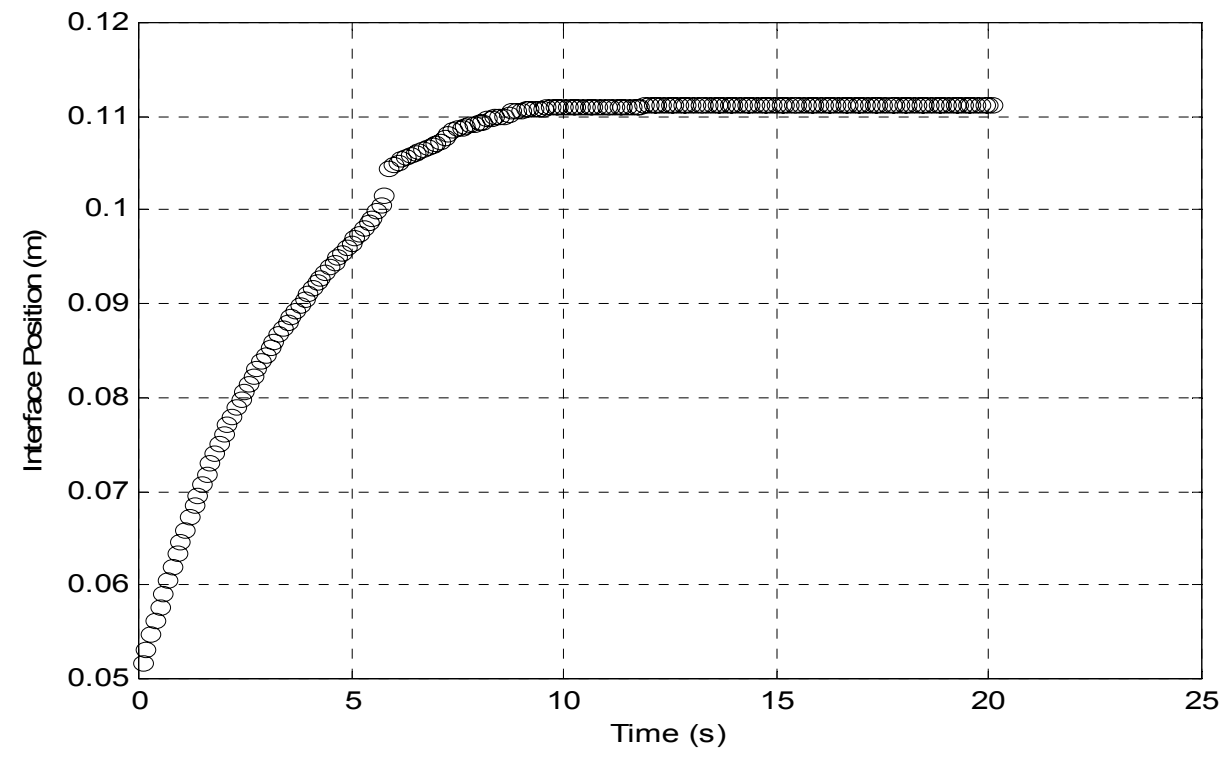

Figure 4.17: Plot of interface position with time for $\mathrm{R}=0.45$ and initial settled layer height of $5 \mathrm{~cm}$.

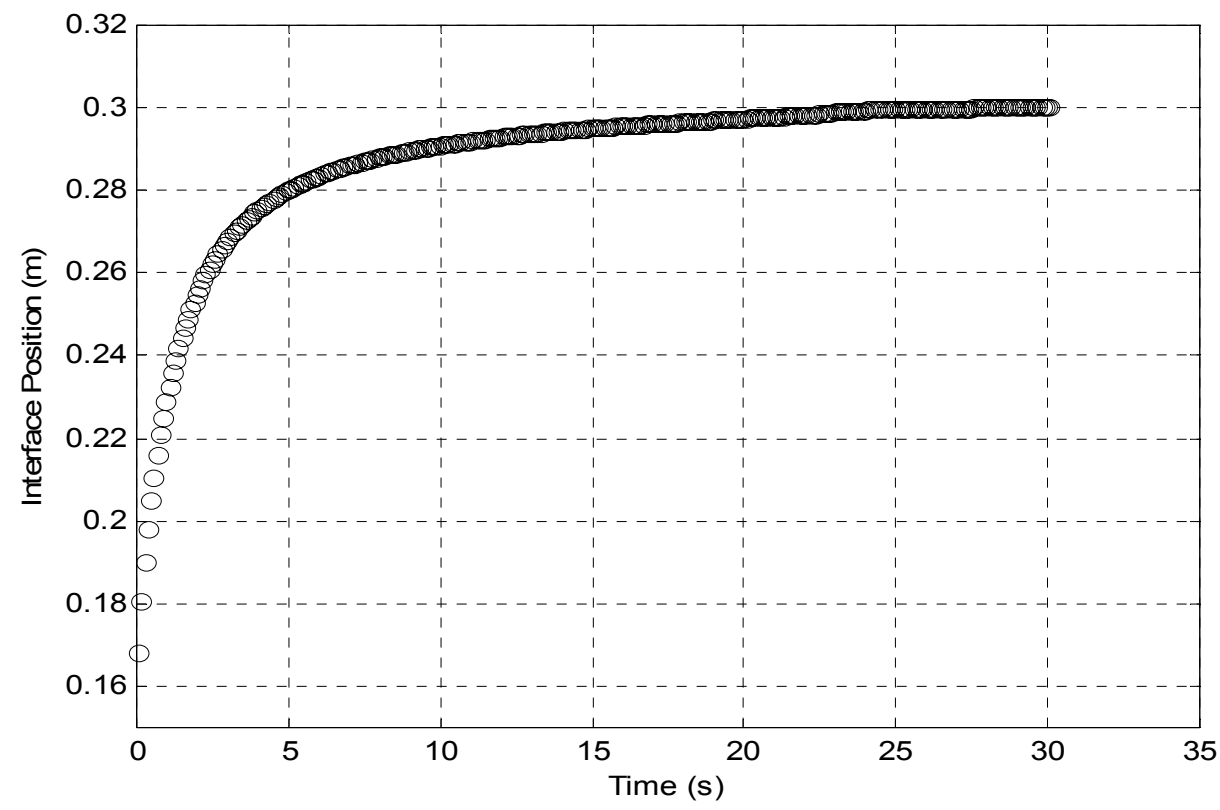

Figure 4.18: Plot of interface position with time for $\mathrm{R}=0.45$ and initial settled layer height of $15 \mathrm{~cm}$. 
In figure 4.17 the initial settled layer height is $5 \mathrm{~cm}(0.05 \mathrm{~m})$. It can be seen that the final equilibrium interface position attained is about $11 \mathrm{~cm}$ which is about twice the initial height. In this simulation, the height ratio (ratio of top to bottom layer heights) keeps increasing and so does the density ratio $R$. Figure 4.18 shows the interface position with time for an initial bottom layer height of $0.15 \mathrm{~m}$ or $15 \mathrm{~cm}$. The final height, again, is nearly twice the initial height. In this simulation, it is assumed that the mixing in the bottom layer occurs instantaneously. However the mixing in the layers can not be instantaneous. So the time taken for the attainment of the final equilibrium interface position is a lot more than the time shown in figure 4.17 and 4.18 .

However, figure 4.17 and 4.18 show that gravity waves can actually cause mixing of particles and thus cause resuspension of particles.

In this chapter, some of the mechanisms that could be responsible for resuspension in the shaking tank experiment were discussed. Some of the reasons for the consideration of these mechanisms and the reason why these mechanisms can be refuted have been discussed in this chapter. Though turbulence could be one of the reasons for resuspension of particles, gravity waves seem to be more likely to cause the resuspension reported in the previous chapter of this thesis. However, the exact reason for the resuspension of particles in the shaking tank experiment is not yet clear. 


\section{Chapter 5: Summary and Future Work}

\subsection{SUMMARY}

An ultrasonic measurement technique was devised in order to make concentration measurements in a tank containing aqueous fly-ash suspension. The measurements were made at two different elevations: 3 inches below the top and 3 inches above the bottom of the tank. The transducers were validated and the accuracy of the measurement was found to be $0.1 \%$ by mass. Three different kinds of experiments were conducted. First, the particles were allowed to settle without shaking the tank containing suspension. The agreement of these results with the existing theory was demonstrated. Next, the settling experiments were carried out with the shaking tank. It was found that the particles started settling down and later, the particles got mixed or resuspended until an equilibrium concentration was reached. The equilibrium concentration for the top zone was found to be about $1 \%$ by mass and that of the bottom zone was found to be $0.5 \%$ by mass. Finally, a tank containing a settled layer of particles was oscillated. The particles were found to resuspend and the equilibrium concentration for the top and bottom zone were both found to be $1.5 \%$ by mass.

The mechanisms that could possibly cause this resuspension were analyzed. Turbulence and internal gravity waves are two of the important mechanisms that could cause resuspension. A shaking tank containing two immiscible fluids was analyzed. It was shown that the waves created at the interface and flow field set up in the fluids could cause considerable mixing and 
resuspension. However, the exact reason for the mixing or resuspension is not clear.

\subsection{FUTURE WORK}

More experiments should be conducted with different particle sizes and amplitudes of oscillation of the tank in order to find the dominant non-

dimensional parameter. A simulation of shaking tank containing a continuously stratified suspension should be performed. A more rigorous analysis of the mechanisms that could cause resuspension should be done. 


\title{
Appendices
}

\author{
APPENDIX 1: NOMENCLATURE. \\ $\ddot{x}$ \\ $\dot{x}$ \\ $Y_{0}^{+}$ \\ $U_{r e l}^{2}$ \\ $\phi$ \\ $a$ \\ A \\ $a_{b}$ \\ $A_{c s}$ \\ $a_{g}$ \\ $A_{i}$ \\ C \\ c \\ $C^{\prime}$ \\ $C_{0}$ \\ Acceleration. \\ Velocity. \\ Near-field distance. \\ Relative velocity of fluid with respect \\ to wall or moving body. \\ Velocity potential. \\ Scatterer radius. \\ Amplitude of Oscillation. \\ Beam diameter. \\ Cross sectional area of the tank. \\ Geometric mean diameter. \\ Amplitude of signal $i$. \\ Concentration of particles in \\ suspension. \\ Damping coefficient. \\ Concentration of particles in \\ suspension at $\mathrm{t}=65$ seconds. \\ Initial concentration of particles in \\ suspension.
}




\begin{tabular}{|c|c|}
\hline$C_{f}$ & Final equilibrium concentration. \\
\hline$d$ & Diameter of particles. \\
\hline$D$ & Duty cycle duration. \\
\hline$d B$ & Decibels. \\
\hline$d_{c}$ & $\begin{array}{l}\text { Characteristic dimension of an } \\
\text { obstacle. }\end{array}$ \\
\hline$D_{e}$ & Diameter of transducer element. \\
\hline$d f$ & $\begin{array}{l}\text { Incremental frequency distribution } \\
\text { function. }\end{array}$ \\
\hline$e_{x}$ & Unit vector along $\mathrm{x}$-axis. \\
\hline$f$ & Frequency of oscillation \\
\hline$F$ & Force exerted. \\
\hline$g$ & Acceleration due to gravity \\
\hline$H$ & $\begin{array}{l}\text { Ratio of top layer to bottom layer } \\
\text { heights. }\end{array}$ \\
\hline$I$ & Actual intensity of light beam \\
\hline$I_{0}$ & Initial intensity of light beam \\
\hline$I_{l}$ & Intensity measured in pure liquid. \\
\hline$I_{m t}$ & Intensity measured in air. \\
\hline$I_{t p}$ & $\begin{array}{l}\text { Intensity measured in two phase } \\
\text { mixture. }\end{array}$ \\
\hline$K$ & Spring constant. \\
\hline$k$ & Compression wave number. \\
\hline
\end{tabular}


$k_{l}$

$k_{p}$

$L$

$l$

$l_{l}$

$l_{p}$

$m$

$M$

$M_{\text {sys }}$

$N$

$N_{0}$

$n_{i}$

$P$

$P_{0}$

$P_{t o t}$

$R$

$R$

$R^{2}$

$R e$

$R e_{p}$
Attenuation coefficient of suspending liquid Attenuation coefficient of particles.

Length of water tank.

Distance between transducers.

Path length of light beam in liquid.

Path length of light beam across particles.

Mass.

Mass of water in the tank.

Mass of the system.

Number of cycles per burst.

Total number of particles.

Frequency of data value $i$.

Pressure at given concentration.

Pressure at zero concentration.

Total power.

Repetition rate of the acoustic signal.

Ratio of top layer to bottom layer densities.

Linear regression coefficient.

Reynolds' number.

Particle Reynolds' number. 
$R_{\text {loss }}$

$S$

St

$S t_{p}$

$S t_{s A}$

$S t_{s L}$

$T$

$t$

$t_{d}$

$t_{i}$

$T_{\text {loss }}$

$u^{\prime}$

$U_{0}$

$u_{2}$

$u_{c}$

$u_{o}$

V

$v_{h}$

$v_{i}$
Reflection loss in $\mathrm{dB}$.

Stopping distance of a particle.

Stokes number.

Particle Stokes number.

Stokes number of the system based on oscillation amplitude.

Stokes number of the system based on length of tank.

Period of oscillation.

Time.

Time of flight in dispersed phase.

Time of flight in pure phase $i$.

Transmission loss in dB.

Velocity with respect to accelerating reference frame.

Undisturbed fluid velocity away from the obstacle.

Secondary flow velocity.

Settling velocity of particle in concentrated suspensions.

Velocity of oscillation.

Total volume of suspending fluid.

Velocity at the top resuspended layer.

Speed of sound in medium $i$. 


\begin{tabular}{|c|c|}
\hline$v_{m}$ & Speed of sound in the material. \\
\hline$V_{p-p}$ & Peak to peak voltage. \\
\hline$V_{r m s}$ & Root-mean-squared voltage. \\
\hline$x$ & Displacement. \\
\hline$x_{f c}$ & $\begin{array}{l}\text { Position of fluid container during } \\
\text { damped oscillation. }\end{array}$ \\
\hline$Z$ & Acoustic impedance. \\
\hline$\alpha$ & Acoustic Attenuation. \\
\hline$\beta$ & Viscous boundary layer thickness. \\
\hline$\delta$ & Logarithmic decrement. \\
\hline$\Delta h$ & Thickness of resuspended layer. \\
\hline$\delta_{t}$ & Material thickness. \\
\hline$\zeta$ & Damping ratio \\
\hline$\lambda$ & Wavelength of sound waves. \\
\hline$\mu$ & $\begin{array}{l}\text { Absolute viscosity of suspending } \\
\text { fluid. }\end{array}$ \\
\hline$\mu_{c}$ & Absolute viscosity of suspension \\
\hline$v$ & $\begin{array}{l}\text { Kinematic viscosity of suspending } \\
\text { fluid. }\end{array}$ \\
\hline$\rho$ & Ratio of particle to fluid densities. \\
\hline$\rho_{c}$ & Density of suspension. \\
\hline$\rho_{p}$ & Density of particles. \\
\hline$\sigma_{g}$ & Geometric standard deviation. \\
\hline
\end{tabular}


Time of descent for a particle in a suspension.

$\tau_{s s}$

$\tau_{s t}$

$\varphi$

$\omega$
Applied shear stress.

Stokes time or Relaxation time.

Phase angle.

Angular frequency of oscillation. 


\section{APPENDiX 2: CALCULATION OF ULTRASONIC TRANSDUCER EXCITATION.}

Following is an example of how to use the above equations to calculate a duty cycle and number of cycles for a Panametrics V310-SU transducer.

Transducer : Panametrics - V310 - SU

$f=5 \mathrm{MHz}$

Diameter : $0.25^{\prime \prime}$

$V_{p-p}=100 \mathrm{~V}$

$Z=50 \Omega$

$\varphi=-45^{\circ}$

$R=5 \mathrm{kHz}$

Step 1: Calculate Vrms.

$$
\begin{aligned}
& V_{r m s}=\frac{1}{2} V_{p-p} \cos (\varphi) \\
& V_{r m s}=\frac{1}{2}(100)(0.707)=35.35
\end{aligned}
$$

Step 2: Solve for Duty Cycle.

$$
\begin{aligned}
& D=\frac{Z P_{t o t}}{V_{r m s}^{2} \cos (\varphi)} \\
& D=\frac{(50 \Omega)(125 \mathrm{~mW})}{(35.35 \mathrm{~V})^{2}(0.707)}=0.007 \mathrm{~ms} / \mathrm{s}
\end{aligned}
$$

This means 7 milliseconds of excitation in every 1000 milliseconds.

Step 3: Find number of cycles per burst (N).

$$
\begin{aligned}
& N=\frac{f D}{R} \\
& N=\frac{(5 M H z)(7 \mathrm{~ms} / \mathrm{s})}{(5 \mathrm{kHz})}=7^{\text {cycles } / \text { burst }}
\end{aligned}
$$




\section{APPENDIX 3: CALIBRATION OF ULTRASONIC TRANSDUCERS.}

A calibration for acoustic transducers measuring particle concentration is a curve of attenuation against concentration of particles in the suspension. Figure 2.9 shows a typical calibration curve for the transducers. Using this curve, the mass concentration in the suspension can be found from the results of sedimentation experiments.

\section{A3.1 Experimental Procedure}

This experimental procedure mainly consisted of two important parts. The first one is to get the data acquisition system ready and the second is to get a uniform suspension in the tank. As mentioned earlier, the output of the acoustic transducers is fed into an oscilloscope. A LabView code is used to acquire the waveform from the oscilloscope onto the computer. This code has provisions to acquire data at regular intervals from the oscilloscope. This will be used for data acquisition during settling experiments. For calibration tests, though, continuous data acquisition is not required. Once this code is executed, the data acquisition system is ready to acquire data.

Then, measured amounts of fly-ash or kaolin particles are added into the tank filled with water. The tank is stirred thoroughly using a mechanical stirrer so that a uniform suspension, i.e., a suspension having uniform concentration of particles, is obtained. Stirring is not done in any particular fashion. This is essential because velocity could be imparted to particles by stirring. 
Measurements have to be taken immediately after stirring is completed in order that settling of particles is negligible.

\section{A3.2 Calibration Results.}

Then calibration tests were conducted with two different kind of particles: a) fly-ash, and b) kaolin (china clay). Also, calibration was done for both the top and bottom transducers. The voltage outputs from the top and bottom transducers for various concentrations of particles in the suspensions were measured. As seen in the figures below, the measurements were taken from 0 per cent concentration to a maximum concentration of 4 per cent. Typical calibration curves for the top and bottom transducers are shown below.

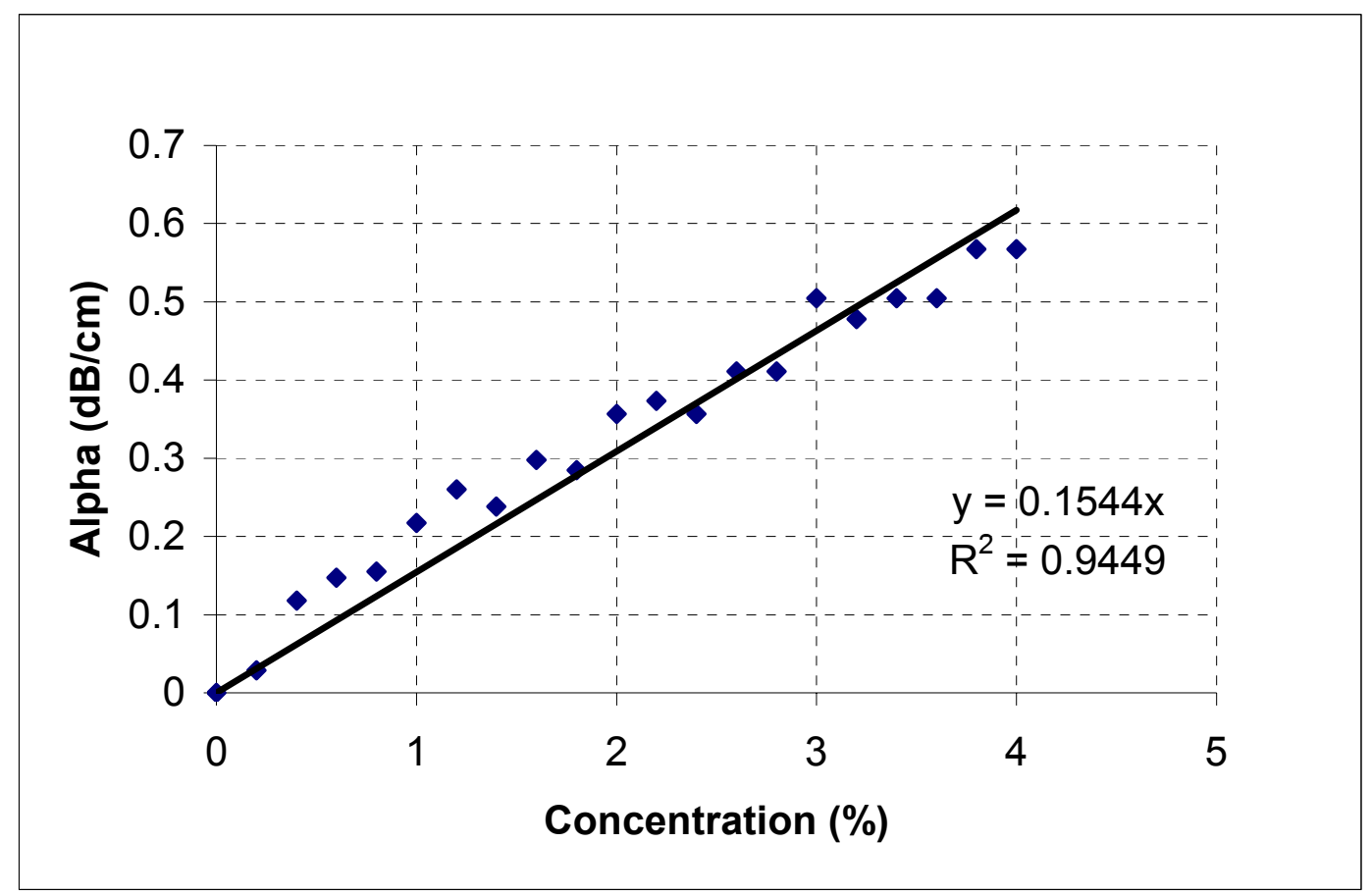

Figure A3.1: Calibration curve for top transducer in fly-ash suspension. 


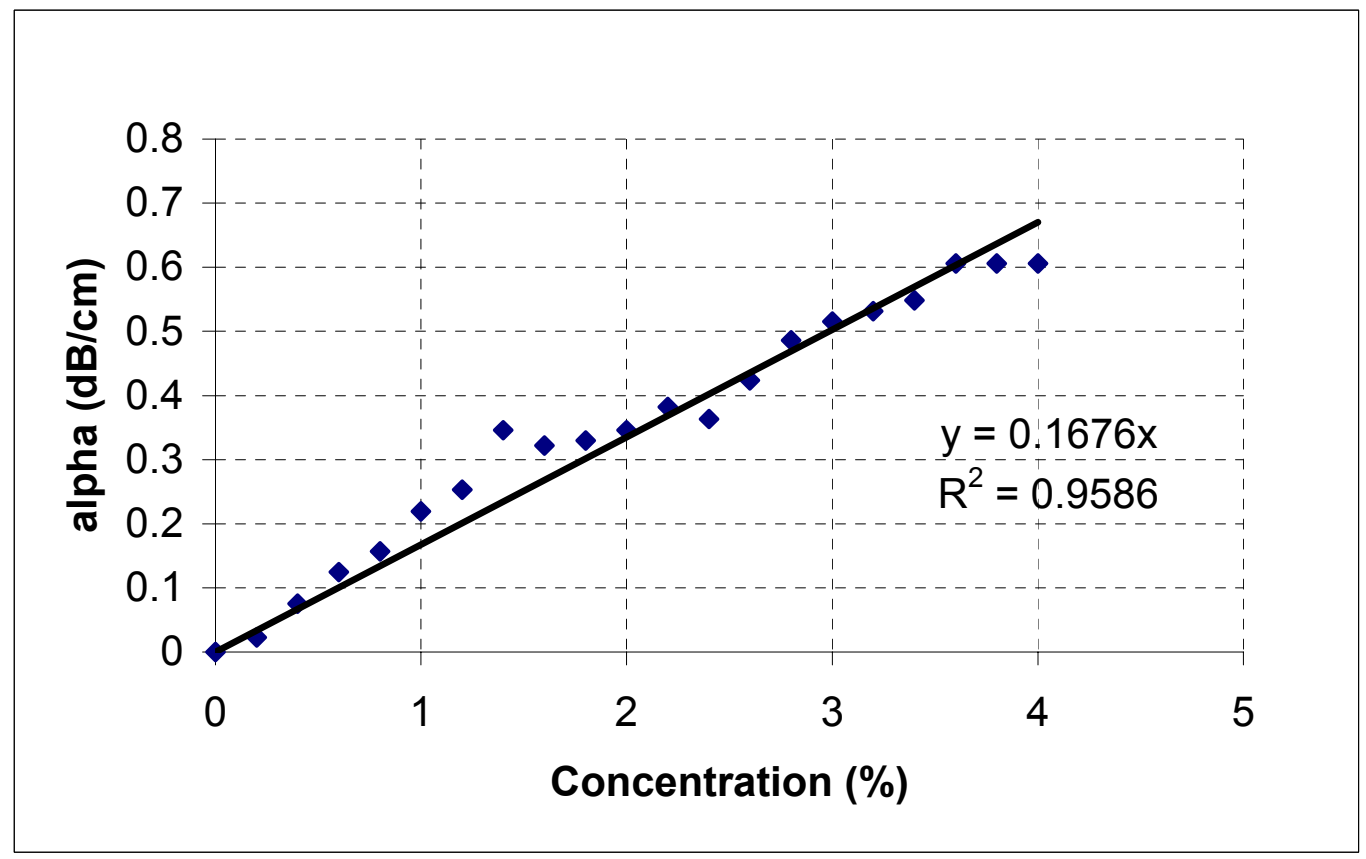

Figure A3.2: Calibration curve for bottom transducer in fly-ash suspension.

Figure A3.1 and A3.2 show plots of attenuation against concentration of particles in the concentration. These are calibration curves for an aqueous suspension of fly-ash particles. Attenuation at each concentration can be found in the following way. The acoustic pressure is attenuated with distance according to the following equation.

$$
\frac{P}{P_{0}}=\exp (-\alpha \cdot l)
$$

$P=$ Pressure at any concentration,

$P_{0}=$ Pressure at zero concentration,

$\alpha=$ attenuation coefficient, and

$l=$ distance between the transducers. 
It is known that the voltage output from the transducers is directly proportional to the acoustic pressure. This means that the ratio of $P$ and $P_{0}$ can be replaced by the ratio of $V$ and $V_{0}$. Thus equation can be rewritten as:

$$
\frac{V}{V_{0}}=\exp (-\alpha \cdot l) \text {. }
$$

If the voltage ratio is known, then the attenuation coefficient, $\alpha$, can be found using the following equation:

$$
\alpha=-\frac{1}{l}\left(\ln \left(\frac{V}{V_{0}}\right)\right) .
$$

The attenuation curve is found using the above equation. The calibration curve for the top and bottom transducers were linear with a linear regression coefficient square $\left(R^{2}\right)$ value of 0.94 and 0.96 respectively. From acoustic theory, it is known that the attenuation is directly proportional to the concentration. The experimental results were found to be consistent with the theory. Using this calibration curve, the error introduced in the voltage measurement because of shaking was found to be 0.2 per cent by mass, approximately. This means that the transducers can not measure a concentration less than 0.2 per cent by mass accurately. The calibration curves for kaolin suspension are shown below. The same experiment was conducted for a suspension of kaolin (china clay). The curves look the same except that the slope is different. This is due to the fact that kaolin particles have a different acoustic absorption than fly-ash particles. 


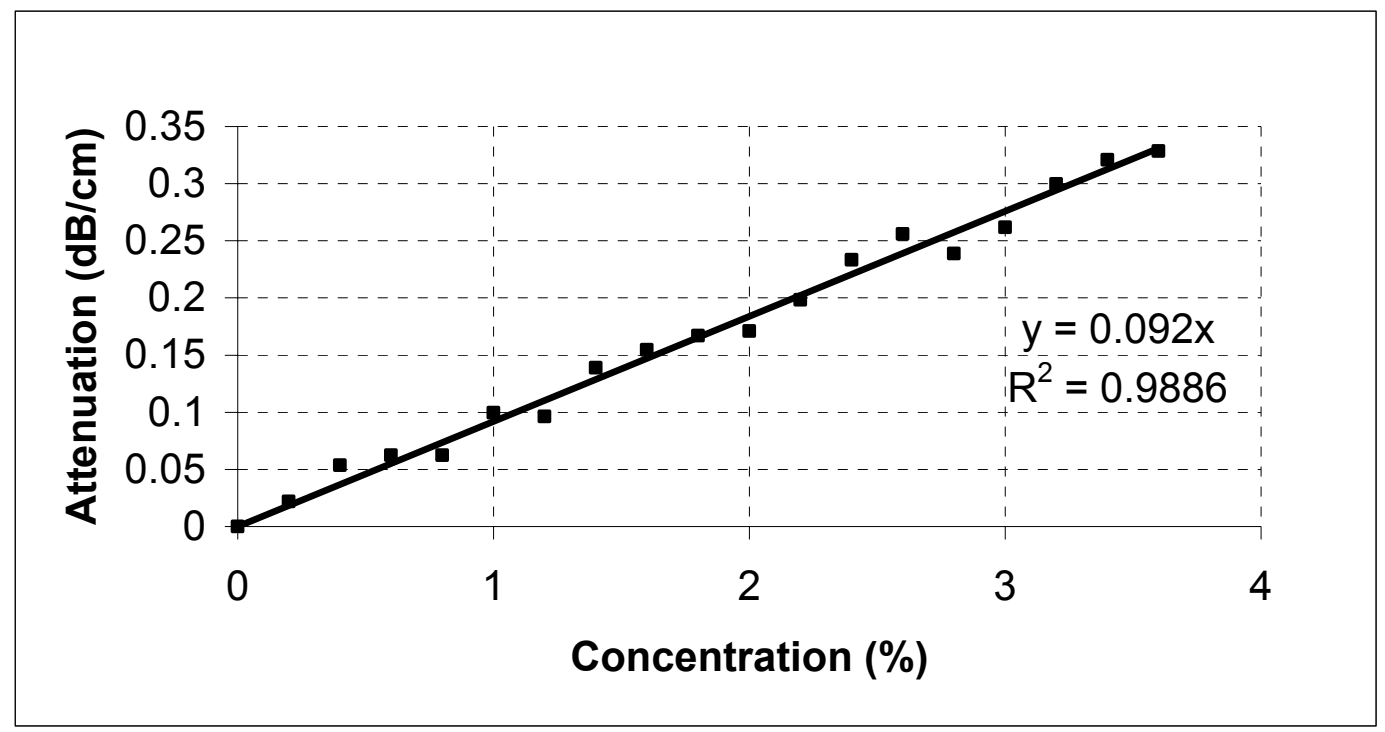

Figure A3.3: Calibration curve for top transducer in kaolin suspension.

As can be seen from figure A3.3 and A3.4, the calibration test was conducted for a kaolin particle concentration range of 0 per cent to 3.6 per cent by mass. The $\mathrm{R}^{2}$ values were found to be 0.98 and 0.99 respectively for top and bottom transducers. 


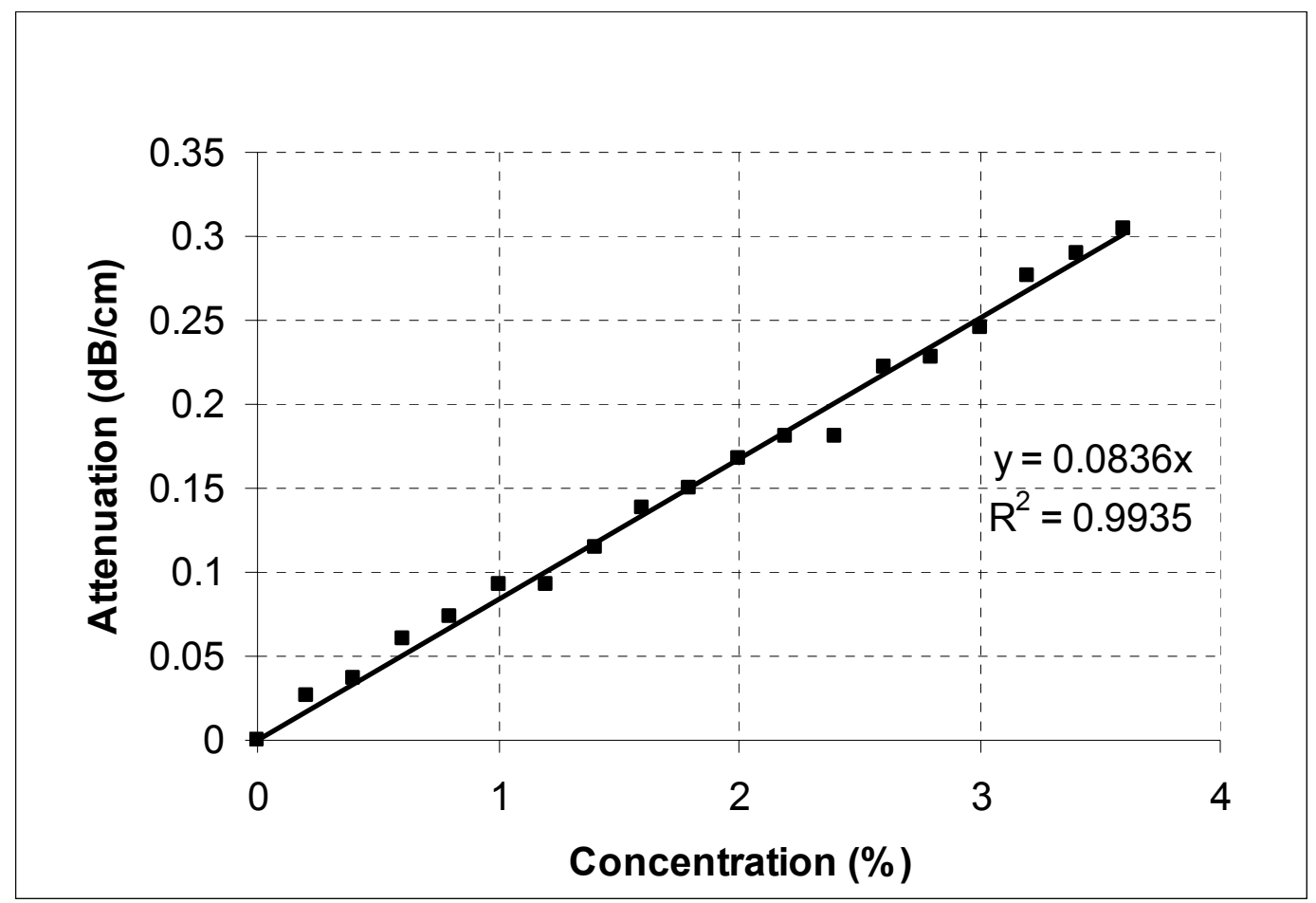

Figure A3.4: Calibration curve for bottom transducer in kaolin suspension.

Figure A3.5 and A3.6 show three calibration curves for top and bottom transducers. It can be seen clearly that the slopes of the three curves in both the cases are different from each other. This difference is attributed to the difference in the PSD (Particle Size Distribution) of the sample taken. One reason for this is acoustic attenuation is different for different PSD as detailed in the previous chapter. Also, settling of particles during the calibration test could be another reason. Though care is taken to make measurements immediately after the mixing is done, yet particles settle down before the measurements are made. This settling rate is strong function of the particle size distribution. Bigger particles tend to settle down faster than smaller particles. If a sample contains more large particles than small particles, then more particles in this sample would settle and hence, the 
acoustic attenuation caused by the suspension will be smaller. This would result in a calibration curve with a smaller slope.

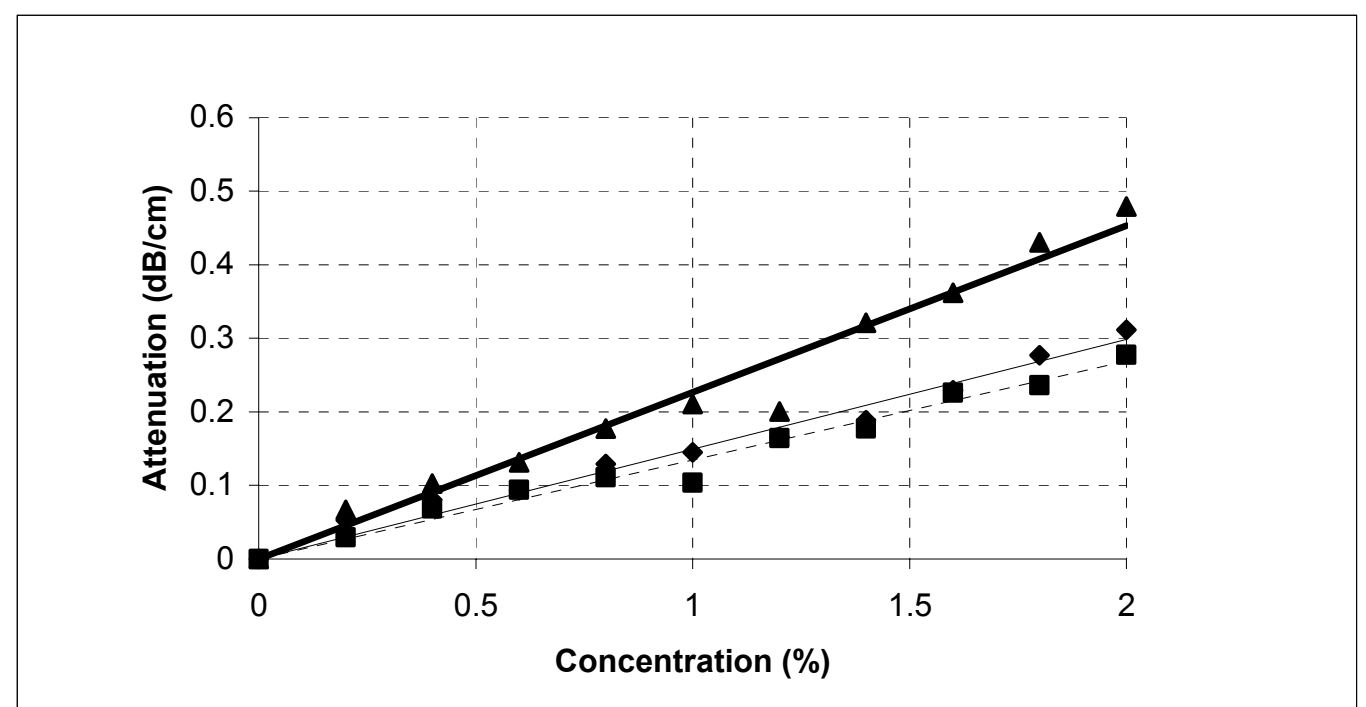

Figure A3.5: Calibration curves for Top Transducer in fly-ash suspension. 


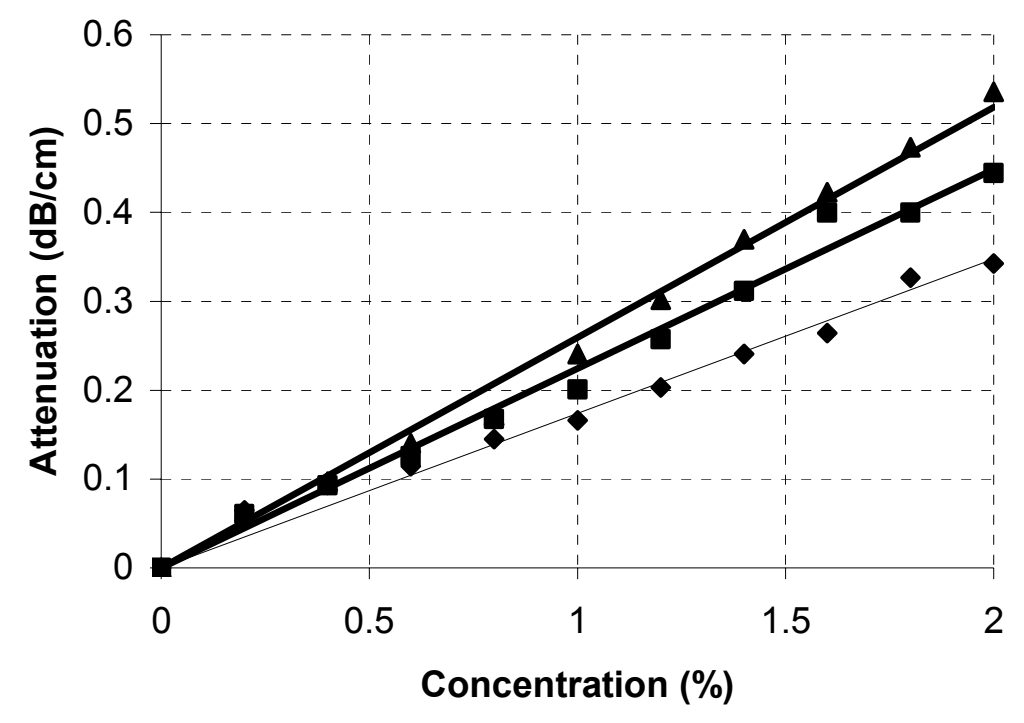

Figure A3.6: Calibration curves for Bottom Transducer in fly-ash suspension.

In general, it can be seen that acoustic attenuation at the bottom zone, i.e., the attenuation measured by the bottom transducer is more than that of the top transducer. There are a couple of reasons that could bring about this difference in transducer measurements. One of the reasons is that the bigger particles do not mix as well as the small particles and stay in the bottom half. The acoustic attenuation of bigger particles is much more than that of smaller particles. Also the settling velocity of the particles at the top zone is greater compared to the settling velocity of particles at the bottom zone. This is due to hindered settling. A repulsive force is exerted on the settling particles by the settled particles (sediment layer) at the bottom. So the particles from the top zone settle down to the bottom zone and increase the acoustic attenuation. 
From these observations, it can be concluded that the calibration curves between concentration and attenuation is linear and that calibration test has to be performed before every sedimentation experiment in order to take of the PSD effects. It is assumed that wetting of fly-ash during the calibration test does not have any effect on their sedimentation properties during the settling experiments. 


\section{References}

Abuaf, N., Wilkins Jr.,B. and Ward, H. C.: Rev. Sci. Instruments 50 (1979) 1260. Radio frequency probe for bubble size and velocity measurements.

Alba, F.: U.S. Pat. No. 5121629 (1992). Method and apparatus for determining particle size distribution and concentration in a suspension using ultrasonics.

Alba, F: European Pat. No. EP 0605409 (1996). Also granted in Japan. Method and apparatus for determining particle size distribution and concentration in a suspension using ultrasonics.

Andrieu, J. P., Scrivener, O., Reitzer, H.: Int. Chem. Eng. 32 (1992) 658. Sedimentation of solid particles in pulsating flow-fields.

Batchelor, G. K.: J. Fluid Mech. 52 (1972) 245. Sedimentation in a dilute dispersion of spheres.

Carlson, J and Grennberg, A.: IEEE Ultrasonics Symposium (1999) 757. Ultrasonic measurements of particles concentration in a multiphase flow.

Carter, J. A.: Study of fluid oscillation and its effect on low Reynolds number particle sedimentation (2000). Thesis.

Catalan, L, Ulrich, E., Hudgins, R. R. and Silveston, P. L.: J. Environmental Eng. 117 (1991) 457. Internal wave action in gravity clarifier.

Chang, Y. W.: Earthquake Engineering \& Structural Dynamics 23 (1994) 799. Discussion on a paper by A.S. Veletsos and P. Shivakumar.

Chaouki, J., Larachi, F. and Dudukovic, M. P.: Non-invasive monitoring of multiphase flows (1997). Elsevier. New York.

Chen, B. F. and Chiang, H. W.: J. Eng. Mech. 125 (1999) 70. Complete 2D and fully nonlinear analysis of ideal fluid in tanks.

Coe, H. S. and Clevenger, G. H.: Trans. Am. Inst. Min. Met. Eng. 55 (1916) 356. Methods for determining the capacities of slime-settling tanks. 
Coulson, J. M. and Richardson, J. F.: Chemical Engineering (1991). Fourth Edition. Pergamon Press. New York.

Crisanti, A., Falcioni, M., Provenzale, A. and Vulpiani, A.: Physica D: Nonlinear phenomena 46 (1990) 79. Passive advection of particles denser than the surrounding fluid.

Cushman, C R et al.: U.S. Pat. No. 3779070 (1973). Particle size and percent solids monitor.

Egolf, C. B. and McCabe, W. L.: Trans. Am. Inst. Chem. Eng. 33 (1937) 620. Rate of sedimentation of flocculated particles.

Einstein, A.: Ann. Phys. 19 (1906) 289. Eine neue Bestimmung der Molekuldimensionen.

Fung, J. C. H.: J. Aerosol Sci. 28 (1997) 753. Gravitational settling of small spherical particles in unsteady cellular flow fields.

Gadala-Maria, F. and Acrivos, A.: J. Rheology 26 (1980) 799. Shear-induced structure in a concentrated suspension of solid spheres.

Groen, J. S., Mudde, R. F. and Van Den Akker, H. E. A.: Trans. Inst. Chem. Engrs. 73 (1995) 615. Time dependent behavior of the flow in bubble column.

Hackley and Texter: Handbook on ultrasonic and dielectric characterization techniques for suspended particles.

Hamilton, M. F. and Blackstock, D. T.: Nonlinear Acoutics. (1998). Academic Press. San Diego. CA.

Happel, J. and Brenner, H.: Low Reynolds Number Hydrodynamics (1983), Nijhoff, Dodrecht, The Netherlands.

Hasegawa, E., Nagashima, F. and Takashima, H.: JSME Int. J. 30 (1987) 1750. Instability of an interface between two immiscible fluids under a vertical oscillation.

Hashimoto, H. and Sudo, S.: Bulletin of JSME 28 (1985) 1910. Dynamic behavior of stratified fluids in a rectangular container subject to vertical vibration. 
Hawksley, P. G.: Inst. of Phys. Symposium (1950) 114. The effect of concentration on the settling of suspensions and flow through porous media.

Hay, A E and Mercer, D G.: J. Acoust. Soc. Am. 85 (1989). A note on the viscous attenuation of sound in suspensions.

Hay, A E and Mercer, D G: J. Acoust. Soc. Am. 78 (1985) 1761. On the theory of sound scattering and viscous absorption in aqueous suspensions at medium and short wavelengths.

Hinds, Williams C.: Aerosol Technology, Second Edition, Wiley-Interscience.

Ho, B. P. and Leal, L. G.: J. Fluid Mech. 65 (1974) 365. Inertial migration of rigid spheres in two dimensional unidirectional flows.

Hsu, C. T., Lu, X. and Kwan, M. K.: J. Eng. Mech. (2000) 186. LES and RANS studies of oscillating flows over flat plate.

Kaye, B. H. and Boardman, R. P.: Third Congress of the European Federation of Chemical Engineering (1962). Symposium on the Interaction between Fluids and Particles 17. Cluster formation in dilute suspensions.

Kermack, W. O., M'Kendrick, A. G. and Ponder, E.: Proc. Royal Soc. Edinburgh 49 (1929) 170. The stability of suspensions. III. The velocities of sedimentation and of cataphoresis of suspensions in a viscous fluid.

Khan, A. R. and Richardson, J. F.: Chem. Eng. Comm. 78 (1989) 111. Fluidparticle interactions and flow characteristics of fluidized beds and settling suspensions of spherical particles.

Khan, A. R. and Richardson, J. F.: Chem. Eng. Sci. 45 (1990) 255. Pressure gradient and friction factor for sedimentation and fluidization of uniform spheres in liquids.

Knudsen, V O.: J. Acous. Soc. Am.18 (1946) 90.

Kynch, G. J.: Trans. Faraday Soc. 48 (1952) 166. A theory of sedimentation.

Lamb, H.: Hydrodynamics. Sixth Edition. Dover Publications. New York.

Lance, M. and Bataille, J.: J. Fluid Mech. 222 (1991) 95. Turbulence in the liquid phase of a uniform bubbly air water flow. 
Landau, L. D. and Lifshitz, E. M.: Fluid mechanics. Translated from the Russian by Sykes, J. B. and Reid, W. H. (1959). Pergamon Press; Addison-Wesley Pub. Co., London

Leighton, D. and Acrivos, A.: Chem. Eng. Sci. 41 (1986) 1377. Viscous Resuspension.

Leighton, D. and Acrivos, A.: J. Fluid Mech. 181 (1987) 415. The shear induced migration of particles in concentrated suspensions.

Levesley, J. A. and Bellhouse, B. J.: Trans. Inst. Chem. Eng. 75 (1997) 288. The retention and suspension of particles in a fluid using oscillatory flow.

Ma, L. and Chang, C. S.: J. Geotechnical Eng. 119 (1993) 1679. Pressures exerted on soil due to rocking of liquid storage tanks.

Mackley, M. R., Smith, K. B. and Wise, N. P.: Trans Inst. Chem. Eng. 71 (1993) 649. The mixing and separation of particle suspensions using oscillatory flow in baffled tubes.

Maxey, M. R. and Corrsin, S.: J. Atmospheric Sci. 43 (1986) 1112. Gravitational settling of particles in randomly oriented cellular flow fields.

Maxey, M. R. and Shin, H.: Physical Review E. Statistical Physics, Plasmas, Fluids, and Related Interdisciplinary Topics 56 (1997) 5431. Chaotic motion of non-spherical particles settling in a cellular flow field.

Mazur, P. and van Saarloos, W.: Physica A 115 (1982) 21. Many-sphere hydrodynamics and mobilities in a suspension.

Moujaes, S. and Dougall, R. S.: Can. J. Chem. Eng. 65 (1987) 705. Experimental investigation of concurrent flow in a vertical rectangular channel.

Richardson, J. F. and Zaki, W. N.: Trans. Inst. Chem. Eng. 32 (1954) 35. Sedimentation and fluidization. Part I.

Richardson, J. F. and Meikle, R. A.: Trans. Inst. Chem. Eng. 39 (1961) 357. Sedimentation and fluidization. Part IV. Drag force on individual particles in an assemblage. 
Richardson, J. F. and Shabi, F. A.: Trans. Inst. Chem. Eng. 38 (1960) 33. the determination of concentration distribution in a sedimenting suspension using radioactive solids.

Riebel, U.: U.S. Pat. No. 4706509 (1987), DE Pat. No. 3438798 (1986). “Method of and an apparatus for ultrasonic measuring of the solids concentration and particle size distribution in a suspension.

Robinson, C. S.: Ind. Eng. Chem. 18 (1926) 869. Some factors influencing sedimentation.

Rowe, P. N.: Chem. Eng. Sci. 42 (1987) 2795. A convenient empirical equation for estimation for the Richardson-Zaki exponent.

Schaflinger, U., Acrivos, A. and Zhang, K.: Int. J. Multiphase Flow 16 (1989) 567. Viscous resuspension of a sediment within a laminar and stratified flow.

Schlichting, H.: Boundary layer theory. (1979). Seventh Edition. McGraw-Hill Book Company.

Steinour, H. H.: Ind. Eng. Chem. 36 (1944) 618. Rate of Sedimentation.

Tang, Y.: J. Eng. Mech. 119 (1993) 531. Dynamic response of tank containing two liquids.

Tang. Y and Chang, Y. W.: ANL/RE/PP-80379. Sloshing Response of nonuniform liquid in a laterally excited tank.

Tennekes, H. and Lumley, J. L.: A first course in turbulence (1987). MIT press. Cambridge, Massachusetts.

Tory, E. M.: Sedimentation of small particles in a viscous fluid. (1996) Computational Mechanics Publications. Boston.

Urick, R J.: J. Acous. Soc. Am. 20 (1948) 283. The absorption of sound in suspensions of irregular particles.

Veletsos, A. S. and Shivakumar, P.: Earthquake Eng. and Structural Dynamics 22 (1993) 801. Sloshing response of layered liquids in rigid tanks. 
Wang, L. P., Maxey, M. R., Burton, T. D. and Stock, D. E.: Physics of Fluids A (Fluid Dynamics) 4 (1992) 1789. Chaotic dynamics of particle dispersion in fluids.

Work, L. T. and Kohler, A. S.: Trans. Am. Inst. Chem. Eng. 36 (1940) 701. The sedimentation of suspensions. 


\title{
Simulation of Gravitational Settling Under Electric Fields
}

By

Vinayak Vidyadhar Barve, BE (Mech.)

\author{
Thesis \\ Presented to the Faculty of the Graduate School of \\ The University of Texas at Austin \\ in Partial Fulfillment \\ of the Requirements \\ for the Degree of
}

Master of Science in Engineering

The University of Texas at Austin

December, 2002 


\section{Abstract \\ Simulation of Gravitational Settling Under Electric Fields \\ By \\ Vinayak Vidyadhar Barve, MSE \\ The University of Texas at Austin, 2002.}

Supervisor: O. A. Ezekoye

The effect of electric fields on aerosol settling rates is studied. The electrical coagulation rates for an aerosol - cigarette smoke, are calculated using a trajectory analysis. The electrical coagulation rates were found to be significantly higher than the gravitational coagulation rates. A sectional algorithm - MAEROS, which simulates the aerosol size evolution, is modified to incorporate the evolution process at multiple elevations within a chamber. The electrical coagulation mechanism is added to the multiple elevation code. Simulations reveal that the electric fields significantly enhance the settling rates and the enhancement is more pronounced at lower levels within the chamber. The simulations are further compared with experimental data obtained at 3 different levels. The comparison shows that the electrical coalescence model is not quite so accurate, though it shows similar trends. 


\section{Table of Contents}

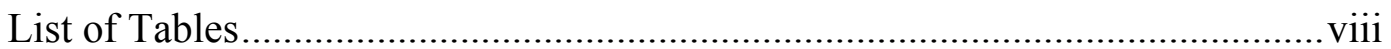

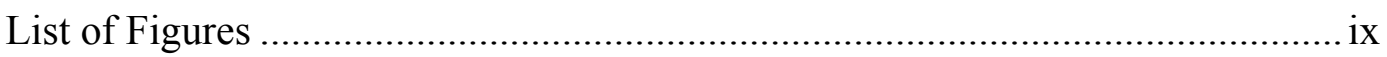

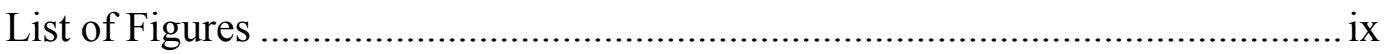

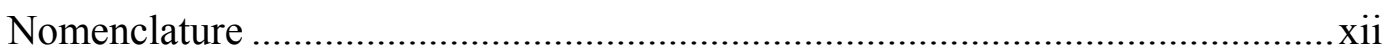

CHAPTER 1: INTRODUCTION

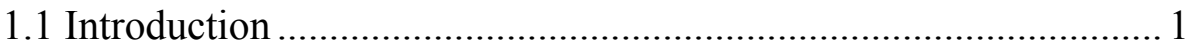

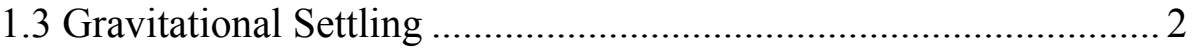

1.4 Electrical Coalescence.....................................................................

1.2 Scope of the Project......................................................................... 4

CHAPTER 2: EXPERIMENTAL SYSTEM 6

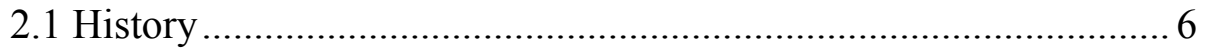

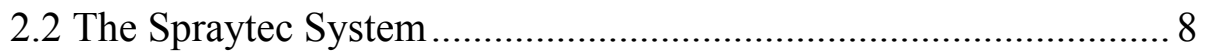

2.3 Experimental Setup and Procedure ………………..................... 10

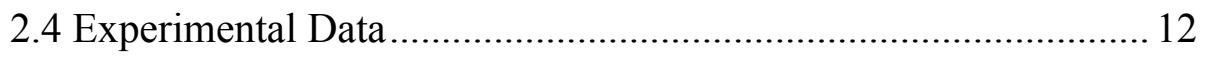

CHAPTER 3: THEORY AND NUMERICAL ANALYSIS 14

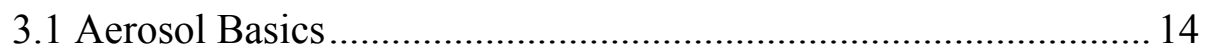

3.2 General Dynamic Equation ....................................................... 14

3.2 Electric Field Induced Forces.................................................... 18

3.3 The Bispherical Polar Coordinate System ................................... 19

3.4 Calculating the Electric Induced Forces......................................... 20

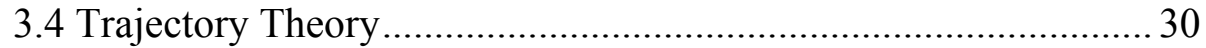

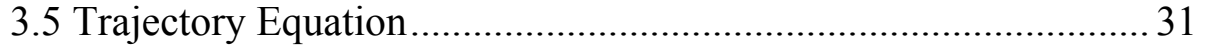

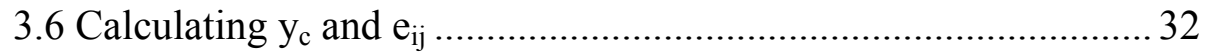

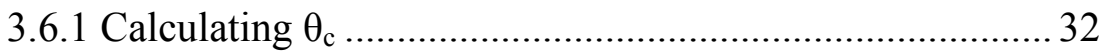

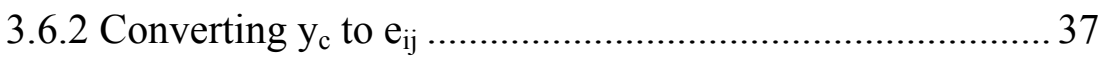


3.7 Numerical Implementation - MAEROS ..................................... 41

CHAPTER 4: RESUltS

4.1 Computational Results ........................................................... 45

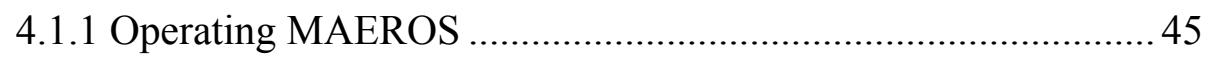

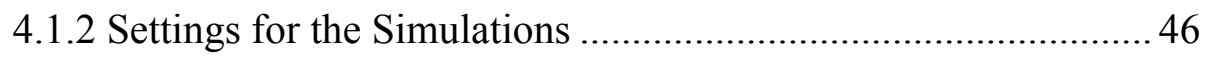

4.1.3 Multiple Elevation Effects .................................................. 48

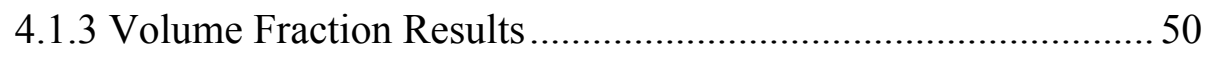

4.1.4 Constant Elevation Results................................................... 52

4.1.5 Particle Size Distribution Data ................................................56

4.2 Comparison with experiments/literature .................................58

CHAPTER 5: CONCLUSIONS AND FUTURE WORK 64

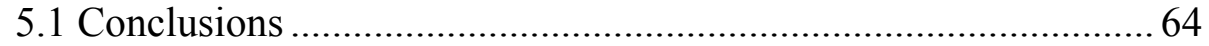

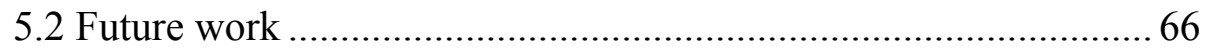

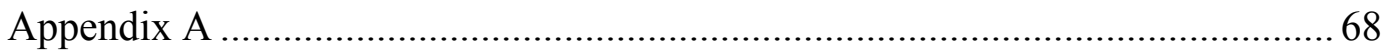

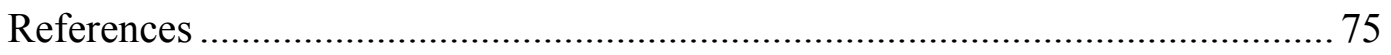

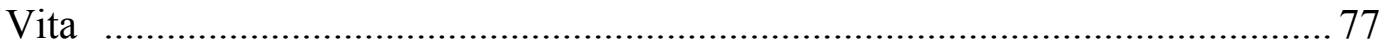




\section{List of Tables}

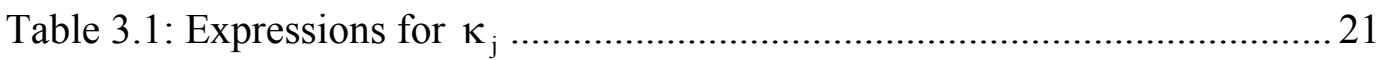

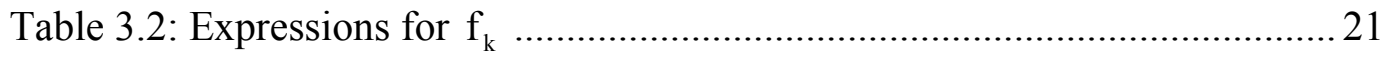

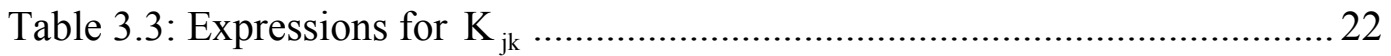

Table 4.1: Diffusion thickness for particles of different sizes based on their

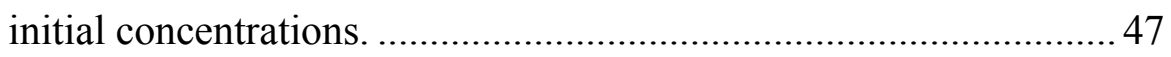

Table 4.2: Errors in the model for the time required to reduce to $50 \%$ of the

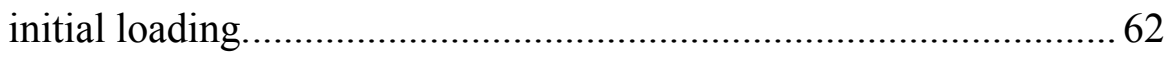

Table 4.3: Errors in the model for the time required to reduce to $20 \%$ of the initial loading... 63 


\section{List of Figures}

Figure 1.1: Induced dipole in adjacent particles associated with electric field

$\overline{\mathrm{E}}$. 3

Figure 2.1: Experimental setup for the laser extinction measurements

(Schmidt, 2000). 7

Figure 2.2: Dimensional and components of the unit reactor (Schmidt, 2000), ..... 7

Figure 2.3: Schematic of the laser diffraction system (Malvern)............................. 8

Figure 2.4: Photograph of the experimental setup ................................................ 11

Figure 2.5: Example of Transmission data....................................................... 12

Figure 2.6: Example of Particle Size Distribution (PSD) data................................ 13

Figure 3.1: Coordinate system used for the relative motion of two differentsized particles induced by gravity and electrical forces (Zhang,

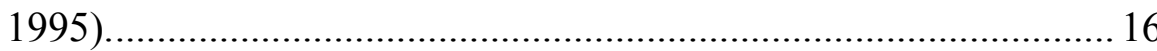

Figure 3.2: The bispherical polar coordinate system (Davis, 1964)..................... 19

Figure 3.3: Plot showing the variation of the induced force with the

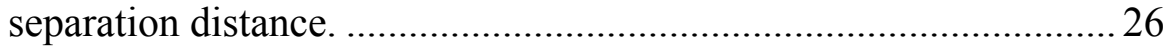

Figure 3.4: Surface densities of charge and forces on two particles induced by an externally applied electric field for different values of angle $\psi$ between the electric field and the line joining the centers of

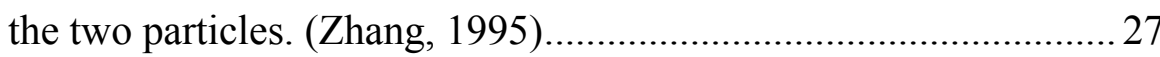

Figure 3.5: Variation of the dimensionless radial force with different orientations. 28 
Figure 3.6: Variation of the dimensionless tangential force with different orientations.

Figure 3.7: Schematic of the coordinate system and relative trajectories of two particles under effect of gravity and electric field (Wang, 1997)....30

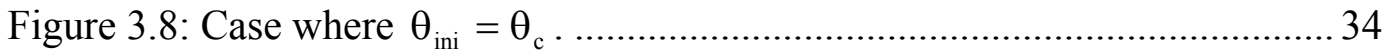

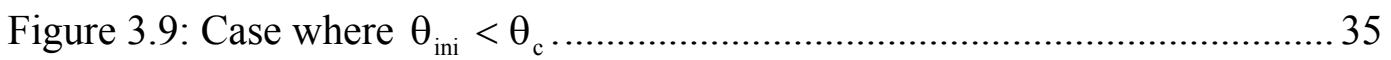

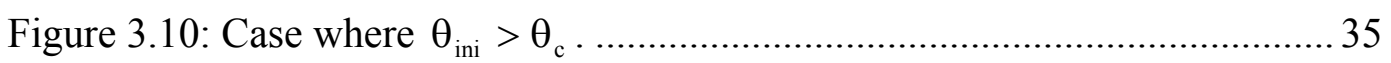

Figure 3.11: Variation of the magnitude of the electrical field force component and the van der Waals force component in the trajectory equation at $\theta=120^{\circ}$ 37

Figure 3.12: Collision efficiencies for an electric field of $25 \mathrm{kV} / \mathrm{m}$. 39

Figure 3.13: Collision efficiencies for an electric field of $50 \mathrm{kV} / \mathrm{m}$..................... 39

Figure 3.14: Collision efficiencies for an electric field of $75 \mathrm{kV} / \mathrm{m}$.................... 40

Figure 3.15: Schematic of the cell being divided into multiple horizontal compartments.

Figure 3.16: Collision efficiencies as calculated by the MAEROS model and the trajectory analysis.

Figure 4.1: Volumetric Loading plot for natural gravitational settling as predicted by the original MAEROS code. 48

Figure 4.3: Volume Fraction Loading for $25 \mathrm{kV} / \mathrm{m}$. 51

Figure 4.4: Volume Fraction Loading for 50 kV/m............................................ 51

Figure 4.5: Volume Fraction Loading for $75 \mathrm{kV} / \mathrm{m}$.......................................... 52

Figure 4.6: Settling plots at $3 \mathrm{~mm}$ elevation for different electric fields ...... 53 
Figure 4.7: Settling plots at $7.5 \mathrm{~mm}$ elevation for different electric fields 54

Figure 4.8: Settling plots at $11.5 \mathrm{~mm}$ elevation for different electric fields 54

Figure 4.9: Plot of time required to reduce to $50 \%$ loading for different electric fields. 55

Figure 4.10: Initial Particle Size Distribution 56

Figure 4.11: Particle Size Distribution for elevation of $7.5 \mathrm{~mm}$ after $20 \mathrm{~s}$ under no electric field.

Figure 4.12: Particle Size Distribution for elevation of $7.5 \mathrm{~mm}$ after $40 \mathrm{~s}$ under no electric field

Figure 4.13: Particle Size Distribution for elevation of $7.5 \mathrm{~mm}$ after $60 \mathrm{~s}$ under no electric field. 58

Figure 4.14: Comparison volume fraction loading plots with no electric field. ... 60 Figure 4.15: Comparison volume fraction loading plots with electric filed of $25 \mathrm{kV} / \mathrm{m}$. 60

Figure 4.16: Comparison volume fraction loading plots with electric filed of $50 \mathrm{kV} / \mathrm{m}$.

Figure 4.17: Comparison volume fraction loading plots with electric filed of $75 \mathrm{kV} / \mathrm{m}$ 


\title{
Nomenclature
}

\author{
$a_{i} \quad$ radius of larger particle of size $i$ \\ $a_{j}$ radius of smaller particle of size $j$ \\ A composite Hamaker constant \\ $\mathrm{C}_{\mathrm{c}}$ Cunningham correction factor \\ $\mathrm{C}_{\mathrm{v}}$ volumetric concentration \\ $\mathrm{C}_{11}, \mathrm{C}_{12}, \mathrm{C}_{22}$ coefficients of capacitance \\ $d_{i}$ diameter of particle of size $i$ \\ $\mathrm{D}_{\mathrm{ij}}^{(0)} \quad$ relative mobility for 2 particles due to an equal and opposite force \\ $\mathrm{D}_{1}, \mathrm{D}_{2}$ distances of the centers of the particles from the origin \\ $\mathrm{E}_{0}$ magnitude of the external electric field \\ $\mathrm{e}_{\mathrm{ij}}$ collision efficiency \\ $\mathrm{e}_{\mathrm{r}} \quad$ unit vectors in radial direction \\ $\mathrm{e}_{\theta} \quad$ unit vectors in tangential direction \\ $\mathrm{F}_{\mathrm{E}, \mathrm{ij}}^{\mathrm{r}} \quad$ electrical field force components along the line of centers \\ $\mathrm{F}_{\mathrm{E}, \mathrm{ij}}^{\theta} \quad$ electrical field force components normal to the line of centers \\ $\mathrm{g}$ gravitational acceleration $(9.81 \mathrm{~m} / \mathrm{s})$ \\ $\mathrm{H}$ the distance between the centers \\ $\mathrm{I}_{\lambda} \quad$ intensity of the beam at the measurement instant \\ $\mathrm{I}_{\lambda, 0} \quad$ intensity of the "empty" laser \\ k Boltzmann's constant \\ $\mathrm{K}$ optical property dependant on the refractive index of the aerosol \\ $\ell$ mean free path \\ $\mathrm{L}_{\text {path }} \quad$ laser beam path length through the aerosol \\ G,L relative mobility functions in the radial direction
}




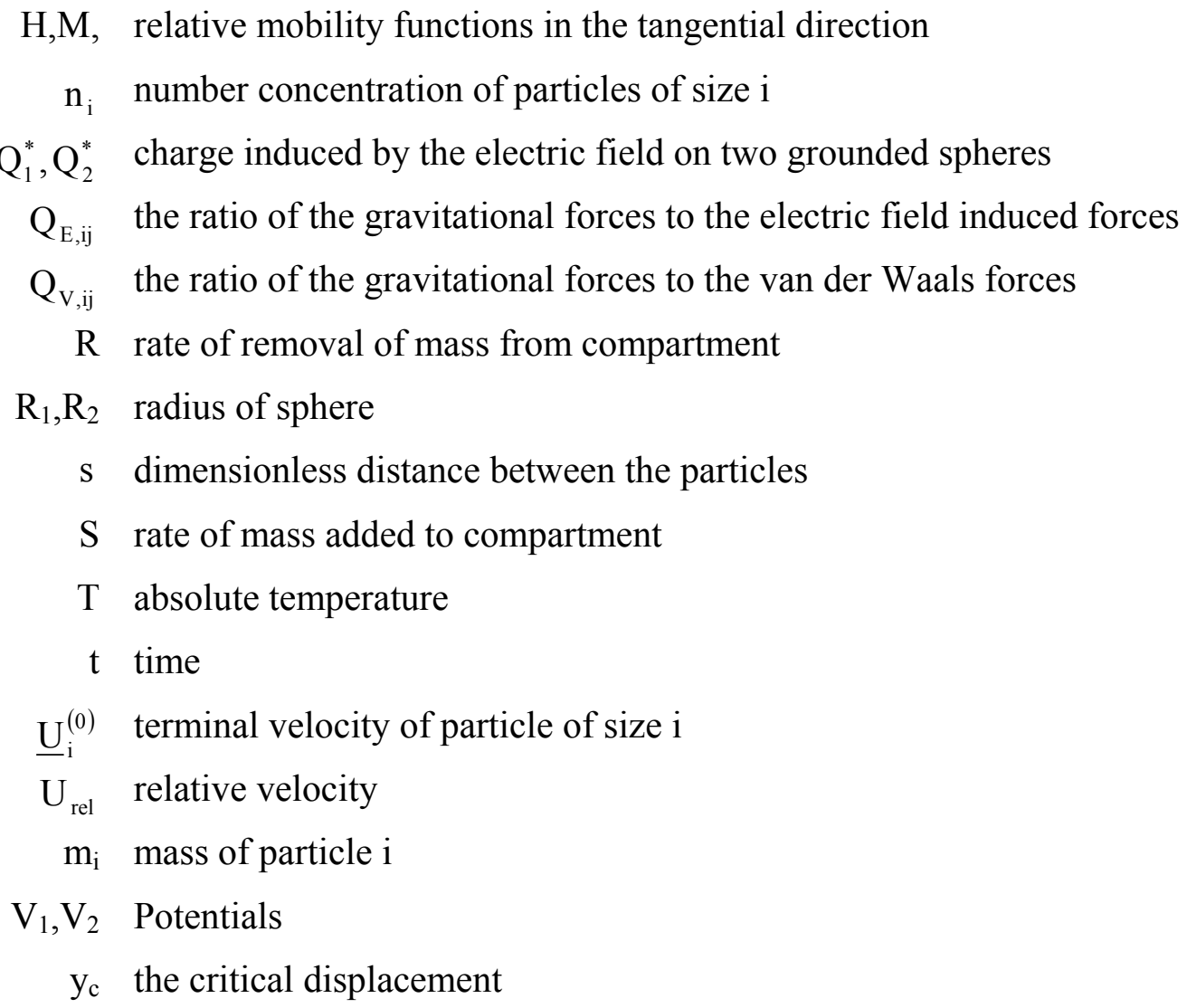

\section{Greek letters}

$\beta$ collision frequency function or the agglomeration kernel

$\delta \quad$ diffusion thickness

$\Delta$ diffusion coefficient

$\in$ permittivity of the surrounding fluid

$\Theta$ angle

$\theta_{\text {ini }}$ initial angle of separation used for the integration

$\theta_{c}$ maximum initial angle of separation at which the two particles collide

$\lambda_{\text {laser }}$ wavelength of the laser light

$\mu_{0} \quad$ viscosity of the air

$\phi_{\mathrm{ij}}$ potential function 
$\begin{array}{ll}\rho_{0} & \text { density of air } \\ \rho_{d} & \text { density of the particle } \\ \psi & \text { angle between the electric field and the particle center line }\end{array}$ 


\section{CHAPTER 1: INTRODUCTION}

\subsection{Introduction}

Air borne particles have important consequences for health, visibility, climate and human welfare in general. Small aerosol particles are a major source of respiratory illnesses since these particles are not captured by the natural human filtration system and reach the lungs. Therefore, the efficient capture and removal of aerosols from the air has immediate benefits for human health. Radioactive aerosols associated with the production of fissionable materials pose as an even greater hazard to human life and the environment as a whole. For the DOE, the

production of aerosols during treatment and sludge retrieval of the waste tanks is a major concern. Conventional filtration systems using filter fiber require periodic manual removal and replacement. Also these systems are associated with energy losses in the form of pressure drops. A replacement for these conventional systems would involve a non-intrusive and inexpensive method of aerosol removal. The system should also have a low maintenance frequency. One of the possible methods would be, to use an electric field to enhance particle coagulation to form larger particles which would settle out of the system.

Electric fields are used extensively in industry for various separation processes. Waterman (1965) reviewed the process of using electric fields to improve coalescence and found these techniques to be quite effective in the removal of water from oil. Williams and Bailey (1986) examined coalescence of conducting drops in the presence of electrical fields theoretically and 
experimentally. They observed that with an electrical field, the mean diameter of the drops increases rapidly as compared to a case without any electric field. Schmidt (2000) performed experiments with different aerosols and observed that the application of electric fields had the effect of reducing sedimentation time. It was also observed that greater the electric field, smaller the sedimentation time. A calculation of the particle size distribution suggested that this was due to the electric field enhancing particle coalescence. Studies to predict the collision frequencies of settling mechanisms have been carried out in the past. Melik and Fogler (1984) used a trajectory analysis to predict aggregation rates for Brownian particles with weak gravitational forces. Wen (1991) used a pair distribution function to analyze a system of weak Brownian and strong gravitational coagulation. For settling in the presence of electric fields, Zhang (1995) used a trajectory analysis to follow the relative motion of two drops and predict pair wise collision and coalescence rates. On solving a dynamic population balance, he found that collision rate and drop growth increase significantly in the presence of electric fields. Wang (1996) used a similar trajectory analysis for estimating the aggregation rates of electric field enhanced gravitational settling of charged particles.

\subsection{Gravitational Settling}

Aerosol particles are in continuous relative motion resulting in the particles colliding and coalescing to form larger particles. Thus, the particle size

distribution of a naturally evolving aerosol is continuously shifting towards the 
larger size. In the presence of gravitational acceleration, the particles have a bulk downward motion. Larger particles settle out faster than the smaller particles since the settling rates depend on the square of the particle diameter (Williams, 1986). As a result, a larger particle would catch up with smaller particles in their path leading to collisions and agglomeration. This phenomenon is called gravitational coagulation. Different methods have been used to analyze the coupling of gravitational and Brownian coagulation mechanisms.

\subsection{Electrical Coalescence}

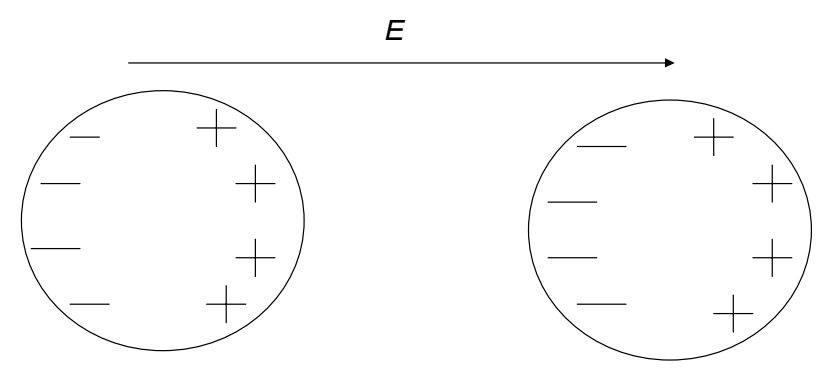

Figure 1.1: Induced dipole in adjacent particles associated with electric field $\overline{\mathrm{E}}$.

An electric field induces a dipole in uncharged aerosol particles. The charges induced on the closest sides of the neighboring particles are of opposite 
sign. These particles experience an attractive force which can eventually lead to the two particles colliding causing agglomeration or coalescence. The attractive forces overcome the hydrodynamic forces between the particles and increase the collision frequency. This modifies the naturally evolving particle size distribution. The increased agglomeration shifts the particle size distribution towards larger sizes faster. Since the larger particles settle faster, the aerosol clears out faster. This phenomenon of increasing the particle collisions is called electrical coalescence.

\subsection{Scope of the Project}

Schmidt (2000) used extinction measurements to determine the settling rates of cigarette smoke. These measurements were done at various elevations within the cell to give a better understanding of the process and detect the stratification that occurs during the settling. These experiments were then repeated in the presence of an electric field. It was observed that settling rates were enhanced by using an electric field due to electrical coalescence.

This project sought to develop a comprehensive prediction model for predicting the settling process under the effect of an electric field. Using a trajectory analysis as suggested by Zhang (1995), expressions for electrical coalescence are derived. An attempt was made to verify this trajectory analysis by applying it to a real-life problem. Towards this, the stratification effects were added to the trajectory analysis. 
For predicting the settling process, MAEROS - a zero dimensional aerosol code was used. The code was first modified to account for the stratification effects. Following that, the electrical coalescence model was added to simulate the settling in the presence of electric fields. The theory behind the electrical coalescence model and the stratification effects are discussed in chapter 3. Also, settling experiments were conducted using laser scattering measurements to provide continuous particle size distribution data. The experimental set-up and diagnostics is described in chapter 2. Further, to test the model, a comparison of the modified MAEROS code with experimental data was performed. The modeling results and comparisons to the experiments are discussed in Chapter 4. Chapter 5 contains the conclusions and some possible future work. 


\section{CHAPTER 2: EXPERIMENTAL SYSTEM}

\subsection{History}

For observing the effect of electric fields on the settling process, experiments were conducted by Schmidt (2000). A cell (rectangular box) made of acrylic and two copper plates, was used for the experiments. The schematic of the cell is shown in Figure 2.1. The internal dimensions of the cell were $10 \mathrm{~cm} \times 10$ $\mathrm{cm} \times 2 \mathrm{~cm}$ with the copper plates set into the inner top and bottom surfaces of the enclosure. The copper plates were connected to a Bertran 205B High Voltage supply to create the electric field. Two opposite sides of the cell had windows machined into them so that non-reflective glass could be inserted for laser extinction measurements.

The experiments involved filling the cell with the aerosol and allowing the aerosol to settle over time. The laser extinction measurements were done at three different elevations $-11.43 \mathrm{~mm}, 7.62 \mathrm{~mm}$ and $3.05 \mathrm{~mm}$ from the upper copper plate. These experiments were then repeated in the presence of different electric fields. The extinction measurements are indicative of the volumetric loading.

The settling of different aerosols like oil fog, smoke from burning tissue paper and cigarette smoke, was examined. For the cigarette smoke experiments, a brass cigarette holder was made, such that all the smoke from the burning cigarette is forced through the cigarette filter. It was found that the cigarette smoke experiments using Maverick brand of cigarettes were most repeatable. 


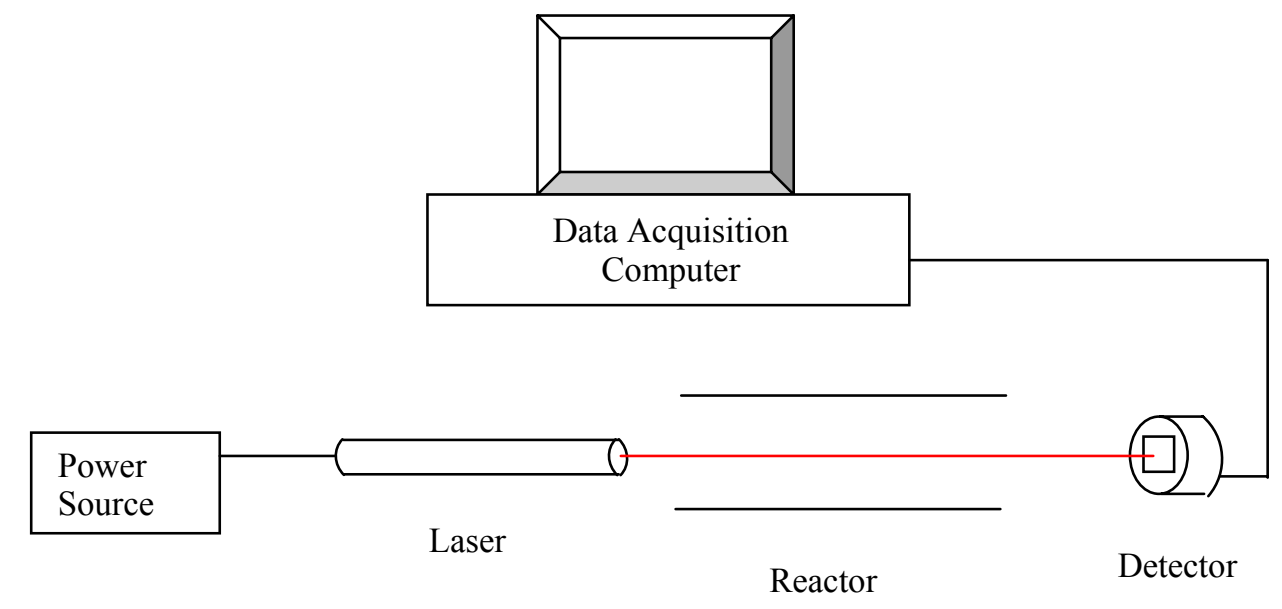

Figure 2.1: Experimental setup for the laser extinction measurements (Schmidt, 2000).

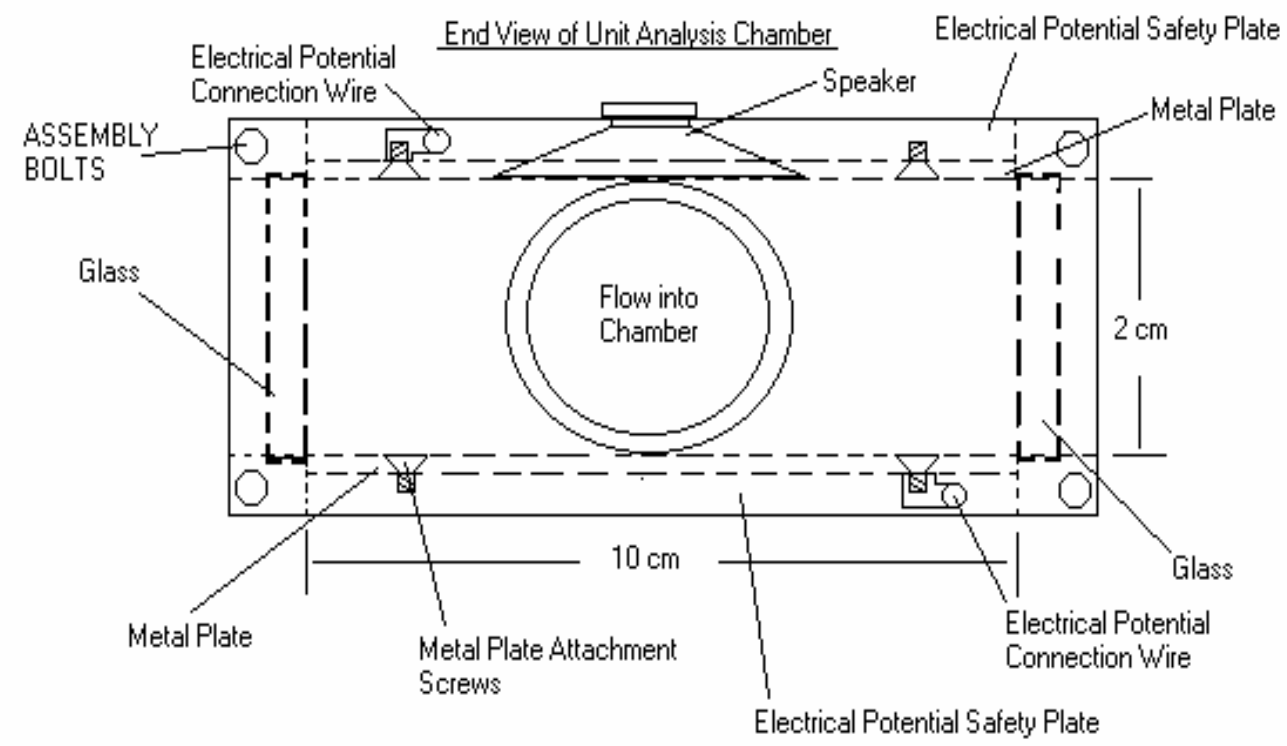

Figure 2.2: Dimensional and components of the unit reactor (Schmidt, 2000). 


\subsection{The Spraytec System}

Similar experiments were repeated at Oak Ridge National Laboratories (ORNL). For data acquisition, a Malvern Instruments Spraytec RTS 5006 system was used. This system is a laser based system allowing continuous particle size distribution (PSD) measurement. The purpose of repeating the experiments was that this system could, in theory, give us the PSD as the aerosol settles. This would help in explaining the process of electrical coalescence.

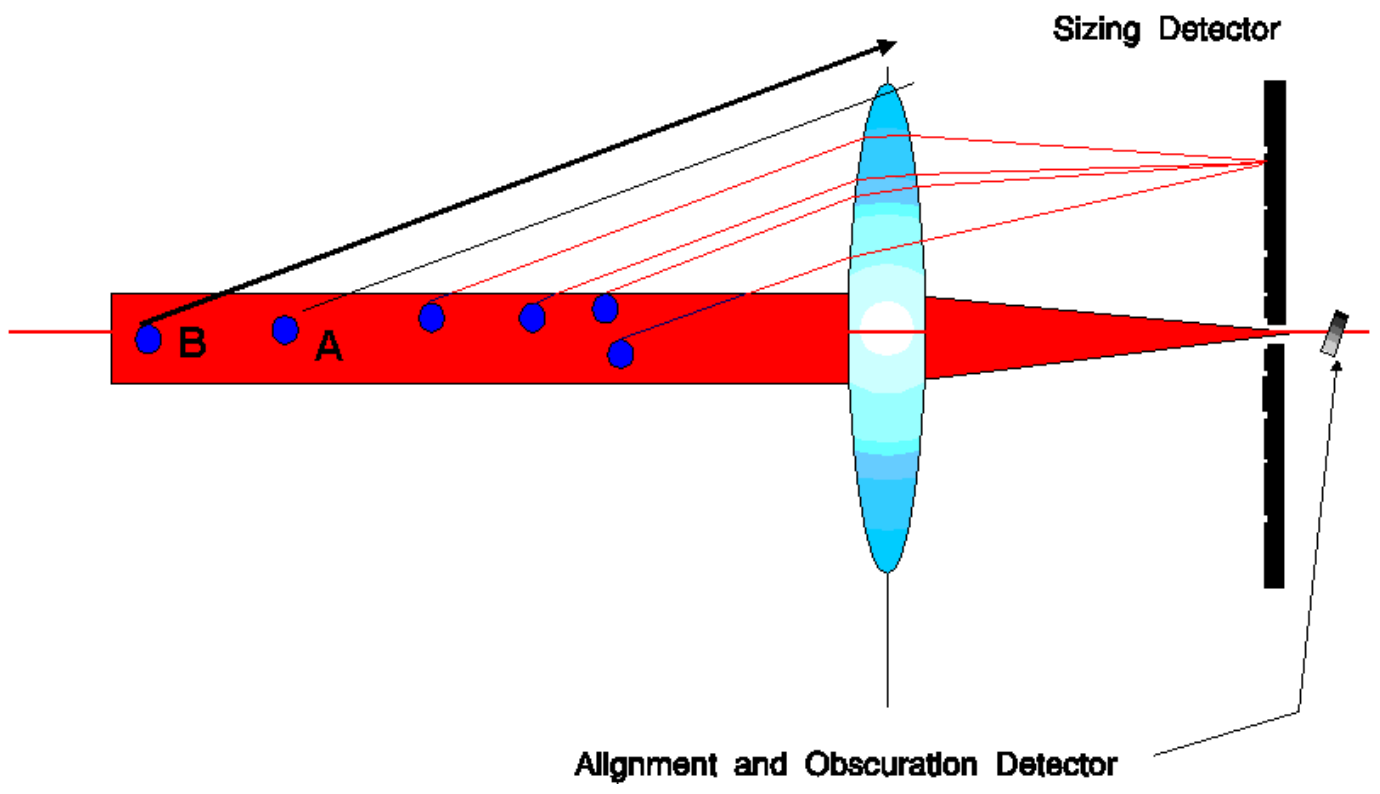

Figure 2.3: Schematic of the laser diffraction system (Malvern).

The laser has a wavelength of $670 \mathrm{~nm}$ and a laser beam diameter of $1 \mathrm{~cm}$. The system uses laser diffraction or a Low Angle Laser Light Scattering (LALLS) 
method to calculate the PSD (Malvern). This is based on the principle that when a particle passes through a laser beam it causes light to be scattered at an angle that is inversely proportional to its size. The scattered light is collected by a detector and the size distribution is calculated based on the diffraction pattern. Figure 2.3 is a schematic of the system. It consists of a Fourier lens which is used to focus the laser beam onto the center of the detector. The detector consists of a large number of photosensitive segments, which are arranged concentrically. There is a pinhole at the center of the detector beyond which lies the obscuration detector. If no particles pass through the laser beam, all the light falls onto the obscuration detector. However, if a particle passes through the laser beam, it scatters light. The Fourier lens has the property of collecting the scattered light, and refracting it onto the detector array based on the scattering angle. The detector elements are arranged such that each concentric element lies in the focal plane of the lens and correspond to a particular scattering angle. As a result the light scattered by a particle will continue to fall on the same part of the detector array for as long as that particle is in the laser beam, regardless of the position of the particle within the beam. Since the scattering angle depends on particle size, and each particle passing through the beam would scatter light, the sum of the light scatter on any particular detector indicates the number count for particles of that size. The analysis of the light scatter on each detector would give the PSD.

The obscuration detector is used to monitor the concentration of the particles passing through the laser beam. If there are no particles in the laser beam, all the light falls onto the obscuration detector. As particles enter the beam, 
they block this light and scatter light onto the sizing elements of the detector array. By measuring the intensity of the "empty" laser beam before a measurement is undertaken (the background measurement), and taking a ratio of the value obtained with the value obtained as a stream of particles enters the beam, we are able to monitor the obscuration. The obscuration is used to calculate the concentration based on Beer-Lamberts law

$$
\frac{\mathrm{I}_{\lambda}}{\mathrm{I}_{\lambda, 0}}=\exp \left(-\frac{\mathrm{C}_{\mathrm{v}} \mathrm{KL}_{\mathrm{path}}}{\lambda}\right)
$$

where $I_{\lambda}$ is the intensity of the beam at the measurement instant, $I_{\lambda, 0}$ is the intensity of the "empty" laser, $\mathrm{C}_{\mathrm{v}}$ is the volumetric concentration, $\mathrm{K}$ is an optical property dependant on the refractive index of the aerosol, $\mathrm{L}_{\mathrm{path}}$ is the laser beam path length through the aerosol sample and $\lambda$ is the wavelength of the laser light.

The data from the detector array is analyzed by the RTSizer software to give the PSD. The analysis is based on Mie theory and the deconvolution (inference of particle size) of the scattering patterns.

\subsection{Experimental Setup and Procedure}

The cell was attached to a jack using Quick-Grip bar clamps. The jack could be moved in the vertical direction. This allowed experiments to be conducted at different levels from the base of the cell. The jack was bolted onto the base of the laser, which in turn was attached to an optical table. Figure 2.4 is a photograph of the experimental set-up. 


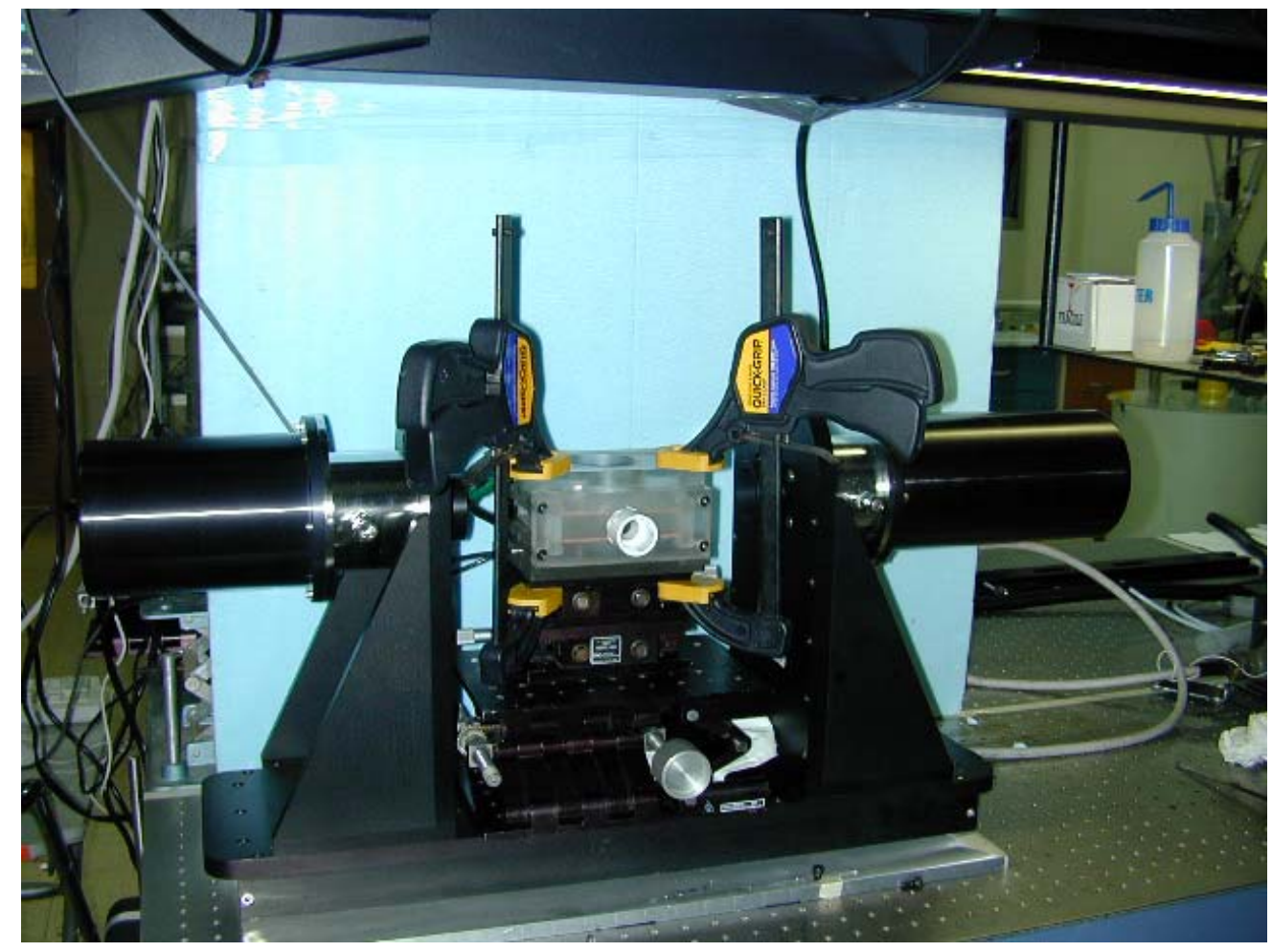

Figure 2.4: Photograph of the experimental setup

Once the cell was setup at the required elevation, it was filled with cigarette smoke. The cigarette was first held in the brass cylinder, and then ignited. Compressed air was used to force the smoke through the filter into the cell. These experiments were done at 3 elevations $-5 \mathrm{~mm}, 10 \mathrm{~mm}$ and $15 \mathrm{~mm}$ from the upper copper plate. Once the cell was completely filled with smoke, the inlet valve was closed and the RTSizer software was initiated to collect data. Data was collected till the transmission readings stagnated (became constant). A 100\% transmission reading was never reached due to deposition of the smoke on the windows. These experiments were repeated with $1 \mathrm{kV}$ and $2 \mathrm{kV}$ applied to the 
copper plates. For the $1 \mathrm{kV}$ and $2 \mathrm{kV}$ experiments, the electric field was put on simultaneously as the RTSizer software was initiated. The windows of the cell were cleaned between each experiment. Every time the windows were cleaned, a background reading was taken.

\subsection{Experimental Data}

The RTSizer software provides the transmission data as well as the particle size distribution. Figure 2.4 is a plot of the transmission measurement versus time for the case of $0 \mathrm{~V}$ at an elevation of $15 \mathrm{~mm}$ from the upper copper plate.

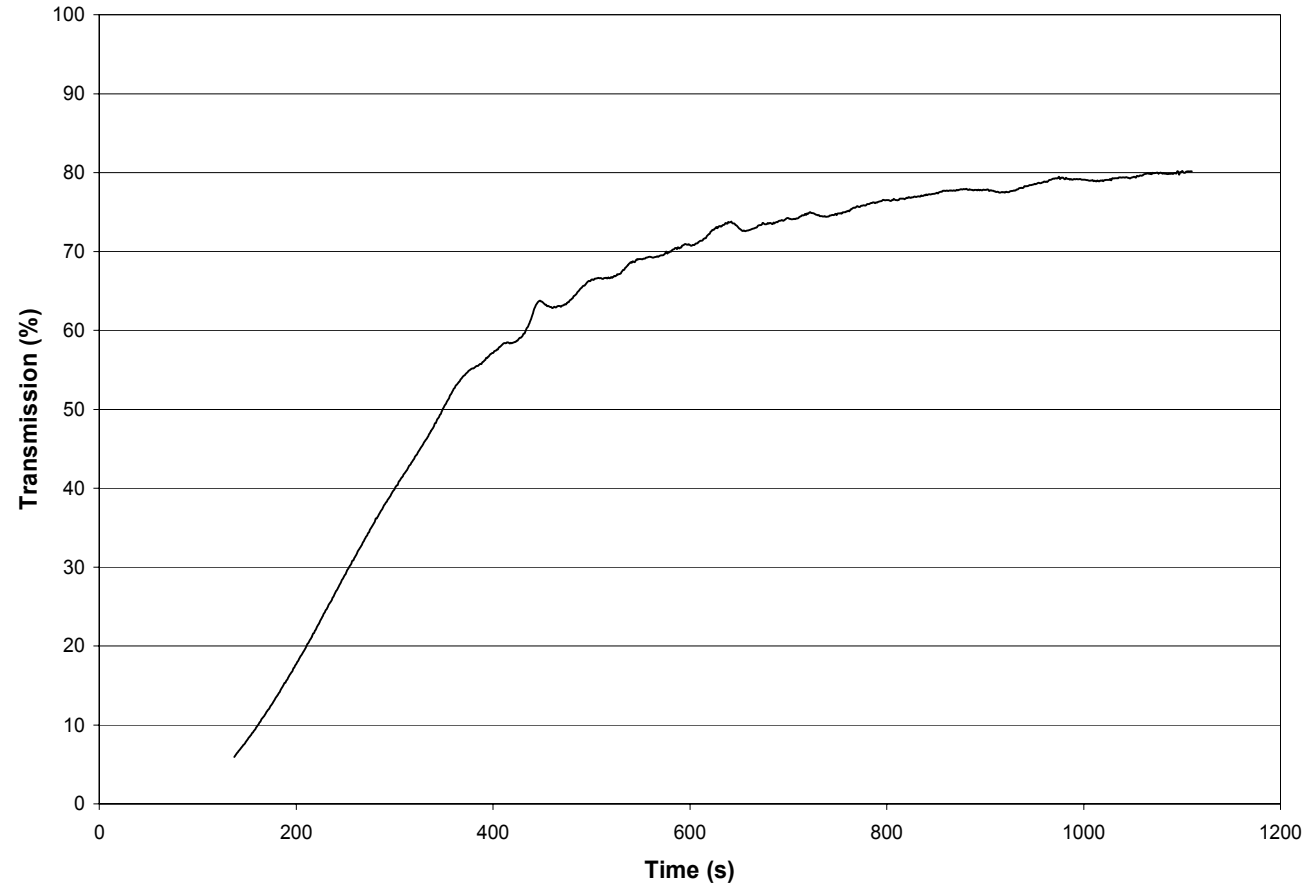

Figure 2.5: Example of Transmission data. 


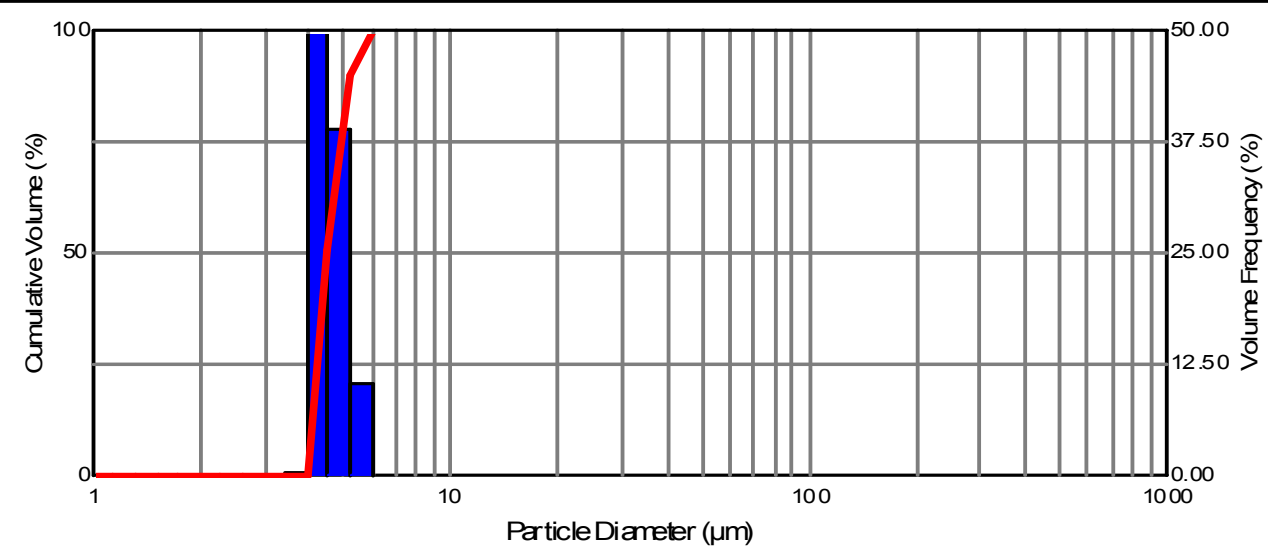

Filename $=$

Figure 2.6: Example of Particle Size Distribution (PSD) data.

The transmission increases as the aerosol clears out and finally flattens out. The PSD data, for the case mentioned above is displayed in Figure 2.5. The size distribution is shown in the cumulative volume (\%) format with the particle size plotted on a log scale. The cumulative volume (\%) in Figure 2.5 indicates the percentage of the total aerosol volume that lies in sizes lower than the particular diameter (red line). The blue bars indicate the volume frequency (\%) which corresponds to the percentage of particles that lie within the size range. The diameter indicated by this measurement is the equivalent scattering diameter. 


\section{CHAPTER 3: THEORY AND NUMERICAL ANALYSIS}

\subsection{Aerosol Basics}

Derivation of the collision rate function for aerosols is analogous to the kinetic theory derivation for particle collision frequencies in a gas. Consider a particle with radius $a_{i}$ moving towards a particle of radius $a_{j}\left(a_{i}>a_{j}\right)$ having number density $\mathrm{n}_{\mathrm{j}}$ with a relative velocity $\mathrm{U}_{\text {rel }}$. The path swept by the larger particle can be described as an "agglomeration volume" consisting of a cylinder with a radius equal to the sum of the radii of the two particles (Ezekoye and Wibowo, 1999). The agglomeration volume swept per unit time will then be $\pi\left(a_{i}+a_{j}\right)^{2} U_{\text {rel }}$. The number of collisions per unit time per unit volume would then be of the form

$\beta \mathrm{n}_{\mathrm{i}} \mathrm{n}_{\mathrm{j}}=\pi\left(\mathrm{a}_{\mathrm{i}}+\mathrm{a}_{\mathrm{j}}\right)^{2} \mathrm{U}_{\mathrm{rel}} \mathrm{n}_{\mathrm{i}} \mathrm{n}_{\mathrm{j}}$

where $\beta$ is the collision frequency function or the agglomeration kernel and $n_{j}$ is the number density of the particle of type $j$.

\subsection{General Dynamic Equation}

The evolution of the number density of particles in the absence of sedimentation is given by the General Dynamic Equation (Smoluchowski's equation). The number concentration of particles $\left(n_{k}\right)$ with size parameter $k$, is given as (Schmidt, 2000)

$$
\frac{\mathrm{dn}_{\mathrm{k}}}{\mathrm{dt}}=\frac{1}{2} \sum_{\mathrm{i}+\mathrm{j}-\mathrm{k}} \beta\left(\mathrm{m}_{\mathrm{i}}, \mathrm{m}_{\mathrm{j}}\right) \mathrm{n}_{\mathrm{i}} \mathrm{n}_{\mathrm{j}}-\mathrm{n}_{\mathrm{k}} \sum_{\mathrm{i}-1} \beta\left(\mathrm{m}_{\mathrm{i}}, \mathrm{m}_{\mathrm{k}}\right) \mathrm{n}_{\mathrm{i}}-\mathrm{n}_{\mathrm{k}} \mathrm{R}\left(\mathrm{m}_{\mathrm{k}}\right)
$$


The first term represents the rate of conversion of particles of size $i$ and $j$ into size $\mathrm{k}$ such that the sum the volumes of the $\mathrm{i}$ and $\mathrm{j}$ particles sums to the volume of the $\mathrm{k}$ particle. The second term represents the conversion of $\mathrm{k}$ sized particles to larger particles through collisions with the rest of the distribution. The third term represents the removal of $\mathrm{k}$ sized particles due to deposition and leakage. $\beta$ is the collision frequency function (agglomeration kernel) and depends on the mass $\left(\mathrm{m}_{\mathrm{i}}, \mathrm{m}_{\mathrm{j}}, \mathrm{m}_{\mathrm{k}}\right)$ of the colliding particles. Typically, Brownian and gravitational coalescence/agglomeration processes are known to be quite important in modifying the number concentration. In general, the GDE depends on the collision rate of different sized particles, which can be formulated in terms of $\beta$ or in terms of the relative velocity between particles. This relative velocity depends on the Gravitational acceleration and Brownian motion. Zhang (1995) expressed the collision rate as

$\beta \mathrm{n}_{\mathrm{i}} \mathrm{n}_{\mathrm{j}}=\pi\left(\mathrm{a}_{\mathrm{i}}+\mathrm{a}_{\mathrm{j}}\right)^{2} \mathrm{n}_{\mathrm{i}} \mathrm{n}_{\mathrm{j}}\left|\underline{\mathrm{U}}_{\mathrm{i}}^{(0)}-\underline{\mathrm{U}}_{\mathrm{j}}^{(0)}\right| \mathrm{e}_{\mathrm{ij}}$

where $\underline{U_{i}^{(0)}}$ and $\underline{U_{j}^{(0)}}$ are the velocities of particles of size $i$ and $j$ respectively due to gravity alone and $\mathrm{e}_{\mathrm{ij}}$ is the collision efficiency which is defined as a ratio of actual collision rate to that for rectilinear particle motion due to gravity alone. Collision efficiency other than unity represents the effect of electric field induced forces. 


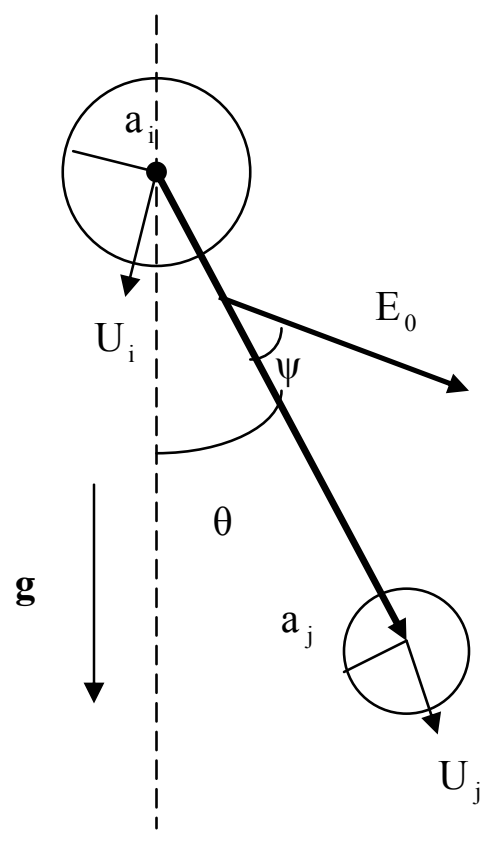

Figure 3.1: Coordinate system used for the relative motion of two different-sized particles induced by gravity and electrical forces (Zhang, 1995).

The relative velocity, $\mathrm{U}_{\text {rel }}$, can be decomposed into components along and perpendicular to the line-of-centers (Zhang, 1995) as

$$
\begin{gathered}
\mathrm{U}_{\text {rel }}=\mathrm{V}_{\mathrm{ij}}^{(0)}\left(-\mathrm{L} \cos \underline{\underline{\mathrm{e}_{\mathrm{r}}}}+\mathrm{M} \sin \theta \underline{\mathrm{e}_{\theta}}\right)-\frac{\mathrm{D}_{\mathrm{ij}}^{(0)}}{\mathrm{kT}}\left(\mathrm{GF}_{\mathrm{E}, \mathrm{ij}}^{\mathrm{r}} \underline{\mathrm{e}_{\mathrm{r}}}-\mathrm{HF}_{\mathrm{E}, \mathrm{ij}} \underline{\mathrm{e}_{\theta}}\right)-\frac{\mathrm{D}_{\mathrm{ij}}^{(0)}}{\mathrm{kT}} \mathrm{GF}_{\mathrm{V}, \mathrm{ij}} \underline{\mathrm{e}_{\mathrm{r}}}(3.4) \\
\text { gravity } \\
\text { electric field }
\end{gathered}
$$

where $\underline{\mathrm{e}_{\mathrm{r}}}$ and $\underline{\mathrm{e}_{\theta}}$ are unit vectors in radial and tangential direction, in a spherical polar coordinate system shown in Figure 3.1, $\mathrm{k}$ is the Boltzmann's constant and $\mathrm{T}$ is the absolute temperature. $\mathrm{F}_{\mathrm{V}, \mathrm{ij}}$ represents the force due to van der Waals forces and is given as (Hamaker, 1937) $\mathrm{F}_{\mathrm{V}, \mathrm{j}}=\nabla \phi_{\mathrm{ij}}$ 
where the potential $\phi_{\mathrm{ij}}$ is given as

$\phi_{\mathrm{ij}}=\frac{\mathrm{A}}{6}\left\{\frac{8 \lambda}{\left(\mathrm{s}^{2}-4\right)(1+\lambda)^{2}}+\frac{8 \lambda}{\mathrm{s}^{2}(1+\lambda)-4(1-\lambda)^{2}}+\ln \left[\frac{\left(\mathrm{s}^{2}-4\right)(1+\lambda)^{2}}{\mathrm{~s}^{2}(1+\lambda)^{2}-4(1-\lambda)^{2}}\right]\right\}$

and $\mathrm{A}$ is the composite Hamaker constant. $\mathrm{D}_{\mathrm{ij}}^{(0)}$ is the relative mobility between two widely spaced particles due to an equal and opposite force, and is given as $\mathrm{D}_{\mathrm{ij}}^{(0)}=\frac{\mathrm{kT}(1+\lambda)}{6 \pi \mu_{0} \mathrm{a}_{\mathrm{i}} \lambda}$

The terms $\mathrm{L}$ and $\mathrm{G}$ are the relative mobility functions in the radial direction, while $\mathrm{M}$ and $\mathrm{H}$ are the relative mobility functions in the tangential direction. The mobility functions are used to describe effects of hydrodynamic interactions between two particles, and they depend on the size ratio of the particles $\left(\lambda=\mathrm{a}_{\mathrm{j}} / \mathrm{a}_{\mathrm{i}}\right)$ and the dimensionless distance between the particles $\mathrm{s}$ $\left(s=2 r /\left(a_{i}+a_{j}\right)\right)$. The analytical expressions for the relative mobility functions are given by Zhang et al (1991) to be of the form

$$
\begin{aligned}
& \mathrm{L}(\mathrm{s})=1-2 \frac{\lambda^{3}-1}{(1+\lambda)\left(\lambda^{2}-1\right) \mathrm{s}}+\mathrm{O}\left(\frac{1}{\mathrm{~s}^{6}}\right) \\
& \mathrm{G}(\mathrm{s})=1-4 \frac{\lambda}{(1+\lambda)^{2} \mathrm{~s}}-16 \frac{\lambda\left(\lambda^{3}+1\right)}{(1+\lambda)^{5} \mathrm{~s}^{4}}+\mathrm{O}\left(\frac{1}{\mathrm{~s}^{6}}\right) \\
& \mathrm{M}(\mathrm{s})=1-\frac{\lambda^{3}-1}{(1+\lambda)\left(\lambda^{2}-1\right) \mathrm{s}}+\mathrm{O}\left(\frac{1}{\mathrm{~s}^{6}}\right) \\
& \mathrm{H}(\mathrm{s})=1-2 \frac{\lambda}{(1+\lambda)^{2} \mathrm{~s}}+\mathrm{O}\left(\frac{1}{\mathrm{~s}^{6}}\right)
\end{aligned}
$$

The terminal velocity of a particle is calculated by balancing the gravitational force acting on a particle with the drag force after accounting for the buoyancy forces (Stoke's law)

$$
\frac{4}{3} \pi \mathrm{a}_{\mathrm{i}}^{3}\left(\rho_{\mathrm{d}}-\rho_{0}\right) \mathrm{g}=6 \pi \mu_{0} \mathrm{U}_{\mathrm{i}}^{(0)} \mathrm{a}_{\mathrm{i}}
$$


where, $\rho_{d}$ and $\rho_{0}$ is the density of the particle and air respectively, $g$ is the gravitational acceleration, $\mu_{0}$ is the viscosity of the air, and $\mathrm{U}_{\mathrm{i}}^{(0)}$ is the terminal velocities of particle of size $\mathrm{i}$. The terminal velocity is now expressed as $\mathrm{U}_{\mathrm{i}}^{(0)}=\frac{2\left(\rho_{\mathrm{d}}-\rho_{0}\right) \mathrm{a}_{\mathrm{i}}^{2} \mathrm{~g}}{9 \mu_{0}}$

From equation 3.13 , we see that the terminal velocity varies as the square of the particle radius. The relative velocity of two particles due to gravity would then be given by

$$
\mathrm{V}_{\mathrm{ij}}^{(0)}=\left|\underline{\mathrm{U}_{\mathrm{i}}^{(0)}}-\underline{\mathrm{U}_{\mathrm{j}}^{(0)}}\right|=\frac{2\left(\rho_{\mathrm{d}}-\rho_{0}\right) \mathrm{a}_{\mathrm{i}}^{2}\left(1-\lambda^{2}\right) \mathrm{g}}{9 \mu_{0}}
$$

\subsection{Electric Field Induced Forces}

Electric field induced forces on two conducting particles with radii of $\mathrm{a}_{\mathrm{i}}$ and $\mathrm{a}_{\mathrm{j}}$ can be decomposed into two components, along and normal to the line of centers of the particles, $\mathrm{F}_{\mathrm{E}, \mathrm{ij}}^{\mathrm{r}}$ and $\mathrm{F}_{\mathrm{E}, \mathrm{ij}}^{\theta}$ respectively,

$\mathrm{F}_{\mathrm{E}, \mathrm{j}}^{\mathrm{r}}=4 \pi \in \mathrm{a}_{\mathrm{j}}^{2} \mathrm{E}_{0}^{2}\left(\mathrm{~F}_{1} \cos ^{2} \psi+\mathrm{F}_{2} \sin ^{2} \psi\right)$

$\mathrm{F}_{\mathrm{E}, \mathrm{ij}}^{\theta}=4 \pi \in \mathrm{a}_{\mathrm{j}}^{2} \mathrm{E}_{0}^{2} \mathrm{~F}_{3} \sin 2 \psi$

where $\mathrm{E}_{0}$ is the magnitude of the external electric field, $\psi$ is the angle between the electric field and the particle center line and $\in$ is the permittivity of the surrounding fluid. The force coefficients, $F_{1}, F_{2}$ and $F_{3}$ are complicated series of expressions that depend on the relative geometry of the two particles i.e. their size ratio and separation. These coefficients are calculated using a bispherical coordinate method (Davis, 1964). 


\subsection{The Bispherical Polar Coordinate System}

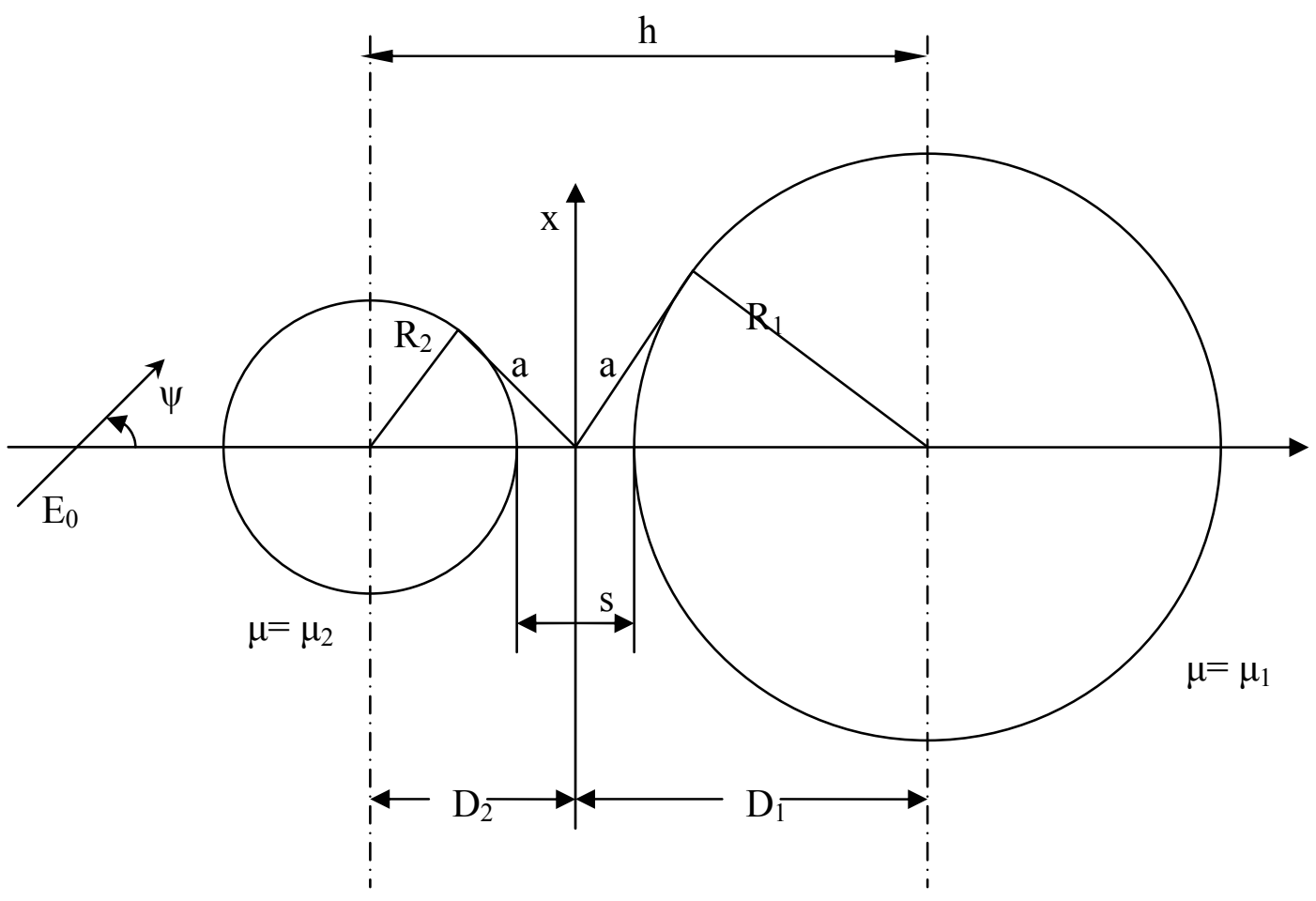

Figure 3.2: The bispherical polar coordinate system (Davis, 1964).

Davis (1964) characterized the two spheres in Figure 3.2 by

$\mu_{1}=\ln \left(\frac{\mathrm{D}_{1}+\mathrm{a}}{\mathrm{R}_{1}}\right)$ and $\mu_{2}=\ln \left(\frac{\mathrm{D}_{2}+\mathrm{a}}{\mathrm{R}_{2}}\right)$

where

a determines the linear scale and is expressed as

$\mathrm{a}=\left(\mathrm{D}_{1}^{2}+\mathrm{R}_{1}^{2}\right)^{1 / 2}=\left(\mathrm{D}_{2}^{2}+\mathrm{R}_{2}^{2}\right)^{1 / 2}$ 
with $R_{1}$ and $R_{2}$ as the radii of the two spheres. $D_{1}$ and $D_{2}$ are distances of the centers of the particles from the origin and are given by

$$
\begin{aligned}
& \mathrm{D}_{1}=\frac{1}{2 \mathrm{~h}}\left(\mathrm{~h}^{2}+\mathrm{R}_{1}^{2}-\mathrm{R}_{2}^{2}\right) \\
& \mathrm{D}_{2}=\frac{1}{2 \mathrm{~h}}\left(\mathrm{~h}^{2}+\mathrm{R}_{2}^{2}-\mathrm{R}_{1}^{2}\right)
\end{aligned}
$$

with $\mathrm{h}$ as the distance between the centers

$$
\mathrm{h}=\mathrm{R}_{1}+\mathrm{R}_{2}+\mathrm{s}=\mathrm{D}_{1}+\mathrm{D}_{2}
$$

\subsection{Calculating the Electric Induced Forces}

Davis (1964) calculated the force coefficients by first deriving a potential function which matches the boundary conditions. The boundary conditions are, (i) at great distances from the spheres, the potential function must correspond to that of the electrical field and (ii) the two spheres carry no net charge. The force acting on a sphere was then computed by integrating the electrical stress on the sphere surface.

The force coefficients, thus calculated are expressed as sums of series as

$$
\mathrm{F}_{\mathrm{j}}=\mathrm{\kappa}_{\mathrm{j}} \sum_{\mathrm{k}=1}^{24} \mathrm{~K}_{\mathrm{jk}} \mathrm{f}_{\mathrm{k}} \quad \mathrm{j}=1,2,3
$$

where

$f_{k} \quad$ are series of type $T_{m}$ or $U_{m}$

$\mathrm{K}_{\mathrm{jk}} \quad$ are relatively simple polynomial expressions

$\kappa_{\mathrm{j}} \quad$ are simple factors.

The series $\mathrm{T}_{\mathrm{m}}$ and $\mathrm{U}_{\mathrm{m}}$ are expressed as 


$$
\begin{aligned}
T_{m}(\xi) & =\sum_{n=0}^{\infty} \frac{(2 \mathrm{n}+1)^{m} e^{(2 n+1) \xi}}{\left(e^{(2 n+1)\left(\mu_{1}+\mu_{12}\right)}-1\right)^{2}} \text { with } m=0,1,2,3 . \\
U_{m}(\xi) & =\sum_{n=0}^{\infty} \frac{(2 n+1)^{m} e^{(2 n+1) \xi}}{\left(e^{(2 n+1)\left(\mu_{1}+\mu_{2}\right)}-1\right)\left(e^{(2 n+3)\left(\mu_{1}+\mu_{2}\right)}-1\right)} \text { with } m=0,1,2,3 .
\end{aligned}
$$

The expressions for $\kappa_{\mathrm{j}}, \mathrm{K}_{\mathrm{jk}}$ and $\mathrm{f}_{\mathrm{k}}$ are given in Tables 3.1, 3.2 and 3.3 respectively.

Table 3.1: Expressions for $\kappa_{\mathrm{j}}$

\begin{tabular}{|c|c|}
\hline $\mathbf{j}$ & $\mathbf{\kappa}_{\mathbf{j}}$ \\
\hline 1 & $\left(\frac{\mathrm{a}}{\mathrm{R}_{2}}\right)^{2}$ \\
\hline 2 & $\frac{1}{2}\left(\frac{\mathrm{a}}{\mathrm{R}_{2}}\right)^{2}$ \\
\hline 3 & $\frac{1}{8}\left(\frac{\mathrm{a}}{\mathrm{R}_{2}}\right)^{2}\left(\mathrm{e}^{2 \mu_{2}}-1\right)$ \\
\hline
\end{tabular}

Table 3.2: Expressions for $\mathrm{f}_{\mathrm{k}}$

\begin{tabular}{|c|c|}
\hline $\mathbf{k}$ & $\mathbf{f}_{\mathbf{k}}$ \\
\hline 1 & $\mathrm{~T}_{0}\left(2 \mu_{1}+\mu_{2}\right)$ \\
\hline 2 & $\mathrm{~T}_{1}\left(2 \mu_{1}+\mu_{2}\right)$ \\
\hline 3 & $\mathrm{~T}_{2}\left(2 \mu_{1}+\mu_{2}\right)$ \\
\hline 4 & $\mathrm{~T}_{3}\left(2 \mu_{1}+\mu_{2}\right)$ \\
\hline 5 & $\mathrm{~T}_{0}\left(\mu_{1}+\mu_{2}\right)$ \\
\hline 6 & $\mathrm{~T}_{1}\left(\mu_{1}+\mu_{2}\right)$ \\
\hline 7 & $\mathrm{~T}_{2}\left(\mu_{1}+\mu_{2}\right)$ \\
\hline 8 & $\mathrm{~T}_{3}\left(\mu_{1}+\mu_{2}\right)$ \\
\hline 9 & $\mathrm{~T}_{0}\left(\mu_{2}\right)$ \\
\hline
\end{tabular}




\begin{tabular}{|c|c|}
\hline 10 & $\mathrm{~T}_{1}\left(\mu_{2}\right)$ \\
\hline 11 & $\mathrm{~T}_{2}\left(\mu_{2}\right)$ \\
\hline 12 & $\mathrm{~T}_{3}\left(\mu_{2}\right)$ \\
\hline 13 & $\mathrm{U}_{0}\left(2 \mu_{1}+\mu_{2}\right)$ \\
\hline 14 & $\mathrm{U}_{1}\left(2 \mu_{1}+\mu_{2}\right)$ \\
\hline 15 & $\mathrm{U}_{2}\left(2 \mu_{1}+\mu_{2}\right)$ \\
\hline 16 & $\mathrm{U}_{3}\left(2 \mu_{1}+\mu_{2}\right)$ \\
\hline 17 & $\mathrm{U}_{0}\left(\mu_{1}+\mu_{2}\right)$ \\
\hline 18 & $\mathrm{U}_{1}\left(\mu_{1}+\mu_{2}\right)$ \\
\hline 19 & $\mathrm{U}_{2}\left(\mu_{1}+\mu_{2}\right)$ \\
\hline 20 & $\mathrm{U}_{3}\left(\mu_{1}+\mu_{2}\right)$ \\
\hline 21 & $\mathrm{U}_{0}\left(\mu_{2}\right)$ \\
\hline 22 & $\mathrm{U}_{1}\left(\mu_{2}\right)$ \\
\hline 23 & $\mathrm{U}_{2}\left(\mu_{2}\right)$ \\
\hline 24 & $\mathrm{U}_{3}\left(\mu_{2}\right) \mathrm{s}$ \\
\hline
\end{tabular}

Table 3.3: Expressions for $\mathrm{K}_{\mathrm{jk}}$

\begin{tabular}{|c|c|}
\hline $\mathbf{k}$ & $\mathbf{K}_{\mathbf{1 k}}(\mathbf{j}=\mathbf{1})$ \\
\hline 1 & 0 \\
\hline 2 & $\mathrm{v}_{2}^{2}$ \\
\hline 3 & $-2 \mathrm{v}_{2}$ \\
\hline 4 & 1 \\
\hline 5 & 0 \\
\hline 6 & $-2 \mathrm{v}_{1} \mathrm{v}_{2}$ \\
\hline 7 & $2\left(\mathrm{v}_{1}-\mathrm{v}_{2}\right)$ \\
\hline 8 & 2 \\
\hline 9 & 0 \\
\hline 10 & $\mathrm{v}_{1}^{2}$ \\
\hline 11 & $2 \mathrm{v}_{1}$ \\
\hline
\end{tabular}




\begin{tabular}{|l|c|}
\hline 12 & 1 \\
\hline 13 & $-\gamma \alpha \mathrm{v}_{2}\left(\mathrm{v}_{2}-2\right)$ \\
\hline 14 & $-\gamma \alpha\left(\mathrm{v}_{2}^{2}-4 \mathrm{v}_{2}+2\right)$ \\
\hline 15 & $-\gamma \alpha\left(-2 \mathrm{v}_{2}+3\right)$ \\
\hline 16 & $-\gamma \alpha$ \\
\hline 17 & $-\gamma\left(2 \mathrm{v}_{1} \alpha-\mathrm{v}_{1} \mathrm{v}_{2}(\alpha+1)-2 \mathrm{v}_{2}\right)$ \\
\hline 18 & $-\gamma\left[\mathrm{v}_{1}(3 \alpha+1)-\mathrm{v}_{2}(\alpha+3)+\left(2-\mathrm{v}_{1} \mathrm{v}_{2}\right)(\alpha+1)\right]$ \\
\hline 19 & $-\gamma(\alpha+1)\left(\mathrm{v}_{1}-\mathrm{v}_{2}+3\right)$ \\
\hline 20 & $-\gamma(\alpha+1)$ \\
\hline 21 & $-\gamma \mathrm{v}_{1}\left(\mathrm{v}_{1}+2\right)$ \\
\hline 22 & $-\gamma\left(\mathrm{v}_{1}^{2}+4 \mathrm{v}_{1}+2\right)$ \\
\hline 23 & $-\gamma\left(2 \mathrm{v}_{1}+3\right)$ \\
\hline 24 & $-\gamma$ \\
\hline
\end{tabular}

Table 3.3 (contd.)

\begin{tabular}{|c|c|c|}
\hline $\mathbf{k}$ & $\mathbf{K}_{\mathbf{2} \mathbf{j}}(\mathbf{j}=\mathbf{2})$ & $\mathbf{K}_{\mathbf{3 k}}(\mathbf{j}=\mathbf{3})$ \\
\hline 1 & 0 & 0 \\
\hline 2 & -1 & 0 \\
\hline 3 & 0 & 0 \\
\hline 4 & 1 & 0 \\
\hline 5 & 0 & 0 \\
\hline 6 & 2 & 0 \\
\hline 7 & 0 & 0 \\
\hline 8 & -2 & 0 \\
\hline 9 & 0 & 0 \\
\hline 10 & -1 & 0 \\
\hline
\end{tabular}




\begin{tabular}{|c|c|c|}
\hline 11 & 0 & 0 \\
\hline 12 & 1 & $2 \alpha\left(2 \mathrm{v}_{2}-1\right)$ \\
\hline 13 & $3 \gamma \alpha$ & $4 \alpha\left(\mathrm{v}_{2}-1\right)$ \\
\hline 14 & $\gamma \alpha$ & $-2 \alpha$ \\
\hline 15 & $-3 \gamma \alpha$ & 0 \\
\hline 16 & $-\gamma \alpha$ & $\left(\mathrm{v}_{1}-\mathrm{v}_{2}+6\right)(1-\alpha)$ \\
\hline 17 & $-3 \gamma(\alpha+1)$ & $2(1-\alpha)$ \\
\hline 18 & $-\gamma(\alpha+1)$ & $2\left(2 \mathrm{v}_{1}+1\right)$ \\
\hline 19 & $3 \gamma(\alpha+1)$ & $4\left(\mathrm{v}_{1}+1\right)$ \\
\hline 20 & $\gamma(\alpha+1)$ & 2 \\
\hline 21 & $3 \gamma$ & 0 \\
\hline 22 & $\gamma$ & \\
\hline 23 & $-3 \gamma$ & $\mathrm{v}_{2}(3+\alpha)-2(1-\alpha)$ \\
\hline 24 & $-\gamma$ & $\left.\mathrm{v}_{2}\right)+2(1-\alpha)$ \\
\hline
\end{tabular}

where

$$
\begin{aligned}
& \alpha=\mathrm{e}^{2 \mu_{1}} \\
& \gamma=\frac{1}{2}\left(\mathrm{e}^{2 \mu_{2}}+1\right)
\end{aligned}
$$

The terms $\mathrm{v}_{1}$ and $\mathrm{v}_{2}$ in Table 3.3 represent the potentials and are given by

$$
\begin{aligned}
& \mathrm{v}_{1}=-\left(\mathrm{P}_{11} \mathrm{Q}_{1}^{*}+\mathrm{P}_{12} \mathrm{Q}_{2}^{*}\right) \\
& \mathrm{v}_{2}=-\left(\mathrm{P}_{12} \mathrm{Q}_{1}^{*}+\mathrm{P}_{22} \mathrm{Q}_{2}^{*}\right)
\end{aligned}
$$


where the charge induced by the electric field of strength $E_{0}$ on two grounded spheres is expressed as

$$
\begin{aligned}
& \mathrm{Q}_{1}^{*}=2 \in \mathrm{E}_{0} \mathrm{a}^{2} \cos \psi\left[\mathrm{S}_{1}\left(\mu_{2}\right)+\mathrm{S}_{1}(0)\right] \\
& \mathrm{Q}_{2}^{*}=2 \in \mathrm{E}_{0} \mathrm{a}^{2} \cos \psi\left[\mathrm{S}_{1}\left(\mu_{1}\right)+\mathrm{S}_{1}(0)\right]
\end{aligned}
$$

Here, the series $\mathrm{S}_{\mathrm{m}}$ is defined as

$$
\mathrm{S}_{\mathrm{m}}(\xi)=\sum_{\mathrm{n}=0}^{\infty} \frac{(2 \mathrm{n}+1)^{\mathrm{m}} \mathrm{e}^{(2 \mathrm{n}+1) \xi}}{\mathrm{e}^{(2 \mathrm{n}+1)\left(\mu_{1}+\mu_{2}\right)}-1} \text { with } \mathrm{m}=0,1,2,3 \text {. }
$$

The expressions for $\mathrm{P}_{11}, \mathrm{P}_{12}$ and $\mathrm{P}_{22}$ in equation 3.22 and 3.23 are

$$
\begin{aligned}
& \mathrm{P}_{11}=\mathrm{C}_{22} / \Delta \\
& \mathrm{P}_{12}=-\mathrm{C}_{12} / \Delta \\
& \mathrm{P}_{22}=\mathrm{C}_{11} / \Delta
\end{aligned}
$$

where $\Delta=\mathrm{C}_{11} \mathrm{C}_{22}-\mathrm{C}_{12}^{2}$.

$\mathrm{C}_{11}, \mathrm{C}_{12}$ and $\mathrm{C}_{22}$ are the coefficients of capacitance and are given by the expressions

$$
\begin{aligned}
& \mathrm{C}_{11}=2 \in \mathrm{aS}_{0}\left(\mu_{2}\right) \\
& \mathrm{C}_{12}=-2 \in \mathrm{aS}_{0}(0) \\
& \mathrm{C}_{22}=2 \in \mathrm{aS}_{0}\left(\mu_{1}\right)
\end{aligned}
$$

The above expressions were coded into a computer program called 'force.c' (attached as Appendix A). The convergence criterion used in the calculation of all three summation series $-T_{m}, U_{m}$ and $S_{m}$ was $\left|S_{n+1}-S_{n}\right| / S_{n} \leq 10^{-7}$, where $S_{n}$ is the nth partial sum of the series (Zhang, 1995).

The input for the program is the size ratio and the separation. The output from the program are the force coefficients $F_{1}, F_{2}$ and $F_{3}$. 
For a given electric field, the induced electrostatic force between two conducting particles is known approximately to be inversely proportional to the fourth power of the separation distance (Waterman, 1965). The electric induced forces increase as the square of the particle size, implying that the electric field would have a greater effect on larger particles (Zhang, 1995).

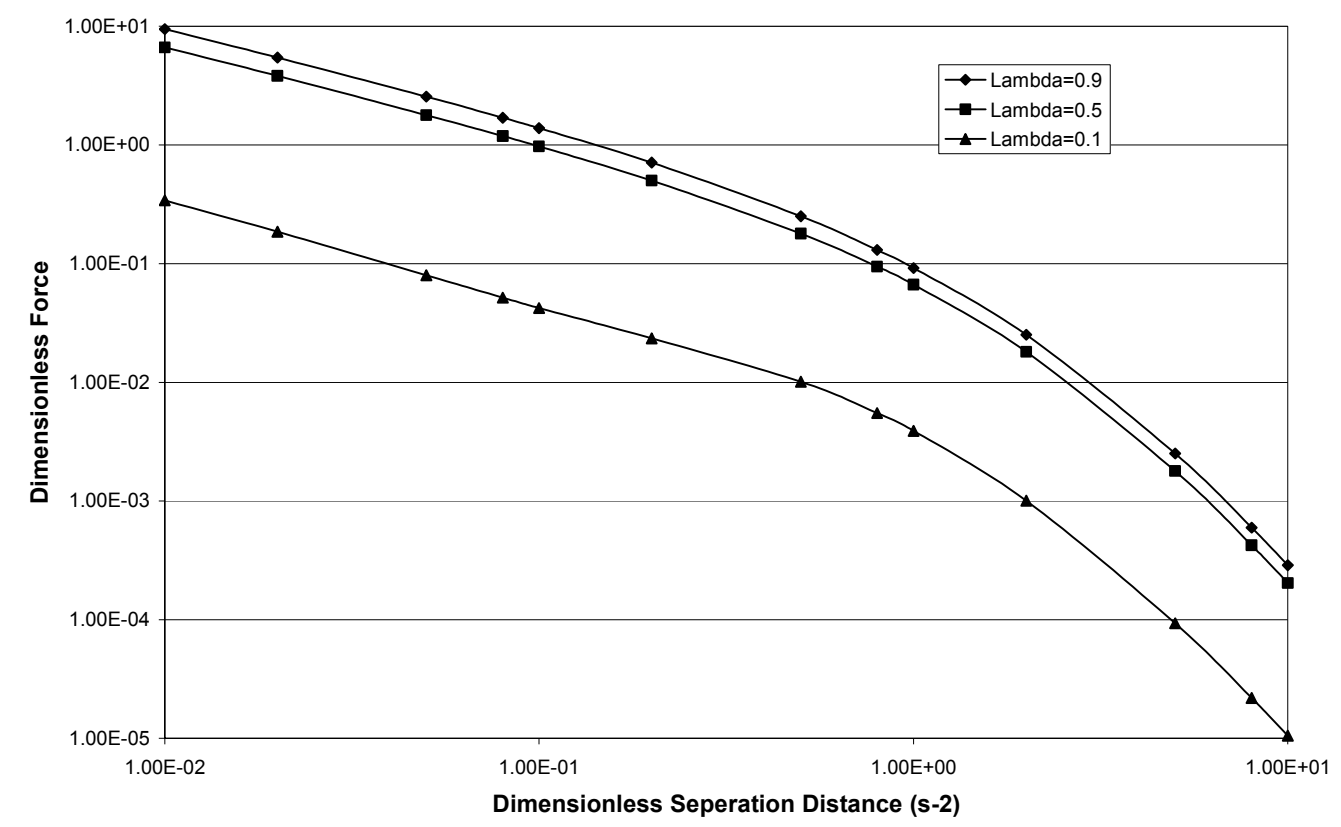

Figure 3.3: Plot showing the variation of the induced force with the separation distance.

Figure 3.3 is a plot of the electric field induced forces (made nondimensional by dividing by $\left.4 \pi \in\left[\left(a_{i}+a_{j}\right) / 2\right]^{2} E_{0}^{2}\right)$ versus the dimensionless distance for different size ratios $(\lambda)$ and $\psi=0$. The dimensionless distance is taken as $\mathrm{s}-2$ where $\mathrm{s}=2 \mathrm{r} /\left(\mathrm{a}_{\mathrm{i}}+\mathrm{a}_{\mathrm{j}}\right)$ and $\mathrm{r}$ is the dimensional distance between the 
particles. The particles would be touching each other when $r=a_{i}+a_{j}$ (i.e. $s=2$ ). Hence, the dimensionless distance is taken as $\mathrm{s}-2$.
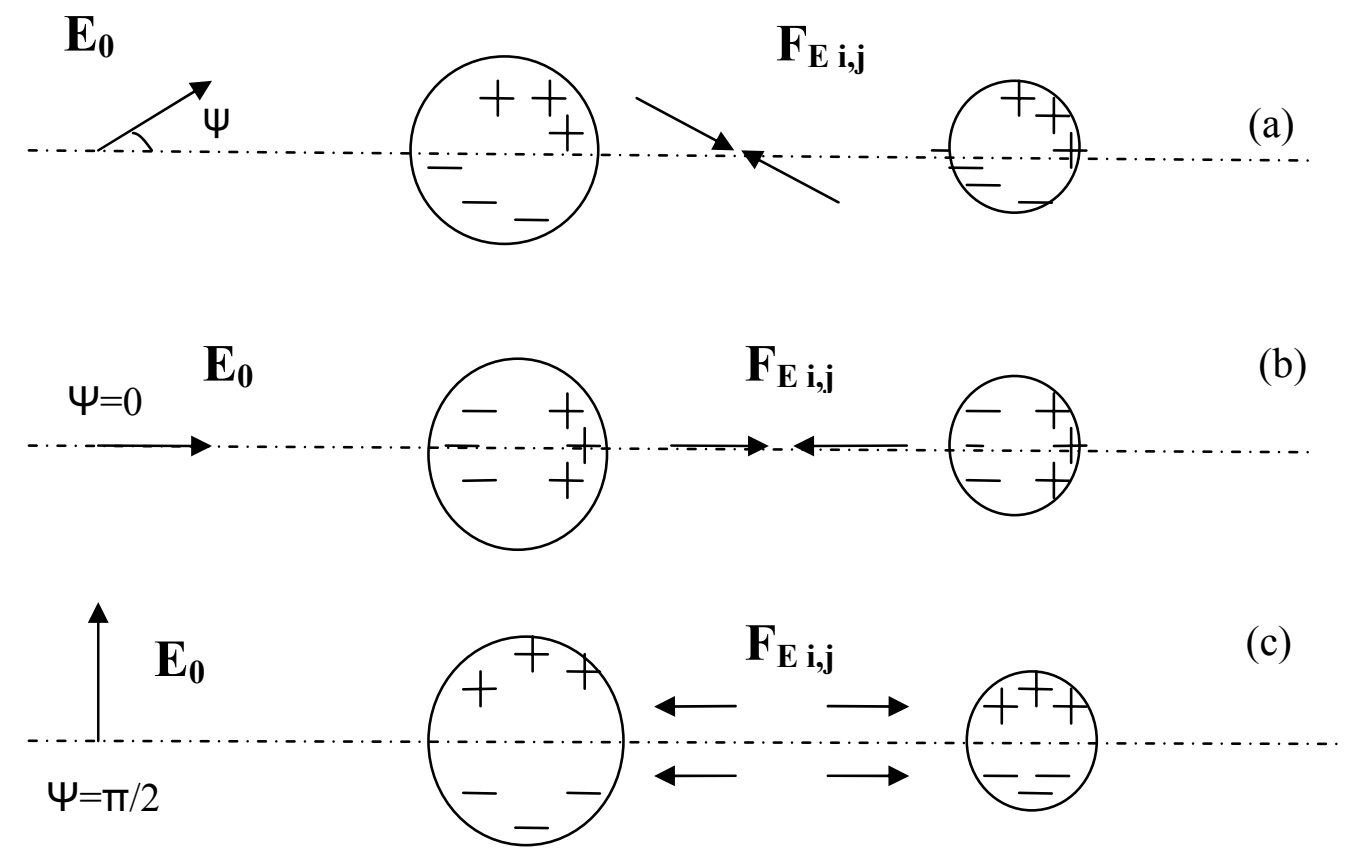

Figure 3.4: Surface densities of charge and forces on two particles induced by an externally applied electric field for different values of angle $\psi$ between the electric field and the line joining the centers of the two particles. (Zhang, 1995)

The magnitude and direction of the electric field induced forces between two particles also depends on the orientation of the electric field with respect to the line-of centers of the two particles. This is elicited in Figure 3.4. Case a and b, where the angle $\psi$ is less than $\pi / 2$, the induced forces are attractive. However, at angle of $\pi / 2$, the forces become repulsive. 
Figure 3.5 shows the variation of the dimensionless electric field induced forces along the line of centers $\left(\mathrm{F}_{\mathrm{E}, \mathrm{ij}}^{\mathrm{r}}\right)$ with the orientation of the electric field $(\psi)$ for different separation distances. The force is maximum at $\psi=0$ and decreases with increasing $\psi$.

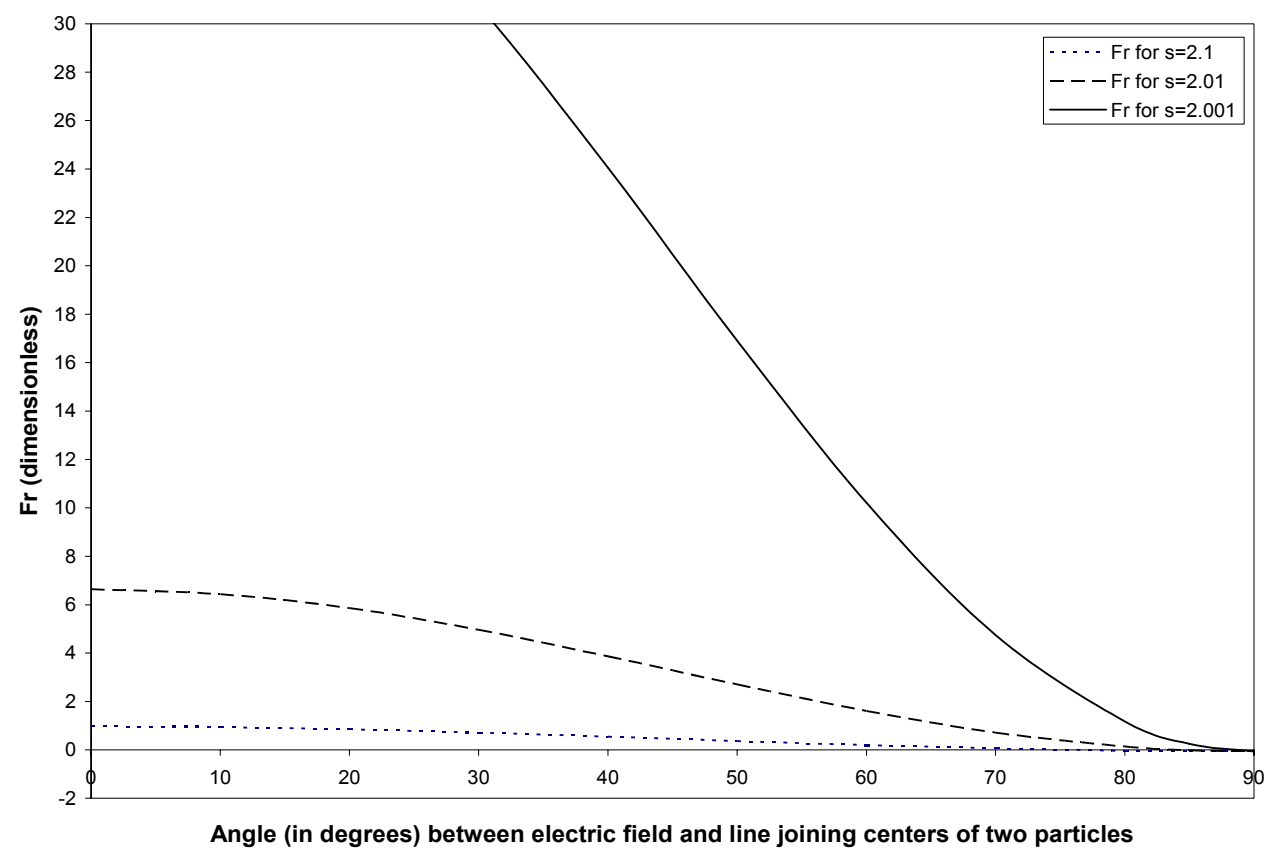

Figure 3.5: Variation of the dimensionless radial force with different orientations. 


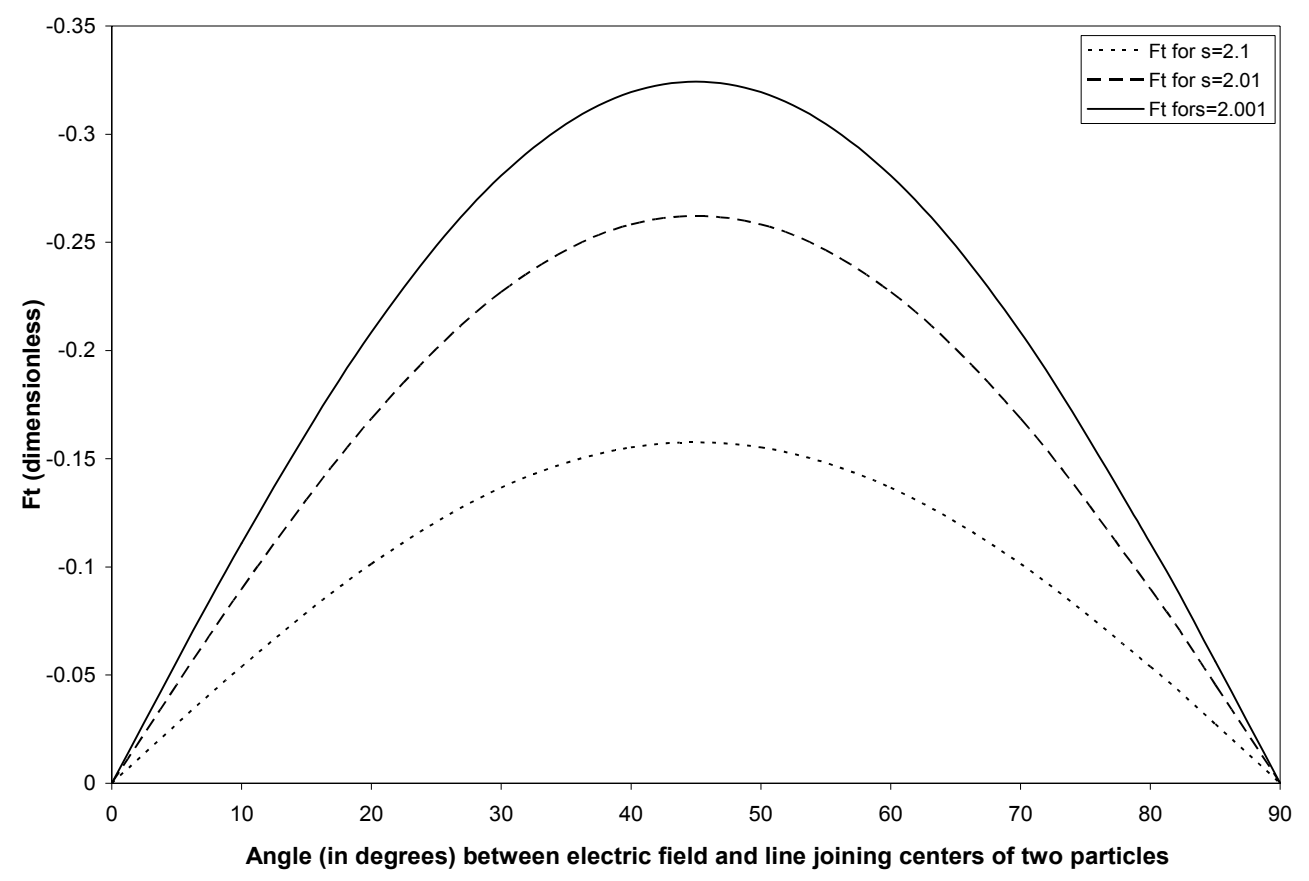

Figure 3.6: Variation of the dimensionless tangential force with different orientations.

Figure 3.6 is a plot of the dimensionless electric field induced forces normal to the line of centers versus the orientation of the electric field $(\psi)$ for different separation distances. These forces are much weaker as compared to the forces along the line of centers. The forces tend to 0 at $\psi=0$ and $\psi=\pi / 2$, and attains a maximum at $\psi=\pi / 4$.

The plots obtained in figures 3.3, 3.5 and 3.6 match the plots obtained by Zhang (1995). 


\subsection{Trajectory Theory}

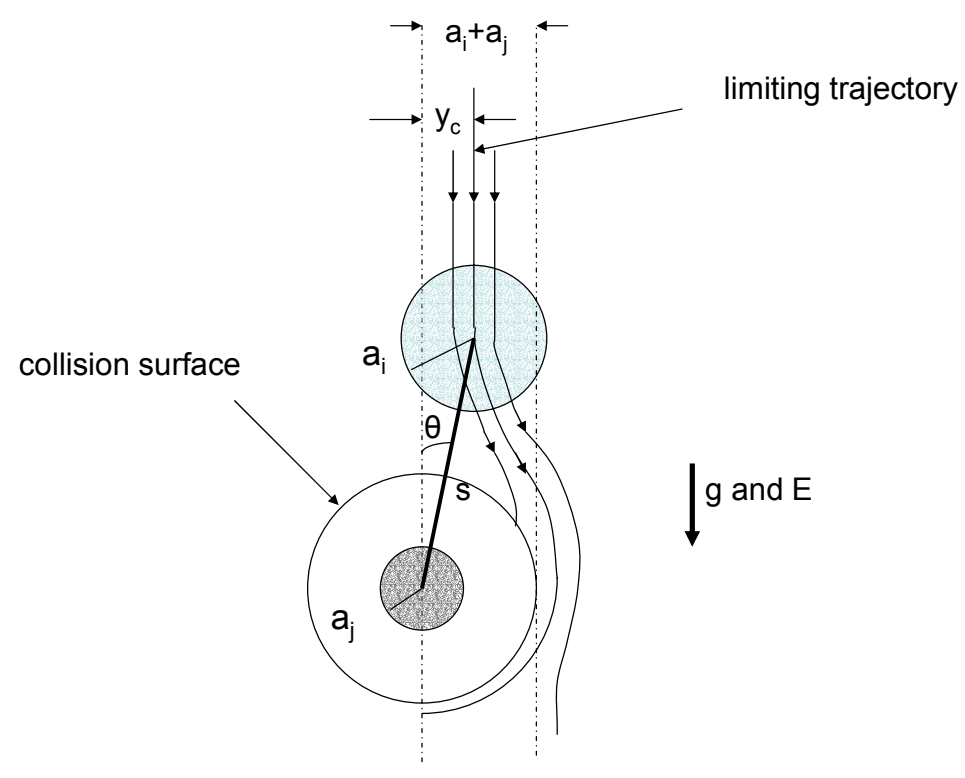

Figure 3.7: Schematic of the coordinate system and relative trajectories of two particles under effect of gravity and electric field (Wang, 1997).

For calculating the collision efficiency $\mathrm{e}_{\mathrm{ij}}$ (in the expression for collision rate in equation 3.3), a trajectory analysis was used.

Since the settling/terminal velocity (equation 3.13) depends on the square of the radius of the particles, the larger particles have larger settling velocities. This would mean that larger particles would catch-up with smaller particles as the aerosol settles. This idea is the basis of the trajectory analysis. Figure 3.7 shows a larger particle approaching a smaller particle. The two particles would collide if the larger particle had a horizontal displacement, from the vertical axis of symmetry, equal to or less than $\mathrm{y}_{\mathrm{c}}$, the critical displacement. In effect, the 
particle would have to lie within a cylinder of radius $y_{c}$ around the axis of symmetry for the particles to collide. Particles lying outside this cylinder would move away without colliding. In the case with the electrical field in the same direction as gravity, as the larger particle approaches the smaller particle, there is an attractive force between the particles. When the two particles come side by side, this force becomes repulsive (Figure 3.4 case c). Once the particles start moving away, the force again becomes attractive. As a result, the particle traveling along the limiting trajectory should collide with the smaller particle at $\theta=\pi$. Zhang (1995) expressed the collision efficiency as

$\mathrm{e}_{\mathrm{ij}}=\frac{\mathrm{y}_{\mathrm{c}}{ }^{2}}{\left(\mathrm{a}_{\mathrm{i}}+\mathrm{a}_{\mathrm{j}}\right)^{2}}$

Thus, to calculate the collision efficiency, one would need to determine $\mathrm{y}_{\mathrm{c}}$.

\subsection{Trajectory Equation}

The relative velocity equation (i.e. equation 3.4) is made non-dimensional by dividing throughout by $V_{i j}^{(0)}$. A ratio of the radial component to the tangential component gives the trajectory equation.

$$
\frac{\mathrm{ds}}{\mathrm{d} \theta}=\mathrm{s} \frac{-\mathrm{L} \cos \theta-\frac{\mathrm{G}}{\mathrm{Q}_{\mathrm{E}, \mathrm{j}}}\left(\frac{2 \lambda}{1+\lambda}\right)^{2}\left(\mathrm{~F}_{1} \cos ^{2} \psi+\mathrm{F}_{2} \sin ^{2} \psi\right)-\frac{\mathrm{G}}{\mathrm{Q}_{\mathrm{V}, \mathrm{ij}}} \frac{\mathrm{a}_{\mathrm{i}}+\mathrm{a}_{\mathrm{j}}}{2} \nabla\left(\frac{\phi_{\mathrm{ij}}}{\mathrm{A}}\right)}{\mathrm{M} \sin \theta+\frac{\mathrm{H}}{\mathrm{Q}_{\mathrm{E}, \mathrm{ij}}}\left(\frac{2 \lambda}{1+\lambda}\right)^{2} \mathrm{~F}_{3} \sin 2 \psi}
$$


The terms $Q_{E, i j}$ and $Q_{V, i j}$ are the ratio of the gravitational forces to the electric field induced forces and the van der Waals forces respectively. These are expressed as

$$
\begin{aligned}
Q_{E, i j} & =\frac{V_{i j}^{(0)}}{\frac{D_{i j}^{(0)}}{k T} 4 \pi \in\left(\frac{a_{i}+a_{j}}{2}\right)^{2} E_{0}^{2}}=\frac{4\left|\rho_{d}-\rho_{a i r}\right| \lambda(1-\lambda) g}{3 \in(1+\lambda)^{2}} \frac{a_{i}}{E_{0}^{2}} \\
Q_{V, i j} & =\frac{V_{i j}^{(0)} \frac{a_{i}+a_{j}}{2}}{\frac{D_{i j}^{(0)}}{k T} A}=\frac{2 \pi \lambda\left(1+\lambda^{2}\right)\left|\rho_{d}-\rho_{0}\right| g}{3 A} a_{i}^{4}
\end{aligned}
$$

From equations 3.41 and 3.42 , it can be seen that for the term $Q_{E, i j}$ to significantly affect the trajectory, the electric field strength should be large and the particle sizes should be small.

\subsection{Calculating $\mathbf{y}_{\mathrm{c}}$ and $\mathrm{e}_{\mathrm{ij}}$}

\subsubsection{Calculating $\theta_{c}$}

For calculating $\mathrm{y}_{\mathrm{c}}$, the trajectory equation (equation 3.40 ) has to be numerically integrated. The integration was done using the 'ode23s' routine in MATLAB. This routine is based on a modified Rosenbrock formula of order 2 which is useful in solving stiff problems.

In equation 3.6, the mobility functions $\mathrm{L}, \mathrm{M}, \mathrm{G}$ and $\mathrm{H}$ as well as the force components $F_{1}, F_{2}$ and $F_{3}$ depend on size ratio $\lambda$. The term $Q_{E, i j}$, depends on the electric field strength, the size ratio and also, the larger particle radius. As a result, the trajectory depends on the size ratio $\lambda$, larger particle radius $a_{i}$ and the electric 
field strength $E_{0}$. The trajectories were calculated for different combinations of $\lambda$ and $a_{i}$ for three values of $E_{0}-25 \mathrm{kV} / \mathrm{m}, 50 \mathrm{kV} / \mathrm{m}$ and $75 \mathrm{kV} / \mathrm{m}$. These values correspond to the electric field strengths acting across the $2 \mathrm{~cm}$ gap of the experimental cell for potentials of $0.5 \mathrm{kV}, 1 \mathrm{kV}$ and $1.5 \mathrm{kV}$ respectively.

For the integration, the range for $\theta$ has to be specified along with the corresponding initial value of the dimensionless separation distance $s$. The values used for the theta range were from $\theta_{\text {ini }}$ to $\pi$ and, the initial separation distance $\mathrm{s}$ was set to 70 , since for this separation all the force components $\left(F_{1}, F_{2}\right.$ and $\left.F_{3}\right)$ reduced to zero. The mobility functions - L, G, M and $\mathrm{H}$ were calculated using equations 3.8, 3.9, 3.10 and 3.11 respectively. The force components $F_{1}, F_{2}$ and $\mathrm{F}_{3}$, were calculated using an interpolation function. For the interpolation, a grid of the dimensionless separation distance $\mathrm{s}$ and the corresponding force components was prepared using the computer program 'force.c'. Also in equation 3.36, $\psi$ becomes the same as $\theta$, since the electric field and the gravitational acceleration are acting in the same direction. For the calculations the value for $\mathrm{kT} / \mathrm{A}$ is taken as 0.1 (Davis, 1984).

The values for $\theta_{\text {ini }}$ were varied and the trajectories calculated to find the maximum initial angle of separation $\theta_{c}$ for which the 2 particles collide. The angle $\theta_{\text {ini }}$ was varied in steps of 0.0001 radians $\left(5.7296^{\circ}\right)$ to find $\theta_{\mathrm{c}}$. This method gives a variation of $1.04 \%$ in the calculation of the collision efficiency $\mathrm{e}_{\mathrm{ij}}$ for the case explained in Figures 3.8, 3.9 and 3.10. Figures 3.8, 3.9 and 3.10 are plots of $\mathrm{s}$ versus $\theta$, where the electric field strength was $25 \mathrm{kV} / \mathrm{m}$, the size ratio $\lambda$ was 0.3 and the large particle radius $\mathrm{a}_{\mathrm{i}}$ was 1.0107 microns. Figure 3.8 is the plot where 
$\theta_{\text {ini }}=\theta_{\text {c }}$ (i.e. 0.0193 radians). The particles collide at an angle close to $\pi$ radians. Figure 3.9 illustrates the case where $\theta_{\text {ini }}<\theta_{c}$ (i.e. $\theta_{\text {ini }}=0.0192$ radians). The particles collide at and angle much before $\pi$ radians. Figure 3.10 is the plot where $\theta_{\text {ini }}>\theta_{\text {c }}$ (i.e. $\theta_{\text {ini }}=0.0194$ radians). Here, the particles come close, but then move away and never collide.

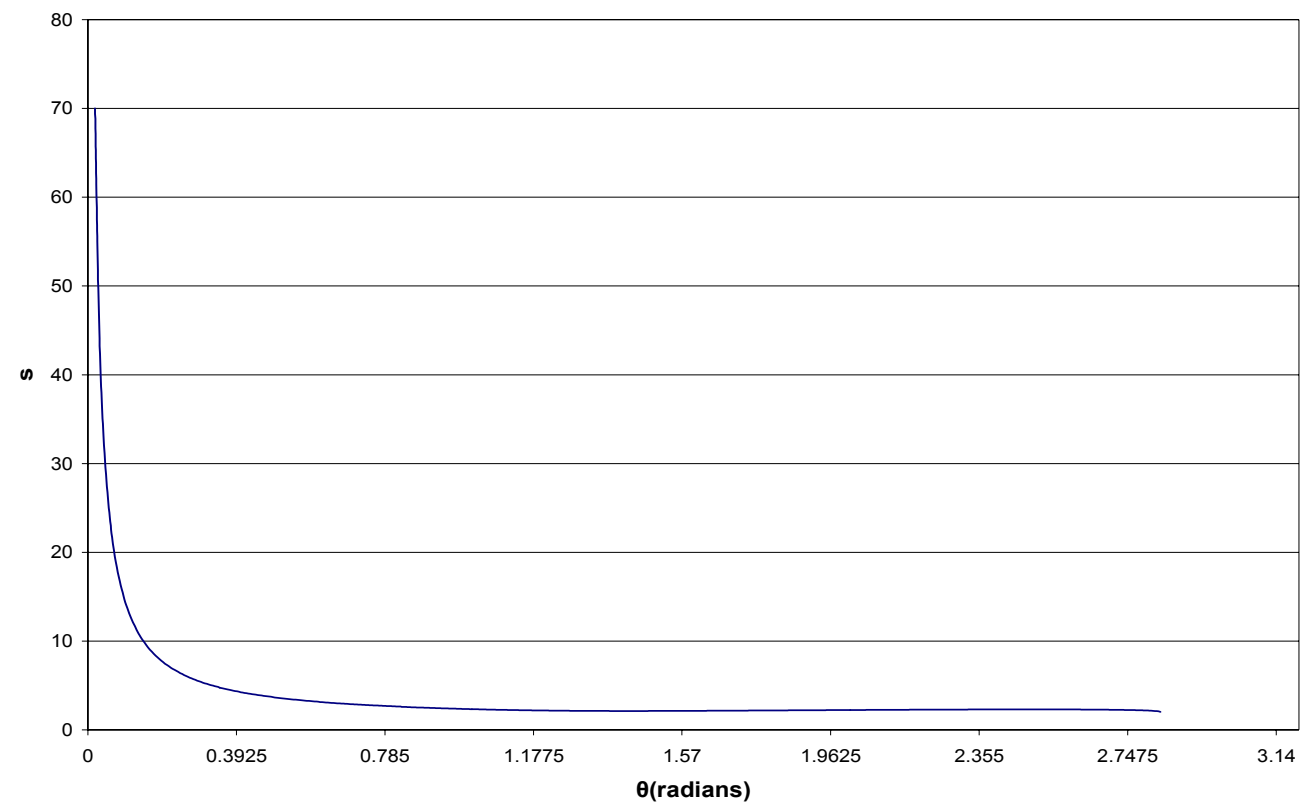

Figure 3.8: Case where $\theta_{\text {ini }}=\theta_{\mathrm{c}}$. 


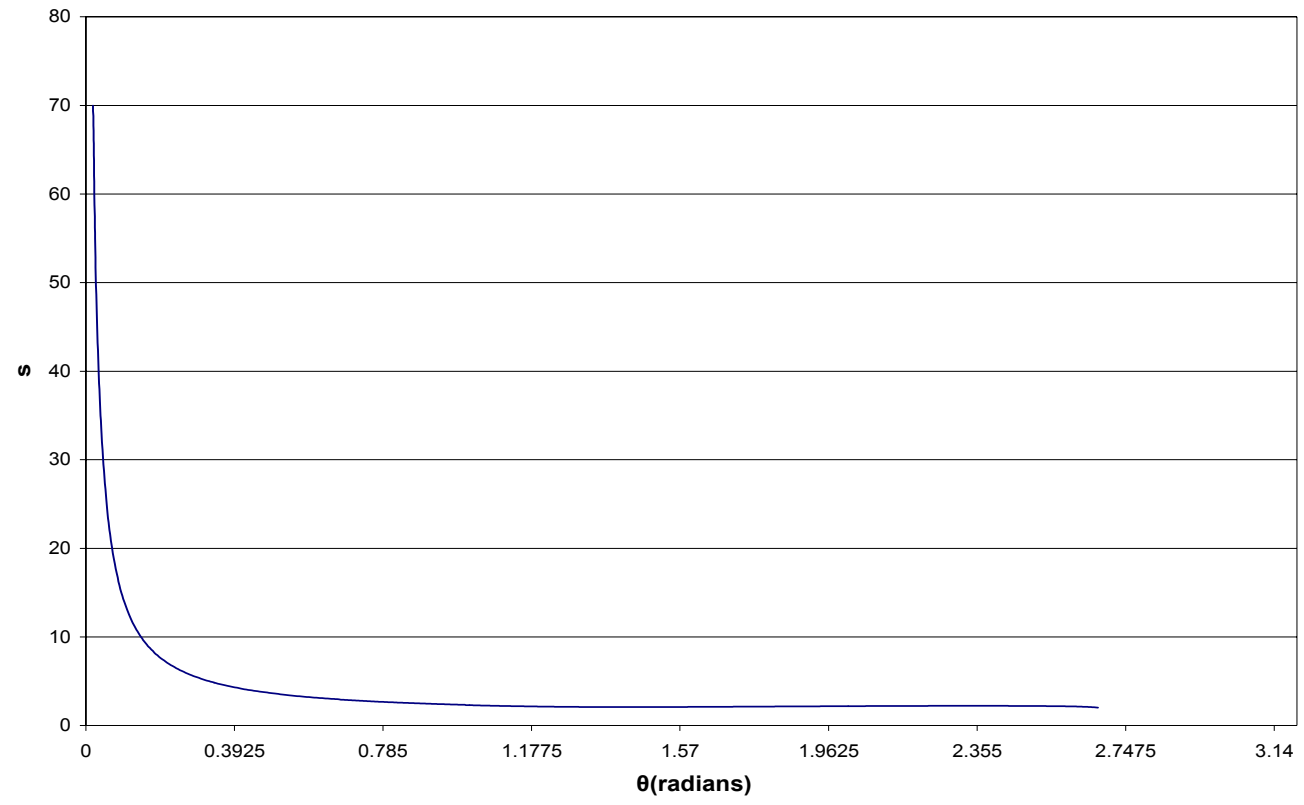

Figure 3.9: Case where $\theta_{\text {ini }}<\theta_{\mathrm{c}}$.

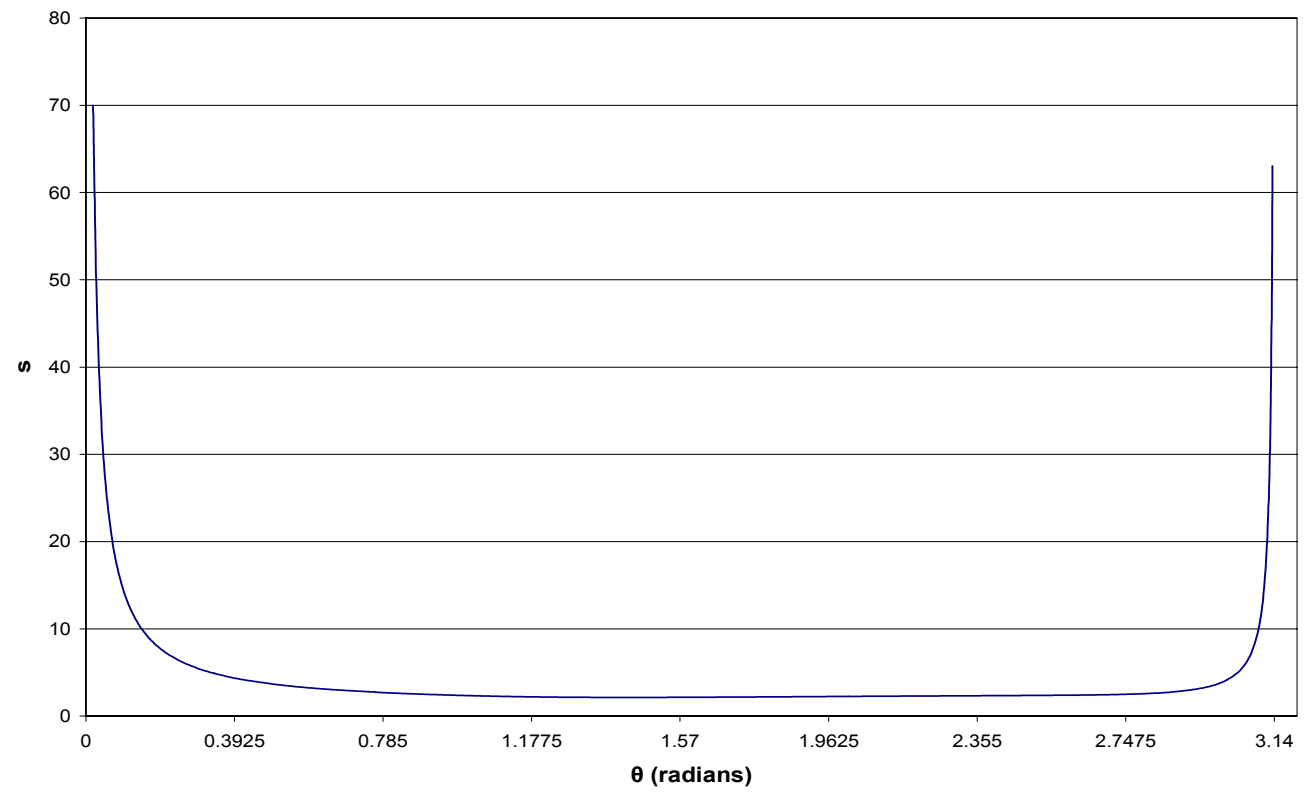

Figure 3.10: Case where $\theta_{\text {ini }}>\theta_{\mathrm{c}}$. 
To examine the significance of the van der Waals forces on the collision efficiencies, calculations were done to compare the collision efficiencies with and without the van der Waals force term in the trajectory equation. The values for the collision efficiencies for the two cases did not differ. The magnitudes of the components due to van der Waals forces and electrical field forces in equation 3.40 were compared. Figure 3.7 is a representative plot of the variation of the components with the dimensionless separation distance at $\theta=120^{\circ}$ on a logarithmic scale. The van der Waals force component is small as compared to the electrical field component till the dimensionless distance (i.e. ratio of the actual separation distance to the average particle radii) reduces to the order of $10^{-3}$. For shorter separation distances, the van der Waals force components continue to grow and become comparable to the electrical field component. Integration of the trajectory equation was done with a step size for varying $\theta$ as 0.005 radians or $0.2865^{\circ}$. With this step size, the van der Waals force component had no effect on the values collision efficiencies. As a result, for the final calculations, the van der Waals force term was neglected to reduce the computational effort. 


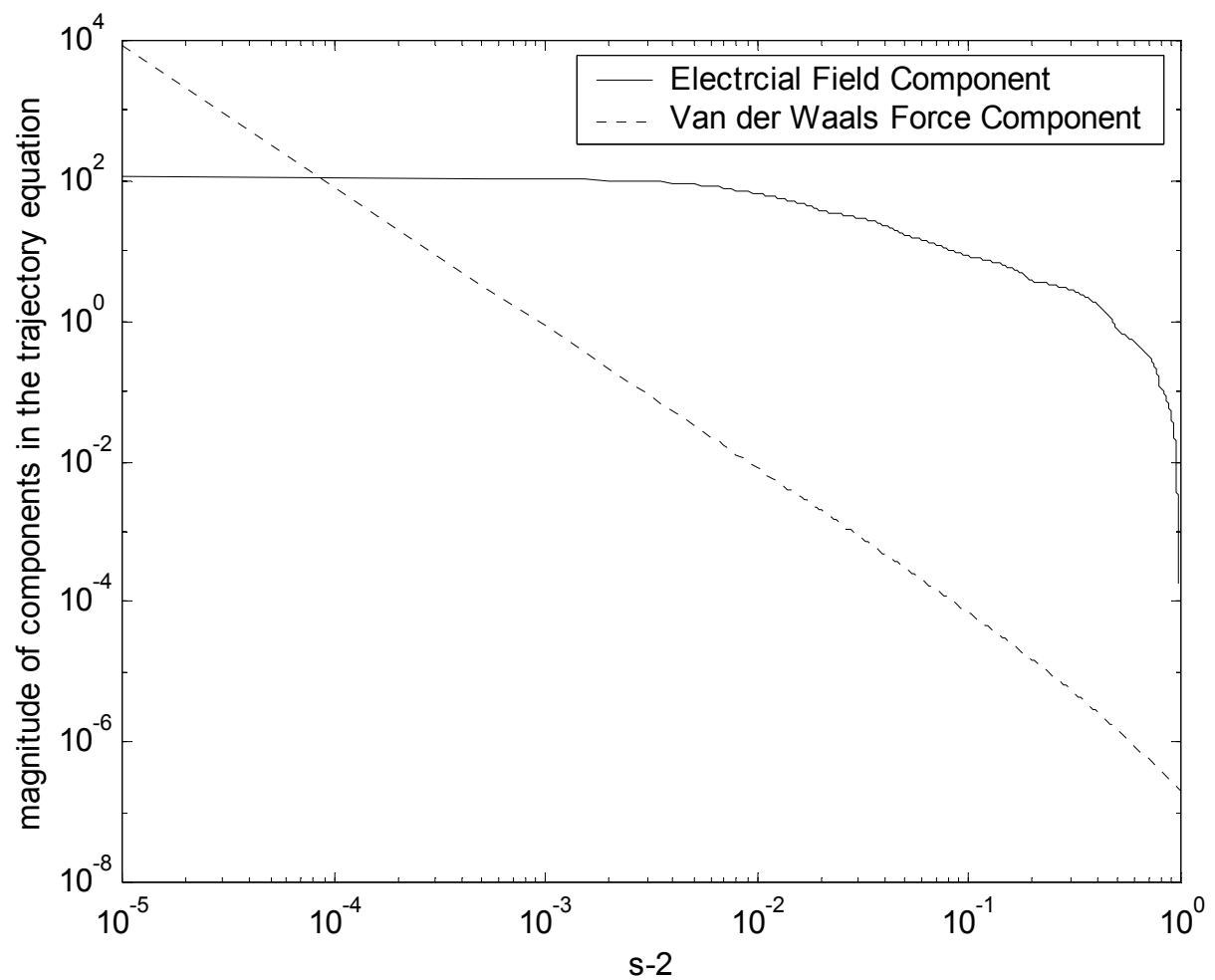

Figure 3.11: Variation of the magnitude of the electrical field force component and the van der Waals force component in the trajectory equation at $\theta=120^{\circ}$.

\subsubsection{Converting $y_{c}$ to $e_{i j}$}

From equation 3.39 , the collision efficiency is given as

$e_{i j}=\frac{y_{c}{ }^{2}}{\left(a_{i}+a_{j}\right)^{2}}$

Putting $y_{c}=r \sin \theta_{c}$

, the expression for collision efficiency is 
$\mathrm{e}_{\mathrm{ij}}=\frac{\mathrm{r}^{2} \sin ^{2} \theta}{\left(\mathrm{a}_{\mathrm{i}}+\mathrm{a}_{\mathrm{j}}\right)^{2}}$

Substituting $r=s\left(a_{i}+a_{j}\right) / 2$ into equation 3.40 for $e_{i j}$, we get

$\mathrm{e}_{\mathrm{ij}}=\left(\frac{\mathrm{s} \sin \theta_{\mathrm{c}}}{2}\right)^{2}$

The collision efficiency (for a fixed electric field strength) calculated from the trajectory equation was dependant on the size ratio of the aerosol particles and the size of one of the particles (the larger particle was used for the calculations). These dependencies are shown in Figures 3.11, 3.12 and 3.13.

The collision efficiency increases rapidly as the particles get smaller for a constant particle size ratio. Also, as the size ratio increases, the values for the collision efficiency increase. At a size ratio of 1 i.e. both particles of the same size the relative velocity due to gravitational acceleration between the particles is 0 , and so no collisions will occur. 


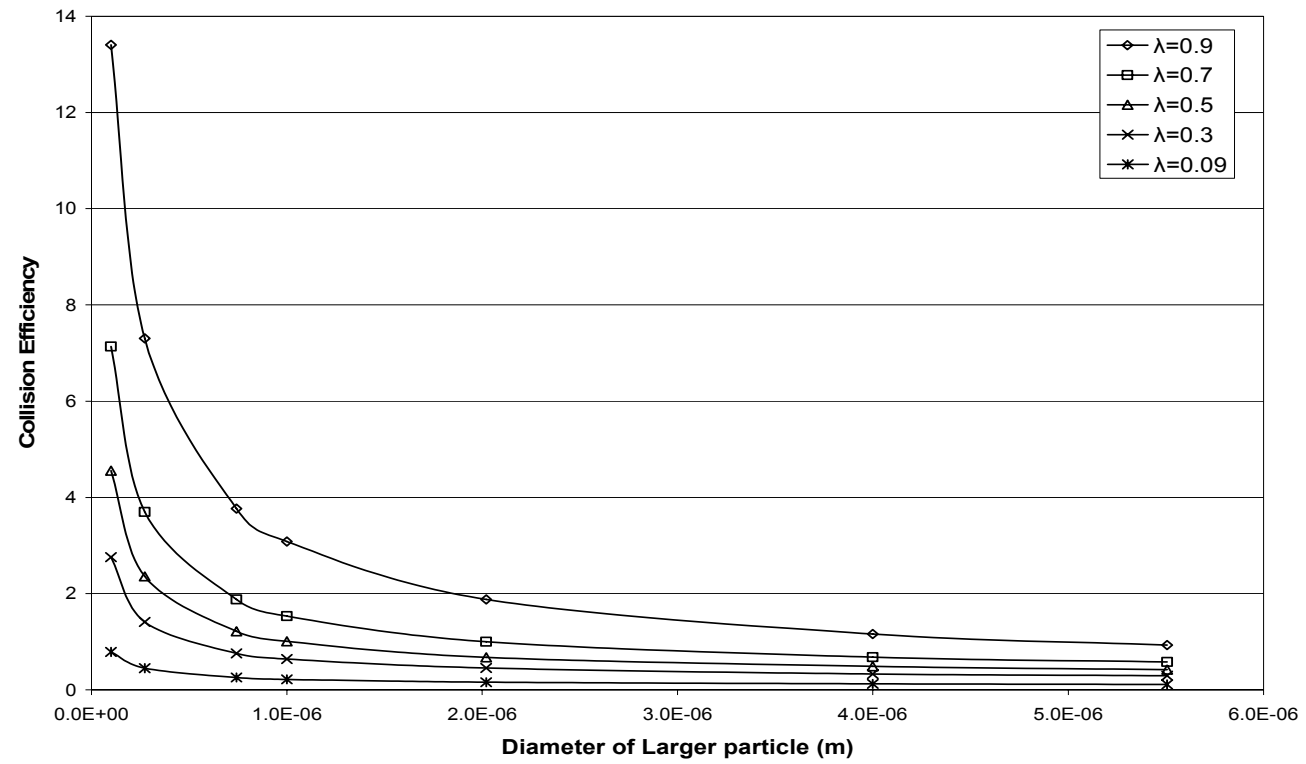

Figure 3.12: Collision efficiencies for an electric field of $25 \mathrm{kV} / \mathrm{m}$.

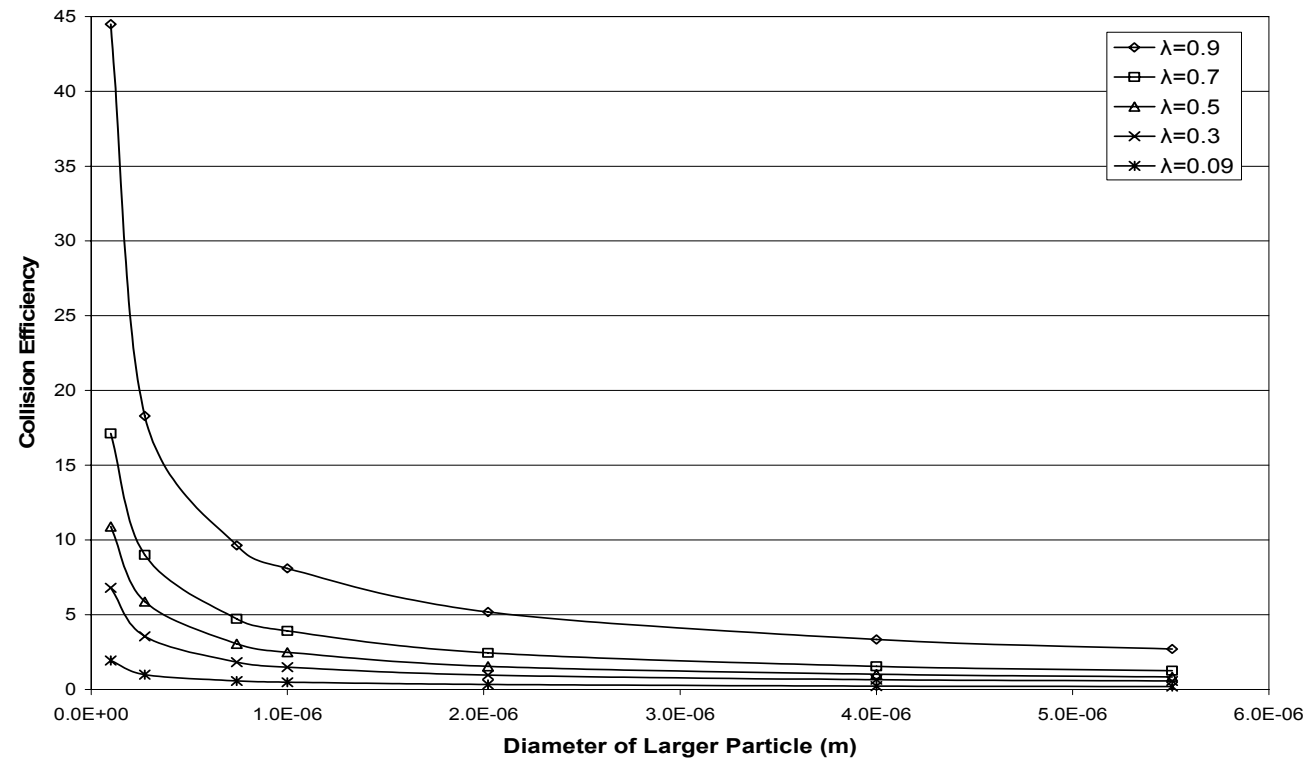

Figure 3.13: Collision efficiencies for an electric field of $50 \mathrm{kV} / \mathrm{m}$. 


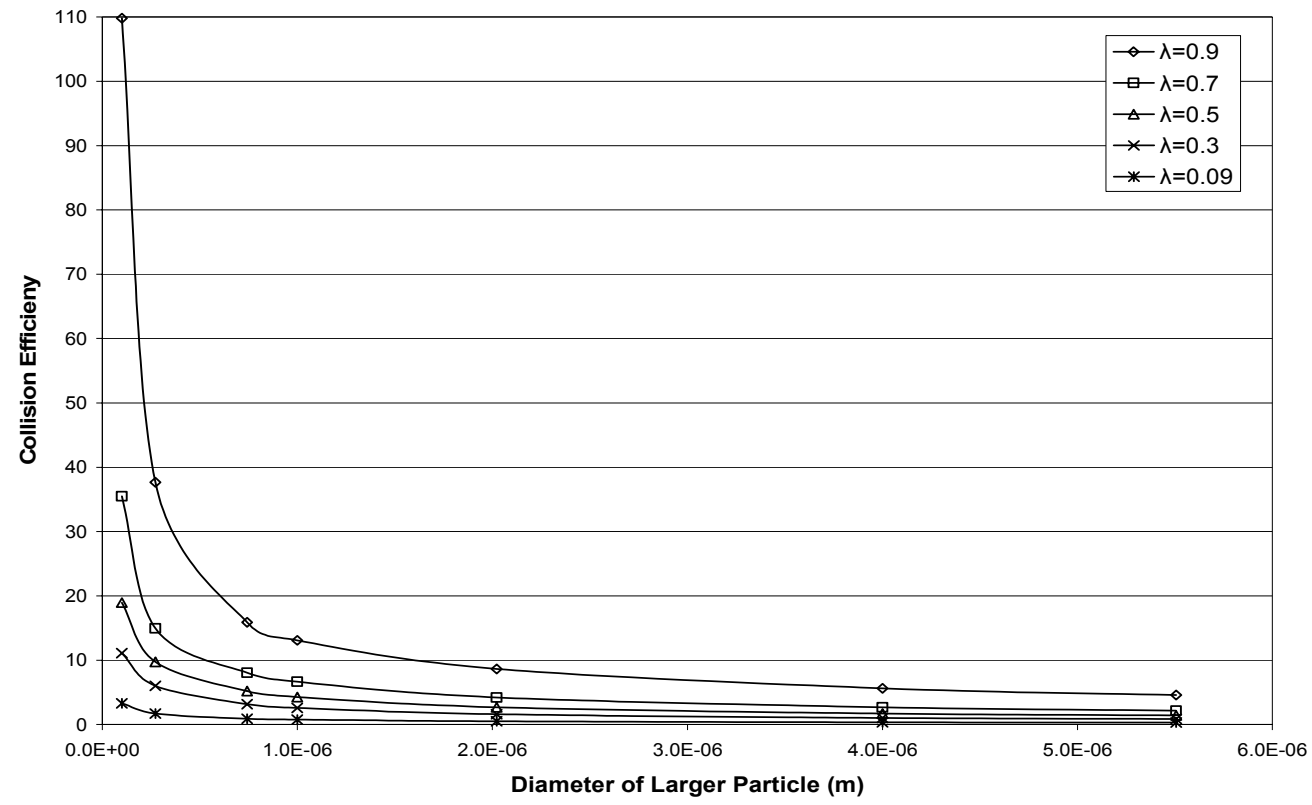

Figure 3.14: Collision efficiencies for an electric field of $75 \mathrm{kV} / \mathrm{m}$.

The collision efficiencies are curve fitted to obtain algebraic expressions dependant on the diameter of the larger particle $d_{i}$, and the size ratio $\lambda$ of the 2 particles.

The expressions obtained were as

For the case of electric field $=25 \mathrm{kV} / \mathrm{m}$

$\mathrm{e}_{\mathrm{ij}}=0.0003 \mathrm{~d}_{\mathrm{i}}^{-\mathrm{x}}$,where the exponent

$x=-14.391 \lambda^{6}+58.094 \lambda^{5}-88.259 \lambda^{4}+64.468 \lambda^{3}-23.619 \lambda^{2}+4.216 \lambda+0.2593$

For the case of electric field $=50 \mathrm{kV} / \mathrm{m}$

$\mathrm{e}_{\mathrm{ij}}=0.0003 \mathrm{~d}_{\mathrm{i}}^{-\mathrm{x}}$, where the exponent

$x==5.6923 \lambda^{5}-14.307 \lambda^{4}+13.839 \lambda^{3}-6.4955 \lambda^{2}+1.5618 \lambda+0.4734$ 
For the case of electric field $=75 \mathrm{kV} / \mathrm{m}$

$\mathrm{e}_{\mathrm{ij}}=0.0005 \mathrm{~d}_{\mathrm{i}}^{-\mathrm{x}}$,where the exponent

$x=-0.1637 \lambda^{4}+1.1742 \lambda^{3}-1.5267 \lambda^{2}+0.85 \lambda+0.473$

\subsection{Numerical Implementation - MAEROS}

For modeling the settling process a sectional code MAEROS, written by Gelbard and Seinfeld (1980) was used. This code calculates aerosol composition and mass concentration as a function of particle size and time. The processes incorporated are coagulation due to Brownian motion and gravity, and particle deposition due to gravitational settling. The numerical method of MAEROS is based on dividing the particle size domain into a number of sections and imposing conservation of mass for the processes given above. The coagulation coefficient is taken as the sum of the coagulation coefficients for Brownian motion and gravitational settling. These coagulation rates are integrated over time using the Runge-Kutta-Fehlberg method to give the particle concentrations.

When particles are settling due to gravity, the upper part of the chamber clears out faster than the lower part and stratification is observed. This occurs because, the particles in the upper portion of the chamber move downwards and replace the particles in the lower portion which have already settled on the floor of the chamber. The supply of particles to the lower portions means that these portions take longer to clear out. To simulate this stratification, the MAEROS code was modified to account for the elevation effects. As shown in Figure 3.14, the entire volume was divided into a number of equal horizontal compartments. 
The division was done such that each compartment had the same length and breadth as that of the entire volume, and only the height is modified. The conservation of mass equation was solved for each of these compartments with the coagulation process. In effect, each compartment now had a source and a sink.

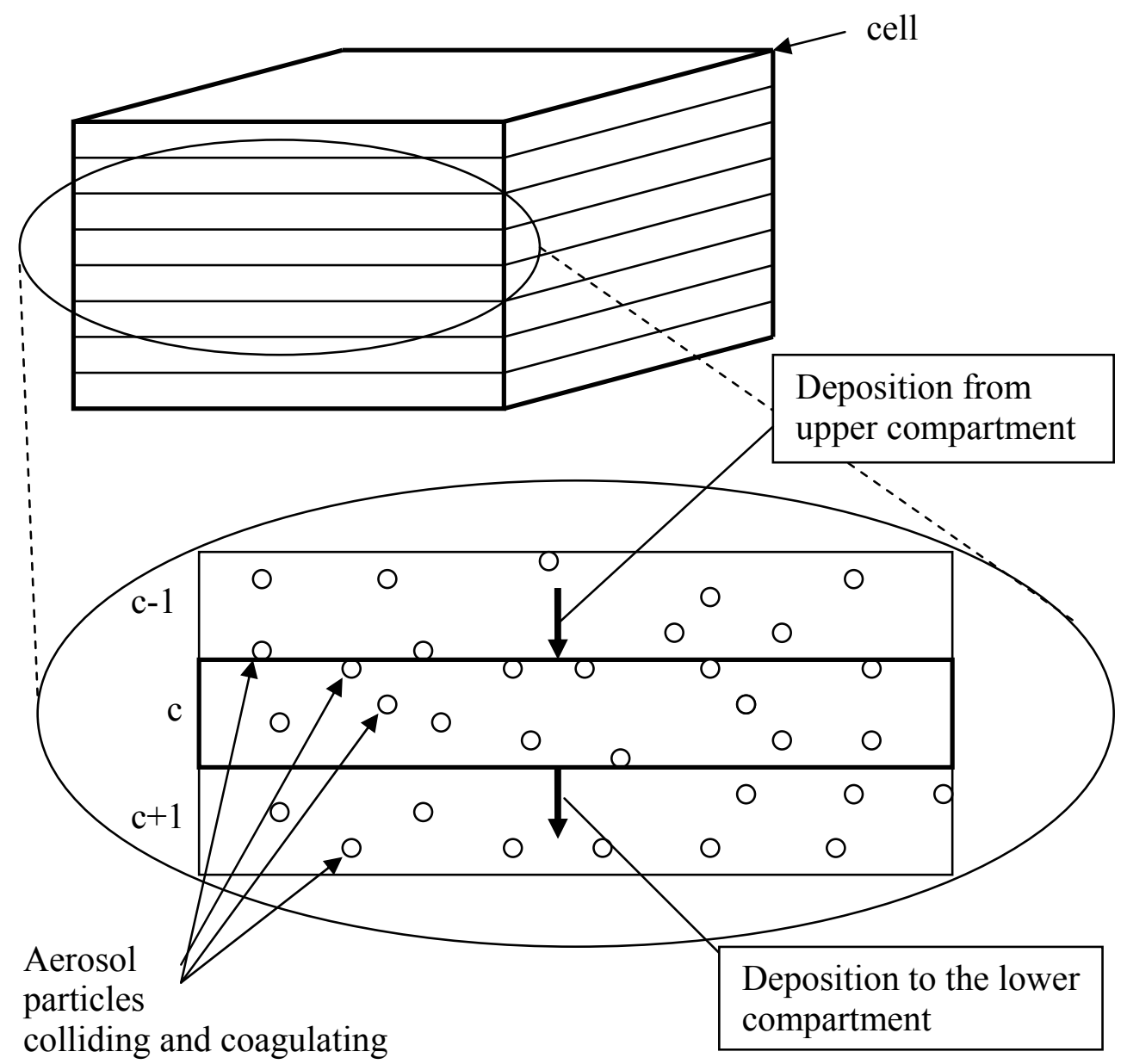

Figure 3.15: Schematic of the cell being divided into multiple horizontal compartments. 
The source is the deposition occurring from the compartment above it, and the sink in the deposition occurring to the compartment below it. For each compartment, Equation 3.2 would get modified as $\frac{\mathrm{dn}_{\mathrm{k}, \mathrm{c}}}{\mathrm{dt}}=\frac{1}{2} \sum_{\mathrm{i}+\mathrm{j}-\mathrm{k}} \beta\left(\mathrm{v}_{\mathrm{i}}, \mathrm{v}_{\mathrm{j}}\right) \mathrm{n}_{\mathrm{i}, \mathrm{c}} \mathrm{n}_{\mathrm{j}, \mathrm{c}}-\mathrm{n}_{\mathrm{k}, \mathrm{c}} \sum_{\mathrm{i}-1} \beta\left(\mathrm{v}_{\mathrm{i}, \mathrm{c}}, \mathrm{v}_{\mathrm{k}, \mathrm{c}}\right) \mathrm{n}_{\mathrm{i}, \mathrm{c}}-\mathrm{n}_{\mathrm{k}, \mathrm{c}} \mathrm{R}\left(\mathrm{v}_{\mathrm{k}}\right)+\mathrm{n}_{\mathrm{k}, \mathrm{c}-1} \mathrm{~S}\left(\mathrm{v}_{\mathrm{k}}\right)$

where the last on the right hand side accounts for the particles being added to the compartment (c) from the upper compartment (c-1). The uppermost compartment would have no source. The deposition occurring from the lowermost compartment would be the deposition to the floor.

The code could thus predict the particle concentration for different elevations within the chamber. This was useful since laser extinction measurements provide data at the particular elevation at which the laser is set. The above modification would help us to compare the model with the experimental observations.

To account for the effects of the electric field, the gravitational coagulation kernel within MAEROS is modified. This is done by multiplying the gravitational coagulation kernel (already present in MAEROS) by the collision efficiency. MAEROS calculated the collision efficiencies as

$$
e_{i j}=2\left(\frac{a_{i}}{a_{i}+a_{j}}\right)^{2}
$$

In the trajectory equation 3.36 , if the contributions of the electrical and van der Waals forces are neglected, the collision efficiency can be calculated as (Zhang, 1991) 
$e_{i j}=\exp \left(-2 \int_{2}^{\infty} \frac{M-l}{s L} d s\right)$

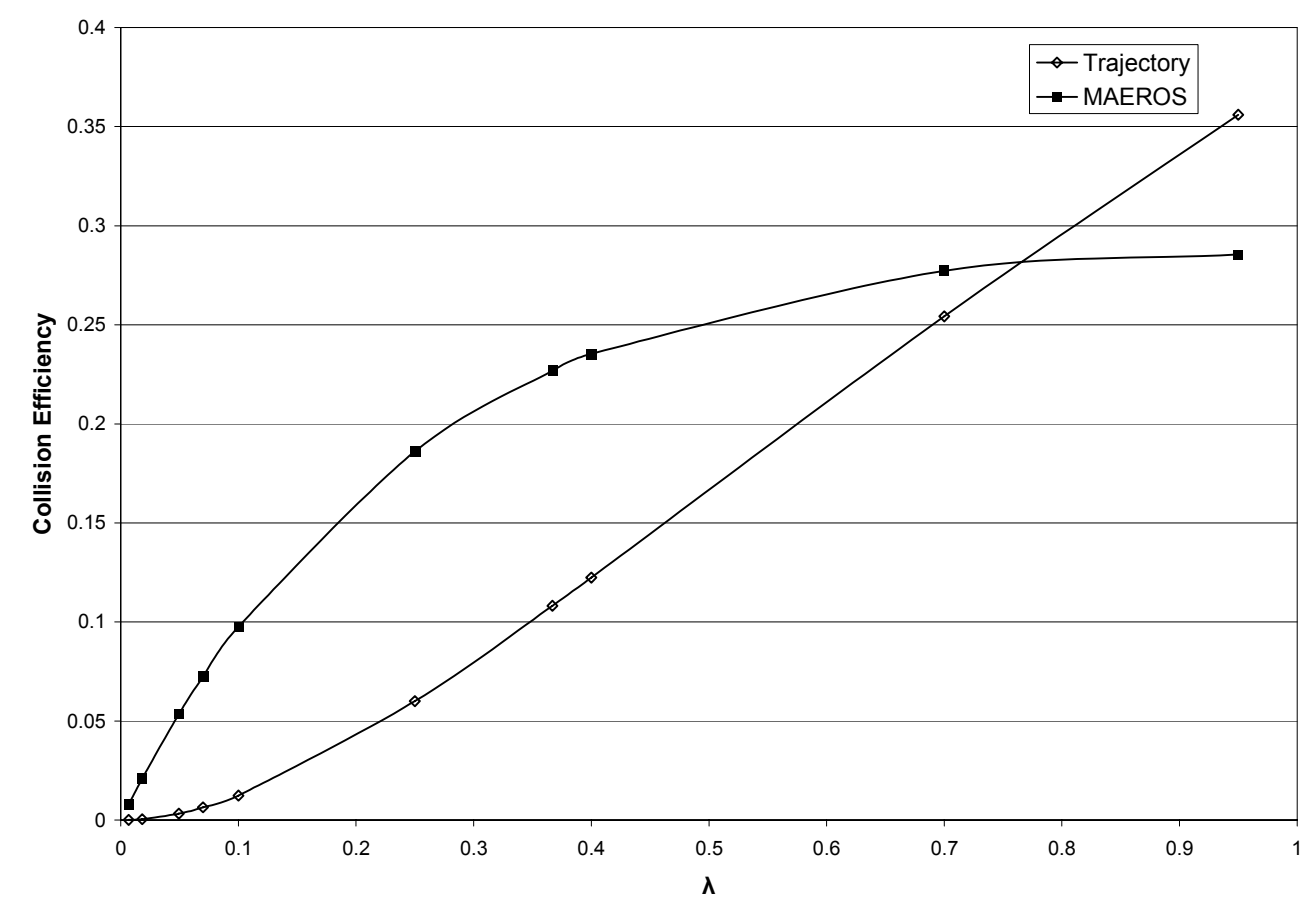

Figure 3.16: Collision efficiencies as calculated by the MAEROS model and the trajectory analysis.

The collision efficiencies as calculated by the MAEROS model and the trajectory analysis for different size ratios have been plotted in Figure 3.15. The simulations with both the models for the collision efficiencies showed negligible difference (less than $0.13 \%$ over a simulation time of 200 seconds). As a result, the MAEROS model was continued with, for calculating the collision efficiencies in the absence of an electric field. The algebraic expressions 3.42, 3.43 and 3.44 were used to calculate the collision efficiencies for the cases with electric fields. 


\section{CHAPTER 4: RESULTS}

\subsection{Computational Results}

The modified version of MAEROS was used to simulate the settling process. The settings for the simulation were based on the experiments conducted by Schmidt (2000).

\subsubsection{Operating MAEROS}

The code MAEROS simulates the evolution of aerosol with respect to the particle size. The equations used in the simulation are explained in Gelbard and Seinfeld (1980). This code was modified to account for the elevation within the cell and also to incorporate electrical coalescence effects.

The code requires an Input file. This file contains the following information

1) Dimensions of the chamber

2) The aerosol size range and the number of sections that this range should be divided. The initial concentration has to be specified and the distribution is assumed to be log-normal. The mean diameter and the geometric standard deviation have to be specified.

3) The time steps during the evolution, at which the aerosol size distribution data is required. 
4) Physical data about the fluid (air), such as Molecular Weight, Temperature and Pressure.

The output from the program gives the mass, mass concentration and the number concentration of the aerosol in the different size ranges at the time steps indicated in the input file. The mass loading at an instant is divided by the initial mass loading to give the volume fraction loading (since the temperature is constant). The simulation is analyzed using the values of the volume fraction loading.

\subsubsection{Settings for the Simulations}

The settings in the Input file replicated the experimental setup used by Schmidt (2000). The chamber dimensions were set to those of the cell i.e. $10 \mathrm{~cm} \mathrm{x}$ $10 \mathrm{~cm} \times 2 \mathrm{~cm}$. The particle size range was set from $0.5 \times 10^{-7} \mathrm{~m}$ to $10 \times 10^{-6} \cdot \mathrm{m}$ This range includes all aerosol sizes encountered. The initial concentration was set to $0.04 \mathrm{~kg} / \mathrm{m}^{3}$. Chen (1990) had found cigarette smoke to have particle density of $1.12 \mathrm{~kg} / \mathrm{m}^{3}$ and a median particle diameter of $0.2 \times 10^{-6} \mathrm{~m}$. The cell was divided into 40 horizontal chambers, so that each chamber had a height of $0.5 \mathrm{~mm}$. Data from the $6^{\text {th }}, 15^{\text {th }}$ and the $23^{\text {rd }}$ chamber were analyzed so that comparisons with experiments (Schmidt, 2000) could be made. These chambers would correspond to the elevations of $3.05 \mathrm{~mm}, 7.62 \mathrm{~mm}$ and $11.43 \mathrm{~mm}$ (from the upper copper plate), used by Schmidt (2000). The code was run for time steps of 20 seconds over a total duration of 200 seconds. 
MAEROS also accounts for the deposition on the walls and the floor of the cell due to diffusion. For the diffusion calculations, the diffusion thickness of the system has to be indicated in the Input File. The diffusion thickness was estimated to be

$$
\delta=\sqrt{\mathrm{Dt}}
$$

where $\mathrm{D}$ is the diffusion coefficient and $\mathrm{t}$ is the time over which the diffusion occurs. Reist (1993) calculated the diffusion coefficient as

$$
\mathrm{D}=\mathrm{kT} \frac{\mathrm{C}_{\mathrm{c}}}{3 \pi \mu \mathrm{d}}
$$

where $\mathrm{C}_{\mathrm{c}}$ is the Cunningham correction factor and is given as

$$
\mathrm{C}_{\mathrm{c}}=1+\frac{2 \ell}{\mathrm{d}}\left[1.257+0.4 \exp \left(-\frac{1.1 \mathrm{~d}}{2 \ell}\right)\right]
$$

$\mathrm{d}$ is the diameter of the particle, $\ell$ is the mean free path, $\mathrm{k}$ is the Boltzmann's constant, $\mathrm{T}$ is the absolute temperature and $\mu$ is the viscosity of the fluid.

Calculations were made for the aerosol based on the initial size distribution, and a value of $0.0219 \mathrm{~m}$. was used for the simulation. Table 4.1 is a list of particle sizes and the corresponding diffusion thickness.

Table 4.1: Diffusion thickness for particles of different sizes based on their initial concentrations.

\begin{tabular}{|c|c|c|c|c|c|}
\hline $\mathbf{d ~ ( m . )}$ & $\mathbf{n}\left(\# / \mathbf{m}^{3}\right)$ & $\lambda(\mathbf{m})$ & $\mathbf{C}_{\mathbf{c}}$ & $\mathbf{D}\left(\mathbf{m}^{2} / \mathbf{s}\right)$ & $\boldsymbol{\delta}(\mathbf{m})$ \\
\hline $2.00 \mathrm{E}-07$ & $4.50 \mathrm{E}+15$ & 0.00125 & $2.07 \mathrm{E}+04$ & $2.41 \mathrm{E}-06$ & 0.021944 \\
\hline $3.00 \mathrm{E}-07$ & $6.29 \mathrm{E}+14$ & 0.003976 & $4.39 \mathrm{E}+04$ & $3.40 \mathrm{E}-06$ & 0.026086 \\
\hline $7.00 \mathrm{E}-07$ & $1.16 \mathrm{E}+16$ & $3.96 \mathrm{E}-05$ & $1.88 \mathrm{E}+02$ & $6.24 \mathrm{E}-09$ & 0.001117 \\
\hline $1.00 \mathrm{E}-06$ & $5.96 \mathrm{E}+11$ & 0.377649 & $1.25 \mathrm{E}+06$ & $2.91 \mathrm{E}-05$ & 0.076271 \\
\hline
\end{tabular}


Since, the diffusion thickness would depend on the particle size, a representative single value for the aerosol would vary as particle sizes vary during the settling process. However, using the different diffusion thickness listed above did not affect the simulations. It was found that this diffusion thickness value was insignificant in the simulation, since gravitational settling and Brownian diffusion were the dominant processes.

\subsubsection{Multiple Elevation Effects}

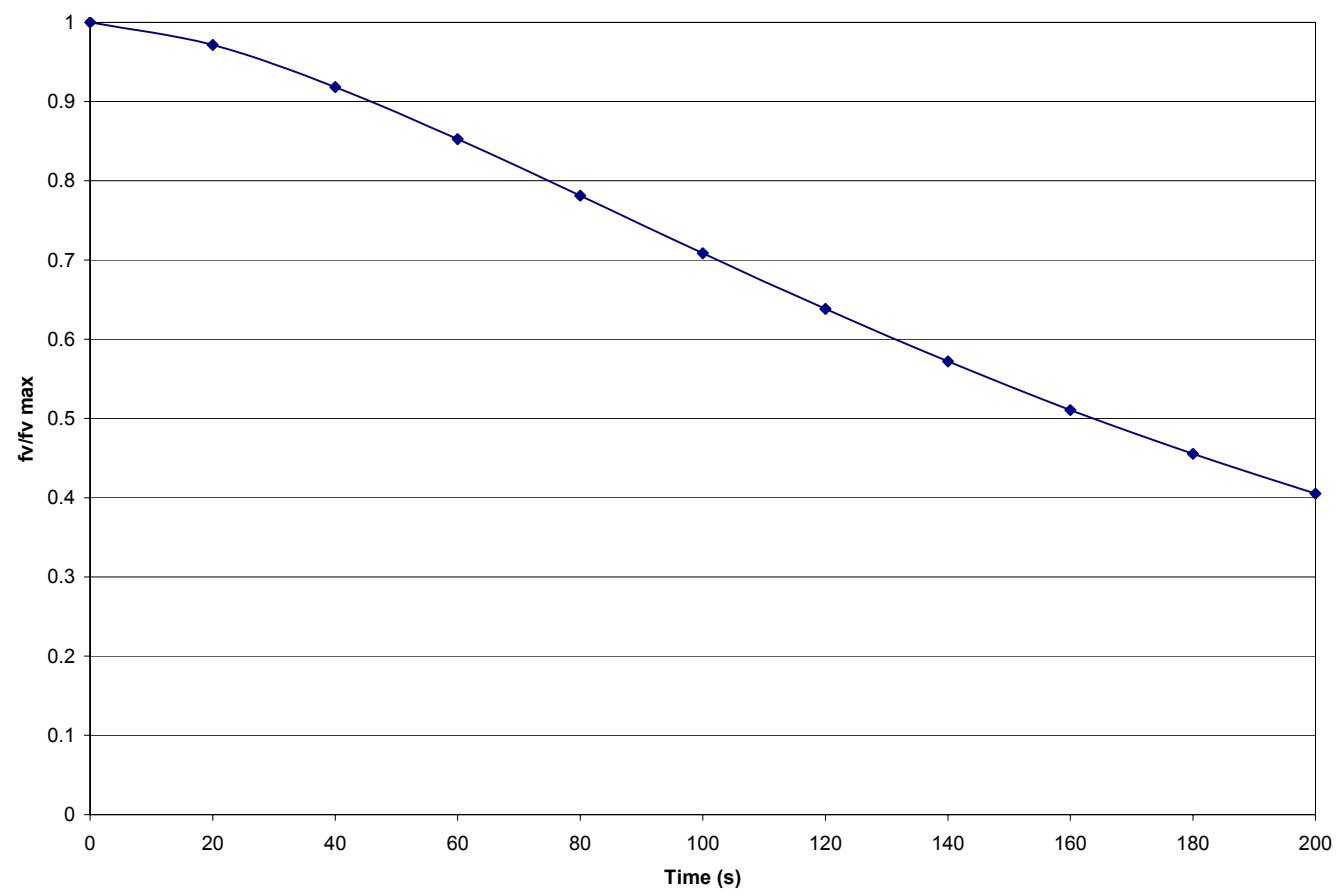

Figure 4.1: Volumetric Loading plot for natural gravitational settling as predicted by the original MAEROS code. 
Figures 4.1 and 4.2 are the volume fraction plots for a case of natural settling with the original MAEROS code and with the compartmentalized MAEROS code respectively. The input parameters were the same as those indicated in section 4.1.2, with the entire volume being left undivided for the former, and the volume compartmentalized into 40 compartments for the latter.

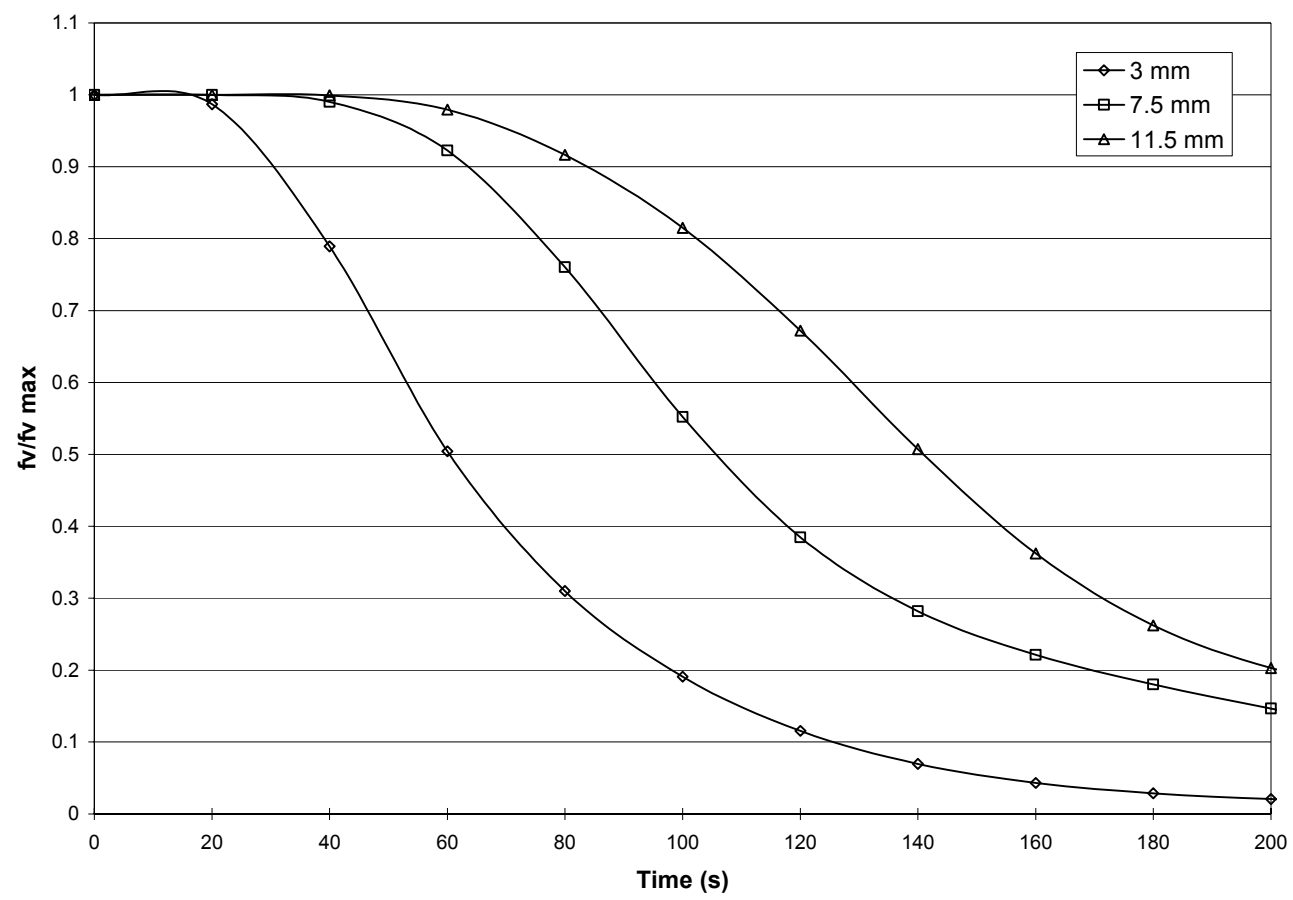

Figure 4.2: Volume Fraction Loading for $0 \mathrm{~V}$.

The original code provides a single loading value for the entire volume which reduces linearly over time. For the modified (compartmentalized) MAEROS run, the volume fraction results for elevations of $3 \mathrm{~mm}, 7.5 \mathrm{~mm}$ and 
$11.5 \mathrm{~mm}$ from the upper copper plate are shown in Figure 4.2. It was observed that the volume fraction reduces faster for the upper level $(3 \mathrm{~mm})$ than that for the central level $(7.5 \mathrm{~mm})$, which in turn reduces faster than that from the lower level $(11.5 \mathrm{~mm})$. Also, the volume fraction reduction does not show a linear pattern as observed in the Figure 4.1. This is due to the mass conservation equation being solved for every compartment. The volume fractions, initially, do not drop, since each of the compartments are loosing and gaining mass at the same time. With time, the upper compartments loose more mass than they receive and so we see a reduction in the loading. As the settling process continues, more and more compartments loose more mass than they receive, and this process moves

progressively through the compartments in the downward direction. In effect a wave seems to be passing through the volume from the top to the bottom clearing up the aerosol. Thus, the stratification process seen during the settling process is simulated. All further analysis is done using the modified (compartmentalized) MAEROS code.

\subsubsection{Volume Fraction Results}

Figures 4.3, 4.4 and 4.5 are the volume fraction loading plots for the cases with electric filed strengths of $25 \mathrm{kV} / \mathrm{m}, 50 \mathrm{kV} / \mathrm{m}$ and $75 \mathrm{kV} / \mathrm{m}$ respectively. Figure 4.2 is the volume fraction plot for the case with no electric field. 


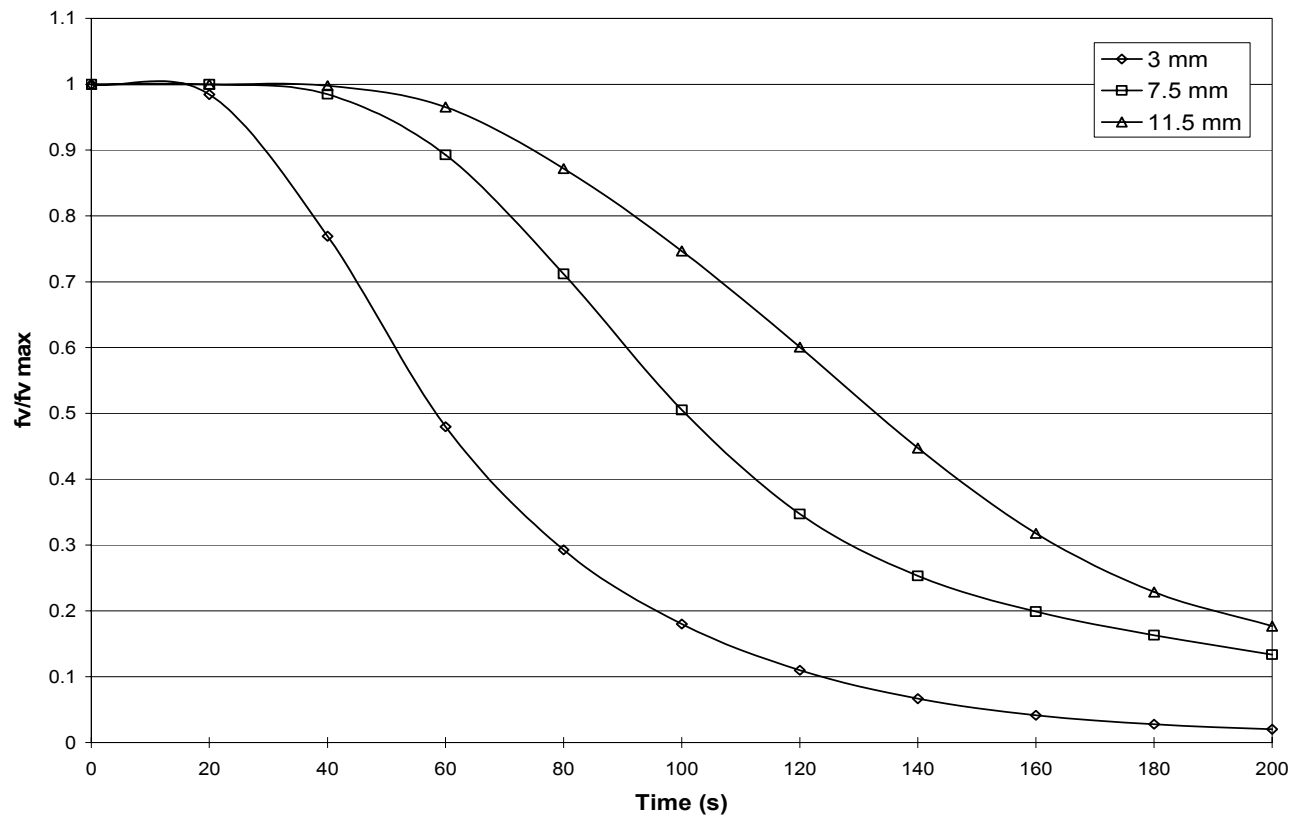

Figure 4.3: Volume Fraction Loading for $25 \mathrm{kV} / \mathrm{m}$.

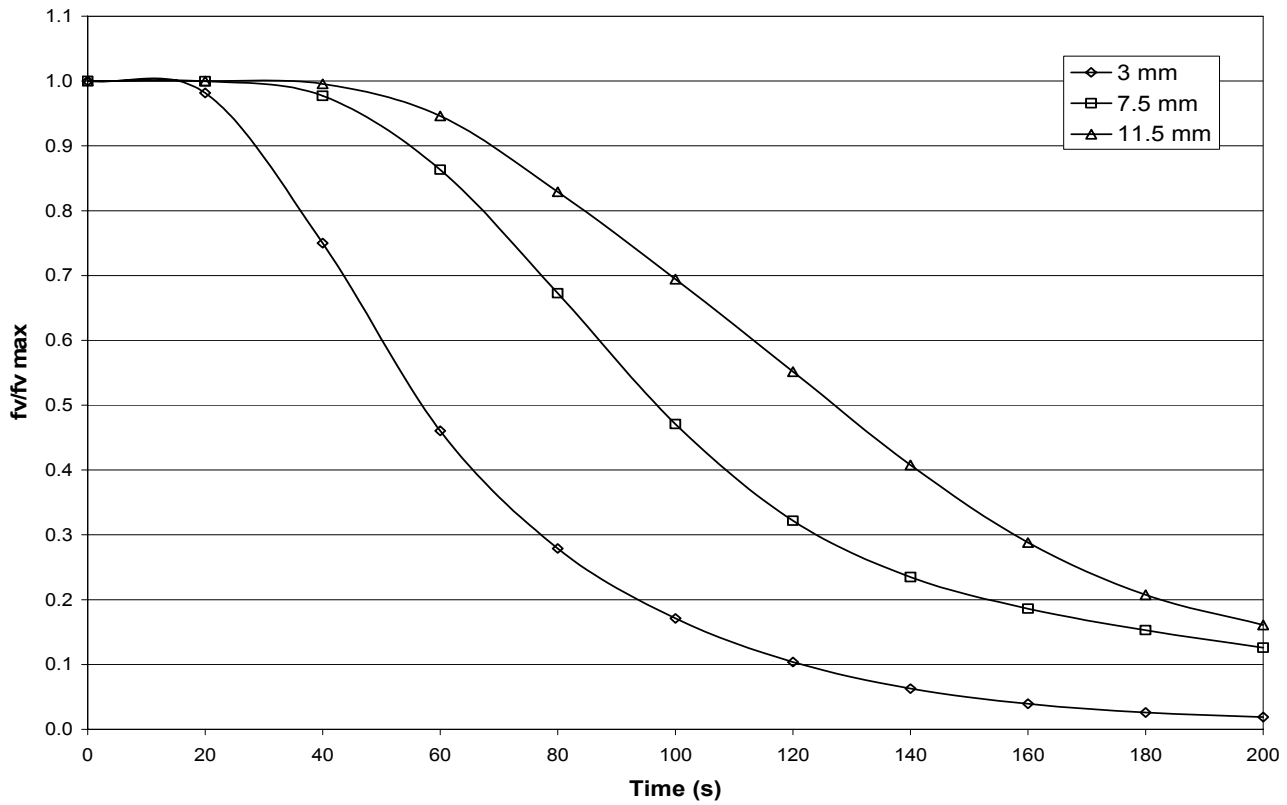

Figure 4.4: Volume Fraction Loading for $50 \mathrm{kV} / \mathrm{m}$. 


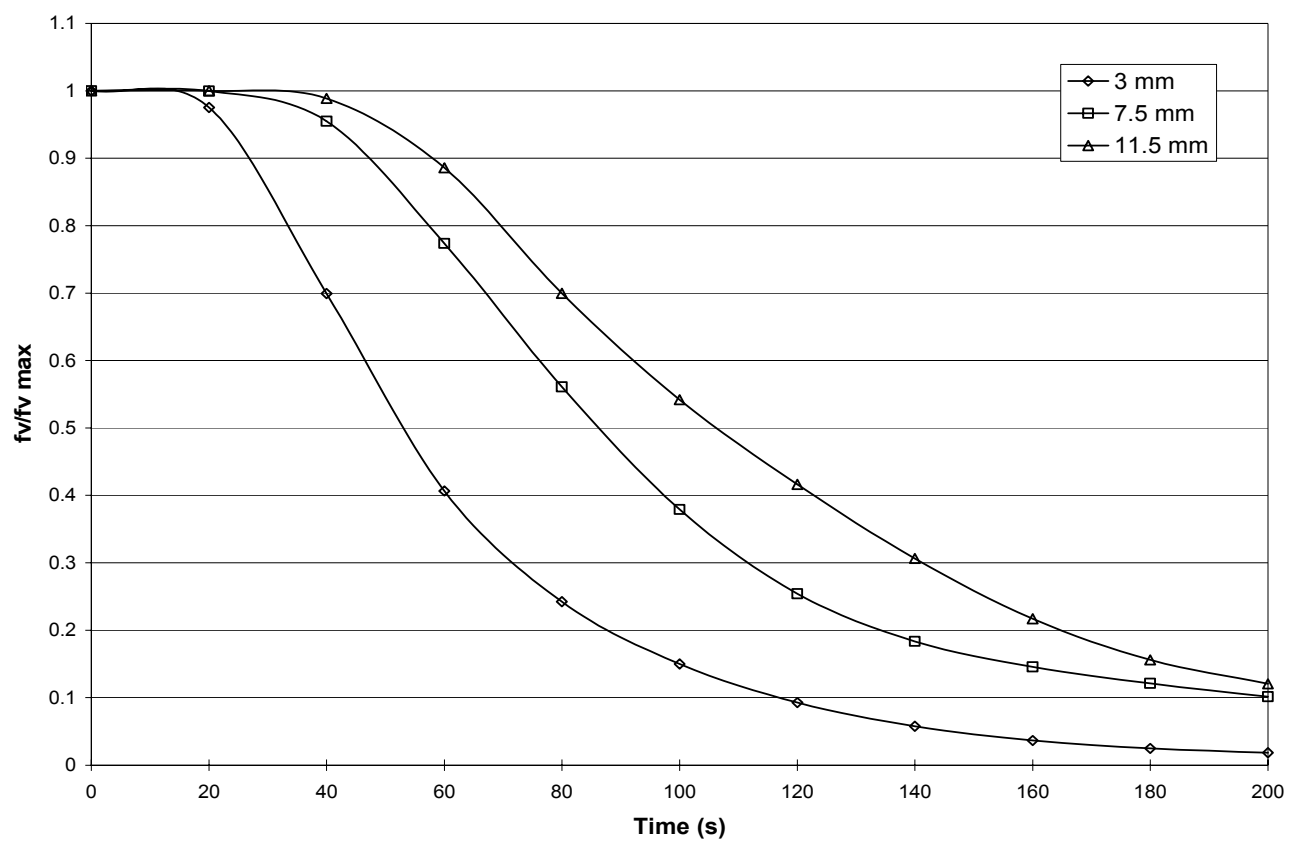

Figure 4.5: Volume Fraction Loading for $75 \mathrm{kV} / \mathrm{m}$.

A comparison of the plots reveals that, with increasing electric fields, a progressive reduction in sedimentation times is observed. This is expected, since the presence of electric fields increases the collision efficiencies. This results in larger particles which have higher terminal velocities, causing the aerosol to clear out quicker. These trends were observed experimentally too.

\subsubsection{Constant Elevation Results}

Figures $4.6,4.7$ and 4.8 are settling plots for the elevations of $3 \mathrm{~mm}, 7.5$ $\mathrm{mm}$ and $11.5 \mathrm{~mm}$ under different electric field strengths respectively. It appears that the effects of the electric field are more pronounced at lower elevations within the cell. This is because, in essence, the electrical field accelerates the 
settling process. As a result the settling rate increases with time. Since the lower levels sustain a high mass loading for longer periods than the upper levels, the effect of the increasing settling rates becomes more observable at the lower elevations.

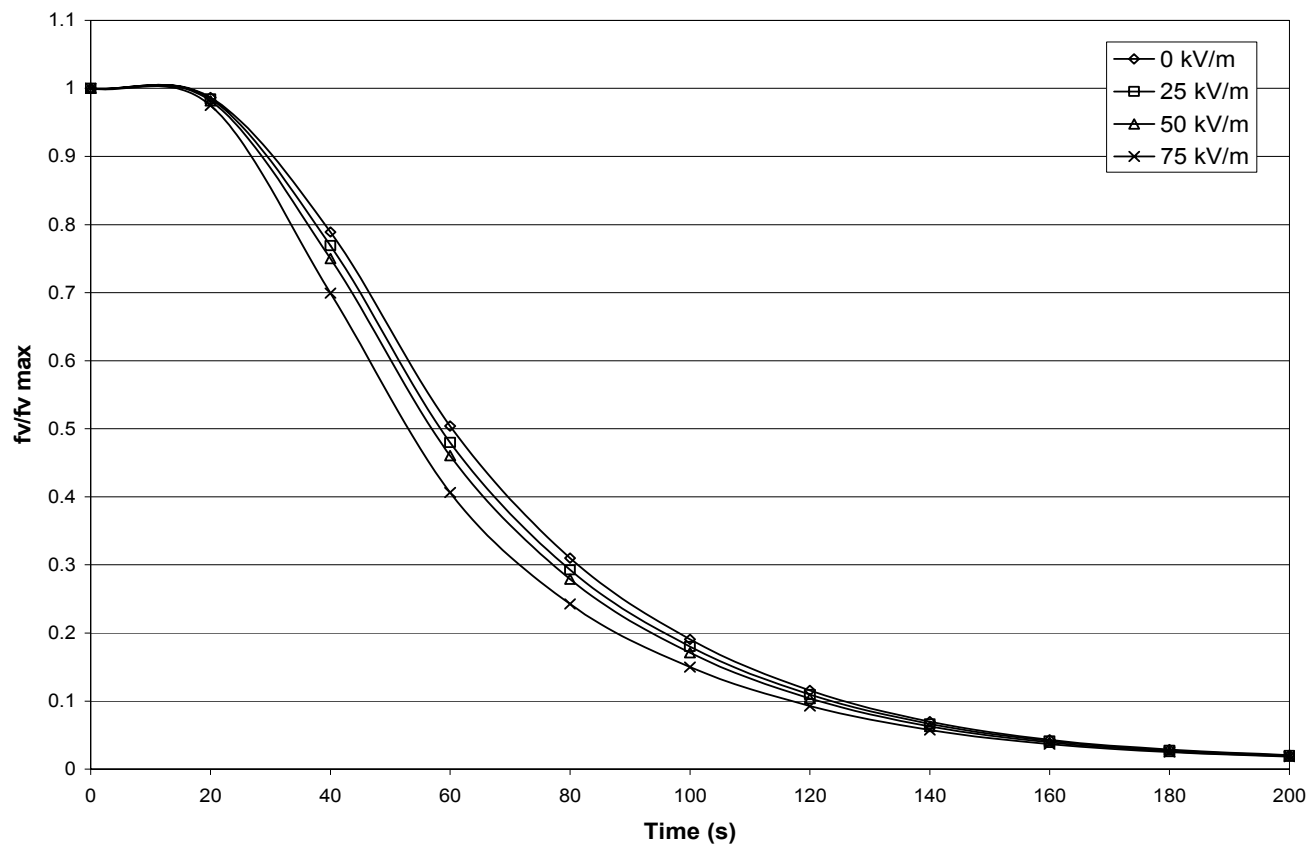

Figure 4.6: Settling plots at $3 \mathrm{~mm}$ elevation for different electric fields 


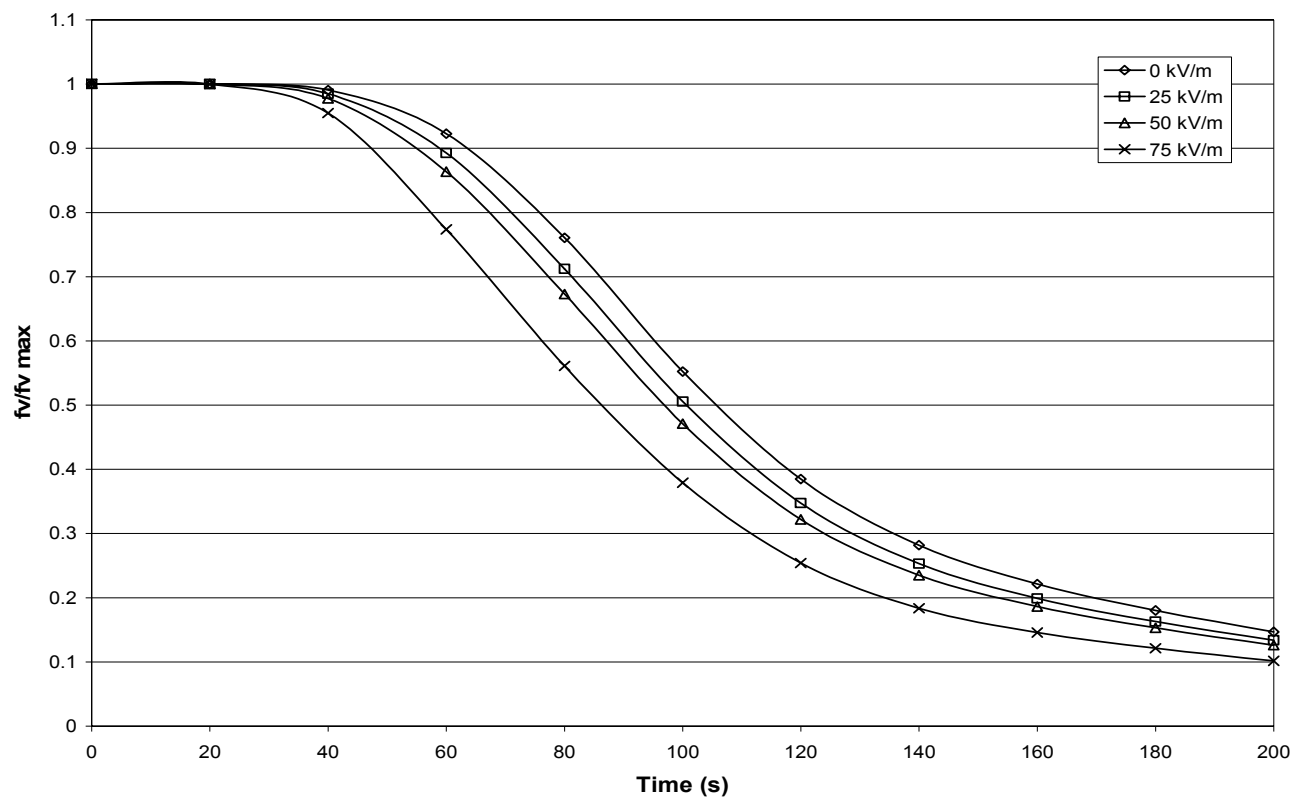

Figure 4.7: Settling plots at $7.5 \mathrm{~mm}$ elevation for different electric fields

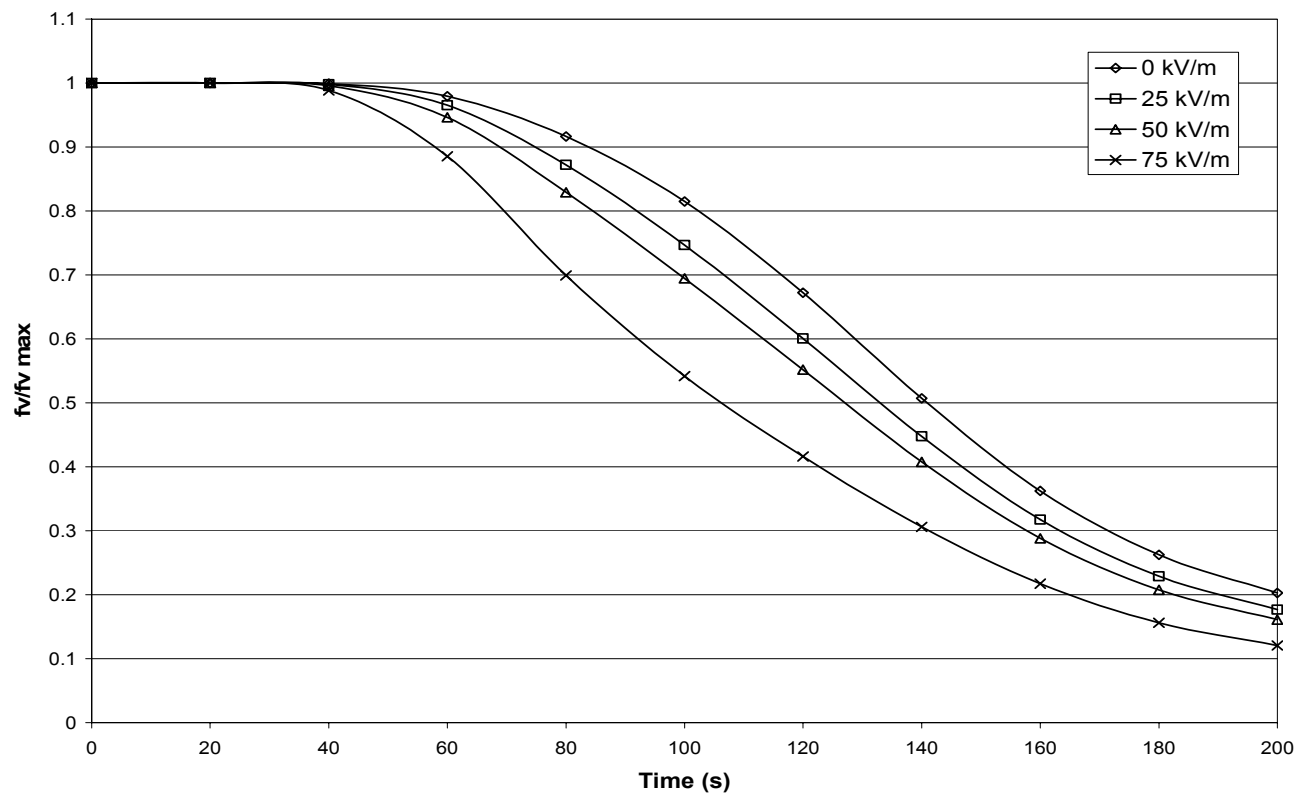

Figure 4.8: Settling plots at $11.5 \mathrm{~mm}$ elevation for different electric fields 


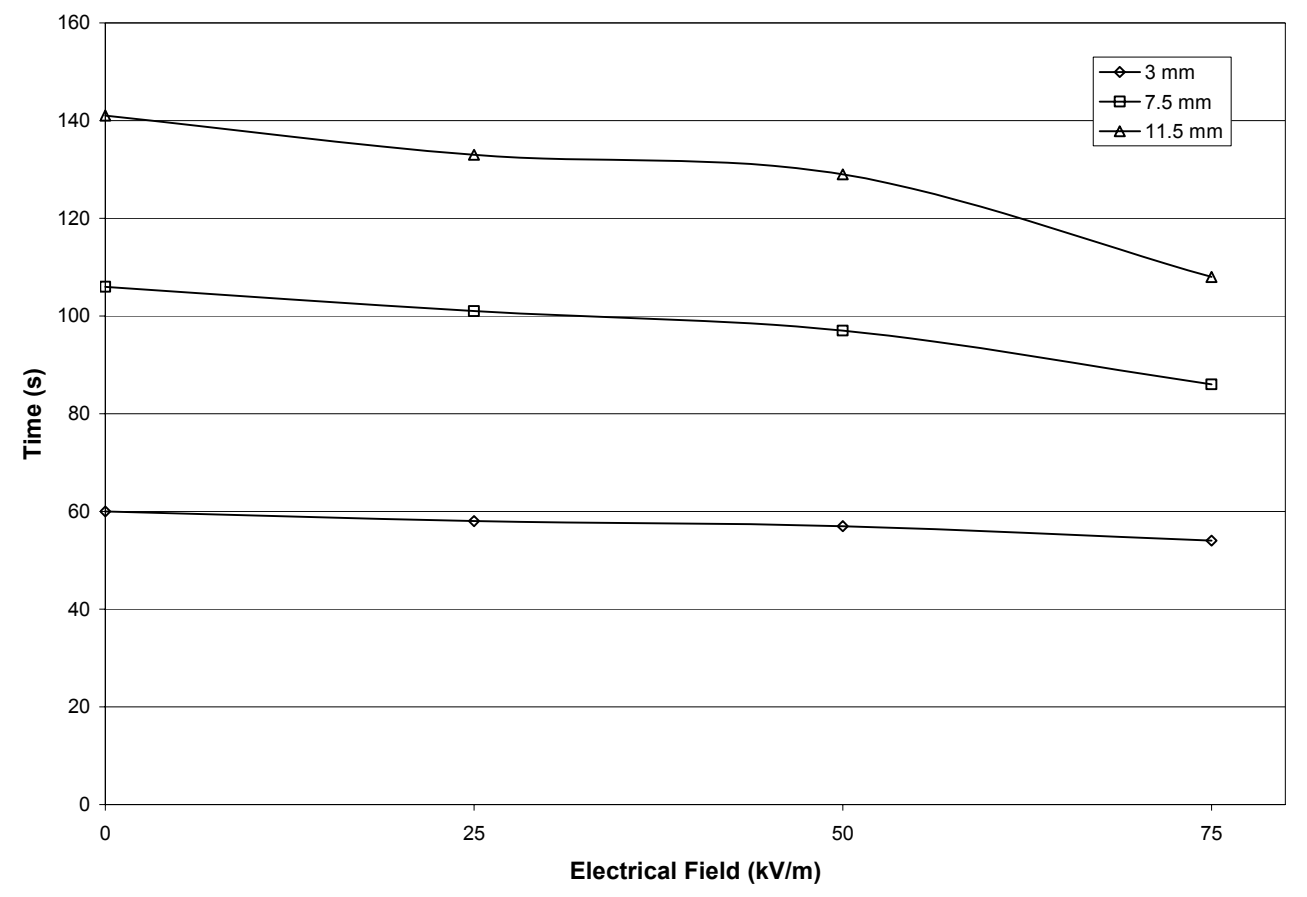

Figure 4.9: Plot of time required to reduce to $50 \%$ loading for different electric fields.

Figure 4.9 is a plot of the $50 \%$ loading times (time required for the volume fraction to reduce to 0.5) versus the Electric Field Strength for the 3 elevations. An electric field strength of $75 \mathrm{kV} / \mathrm{m}$ reduced the $50 \%$ loading times by $10 \%, 18.86 \%$ and $23.4 \%$ for the elevations of $3 \mathrm{~mm} ., 7.5 \mathrm{~mm}$. and $11.5 \mathrm{~mm}$. In effect, the wave front that clears the aerosol in the cell seems to be accelerating. 


\subsubsection{Particle Size Distribution Data}

The particle size distribution as predicted by MAEROS was analyzed. Figure 4.10 is the initial particle size distribution. Figures 4.11, 4.12 and 4.13 are the particle size distribution after 20 seconds, 40 seconds and 60 seconds respectively, with no electric field at an elevation of $7.5 \mathrm{~mm}$ from the top plate. The shift of the particle size distribution towards the larger particle sizes occurs due to particles agglomerating or coalescing to form larger particles. Similar trends are observed for the cases with the electric field.

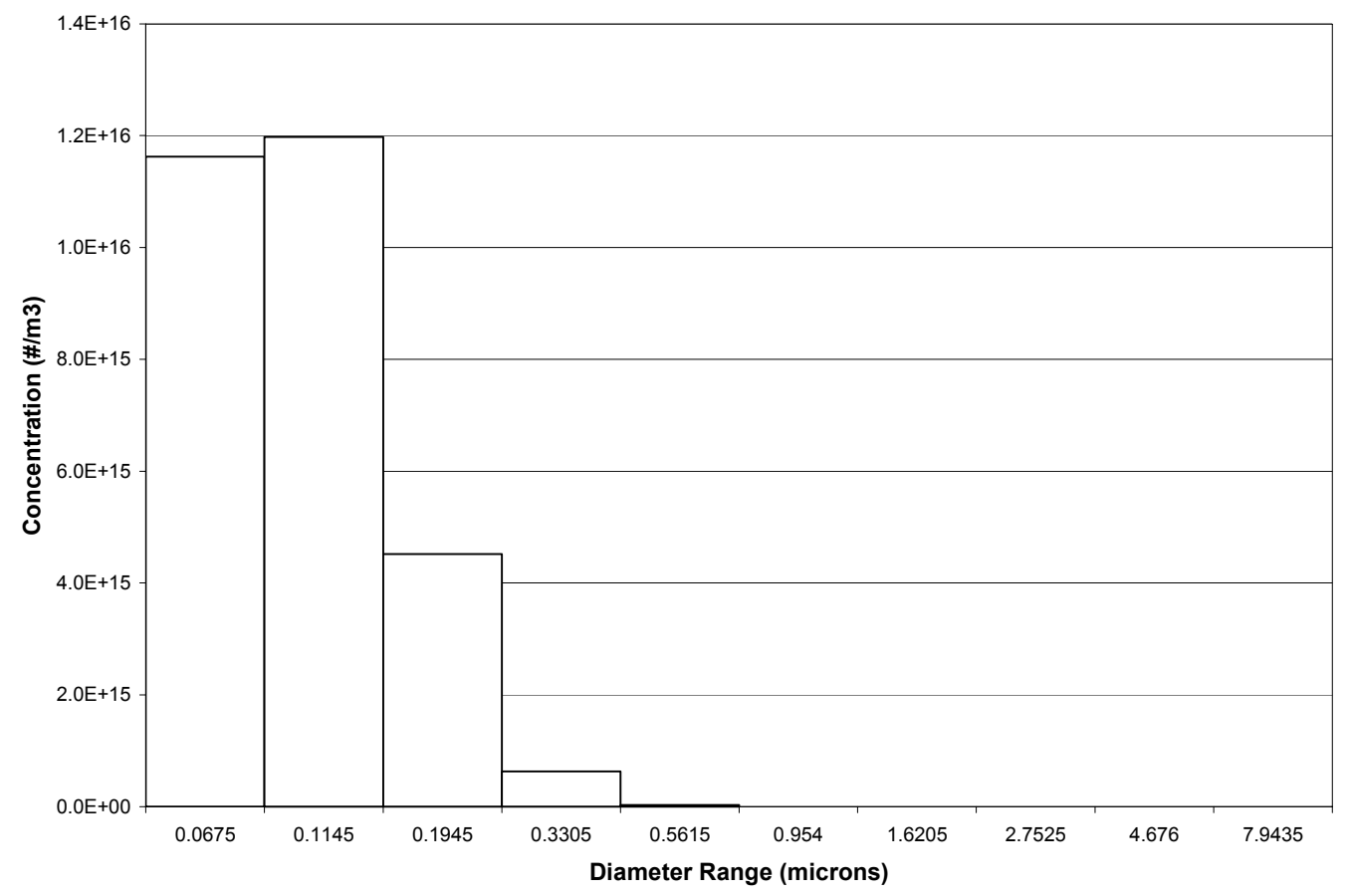

Figure 4.10: Initial Particle Size Distribution 


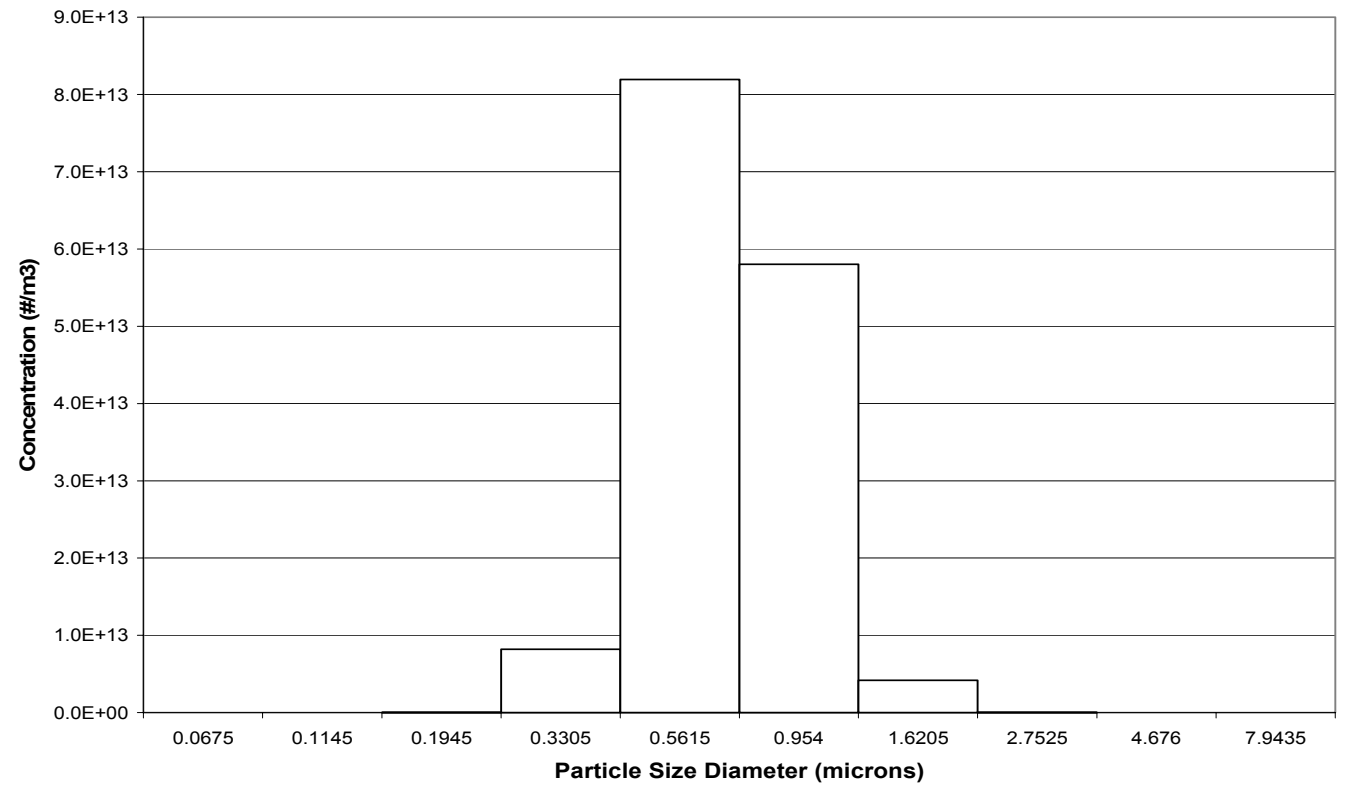

Figure 4.11: Particle Size Distribution for elevation of $7.5 \mathrm{~mm}$ after $20 \mathrm{~s}$ under no electric field.

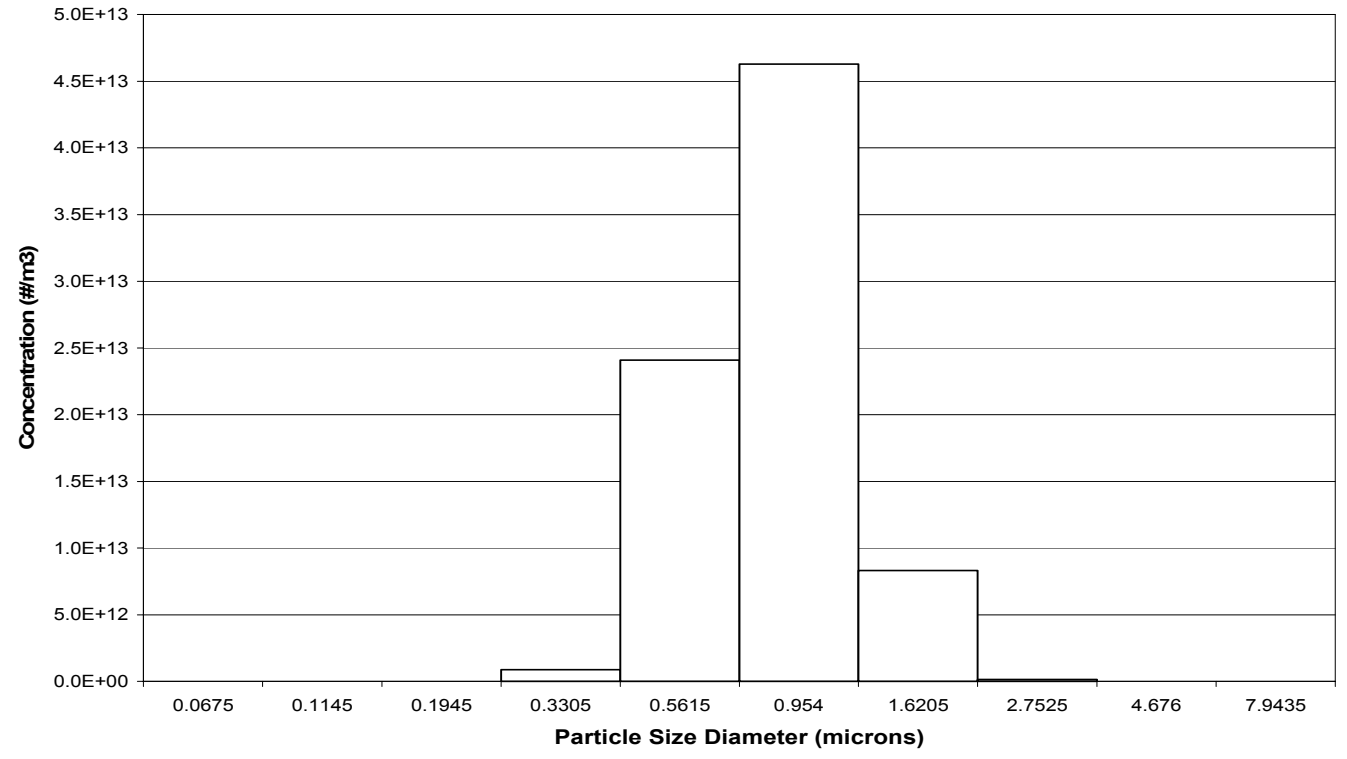

Figure 4.12: Particle Size Distribution for elevation of $7.5 \mathrm{~mm}$ after $40 \mathrm{~s}$ under no electric field. 


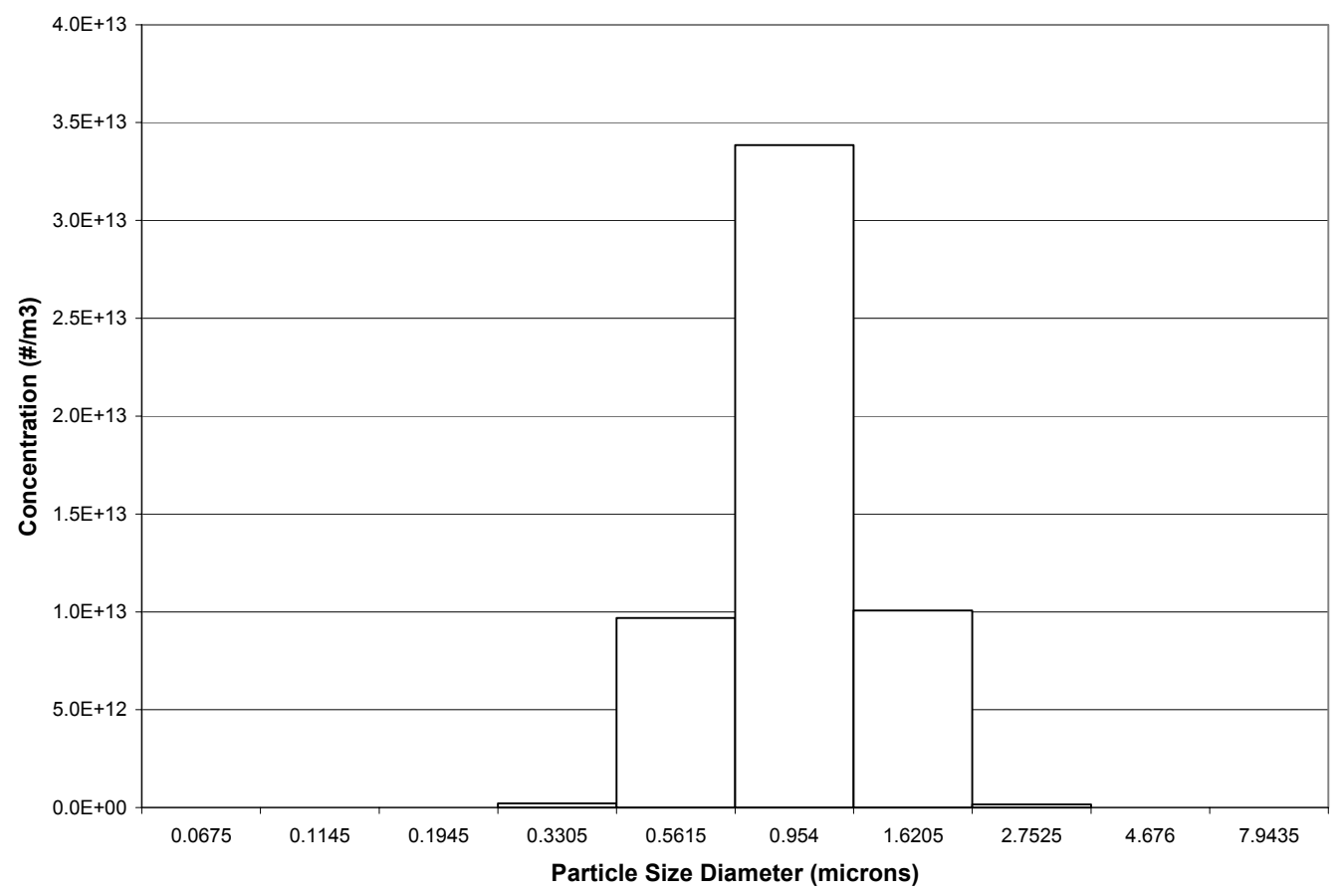

Figure 4.13: Particle Size Distribution for elevation of $7.5 \mathrm{~mm}$ after $60 \mathrm{~s}$ under no electric field.

\subsection{Comparison with experiments/literature}

The experimental data collected at ORNL had certain shortcomings

1) The laser had a beam diameter of the laser was $1 \mathrm{~cm}$ and so the data collected was indicative of the processes taking place in the aerosol over 1 $\mathrm{cm}$. The cell height was $2 \mathrm{~cm}$, the laser beam would occupy half the cell. As a result the measurements were not indicative of the particular elevation, but that of $0.5 \mathrm{~cm}$ below and above the indicated elevation. 
2) The PSD data provided by the RTSizer had a lower particle size limit of 0.5 microns. This meant that the particles less than 0.5 microns would not be used in the calculations and the PSD could have significant errors.

3) For the efficient functioning of the Spraytec system, the transmission ratio should be over $70 \%$ (Corcoran, 2000). This is because with high particle concentrations multiple scattering become a concern. However, the transmission ratios over a vast duration of the experiment were less than $10 \%$, since the cell would be completely filled with cigarette smoke. Therefore, the PSD data was not available during initial stages of the experiment.

4) The experiments were not repeatable.

5) There was deposition of smoke particles on the window of the cell. As a result the light scattering would be modified and the measurements taken would not be indicative of the aerosol within the cell. Since the deposition would occur during the experiment, there was no method for correcting the data for this problem.

As a result, the simulations were done to match the experiments conducted by Schmidt (2000). Figures 4.14, 4.15, 4.16 and 4.17 are the volume fraction comparison plots of the computational model results with the experimental data from Schmidt (2000) under electric fields of $0 \mathrm{~V} / \mathrm{m}, 25 \mathrm{kV} / \mathrm{m}, 50 \mathrm{kV} / \mathrm{m}$ and 75 $\mathrm{kV} / \mathrm{m}$ respectively. 


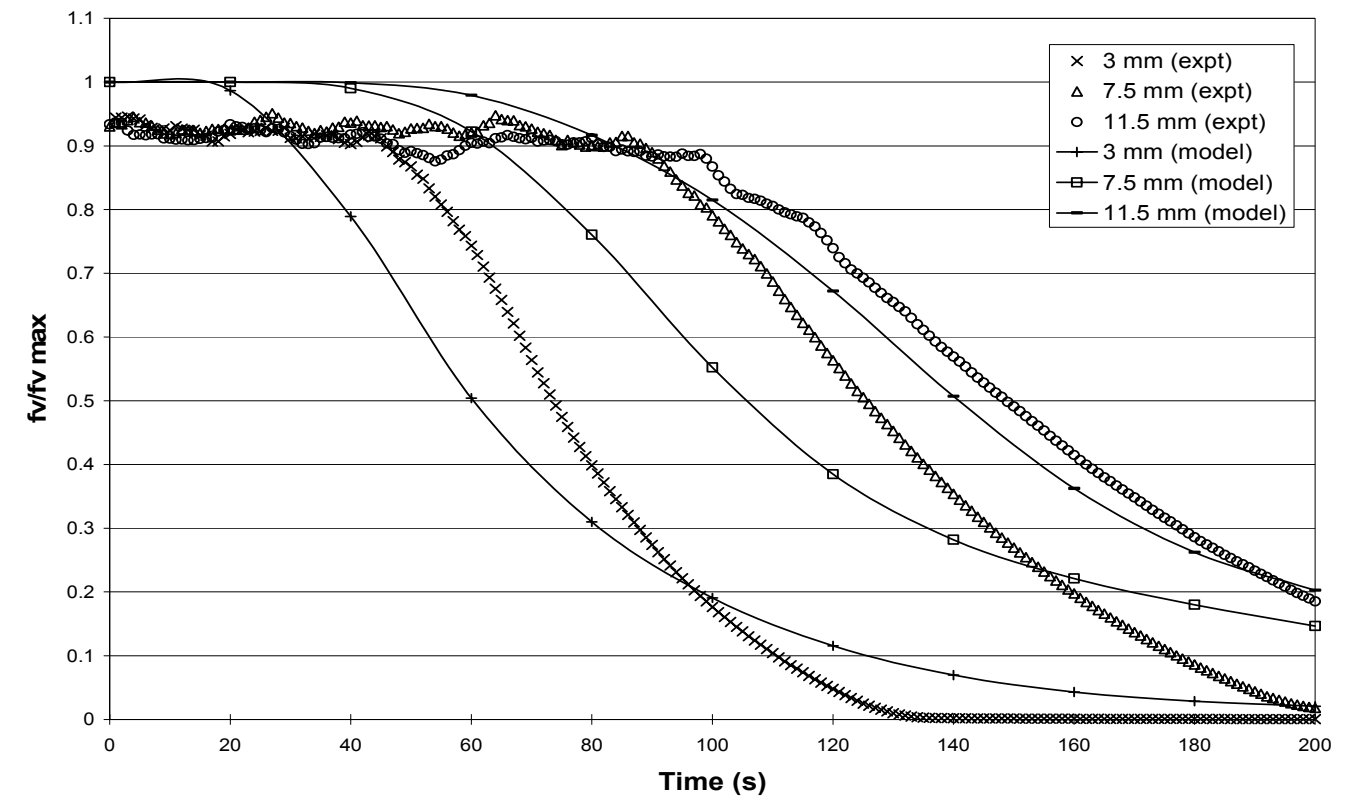

Figure 4.14: Comparison volume fraction loading plots with no electric field.

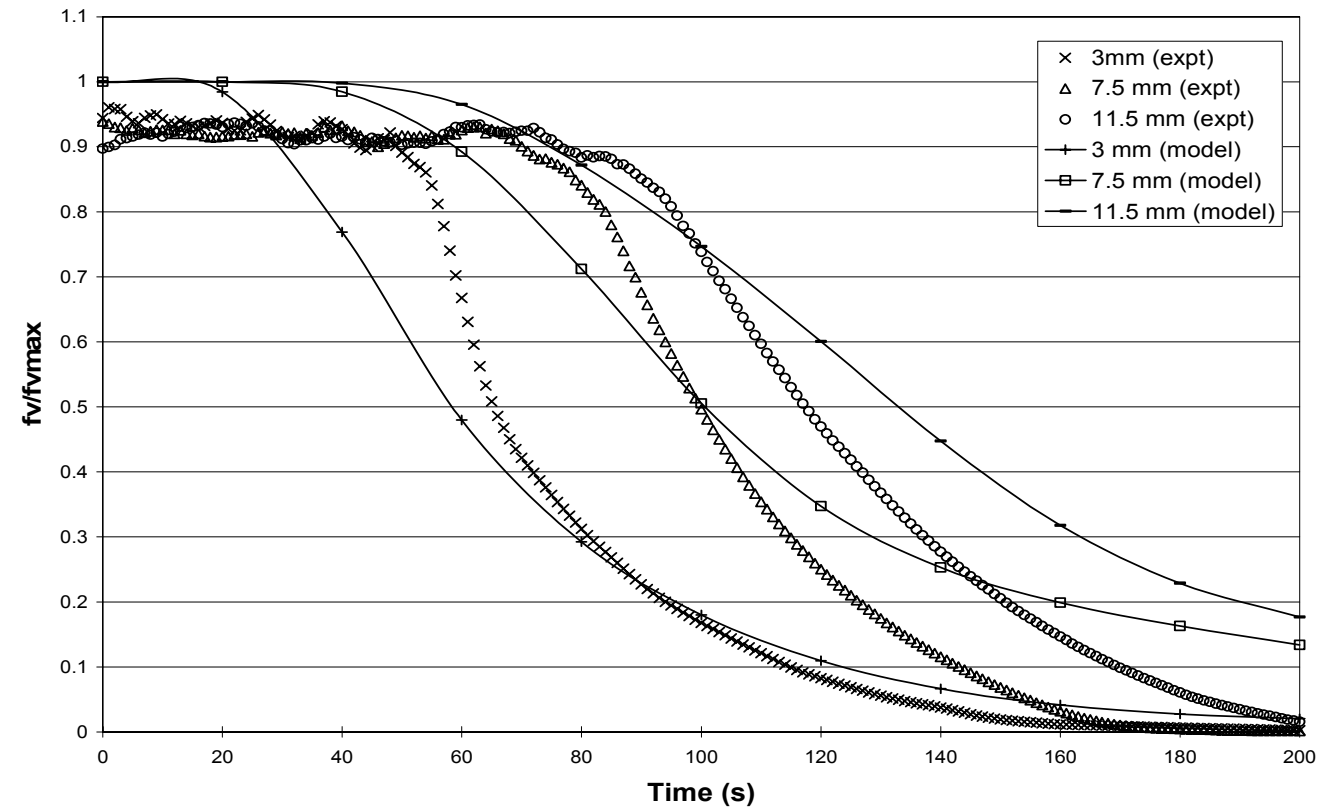

Figure 4.15: Comparison volume fraction loading plots with electric filed of 25 $\mathrm{kV} / \mathrm{m}$. 


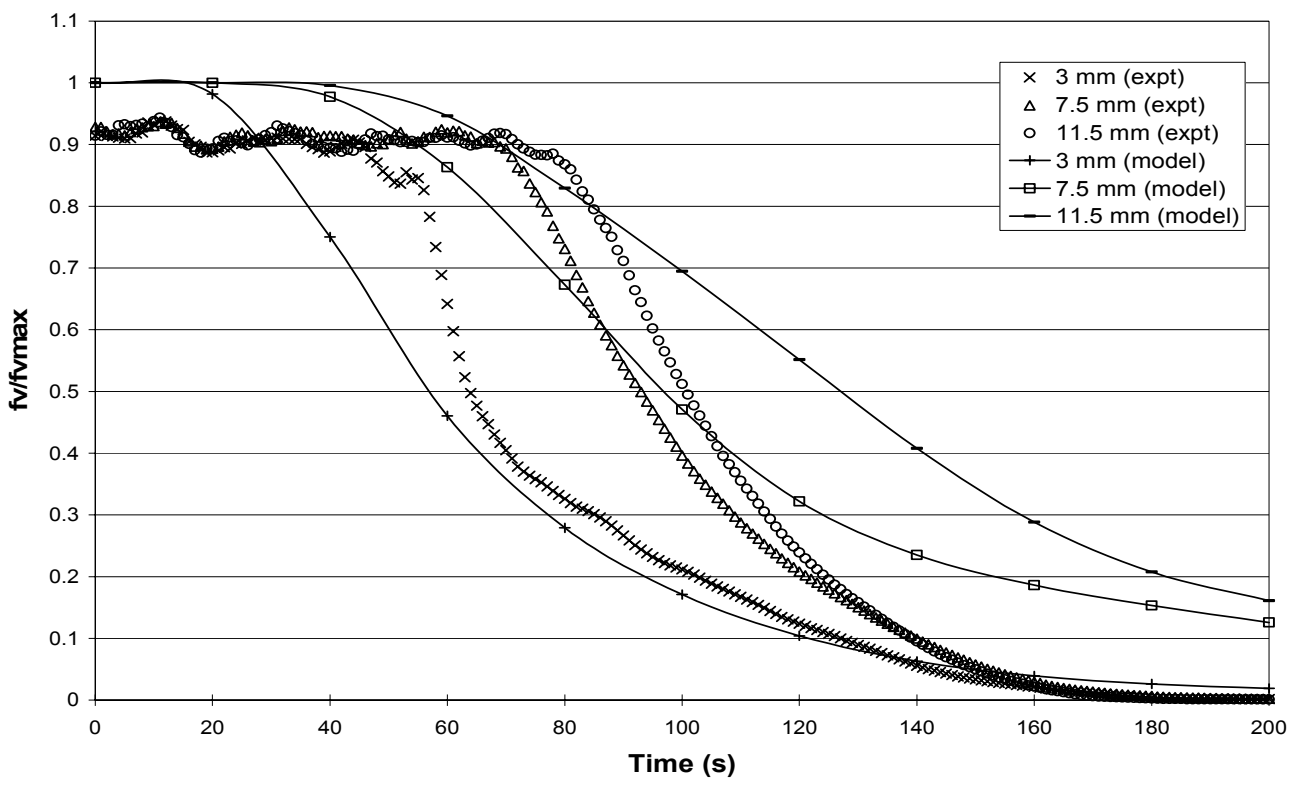

Figure 4.16: Comparison volume fraction loading plots with electric filed of 50 $\mathrm{kV} / \mathrm{m}$.

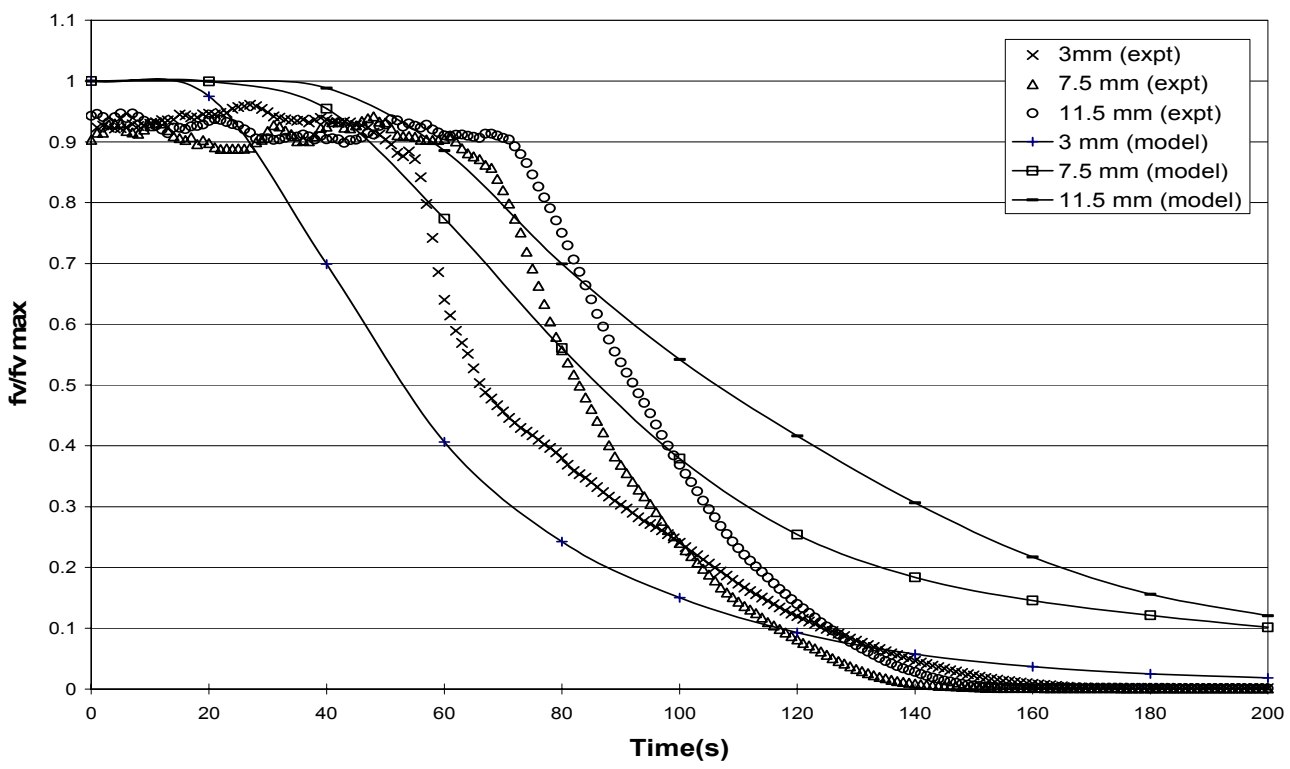

Figure 4.17: Comparison volume fraction loading plots with electric filed of 75 $\mathrm{kV} / \mathrm{m}$. 
The computational model and experimental data have similar settling trends. However, the model and the experimental data deviate from one another randomly as the electric field increases. The deviation is enhanced at later stages during the simulation. This can be observed from Table 4.2 which lists the percentage error of the model for the time required to reduce to $50 \%$ of the initial loading. Table 4.3 is a table listing the percentage error for the time required to reduce to $20 \%$ of the initial loading. A comparison of the corresponding values in tables 4.2 and 4.3 shows no regular trend in the errors.

Table 4.2: Errors in the model for the time required to reduce to $50 \%$ of the initial loading.

\begin{tabular}{|c|c|c|c|}
\hline \multirow{2}{*}{$\begin{array}{c}\text { Electrical Field } \\
(\mathbf{k V} / \mathbf{m})\end{array}$} & $\mathbf{3}$ Error (\%) \\
\cline { 2 - 4 } & $\mathbf{3 m}$ & $\mathbf{7 . 5} \mathbf{~ m m}$ & $\mathbf{1 1 . 5} \mathbf{~ m m}$ \\
\hline 0 & 17.8 & 15.8 & 5.37 \\
\hline 25 & 10.77 & 1 & 12.7 \\
\hline 50 & 10.9 & 4.3 & 29 \\
\hline 75 & 18.2 & 3.6 & 17.4 \\
\hline
\end{tabular}


Table 4.3: Errors in the model for the time required to reduce to $20 \%$ of the initial loading.

\begin{tabular}{|c|c|c|c|}
\hline \multirow{2}{*}{$\begin{array}{c}\text { Electrical field } \\
(\mathbf{k V} / \mathbf{m})\end{array}$} & $\mathbf{3} \mathbf{~ m m}$ & $\mathbf{7 . 5} \mathbf{~ m m}$ & $\mathbf{1 1 . 5} \mathbf{~ m m}$ \\
\cline { 2 - 4 } & 1 & 6.9 & 1.5 \\
\hline 0 & 2.1 & 26.9 & 27.3 \\
\hline 50 & 9.7 & 25.4 & 46.4 \\
\hline 75 & 16.9 & 28.8 & 45.6 \\
\hline
\end{tabular}

The differences in the model and the experimental data could be due to the model accounting for the combination of Brownian and gravitational coagulation by adding them to give the total coagulation kernel. This is based on the premise that the velocity acquired by a particle due to Brownian diffusion is independent of the velocity due to gravitational acceleration. However, the gravitational acceleration acts primarily to bring particles in proximity while the Brownian motion actually overcomes the inter-particle forces. This would mean that the two effects are not important simultaneously and taking a sum of the coagulation rates would not be consistent. The modification of the gravitational kernel to account for the electric field effects could have further magnified the inconsistencies in the model. 


\section{CHAPTER 5: CONCLUSIONS AND FUTURE WORK}

\subsection{Conclusions}

Experiments conducted by Schmidt (2000) had shown that electric fields shorten the settling times of an aerosol. The purpose of this project was to devise a numerical model to simulate the settling process in the presence of electrical fields. To account for electric field effects on the settling process, a trajectory analysis was used. This required calculating the force coefficients on the particles due to electric fields. A computer program based on the bispherical polar coordinate method (Davis, 1964) was written to calculate the force coefficients. Using these force coefficients, a trajectory analysis was performed. This involved integrating the trajectory equation to find the limiting displacement (from the vertical axis of symmetry between the particle pair), that a particle would have to lie within, for the two particles to collide. This critical displacement along with the particle pair size gives the collision efficiency. The collision efficiencies for particle pairs under electric fields of $25 \mathrm{kV} / \mathrm{m}, 50 \mathrm{kV} / \mathrm{m}$ and $75 \mathrm{kV} / \mathrm{m}$ were calculated. The collision efficiencies were then used to modify the aerosol simulation code MAEROS (Gelbard and Seinfeld, 1980).

The original code MAEROS was also modified to account for elevation effects during the settling process. As an aerosol settles, stratification occurs such that the top layers clear out faster than the lower layers. To simulate this, the volume of the chamber was divided into equal horizontal compartments having the same floor or ceiling area as the chamber but having reduced heights. The conservation of mass equation was solved for each of these horizontal 
compartments. The multiple level simulations were used for comparisons with extinction measurements done at different elevations within a chamber.

Simulations, performed under the settings to match the experimental conditions of Schmidt (2000), showed that the electric field had an effect of reducing settling times. Also the reduced settling times were more pronounced with higher electric fields and more so towards the floor of the cell. The time required to reduce to $50 \%$ of the initial loading was reduced by $10 \%, 18.86 \%$ and $23.4 \%$ for the elevations of $3 \mathrm{~mm}, 7.5 \mathrm{~mm}$ and $11.5 \mathrm{~mm}$. with an electric field of $75 \mathrm{kV} / \mathrm{m}$ acting along the direction of gravity. The reduced settling times were due to enhanced particle agglomeration occurring due to the electric field.

To validate the model, comparisons were made with experimental data from Schmidt (2000). This showed that the model deviated from the experiments as the field increased. Also, this deviation was enhanced at later stages of the simulation. The deviation could be due to the MAEROS model accounting for Brownian and gravitational agglomeration by adding the two agglomeration kernels. Another limitation was that the model assumes the aerosol particles to be perfect spheres. However, cigarette smoke particles are basically many fractals and their characteristics may be quite different from spheres. Also, the model assumes that each collision results in the two particles agglomerating to form a single particle. These assumptions together change the dynamics of the system. 


\subsection{Future work}

To calculate the agglomeration kernel, it would be better if the forces acting on the particles due to Brownian diffusion and gravitational acceleration are added in a theoretically consistent manner to give a more accurate account for the velocity acquired by the particles. Further the forces induces by the electric field would need to be coupled. This resultant force could then be used to calculate the relative velocities between particles, thus giving us the agglomeration kernel. Also, the models used in this study were applicable for pair-wise systems. These models would need to be extended to account for multiparticle effects. Further, incorporating the fractal properties of the particles could make the model more accurate.

The collision efficiency plots in Figure 3.8, 3.9 and 3.10 indicate that the electric field has the greatest effect in enhancing the collision frequencies among pairs of particles of small size ( $<1$ micron). During the agglomeration process, an aerosol has a higher percentage of smaller particles initially, and as the evolution proceeds, the particle size distribution moves from towards larger particle sizes. As a result, the effect of the electric field is largest during the initial stages, and reduces as the settling proceeds. The electric field could be used only in the initial stages, thus saving power and making the method more cost effective. An examination of the exact time periods for this method could be useful in the evaluation of this process as a cost-effective method for aerosol removal.

The effects of orientation of the electric field on the collision with gravity could also be studied. Zhang (1995) found that collision efficiencies with an 
electric field operating normal to the gravitational force were an order of magnitude higher than those with the field acting along gravitational force. Also, an electrical field (of small magnitude) acting in the direction against gravity could be extremely efficient in increasing the collision efficiency. 


\section{Appendix A}

The code 'force.c' is program in C-language used for calculating the force components $F_{1}, F_{2}$ and $F_{3}$ based on the bispherical polar method by Davis (1964). The equations used in the program are listed in sections 3.3 and 3.4.

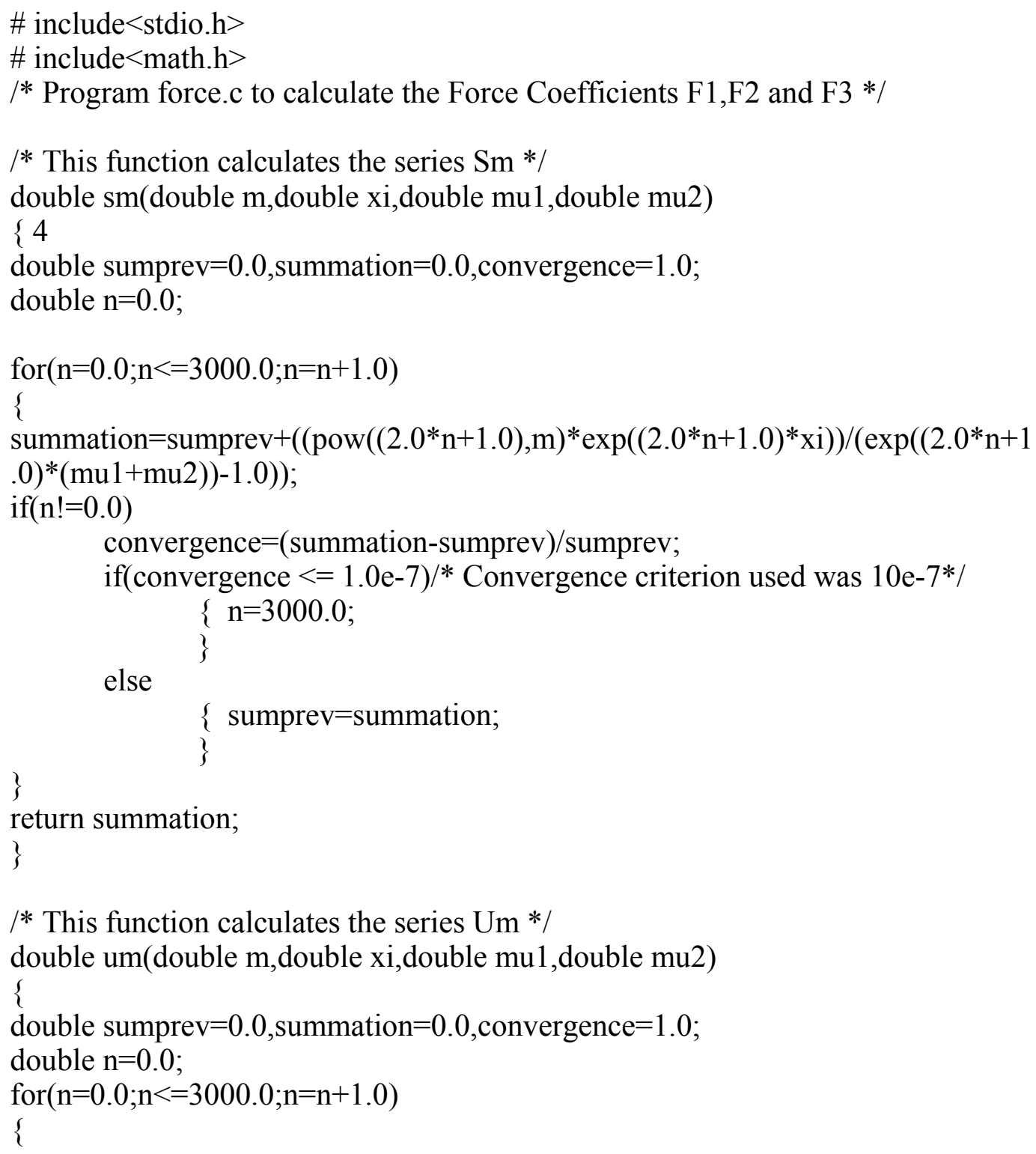




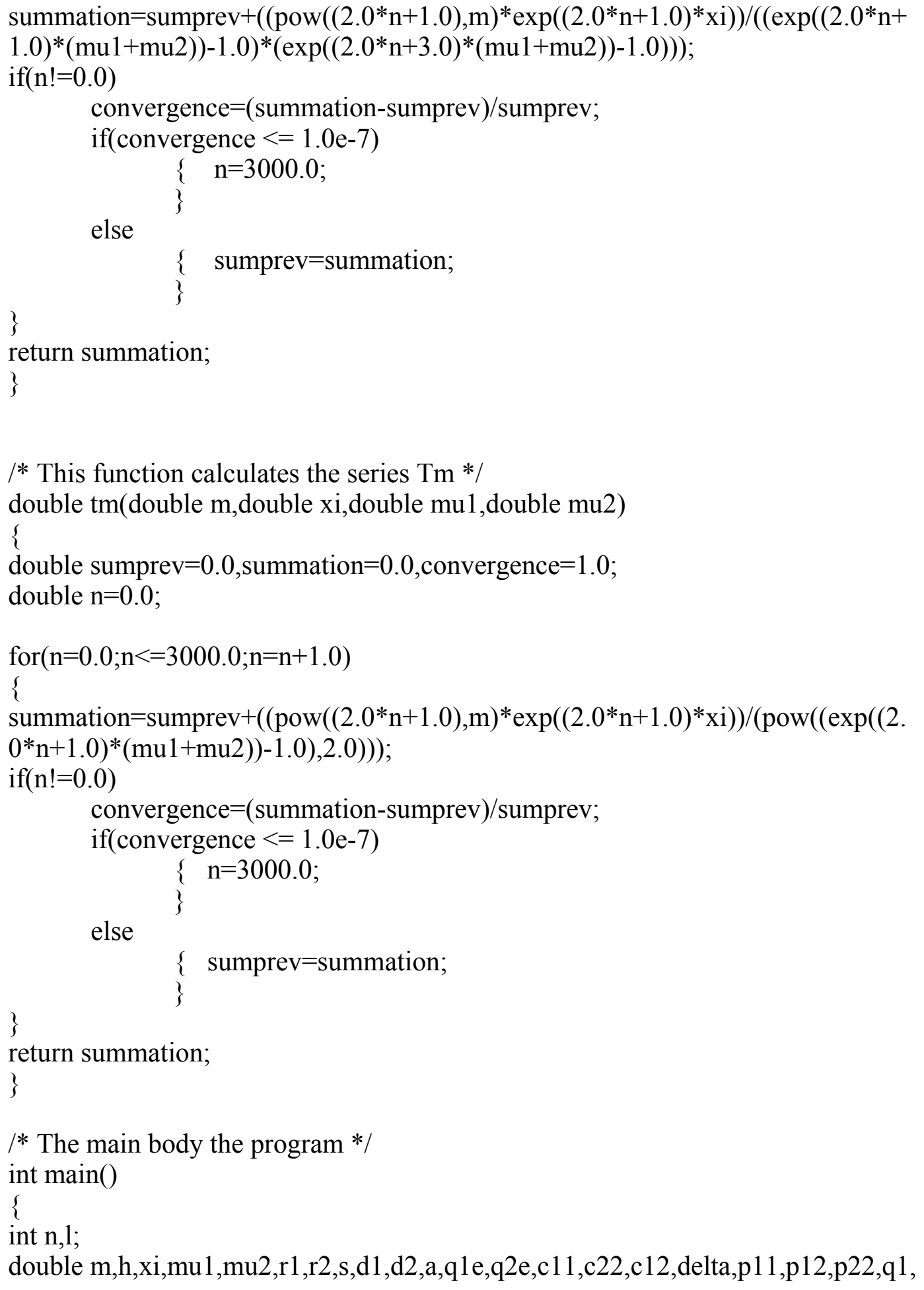


q2,w1,w2,v1,v2,alpha,gamma,kappa1,F1,F2,F3,Fnondim,nondimS,E,epsilon,si,nd ,musum1,musum2,kappa2,kappa3,ratio1;

double szero[3], sone[3],f[24];

double K[3][24];

fflush;

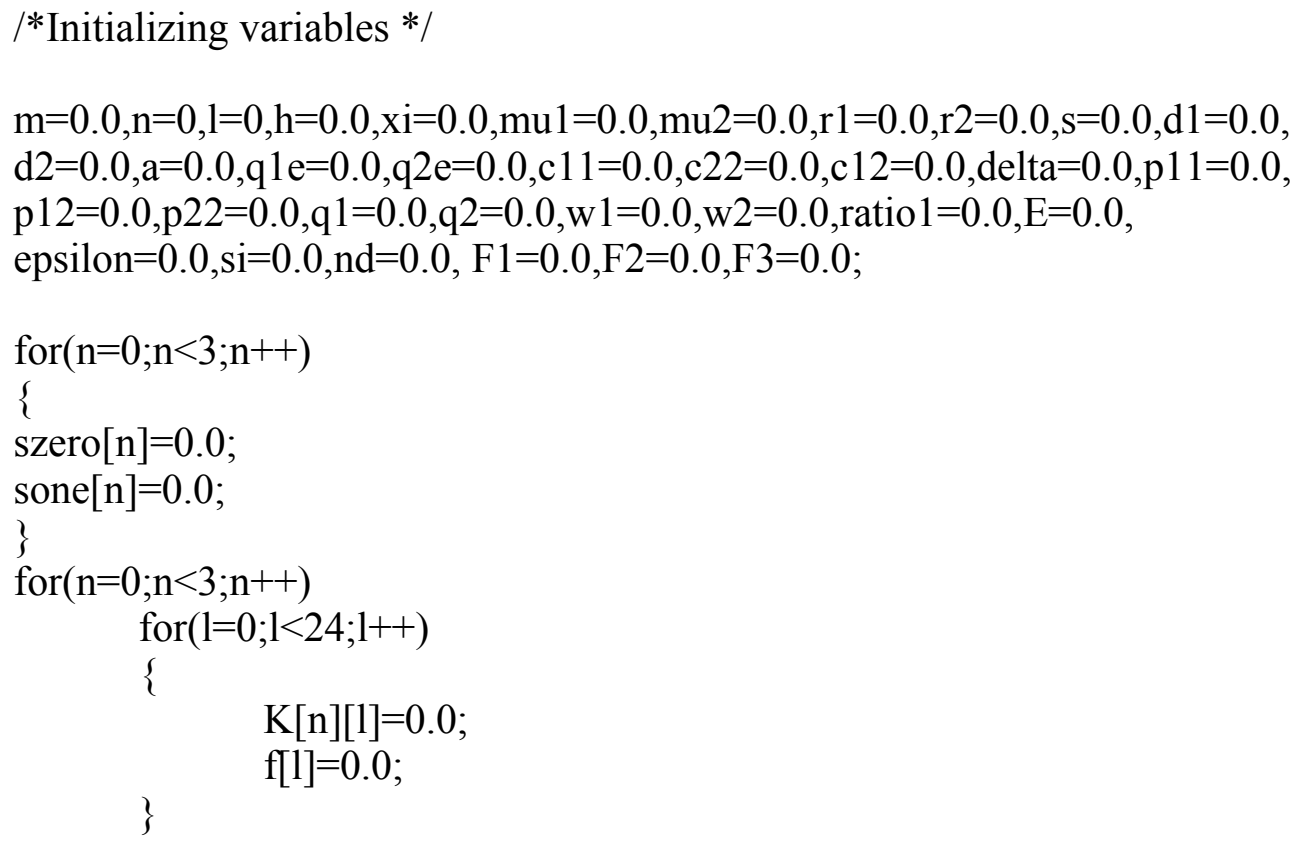

$\mathrm{r} 2=1 \mathrm{e}-6 ;{ }^{*} \mathrm{~A}$ value for $\mathrm{r} 2$ is set to $1 \mathrm{E}-6$. However this does not affect the calculations for $\mathrm{F} 1, \mathrm{~F} 2$ and $\mathrm{F} 3$ */

printf("Enter LAMBDA(R2/R1) ratio :");

scanf("\%lf",\&ratio1);

printf("Enter Non Dimensional S:");

scanf("\%lf",\&nondimS);

/* Accept Size ratio LAMBDA and the dimensionless separation distance are inputs */

$\mathrm{r} 1=\mathrm{r} 2 /$ ratio 1 ;

$\mathrm{s}=(\mathrm{r} 1+\mathrm{r} 2) *$ nondimS $/ 2 ; / *$ the dimensionless distance is converted to a dimensional separation */

/* Setting the values of the variables based on the bispherical polar method*/

$\mathrm{h}=\mathrm{r} 1+\mathrm{r} 2+\mathrm{s}$;

$\mathrm{d} 1=(\mathrm{h} * \mathrm{~h}+\mathrm{r} 1 * \mathrm{r} 1-\mathrm{r} 2 * \mathrm{r} 2) /(2 * \mathrm{~h})$;

$\mathrm{d} 2=(\mathrm{h} * \mathrm{~h}+\mathrm{r} 2 * \mathrm{r} 2-\mathrm{r} 1 * \mathrm{r} 1) /(2 * \mathrm{~h})$; 


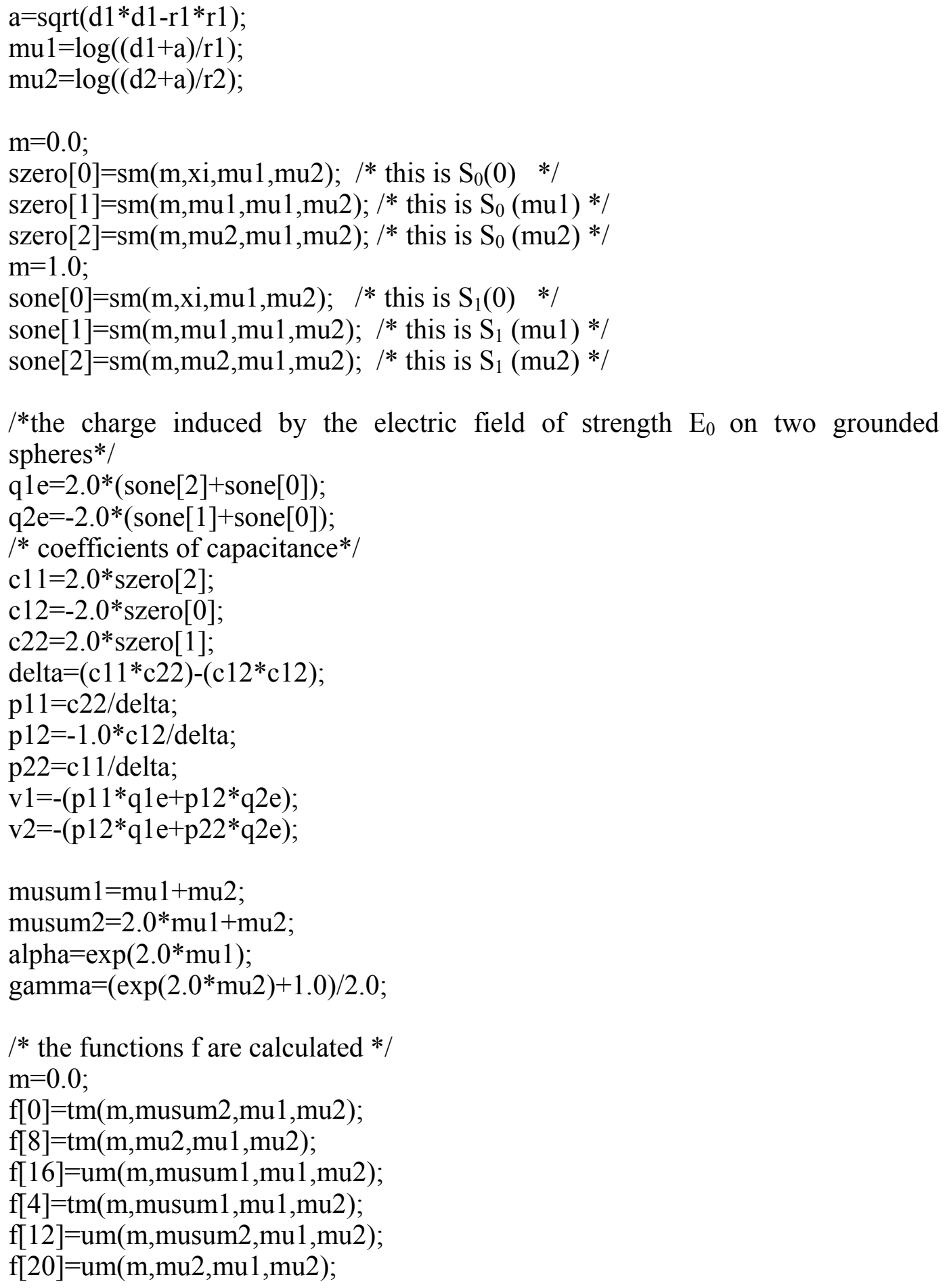


$\mathrm{m}=1.0$;

$\mathrm{f}[1]=\mathrm{tm}(\mathrm{m}$, musum $2, \mathrm{mu} 1, \mathrm{mu} 2)$;

$\mathrm{f}[9]=\operatorname{tm}(\mathrm{m}, \mathrm{mu} 2, \mathrm{mu} 1, \mathrm{mu} 2)$;

$\mathrm{f}[17]=\mathrm{um}(\mathrm{m}$, musum $1, \mathrm{mu} 1, \mathrm{mu} 2)$;

$\mathrm{f}[5]=\operatorname{tm}(\mathrm{m}$, musum $1, \mathrm{mu} 1, \mathrm{mu} 2)$;

$\mathrm{f}[13]=\mathrm{um}(\mathrm{m}$, musum $2, \mathrm{mu} 1, \mathrm{mu} 2)$;

$\mathrm{f}[21]=\mathrm{um}(\mathrm{m}, \mathrm{mu} 2, \mathrm{mu} 1, \mathrm{mu} 2)$;

$\mathrm{m}=2.0$;

$\mathrm{f}[2]=\mathrm{tm}(\mathrm{m}$, musum $2, \mathrm{mu} 1, \mathrm{mu} 2)$;

$\mathrm{f}[10]=\operatorname{tm}(\mathrm{m}, \mathrm{mu} 2, \mathrm{mu} 1, \mathrm{mu} 2)$;

$\mathrm{f}[18]=\mathrm{um}(\mathrm{m}, \mathrm{musum} 1, \mathrm{mu} 1, \mathrm{mu} 2)$;

$\mathrm{f}[6]=\operatorname{tm}(\mathrm{m}$, musum $1, \mathrm{mu} 1, \mathrm{mu} 2)$;

$\mathrm{f}[14]=\mathrm{um}(\mathrm{m}, \mathrm{musum} 2, \mathrm{mu} 1, \mathrm{mu} 2)$;

$\mathrm{f}[22]=\mathrm{um}(\mathrm{m}, \mathrm{mu} 2, \mathrm{mu} 1, \mathrm{mu} 2)$;

$\mathrm{m}=3.0$;

$\mathrm{f}[3]=\operatorname{tm}(\mathrm{m}$, musum $2, \mathrm{mu} 1, \mathrm{mu} 2)$;

$\mathrm{f}[11]=\operatorname{tm}(\mathrm{m}, \mathrm{mu} 2, \mathrm{mu} 1, \mathrm{mu} 2)$;

$\mathrm{f}[19]=\mathrm{um}(\mathrm{m}, \mathrm{musum} 1, \mathrm{mu} 1, \mathrm{mu} 2)$;

$\mathrm{f}[7]=\operatorname{tm}(\mathrm{m}$, musum $1, \mathrm{mu} 1, \mathrm{mu} 2)$;

$\mathrm{f}[15]=\mathrm{um}(\mathrm{m}, \mathrm{musum} 2, \mathrm{mu} 1, \mathrm{mu} 2)$;

$\mathrm{f}[23]=\mathrm{um}(\mathrm{m}, \mathrm{mu} 2, \mathrm{mu} 1, \mathrm{mu} 2)$;

/* $\mathrm{K}[\mathrm{j}][\mathrm{k}]$ values based on Table 3 . In $\mathrm{C}$ programming, arrays are indexed starting from 0 , so index 0 corresponds to 1 in the system expressed in table $3 * /$

$\mathrm{K}[0][0]=0.0$;

$\mathrm{K}[0][1]=\operatorname{pow}(\mathrm{v} 2,2.0)$;

$\mathrm{K}[0][2]=-2.0 * \mathrm{v} 2$;

$\mathrm{K}[0][3]=1.0$;

$\mathrm{K}[0][4]=0.0$

$\mathrm{K}[0][5]=-2.0 * \mathrm{v} 1 * \mathrm{v} 2$;

$\mathrm{K}[0][6]=2.0 *(\mathrm{v} 1-\mathrm{v} 2)$;

$\mathrm{K}[0][7]=2.0$

$\mathrm{K}[0][8]=0.0$;

$\mathrm{K}[0][9]=\operatorname{pow}(\mathrm{v} 1,2.0)$;

$\mathrm{K}[0][10]=2.0 * \mathrm{v} 1$;

$\mathrm{K}[0][11]=1.0$;

$\mathrm{K}[0][12]=-1.0 *$ gamma*alpha*v2*(v2-2.0);

$\mathrm{K}[0][13]=-1.0 *$ gamma*alpha* $($ pow $(\mathrm{v} 2,2.0)-4.0 * \mathrm{v} 2+2.0)$;

$\mathrm{K}[0][14]=-1.0 *$ gamma*alpha*(-2.0*v2+3.0);

$\mathrm{K}[0][15]=-1.0 *$ gamma*alpha; 
$\mathrm{K}[0][16]=-1.0 *$ gamma* $(2.0 * \mathrm{v} 1 *$ alpha-v $1 * \mathrm{v} 2 *($ alpha +1.0$)-2.0 * \mathrm{v} 2)$;

$\mathrm{K}[0][17]=-1.0 *$ gamma* $(\mathrm{v} 1 *(3.0 *$ alpha +1.0$)-\mathrm{v} 2 *($ alpha +3.0$)+(2.0-$

$\left.\mathrm{v} 1{ }^{*} \mathrm{v} 2\right) *($ alpha +1.0$\left.)\right)$;

$\mathrm{K}[0][18]=-1.0 *$ gamma*(alpha +1.0$) *(\mathrm{v} 1-\mathrm{v} 2+3.0)$;

$\mathrm{K}[0][19]=-1.0 *$ gamma*(alpha +1.0$)$;

$\mathrm{K}[0][20]=-1.0 *$ gamma*v1*(v1+2.0);

$\mathrm{K}[0][21]=-1.0 *$ gamma* $(\operatorname{pow}(\mathrm{v} 1,2.0)+4.0 * \mathrm{v} 1+2.0)$;

$\mathrm{K}[0][22]=-1.0 *$ gamma* $(2.0 * \mathrm{v} 1+3.0)$;

$\mathrm{K}[0][23]=-1.0 *$ gamma;

$\mathrm{K}[1][0]=0.0$;

$\mathrm{K}[1][1]=-1.0$;

$\mathrm{K}[1][2]=0.0$

$\mathrm{K}[1][3]=1.0$;

$\mathrm{K}[1][4]=0.0$

$\mathrm{K}[1][5]=2.0$;

$\mathrm{K}[1][6]=0.0$;

$\mathrm{K}[1][7]=-2.0$;

$\mathrm{K}[1][8]=0.0$;

$\mathrm{K}[1][9]=-1.0$;

$\mathrm{K}[1][10]=0.0$;

$\mathrm{K}[1][11]=1.0$;

$\mathrm{K}[1][12]=3.0 *$ gamma*alpha;

$\mathrm{K}[1][13]=$ gamma*alpha;

$\mathrm{K}[1][14]=-3.0 *$ gamma*alpha;

$\mathrm{K}[1][15]=-1.0 *$ gamma*alpha;

$\mathrm{K}[1][16]=-3.0 *$ gamma*(alpha +1.0$)$;

$\mathrm{K}[1][17]=-1.0 *$ gamma*(alpha +1.0$)$;

$\mathrm{K}[1][18]=3.0 *$ gamma*(alpha +1.0$)$;

$\mathrm{K}[1][19]=$ gamma* $($ alpha +1.0$)$;

$\mathrm{K}[1][20]=3.0 *$ gamma;

$\mathrm{K}[1][21]=$ gamma;

$\mathrm{K}[1][22]=-3.0 *$ gamma;

$\mathrm{K}[1][23]=-1.0 *$ gamma;

$\mathrm{K}[2][0]=0.0$;

$\mathrm{K}[2][1]=0.0$;

$\mathrm{K}[2][2]=0.0$;

$\mathrm{K}[2][3]=0.0$;

$\mathrm{K}[2][4]=0.0$;

$\mathrm{K}[2][5]=0.0$;

$\mathrm{K}[2][6]=0.0$; 


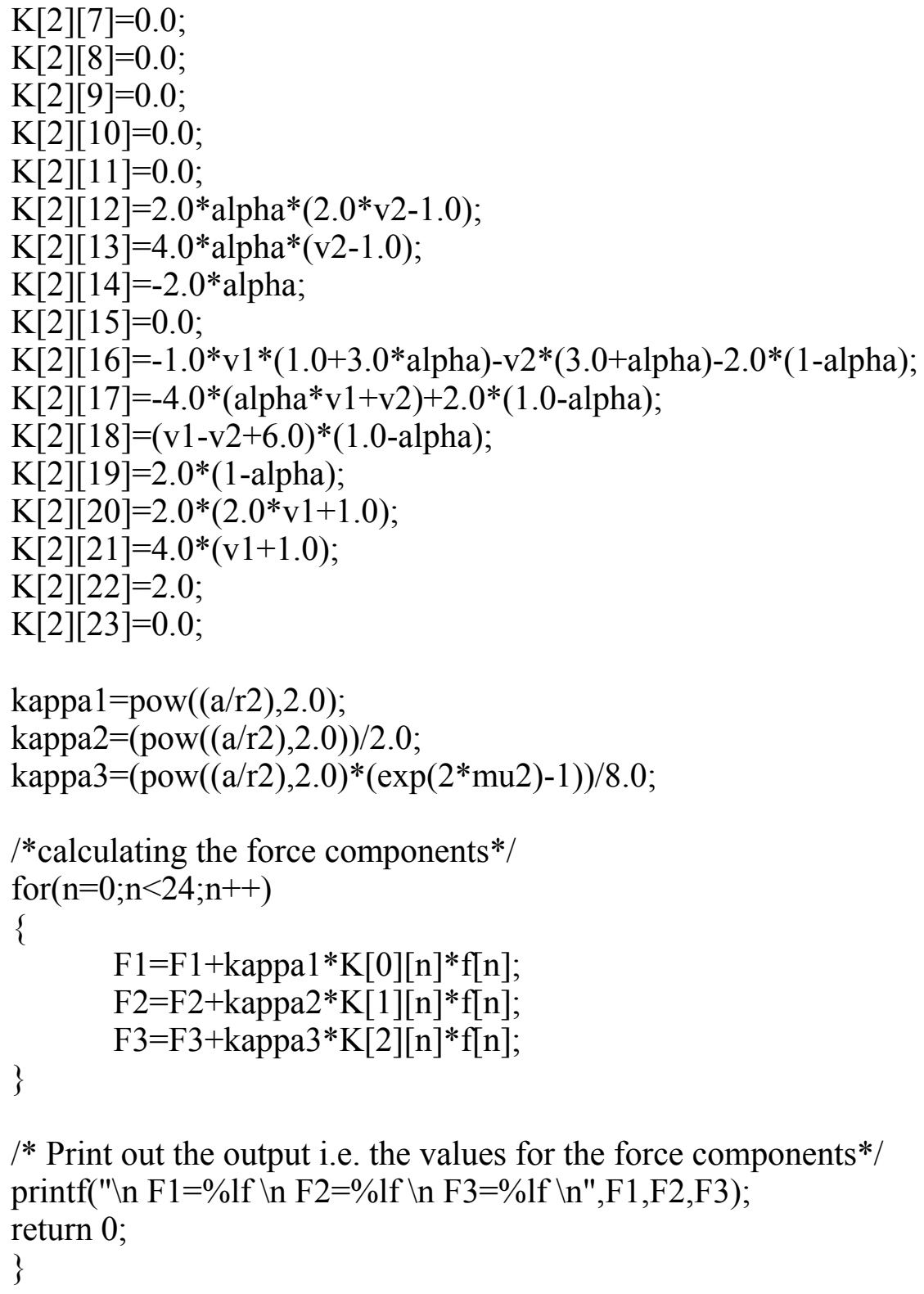




\section{References}

Corcoran, T.E., Hitron R., Humphrey, W., Chigier, N. (2000) Optical Measurement of Nebulizer Sprays: A Quantitative Comparison of Diffraction, Phase Doppler Interferometry, and Time of Flight Techniques, J. Aerosol Sci., 31, 35-50.

Chen, B.T., Jamenyi, J., Yeh, H.C., Mauderly and Cuddihy, R.G. (1990) Physical Characterization of Cigarette Smoke Aerosol Generated from a Walton Smoke Machine, Aerosol Sci. Tech., 12, 364.

Davis, M.H. (1964) Two Charged Spherical Conductors in a Uniform Electric Field: Forces and Field Strength, Quart. J. Mech. Appl. Math, 17, 499.

Davis, R.H. (1984) Rate of Coagulation of a Dilute Polydisperse system of Sedimenting Spheres, J. Fluid Mech., 145, 179.

Ezekoye, O.A. (1999) Simulation of Acoustic Agglomeration Processes using a Sectional a Algorithm, J. Aerosol Sci., 30, 1117

Gelbard, F. and Seinfeld, J.H. (1980) Simulation of Multicomponent Aerosol Dynamics, J. Colloid Interface Sci., 78, 485.

Hamaker, H.C. (1937) The London- Van Der Waals Attraction Between Spherical Particles, Physica, 4, 1058.

Melik, D.H. and Fogler, H.S. (1984) Effect of Gravity on Brownian Flocculation, J. Colloid Interface Sci., 101, 84.

Reist, P.C. (1993), Aerosol Science and Technology, $2^{\text {nd }}$ ed, McGraw-Hill, New York.

Schmidt, J.J. (2000) Experimental Study of Electro-Coalescence in a Unit Reactor, Master's Thesis, University of Texas.

Wang, H., Zeng, S., Loewenberg, M., Davis, R.H. (1997) Particle Aggregation due to Combined Gravitational and Electrophoretic Motion, J. Colloid Interface Sci., 187, 213-220. 
Waterman, L.C., (1965) Electrical Coalescers, Chem. Eng. Prog., 61, 51.

Wen, C.S., Zhang, L. and Lin, H. (1991) The Rate of Coagulation of Particles in a Sedimenting Dispersion at Large Peclet Number, J. Colloid Interface Sci., $142,257$.

Williams, T.J. and Bailey, A.G. (1986) Changes in the Size Distribution of a Water-in-Oil Emulsion Sue to electric Field Induced Coalescence, IEEE Trans. Ind. Appl., IA-22, 536.

Zhang, X. and Davis, R.H. (1991) The Rate of Collisions due to Brownian or Gravitational Motion of Small Drops, J. Fluid Mech., 230, 479-504.

Zhang, X., Basaran, O.A. and Wham, R.M. (1995) Theoretical Prediction of Electric Field-Enhanced Coalescence of Spherical Drops, AIChE J., 41, 1929. 


\title{
THE EFFECT OF ELECTRIC FIELD ON MOVING AQUEOUS AEROSOL SUSPENDED IN A DYNAMIC FLUID CHAMBER
}

\author{
A Thesis \\ Presented for the \\ Master of Science \\ Degree \\ The University of Tennessee, Knoxville
}




\begin{abstract}
The primarily aim of this research work was to develop a better understanding of electrically driven drop-drop and drop-electrode interactions in an aerosol flow chamber. A particular application of this technology is for treatment processing of radioactive droplets generated in tank waste at Department of Energy sites.

In this research, experimental studies were conducted to explore methods of enhancing the agglomeration of micron sized particles. In such studies, the removal of aerosols by means of DC electric fields was investigated. As a high strength electric field is of importance when building a full-scale agglomerator, agglomeration experiments were carried out with the use of a DC electric field.
\end{abstract}


An experimental setup was designed and built in which to carry out various aerosol experiments to produce the needed data for this research. The experimental setup consisted of two parallel electrodes, a laser-light-scattering particle sizing system, a rectangular test chamber, dry-ice trap, and a variable high voltage DC power supply. The experimental data were used to accomplish the following tasks:

- Quantify removal of drops from an air stream by an electric field.

- Measure drop size distribution along the flow chamber and determine the effect of an electric field on drop coalescence.

The data indicate that the droplet removal efficiency increases approximately linearly with voltage over the range of voltages investigated, except for one case. In this case, there was no appreciable increase in droplet removal efficiency in the range of 24$26 \mathrm{kV}$, suggesting a saturation effect may be occurring. The analysis also shows that in general the removal efficiency is a function of initial droplet concentration. For instance, for a concentration of $24.23 \mathrm{~g} / \mathrm{m}^{3}$, the maximum removing efficiency is approximately 85.5 percent while for the concentration of $8.32 \mathrm{~g} / \mathrm{m}^{3}$ the maximum removing efficiency is 65.0 percent under the same experimental conditions.

The analysis of the droplet distribution reveals that the mean size of droplets does not always change with voltage; however, the distribution of the number of droplets does change. Appreciable changes in the number of droplets were observed for droplet sizes in a typical range of 4 to $10 \mu \mathrm{m}$. 
The overall experience and knowledge gained in this research will be useful in developing effective applications of superimposed electric fields for the elimination of mist from air or gaseous streams. 


\section{TABLE OF CONTENTS}

CHAPTER

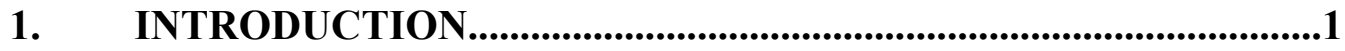

1.1 Background ......................................................................

1.2 Problem Statement and Research Objectives ................................. 3

1.3 Thesis Organization ........................................................................5

2. REVIEW OF PERTINENT LITERATURE.................................6

2.1 Cyclone Collector ..................................................................6

2.2 Fabric Filters ....................................................................... 7

2.3 Electrostatic Precipitators .........................................................8

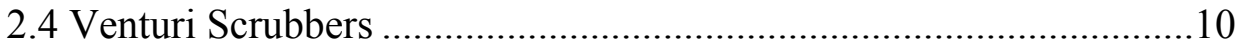

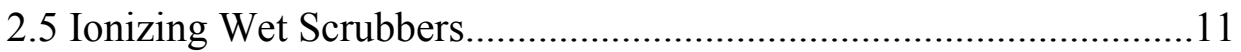

2.6 Collision Scrubbers ..................................................................... 12

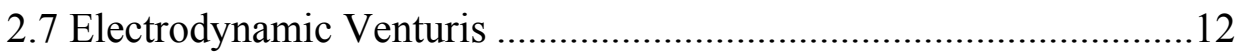

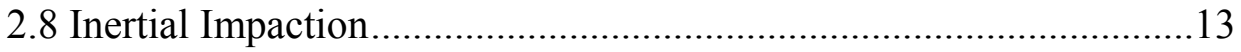

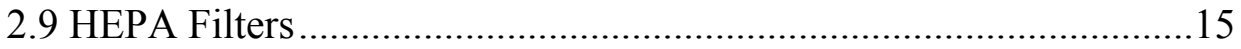

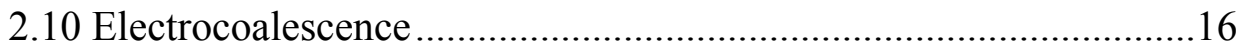

2.11Electro-filtration of Aerosols ......................................................19

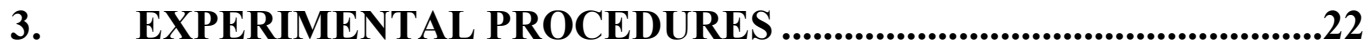

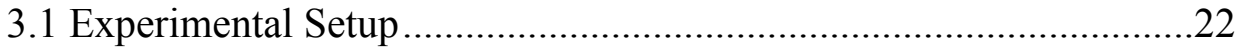

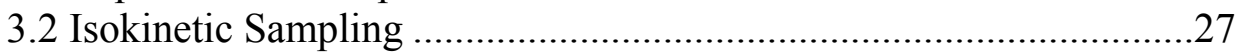

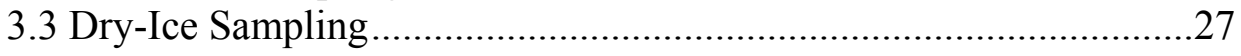

3.4 Particle Size Distribution Measurement and Control ........................32

4. THEORETICAL CONSIDERATIONS .........................................37

4.1 Equation of Motion for a Single Aerosol Particle ............................37

4.2 Particle Motion in an Electric Field ..................................................39

4.3 Electric field Between Parallel Plates ..............................................43

4.4 Theory of Electro-Coalescence ...................................................45

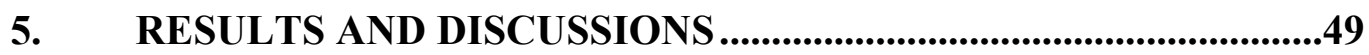

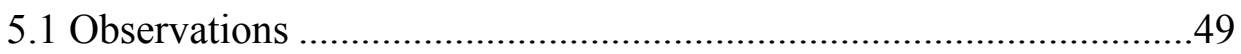

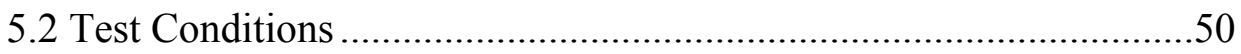

5.3 Effects of Electric Field Strength on Droplet Concentration..............51

5.4 Effects of Electric Field Strength on Light Transmission .................54

5.5 Effect of Electric Field on Droplet Numbers ..................................57

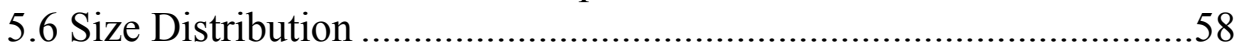

5.7 Multiple Elevation Results........................................................63

5.8 Multiple Mist Densities at Constant Flow Rates ............................65

5.9 Multiple Flow Rates at Constant Mist Concentration......................68 
5.10 Removing Efficiency of the Droplets .....

6. SUMMARY and CONCLUSIONS ......................................................76

7. RECOMMENDATIONS.............................................................79

REFERENCES.............................................................................88

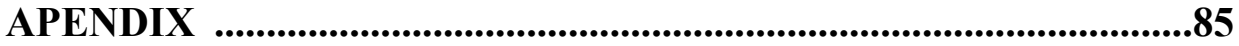

VITA 


\section{LIST OF TABLES}

Table 5.1 Percent error of measured and calculated mass of collected water rom room's air at variable flow rates

Table 5.2 Comparison of percent light transmission at various heights and applied voltages

Table 5.3 Effect of applied voltage on droplet concentration for

Reynolds Number of 1350 .66

Table 5.4 Effect of applied voltage on droplet concentration for

Reynolds Number of 1950

Table 5.5 Constant droplet concentration at

Reynolds Numbers of 940,1350 , and 1680

Table 5.6 Exit mist concentrations for

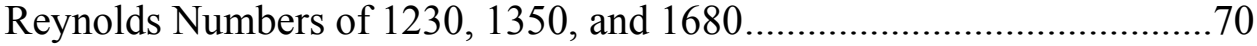

Table 5.7 Exit mist concentration for

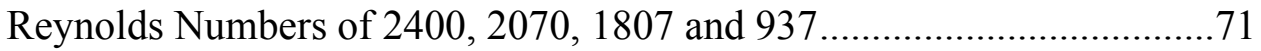

Table 5.8 Exit mist concentrations for

Reynolds Numbers of 2200, 1680, 1350 and 940

Table A.1a Droplet Diameter vs. Cumulative (\%) and Volume Frequency (\%)

Respectively for conditions of Figure A la

Table A.1b Droplet Diameter vs. Cumulative (\%) and Volume Frequency (\%)

Respectively for conditions of Figure Alb....

Table A.1c Droplet Diameter vs. Cumulative (\%) and Volume Frequency (\%)

Respectively for conditions of Figure A1c

Table A.1d Droplet Diameter vs. Cumulative (\%) and Volume Frequency (\%)

Respectively for conditions of Figure A1d

Table A.1e Droplet Diameter vs. Cumulative (\%) and Volume Frequency (\%)

Respectively for conditions of Figure Ale

Table A.1f Droplet Diameter vs. Cumulative (\%) and Volume Frequency (\%) Respectively for conditions of Figure A1f.....

Table A.2a Droplet Diameter vs. Cumulative (\%) and Volume Frequency (\%)

Respectively for conditions of Figure A2a

Table A.2b Droplet Diameter vs. Cumulative (\%) and Volume Frequency (\%)

Respectively for conditions of Figure A2b....

Table A.2c Droplet Diameter vs. Cumulative (\%) and Volume Frequency (\%)

Respectively for conditions of Figure A2c

Table A.2d Droplet Diameter vs. Cumulative (\%) and Volume Frequency (\%)

Respectively for conditions of Figure A2d.....

Table A.2e Droplet Diameter vs. Cumulative (\%) and Volume Frequency (\%) Respectively for conditions of Figure A2e

Table A.2f Droplet Diameter vs. Cumulative (\%) and Volume Frequency (\%)

Respectively for conditions of Figure A2f.

Table A.3a Droplet Diameter vs. Cumulative (\%) and Volume Frequency (\%)

Respectively for conditions of Figure A3a....

Table A.3b Droplet Diameter vs. Cumulative (\%) and Volume Frequency (\%) 
Respectively for conditions of Figure A3b

Table A.3c Droplet Diameter vs. Cumulative (\%) and Volume Frequency (\%)

Respectively for conditions of Figure A3c

Table A.3d Droplet Diameter vs. Cumulative (\%) and Volume Frequency (\%)

Respectively for conditions of Figure A3d

Table A.3e Droplet Diameter vs. Cumulative (\%) and Volume Frequency (\%)

Respectively for conditions of Figure A3e

Table A.3f Droplet Diameter vs. Cumulative (\%) and Volume Frequency (\%) Respectively for conditions of Figure A3f.

Table A.4a Droplet Diameter vs. Cumulative (\%) and Volume Frequency (\%)

Respectively for conditions of Figure A4a

Table A.4b Droplet Diameter vs. Cumulative (\%) and Volume Frequency (\%) Respectively for conditions of Figure A4b

Table A.4c Droplet Diameter vs. Cumulative (\%) and Volume Frequency (\%)

Respectively for conditions of Figure A4c

Table A.4d Droplet Diameter vs. Cumulative (\%) and Volume Frequency (\%)

Respectively for conditions of Figure A4d....

Table A.4e Droplet Diameter vs. Cumulative (\%) and Volume Frequency (\%)

Respectively for conditions of Figure A4e

Table A.4f Droplet Diameter vs. Cumulative (\%) and Volume Frequency (\%)

Respectively for conditions of Figure A4f.

Table A.5a Droplet Diameter vs. Cumulative (\%) and Volume Frequency (\%) Respectively for conditions of Figure A5a.....

Table A.5b Droplet Diameter vs. Cumulative (\%) and Volume Frequency (\%)

Respectively for conditions of Figure A $5 b$

Table A.5c Droplet Diameter vs. Cumulative (\%) and Volume Frequency (\%)

Respectively for conditions of Figure A $5 \mathrm{c}$

Table A.5d Droplet Diameter vs. Cumulative (\%) and Volume Frequency (\%)

Respectively for conditions of Figure A5d.

Table A.5e Droplet Diameter vs. Cumulative (\%) and Volume Frequency (\%)

Respectively for conditions of Figure A5e

Table A.5f Droplet Diameter vs. Cumulative (\%) and Volume Frequency (\%) Respectively for conditions of Figure A5f.

Table A.6a Droplet Diameter vs. Cumulative (\%) and Volume Frequency (\%) Respectively for conditions of Figure A6a

Table A.6b Droplet Diameter vs. Cumulative (\%) and Volume Frequency (\%) Respectively for conditions of Figure A6b.

Table A.6c Droplet Diameter vs. Cumulative (\%) and Volume Frequency (\%)

Respectively for conditions of Figure A6c

Table A.6d Droplet Diameter vs. Cumulative (\%) and Volume Frequency (\%)

Respectively for conditions of Figure A6d....

Table A.6e Droplet Diameter vs. Cumulative (\%) and Volume Frequency (\%)

Respectively for conditions of Figure A6e

Table A.6f Droplet Diameter vs. Cumulative (\%) and Volume Frequency (\%) Respectively for conditions of Figure A6f.

Table A.7a Droplet Diameter vs. Cumulative (\%) and Volume Frequency (\%) 
Respectively for conditions of Figure A7a

Table A.7b Droplet Diameter vs. Cumulative (\%) and Volume Frequency (\%)

Respectively for conditions of Figure A7b ...........................................125

Table A.7c Droplet Diameter vs. Cumulative (\%) and Volume Frequency (\%)

Respectively for conditions of Figure A7c ..........................................126

Table A.7d Droplet Diameter vs. Cumulative (\%) and Volume Frequency (\%)

Respectively for conditions of Figure A7d ..........................................126

Table A.7e Droplet Diameter vs. Cumulative (\%) and Volume Frequency (\%)

Respectively for conditions of Figure A7e

Table A.7f Droplet Diameter vs. Cumulative (\%) and Volume Frequency (\%)

Respectively for conditions of Figure A7f.....

Table A.8a Effect of Applied Voltage on Droplet Concentration

for a Reynolds Number of 1350 (Run 1) ……………........................128

Table A.8b Effect of Applied Voltage on Droplet Concentration for a Reynolds Number of 1350 (Run 2) ............................................128

Table A.8c Effect of Applied Voltage on Droplet Concentration for a Reynolds Number of 1350 (Ave.)

Table A.9a Effect of Applied Voltage on Droplet Concentration for a Reynolds Number of 1950 (Run 1)

Table A.9b Effect of Applied Voltage on Droplet Concentration for a Reynolds Number of 1950 (Run 2)

Table A.9c Effect of Applied Voltage on Droplet Concentration for a Reynolds Number of 1950 (Ave.)

Table A.10a Constant Droplet Concentration at Reynolds Number of 940,1350 and 1680 (Run 1)

Table A.10b Constant Droplet Concentration at Reynolds Number of 940,1350 and 1680 (Run 2)

Table A.10c Constant Droplet Concentration at Reynolds Number of 940,1350 and 1680 (Ave.)

Table A.11a Exit Mist Concentration for a Reynolds Number of 1230,1350 and 1680 (Run 1)

Table A.11b Exit Mist Concentration for a Reynolds Number of 1230,1350 and 1680 (Run 2) .....................131

Table A.11c Exit Mist Concentration for a Reynolds Number of 1230,1350 and 1680 (Ave.)

Table A.12a Exit Mist Concentration for a Reynolds Number of 2400, 2070, 1810, and 940 (Run 1) ...........132

Table A.12b Exit Mist Concentration for a Reynolds Number of 2400, 2070, 1810, and 940 (Run 2) ...........132

Table A.12c Exit Mist Concentration for a Reynolds Number of 2400, 2070, 1810, and 940 (Ave.)

Table A.13a Exit Mist Concentration for a Reynolds Number of 2200, 1680,1350, and 940 (Run 1)

Table A.13b Exit Mist Concentration 
for a Reynolds Number of 2200, 1680,1350, and 940 (Run 2)

Table A.13c Exit Mist Concentration for a Reynolds Number of 2200, 1680,1350, and 940 (Ave.) .133 


\section{LIST OF FIGURES}

Figure 3.1 Schematic diagram of Electro-Coalescence Unit System .......................23

Figure 3.2 Schematic diagram of the experimental setup ....................................24

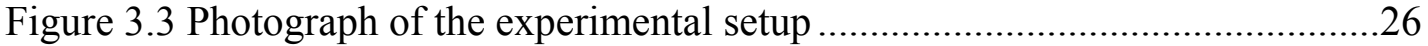

Figure 3.4 Schematic diagram of macroscopic mass balance process......................28

Figure 3.5 Dimension and components of the Malvern

Spraytec RTS 5000 Series (Malvern Instrument) .................................33

Figure 3.6 Spraytec RTS 5000 System Layout (Malvern Instrument .......................33

Figure 4.1 Electric field around an aerosol uncharged particle ..............................40

Figure 4.2 Electric field around a partially charged aerosol particle.......................40

Figure 4.3 Electric field between parallel plates with plate spacing, d......................43

Figure 4.4 Possible relative trajectories of two drops............................................48

Figure 5.1 Droplets concentration vs. applied voltage at

Reynolds Number of 1350 ..................................................................52

Figure 5.2 Droplets concentration vs. applied voltage at

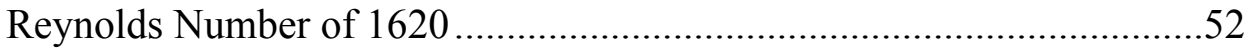

Figure 5.3 Droplets concentration vs. applied voltage at

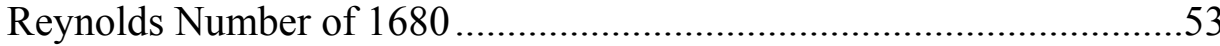

Figure 5.4 Droplets concentration vs. applied voltage at

Reynolds Number of 1950

Figure 5.5 Droplets concentration vs. applied voltage at

Reynolds Number of 2200

Figure 5.6 Droplets concentration vs. percent light transmission at

Reynolds Number of 1350 under varying applied voltage .....................55

Figure 5.7 Droplets concentration vs. percent light transmission at

Reynolds Number of 1620 under varying applied voltage ......................55

Figure 5.8 Droplets concentration vs. percent light transmission at

Reynolds Number of 1680 under varying applied voltage ....................56

Figure 5.9 Droplets concentration vs. percent light transmission at

Reynolds Number of 1950 under varying applied voltage .....................56

Figure 5.10 Droplets concentration vs. percent light transmission at

Reynolds Number of 2200 under varying applied voltage

Figure 5.11 Number droplets vs. particle diameter in micron at inlet

Mist concentration of $12.4 \mathrm{~g} / \mathrm{m}^{3}$ and Reynolds Number of 1624

Figure 5.12 Number droplets vs. particle diameter in micron at inlet

Mist concentration of $8.72 \mathrm{~g} / \mathrm{m}^{3}$ and Reynolds Number of 2195

Figure 5.13 Droplet size distribution for Reynolds Number of 1345

a. Droplet concentration of $12.11 \mathrm{~g} / \mathrm{m}^{3}$ at applied voltage of $0.0 \mathrm{kV}$

b. Droplet concentration of $5.96 \mathrm{~g} / \mathrm{m}^{3}$ at applied voltage of $26 \mathrm{kV}$

Figure 5.14 Droplet size distribution for Reynolds Number of 1345.

a. Droplet concentration of $24.23 \mathrm{~g} / \mathrm{m}^{3}$ at applied voltage of $0.0 \mathrm{kV}$

b. Droplet concentration of $8.17 \mathrm{~g} / \mathrm{m}^{3}$ at applied voltage of $26 \mathrm{kV}$

Figure 5.15 Percent light transmission vs. height of column at Reynolds

Number of 1624 and inlet droplet concentration of $27.27 \mathrm{~g} / \mathrm{m}^{3}$ 
Figure 5.16 Collected Droplets $\left(\mathrm{g} / \mathrm{m}^{3}\right)$ vs. applied voltage $(\mathrm{kV})$

at Reynolds Number of 1346 .

Figure 5.17 Collected Droplets $\left(\mathrm{g} / \mathrm{m}^{3}\right)$ vs. applied voltage $(\mathrm{kV})$

at Reynolds Number of 1946

Figure 5.18 Collected Droplets $\left(\mathrm{g} / \mathrm{m}^{3}\right)$ vs. applied voltage $(\mathrm{kV})$

at Reynolds Numbers of 937, 1346 and 1682

Figure 5.19 Collected Droplets $\left(\mathrm{g} / \mathrm{m}^{3}\right)$ vs. applied voltage $(\mathrm{kV})$

at Reynolds Numbers of 1225, 1346 and 1682 ....

Figure 5.20 Collected Droplets $\left(\mathrm{g} / \mathrm{m}^{3}\right)$ vs. applied voltage $(\mathrm{kV})$

at Reynolds Numbers of 2400, 2070, 1807 and 937

Figure 5.21 Collected Droplets $\left(\mathrm{g} / \mathrm{m}^{3}\right)$ vs. applied voltage $(\mathrm{kV})$

at Reynolds Numbers of 2195, 1682, 1346 and 937

Figure 5.22 Percent removal efficiency vs. applied voltages $(\mathrm{kV})$ at Reynolds Number of 1346

Figure 5.23 Percent removal efficiency vs. applied voltages $(\mathrm{kV})$ at Reynolds Number of 1946

Figure A.1a Droplet size distribution for Reynolds Number of

1345 and concentration of $12.1 \mathrm{~g} / \mathrm{m}^{3}$ at $\mathrm{V}=0.0 \mathrm{kV}$ .86

Figure A.1b Droplet size distribution for experimental conditions of Figure Ala, with $\mathrm{V}=10.0 \mathrm{kV}$; Droplet Concentration at exit $=9.2 \mathrm{~g} / \mathrm{m}^{3}$....

Figure A.1c Droplet size distribution for experimental conditions of Figure Ala, with $\mathrm{V}=15.0 \mathrm{kV}$; Droplet Concentration at exit $=8.0 \mathrm{~g} / \mathrm{m}^{3}$.

Figure A.1d Droplet size distribution for experimental conditions of Figure A1a, with $\mathrm{V}=18.0 \mathrm{kV}$; Droplet Concentration at exit $=6.60 \mathrm{~g} / \mathrm{m}^{3}$

Figure A.1e Droplet size distribution for experimental conditions of Figure A1a, with V $=20.0 \mathrm{kV}$; Droplet Concentration at exit $=6.16 \mathrm{~g} / \mathrm{m}^{3}$....

Figure A.1f Droplet size distribution for experimental conditions of Figure A1a, with $\mathrm{V}=26.0 \mathrm{kV}$; Droplet Concentration at exit $=5.0 \mathrm{~g} / \mathrm{m}^{3}$.

Figure A.2a Droplet size distribution for Reynolds Number of 1350 and concentration of $24.23 \mathrm{~g} / \mathrm{m}^{3}$ at $\mathrm{V}=0.0 \mathrm{kV}$

Figure A.2b Droplet size distribution for experimental conditions of Figure A2a, with $\mathrm{V}=10.0 \mathrm{kV}$; Droplet Concentration at exit $=18.97 \mathrm{~g} / \mathrm{m}^{3}$

Figure A.2c Droplet size distribution for experimental conditions of Figure A2a, with $\mathrm{V}=15.0 \mathrm{kV}$; Droplet Concentration at exit $=15.8 \mathrm{~g} / \mathrm{m}^{3}$

Figure A.2d Droplet size distribution for experimental conditions of Figure A2a, with $\mathrm{V}=18.0 \mathrm{kV}$; Droplet Concentration at exit $=13.7 \mathrm{~g} / \mathrm{m}^{3} \ldots \ldots \ldots \ldots . .90$

Figure A.2e Droplet size distribution for experimental conditions of Figure A2a, with $\mathrm{V}=20.0 \mathrm{kV}$; Droplet Concentration at exit $=11.6 \mathrm{~g} / \mathrm{m}^{3}$

Figure A.2f Droplet size distribution for experimental conditions of Figure A2a, with $\mathrm{V}=26.0 \mathrm{kV}$; Droplet Concentration at exit $=5.12 \mathrm{~g} / \mathrm{m}^{3}$.

Figure A.3a Droplet size distribution for Reynolds Number of 1350 and concentration of $16.3 \mathrm{~g} / \mathrm{m}^{3}$ at $\mathrm{V}=0.0 \mathrm{kV}$

Figure A.3b Droplet size distribution for experimental conditions of Figure A3a, with V $=10.0 \mathrm{kV}$; Droplet Concentration at exit $=11.7 \mathrm{~g} / \mathrm{m}^{3}$.

Figure A.3c Droplet size distribution for experimental conditions of Figure A3a, with $\mathrm{V}=15.0 \mathrm{kV}$; Droplet Concentration at exit $=9.3 \mathrm{~g} / \mathrm{m}^{3}$. .93 
Figure A.3d Droplet size distribution for experimental conditions of Figure A3a, with $\mathrm{V}=18.0 \mathrm{kV}$; Droplet Concentration at exit $=8.6 \mathrm{~g} / \mathrm{m}^{3}$.

Figure A.3e Droplet size distribution for experimental conditions of Figure A3a, with $\mathrm{V}=20.0 \mathrm{kV}$; Droplet Concentration at exit $=8.1 \mathrm{~g} / \mathrm{m}^{3}$.

Figure A.3f Droplet size distribution for experimental conditions of Figure A3a, with $\mathrm{V}=26.0 \mathrm{kV}$; Droplet Concentration at exit $=5.4 \mathrm{~g} / \mathrm{m}^{3}$.

Figure A.4a Droplet size distribution for Reynolds Number of 1350 and Droplet Concentration of $8.3 \mathrm{~g} / \mathrm{m}^{3}$ at $\mathrm{V}=0.0 \mathrm{kV}$ .95

Figure A.4b Droplet size distribution for experimental conditions of Figure A4a, with $\mathrm{V}=10.0 \mathrm{kV}$; Droplet Concentration at exit $=7.3 \mathrm{~g} / \mathrm{m}^{3}$. .95

Figure A.4c Droplet size distribution for experimental conditions of Figure A4a, with $\mathrm{V}=15.0 \mathrm{kV}$; Droplet Concentration at exit $=6.0 \mathrm{~g} / \mathrm{m}^{3}$. .96

Figure A.4d Droplet size distribution for experimental conditions of Figure A4a, with $\mathrm{V}=18.0 \mathrm{kV}$; Droplet Concentration at exit $=5.4 \mathrm{~g} / \mathrm{m}^{3}$. 96

Figure A.4e Droplet size distribution for experimental conditions of Figure A4a, with $\mathrm{V}=20.0 \mathrm{kV}$; Droplet Concentration at exit $=4.6 \mathrm{~g} / \mathrm{m}^{3}$.

Figure A.4f Droplet size distribution for experimental conditions of Figure A4a, with $\mathrm{V}=26.0 \mathrm{kV}$; Droplet Concentration at exit $=3.0 \mathrm{~g} / \mathrm{m}^{3}$.

Figure A.5a Droplet size distribution for Reynolds Number of 1950 and Droplet Concentration of $13.3 \mathrm{~g} / \mathrm{m}^{3}$ at $\mathrm{V}=0.0 \mathrm{kV}$

Figure A.5b Droplet size distribution for experimental conditions of Figure A5a, with $\mathrm{V}=10.0 \mathrm{kV}$; Droplet Concentration at exit $=9.7 \mathrm{~g} / \mathrm{m}^{3}$. .98

Figure A.5c Droplet size distribution for experimental conditions of Figure A5a, with $\mathrm{V}=15.0 \mathrm{kV}$; Droplet Concentration at exit $=9.0 \mathrm{~g} / \mathrm{m}^{3}$....

Figure A.5d Droplet size distribution for experimental conditions of Figure A5a, with $\mathrm{V}=18.0 \mathrm{kV}$; Droplet Concentration at exit $=8.0 \mathrm{~g} / \mathrm{m}^{3}$

Figure A.5e Droplet size distribution for experimental conditions of Figure A5a, with $\mathrm{V}=20.0 \mathrm{kV}$; Droplet Concentration at exit $=7.5 \mathrm{~g} / \mathrm{m}^{3}$.

Figure A.5f Droplet size distribution for experimental conditions of Figure A5a, with $\mathrm{V}=26.0 \mathrm{kV}$; Droplet Concentration at exit $=3.3 \mathrm{~g} / \mathrm{m}^{3}$.

Figure A.6a Droplet size distribution for Reynolds Number of 1950 and Droplet Concentration of $10.1 \mathrm{~g} / \mathrm{m}^{3}$ at $\mathrm{V}=0.0 \mathrm{kV}$ 101

Figure A.6b Droplet size distribution for experimental conditions of Figure A6a, with $\mathrm{V}=10.0 \mathrm{kV}$; Droplet Concentration at exit $=8.8 \mathrm{~g} / \mathrm{m}^{3}$.

Figure A.6c Droplet size distribution for experimental conditions of Figure A6a, with $\mathrm{V}=15.0 \mathrm{kV}$; Droplet Concentration at exit $=7.5 \mathrm{~g} / \mathrm{m}^{3}$.

Figure A.6d Droplet size distribution for experimental conditions of Figure A6a, with $\mathrm{V}=18.0 \mathrm{kV}$; Droplet Concentration at exit $=6.3 \mathrm{~g} / \mathrm{m}^{3}$.

Figure A.6e Droplet size distribution for experimental conditions of Figure A6a, with $\mathrm{V}=20.0 \mathrm{kV}$; Droplet Concentration at exit $=5.2 \mathrm{~g} / \mathrm{m}^{3}$.

Figure A.6f Droplet size distribution for experimental conditions of Figure A6a, with $\mathrm{V}=26.0 \mathrm{kV}$; Droplet Concentration at exit $=3.3 \mathrm{~g} / \mathrm{m}^{3}$.

Figure A.7a Droplet size distribution for Reynolds Number of 1950 and Droplet Concentration of $8.1 \mathrm{~g} / \mathrm{m}^{3}$ at $\mathrm{V}=0.0 \mathrm{kV}$

Figure A.7b Droplet size distribution for experimental conditions of Figure A7a, with $\mathrm{V}=10.0 \mathrm{kV}$; Droplet Concentration at exit $=7.3 \mathrm{~g} / \mathrm{m}^{3}$. 104 
Figure A.7c Droplet size distribution for experimental conditions of Figure A7a, with V $=15.0 \mathrm{kV}$; Droplet Concentration at exit $=5.9 \mathrm{~g} / \mathrm{m}^{3}$

Figure A.7d Droplet size distribution for experimental conditions of Figure A7a, with V $=18.0 \mathrm{kV}$; Droplet Concentration at exit $=4.6 \mathrm{~g} / \mathrm{m}^{3} \ldots \ldots \ldots \ldots \ldots . . .105$

Figure A.7e Droplet size distribution for experimental conditions of Figure A7a, with V $=20.0 \mathrm{kV}$; Droplet Concentration at exit $=4.4 \mathrm{~g} / \mathrm{m}^{3}$. 106

Figure A.7f Droplet size distribution for experimental conditions of Figure A7a, with $\mathrm{V}=26.0 \mathrm{kV}$; Droplet Concentration at exit $=3.3 \mathrm{~g} / \mathrm{m}^{3}$ 106 


\section{CHAPTER 1}

\section{INTRODUCTION}

The Clean Air Act Amendments of 1990 (CAAA) changed the air quality regulations in the United States. A major component of the CAAA concerns the emissions of hazardous air pollutants (HAPs). After the rules have been implemented by U.S Environmental Protection Agency (EPA), companies must modify their operations and emission control systems to meet the regulatory challenges (Bacon, 1997). These changes are mainly concerned with the control of hazardous aerosol particle sizes and human exposure to such particles. Thus, it is important to characterize these particles in both size and dynamics as accurately as possible. Such characterization will help develop new filtering technologies that could significantly reduce/control the emission of hazardous aerosol particles in a cost effective manner.

For instance, the growing concern regarding the production of radioactive aerosols during treatment and sludge retrieval operations in high-level radioactive waste tanks has led the Department of Energy to an increasing interest in reducing the emissions, worker exposure, cost and increased waste volume from such tanks. Conventional mist eliminators such as High Efficiency Particulate Attenuation (HEPA) filtration were not sufficient to treat off-gas in a recent In-Tank Evaporation process (ITE) at the Oak Ridge National Laboratory, Melton Valley Storage Tank waste, resulting in contamination of system ductwork and wetting and plugging of HEPA filters.

It should be emphasized that the human exposure to hazardous aerosol particles in general and sub-micron particles in particular has been a major health concern over the 
past decade. In fact, sub-micron particles including droplets constitute the most serious environmental and health related problems. One problem is that such small particles are able to penetrate into the human lung system where the exchange of gases between blood and air takes place (Brown, 1981). The human lower pulmonary system is unfortunately most efficient in retaining particles in 1 micron range (Davies, 1953 \& Cadle, 1965 ). The deposition of small particles in the pulmonary system tends to intensify such respiratory disease as bronchitis, emphysema and lung cancer (Cadle, 1965). In addition, submicron particles will stay in the atmosphere for a considerable period of time, scattering the sunlight and dissipating over large area (Kildesq, 1995).

\section{$\underline{1.1 \text { Background }}$}

It is known that the removal of small particles from process or waste streams is costly and often very time consuming (Barker et al.,1991). Elimination of liquid droplets and/or solid particles from gas or vapor streams is a common operation in a variety of chemical and environmental processes. Despite the utilized equipment or separation mechanisms, the mist eliminators are designed to operate as coalescers or agglomerators and thereby form larger droplets that can be removed from the process by gravity or shear forces. Droplets of size smaller than 10 microns are in general difficult to remove from gaseous streams. Current particle removing techniques (cyclone, electrostatic precipitator, scrubbers, etc.) have been used to remove larger droplets/particles from a carrier gas with high efficiency (Fuchs, 1964). An electrically driven process including mechanisms such as electrocoalescence and electrophoresis is the focus of this research. The effect of electric fields on particle agglomeration has been investigated by Loffler \& 
Gutsch (1993) and Zhang et al. (1995), while Lucker et al. (1995) studied electric-field bed filtration.

In order to design an efficient aerosol removal system, we need a fundamental knowledge of droplet-droplet interactions. The selection and evaluation of an air pollution-control system depends on many criteria such as gas flow rate, gas temperature, moisture content, chemical properties of the gas, properties of particulate matter, available space, system operating pressure, waste generation and economics.

\section{2 Problem Statement and Research Objectives}

Over the past three decades an extensive effort has been directed to finding new applications of electrical energy in the field of heat and mass transfer operations and phase separation processes. In many situations electrically driven processes, including electrocoalescence and electric filtration, can be considered competitive alongside mechanically or thermally driven operations (Ptasinski, 1992) and powerful for improving aerosol elimination. Electrocoalescence increases the mean droplet size, which is a key for improving the removal efficiency by HEPA filtration.

The primary aim of this research work is at developing a better understanding of electrically driven drop-drop and drop-electrode interactions in an aerosol flow chamber. The overall gained experience and knowledge will be useful in developing effective applications of superimposed electric fields for the elimination of mist from air or gaseous streams. 
In this research, experimental studies have been undertaken for enhancing the agglomeration of small droplets. In such studies, the agglomeration of aerosols by means of DC electric fields has been investigated.

Experimental measurements have been conducted in order to accomplish the following tasks:

- Quantify removal of drops by an electric field.

- Measure drop size distribution along the flow chamber and determine the effect of an electric field on drop coalescence.

In this work, a homogeneous DC electric field between two parallel plates was used. It is believed that a well-functioning agglomerator under a DC electric field might have a considerable impact on the control of aerosol emissions from waste treatment operations. This is due to the fact that polarized aerosol particles and droplets are attracted to each other in a DC electric field. This behavior enhances the collision and coalescence rate of particles leading to larger aggregates or droplets, which can be removed easier by a conventional filter. Other mechanisms of particle/drop interactions with the electric field are also possible, such as electrophoresis and dielectrophoresis. The results of these interactions with respect to particle/drop removal by collision with the electrodes, are also investigated in this work. 


\subsection{Thesis Organization}

This thesis is organized into six chapters. After this introductory chapter, Chapter 2 presents a review of pertinent literature. Experimental procedures used in this research are described in Chapter 3. The equation of motion for a single particle with and without an electric field, and the macroscopic mass balance equation are discussed in Chapter 4 . Chapter 5 presents results and discussion. Conclusions are presented in Chapter 6 . Avenues for future work are suggested in Chapter 7. 


\section{CHAPTER 2}

\section{REVIEW OF PERTINENT LITERATURE}

\section{$\underline{2.1 \text { Cyclone Collector }}$}

The cyclone collector is one of the most common and oldest devices for controlling the emissions of large-diameter particulates. The separator generates two vortices as the gas stream enters the top of the cyclone. Centrifugal action throws the heavier particles to the side of the cyclone. Frictional and gravitational forces act on the particles closest to the wall, causing them to slide down into a collector, while particles of less mass remain closer to the center of the vortex. Inside the lower segment of the cyclone, the direction of the gas-flow vortex is reversed and the inner ascending vortex is formed. The inner vortex is a relatively particulate free air (Wark and Warner, 1981). Cyclone systems are classified as follows: simple, multiple, or multi tube. A simple system is a single cyclone, where a multiple cyclone refers to the series or parallel arrangement of more than one cyclone. A multi-tube system is a group of smaller diameter vane axial parallel cyclones within a common housing which contribute to a common inlet and discharges to a common track. Multi-tube cyclones are used for large gas flow rates (Bacon, 1997). The removal efficiency of cyclones for submicron particulates is low and they require very high energy input. However, they are efficient for emissions of large particulates and effective with no liquid waste streams generated. Cyclones are frequently used as pretreatment devices for high efficiency particulate control devices such as ESPs, venturi scrubers, and baghouse. Cyclone collectors are 
used primarily when product recovery is an important issue for a facility's production efficiency.

\section{$\underline{\text { 2.2 Fabric Filters }}$}

Filters are used in a wide variety of materials and geometries to remove particulates from gas streams. Fabric filters or baghouses consist of a large number of filter bags, arranged so that continuous removal of the collected material is possible, and are very effective removal devices for fine particulate matter (Perkins, 1974). The basic components of a baghouse include a filter medium, a cage to support the bag, a gas tight enclosure and a mechanism to remove accumulated particulates. Gases enter one of several separate baghouse compartments, where they are directed upward through a cell plate and into contact with the tubular bag. The tubular bags are contained in multiple modular units comprising the total baghouse system. Initial particulate collection forms a thick porous cake on the bags, which acts as the filtration medium while the bags support the particulate cake. The accumulated dust cake is removed from the bags periodically.

There are three categories of baghouses depending on their flow rate capacity such as low, medium, and large. The low capacity fabric filters are capable of handling hundreds to a few thousand cfm of process gas. The medium capacity fabric filters are modular units designed to handle about $10,000 \mathrm{cfm}$. The high capacity fabric filters can handle 50,000 to a million cfm (Bacon, 1997). Baghouses are excellent for controlling the small diameter particulate matter from exhaust stream. For example, medium baghouses have efficiencies of 99 percent or better when collecting 0.5 micron particles and can remove substantial quantities of 0.01 micron. The operating temperatures of 
baghouses can vary from 175 to $550{ }^{\circ} \mathrm{F}$ depending on the nature of baghouse fabric (e.g; cotton, wool, or glass). One of the disadvantages of fabric filters is the necessity of relatively frequent cleaning to avoid an excessive pressure drop (Wark \& Warner, 1981). Normally, these filters need to be cleaned up when the pressure drop approaches 5 to $6 \mathrm{in}$. water. A reasonable operating pressure drop is about 2 to 3 in. water. These filters are also temperature and moisture sensitive. As a result, the moisture in the inlet gas can form a cake, leading to plugging. This might cause fire and/or explosion in the filters if they are not properly cleaned up. In comparison to other competitive types of removal devices, the overall size of the equipment is large and a large physical space is required.

\section{$\underline{\text { 2.3 Electrostatic Precipitators (ESP) }}$}

Electrostatic phenomena have been known for the past few hundred years. It is a technique to remove suspended particles in a gas using an electric force (White, 1962, Masuda and Hosokawa, 1997 and Boem, 1982). Recently, It also has been used for cleaning indoor air (Mizuno, 1981). Particulate and aerosol collection by electrostatic precipitation is based on the combined attraction between particles of one electrical charge and a collecting electrode of opposite polarity (Wark \& Warner, 1981). The ESP technology used for particulate and trace metal removal can be employed as either a dry or wet system (Bacon et al., 1997). Dry and wet ESPs can vary in shape from concentric to square. The concentric ESPs use high intensity ionization electrodes mounted on the central tube. Uniform flow through the tube can be accomplished by a distributor device. As the gas enters the tube, it passes through a charging zone. Particles are charged by ions diffusing from so-called corona dischargers. As the gas flows farther down the tube, 
the charged particles are forced to the tube walls by an electrostatic field. The clean gas exits the tube into a clean air compartment which is located at the bottom of the vessel. Square ESPs are build on the same principle as concentric ESPs but they are configured differently. They can use an alternating array of negatively charged grids and positively charged collection plates. The discharged electrodes are usually metal wires suspended along the center by weights or rigid frames. The dry ESP employs an alternating rapping action to remove particulate matter collected on the surface of opposite charge. Wetelectrostatic precipitators are washed with water sprays to remove collected particles from collection surfaces. The high voltage single stage electrostatic precipitator is used to collect both solid and liquid (mist and fog) particulate matter. Low voltage double stage precipitators are limited fully to the collection of particulates or drops.

The advantages of ESPs include high capacity to handle large gas volumes, high collection efficiencies even for submicron size particles, low energy consumption and ability to operate with relatively high temperature gases. Their collection efficiency for trace metals, mist, and fog is very high. Electrostatic precipitators have been built for volumetric rates from 100 to 4,000,000 cfm, and they are used to remove particles from 0.05 to 200 microns with efficiencies in the range of 98 to 99 percent range in some cases. The pressure drop is quite small, extending from 0.1 to 0.5 inches of water. Gas temperatures up to $1200{ }^{0} \mathrm{~F}$ and pressures up to $150 \mathrm{psi}$ can be accommodated.

Electrostatic precipitators also have problems. Ionization of the gas occurs only in a limited temperature range so that the device can suffer a loss in efficiency if sudden changes occur in the operating conditions. Build-up of collected material can cause "spark-over" between the electrodes which in turn causes a high current flow and 
excessive power use. Build-up of collected material on the negative electrode can suppress the corona discharge and reduce efficiency. The resistivity of the gasparticulate combination also affects the corona and the collection efficiency. Reentrainment of the collected material can interfere with the particle charging and result in direct release of particulate at the exit stream (Perkins, 1974). The other disadvantages of electrostatic precipitators include high costs of operation, maintenance, equipment and energy requirements. In addition, they require large metallic collection surface and expensive corrosion resistant alloys.

\subsection{Venturi Scrubbers}

A venturi is defined as a rectangular or circular flow channel which converges to a narrow throat section and then diverges back to its original cross-section area. In the converging section, the fluid flow is converted into kinetic energy, with a decrease in static pressure and rise in velocity. Venturi scrubbers are designed to serve as control devices for applications requiring very high collection efficiencies of particles between 0.5 and 5.0 micron in diameter. The collection efficiency depends on the length of the throat. A liquid, usually water, is introduced upstream of the throat and flows down the converging sides into the throat where it is atomized by the gas stream. Once the liquid is atomized, it begins to collect particles from the gas impacting into the liquid as a result of the difference in velocities of the gas stream and the atomized droplets. As the mixture decelerates in the diverging section, further contact occurs, causing the droplets to agglomerate. Once the particles have been trapped by the liquid, a separator such as cyclone or mist eliminator can readily remove the scrubbing liquid from the cleaned gas 
stream. Venturi scrubbers are excellent fine particulate control devices to a corrosive process stream. Also, they can operate in high temperature and high moisture environments. The disadvantages of this control device are the expensive materials of construction and costly operation and maintenance.

\section{$\underline{2.5 \text { Ionizing Wet Scrubbers }}$}

The ionizing wet scrubber uses the principle of electrostatic particle charging, inertial impaction, and gas absorption to collect submicron solid and liquid particles. It uses high voltage ionization to electrostatically charge particulate matter in the gas stream before the particles enter a packed bed scrubber section. At that point, they are removed by attraction of the charged particles by neutral surfaces. Collection of particles of three to five microns and larger can take place by inertial impaction. The smaller particles flow through the scrubber where they pass over the surfaces of packing material and scrubbing liquid droplets. The electrostatic charge of the particles causes them to be attracted to neutral surfaces. The scrubbing liquid and packing material act as collection surfaces. The shell and most internal parts are made of fiberglass and thermoplastic materials to prevent corrosion in the presence of acid gases (Bacon et al., 1997). Collection of submicron particles, mist, and fog by this technique is very effective. Operation and maintenance costs are relatively low, whereas the capital costs are very high and liquid waste streams are generated. 


\section{$\underline{\text { 2.6 Collision Scrubbers }}$}

The collision scrubber has better efficiency than the wet scrubber. It has three scrubber throats arranged in a "T". The gas enters the collision scrubber then splits into two gas streams prior to entering a pair of scrubber throats. At the entrance of each throat, water is injected and then it is atomized by the gas stream. The droplets collect particles as they are accelerated toward the center of the throat. Because of their inertia, they travel into the opposing gas stream with high velocity and turning into finer droplets, which more effectively collect submicron particles and produce a larger liquid surface area for gas absorption. A multi-stage collision scrubber takes the gas/water mixture and removes the water, letting the clean gas to exit the system. The collected water returns to the scrubber throat by a centrifugal pump (Bacon et al., 1997). The collision scrubber can collect trace metal and submicron particulates from process exhaust streams, but its capital and operating costs are high and liquid waste streams are generated.

\section{$\underline{\text { 2.7 Electrodynamic Venturis }}$}

This air pollution control device consists of a sequence of steps which include saturation, expansion, condensation, ionization, and filtration. Saturation takes place in a quench/spray tower. The additional steps take place in the main unit. The saturated gas stream leaving the spray tower enters the main unit, then travels into a converging/ diverging section causing an adiabatic expansion of the gas which results in a change in the state of energy (Bacon et al., 1997). As the kinetic energy of the gas increases in the converging section, the static pressure energy decreases. The reduced pressure of the gas saturated with water vapor creates supersaturation in the diverging section. The 
suspended particulates in the gas act as condensation nuclei and a film of a water is formed on the surface of the particles. The presence of a water film ensures that the composite particle is capable of receiving and retaining an electric charge. For ionization, the divergent section of the main unit is designed to minimize pressure loss and ensure suitable ionization conditions. On the axial centerline of the main unit, an electrode is mounted and creates a corona within the flow of gas stream. In the final filtration and collection step, two separate mechanisms take place, physical impaction and electrostatic attraction. A hollow cone spray nozzle is located at the center and positioned adjacent to the recovery section of the main unit. The water curtain by the nozzle is positively charged under the influence of the electric field which is generated by the electrode. The carrier gas passes through the charged droplet curtain. The mist eliminator collects the particulate bearing water droplets. Then, large particulates are separated from gas stream by the physical impaction of the spray droplets enhanced by the electrostatic attraction effect. Finally, at the filtration step, the total water droplets are removed from the gas stream and clean gas exits the system (Bacon et al. 1997). The electrodynamic venturi can remove submicron particulate matter from the exhaust streams effectively. On the other hand, the capital costs are relatively high, and it generates a lot of liquid waste.

\section{$\underline{2.8 \text { Inertial Impaction }}$}

Inertial impaction units capture tiny liquid droplets entrained in a gas or vapor by impingement on solid surfaces. In vane-type units, the gas flows either horizontally or upward and vanes direct the flowing gas back and forth in a twisting pattern. Mist 
droplets are carried along by the gas. Because of their momentum due to their higher density, the droplets tend to move in straight lines than the bulk of the gas. At every change in the gas direction, some droplets strike and stick to the surface. The captured droplets coalesce on the vanes, forming larger drops which have enough weight to drip down. With horizontal flow, a drainage path is provided from the bottom of the vanes. With vertical flow, collected liquid drips from the bottom of the vane unit and falls on the rising gas (Hansen et al., 1983).

Separation of liquid droplets from gas or vapor stream is one of the most common operations in chemical plants. Separation of liquid is not only to recover valuable products or to protect the equipment from corrosive liquids, also it is necessary to improve emissions controls. Knitted wire mesh mist eliminators are used extensively in many industrial plants. Their widespread application is due to the low cost and efficient removal of entrained liquid droplets from vapor and gas streams. They can be classified as "impingement type separators" and their working principle is based on inertial force (Brunazzi, 1999). They are designed to operate as coalescers or agglomerators, i.e..to combine small liquid particles into larger drops which can be readily removed from the process by gravity (Hansen et al., 1983). They are efficient for capturing droplets in the range of 5-100 microns. One of principle limitation of mesh pad is flooding, meaning choking with liquid due to excessive quantities of mist. 


\subsection{HEPA Filters}

High Efficiency Particulate Attenuation (HEPA) filters are used to prevent the emissions of particulates and mists into the environment. Filters are often made of 0.013inch thick glass fiber materials. These filters are efficient to capture $99.97 \%$ of 0.3 micron diameter aerosol particles. Water is the major problem for HEPA. Usually, a preheater is installed in the air ducts of waste stream to prevent condensation which damage the HEPA. Construction of HEPA with sintered stainless steel will provide a filter which is not subject to water damages and can be installed with built in water jets to wash the filter to reduce radiation and to eliminate the dirt accumulation. Preliminary tests showed that the use of such filters gives the same efficiency as filtration with a fiberglass filter medium. This medium resulted in data indicating that it was efficient to capture $99.97 \%$ of aerosol with 0.3 micron in diameter. Test data indicated that removal of water from the clean side of the filter is required for normal filter operation. Water on the clean side of filter flows by gravity and air movement out of the bottom of the cylindrical filter. Using a vacuum pump, clean air will be drawn out of the cylindrical filter. Water sprayed on the dirty side of a vertical filter flushes the accumulated aerosol down the dirty side. One of the advantages is the formation of a very thin filter medium which stops particle transmission at the exposed surface of the medium. The collected particles can be easily removed from the relatively smooth surface compared to a thicker medium, such as wire mesh. In addition, the stainless steel construction makes the filter resistant to mechanical damage, high pressure, and temperature. On the other hand, washing the dirty side of filter increases the waste volume. 


\section{$\underline{2.10 \text { Electrocoalescence }}$}

The problem of efficient removal of dispersed droplets from gas or vapor stream is important and extensive in many industrial processes. One of the many proposed different improvements of phase separation (Berger, 1986), the application of an electric field seems to be the most promising. Fundamentally, the strong electric fields induce a dipole in the aerosol particles. Adjacent particles experience an attractive force, which can lead to agglomeration or coalescence. Electrical coalescers have been successfully used for many years from the late 1950's and early 1960's for removal of water during desalting of crude oil in the petroleum industry (Waterman, 1965). Recently, electrical coalescence has been considered as the most promising technique in such fields as the liquid membrane extraction process, where the breaking of a liquid emulsion is a key step in this technology (Hsu, 1985, Hauertmann, 1989, \& Kataoka, 1990), direct liquid-liquid emulsion heat transfer, separation of two liquid phases formed as the product, and emulsion phase contactor (EPC) for desulfurization of crude oil (Tsouris et al., 1998).

The coalescence of suspended aqueous droplets in an insulating liquid under electric field has been influenced by the field strength, the droplet size, and the viscous resistance (Zhang, 1995). The mechanism of electrical coalescene is poorly understood because of the complexity of hydrodynamic and electrical phenomena.

A number of mechanisms have been proposed and they involve such effects as electromigration (electrophoresis), drop-drop coalescence, drop-electrode collision, dielectrophoresis, and dipole coalescence (Waterman, 1965 \& Bailes, 1982). The formation of droplets oriented along the electric field lines originates from the induced 
polarization forces $((\mathrm{Hsu} \& \mathrm{Li}, 1985)$. It suggests that electric field should be an effective means for droplets coalescence.

When two droplets move toward each other, the strength of electric field at the closest point between them can reach several times of applied electric field, as a result of shared interaction between the induced charges on the drop surfaces (Latham \& Roxburgh, 1966). Davis developed a separation technique to solve the Laplace equation which dominates the electric field outside the droplets for electrohydrostatic problems of two conducting sphere (Davis, 1964). The induced charges on the surfaces of two different sized droplets were determined under an arbitrary electric field and different separation of two drops. The induced electric field forces on two conducting droplets with radii of $\mathrm{a}_{1}$ and $\mathrm{a}_{2}$ and net charges of $\mathrm{Q}_{1}$ and $\mathrm{Q}_{2}$ can be decomposed into two components, along and normal to the line-of-centers of the droplets, $\mathrm{F}_{\mathrm{E}, 12}^{\mathrm{r}}$ and $\mathrm{F}_{\mathrm{E}, 12}^{\theta}$, respectively.

$$
\begin{gathered}
\mathrm{F}_{\mathrm{E}, 12}^{\mathrm{r}}=4 \pi \varepsilon \mathrm{a}_{2}^{2} E_{0}^{2}\left(F_{1} \cos ^{2} \Psi+\mathrm{F}_{2} \sin ^{2} \Psi\right)+\mathrm{E}_{0} \cos \Psi\left(\mathrm{F}_{3} \mathrm{Q}_{1}+\mathrm{F}_{4} \mathrm{Q}_{2}\right) \\
+\frac{1}{4 \pi \varepsilon \mathrm{a}_{2}^{2}}\left(\mathrm{~F}_{5} \mathrm{Q}_{1}^{2}+\mathrm{F}_{6} \mathrm{Q}_{1} \mathrm{Q}_{2}+\mathrm{F}_{7} \mathrm{Q}_{2}^{2}\right)+\mathrm{E}_{0} \mathrm{Q}_{2} \cos \Psi \\
\mathrm{F}_{\mathrm{E}, 12}^{\theta}=4 \pi \varepsilon \mathrm{a}_{2}^{2} \mathrm{E}_{0}^{2} \mathrm{~F}_{8} \sin 2 \Psi+\mathrm{E}_{0} \sin \Psi\left(\mathrm{F}_{9} \mathrm{Q}_{1}+\mathrm{F}_{10} \mathrm{Q}_{2}\right)+\mathrm{E}_{0} \mathrm{Q}_{2} \sin \Psi
\end{gathered}
$$

where $\mathrm{E}_{0}$ is the magnitude of the external electric field and $\Psi$ is the angle between the electric field and the droplet center line. The force coefficients, $F_{i}(i=1,2, \ldots, 10)$, are complicated series of expressions that depend on the relative geometry of the two droplets. The solution for the force coefficients, $\mathrm{F}_{\mathrm{i}}$, diverge when the distance between the two drops tends to zero, indicating a dramatic increase in the electric-field-induced 
forces between two drops in Latham \& Roxburgh (1966) experiment. Erker and Baygents have examined the electrohydrodynamic interactions between two dielectric spherical drops using the bispherical coordinates technique to solve the Laplace equation for the electric potential and the Stokes equations for the velocity field (Erker \& Baygents, 1996). Their results showed that the induced electrical interaction between two drops by an imposed electric field is always attractive when the drops are in close contact. Recently, Keh \& Chen (1989a \& 1989b) have studied the electrohydrodynamics of two charged, dielectric spheres for the electrophoretic motion along and normal to the drop center line, Loewenberg \& Davis (1995) studied a similar problem for near contact axisymmetric electrophoretic motion. Their results showed that the electrophoresis motion is at least three times greater than buoyancy-driven motion when they are in close contact. Depending on their polarity and free charge, two droplets may respond differently to an electric field. They concluded that the motion of the drops can be changed when they are in close contact.

In an external electric field, the enhanced coalescence of conducting spheres was examined by Williams \& Bailey (1983 \& 1986) theoretically and experimentally. They used an approximate solution which is not valid when two drops are close together. They did not account the viscous interactions which is significant for the coalescence rate. To resolve this problem, Zhang (1995) used a trajectory method to predict the coalescence rate of spherical conducting drops without charge. In their work, they accounted for viscous and electrostatic interactions of the droplets. They concluded that the rate of coalescence can be increased by application of electric field especially when the field acts perpendicular to the gravity. 
A natural practical extension of drop electro-coalescence is electro-filtration. A more detailed discussion of the basic operation of electro-filtration processes will be provided in the following section. Zhang et al. (1995) added theoretical justification to quantitatively predict collision and coalescence rates of conducting, charge-free spherical drops and their growth induced by combined gravitational and electric-field-induced forces.

\section{$\underline{\text { 2.11 Electro-filtration of Aerosols }}$}

The removal of sub-micron particles from processed waste streams is ineffective and requires a significant amount of energy. Several reports on investigations of electrically enhanced aerosol filtration in fiber filters have been published. Silverman (1954), Thomas and Woodfin (1959) empirically showed that fiber filtration efficiency was enhanced by the application of an electric field. They used a bank of metal screens (Silverman et al., 1954) and several layers of nylon hosiery (Thomas \& Woodfin, 1959) placed between high voltage electrodes as a filter. They measured filtration efficiency with and without electric field. The filtration efficiency was much higher with electric field.

Bogardus (1974) and Fielding (1975) studied the performance of commercial fiber filters when placed in an electric field. Their experiment showed a great improvements of filtration efficiency over non-electrified filters.

Granular filtration (deep-bed filtration) is mechanically simple and consumes little energy. However, it relies principally on the mechanisms of interception, sedimentation, diffusion, inertia, and surface forces, which yield low collision efficiency 
between particle and collectors (Barker, 1988). It is well known that the efficiency of aerosol filtration in fiber mats (Silverman et al., 1954) and in granular bed (Harriott et al., 1980) can be improved by the application of an electric field to the filter media. Filtration efficiency greater than $99 \%$ are repoted in the literature (Watermam, 1951).

A granular electrofilter is simply a bed of coarse particles (diameter $\approx 1 \mathrm{~mm}$ ), with electrodes arranged so that a strong electric field can be applied to the bed. The electric field polarizes the packing granules, and the combined electric field causes the charged suspended particles to deviate from the fluid streamline and be collected on the oppositely charged surface of the granules. In a different concept of electrofiltration, the electric charge is applied directly to the granules (Muller, 1982). In this case, the granular electrofilter operates much like an electrostatic precipitator, but with more complex flow paths. This type of electrofiltration is more suitable with less densely packed beds, such as fiber filled beds. The filter media is also restricted to a conducting medium.

Applied electric field across fibrous filters have enhanced performance with gas filtration, and successful mathematical analyses have been presented (Henry, 1986, Van Osdell, 1986, \& Accomazzo, 1986). There are two differences between fibrous and granular filters: first, the geometry of the collecting solids, and second, in fibrous filtration of gaseous streams, the drag on suspending particles is more likely to be dominated by inertial rather than viscous forces.

Local electric fields are essentially in the direction of the applied field and important deviations occur near the granule or fiber packing. If the granules/fiber packing are non-conducting, the field lines will be diverted around the granules or fibers. 
If the packing is a better conductor than the fluid, the field lines will be diverted toward the granules or fibers. Since the electric forces on the particulates act along the electric field lines, it is desirable to have the lines diverted into (rather than around) the granules or fibers. This is similar to the concept of using conducting granules or fibers which can be considered to have a very high dielectric constant than the fluid. When both fluid and packing are conductors, it is desirable for the granules or fibers to have a higher conductivity than the fluid. In the case, the packing materials are highly conductive, more or less packed fibers may be needed to prevent unnecessary current through the bed. Cleaner granules could be obtained by vigorously backwashing, but the particles could be obtained in a more concentrated suspension by slow backwashing when the field is off (Barker et al., 1991).

In summary, many parameters can have a major effect on the electric filtration process such as geometry of packing and density, orientation and strength of the electric field, particle charge, dielectric constant and conductivity of the packing material and fluid, and particle size. It is in our best interest to take all of the major parameters into account in order to achieve the highest separation efficiency. 


\section{CHAPTER 3}

\section{EXPERIMENTAL PROCEDURES}

\subsection{Experimental Setup}

The primary objective of this research project is to develop a better understanding of electrically driven drop-drop and drop-electrode interactions and agglomeration of droplets in an aerosol flow chamber. To accomplish this objective, an experimental setup was designed to carry out a series of experiments to:

- Characterize the size distribution of aerosol droplets under the influence of a strong DC electric field

- Measure the removal of drops by an electric field.

Using the experimental setup described below, a sequence of procedures was followed in each experiment in order to ensure repeatable results.

The experimental setup for this study is shown schematically in Figures 3.1 and 3.2. The aerosol observation chamber unit was designed as a small, controlled environment in which the flow-through water aerosol could be excited into additional motion by a relatively strong electric field. The chamber unit is made of clear Lexan with a cross-sectional area of 10x10 cm and approximately $94 \mathrm{~cm}$ long. Two 9x60 cm steel electrodes are mounted vertically inside the chamber on opposite sides of the test window. The rectangular test view window is made of flat optical glass (non-reflecting) whose dimensions are $2 \times 60 \mathrm{~cm}$ (Figure 3.1). A heating tape is attached to the glass to prevent accumulation of moisture during operation. 


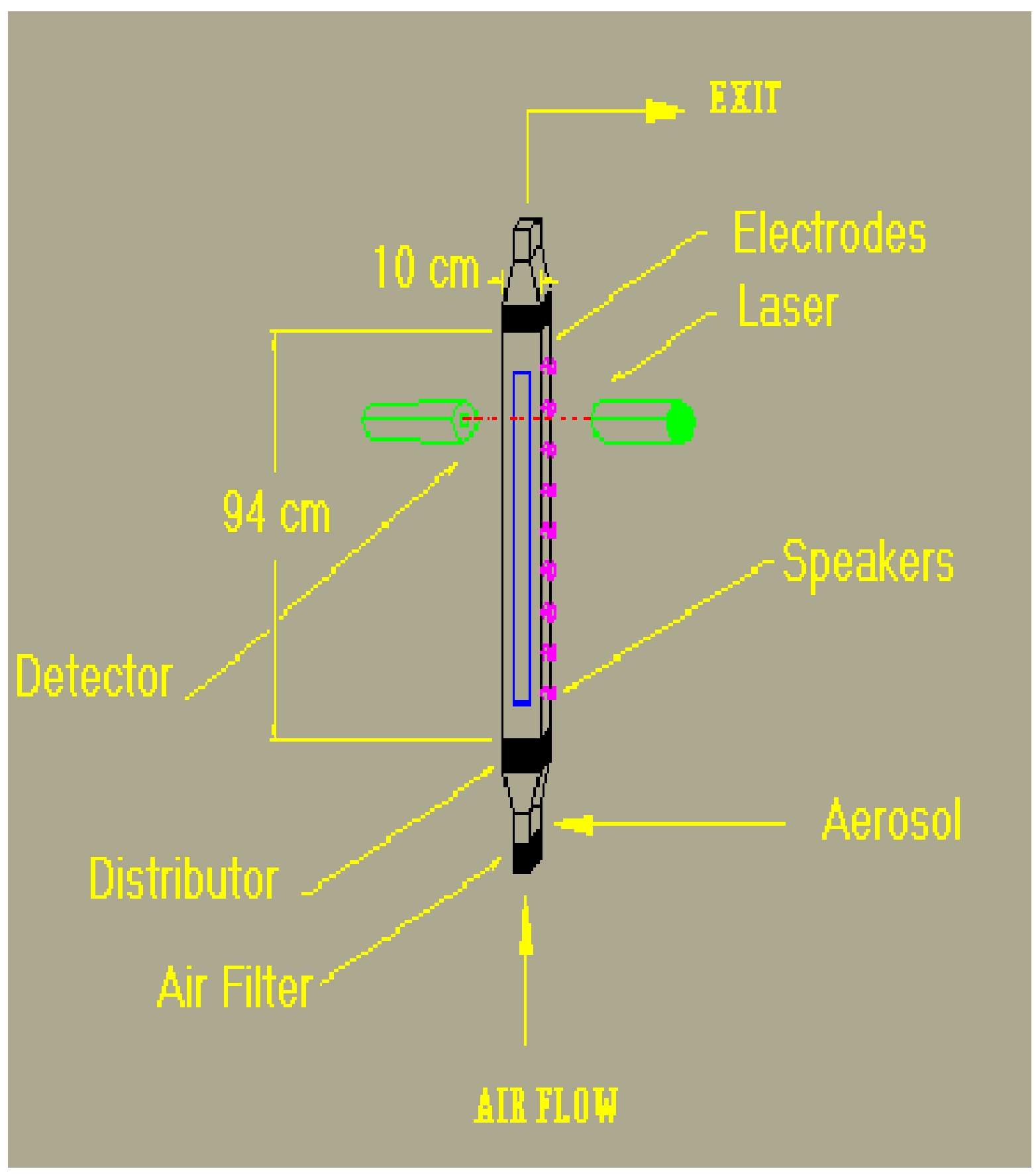

Figure 3.1: Schematic diagram of Electro-Coalescence Unit System 


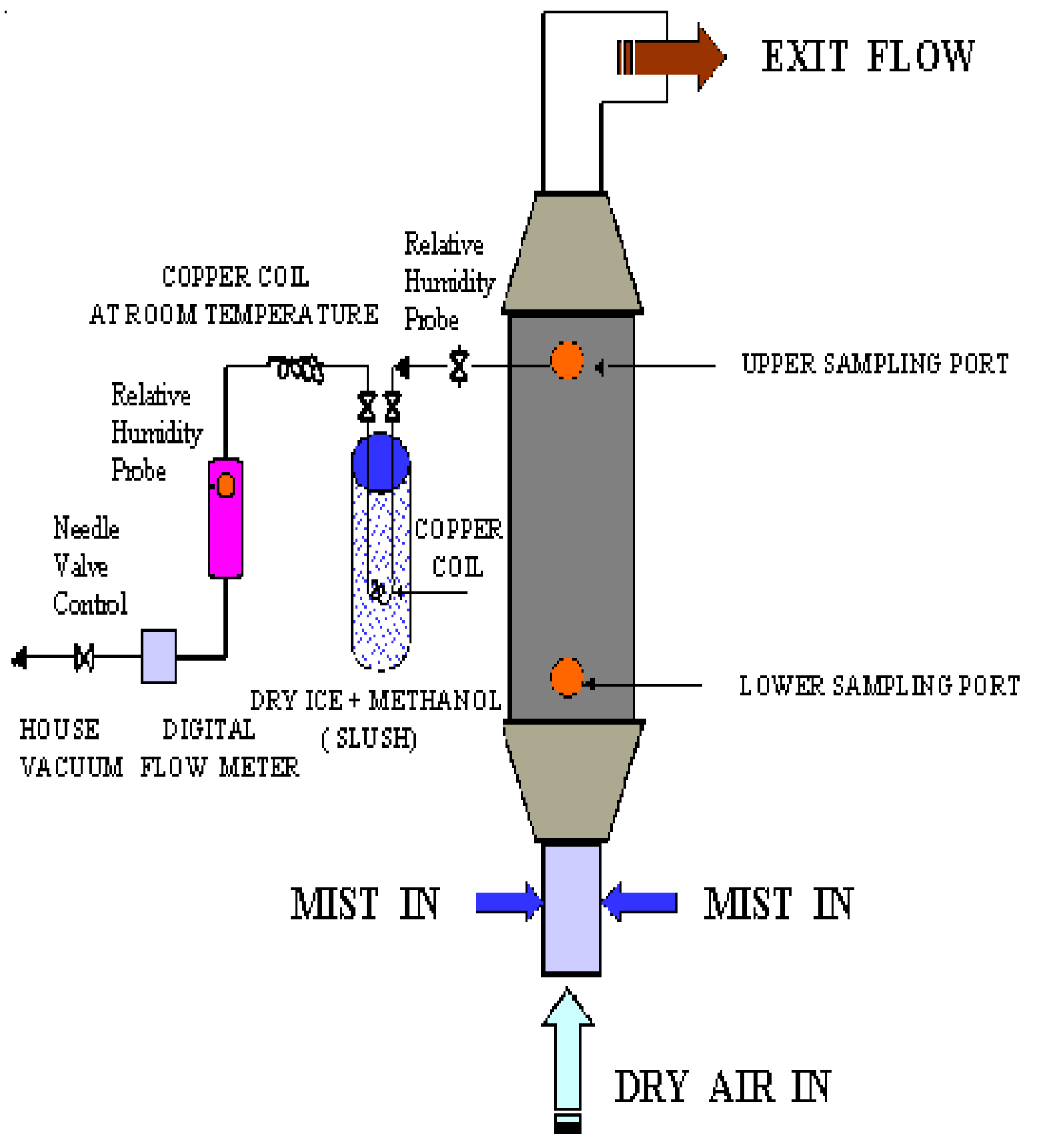

Figure 3.2: Schematic diagram of the experimental setup. 
The top section of chamber is connected by flexible ductwork to the exhaust in a hood Two sampling ports are available on the test section of the chamber. At the bottom of the tunnel, mist from two ultrasonic humidifiers is introduced into the air stream by a diffuser, as shown in Figure 3.2. The bottom part of test section is connected to the steel duct which is heated before the experiment for condensation prevention. The supplied house air is passed through a flow meter and air filter before delivering the mist through the test section of the chamber. At the entrance of the test section, a honeycomb baffle is placed for constant and evenly distributed air flow. It should be noted that distilled water was used for the two ultrasonic humidifiers (Electro-Tech System, Model 5612) that generated the aerosol flowing through the test chamber. A probe is used to measure the relative humidity/temperature of flowing air at the exit of the test section. A high voltage of up to $26 \mathrm{kV}$ is applied to the positive electrode during the experiment by a DC power supply. The aerosol size distribution in the test chamber is determined by laser-light scattering using a Malvern Spraytec RTS 5006 droplet sizing system which is mounted on a movable stage. A moisture trap of dry ice and methanol slush is prepared in a dewar flask to remove the moisture from the sampled fluid. By using this technique, a controlled flow rate of gas is drawn from the sampling port by the house vacuum through a copper coil which is immersed in the slush. A scale (Mettler PJ 3600 Delta Range) is used to determine the weight of collected mist. Sampling of the gas was carried out 


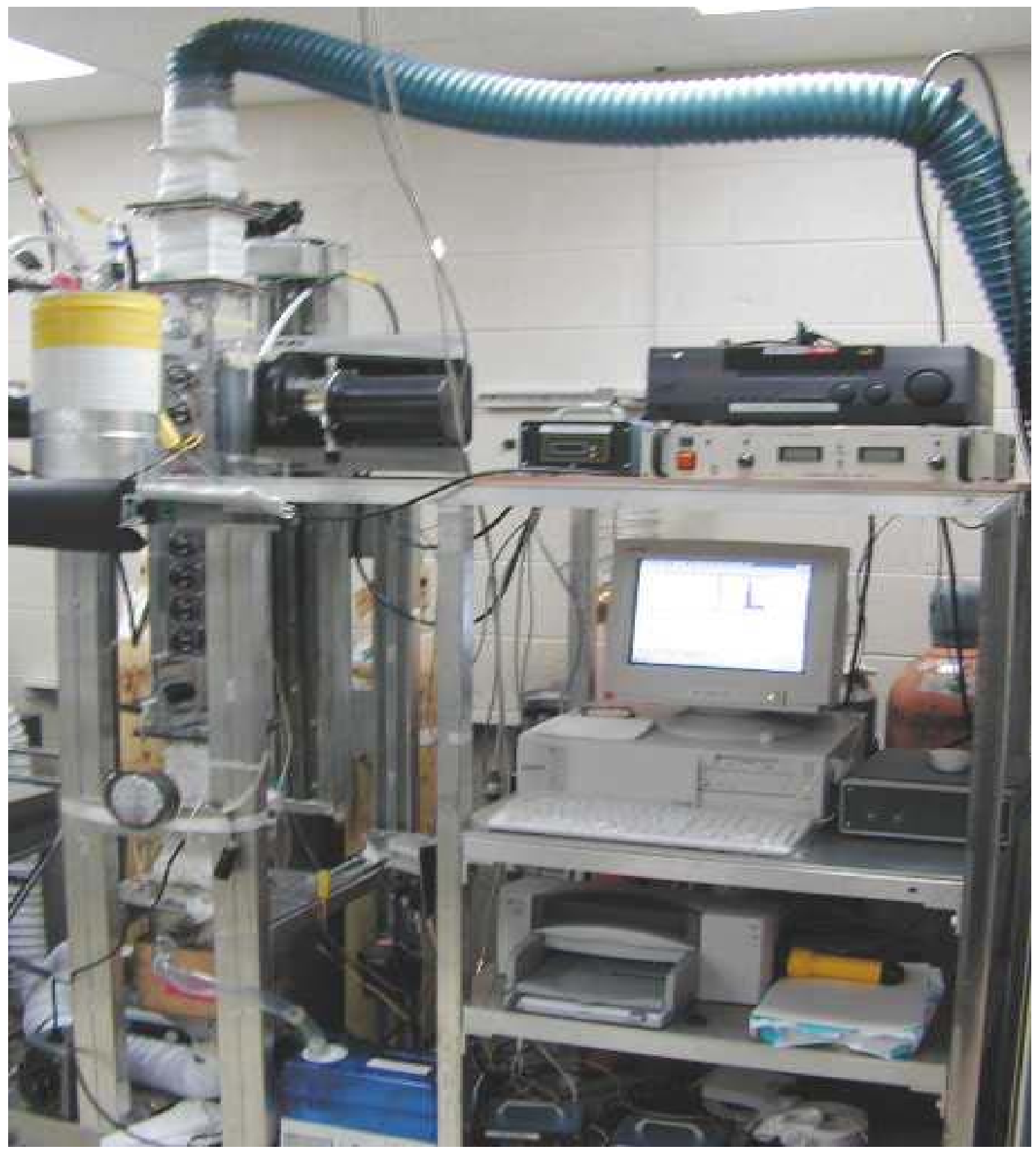

Figure 3.3: Photograph of experimental setup used in this study. 
isokinetically as discussed in the following section. A photograph of the laboratory test set up is provided as Figure 3.3.

\subsection{Isokinetic Sampling}

In aerosol sampling, the measured concentration and size distribution should represent as closely as possible the concentration and size distribution of the original aerosol. There are several reasons why the measured concentration can differ from the true concentration. One reason is that gravitational or inertial deposition of the sample as it flows into the sampling tube can result in loss of the larger-sized particles. Also, deposition or selective collection at the entrance of the sampling tube can yield either greater or lesser amounts of larger particles. If the sampling probe is aligned with the flow and the sample velocity is equal to the stream velocity, sampling is said to be isokinetic, and the collected sample should match the actual concentration and size distribution. For this purpose, the flow rate of the sampling stream was set such that the velocity at the sampling line entrance was equal to the average velocity in the chamber. In addition, the copper sampling probe was heated to evaporate the deposited water droplets at the edge and inside the probe, so that the experimental error can be reduced.

\subsection{Dry-Ice Sampling}

For capturing the water droplets from isokinetic sampling probe, a slush of dryice and methanol was prepared in a dewar flask. During the sampling events, a 


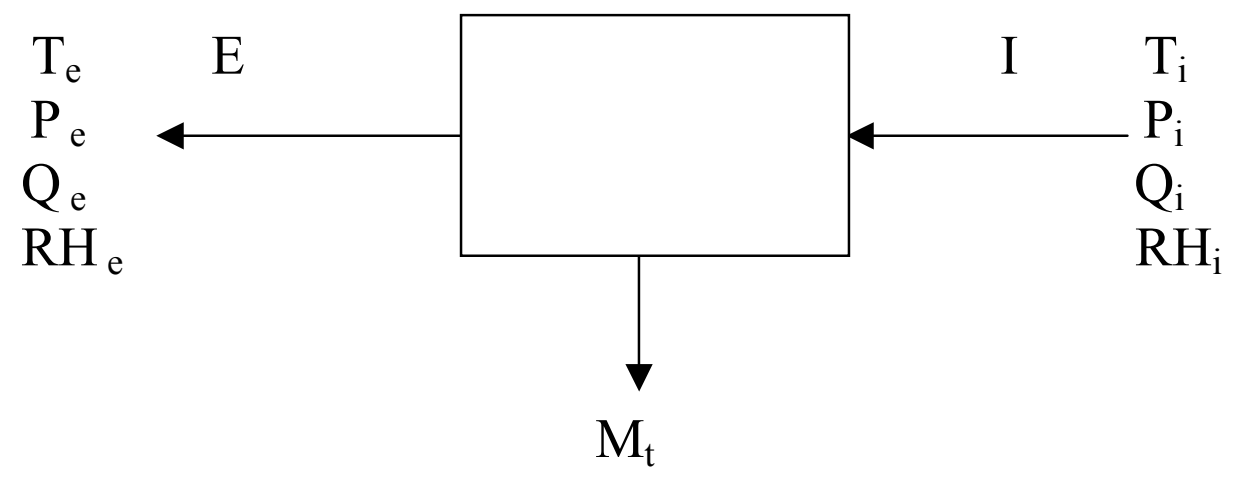

Figure 3.4: Schematic diagram of macroscopic mass balance process

controlled flow rate of gas was pulled out by house vacuum through a copper coil $(2.0 \mathrm{~m}$ long and $1.25 \mathrm{~cm} \mathrm{ID)} \mathrm{immersed} \mathrm{in} \mathrm{the} \mathrm{slush.} \mathrm{The} \mathrm{copper} \mathrm{coil} \mathrm{was} \mathrm{weighed} \mathrm{before} \mathrm{and}$ after sampling on a sensitive balance $( \pm 0.001 \mathrm{~g})$. A long sampling time was used to achieve a higher mass loading in the copper coil for a low flow rate. In contrast, a short sampling time was used for a higher flow rate. A battery-powered relative humidity/temperature probe (ALNOR APM-360) was utilized to monitor the inlet and exit relative humidity for mass balance calculations. The exit air was first passed through a $3.0 \mathrm{~m}$ long copper coil before going into the chamber to reach room temperature before the relative humidity and temperature were measured. A pressure device (SENSOTEC SC Series) was also connected to the relative humidity/temperature probe chamber to monitor the pressure drop in the dry-ice system.

The net droplet mass is calculated using the accumulated water weight in the copper coil (A), mass of the water vapor in the humid air exiting from the copper coil (B), and the mass of water vapor contained in the humid air flowing through the test chamber at the sampling port (C). Thus, the mathematical description of the net droplet mass in the air stream is as follows: 


\section{Net droplet mass $=A+B-C$}

The masses of $\mathrm{B}$ and $\mathrm{C}$ are calculated using relative humidity and temperature measurements and $\mathrm{A}$ is directly obtained from the balance. The equations for a mass balance around the dry-ice trap (Figure 3.4) used in this study are similar to the equations obtained in studies of a dehumidification process. Assuming that dispersion is negligible, a material mass balance equation can be written as

$$
\text { Accumulation rate }=\text { Flow in }- \text { Flow out }
$$

Where

$$
\text { Accumulation due to mist collection }=\mathrm{M}_{\mathrm{t}}
$$

$$
\text { Flow in }=\mathrm{I}
$$

And

$$
\text { Flow out }=\mathrm{E}
$$

Or

$$
\mathrm{M}_{\mathrm{t}}=\mathrm{I}-\mathrm{E}
$$

Symbolic notation has been chosen to be consistent as possible with the dehumidification process. $T_{i}$ is the temperature of inlet, $P_{i}$ is the inlet pressure, $Q_{i}$ is the inlet flow rate and $(\mathrm{RH})_{\mathrm{i}}$ is the relative humidity of inlet air. $\mathrm{M}_{\mathrm{t}}$ is the total mass accumulated. $\mathrm{T}_{\mathrm{e}}$ is the temperature of exit air, $\mathrm{P}_{\mathrm{e}}$ is the exit pressure, $\mathrm{Q}_{\mathrm{e}}$ is the exit flow rate and $(\mathrm{RH})_{\mathrm{i}}$ is the relative humidity of exit air.

The ideal gas law can be derived from the theory of gases by assuming that gas molecules have a negligible volume, exert no forces on one another, and collide elastically with the walls of their container (Felder and Rousseau). The law usually appears in the form 


$$
\mathrm{PV}=\mathrm{nRT}
$$

Where

$$
\begin{aligned}
& \mathrm{P}=\text { absolute pressure of a gas } \\
& \mathrm{V}=\text { volume or volumetric flow rate of the gas } \\
& \mathrm{n}=\text { number of moles or molar flow rate of the gas } \\
& \mathrm{R}=\text { the gas constant }, \text { whose value depends on the units of } \mathrm{P}, \mathrm{V}, \mathrm{n} \text {, and } \mathrm{T} \\
& \mathrm{T}=\text { absolute temperature of the gas }
\end{aligned}
$$

Assuming ideal gas behavior,

$$
\begin{aligned}
& \mathrm{P}_{\mathrm{I}}^{\mathrm{A}} \mathrm{V}_{\mathrm{I}}=\mathrm{n}_{\mathrm{I}}^{\mathrm{A}} \mathrm{RT}_{\mathrm{I}} \\
& \mathrm{P}_{\mathrm{I}}^{\mathrm{W}} \mathrm{V}_{\mathrm{I}}=\mathrm{n}_{\mathrm{I}}^{\mathrm{W}} \mathrm{RT}_{\mathrm{I}}
\end{aligned}
$$

Where the subscript I represents inlet and superscript A and W represent air and water vapor respectively, through the dry-ice trap.

The density of a fluid can be used to convert a known volumetric flow rate of a process stream to the mass flow rate of that stream or vice versa. Therefore:

$$
\begin{aligned}
& \mathrm{M}_{\mathrm{I}}=\rho_{\mathrm{I}} \mathrm{Q}_{\mathrm{I}} \\
& \mathrm{M}_{\mathrm{E}}=\rho_{\mathrm{E}} \mathrm{Q}_{\mathrm{E}}
\end{aligned}
$$

Where $\mathrm{M}_{\mathrm{I}}$ and $\mathrm{M}_{\mathrm{E}}$ are the mass flow rate and $\rho_{\mathrm{I}}$ and $\rho_{E}$ are the densities of inlet and outlet fluid respectively. Recalling the material balance equation in terms of mass;

$$
M_{t}=M_{I}-M_{E}
$$

In terms of moles

$$
\mathrm{n}_{\mathrm{t}}=\mathrm{n}_{\mathrm{t}}^{\mathrm{A}}+\mathrm{n}_{\mathrm{t}}^{\mathrm{W}}=\mathrm{n}_{\mathrm{I}}-\mathrm{n}_{\mathrm{E}}=\mathrm{n}_{\mathrm{I}}^{\mathrm{A}}+\mathrm{n}_{\mathrm{I}}^{\mathrm{W}}-\left(\mathrm{n}_{\mathrm{E}}^{\mathrm{A}}-\mathrm{n}_{\mathrm{E}}^{\mathrm{W}}\right)
$$

Substituting the equation 3.8 into the above equation and using the ideal gas law, 


$$
\mathrm{n}_{\mathrm{t}}^{\mathrm{A}}+\mathrm{n}_{\mathrm{t}}^{\mathrm{W}}=\left(\frac{\mathrm{P}_{\mathrm{I}}^{\mathrm{A}} \mathrm{V}_{\mathrm{I}}}{\mathrm{RT}_{\mathrm{I}}}\right)+\left(\frac{\mathrm{P}_{\mathrm{I}}^{\mathrm{W}} \mathrm{V}_{\mathrm{I}}}{\mathrm{RT}_{\mathrm{I}}}\right)-\left(\frac{\mathrm{P}_{\mathrm{E}}^{\mathrm{W}} \mathrm{V}_{\mathrm{E}}}{\mathrm{RT}_{\mathrm{E}}}\right)
$$

$$
\mathrm{n}_{\mathrm{t}}^{\mathrm{W}}=\left(\frac{\mathrm{P}_{\mathrm{I}}^{\mathrm{W}} \mathrm{V}_{\mathrm{I}}}{\mathrm{RT}_{\mathrm{I}}}\right)-\left(\frac{\mathrm{P}_{\mathrm{E}}^{\mathrm{W}} \mathrm{V}_{\mathrm{E}}}{\mathrm{RT}_{\mathrm{E}}}\right)
$$

The above equation gives the moles of water at large sampling time. Also, this can be written in terms of relative humidity as

$$
n_{t}^{W}=\left(\frac{R H_{I} \cdot P_{I}^{*}\left(T_{I}\right) Q_{I}}{R T_{I}}\right)-\left(\frac{R H_{E} \cdot P_{E}^{*}\left(T_{E}\right) Q_{E}}{R T_{E}}\right)
$$

where $\mathrm{RH}_{\mathrm{I}}$ and $\mathrm{RH}_{\mathrm{E}}$ are the relative humidity, $\mathrm{P}_{\mathrm{I}}^{*}$ and $\mathrm{P}_{\mathrm{E}}^{*}$ are the water vapor pressure of inlet and outlet flow, respectively. The relationship between relative humidity and vapor pressure is described such that a gas at temperature $\mathrm{T}$ and pressure $\mathrm{P}$ contains a vapor whose partial pressure is $p_{v}$ and whose vapor pressure is $p_{v}^{*}(\mathrm{~T})$. It is given as

$$
\mathrm{RH}=\frac{p_{v}}{p_{v}^{*}(\mathrm{~T})} \times 100 \%
$$

A relative humidity of $50 \%$, for example, signifies that the partial pressure of water vapor equals 0.50 of the vapor pressure of water at the system temperature. Equation (3.10) also can be written for a period of experiment as follows:

$$
\mathrm{n}_{\mathrm{t}}^{\mathrm{w}}=\frac{\mathrm{RH}_{\mathrm{I}} \cdot \mathrm{P}_{\mathrm{I}}^{*}\left(\mathrm{~T}_{\mathrm{I}}\right) \mathrm{Q}_{\mathrm{I}}}{\mathrm{RT}_{\mathrm{I}}}-\frac{\mathrm{P}_{\mathrm{E}}^{*} \mathrm{Q}_{\mathrm{E}}}{\mathrm{RT}_{\mathrm{E}}} \int_{t_{i}}^{t_{f}} \mathrm{RH}_{\mathrm{I}} d t
$$

In terms of mass, it can be written as 
$\mathrm{M}_{\mathrm{t}}^{\mathrm{w}}=\frac{\mathrm{M}}{\mathrm{R}}\left[\frac{\mathrm{P}_{\mathrm{I}}^{*}\left(\mathrm{~T}_{\mathrm{I}}\right) \mathrm{Q}_{\mathrm{I}}}{\mathrm{T}_{\mathrm{I}}}-\frac{\mathrm{P}_{\mathrm{E}}^{*}\left(\mathrm{~T}_{\mathrm{E}}\right) \mathrm{Q}_{\mathrm{E}}}{\mathrm{T}_{\mathrm{E}}} \int_{t_{i}}^{t_{f}} \mathrm{RH}_{\mathrm{E}} d t\right]$

For the case $T_{I}=T_{E}=T$, the above equation can be written as

$$
\mathrm{M}_{\mathrm{t}}^{\mathrm{W}}=\frac{\mathrm{M}}{\mathrm{RT}}\left[\mathrm{P}_{\mathrm{I}}^{*}\left(\mathrm{~T}_{\mathrm{I}}\right) \mathrm{Q}_{\mathrm{I}}-\mathrm{P}_{\mathrm{E}}^{*}\left(\mathrm{~T}_{\mathrm{E}}\right) \mathrm{Q}_{\mathrm{E}} \int_{\mathrm{t}_{\mathrm{i}}}^{\mathrm{t}} \mathrm{RH}_{\mathrm{E}} \mathrm{dt}\right]
$$

Where:

$$
\begin{aligned}
& \mathrm{M}=\text { molecular weight of water } \\
& \mathrm{R}=\text { the gas constant } \\
& \mathrm{t}_{\mathrm{i}}=\text { initial time of dry-ice trap experiment } \\
& \mathrm{t}_{\mathrm{f}}=\text { final time of dry-ice trap experiment }
\end{aligned}
$$

The above equation gives the theoretical value of collected mass of water vapor in the air.

\subsection{Particle Size Distribution Measurements and Control}

The aerosol size distribution in the test chamber is determined by laser-light scattering using a Malvern Spraytec RTS 5006 (Figure 3.5) droplet sizing system which is mounted on a movable stage for measurements at various heights along the column. The system is designed to continuously measure and present in real time particle size distribution information from a stream of aerosol. 


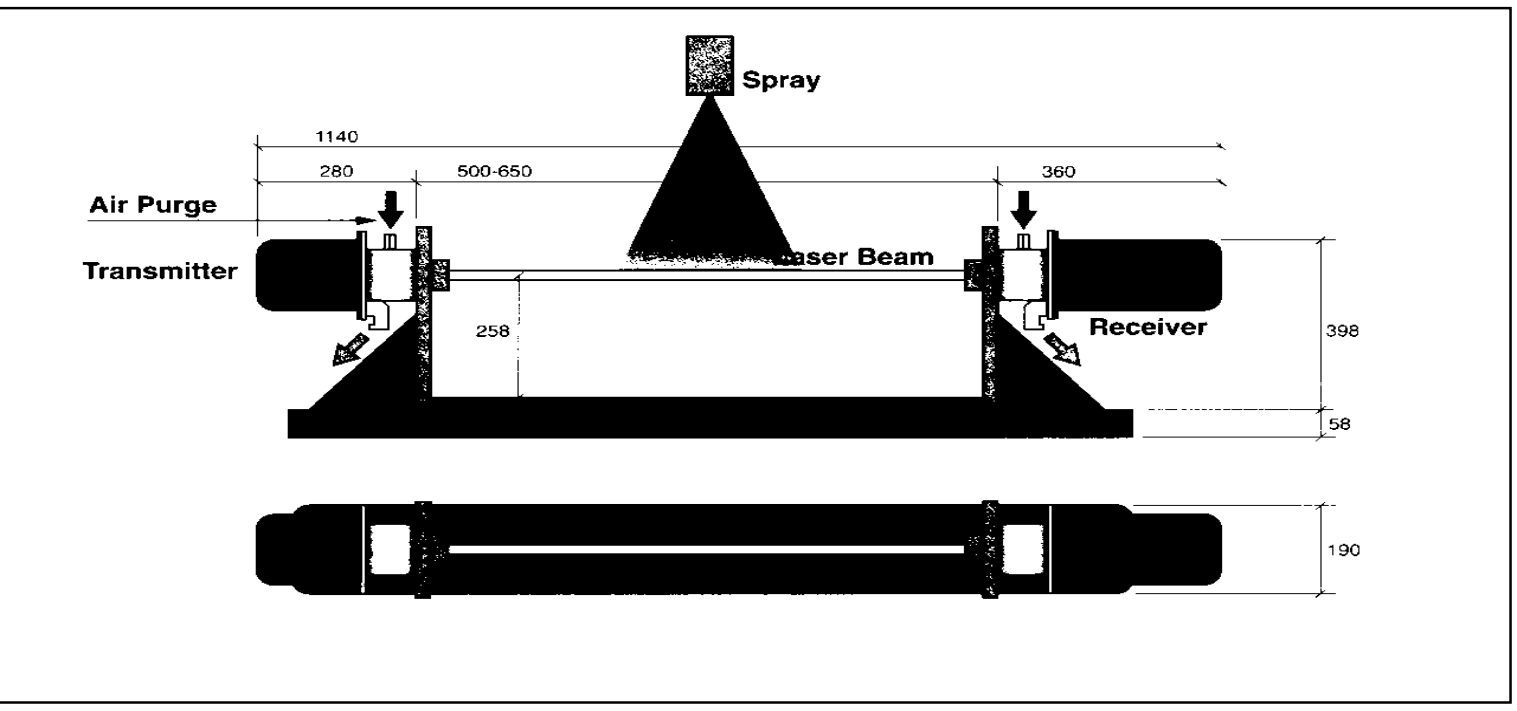

Figure 3.5: Dimension and components of the Malvern Spraytec RTS 5000 Series (Malvern Instrument)

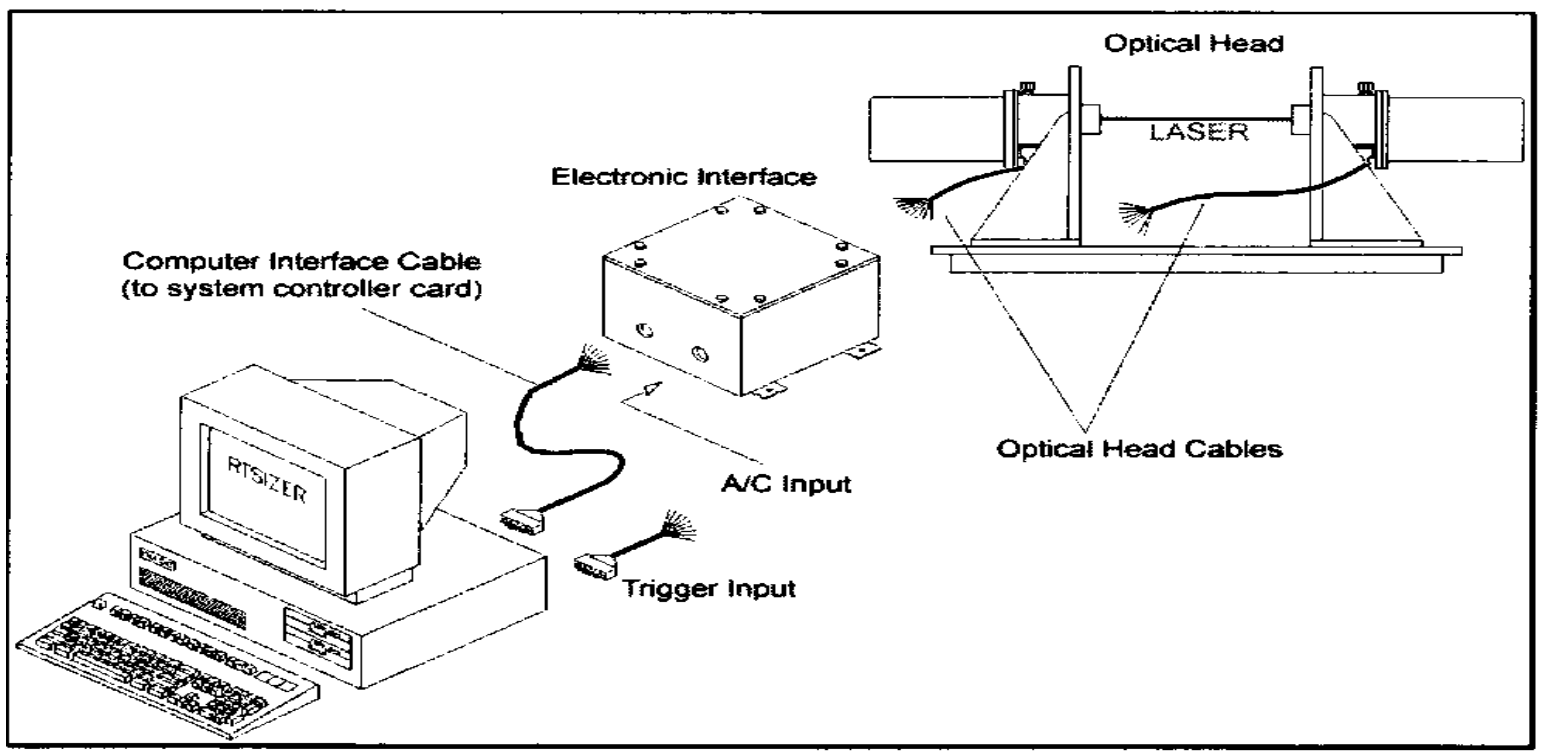

Figure 3.6: Spraytec RTS 5000 System Layout (Malvern Instrument) 
The main components of the RTS 5006 system include the optical head, interface box, computer and software (Figure 3.6). The particle sensing region or sample volume forward direction is collected by the receiver lens and focused onto an annular ring detector. The detector is scanned at high speed by the interface card which records the signal levels on each ring. Each ring on the detector measures the total signal intensity. Each particle scatters light on all rings of the detector. Therefore, the measured signal is the summation of the light scattered from all particles. Once a significant number of detector scans are acquired, the software uses a non-linear inversion technique to solve for the relative particle concentration. No assumptions are required about the shape of the size distribution, which is obtained directly from the experimental scattering information.

The chamber design for the experiments (See Figure 3.1) was guided by the goal to achieve a small and controlled volume in which the droplets could be subjected to a relatively high electric field. A number of design criteria had to be taken into consideration.

1. It was desired to generate a homogeneous electric field within the observation chamber to simplify the geometry. Such a field can be generated between two parallel flat steel electrodes mounted vertically inside the chamber on opposing sides of the test section.

2. Care was taken that the two mist feeding ports, two sampling ports, and the two flat optical windows would minimize possible disturbances of air flow due to sharp edges within the enclosure. 
3. To improve visualization of droplets and reduce the error in drop size measurements, two removable flat optical windows were designed so that they could be easily removed and cleaned after each experiment.

4. The mist feeding system was designed to distribute the produced mist uniformly by using a rubber diffuser which connects the two outputs from two ultrasonic humidifiers just below the honeycomb baffle.

5. Moisture accumulation on the optical window was prevented by heating, using ultra thin heating tape.

6. The construction was such to avoid vibrations of the setup during the experiments.

The following steps were carried out before drop size distribution measurements in each run:

- Reference Noise Measurement: This is a permanent acquisition module that allows us to record the reference noise. The reference noise is a dark background measurement, or a measurement of the electronic noise present in the system.

- Background Measurement: This is a permanent acquisition module that allows us to record a background measurement. The background is a measure of stray light in the optical system and most importantly, particulate contamination of the windows. A good background measurement is critical to insure accurate and precise drop size distribution measurements. RTSizer uses the background to compensate for any particulate contamination by using the background as a baseline that is subtracted from all drop size distribution measurements. Background was recorded for each different experiment. 
- Time History: The time history is an advantage offered by the RTSizer. The computer records and saves drop size distribution data continuously. Data are stored in a circular data base file with a fixed maximum size. Once the maximum size is reached, the oldest data acquired is overwritten by new data. The time history allows us to determine variables such as light transmission, particle diameter $(\mu \mathrm{m})$ vs. cumulative volume (\%) and volume frequency $(\%)$ for each run. 


\section{CHAPTER 4}

\section{THEORETICAL CONSIDERATIONS}

The theoretical approach traditionally used to describe the particle behavior under an electric field is conceptually simple, although detailed application of the concept is complex. Specifically, the approach consists of the equation of motion for a single spherical aerosol particle in the absent and present of an electric field, a basic electric field mechanism for particle removal from a flowing suspension, electro-coalescence of particles, and macroscopic material balance, which may be based on drop population dynamics.

\section{$\underline{4.1}$ Equation of motion for a single aerosol particle}

This problem was originally studied by Stokes and the expression for force since has become known as "Stokes' law" (Reist, 1993). Modifications have been made over the years by Basset (1888), Boussinesq (1885), and Oseen (1927). All three studied a spherical particle with motion induced by an arbitrary acceleration in a Newtonian fluid and arrived at their conclusion by neglecting convective acceleration in the NavierStokes equations which is called Basset-Boussinesq-Oseen (BBO) equation. More comprehensive studies were performed by Corrsin and Lumley (1956), and Maxey and Riley (1983). A familiar form of the equation in terms of a force balance on a spherical particle is

$\mathrm{F}_{\text {drag }}+\mathrm{F}_{\text {bouyancy }}=\mathrm{F}_{\text {gravity }}$ 
The gravity force may be replaced by Newton's second law, $\mathrm{F}=\mathrm{m}_{\mathrm{p}} \mathrm{g}$. The Stokes drag force is correlated to other physical variables through a drag coefficient, $C_{D}$. The buoyancy force known as the force due to the added mass of the fluid (Maxey and Riley (1983), Panton (1996)).

The overall force balance is written as:

$\frac{\rho_{\mathrm{g}} \mathrm{AV}_{\mathrm{t}}^{2} \mathrm{C}}{2}+\mathrm{m}_{\mathrm{p}}\left(\frac{\rho_{\mathrm{g}}}{\rho_{\mathrm{p}}}\right) g=\mathrm{m}_{\mathrm{p}} g$

Where $\rho_{\mathrm{g}}$ is the fluid density, $\rho_{\mathrm{p}}$ is the particulate density, $\mathrm{V}_{\mathrm{t}}$ is the terminal or settling velocity, $\mathrm{m}_{\mathrm{p}}$ is the mass of the particle, $\mathrm{A}$ is the frontal cross-sectional area, and $\mathrm{g}$ is the local acceleration of gravity. In terms of terminal velocity,

$\mathrm{V}_{\mathrm{t}}=\left[\frac{2 \mathrm{~m}_{\mathrm{p}} g\left(\rho_{\mathrm{p}}-\rho_{\mathrm{g}}\right)}{\mathrm{A} C_{\mathrm{D}} \rho_{\mathrm{p}} \rho_{\mathrm{g}}}\right]^{1 / 2}$

The drag coefficient $C_{D}$ is a function of the shape of the object. Assuming spherical particles, equation (4.3) becomes

$\mathrm{V}_{\mathrm{t}}=\left[\frac{4 \mathrm{gd}_{\mathrm{p}}\left(\rho_{p}-\rho_{g}\right)}{3 \rho_{\mathrm{g}} C}\right]^{1 / 2}$

Where $d_{p}$ is the particle diameter. The drag coefficient for the spherical particles are usually plotted as a function of Reynolds number, Re, and is available from literature. By definition, $\operatorname{Re}=\rho_{\mathrm{g}} \mathrm{Vd} / \mu$, where $\mathrm{d}$ is a characteristic length such as a diameter and $\mu$ is 
the fluid dynamic viscosity. A Stokes time scale can be derived from drag force equation, as

$$
\tau=\frac{\left(\rho_{\mathrm{p}-} \rho_{g}\right) \mathrm{d}_{\mathrm{p}}{ }^{2}}{18 \mu}
$$

Since for air $\rho_{\mathrm{p}} \gg \rho_{\mathrm{g}}, \tau$ is usually written as

$$
\tau=\frac{1}{18} \frac{\mathrm{d}_{\mathrm{p}}^{2}}{\mu} \rho_{\mathrm{p}}
$$

The factor $\tau$ has units of seconds. It represents a relaxation time for the aerosol particle.

\subsection{Particle motion in an electric field}

When an uncharged spherical conducting aerosol particle is placed in a uniform electric field, the field near the particle is distorted, as illustrated in Fig. 4.1, so that gas ion following the field lines would immediately begin to charge the particle. The dashed lines indicate the limits of the field which passes through the sphere. When the particle becomes charged, it repels some of the incoming ions. This repulsion results in an alteration of the field configuration which accordingly reduces the charging rate. A point will be reached where no further charging of the particle takes place. This point is known as the saturation charge of the particle. When one-half the saturation charge on the particle is reached, the electric field surrounding the particle is similar to that shown in Fig. 4.2 (Rohmann, 1923; Pauthenier and Moreau-Hanot, 1923). 


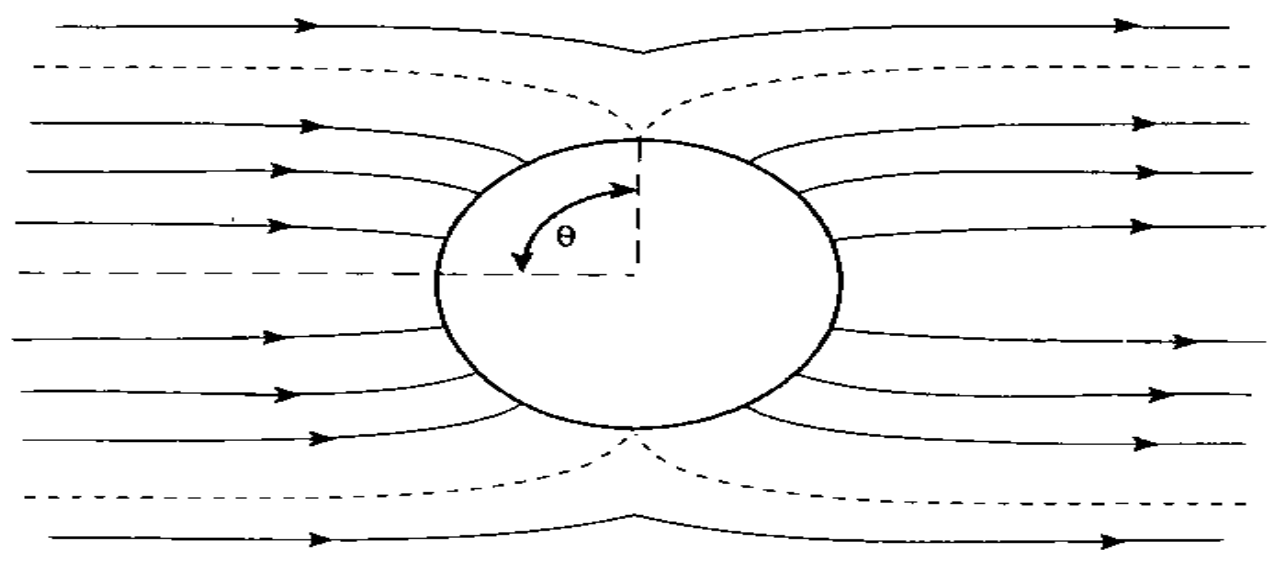

Figure 4.1 Electric field around an aerosol uncharged particle (Reist, 1993).

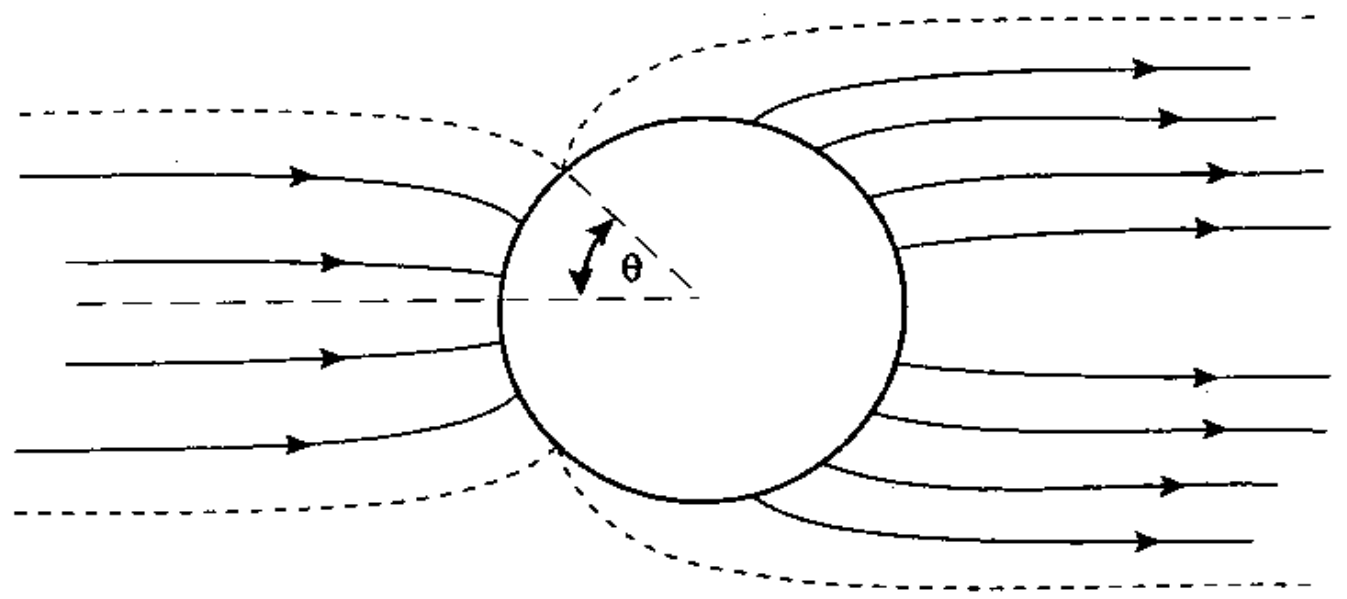

Figure 4.2 Electric field around a partially charged aerosol particle (Reist, 1993) 
The main reason for evaluating the charge on aerosol particles and the electric fields that act on these charges is to develop models which describe the effect on particle motion of the electric force. The equation of motion for a spherical aerosol particle in a homogeneous air stream at the presence of only gravity can be written as

$\mathrm{m} \frac{\mathrm{dv}}{\mathrm{dt}}=\mathrm{F}_{\mathrm{D}}+\mathrm{F}_{\mathrm{G}}$

where $\mathrm{m}$ is the mass of the particle and $\mathrm{v}$ is the velocity of the center of gravity of the aerosol particle. $F_{D}$ is the steady Stokes drag force on the particle represented as

$\mathrm{F}_{\mathrm{D}}=3 \pi \mu \mathrm{d}(\mathrm{u}-\mathrm{v})$

where $u$ is the velocity of the air stream near the particle. Therefore, (u-v) is the particle velocity relative to the medium velocity. The force of gravity can be written as

$\mathrm{F}_{\mathrm{G}}=m g$

Dividing the equation 4.7 by $3 \pi \mu \mathrm{d}$ and rearranging terms gives

$\tau \frac{\mathrm{dv}}{\mathrm{dt}}=(\mathrm{u}-\mathrm{v})+\tau \mathrm{g}$

where

$\tau=\frac{\mathrm{m}}{3 \pi \mu \mathrm{d}}$

The equation of motion for an aerosol particle at the present of an electric force can be written as

$\mathrm{m} \frac{\mathrm{dv}}{\mathrm{dt}}=\mathrm{F}_{\mathrm{D}}+\mathrm{F}_{\mathrm{G}}+\mathrm{F}_{\mathrm{E}}$

which becomes

$\tau \frac{\mathrm{dv}}{\mathrm{dt}}=(\mathrm{u}-\mathrm{v})+\tau \mathrm{g}-\mathrm{EqB}$ 
where $\mathrm{E}$ is the electric field, $\mathrm{q}$ is the particle charge and $\mathrm{B}$ is the particle mobility and can be written as

$$
\mathrm{B}=\frac{\mathrm{C}_{\mathrm{c}}}{3 \pi \mu \mathrm{d}}
$$

Where $\mathrm{C}_{\mathrm{c}}$ is the Cunningham correction factor and is available from literature.

Let $\mathrm{u}_{0}=\mathrm{u}+\tau g$

Then equation 4.14 becomes

$\tau \frac{\mathrm{dv}}{\mathrm{dt}}+\mathrm{v}=\mathrm{u}_{0}-\mathrm{EqB}$

In terms of a dimensionless velocity $\mathrm{v}^{\prime}=\frac{\mathrm{v}}{\mathrm{u}_{0}}$, we can write the above equation as

$\tau \frac{\mathrm{dv} \mathrm{v}^{\prime}}{\mathrm{dt}}+\mathrm{v}^{\prime}=1-\Gamma$

the dimensionless parameter $\Gamma$ (can be either positive or negative) is equal to $\mathrm{EqB} / \mathrm{u}_{0}$ and indicates the ratio of the particle velocity in an electric field to the constant velocity $\mathrm{u}_{0}$. If $|\Gamma|>>1$, then electric forces predominate, whereas when $|\Gamma|<<1$, gravity and inertial effects predominate. When $|\Gamma|>>1, \tau$ is very small and the inertia term $\tau \mathrm{dv}^{\prime} / \mathrm{dt}$ can be ignored. The equation 4.16 can be simplified as

$v^{\prime} \cong-\Gamma$

$\mathrm{v} \cong-\mathrm{EqB}$

where $\mathrm{v}$ is the electrical drift velocity. Assuming a saturation or field charge, the equation 4.18 can be written as 


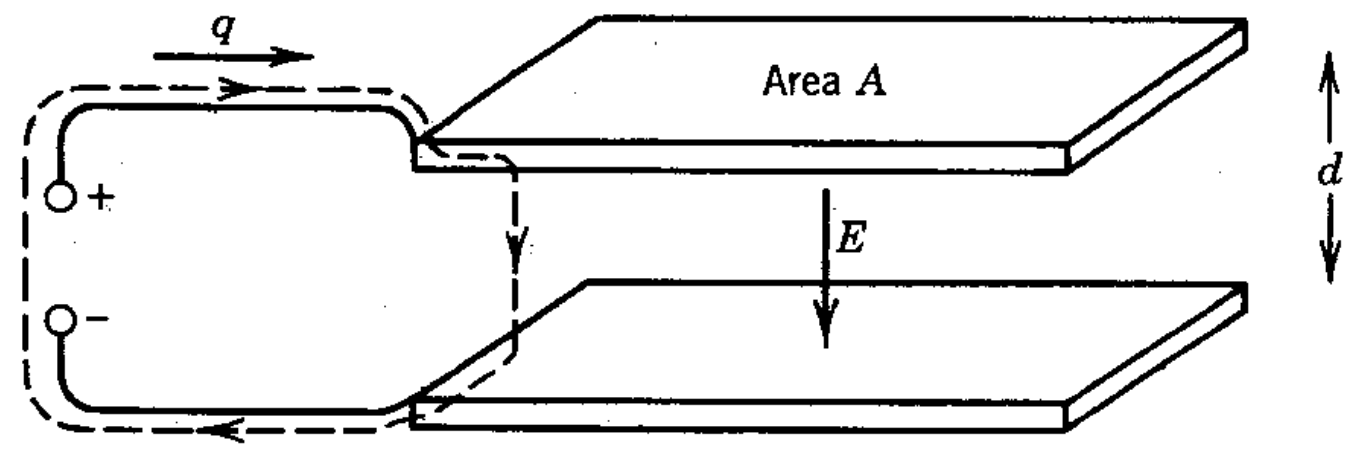

Figure 4.3: Electric field between parallel plates with plate spacing, d (Crowley, 1986)

$w=\mathrm{E}\left(\frac{x \mathrm{E}_{0} \mathrm{~d}^{2}}{4}\right)\left(\frac{\mathrm{C}_{\mathrm{c}}}{3 \pi \mu \mathrm{d}}\right)$

or

$w=\frac{x \mathrm{EE}_{0} \mathrm{dC}_{\mathrm{c}}}{12 \pi \mu}$

where $x=3 \varepsilon_{0} /\left(\varepsilon_{0}+2\right)$ and $\varepsilon_{0}$ is the dielectric constant of the spherical aerosol particle and $w$ is the particle velocity in an electric field. The term E0 is the field generating the particle charge and $\mathrm{E}$ is the collecting field strength.

\section{$\underline{4.3 \text { Electric filed between parallel plates }}$}

Electrostatics involves charges in motion as well as at rest. The most fundamental quantities in electrostatics are voltage, charge, and current. Voltages or electromotive forces (EMF) are the most important fundamental quantities for engineers. The word 
force reflects the fact that voltage is related to the force applied to a charge and is derived from the electrostatic force law

$\mathrm{F}=\mathrm{qE}$

The F vector is the force experienced by a unit charge. This force can be expressed by

$$
\oint \mathrm{qE} \bullet \mathrm{dr}=0
$$

When a voltage is applied across a gap between two parallel plates (Figure 4.1), the electric field can be specified by (Crowley, 1986),

$$
\oint \mathrm{E} \bullet \mathrm{dr}=\int_{-t e r \text { min } a l}^{+t e r \text { min } a l} \mathrm{E} \bullet \mathrm{dr}+\int_{\text {wires }} \mathrm{E} \bullet \mathrm{dr}+\int_{\text {gap }} \mathrm{E} \bullet \mathrm{dr}=0
$$

The integral inside the wires vanishes because the electric field inside a good conductor is very small. The electric field $\mathrm{E}$ is uniform inside the capacitor, so only two line integrals are left to evaluate.

The integral becomes

$$
\int_{g a p}^{\mathrm{E}} \bullet \mathrm{dr}=\mathrm{Ed}
$$

The E field between the terminals can be described as the voltage across the terminals:

$$
\mathrm{V}=-\int_{\text {-terminal }}^{+ \text {terminal }} \mathrm{E} \bullet \mathrm{dr}
$$

Thus, in this example, the terminal voltage is given by

$$
\mathrm{V}=\mathrm{Ed}
$$

Breakdown usually occurs at high electric field within the material. It should be noted that there are two criteria for breakdown to become possible, one based on voltage and one on electric field. Generally, both limits must be exceeded before breakdown. For air, the breakdown voltage is 300 volts, and the breakdown electric field is $3 \times 10^{6} \mathrm{v} / \mathrm{m}$. 
Below this voltage, the components can be placed as close as a few microns apart. Above this voltage, corona and sparking must be considered in the design which increase both cost and size. Breakdown actually occurs when the insulation properties of the material break down and the material acts like a conductor. If the air acts like a conductor, the current can actually surge across the gap which is known as sparking. A corona discharge usually occur near a conductor with sharp points, or a small radius of curvature. This breakdown is usually manifested by a blue glow. The corona is said to be negative if a cloud of negative ions is formed and positive if positive ions are formed. Corona characteristics will depend on the predominant gas and impurities present. In our experimental system, only water aerosol was tested at various mist and air flow rates.

Williams and Bailey (1983) describe that in their experiment that the permittivity of the particles is larger than that of the fluid. Also, the inter-particle force depends only on the permittivity of the fluid and the size of the particle. Therefore, the size of the particle, electric field strength, separation distance between particles and the fluid permittivity are very important properties which influence the electro-coalescence.

\subsection{Theory of electro-coalescence}

A number of external physical factors can act to enhance the coagulation of aerosols. One important factor is electrical effects such as the attraction or mutual repulsion of charged or polarized particles. In an electric field, particles diffuse toward one another until they are close enough for electric forces to come into play. Then the motion of the particles assumes an ordered nature. 
As two drops approach one another, the strength of the electric field in the region between their points of closest separation can attain values many times larger than that acting by the applied electric field on drops, when they are far from each other. This is a result of the mutual interactions between the induced charges on the drop surfaces (Latham \& Roxburgh, 1966).

The model of Zhang et al., (1995) has been developed to quantitatively predict collision and coalescence rates of conducting, charge-free spherical drops and their growth induced by combined gravitational and electric-field-induced forces. Yiacoumi et al., (1996) developed a similar model for particle coagulation in magnetic field.

$$
\begin{gathered}
V_{12}=V_{12}^{0}\left(-L \cos \theta e_{r}+M \sin \theta e_{\theta}\right)-\frac{\mathrm{D}_{12}^{0}}{\mathrm{kT}}\left(G F_{\left.\mathrm{E}, 12{ }^{2} \mathrm{e}^{\mathrm{e}}-H F_{\mathrm{E}, 12}^{\theta} \theta\right)}^{\mathrm{e} \theta}\right)-\frac{\mathrm{D}_{12}^{0}}{\mathrm{kT}} G F_{\mathrm{p}, 12}{ }_{\mathrm{r}}^{\mathrm{e}} \\
\text { gravity } \\
\text { electric field }
\end{gathered}
$$

where $V_{12}^{0}$ is the relative velocity of two widely separated drops/particles due to gravity which is given by the Hadamard-Rybezynski formula (Kim \& Karrila, 1991), $e_{r}$ and $e_{\theta}$ are unit vectors along and normal to the line of centers, respectively, $D_{12}^{0}$ is the relative diffusivity of two widely separated drops, $\mathrm{k}$ is Boltzmann constant, $\mathrm{T}$ is the absolute temperature, $L, M, G$, and $H$ are the relative mobility functions describing the viscous effects on drop motion, and $F_{\mathrm{E}, 12}$ and $F_{\mathrm{P}, 12}$ are electric-field induced forces (See Equations $1 \& 2$ in chapter 2) and interparticle forces, respectively.

Recently, Ezekoye and Wibowo (1999) used a discretized general dynamic equation (Smoluchowski's equation) in the absence of sedimentation and breakup as, 
$\frac{d n_{k}}{d t}=\frac{1}{2} \sum_{i+j-k} \beta\left(v_{i}, v_{j}\right) n_{i} n_{j}-n_{k} \sum_{i-1} \beta\left(v_{i}, v_{k}\right) n_{i}$

The first term represents the rate of conversion of particles of size $i$ and $j$ into size $k$ such that the sum of the volumes of the $i$ and $j$ particles is to the volume of the $k$ particle. The second term represents the conversion of $k$ sized particles to larger particles through collisions with the rest of the distribution. The evaluation of $\beta$ for various types of collisional processes is essential to properly modeling/characterizing the aerosol dynamics and in terms of relative velocity between particles, it can be shown as,

$\beta n_{1} n_{2}=\pi \mathrm{r}^{2} V_{12} n_{1} n_{2}=\pi \mathrm{r}^{2}\left(1_{1}-V_{2}\right) n_{1} n_{2}$

where $\beta$ is the collision rate, $n_{1}$ and $n_{2}$ are number densities of droplets 1 and 2 , respectively, $V_{12}$ is the relative velocity between two droplets (equation 4.27), $\mathrm{r}$ is the radius of the collision volume.

A limiting trajectory can be determined along which two drops approach each other with the largest possible horizontal displacement, $y_{c}$, such that they will collide, as shown in Figure 4.4 which is duplicated from Zhang et al. (1991). Any relative trajectories that are inside this limiting trajectory end with the drops colliding, whereas those outside the limiting trajectory end with the drop separating. The collision or coalescence efficiency can be calculated to describe the effects of electric-field induced and van der Waals attractive forces and viscous resistances, which can be defined as :

$$
\beta=\frac{\mathrm{y}_{\mathrm{c}}^{2}}{\left(a_{1}+a_{2}\right)^{2}}
$$




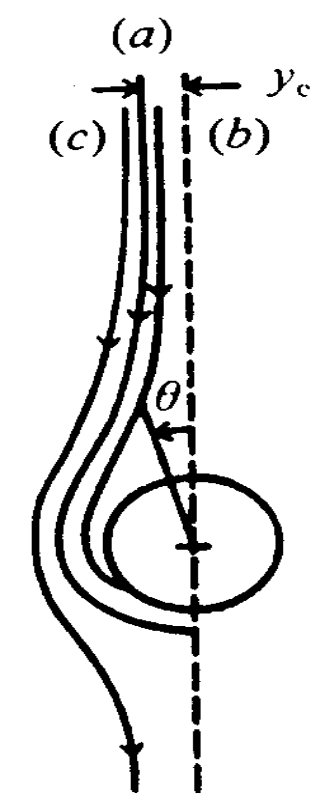

Figure 4.4: Possible relative trajectories of two drops: (a) the limiting trajectory; (b) a trajectory terminating with contact; and (c) a trajectory for which two drops move past one another and separate (Zhang et al., 1991).

where $a_{1}$ and $a_{2}$ are the radii of the two conducting droplets respectively. The resultant coalescence efficiency can be used to compute the evolution of a population of drops in a homogeneous, isotropic dispersion by solving the population balance equation (4.28).

This equation was also solved by Tsouris et al., (1995) to include coagulation of magnetic particles in a magnetic field. This model can be readily employed to describe coalescence of polarized droplets. 


\section{CHAPTER 5}

\section{RESULTS AND DISCUSSION}

\subsection{Observations}

Prior to the discussion of experimental results, it is important to point out the following observations when the experimental set up was turned on to conduct experiments of this research.

- At zero voltage, the mist distribution was uniform through out the test chamber.

- At a given voltage level, the water droplets were deposited first on the lower end of positive electrode, principally. As the experiment progressed over time, the droplet formation was observed to progress up the surfaces of both electrodes. Droplets drained down the electrodes and formed pools on the bottom of the test unit.

- The electric field caused the mist motion to change from a uniform pattern to a somewhat random pattern with some jetting toward the positive electrode. This more or less chaotic droplet motion was especially intense in the voltage range of $20 \mathrm{kV}$ and above.

- In all cases, at high voltages, the droplets build up at the lower level of the test chamber, causing short circuit between positive and negative electrodes, resulting in sparks and thereby shutting down the system. It was therefore necessary to limit the voltage drop across the electrodes to $26 \mathrm{kV}$ in these experiments in the test apparatus. 


\section{$\underline{5.2 \text { Test conditions }}$}

All experimental measurements were obtained at room temperature of approximately $23^{\circ} \mathrm{C}$ and ambient pressure of $750-\mathrm{mm} \mathrm{Hg}$. The air density $\rho_{\text {air }}=1.187 \mathrm{~kg} / \mathrm{m}^{3}$, air dynamic viscosity $\mu_{\text {air }}=1.86 \times 10^{-5} \mathrm{~kg} / \mathrm{m} . \mathrm{s}$, and tunnel equivalent diameter $\mathrm{D}_{\mathrm{e}}=0.1146 \mathrm{~m}$ were assumed for the calculation of Reynolds Numbers in all experiments. The accuracy of dry-ice-trap was tested by measuring the mass of water vapor in the air passed through the trap and the material balance equation presented in Chapter 4 (Equation (3.15)). Assuming that the air behaves as an ideal gas, the mass of collected water from the room's air in the trap and calculated mass by using the material balance equation were compared and the range of error was within 6 percent. This range was confirmed at different flow rates used in the experiments (Table 5.1).

Table 5.1: Percent error of measured and calculated mass of collected water from room's air at variable flow rates.

\begin{tabular}{|c|c|c|c|c|}
\hline Run & $\begin{array}{c}\text { Flow Rate } \\
(1 / \mathrm{min})\end{array}$ & $\begin{array}{c}\text { Measured mass } \\
\text { during 33 min } \\
(\mathrm{g})\end{array}$ & $\begin{array}{c}\text { Calculated mass } \\
\text { during 33 min } \\
(\mathrm{g})\end{array}$ & Percent Error \\
\hline 1 & 3.0 & 0.38 & 0.40 & 5.00 \\
\hline 2 & 4.5 & 0.50 & 0.53 & 5.66 \\
\hline 3 & 5.0 & 0.58 & 0.62 & 6.50 \\
\hline 4 & 5.5 & 0.48 & 0.50 & 4.00 \\
\hline 5 & 6.0 & 0.50 & 0.53 & 5.70 \\
\hline 6 & 7.0 & 0.63 & 0.65 & 3.08 \\
\hline
\end{tabular}


Each experimental run was made at a fixed value of the air flow rate and mist

flow rate. The range of Reynolds Number was from 940 to 2400 in all experiments. The raw experimental data were converted into a mist concentration as described in the dryice procedure (Sections 3.2 and 3.3). The mist concentration was plotted vs. applied voltage and light transmissions for each experiment. The applied voltages were $0,10,15$, 18, 20, 22, 24, and 26 kilovolts. These values were selected based on the power supply and the test chamber limitations.

\subsection{Effects of electric field strength on droplet concentration}

Figures 5.1 through 5.5 show experimental data obtained by the method isokinetic/dry-ice sampling at Reynolds Numbers 1350, 1620, 1680, 1950, and 2200 respectively. The figures indicate that the electric field has an effect on the flowing mist in the wind tunnel. They also reveal that as the applied electric potential increases, the quantity of the mist in the air stream decreases. This is due to the increasing number of collisions between droplets (agglomeration), droplets and electrodes and droplets with the chamber walls as the droplets flow in the tunnel under the influence of electric field. Note in Figure 5.3 that the quantity of droplets collected levels off above an applied voltage of $24 \mathrm{kV}$. This effect is reproducible in this case suggesting that no further reduction of droplets take place. The standard deviation for all experimental data is shown in the form of error bar. 


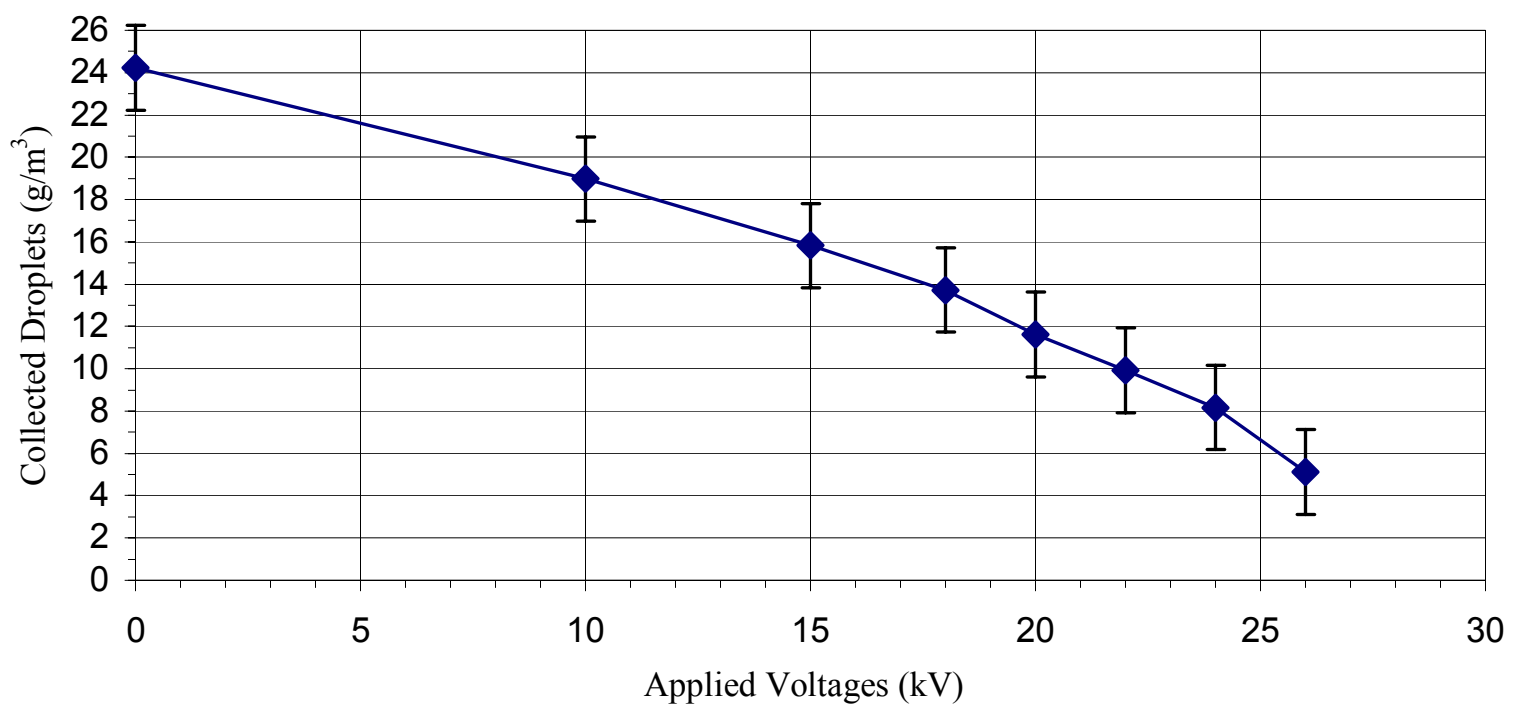

Figure 5.1: Droplets Concentration $\left(\mathrm{g} / \mathrm{m}^{3}\right)$ vs. Applied Voltage $(\mathrm{kV})$ at Reynolds Number of 1350

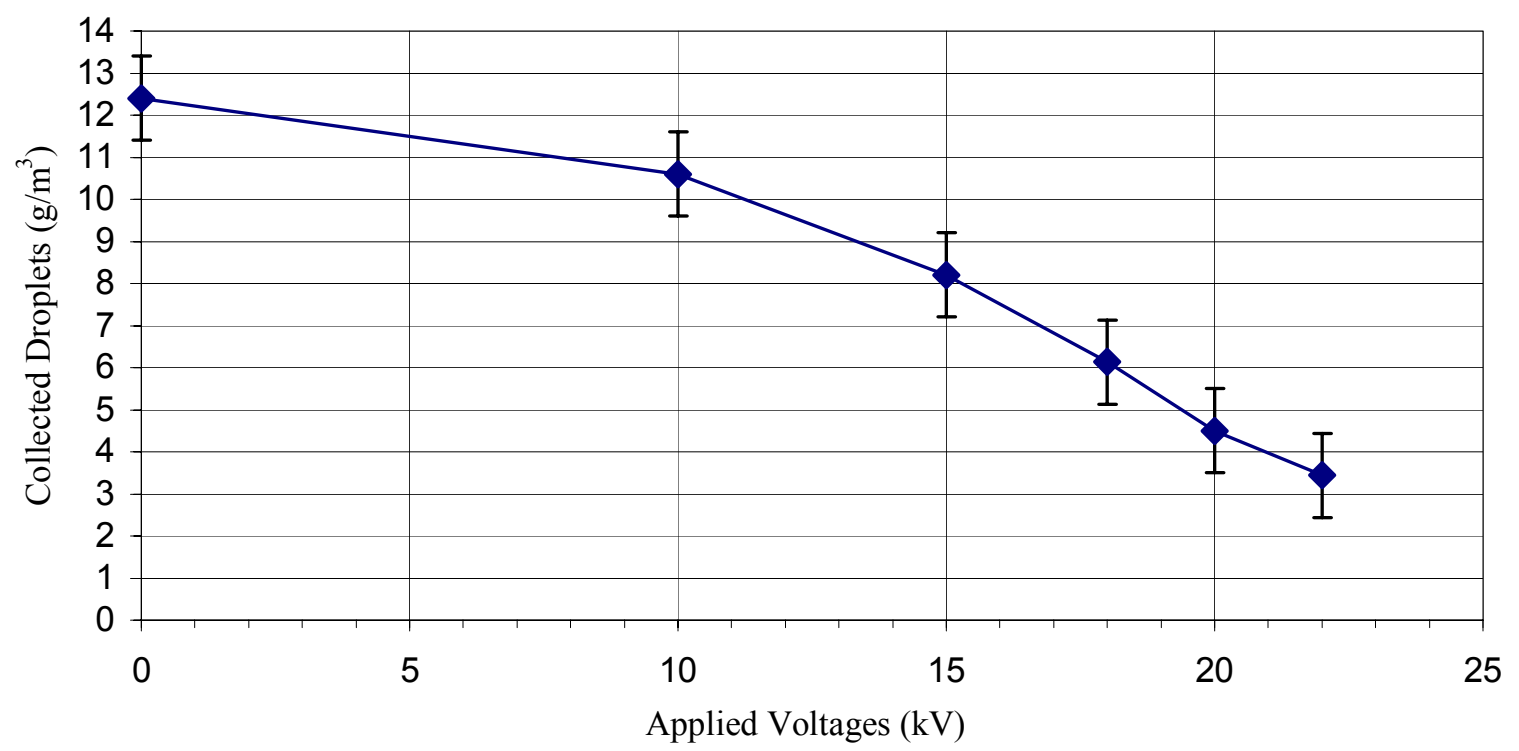

Figure 5.2: Droplets Concentration $\left(\mathrm{g} / \mathrm{m}^{3}\right)$ vs. Applied Voltage $(\mathrm{kV})$ at Reynolds Number of 1620 


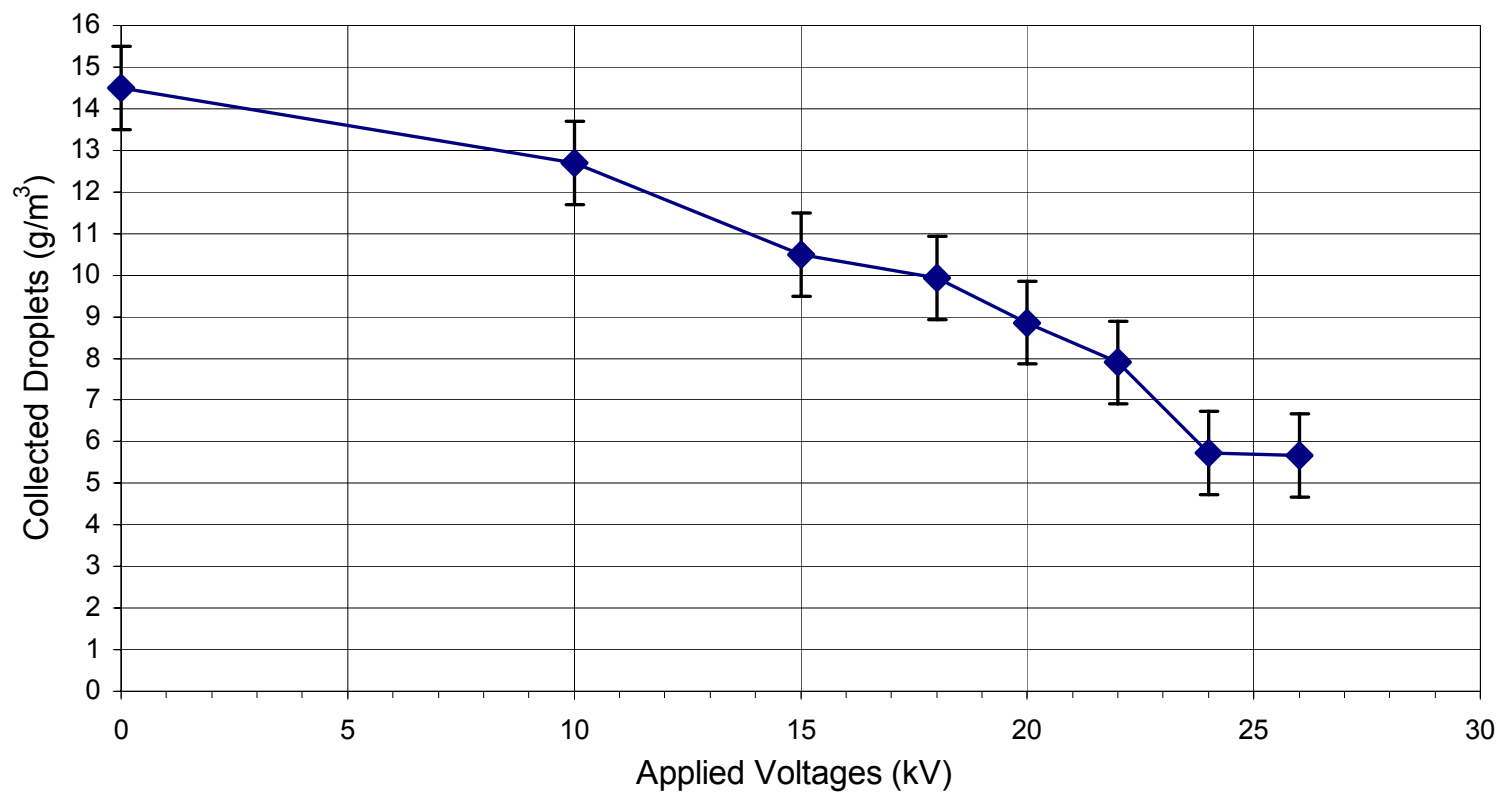

Figure 5.3: Droplets Concentration $\left(\mathrm{g} / \mathrm{m}^{3}\right)$ vs. Applied Voltage $(\mathrm{kV})$ at Reynolds Number of 1680

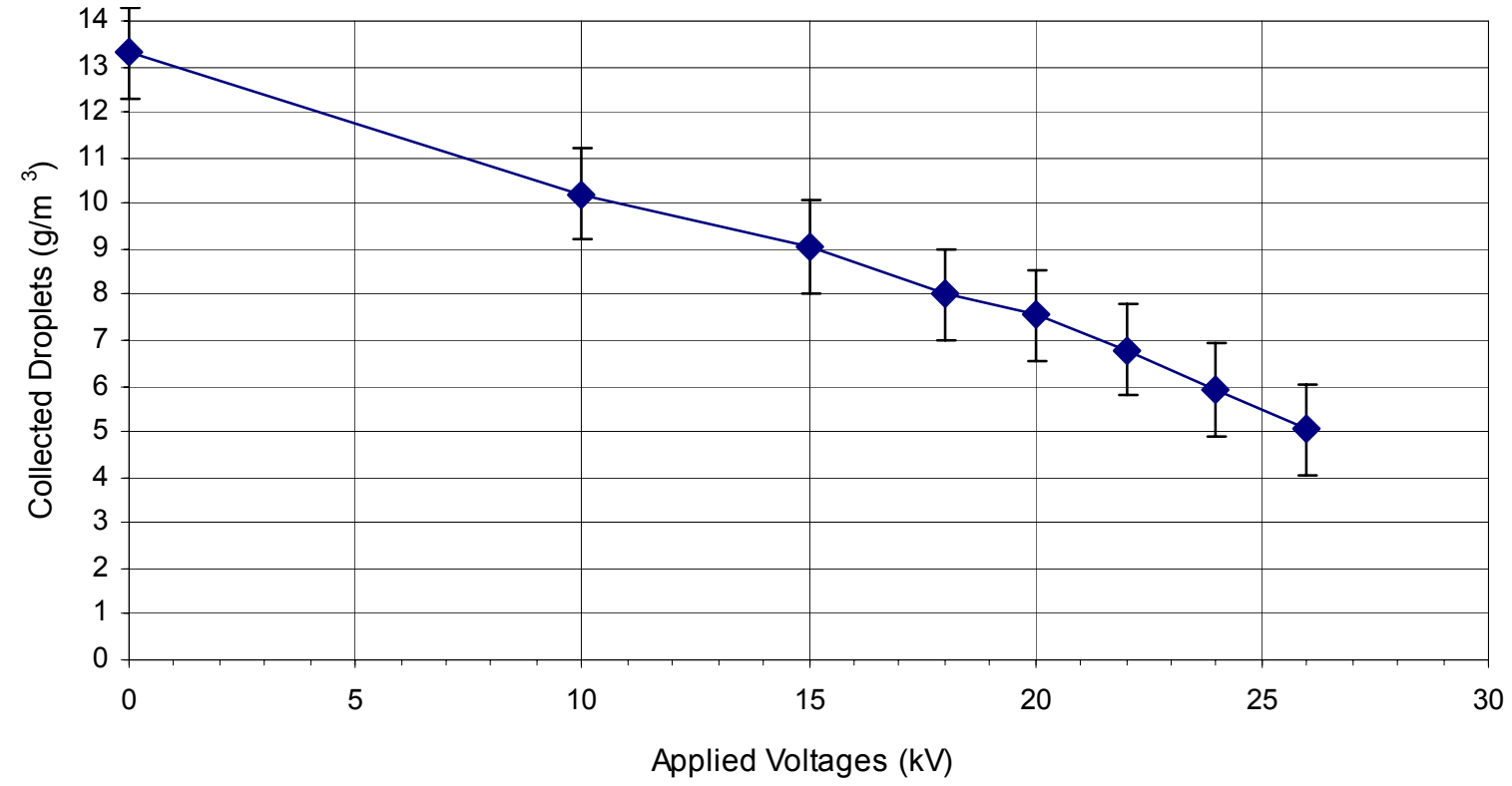

Figure 5.4: Droplets Concentration $\left(\mathrm{g} / \mathrm{m}^{3}\right)$ vs. Applied Voltage $(\mathrm{kV})$ at Reynolds Number of 1950 


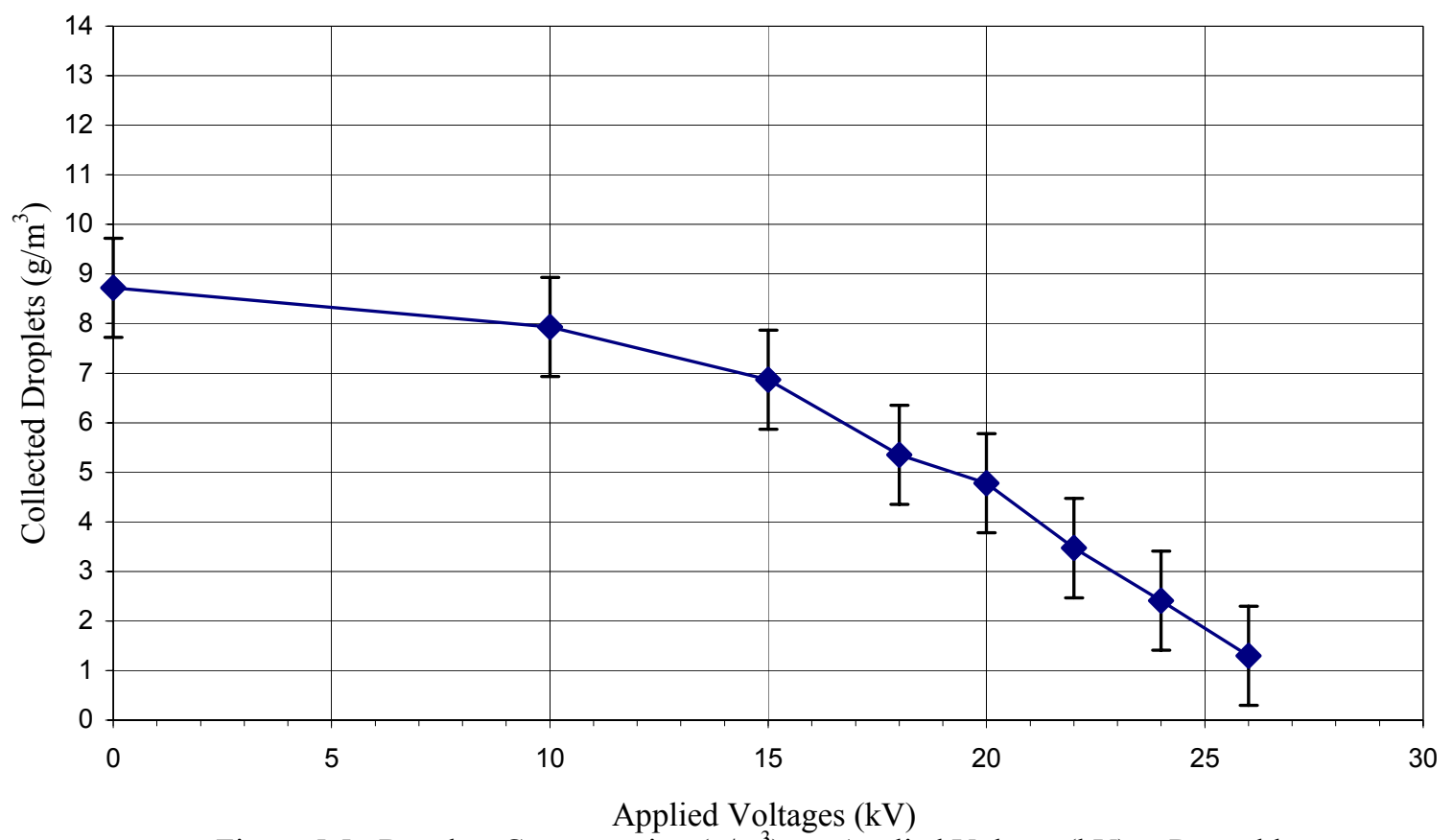

Figure 5.5: Droplets Concentration $\left(\mathrm{g} / \mathrm{m}^{3}\right)$ vs. Applied Voltage $(\mathrm{kV})$ at Reynolds Number of 2200

\subsection{Effects of electric field strength on light transmission reduction}

Figures 5.6 through 5.10 show the relationship between the concentration of collected droplets and light transmission. The investigation of each individual data point reveals that the light transmission is related directly to mist concentration. This could be due to the increasing number of collisions as described in the previous section. 


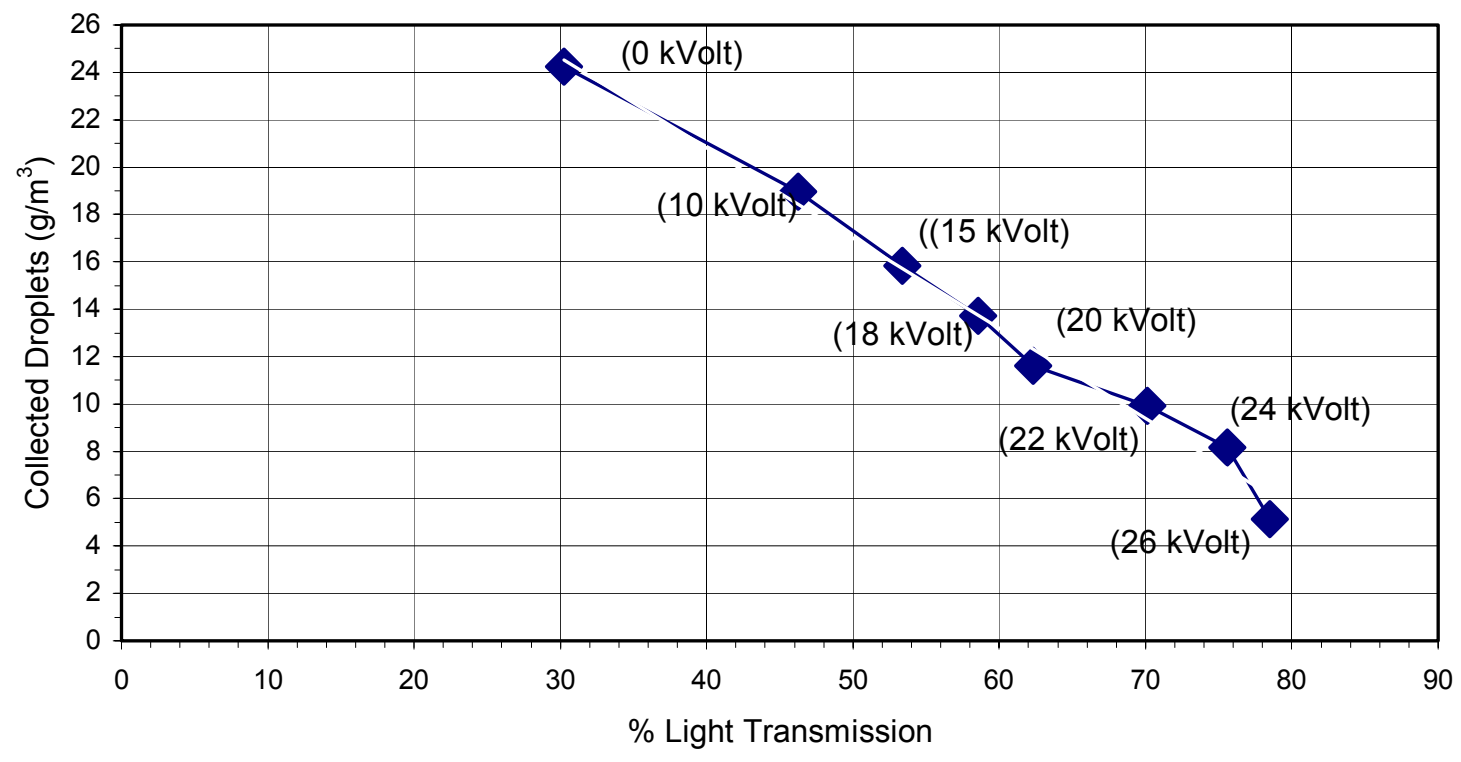

Figure 5.6: Droplets Concentration $\left(\mathrm{g} / \mathrm{m}^{3}\right)$ vs. Percent Light Transmission at Reynolds Number of 1350 under Varying Applied Voltage

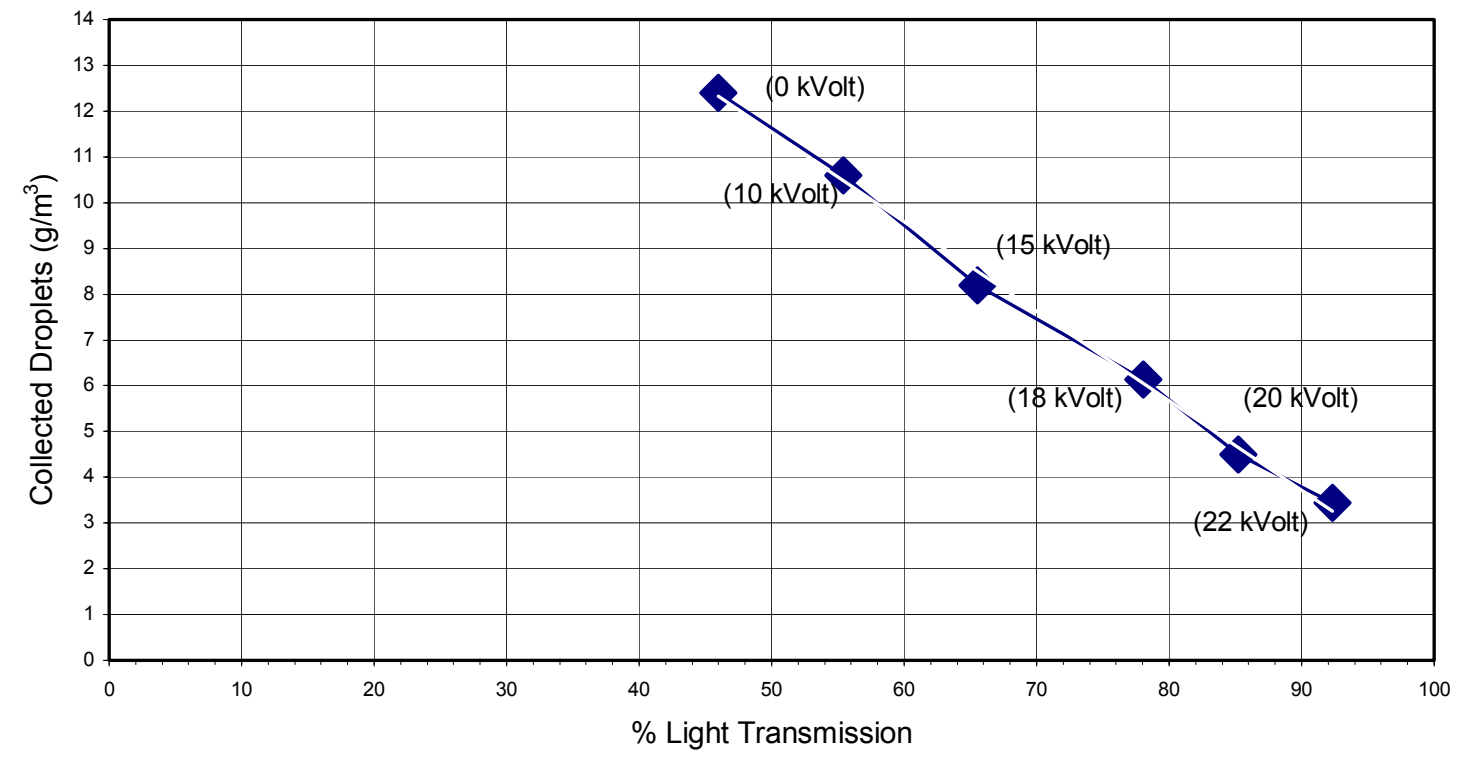

Figure 5.7: Droplets Concentration $\left(\mathrm{g} / \mathrm{m}^{3}\right)$ vs. Percent Light Transmission at Reynolds Number of 1620 under varying Applied Voltage 


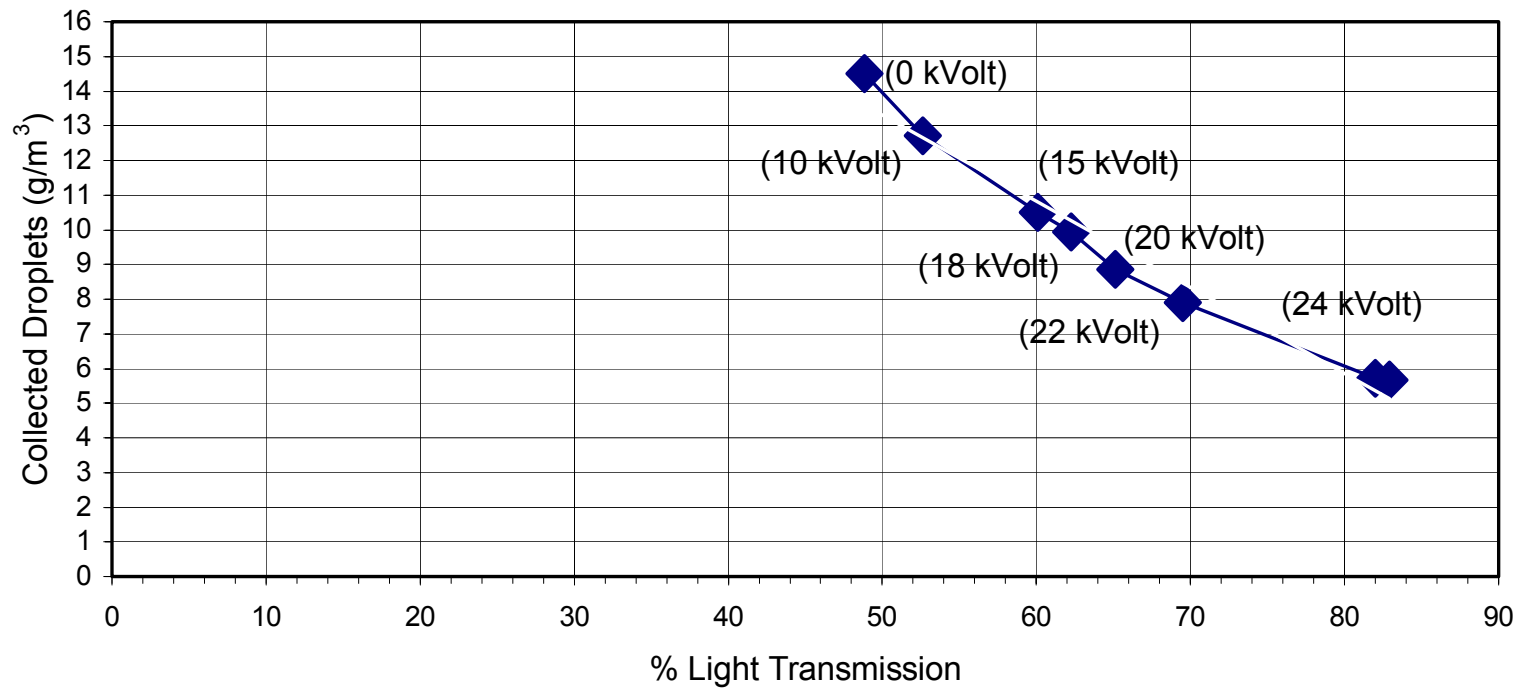

Figure 5.8: Droplets Concentration $\left(\mathrm{g} / \mathrm{m}^{3}\right)$ vs. Percent Light Transmission at Reynolds Number of 1680 under Varying Applied Voltage

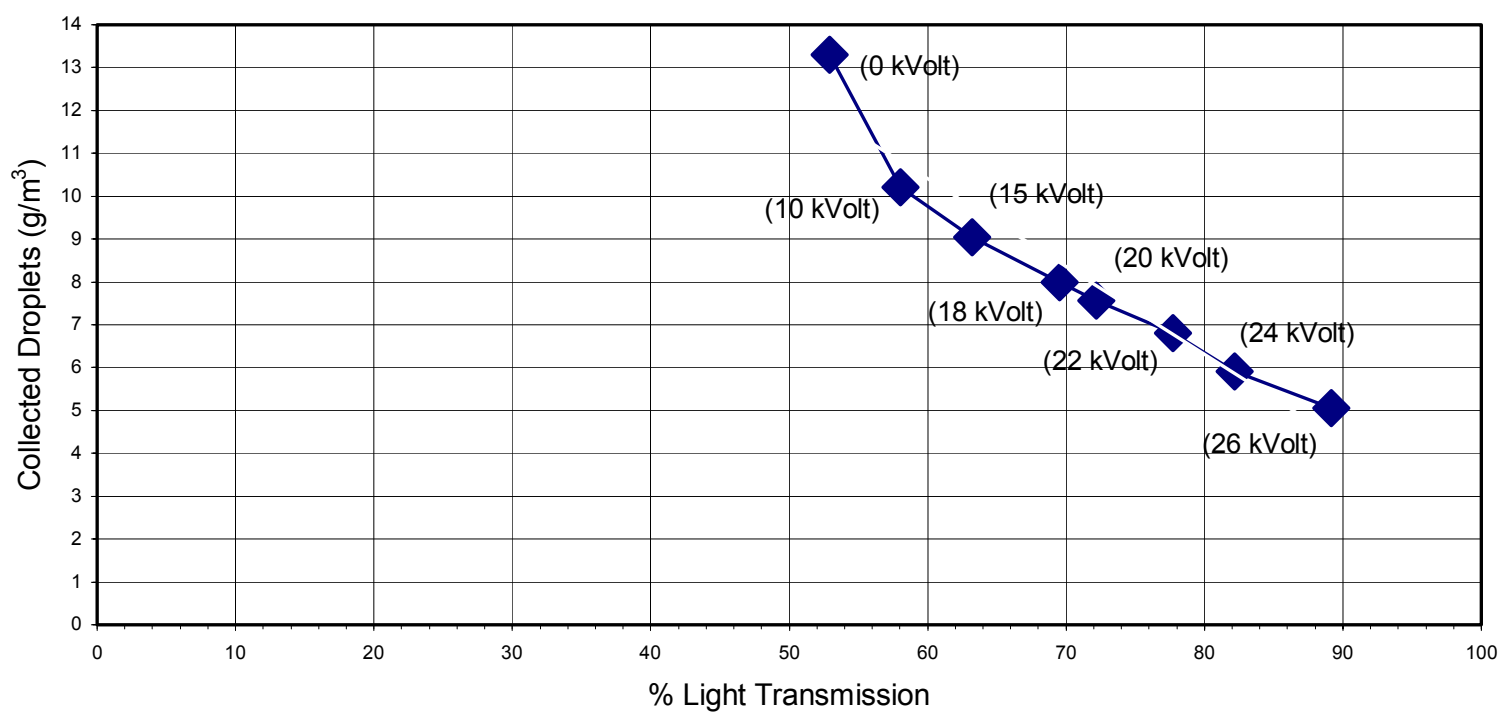

Figure 5.9: Droplets Concentration $\left(\mathrm{g} / \mathrm{m}^{3}\right)$ vs. Percent Light Transmission at Reynolds Number of 1950 under Varing Applied Voltage 


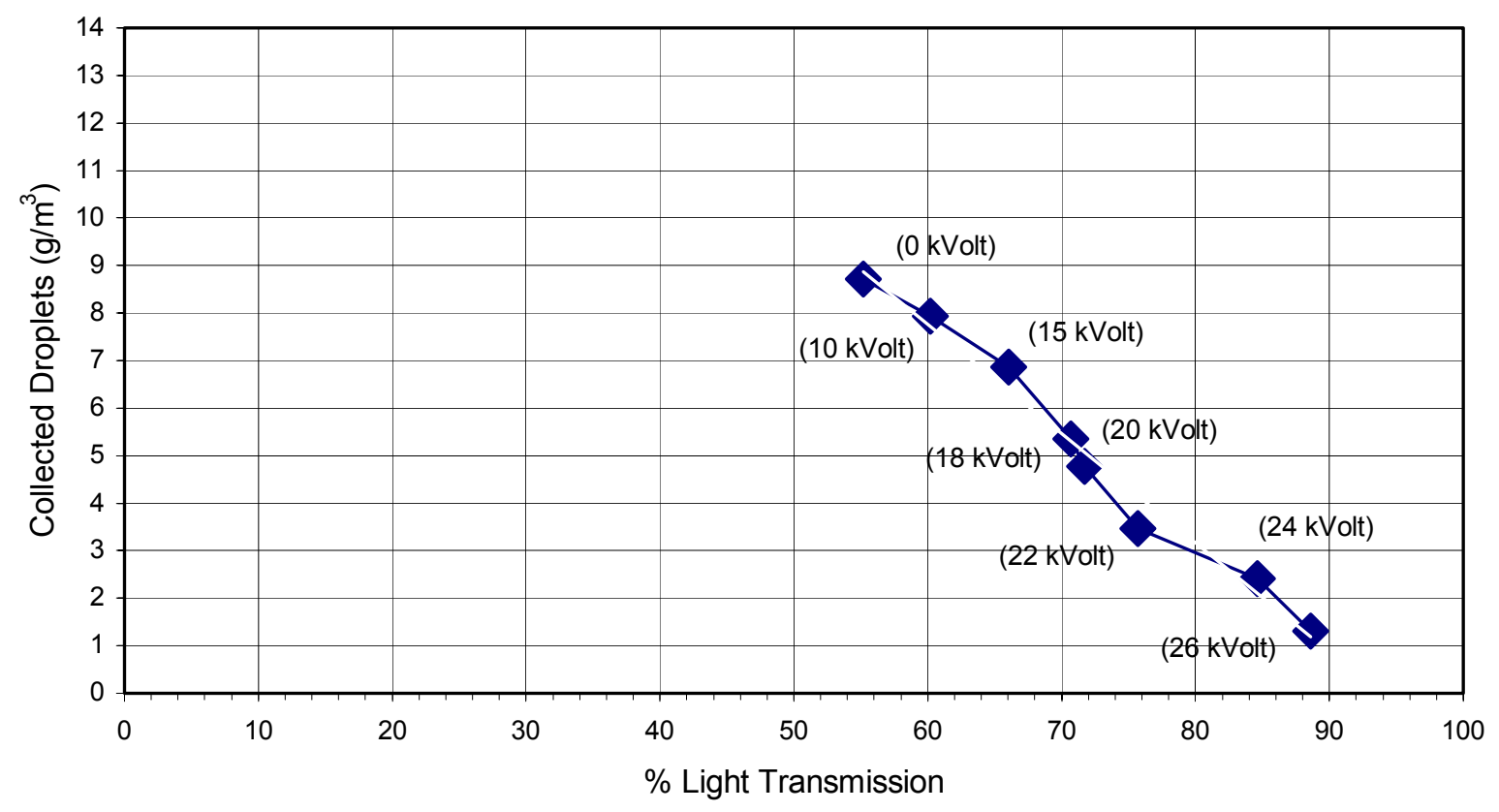

Figure 5.10: Droplets Concentration $\left(\mathrm{g} / \mathrm{m}^{3}\right)$ vs. Percent Light Transmission at Reynolds Number of 2200 under Varying Applied Voltage

\subsection{Effect of electric field on droplet numbers}

A number of experiments were conducted to understand the effect of electric field on both average droplet size and droplet distribution. The average droplet size $\left(\mathrm{d}_{\mathrm{ave}}\right)$ was calculated by averaging all sizes of droplets that passed through the laser path. The number of droplets was determined in the following manner

- Calculated the average droplet size, $\mathrm{d}_{\mathrm{ave}}$

- Determined the average droplet volume, $\mathrm{V}_{\mathrm{ave}}=4 \pi / 3\left(\mathrm{~d}_{\mathrm{ave}} / 2\right)^{3}$

- Measured the mist density obtained from isokentic/dry-ice method 
- Multiplied the volume frequency obtained from RTSizer instrument by mist density to determine the total water volume fraction, $\mathrm{V}_{\mathrm{x}}$, for droplet size.

- Estimated the number of droplets by dividing $\mathrm{V}_{\mathrm{x}}$ over $\mathrm{V}_{\mathrm{ave}}$

Figure 5.11 shows the distributions of number of droplets versus the averaged droplet size at Reynolds number 1620 and the inlet mist concentration of $12.4\left(\mathrm{~g} / \mathrm{m}^{3}\right)$ for a range of applied voltages. The figure reveals that the size range of droplets does not change significantly with voltage; however, the distribution of the number of droplets does change. The changes are rather significant for droplet sizes in the range of 4 to $8 \mathrm{~m}$. For instance, when the applied voltage increases from 0 to $22 \mathrm{kV}$, the number of droplets reduces from $1.3 \times 10^{10}$ to $4.0 \times 10^{8}$ for the droplet size $5.23 \mathrm{~m}$. The main reason for this large reduction is attributed to the collision of droplets with the electrodes and the test chamber walls. As a result of these collisions, many droplets attach to the wall and electrodes, and they eventually flow downward to the chamber floor because of gravitational forces. The same experiment was conducted with a Reynolds number of 2200. Figure 5.12 shows a very similar behavior as seen in figure 5.11. It was also observed that the mist concentration reduces from 12.4 to $8.7\left(\mathrm{~g} / \mathrm{m}^{3}\right)$ when the Reynolds Number increases from 1620 to 2200 .

\subsection{Size distribution}

A number of experiments were conducted to determine whether or not the size of droplets could vary as the Reynolds number and mist concentration change under different voltage levels. In one experiment, the air flow rate was arbitrarily changed to a specific value corresponding to a Reynolds Number of 1230 . At this number, the 
measured droplet concentration using dry-ice trap method was $12.1(\mathrm{~g} / \mathrm{m} 3)$ for a zero voltage.

The droplet size distributions for 0 and $26 \mathrm{kV}$ are shown in Figures 5.13a and 5.13b. A comparison between the two figures indicates that the size distribution range is increased from [3.97-31.56] $\mu \mathrm{m}$ to [3.97-47.78] $\mu \mathrm{m}$. It also reveals that the light transmission percent is increased from 63.5 to 94.4 . The reason for these differences could be attributed to the enhanced agglomeration and droplet removal at higher voltages.

A similar experiment was carried out with a different droplet concentration but with the same flow rate value of the first experiment. In this experiment the droplet concentration was increased by twice as much as the first experiment. The results of this experiment are shown in Figures 5.14a and 5.14b. The results indicate that the change in size distribution in this experiment are more pronounced than those seen in Figure 5.13a and 5.13b. This is due to the fact that at higher mist concentration rates, the relative distances between droplets are shorter in a fixed volume. This causes enhanced mutual interactions between the droplets (Latham \& Roxburgh, 1966). In both experiments, it was observed that the droplet concentration was reduced when the applied voltage increased from 0 to $26 \mathrm{kV}$. A complete set of graphical results at various voltages $(0-26$ $\mathrm{kV}$ ) is shown in the Appendix at Reynolds Number of 1350 for starting droplet concentrations of $12.1 \mathrm{~g} / \mathrm{m}^{3}$ and $24.2 \mathrm{~g} / \mathrm{m}^{3}$ respectively. 


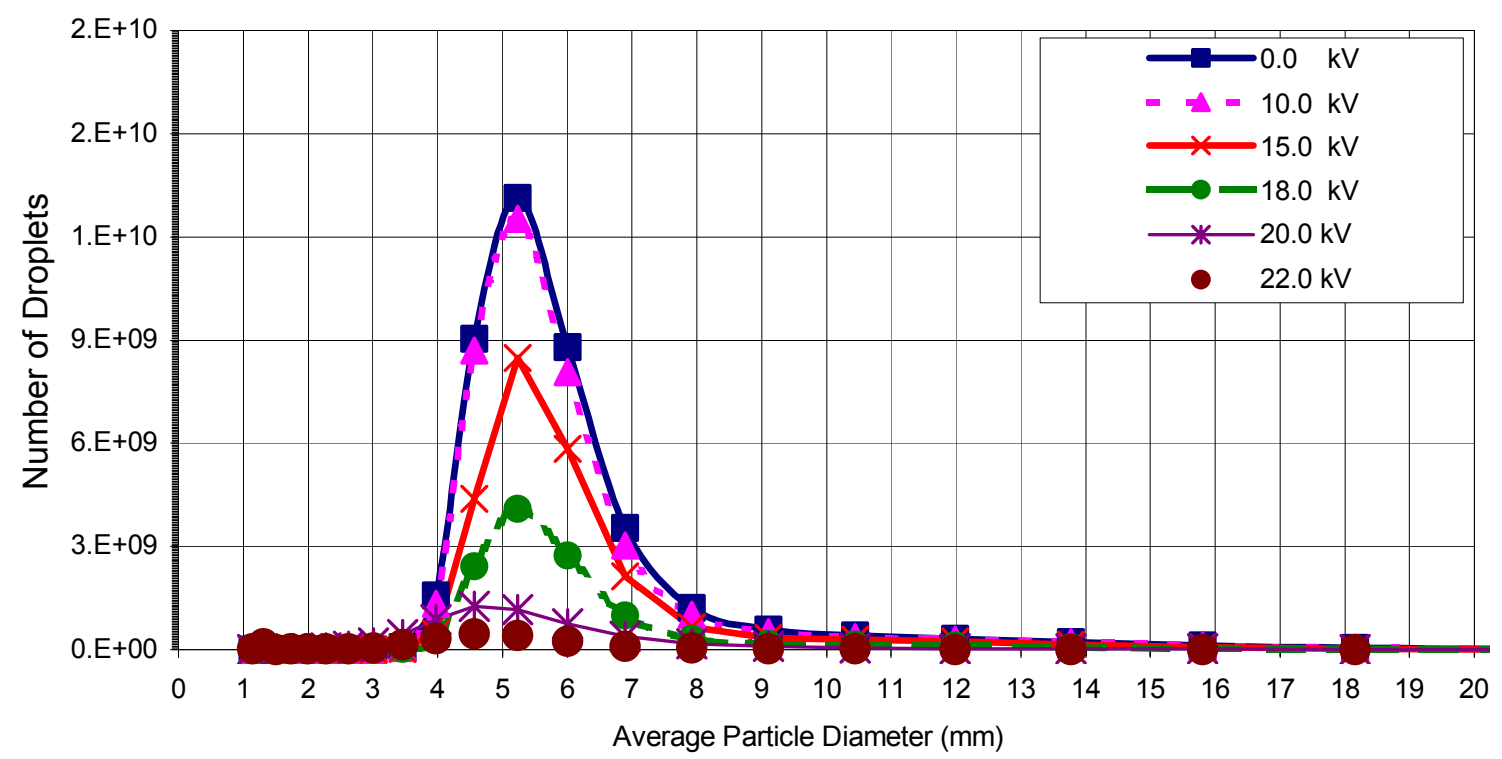

Figure 5.11: Number of Droplets vs. Particle Diameter in Microns at Inlet Mist Concentration of $12.4 \mathrm{~g} / \mathrm{m}^{3}$ and Reynolds Number of 1620

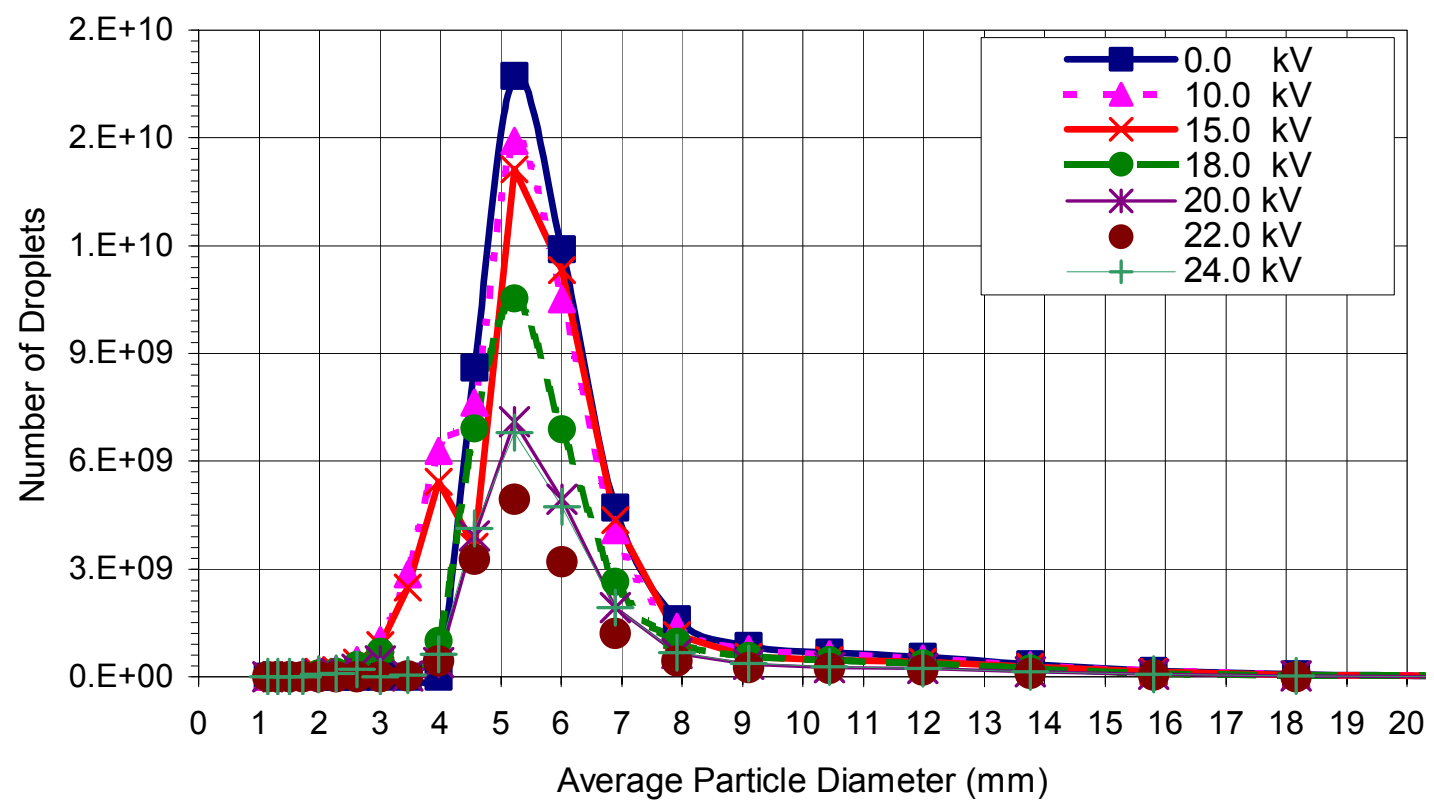

Figure 5.12: Number of Droplets vs. Particle Diameter in Microns at Inlet Mist Concentration of $8.72 \mathrm{~g} / \mathrm{m}^{3}$ and Reynolds Number of 2200 


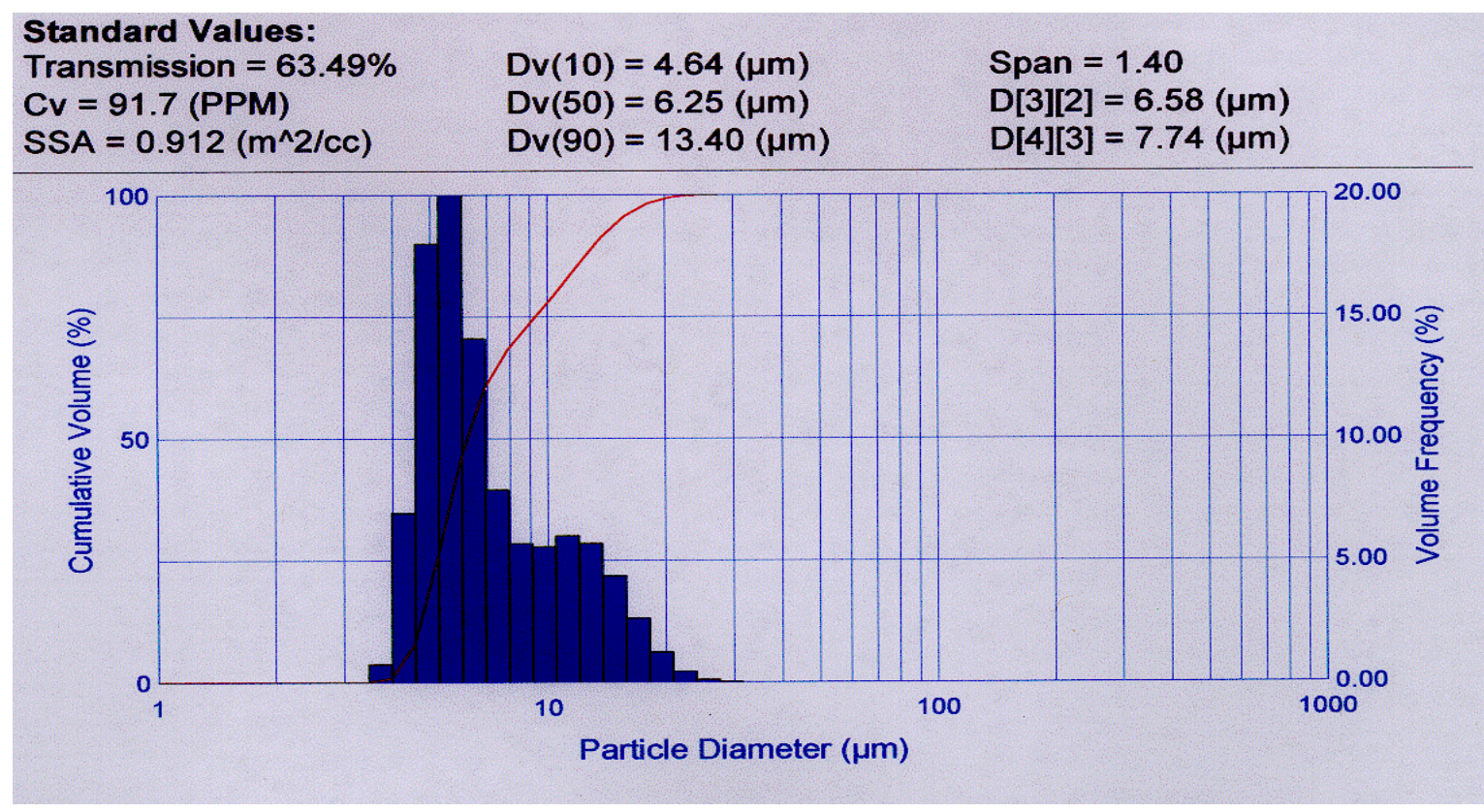

a) Voltage $=0.0 \mathrm{kV}$ and Initial Droplet Concentration $=12.1 \mathrm{~g} / \mathrm{m}^{3}$

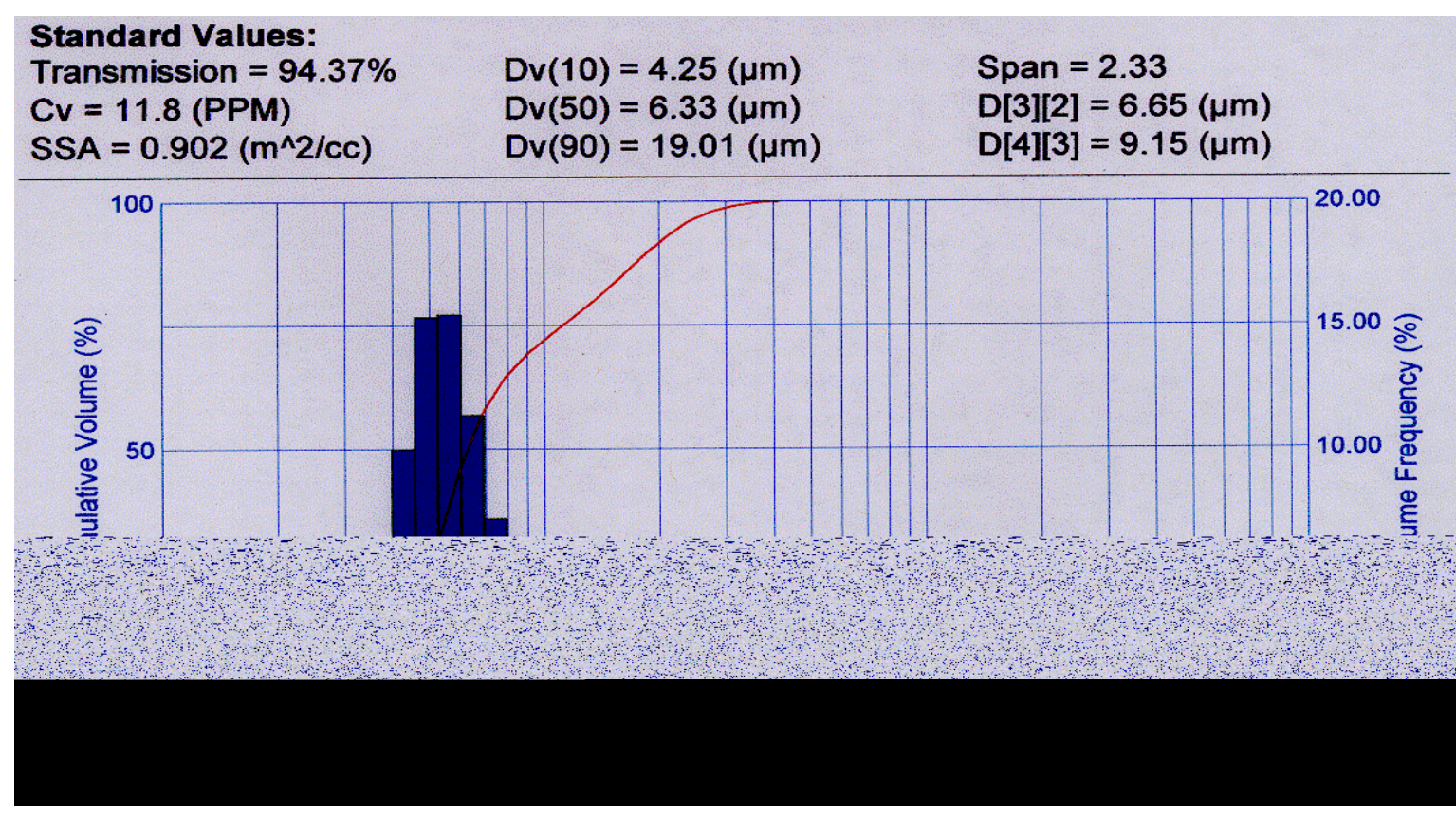

b) Voltage $=26 \mathrm{kV}$ and Droplet Concentration $=5.96 \mathrm{~g} / \mathrm{m}^{3}$

Figure 5.13: Droplet size distribution for Reynolds Number of 1350 
a) Voltage $=0.0 \mathrm{kV}$ and Initial Droplet Concentration $=24.2 \mathrm{~g} / \mathrm{m}^{3}$

\section{Standard Values:}

b) Voltage $=26.0 \mathrm{kV}$ and Droplet Concentration $=8.17 \mathrm{~g} / \mathrm{m}^{3}$

Figure 5.14: Droplet size distribution at Reynolds Number of 1350 


\section{$\underline{5.7 \text { Multiple elevation results }}$}

A number of experiments were carried out to investigate the effect of distance down electrodes on the light transmission with and without electric field using a constant flow rate. The results of this experiment are shown in Figure 5.15 and Table 5.1. In the figure, the light transmission percent generally increases with the elevation. However, at low voltages the increase is not significant as compared to high voltages. This behavior may be explained as follows. When the droplets travel along the test chamber and between the electrodes, they experience a longer residence time at higher elevations as compared to those at lower elevations. As the residence time increases, the chances for coalescence become greater and thereby reducing the droplet concentration that in turn increases the light transmission percent.

The standard deviation for all experimental data is shown in the form of error bars on each two averaged data point as shown in Figure 5.15. A large error bar indicates poor repeatability of the experiments. The lack of repeatability could be attributed to the heat generated in the medium, resulting in local temperature gradients which in turn give rise to gradients in the conductivity and permittivity of the medium. These can induce fluid unwanted movements that cause errors in measured data. Another reason could be the collision of the droplets on the laser observation window that causes moisture appearance on the window and thereby resulting in incorrect measurement. 


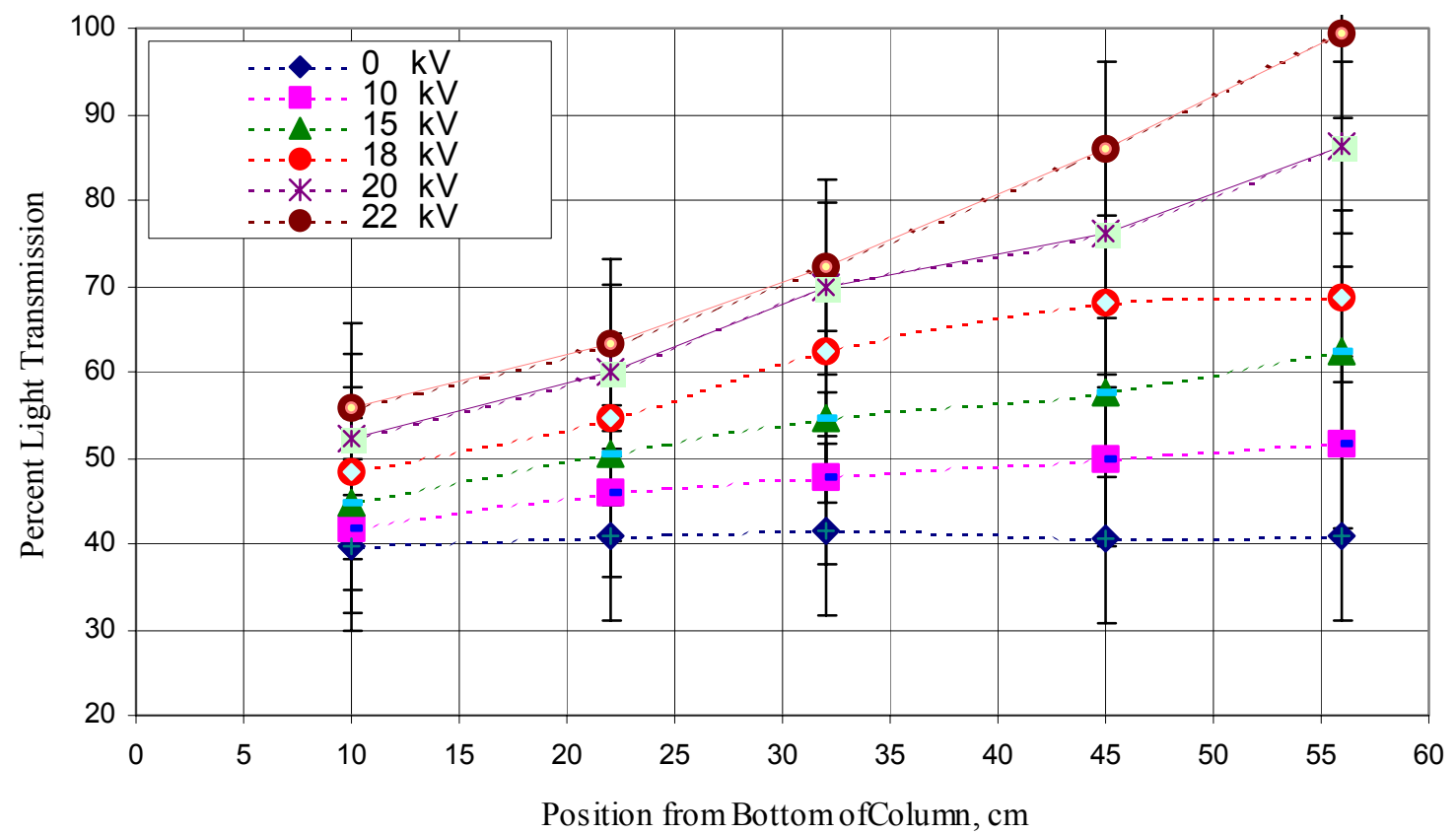

Figure 5.15: Percent Light Transmission vs Height of Column at Reynolds Number of 1620 and inlet mist concentration of $27.3 \mathrm{~g} / \mathrm{m}^{3}$

Table 5.2: Comparison of percent light transmission at various heights and applied voltages.

\begin{tabular}{|c|c|c|c|c|c|}
\hline \multirow{2}{*}{$\begin{array}{c}\text { Electric } \\
\text { Potential } \\
(\mathrm{kV})\end{array}$} & \multicolumn{5}{|c|}{ Height (cm) } \\
\cline { 2 - 7 } & 10 & 22 & 32 & 45 & 56 \\
\hline 10 & 39.8 & 41.0 & 41.6 & 40.6 & 51.0 \\
\hline 15 & 41.8 & 46.0 & 47.6 & 49.8 & 62.3 \\
\hline 20 & 44.7 & 50.3 & 54.6 & 57.7 & 68.7 \\
\hline 20 & 58.2 & 54.6 & 62.4 & 68.0 & 86.2 \\
\hline
\end{tabular}




\subsection{Multiple mist densities at constant flow rates.}

A number of experiments were carried out to understand the influence of electric field on various droplet concentrations at a constant flow rate. In this regard, three different droplet concentrations were used under a variable electric field that changed from 0 to $26 \mathrm{kV}$. The results of these experiments are shown in Figure 5.16 and Table 5.2. The figure indicates that as the voltage increases, the droplet concentrations uniformly decrease from the three different starting levels and converge to a specific levels.

The same experiments were repeated at higher Reynolds numbers and they all indicated the same trend. The results of these experiments are shown in Figures 5.17 and Table 5.3. In Figure 5.17, at the droplet concentration of $8.09 \mathrm{~g} / \mathrm{m}^{3}$, saturation occurs at voltages above $22 \mathrm{kV}$. Therefore, the trend in the data for this particular concentration at voltages above $22 \mathrm{kV}$ was leveled off and did not change in a uniform manner that was the case with the other voltages. The standard deviation for all experimental data is shown in the form of error bars in Figures 5.16 and 5.17. 


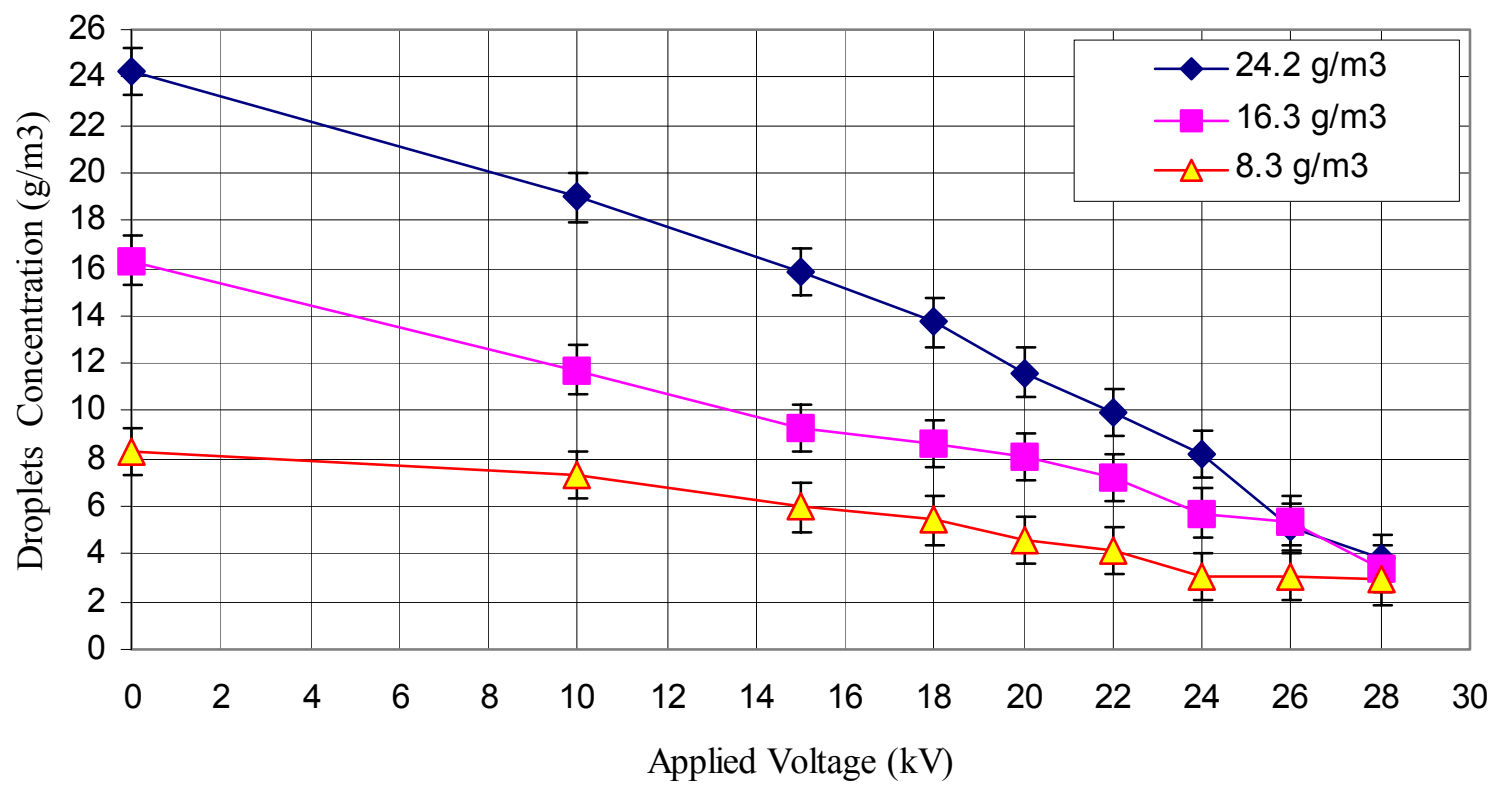

Figure 5.16: Collected Droplets $\left(\mathrm{g} / \mathrm{m}^{3}\right)$ vs. Applied Voltage $(\mathrm{kV})$ at Reynolds Number of 1350

Table 5.3: Effect of Applied Voltage on Droplet Concentration for a Reynolds Number of 1350

\begin{tabular}{|c|c|c|c|c|c|c|c|c|c|}
\hline $\begin{array}{l}\text { Applied Voltage } \\
(\mathrm{Kv})\end{array}$ & 0 & 10 & 15 & 18 & 20 & 22 & 24 & 26 & 28 \\
\hline $\begin{array}{c}\text { Ave. Droplet } \\
\text { Concentration } \\
\left(\mathrm{g} / \mathrm{m}^{3}\right)\end{array}$ & 24.23 & 18.97 & 15.82 & 13.72 & 11.62 & 9.92 & 8.17 & 5.12 & 3.86 \\
\hline $\begin{array}{c}\text { Ave. Droplet } \\
\text { Concentration } \\
\left(\mathrm{g} / \mathrm{m}^{3}\right)\end{array}$ & 16.33 & 11.73 & 9.28 & 8.60 & 8.08 & 7.20 & 5.73 & 5.40 & 3.42 \\
\hline $\begin{array}{c}\text { Ave. Droplet } \\
\text { Concentration } \\
\left(\mathrm{g} / \mathrm{m}^{3}\right)\end{array}$ & 8.32 & 7.30 & 5.97 & 5.41 & 4.60 & 4.16 & 3.04 & 3.04 & 2.90 \\
\hline
\end{tabular}


Table 5.4: Effect of Applied Voltage on Droplet Concentration for a Reynolds Number of 1950

\begin{tabular}{|c|c|c|c|c|c|c|c|c|}
\hline $\begin{array}{c}\text { Applied Voltage } \\
(\mathrm{Kv})\end{array}$ & 0 & 10 & 15 & 18 & 20 & 22 & 24 & 26 \\
\hline $\begin{array}{c}\text { Ave. Droplet Concentration } \\
\left(\mathrm{g} / \mathrm{m}^{3}\right)\end{array}$ & 13.30 & 9.70 & 9.05 & 8.01 & 7.56 & 7.07 & 5.74 & 3.26 \\
\hline $\begin{array}{c}\text { Ave. Droplet Concentration } \\
\left(\mathrm{g} / \mathrm{m}^{3}\right)\end{array}$ & 10.10 & 8.81 & 7.50 & 6.28 & 5.20 & 4.05 & 3.37 & 3.30 \\
\hline $\begin{array}{c}\text { Ave. Droplet Concentration } \\
\left(\mathrm{g} / \mathrm{m}^{3}\right)\end{array}$ & 8.10 & 7.31 & 5.86 & 4.58 & 4.41 & 3.92 & 3.30 & 3.32 \\
\hline
\end{tabular}




\subsection{Multiple flow rates at constant mist concentration}

Experiments were conducted to investigate the effect of variable flow rate on droplet concentration under various electric field strengths. In each set of experiment the starting droplet concentration was the same. The results of these experiments for a starting droplet concentration of $14.5\left(\mathrm{~g} / \mathrm{m}^{3}\right)$ and Reynolds numbers of 940,1350 , and 1680 are shown in Figure 5.18 and Table 5.4. As it is seen in the figure, the droplet concentration uniformly reduces with voltage to a certain value. However, after this point further increases in the voltage no longer change the droplet concentration. This appears to be due to charge saturation, as described in the Section 4.2.

Figure 5.19 and Table 5.5 show the results of a similar experiment but with a higher starting droplet concentration and Reynolds number. The figure indicates that at a Reynolds number of 1230 and voltages beyond $22 \mathrm{kV}$, no further reduction of droplets takes place, in contrast to what was expected. To ensure that the experimental procedure was correct, these experiments were repeated three times with the same outcome. The reason for this behavior is not known at this point.

Figure 5.20 and Table 5.6 show the results of similar experiments with Reynolds numbers of 2400, 2070, 1810, and 940 and a starting droplet concentration of approximately $3.4\left(\mathrm{~g} / \mathrm{m}^{3}\right)$. The results reveal that the droplet concentration decreases with applied voltage for all Reynolds numbers as expected. The results also reveal that maximum reduction in concentration occurs at $22 \mathrm{kV}$ for all Reynolds numbers. The reduction is more significant for the Reynolds number of 940 . The figure also indicates that beyond this voltage no further reduction of droplets take place. 


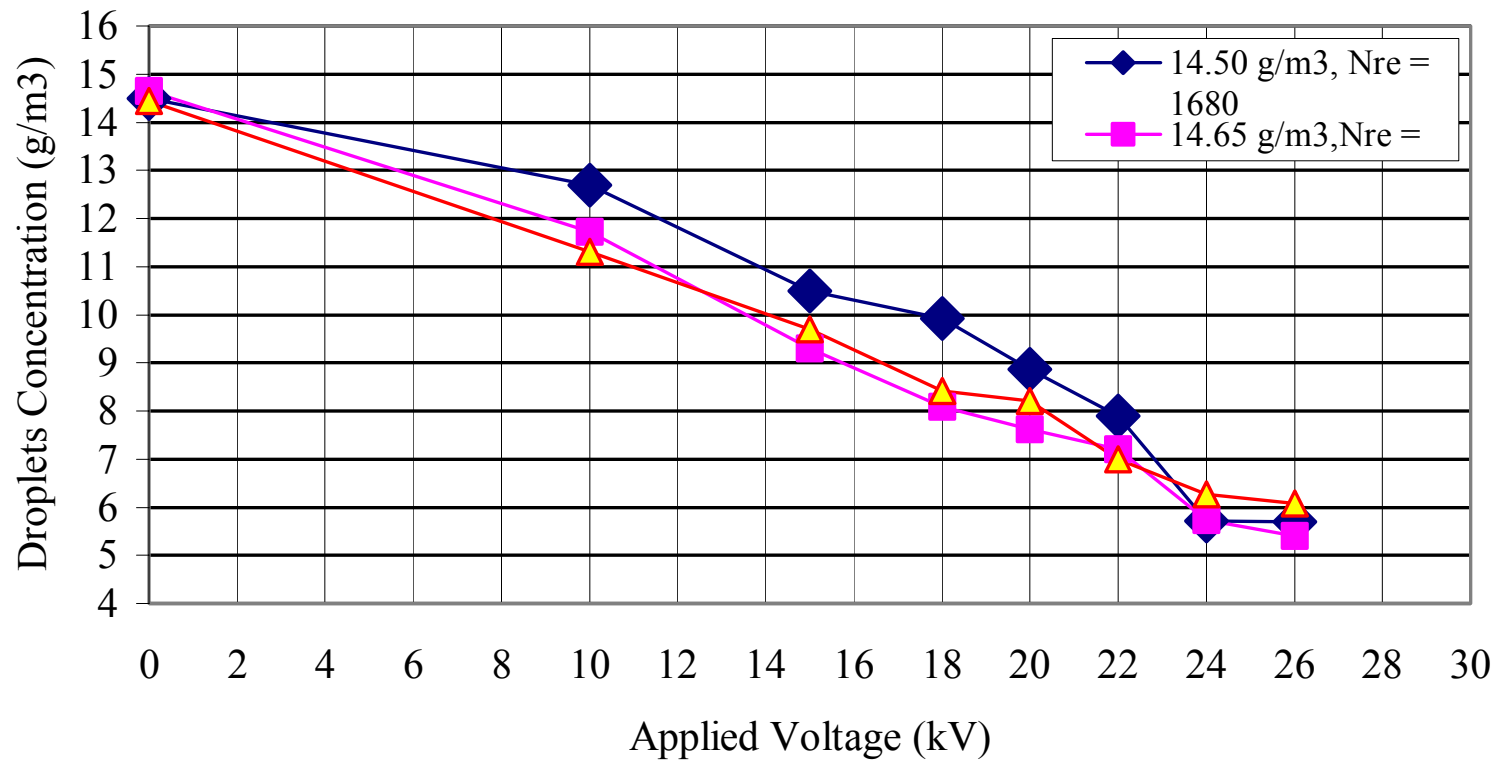

Figure 15.18: Collected Droplets $\left(\mathrm{g} / \mathrm{m}^{3}\right)$ vs. Applied Voltage $(\mathrm{kV})$ at Reynolds Numbers of 940, 1350 and 1680.

Table 5.5: Constant droplet concentration at Reynolds Numbers of 940, 1350 and 1680

\begin{tabular}{|c|c|c|c|c|c|c|c|c|c|}
\hline $\begin{array}{c}\text { Reynolds } \\
\text { Numbers }\end{array}$ & $\begin{array}{c}\text { Applied } \\
\text { Voltage (kV) }\end{array}$ & 0 & 10 & 15 & 18 & 20 & 22 & 24 & 26 \\
\hline 940 & $\begin{array}{c}\text { Ave.Droplet } \\
\text { Concentration } \\
\left(\mathrm{g} / \mathrm{m}^{3}\right)\end{array}$ \\
1350 & $\begin{array}{c}\text { Ave. Droplet } \\
\text { Concentration } \\
\left(\mathrm{g} / \mathrm{m}^{3}\right)\end{array}$ & 14.7 & 11.7 & 9.3 & 8.1 & 7.6 & 7.2 & 5.7 & 5.4 \\
\hline 1680 & $\begin{array}{c}\text { Concentration } \\
\left(\mathrm{g} / \mathrm{m}^{3}\right)\end{array}$ & 14.5 & 11.3 & 9.7 & 8.4 & 8.2 & 7.0 & 6.3 & 6.0 \\
\hline
\end{tabular}




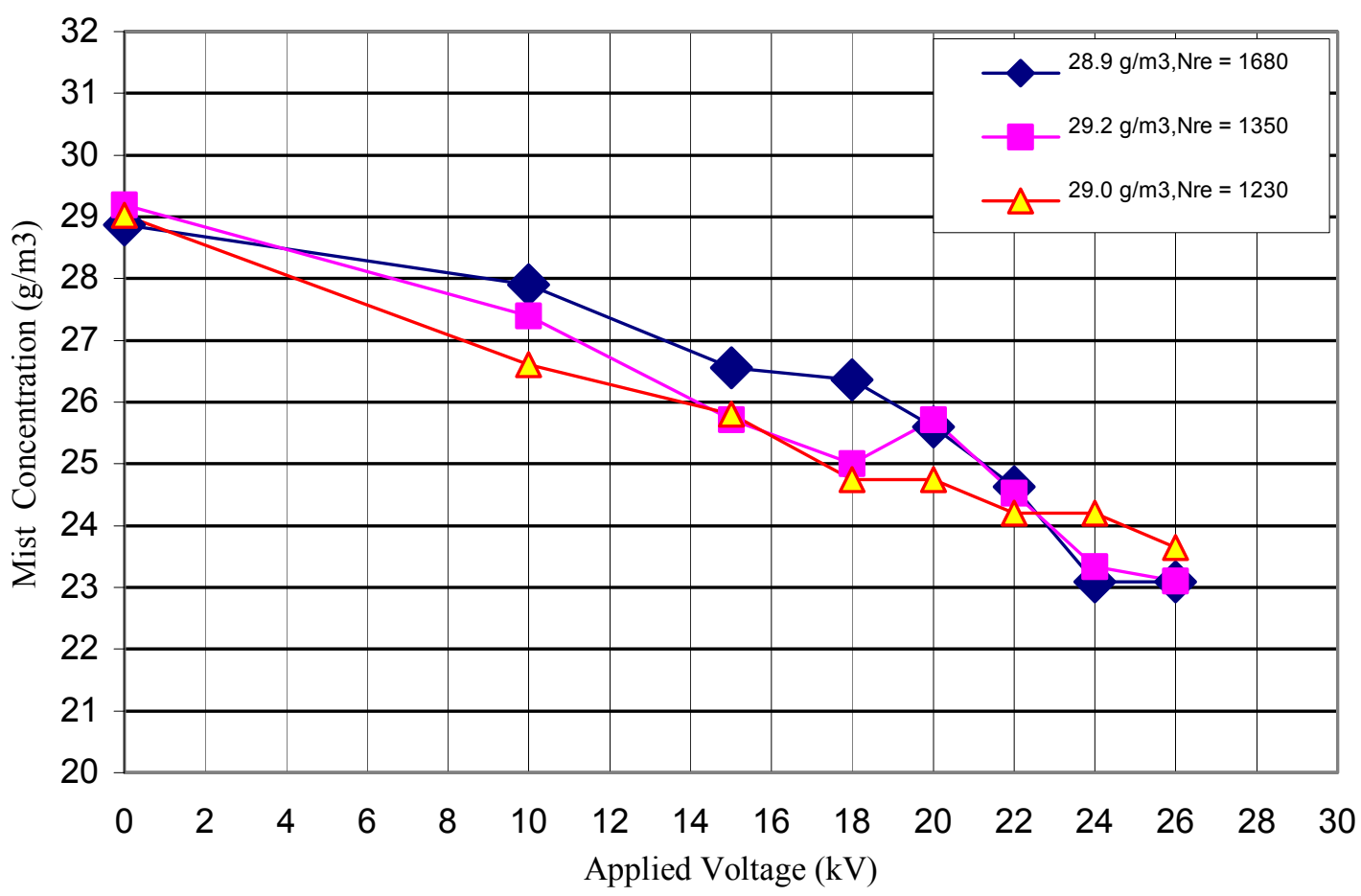

Figure 5.19: Collected Mist (g/ $\left.\mathrm{m}^{3}\right)$ vs. Applied Voltage $(\mathrm{kV})$ at Reynolds Number of 1230,1350 , and 1680

Table 5.6: Exit mist concentrations for Reynolds Numbers of 1230, 1350, and 1680

\begin{tabular}{|c|c|c|c|c|c|c|c|c|c|}
\hline $\begin{array}{c}\text { Reynolds } \\
\text { Numbers }\end{array}$ & $\begin{array}{c}\text { Applied Voltage } \\
(\mathrm{Kv})\end{array}$ & 0 & 10 & 15 & 18 & 20 & 22 & 24 & 26 \\
\hline 1230 & $\begin{array}{c}\text { Ave. Droplet } \\
\text { Concentration } \\
\left(\mathrm{g} / \mathrm{m}^{3}\right)\end{array}$ & 28.87 & 27.90 & 26.56 & 26.36 & 25.60 & 24.63 & 23.09 & 23.09 \\
\hline 1350 & $\begin{array}{c}\text { Ave. Droplet } \\
\text { Concentration } \\
\left(\mathrm{g} / \mathrm{m}^{3}\right)\end{array}$ & 29.20 & 27.40 & 25.71 & 25.00 & 25.71 & 24.52 & 23.34 & 23.10 \\
\hline 1680 & $\begin{array}{c}\text { Ave. Droplet } \\
\text { Concentration } \\
\left(\mathrm{g} / \mathrm{m}^{3}\right)\end{array}$ & 29.03 & 26.61 & 25.80 & 24.74 & 24.74 & 24.20 & 24.20 & 23.65 \\
\hline
\end{tabular}




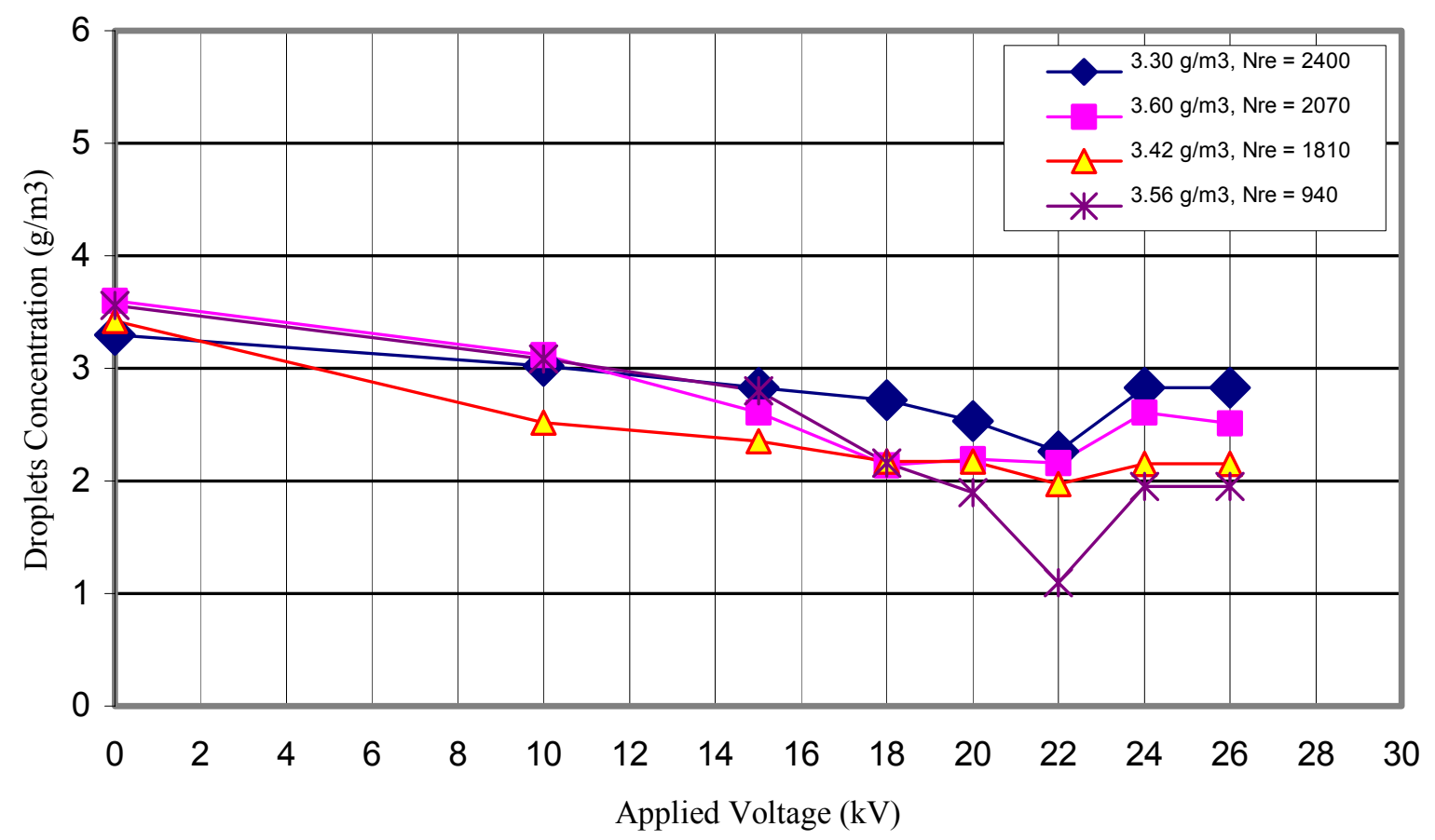

Figure 5.20: Collected Droplets $\left(\mathrm{g} / \mathrm{m}^{3}\right)$ vs. Applied Voltage $(\mathrm{kV})$ at Reynolds Numbers of $2400,2070,1810$, and 940

Table 5.7: Exit mist concentrations for Reynolds Numbers of 2400, 2070, 1810, and 940

\begin{tabular}{|c|c|c|c|c|c|c|c|c|c|}
\hline $\begin{array}{c}\text { Reynolds } \\
\text { Numbers }\end{array}$ & $\begin{array}{c}\text { Applied Voltage } \\
(\mathrm{kV})\end{array}$ & 0 & 10 & 15 & 18 & 20 & 22 & 24 & 26 \\
\hline 2400 & $\begin{array}{c}\text { Ave. Droplet } \\
\text { Concentration } \\
\left(\mathrm{g} / \mathrm{m}^{3}\right)\end{array}$ & 3.30 & 3.02 & 2.83 & 2.72 & 2.53 & 2.26 & 2.83 & 2.83 \\
\hline 2070 & $\begin{array}{c}\text { Ave. Droplet } \\
\text { Concentration } \\
\left(\mathrm{g} / \mathrm{m}^{3}\right)\end{array}$ & 3.60 & 3.12 & 2.61 & 2.14 & 2.19 & 2.16 & 2.61 & 2.51 \\
\hline 1810 & $\begin{array}{c}\text { Ave. Droplet } \\
\text { Concentration } \\
\left(\mathrm{g} / \mathrm{m}^{3}\right)\end{array}$ & 3.42 & 2.52 & 2.35 & 2.17 & 2.17 & 1.97 & 2.15 & 2.15 \\
\hline 940 & $\begin{array}{c}\text { Ave. Droplet } \\
\text { Concentration } \\
\left(\mathrm{g} / \mathrm{m}^{3}\right)\end{array}$ & 3.56 & 3.08 & 2.80 & 2.16 & 1.90 & 1.10 & 1.95 & 1.95 \\
\hline
\end{tabular}


These experiments were also used to identify a Reynolds number that maximizes the removal of droplets so that a minimum concentration is achieved at a certain applied voltage. Figure 5.20 shows that this phenomenon takes place at a Reynolds number of 940 and applied voltage of $22 \mathrm{kV}$. This minimum at $22 \mathrm{kV}$ was repeated in 2 runs.

Similarly, additional experiments were carried out but with different starting droplet concentration and Reynolds numbers. The results of these experiments are shown in Figure 5.21 and Table 5.7. In these experiments, it was noticed that for the Reynolds number 940 , the droplet concentration did not decrease beyond $20 \mathrm{kV}$ as compared to the other Reynolds numbers. Since this was the smallest Reynolds number used in this experiment, we expected to see the droplet concentration decreasing more than at other Reynolds numbers for all voltages. However, this was not the case when the voltage went beyond $20 \mathrm{kV}$. The reason for this behavior is not known.

\subsection{Removal efficiency of the droplets}

A key objective of this research work was to quantify the removing efficiency of droplets from an air stream at constant flow rate under a DC electric field. To achieve this objective several experiments were carried out. In this regard, three different droplet concentrations were used under a variable electric field that change from 0 to $28 \mathrm{kV}$. The results of these experiments are shown in Figure 5.22. The figure reveals that the removal efficiency of all three droplet concentrations vary approximately linearly related with the applied voltage. At the droplet concentration of $8.3 \mathrm{~g} / \mathrm{m}^{3}$, saturation occurred at voltages above $24 \mathrm{kV}$ and the removal efficiency was steady at 63.5 percent. 


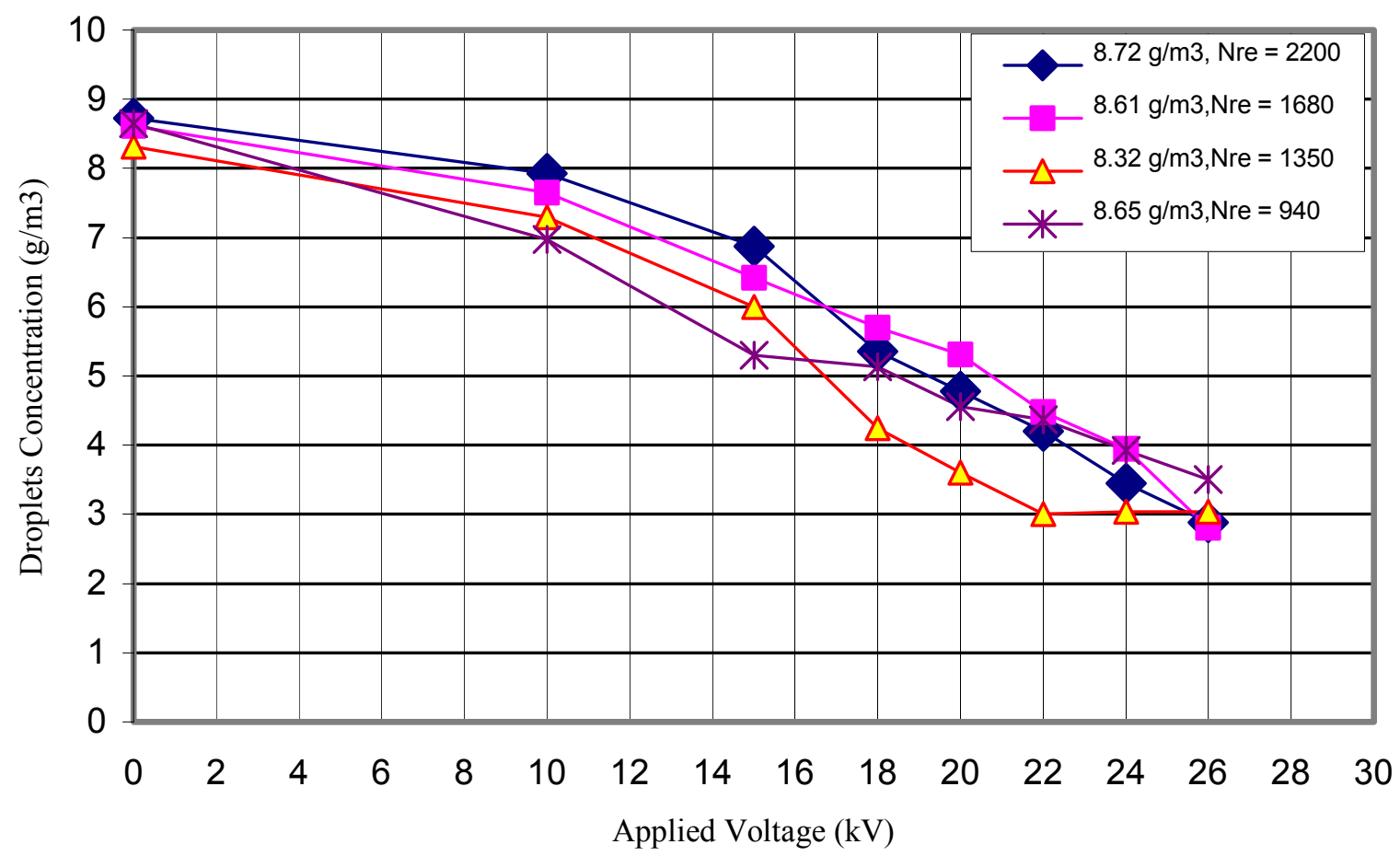

Figure 5.21: Collected Droplets $\left(\mathrm{g} / \mathrm{m}^{3}\right)$ vs. Applied Voltage $(\mathrm{kV})$ at Reynolds Numbers of $2200,1680,1350$, and 940

Table 5.8: Exit mist concentrations for Reynolds Numbers of 2200, 1680, 1350, and 940

\begin{tabular}{|c|c|c|c|c|c|c|c|c|c|}
\hline $\begin{array}{c}\text { Reynolds } \\
\text { Numbers }\end{array}$ & $\begin{array}{c}\text { Applied Voltage } \\
(\mathrm{kV})\end{array}$ & 0 & 10 & 15 & 18 & 20 & 22 & 24 & 26 \\
\hline 2200 & $\begin{array}{c}\text { Ave. Droplet } \\
\text { Concentration } \\
\left(\mathrm{g} / \mathrm{m}^{3}\right)\end{array}$ & 8.72 & 7.93 & 6.87 & 5.35 & 4.78 & 4.20 & 3.45 & 2.88 \\
\hline 1680 & $\begin{array}{c}\text { Ave. Droplet } \\
\text { Concentration } \\
\left(\mathrm{g} / \mathrm{m}^{3}\right)\end{array}$ & 8.61 & 7.65 & 6.42 & 5.70 & 5.31 & 4.48 & 3.95 & 2.80 \\
\hline 1350 & $\begin{array}{c}\text { Ave. Droplet } \\
\text { Concentration } \\
\left(\mathrm{g} / \mathrm{m}^{3}\right)\end{array}$ & 8.32 & 7.30 & 6.00 & 4.25 & 3.60 & 3.00 & 3.04 & 3.04 \\
\hline 940 & $\begin{array}{c}\text { Ave. Droplet } \\
\text { Concentration } \\
\left(\mathrm{g} / \mathrm{m}^{3}\right)\end{array}$ & 8.65 & 6.97 & 5.30 & 5.13 & 4.56 & 4.37 & 3.93 & 3.50 \\
\hline
\end{tabular}




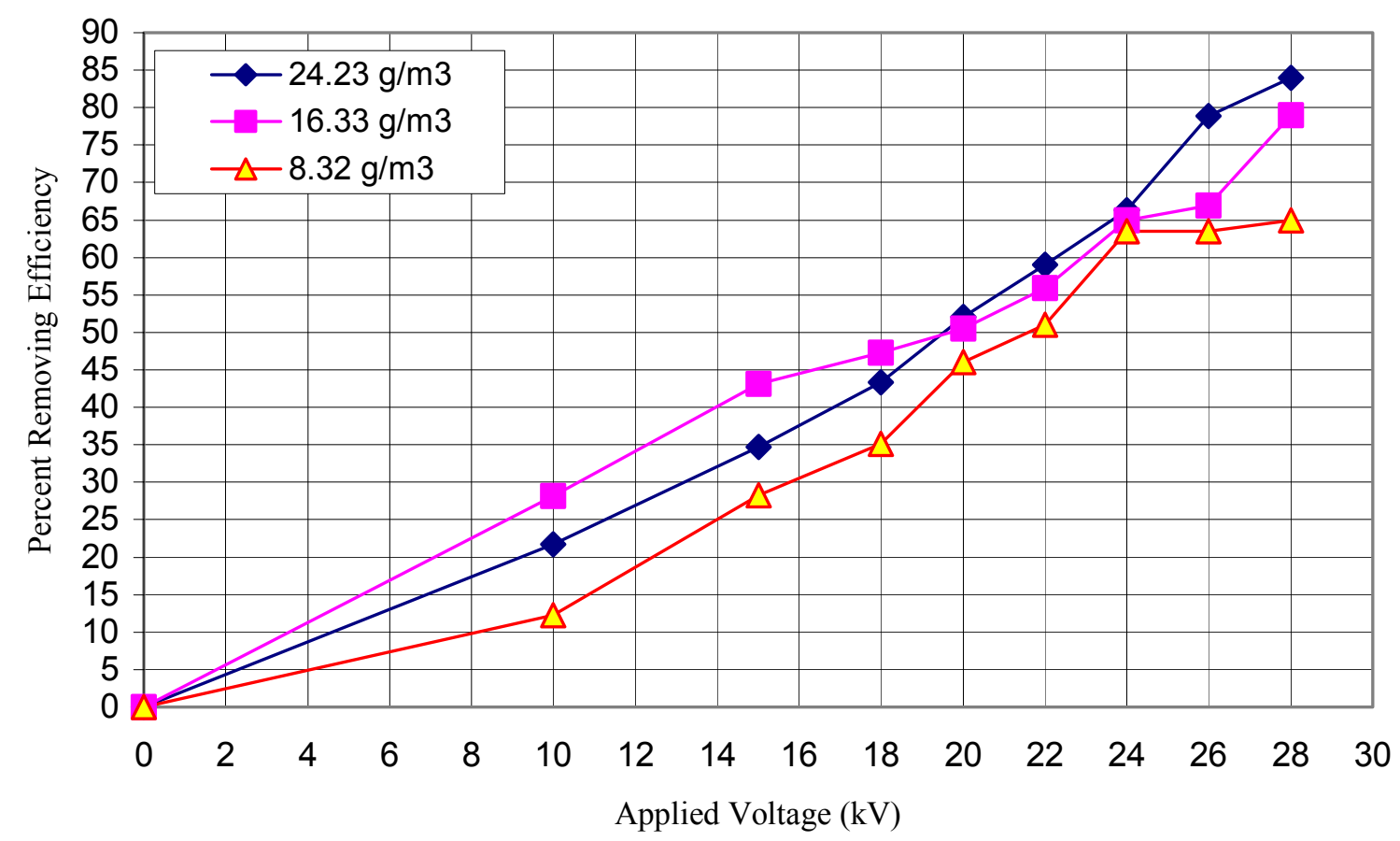

Figure 5.22: Percent removal efficiency vs. applied voltages $(\mathrm{kV})$ at Reynolds Numbers of 1350

At droplet concentrations of 24.2 and $16.3 \mathrm{~g} / \mathrm{m}^{3}$, the removal efficiency of 84.0 and 79.0 percent was achieved, respectively.

The same experiments were repeated at a higher Reynolds number and they all indicated similar results. The results of these experiments are shown in Figure 5.23. In this Figure, at droplet concentrations of 10.1 and $8.1 \mathrm{~g} / \mathrm{m}^{3}$, a maximum efficiency of 67.0 and 59.0 percent was achieved at $24.0 \mathrm{kV}$. Above $24 \mathrm{kV}$, saturation occurred and therefore, the efficiency remained constant with further increase in voltage. At a droplet concentration of $13.3 \mathrm{~g} / \mathrm{m}^{3}$, the maximum removal efficiency of 75.5 percent was achieved. 


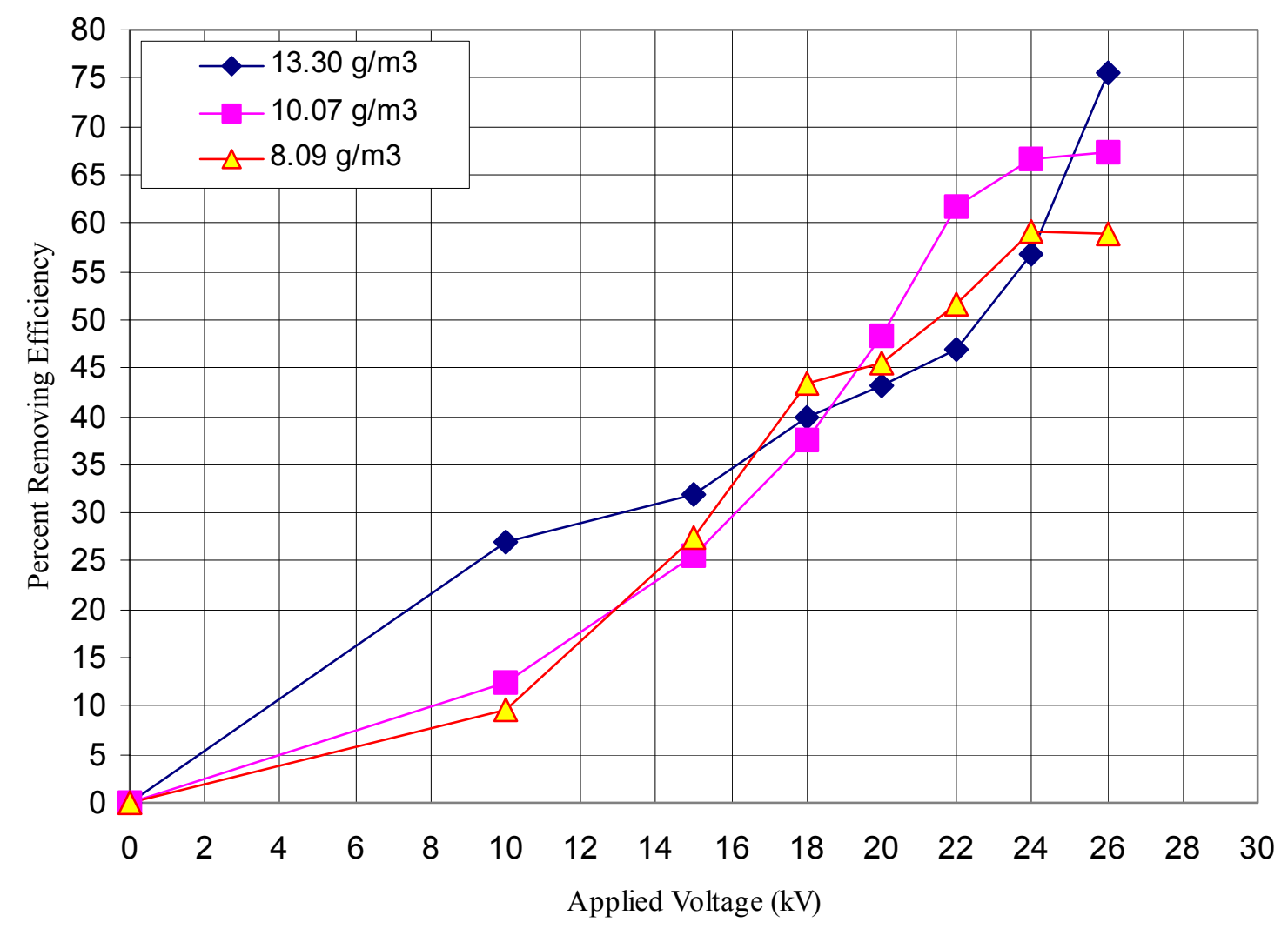

Figure 5.23: Percent removing efficiency vs. applied aoltage $(\mathrm{kV})$ at Reynolds numbers of 1946

Also, it was observed that no saturation was occurred under these experimental conditions.

Comparing the two figures, we can conclude that the higher droplet concentration gives higher removal efficiency presumably due to higher number of droplet collision. 


\section{CHAPTER 6}

\section{SUMMARY and CONCLUSIONS}

A series of experiments was conducted in a carefully designed section in order to quantify the effect of an applied electric potential on the water droplet content in simulated moist air streams. The test chamber is clear Lexan with a cross section of 10 $\mathrm{cm} \times 10 \mathrm{~cm}$ and approximately $94 \mathrm{~cm}$ long mounted vertically, with the air flow from bottom to top through the unit. The two $9 \mathrm{~cm}$ x $60 \mathrm{~cm}$ electrodes are mounted vertically approximately $8 \mathrm{~cm}$ apart on opposite sides of the test window. The applied electric potential gradients were thus normal to the air, mist flow direction. The applied electric potentials ranged from 0 to $26 \mathrm{kV}$ in all experiments.

The mist containing air streams were forced through the test chamber at Reynolds Numbers whose nominal values ranged from 940 to 2200, which correspond to residence times in the test section of 7 seconds to 3 seconds. The droplets are produced by an aerosol generator fed with distilled water, and the size distribution of the resulting mist determined by laser-light scattering using a Malvern Spraytec RTS 5000 Series droplet sizing system mounted on a movable stage. Analysis of the data show that the water droplets in the mist range in diameter from a few micrometers to about 20 micrometers, more or less, in each of the test experiments. The droplet loadings in the air streams, determined by freezing samples withdrawn isokinetically, range from nominal values of 8 to $24 \mathrm{~g}$ mass per cubic meter.

The following conclusions are drawn from experimental results and observations: 
1. The applied electric potentials reduced the mist content of the air exiting the test unit as the potential is increased from 0 to $26 \mathrm{kV}$, in all but one of the cases investigated. In this case there is no further reduction in water droplet content of the air stream when the potential is increased from 24 to $26 \mathrm{kV}$, suggesting that a saturation effect may be occurring in this case.

2. The efficiency of the droplet removal process ranges from about $65 \%$ to $85 \%$ on a mass basis for the series of runs at a nominal Reynolds Numbers of 1350, with the higher efficiencies corresponding to the higher nominal initial droplet loading of $24 \mathrm{~g}$ mass per cubic meter. The removal efficiency of $65 \%$ was achieved for a nominal droplet loading of $8 \mathrm{~g}$ mass per cubic meter.

3. In a strict sense, the effect of the applied electric potential on the water droplet content of the air stream is not linear. However, fitting the data with a linear model may be useful for preliminary engineering design purposes.

4. The water droplets migrate to the electrodes when the electric potential is applied across the plates, as evidenced by water accumulation on the plate surfaces. Although not quantified, it appeared that more water accumulated on the positive electrode than the negative one. Accumulation of mist on the plates results in draining of water down the plates and pooling at the bottom of the unit. Fogging begins near the bottom of the electrode plates and progresses in time up the plates. This migration of mist from the air stream to the plates due to the influence of the electric potential is the principal mechanism of droplet removal from the air stream in these experiments. 
5. Experiments show that the droplet content of the air stream decreases approximately linearly with distance up the test unit. This result suggests that further increases in removal efficiencies can be achieved by the use of electrode plates longer than the $60 \mathrm{~cm}$ used in these experiments.

6. There is limited evidence that the electric potential promotes droplet coalescence in the size range of approximately 10 to 20 micrometers, at least. This process should improve droplet collection efficiency in a filter installed downstream of an electric potential demisting unit in an actual application, although sufficient data are not provided here for quantifying the extent to which the overall droplet collection efficiency may be improved. 


\section{CHAPTER 7}

\section{RECOMMENDATIONS}

A suggestion for improving the repeatability of the experiments is to use a Laser Mass Velocimeter instead of using a dry-ice trap for measuring droplet concentrations in the air stream. Also, it would be helpful to find a better way to clean the observation windows of the test chamber. Additional experiments are required for determining the exact power consumption in order to characterize the relationship between power consumption and droplet removal efficiency.

It would be interesting also to conduct similar experiments with two additional electrodes between the two original electrodes used in these experiments to promote droplet collisions and preventing the short circuits.

Experiments should be conducted to examine the effects of an acoustic field and a combination of an electric field and an acoustic field in the coalescence unit as illustrated in Figure 3.1.

Finally, it is recommended that the effect of diffusion on the particle collision process be examined theoretically. The diffusion of particles relative to each other and its interaction with the electrically induced collisions apparently has not been investigated in the past. This may cause problems for small particles which have larger diffusivity. 


\section{REFERENCES}


1. Accomazzo, M.A. and Grant D.C. "Mechanisms and devices for filtration of critical Process gases”. Fluid Filtration: Gas, 1, ASTM STP 975, 402, 1986.

2. ACS Industries, Inc. Bulletin B-124 March, 1990.

3. Bacon, G., Ramon, L., and Liang, K., "Control Particulate and Metal HAPs" Chem.Eng. Prog., December 1997.

4. Bailes, P. J, and Larkai, S. K. L, "Liquid Phase Separation in Pulsed D.C. Fields," Chem Eng-Lond, TI 60, 115, (1982).

5. Barker, R. E, Granular Electrofiltration. Ph.D. Diss., University of Tennessee, 1988.

6. Barker, R. E, Brunson, R. R, Clinton, S. D, and Watson J. D, Granular Electrofiltration. Separations Technology, 1, 1991.

7. Basset, A.B. Treatise on hydrodynamics. Deighton Bell, London, 1888.

8. Berger, R, Koaleszensprobleme in chemischen Prozessen,"Chem.,Ing.,Tech"., 58, 449, 1986.

9. Boem, J. Electrostatic Precipitation, Elsevier Scientific, 1982.

10. Bogarduos, H. F., R.C. Clark, J. K. Thomspon, and G. H. Fielding, "Enhancement of filter media performance by corona-free electric fields", Presented at the $13^{\text {th }}$ AEC Air Cleaning Conference, August 1974.

11. Brown, R. C., "Capture of dust particles in filters by line-dipole charged filter", $J$. Aerosol Sci., 12, 349, 1981.

12. Brunazzi, E. and Paglianti, A. Design of complex wire mesh mist eliminators. AIChE J. 24,1199,2000. 1999. 
13. Cadle, R. D, Particle Size: Theory and Industrial Applications, Reinhold Publishing Corporation, New York, 1965.

14. Corrsin, S. and Lumley, J. Appl. Sci. Res. A6, 114, 1956.

15. Crowley, J. M., Fundamentals of Applied Electrostatics, John Wiley and Sons, New York, 1986.

16. Davies, R. N, Dust is Dangerous, Faker \& Faker Limited, London, 1953.

17. Davis, M.H. Two charged Spherical Conductors in a Uniform Electric Field Forces and Field Strength. Quart. J. Mech. Appl. Math. 17,499, 1964.

18. Erker, J.A. and Baygents, J.C. NASA Conference publication 3338, 731, 1996.

19. Ezekoye, O. A. and Wibowo, Y. W., Simulation of Acoustic Agglomeration Processes using a Sectional Algorithm, J. Aerosol Sci. 30, 1117-1138, 1999.

20. Felder, R. M. and Rousseau, R. W. Elementry principles of Chemical Processes. John Wiley \& Sons, New York, 1978.

21. Fuchs, N.A, Mechanics of Aerosols, Pergamon, New York, 1964.

22. Fielding, G.H.,H.F. Bogardus, R.C. Clark, and J.K. Thompson, "Electrically Augmented Filtration of Aerosols”, in ACS Symp. Ser. 17, 1975.

23. Hansen, O. V, Reid, J. L, CPI-100 NOV-DEC, 1983.

24. Harriott, G.M. and Saville D.A. "Electrically stimulated aerosol filtration in packed beds", AIChE J, 26, 325, 1971.

25. Hauertmann, H. B, Degener, W. and Schugerl, Electrostatic Coalescence: Reactor Process Control and Important Parameters, lbid.,24, 253, 1989.

26. Henry, F.S. and Ariman T. "Numeric modeling of electrically enhanced fibrous filtration”. ASTM Committee F-21, Fluid Filtration: Gas, 1, 13, 1986. 
27. Hsu, E. C. and Li, N. N. "Membrane Recovery in Liquid Membrane Separation Processes”, Sep. Sci. Technol., 20, 115, 1985.

28. Kataoka, T. and Nishiki, T.” Development of a Continuous Electric Coalescence of W/O Emulsions in Liquid Surfactant Membrane Proces”. lbid., 25, 171, 1990.

29. Keh H.J. and Chen, S.B., J. Colloid Interf. Sci. 130, 542, 1989a.

30. Keh H.J. and Chen, S.B., J. Colloid Interf. Sci. 130, 556, 1989 b.

31. Kildesg, J., Bhatia, V., Lind, L., Johnson, E., and Johansen, A. An Experimental Investigation for Agglomeration of Aerosols in Alternating Electric Fields. Aerosol Science and Technology 23:603-610, 1995.

32. Latham, J. and Roxburgh, I.W. Proc. Roy. Soc. A 295, 84. Maxey, M.R. and Riley, J.J. (1983) Equation of motion for a small rigid sphere in a non-uniform flow. Phys. Fluids. 26(4): 883-888, 1966.

33. Loewvenberg, M and Davis, R.H., J. Fluid Mech. 89, 123, 1995.

34. Loffler, F. and Gutsch, A., J. Aerosol Sci., 24, S505, 1993.

35. Luckner, J., J. Aerosol Sci. 25, S915, 1995.

36. Maxey, M.R. and Riely, J.J., "Equation of motion for a small rigid sphere in a nonuniform flow", Phys. Fluids, 26(4): 883-888, 1983.

37. Mizuno, A., Review of Particle charging Research, Proc. $1^{\text {st }}$ Int. Conf. On Electrostatic Precipitation, pp. 304-325, 1981.

38. Muller, D.J., "Electrostatic granular bed filter development program", Paper presented at $2^{\text {nd }}$ Annual Contractors' Meeting on Contaminant Control in Hot Coal Derived Gas Streams, pp. 75-85, Morgantown, WV, 17-19 February 1982.

39. Panton, R.L, Incompressible Flow. John Wiley and Sons, New York, 1996. 
40. Pauthenier, M. M., and Moreau-Hanot, M.,J. Phys. et Rad., Ser. 7, 3, pp. 590, 1932.

41. Perkins, H.C Air Pollution, McGraw-Hill, Inc. New York, 1974.

42. Ptasinski, K.J. and Kerkhof, P.J.A.M.,Sep. Sci. Technoloy., 27, Sep. 1992.

43. Reist, P.C., Aerosol Science and Technology, McGraw-Hill, New York, 1993.

44. Rohmann, H., Zeit. Fur Phys., 17, pp. 253, 1923.

45. Silverman, L., Billings, C.E. and Dennis, R., "Performance of the model K electro-polar filte”, USAEC Report NYO 1592, Harvard University, 1954.

46. Thomas, J. W., and E. J. Woodfin, "Electrified fibrous air filter", AIEE Transactions, 78, 276, 1959.

47. Tsouris, C., A.P. Borole, and E.N. Kaufman, D.W. Depaoli "An Electrically Driven Gas-Liquid-Liquid Contactor for Bioreactor and Other Applications," Ind. Eng. Chem. Res.,38, 1877, 1999.

48. Tsouris, C., Yiacoumi, S., and Scott, T.C., Chem. Eng. Commun. 137, 147, 1995.

49. Van de Hulst, H. C., Light Scattering by Small Particles, Wiley, New York, 1957.

50. Wark, K. and Warner, C.F., Air Pollution: Its Origin and Control, Harper and Row, New York, 1981.

51. Waterman, L. C. "Electrical Coalescers", Chem. Eng. Prog., 61, 51-57,1965.

52. White, H. J., Industrial Electrostatic Precipitation, Addison-Wesley Publishing Co., 1962.

53. Williams, T.J. and Bailey, A.G. Inst. Phys. Conf. Ser. 66-II, 39, 1983.

54. Zhang, X. and Davis, R.H., The Rate of Collisions Due to Brownian or Gravitational Motion of Small Drops. J. Fluid Mech. 230, 479-504, 1991. 
55. Zhang, X., Davis, R.H. and Ruth, M.F., J. Fluid Mech. 249, 227, 1993.

56. Zhang, X., Basaran, O.A. and Wham, R.M. "Theoretical Prediction of Electric Field-Enhanced Coalescence of Spherical Drops”. AIChE J. 41, 1629, 1995. 


\section{APPENDIX}

Table A.8a: Effect of Applied Voltage on Droplet Concentration for a Reynolds Number of 1350 (Run 1)

\begin{tabular}{|c|c|c|c|c|c|c|c|c|c|}
\hline $\begin{array}{c}\text { Applied Voltage } \\
(\mathrm{Kv})\end{array}$ & 0 & 10 & 15 & 18 & 20 & 22 & 24 & 26 & 28 \\
\hline $\begin{array}{c}\text { Droplet } \\
\text { Concentration } \\
\left(\mathrm{g} / \mathrm{m}^{3}\right)\end{array}$ & 23.70 & 19.30 & 15.82 & 13.5 & 11.57 & 9.88 & 8.15 & 5.10 & 3.78 \\
\hline $\begin{array}{c}\text { Droplet } \\
\text { concentration } \\
\left(\mathrm{g} / \mathrm{m}^{3}\right)\end{array}$ & 15.80 & 11.80 & 9.65 & 8.75 & 8.06 & 7.42 & 5.96 & 5.31 & 3.35 \\
\hline $\begin{array}{c}\text { Droplet } \\
\text { Concentration } \\
\left(\mathrm{g} / \mathrm{m}^{3}\right)\end{array}$ & 8.40 & 7.6 & 5.32 & 5.54 & 4.72 & 4.25 & 3.3 & 3.05 & 3.0 \\
\hline
\end{tabular}

Table A.8b: Effect of Applied Voltage on Droplet Concentration for a Reynolds Number of 1350 (Run 2)

\begin{tabular}{|c|c|c|c|c|c|c|c|c|c|}
\hline $\begin{array}{c}\text { Applied Voltage } \\
(\mathrm{Kv})\end{array}$ & 0 & 10 & 15 & 18 & 20 & 22 & 24 & 26 & 28 \\
\hline $\begin{array}{c}\text { Droplet } \\
\begin{array}{c}\text { Concentration } \\
\left(\mathrm{g} / \mathrm{m}^{3}\right)\end{array}\end{array}$ & 24.76 & 18.65 & 15.80 & 13.94 & 11.67 & 9.96 & 8.18 & 5.15 & 3.94 \\
\hline $\begin{array}{c}\text { Droplet } \\
\text { Concentration } \\
\left(\mathrm{g} / \mathrm{m}^{3}\right)\end{array}$ & 16.86 & 11.66 & 9.00 & 8.45 & 8.0 & 7.0 & 5.50 & 5.30 & 3.40 \\
\hline $\begin{array}{c}\text { Droplet } \\
\text { Concentration } \\
\left(\mathrm{g} / \mathrm{m}^{3}\right)\end{array}$ & 8.25 & 7.01 & 5.50 & 5.28 & 4.50 & 4.10 & 2.8 & 3.0 & 2.80 \\
\hline
\end{tabular}

Table A.8c: Effect of Applied Voltage on Droplet Concentration for a Reynolds Number of 1350 (Ave.)

\begin{tabular}{|c|c|c|c|c|c|c|c|c|c|}
\hline $\begin{array}{c}\text { Applied Voltage } \\
(\mathrm{Kv})\end{array}$ & 0 & 10 & 15 & 18 & 20 & 22 & 24 & 26 & 28 \\
\hline $\begin{array}{c}\text { Ave. Droplet } \\
\text { Concentration } \\
\left(\mathrm{g} / \mathrm{m}^{3}\right)\end{array}$ & 24.23 & 18.97 & 15.82 & 13.72 & 11.62 & 9.92 & 8.17 & 5.12 & 3.86 \\
\hline
\end{tabular}




\begin{tabular}{|c|c|c|c|c|c|c|c|c|c|}
\hline $\begin{array}{c}\text { Ave. Droplet } \\
\text { Concentration } \\
\left(\mathrm{g} / \mathrm{m}^{3}\right)\end{array}$ & 16.33 & 11.73 & 9.28 & 8.60 & 8.08 & 7.20 & 5.73 & 5.40 & 3.42 \\
\hline $\begin{array}{c}\text { Ave. Droplet } \\
\text { Concentration } \\
\left(\mathrm{g} / \mathrm{m}^{3}\right)\end{array}$ & 8.32 & 7.30 & 5.97 & 5.41 & 4.60 & 4.16 & 3.04 & 3.04 & 2.90 \\
\hline
\end{tabular}

Table A.9a: Effect of Applied Voltage on Droplet Concentration for a Reynolds Number of 1950 (Run 1)

\begin{tabular}{|c|c|c|c|c|c|c|c|c|}
\hline $\begin{array}{c}\text { Applied Voltage } \\
(\mathrm{Kv})\end{array}$ & 0 & 10 & 15 & 18 & 20 & 22 & 24 & 26 \\
\hline $\begin{array}{c}\text { Droplet Concentration } \\
\left(\mathrm{g} / \mathrm{m}^{3}\right)\end{array}$ & 12.80 & 9.60 & 9.0 & 7.80 & 7.50 & 7.02 & 5.80 & 3.30 \\
\hline $\begin{array}{c}\text { Droplet Concentration } \\
\left(\mathrm{g} / \mathrm{m}^{3}\right)\end{array}$ & 10.00 & 8.75 & 7.45 & 6.25 & 5.0 & 4.0 & 3.30 & 3.25 \\
\hline $\begin{array}{c}\text { Droplet Concentration } \\
\left(\mathrm{g} / \mathrm{m}^{3}\right)\end{array}$ & 8.20 & 7.50 & 5.98 & 4.45 & 4.40 & 3.80 & 3.20 & 3.05 \\
\hline
\end{tabular}

Table A.9b: Effect of Applied Voltage on Droplet Concentration for a Reynolds Number of 1950 (Run 2)

\begin{tabular}{|c|c|c|c|c|c|c|c|c|}
\hline $\begin{array}{c}\text { Applied Voltage } \\
(\mathrm{Kv})\end{array}$ & 0 & 10 & 15 & 18 & 20 & 22 & 24 & 26 \\
\hline $\begin{array}{c}\text { Droplet Concentration } \\
\left(\mathrm{g} / \mathrm{m}^{3}\right)\end{array}$ & 13.80 & 9.80 & 9.10 & 8.21 & 7.62 & 7.06 & 5.70 & 3.23 \\
\hline $\begin{array}{c}\text { Droplet Concentration } \\
\left(\mathrm{g} / \mathrm{m}^{3}\right)\end{array}$ & 10.20 & 8.85 & 7.52 & 6.33 & 5.43 & 4.06 & 3.42 & 3.35 \\
\hline $\begin{array}{c}\text { Droplet Concentration } \\
\left(\mathrm{g} / \mathrm{m}^{3}\right)\end{array}$ & 8.00 & 7.10 & 5.75 & 4.73 & 4.42 & 4.06 & 3.40 & 3.30 \\
\hline
\end{tabular}

Table A.9c: Effect of Applied Voltage on Droplet Concentration for a Reynolds Number of 1950 (Ave.)

\begin{tabular}{|c|c|c|c|c|c|c|c|c|}
\hline $\begin{array}{c}\text { Applied Voltage } \\
(\mathrm{Kv})\end{array}$ & 0 & 10 & 15 & 18 & 20 & 22 & 24 & 26 \\
\hline $\begin{array}{c}\text { Ave. Droplet Concentration } \\
\left(\mathrm{g} / \mathrm{m}^{3}\right)\end{array}$ & 13.30 & 9.70 & 9.05 & 8.01 & 7.56 & 7.07 & 5.74 & 3.26 \\
\hline $\begin{array}{c}\text { Ave. Droplet Concentration } \\
\left(\mathrm{g} / \mathrm{m}^{3}\right)\end{array}$ & 10.10 & 8.81 & 7.50 & 6.28 & 5.20 & 4.05 & 3.37 & 3.30 \\
\hline
\end{tabular}


Ave. Droplet Concentration $\left(\mathrm{g} / \mathrm{m}^{3}\right)$

Table A.10a: Constant Droplet Concentration at Reynolds Numbers of 940, 1350 and 1680 (Run 1)

\begin{tabular}{|c|c|c|c|c|c|c|c|c|c|}
\hline $\begin{array}{c}\text { Reynolds } \\
\text { Numbers }\end{array}$ & $\begin{array}{c}\text { Applied } \\
\text { Voltage }(\mathrm{kV})\end{array}$ & 0 & 10 & 15 & 18 & 20 & 22 & 24 & 26 \\
\hline 940 & $\begin{array}{c}\text { Droplet } \\
\text { Concentration } \\
\left(\mathrm{g} / \mathrm{m}^{3}\right)\end{array}$ & 14.02 & 12.50 & 10.00 & 10.00 & 8.85 & 7.80 & 5.55 & 5.50 \\
\hline 1350 & $\begin{array}{c}\text { Droplet } \\
\text { Concentration } \\
\left(\mathrm{g} / \mathrm{m}^{3}\right)\end{array}$ & 14.65 & 11.50 & 9.00 & 8.0 & 7.50 & 7.40 & 5.70 & 5.40 \\
\hline 1680 & $\begin{array}{c}\text { Droplet } \\
\text { Concentration } \\
\left(\mathrm{g} / \mathrm{m}^{3}\right)\end{array}$ & 14.50 & 11.25 & 9.65 & 8.25 & 8.00 & 7.00 & 6.10 & 5.8 \\
\hline
\end{tabular}

Table A.10b: Constant Droplet Concentration at Reynolds Numbers of 940, 1350 and 1680 (Run 2)

\begin{tabular}{|c|c|c|c|c|c|c|c|c|c|}
\hline $\begin{array}{c}\text { Reynolds } \\
\text { Numbers }\end{array}$ & $\begin{array}{c}\text { Applied } \\
\text { Voltage }(\mathrm{kV})\end{array}$ & 0 & 10 & 15 & 18 & 20 & 22 & 24 & 26 \\
\hline 940 & $\begin{array}{c}\text { Droplet } \\
\text { Concentration } \\
\left(\mathrm{g} / \mathrm{m}^{3}\right)\end{array}$ & 14.97 & 12.86 & 11.00 & 9.98 & 8.98 & 8.00 & 5.90 & 5.65 \\
\hline 1350 & $\begin{array}{c}\text { Droplet } \\
\text { Concentration } \\
\left(\mathrm{g} / \mathrm{m}^{3}\right)\end{array}$ & 14.75 & 11.90 & 9.60 & 8.20 & 7.40 & 7.00 & 5.71 & 5.43 \\
\hline 1680 & $\begin{array}{c}\text { Droplet } \\
\text { Concentration } \\
\left(\mathrm{g} / \mathrm{m}^{3}\right)\end{array}$ & 14.50 & 11.36 & 9.75 & 8.56 & 8.41 & 7.00 & 6.42 & 6.21 \\
\hline
\end{tabular}

Table A.10c: Constant Droplet Concentration at Reynolds Numbers of 940, 1350 and 1680 (Ave.)

\begin{tabular}{|c|c|c|c|c|c|c|c|c|c|}
\hline $\begin{array}{c}\text { Reynolds } \\
\text { Numbers }\end{array}$ & $\begin{array}{c}\text { Applied } \\
\text { Voltage (kV) }\end{array}$ & 0 & 10 & 15 & 18 & 20 & 22 & 24 & 26 \\
\hline 940 & $\begin{array}{c}\text { Ave.Droplet } \\
\text { Concentration } \\
\left(\mathrm{g} / \mathrm{m}^{3}\right)\end{array}$ & 14.5 & 12.7 & 10.5 & 9.9 & 8.9 & 7.9 & 5.7 & 5.7 \\
\hline
\end{tabular}




\begin{tabular}{|c|c|c|c|c|c|c|c|c|c|}
\cline { 2 - 8 } 1350 & $\begin{array}{c}\text { Ave. Droplet } \\
\text { Concentration } \\
\left(\mathrm{g} / \mathrm{m}^{3}\right)\end{array}$ & 14.7 & 11.7 & 9.3 & 8.1 & 7.6 & 7.2 & 5.7 & 5.4 \\
\hline 1680 & $\begin{array}{c}\text { Ave. Droplet } \\
\text { Concentration } \\
\left(\mathrm{g} / \mathrm{m}^{3}\right)\end{array}$ & 14.5 & 11.3 & 9.7 & 8.4 & 8.2 & 7.0 & 6.3 & 6.0 \\
\hline
\end{tabular}

Table A.11a: Exit Mist Concentrations for Reynolds Numbers of 1230, 1350, and 1680 (Run 1)

\begin{tabular}{|c|c|c|c|c|c|c|c|c|c|}
\hline $\begin{array}{c}\text { Reynolds } \\
\text { Numbers }\end{array}$ & $\begin{array}{c}\text { Applied Voltage } \\
(\mathrm{Kv})\end{array}$ & 0 & 10 & 15 & 18 & 20 & 22 & 24 & 26 \\
\hline 1230 & $\begin{array}{c}\text { Droplet } \\
\text { Concentration } \\
\left(\mathrm{g} / \mathrm{m}^{3}\right)\end{array}$ & 28.00 & 27.10 & 25.80 & 25.20 & 25.30 & 23.90 & 22.88 & 22.86 \\
\hline 1350 & $\begin{array}{c}\text { Droplet } \\
\text { Concentration } \\
\left(\mathrm{g} / \mathrm{m}^{3}\right)\end{array}$ & 29.00 & 27.15 & 24.87 & 24.82 & 25.00 & 24.00 & 23.20 & 22.92 \\
\hline 1680 & $\begin{array}{c}\text { Droplet } \\
\text { oncentration } \\
\left(\mathrm{g} / \mathrm{m}^{3}\right)\end{array}$ & 29.00 & 25.10 & 25.00 & 24.00 & 25.70 & 23.92 & 23.85 & 22.86 \\
\hline
\end{tabular}

Table A.11b: Exit Mist Concentrations for Reynolds Numbers of 1230, 1350, and 1680 (Run 2)

\begin{tabular}{|c|c|c|c|c|c|c|c|c|c|}
\hline $\begin{array}{c}\text { Reynolds } \\
\text { Numbers }\end{array}$ & $\begin{array}{c}\text { Applied Voltage } \\
(\mathrm{Kv})\end{array}$ & 0 & 10 & 15 & 18 & 20 & 22 & 24 & 26 \\
\hline 1230 & $\begin{array}{c}\text { Droplet } \\
\text { Concentration } \\
\left(\mathrm{g} / \mathrm{m}^{3}\right)\end{array}$ & 29.75 & 28.70 & 27.35 & 27.53 & 25.94 & 25.36 & 23.32 & 23.34 \\
\hline 1350 & $\begin{array}{c}\text { Droplet } \\
\text { Concentration } \\
\left(\mathrm{g} / \mathrm{m}^{3}\right)\end{array}$ & 29.42 & 27.71 & 26.55 & 25.21 & 26.45 & 25.08 & 23.51 & 23.30 \\
\hline 1680 & $\begin{array}{c}\text { Droplet } \\
\text { oncentration } \\
\left(\mathrm{g} / \mathrm{m}^{3}\right)\end{array}$ & 29.05 & 28.14 & 26.60 & 25.00 & 23.80 & 24.50 & 24.56 & 24.48 \\
\hline
\end{tabular}

Table A.11c: Exit Mist Concentrations for Reynolds Numbers of 1230, 1350, and 1680 (Ave.)

\begin{tabular}{|c|c|c|c|c|c|c|c|c|c|}
\hline $\begin{array}{c}\text { Reynolds } \\
\text { Numbers }\end{array}$ & $\begin{array}{c}\text { Applied Voltage } \\
(\mathrm{Kv})\end{array}$ & 0 & 10 & 15 & 18 & 20 & 22 & 24 & 26 \\
\hline 1230 & $\begin{array}{c}\text { Ave. Droplet } \\
\text { Concentration } \\
\left(\mathrm{g} / \mathrm{m}^{3}\right)\end{array}$ & 28.87 & 27.90 & 26.56 & 26.36 & 25.60 & 24.63 & 23.09 & 23.09 \\
\hline 1350 & $\begin{array}{c}\text { Ave. Droplet } \\
\text { Concentration } \\
\left(\mathrm{g} / \mathrm{m}^{3}\right)\end{array}$ & 29.20 & 27.40 & 25.71 & 25.00 & 25.71 & 24.52 & 23.34 & 23.10 \\
\hline
\end{tabular}


1680

\begin{tabular}{|c|c|c|c|c|c|c|c|c|}
\hline $\begin{array}{c}\text { Ave. Droplet } \\
\text { Concentration } \\
\left(\mathrm{g} / \mathrm{m}^{3}\right)\end{array}$ & 29.03 & 26.61 & 25.80 & 24.74 & 24.74 & 24.20 & 24.20 & 23.65 \\
\hline
\end{tabular}

Table A.12a: Exit Mist Concentrations for Reynolds Numbers of 2400, 2070, 1810, and 940 (Run 1)

\begin{tabular}{|c|c|c|c|c|c|c|c|c|c|}
\hline $\begin{array}{c}\text { Reynolds } \\
\text { Numbers }\end{array}$ & $\begin{array}{c}\text { Applied Voltage } \\
(\mathrm{kV})\end{array}$ & 0 & 10 & 15 & 18 & 20 & 22 & 24 & 26 \\
\hline 2400 & $\begin{array}{c}\text { Droplet } \\
\text { Concentration } \\
\left(\mathrm{g} / \mathrm{m}^{3}\right)\end{array}$ & 3.10 & 3.00 & 2.78 & 2.65 & 2.45 & 2.20 & 2.80 & 2.75 \\
\hline 2070 & $\begin{array}{c}\text { Droplet } \\
\text { Concentration } \\
\left(\mathrm{g} / \mathrm{m}^{3}\right)\end{array}$ & 3.51 & 2.89 & 2.55 & 2.00 & 2.10 & 2.16 & 2.50 & 2.50 \\
\hline 1810 & $\begin{array}{c}\text { Droplet } \\
\text { Doncentration } \\
\left(\mathrm{g} / \mathrm{m}^{3}\right)\end{array}$ & 3.10 & 2.41 & 2.20 & 2.35 & 2.00 & 1.95 & 2.15 & 2.00 \\
\hline 940 & $\begin{array}{c}\text { Droplet } \\
\text { Concentration } \\
\left(\mathrm{g} / \mathrm{m}^{3}\right)\end{array}$ & 3.56 & 2.95 & 2.75 & 1.95 & 1.85 & 1.00 & 2.06 & 1.85 \\
\hline
\end{tabular}

Table A.12b: Exit Mist Concentrations for Reynolds Numbers of 2400, 2070, 1810, and 940 (Run 2)

\begin{tabular}{|c|c|c|c|c|c|c|c|c|c|}
\hline $\begin{array}{c}\text { Reynolds } \\
\text { Numbers }\end{array}$ & $\begin{array}{c}\text { Applied Voltage } \\
(\mathrm{kV})\end{array}$ & 0 & 10 & 15 & 18 & 20 & 22 & 24 & 26 \\
\hline 2400 & $\begin{array}{c}\text { Droplet } \\
\text { Concentration } \\
\left(\mathrm{g} / \mathrm{m}^{3}\right)\end{array}$ & 3.60 & 3.05 & 2.88 & 2.81 & 2.60 & 2.34 & 2.87 & 2.92 \\
\hline 2070 & $\begin{array}{c}\text { Droplet } \\
\text { Concentration } \\
\left(\mathrm{g} / \mathrm{m}^{3}\right)\end{array}$ & 3.72 & 3.35 & 2.70 & 2.30 & 2.21 & 2.15 & 2.73 & 2.53 \\
\hline 1810 & $\begin{array}{c}\text { Droplet } \\
\text { Doncentration } \\
\left(\mathrm{g} / \mathrm{m}^{3}\right)\end{array}$ & 3.50 & 2.65 & 2.51 & 2.00 & 2.20 & 1.98 & 2.14 & 2.32 \\
\hline 940 & $\begin{array}{c}\text { Droplet } \\
\text { Concentration } \\
\left(\mathrm{g} / \mathrm{m}^{3}\right)\end{array}$ & 3.58 & 3.22 & 2.80 & 2.38 & 1.97 & 1.22 & 1.90 & 2.06 \\
\hline
\end{tabular}

Table A.12c: Exit Mist Concentrations for Reynolds Numbers of 2400, 2070, 1810, and 940 (Ave.)

\begin{tabular}{|c|c|c|c|c|c|c|c|c|c|}
\hline $\begin{array}{c}\text { Reynolds } \\
\text { Numbers }\end{array}$ & $\begin{array}{c}\text { Applied Voltage } \\
(\mathrm{kV})\end{array}$ & 0 & 10 & 15 & 18 & 20 & 22 & 24 & 26 \\
\hline 2400 & $\begin{array}{c}\text { Ave. Droplet } \\
\text { Concentration } \\
\left(\mathrm{g} / \mathrm{m}^{3}\right)\end{array}$ & 3.30 & 3.02 & 2.83 & 2.72 & 2.53 & 2.26 & 2.83 & 2.83 \\
\hline 2070 & $\begin{array}{c}\text { Ave. Droplet } \\
\text { Concentration } \\
\left(\mathrm{g} / \mathrm{m}^{3}\right)\end{array}$ & 3.60 & 3.12 & 2.61 & 2.14 & 2.19 & 2.16 & 2.61 & 2.51 \\
\hline
\end{tabular}




\begin{tabular}{|c|c|c|c|c|c|c|c|c|c|}
\hline 1810 & $\begin{array}{c}\text { Ave. Droplet } \\
\text { Concentration } \\
\left(\mathrm{g} / \mathrm{m}^{3}\right)\end{array}$ & 3.42 & 2.52 & 2.35 & 2.17 & 2.17 & 1.97 & 2.15 & 2.15 \\
\hline 940 & $\begin{array}{c}\text { Ave. Droplet } \\
\text { Concentration } \\
\left(\mathrm{g} / \mathrm{m}^{3}\right)\end{array}$ & 3.56 & 3.08 & 2.80 & 2.16 & 1.90 & 1.10 & 1.95 & 1.95 \\
\hline
\end{tabular}

Table A.13a: Exit Mist Concentrations for Reynolds Numbers of 2200, 1680, 1350, and 940 (Run 1)

\begin{tabular}{|c|c|c|c|c|c|c|c|c|c|}
\hline $\begin{array}{c}\text { Reynolds } \\
\text { Numbers }\end{array}$ & $\begin{array}{c}\text { Applied Voltage } \\
(\mathrm{kV})\end{array}$ & 0 & 10 & 15 & 18 & 20 & 22 & 24 & 26 \\
\hline 2200 & $\begin{array}{c}\text { Droplet } \\
\text { Concentration } \\
\left(\mathrm{g} / \mathrm{m}^{3}\right)\end{array}$ & 8.65 & 7.50 & 6.42 & 5.00 & 4.56 & 4.00 & 3.25 & 2.55 \\
\hline 1680 & $\begin{array}{c}\text { Droplet } \\
\text { Concentration } \\
\left(\mathrm{g} / \mathrm{m}^{3}\right)\end{array}$ & 8.32 & 7.51 & 6.00 & 5.20 & 5.00 & 4.25 & 3.90 & 2.75 \\
\hline 1350 & $\begin{array}{c}\text { Droplet } \\
\text { Concentration } \\
\left(\mathrm{g} / \mathrm{m}^{3}\right)\end{array}$ & 8.25 & 7.58 & 5.50 & 4.20 & 3.42 & 2.85 & 3.00 & 2.72 \\
\hline 940 & $\begin{array}{c}\text { Droplet } \\
\text { Concentration } \\
\left(\mathrm{g} / \mathrm{m}^{3}\right)\end{array}$ & 8.55 & 6.80 & 4.90 & 4.85 & 4.25 & 4.00 & 3.50 & 3.45 \\
\hline
\end{tabular}

Table A.13b: Exit Mist Concentrations for Reynolds Numbers of 2200, 1680, 1350, and 940 (Run 2)

\begin{tabular}{|c|c|c|c|c|c|c|c|c|c|}
\hline $\begin{array}{c}\text { Reynolds } \\
\text { Numbers }\end{array}$ & $\begin{array}{c}\text { Applied Voltage } \\
(\mathrm{kV})\end{array}$ & 0 & 10 & 15 & 18 & 20 & 22 & 24 & 26 \\
\hline 2200 & $\begin{array}{c}\text { Droplet } \\
\text { Concentration } \\
\left(\mathrm{g} / \mathrm{m}^{3}\right)\end{array}$ & 8.80 & 8.38 & 7.32 & 5.72 & 5.00 & 4.43 & 3.66 & 3.25 \\
\hline 1680 & $\begin{array}{c}\text { Droplet } \\
\text { Concentration } \\
\left(\mathrm{g} / \mathrm{m}^{3}\right)\end{array}$ & 8.90 & 7.66 & 6.86 & 6.22 & 5.62 & 4.72 & 4.00 & 2.98 \\
\hline 940 & $\begin{array}{c}\text { Droplet } \\
\text { Concentration } \\
\left(\mathrm{g} / \mathrm{m}^{3}\right)\end{array}$ & 8.40 & 7.00 & 6.60 & 4.36 & 3.82 & 3.18 & 3.15 & 3.30 \\
\hline $\begin{array}{c}\text { Droplet } \\
\text { Concentration } \\
\left(\mathrm{g} / \mathrm{m}^{3}\right)\end{array}$ & 8.76 & 7.05 & 6.10 & 5.40 & 4.90 & 4.76 & 3.90 & 3.62 \\
\hline
\end{tabular}

Table A.13c: Exit Mist Concentrations for Reynolds Numbers of 2200, 1680, 1350, and 940 (Ave.)

\begin{tabular}{|c|c|c|c|c|c|c|c|c|c|}
\hline $\begin{array}{c}\text { Reynolds } \\
\text { Numbers }\end{array}$ & $\begin{array}{c}\text { Applied Voltage } \\
(\mathrm{kV})\end{array}$ & 0 & 10 & 15 & 18 & 20 & 22 & 24 & 26 \\
\hline 2200 & $\begin{array}{c}\text { Ave. Droplet } \\
\text { Concentration } \\
\left(\mathrm{g} / \mathrm{m}^{3}\right)\end{array}$ & 8.72 & 7.93 & 6.87 & 5.35 & 4.78 & 4.20 & 3.45 & 2.88 \\
\hline 1680 & $\begin{array}{c}\text { Ave. Droplet } \\
\text { Concentration } \\
\left(\mathrm{g} / \mathrm{m}^{3}\right)\end{array}$ & 8.61 & 7.65 & 6.42 & 5.70 & 5.31 & 4.48 & 3.95 & 2.80 \\
\hline
\end{tabular}




\begin{tabular}{|c|c|c|c|c|c|c|c|c|c|}
\hline 1350 & $\begin{array}{c}\text { Ave. Droplet } \\
\text { Concentration } \\
\left(\mathrm{g} / \mathrm{m}^{3}\right)\end{array}$ & 8.32 & 7.30 & 6.00 & 4.25 & 3.60 & 3.00 & 3.04 & 3.04 \\
\hline 940 & $\begin{array}{c}\text { Ave. Droplet } \\
\text { Concentration } \\
\left(\mathrm{g} / \mathrm{m}^{3}\right)\end{array}$ & 8.65 & 6.97 & 5.30 & 5.13 & 4.56 & 4.37 & 3.93 & 3.50 \\
\hline
\end{tabular}

